\title{
AVIAN MUSCLE DEVELOPMENT AND GROWTH MECHANISMS: ASSOCIATION WITH MUSCLE MYOPATHIES AND MEAT QUALITY
}

EDITED BY: Sandra G. Velleman and Massimiliano Petracci PUBLISHED IN: Frontiers in Physiology

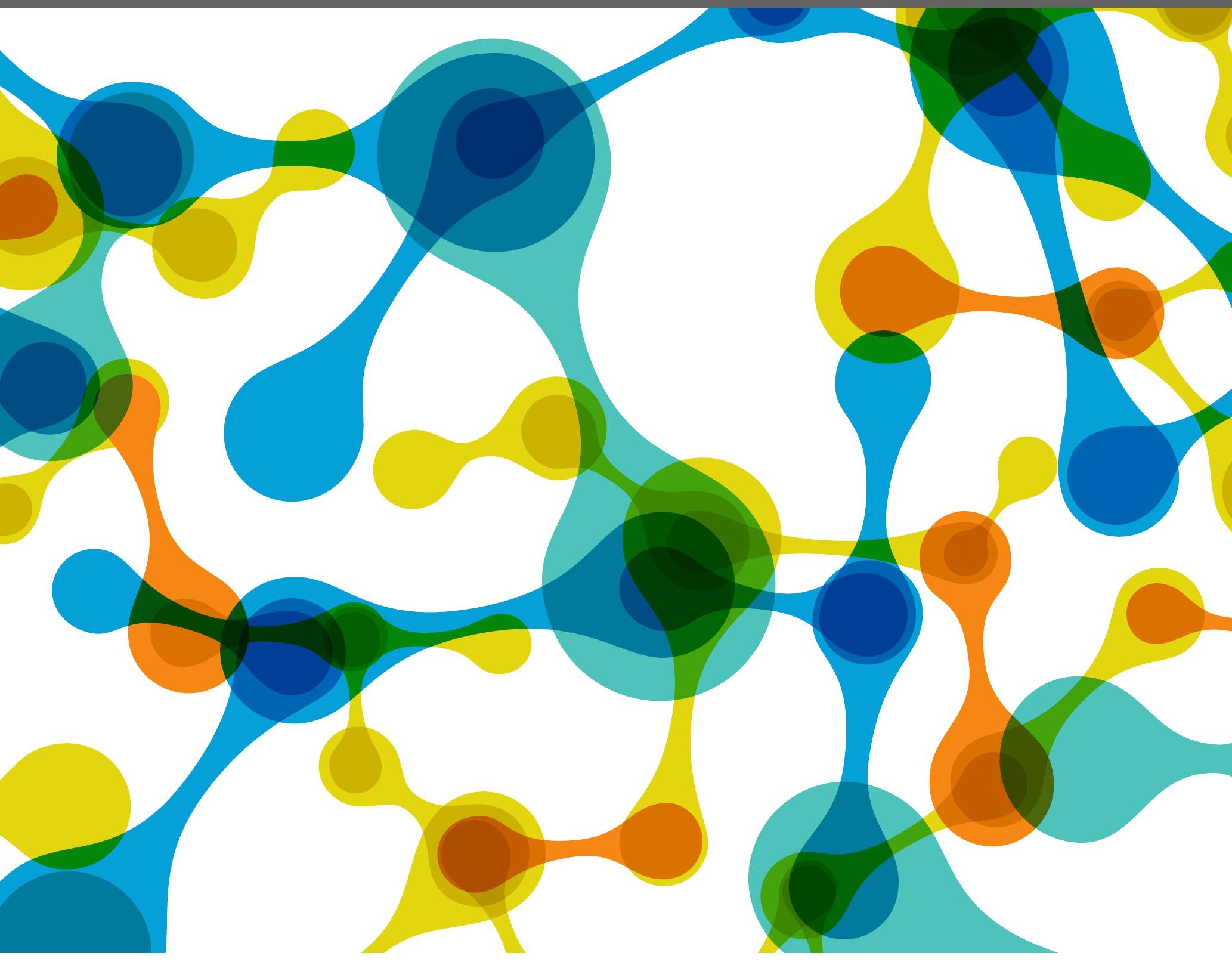




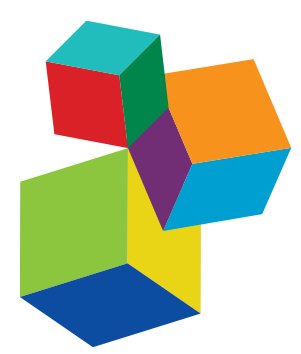

Frontiers eBook Copyright Statement

The copyright in the text of individual articles in this eBook is the property of their respective authors or their respective institutions or funders. The copyright in graphics and images within each article may be subject to copyright of other parties. In both cases this is subject to a license granted to Frontiers.

The compilation of articles constituting this eBook is the property of Frontiers.

Each article within this eBook, and the eBook itself, are published under the most recent version of the Creative Commons CC-BY licence. The version current at the date of publication of this eBook is CC-BY 4.0. If the CC-BY licence is updated, the licence granted by Frontiers is automatically updated to the new version.

When exercising any right under the CC-BY licence, Frontiers must be attributed as the original publisher of the article or eBook, as applicable.

Authors have the responsibility of ensuring that any graphics or other materials which are the property of others may be included in the

CC-BY licence, but this should be checked before relying on the

CC-BY licence to reproduce those materials. Any copyright notices relating to those materials must be complied with.

Copyright and source acknowledgement notices may not be removed and must be displayed in any copy, derivative work or partial copy which includes the elements in question.

All copyright, and all rights therein, are protected by national and international copyright laws. The above represents a summary only.

For further information please read Frontiers' Conditions for Website

Use and Copyright Statement, and the applicable CC-BY licence.

ISSN 1664-8714

ISBN 978-2-88966-314-9

DOI 10.3389/978-2-88966-314-9

\section{About Frontiers}

Frontiers is more than just an open-access publisher of scholarly articles: it is a pioneering approach to the world of academia, radically improving the way scholarly research is managed. The grand vision of Frontiers is a world where all people have an equal opportunity to seek, share and generate knowledge. Frontiers provides immediate and permanent online open access to all its publications, but this alone is not enough to realize our grand goals.

\section{Frontiers Journal Series}

The Frontiers Journal Series is a multi-tier and interdisciplinary set of open-access, online journals, promising a paradigm shift from the current review, selection and dissemination processes in academic publishing. All Frontiers journals are driven by researchers for researchers; therefore, they constitute a service to the scholarly community. At the same time, the Frontiers Journal Series operates on a revolutionary invention, the tiered publishing system, initially addressing specific communities of scholars, and gradually climbing up to broader public understanding, thus serving the interests of the lay society, too.

\section{Dedication to Quality}

Each Frontiers article is a landmark of the highest quality, thanks to genuinely collaborative interactions between authors and review editors, who include some of the world's best academicians. Research must be certified by peers before entering a stream of knowledge that may eventually reach the public - and shape society; therefore, Frontiers only applies the most rigorous and unbiased reviews.

Frontiers revolutionizes research publishing by freely delivering the most outstanding research, evaluated with no bias from both the academic and social point of view. By applying the most advanced information technologies, Frontiers is catapulting scholarly publishing into a new generation.

\section{What are Frontiers Research Topics?}

Frontiers Research Topics are very popular trademarks of the Frontiers Journals Series: they are collections of at least ten articles, all centered on a particular subject. With their unique mix of varied contributions from Original Research to Review Articles, Frontiers Research Topics unify the most influential researchers, the latest key findings and historical advances in a hot research area! Find out more on how to host your own Frontiers Research Topic or contribute to one as an author by contacting the Frontiers Editorial Office: researchtopics@frontiersin.org 


\section{AVIAN MUSCLE DEVELOPMENT AND GROWTH MECHANISMS: ASSOCIATION WITH MUSCLE MYOPATHIES AND MEAT QUALITY}

Topic Editors:

Sandra G. Velleman, The Ohio State University, United States

Massimiliano Petracci, University of Bologna, Italy

Citation: Velleman, S. G., Petracci, M., eds. (2021). Avian Muscle Development and Growth Mechanisms: Association With Muscle Myopathies and Meat Quality. Lausanne: Frontiers Media SA. doi: 10.3389/978-2-88966-314-9 


\section{Table of Contents}

05 Editorial: Avian Muscle Development and Growth Mechanisms: Association With Muscle Myopathies and Meat Quality

Sandra G. Velleman and Massimiliano Petracci

08 Evaluation of Bone Marrow Adipose Tissue and Bone Mineralization on Broiler Chickens Affected by Wooden Breast Myopathy

Barbara de Almeida Mallmann, Elizabeth M. Martin, Kyung Soo Kim, Norma L. Calderon-Apodaca, Mikayla F. A. Baxter, Juan D. Latorre, Xochitl Hernandez-Velasco, Leopoldo Paasch-Martinez, Casey M. Owens, Sami Dridi, Walter G. Bottje, Elizabeth S. Greene and Guillermo Tellez-Isaias RNA-Seq Analysis Reveals Spatial and Sex Differences in Pectoralis Major Muscle of Broiler Chickens Contributing to Difference in Susceptibility to Wooden Breast Disease

Brilynn Brothers, Zhu Zhuo, Michael B. Papah and Behnam Abasht

Distribution and Expression of Vimentin and Desmin in Broiler Pectoralis major Affected by the Growth-Related Muscular Abnormalities Francesca Soglia, Maurizio Mazzoni, Martina Zappaterra, Mattia Di Nunzio, Elena Babini, Martina Bordini, Federico Sirri, Paolo Clavenzani, Roberta Davoli and Massimiliano Petracci

41 Evaluation via Supervised Machine Learning of the Broiler Pectoralis Major and Liver Transcriptome in Association With the Muscle Myopathy Wooden Breast

Chelsea A. Phillips, Benjamin J. Reading, Matthew Livingston,

Kimberly Livingston and Chris M. Ashwell

54 Glucolipotoxicity: A Proposed Etiology for Wooden Breast and Related Myopathies in Commercial Broiler Chickens

Juniper A. Lake and Behnam Abasht

67 Timing is Everything-The High Sensitivity of Avian Satellite Cells to Thermal Conditions During Embryonic and Posthatch Periods Orna Halevy

74 Blood Gas Disturbances and Disproportionate Body Weight Distribution in Broilers With Wooden Breast

Juniper A. Lake, Erin M. Brannick, Michael B. Papah, Cory Lousenberg, Sandra G. Velleman and Behnam Abasht

83 Altered Sarcomeric Structure and Function in Woody Breast Myopathy of Avian Pectoralis Major Muscle Jiao Liu, Eero Puolanne, Matthias Schwartzkopf and Anders Arner

94 Pectoralis Major (Breast) Muscle Extracellular Matrix Fibrillar Collagen Modifications Associated With the Wooden Breast Fibrotic Myopathy in Broilers

Sandra G. Velleman

101 Exploring the Factors Contributing to the High Ultimate $\mathrm{pH}$ of Broiler Pectoralis Major Muscles Affected by Wooden Breast Condition Giulia Baldi, Con-Ning Yen, Morgan R. Daughtry, Jocelyn Bodmer, Brian C. Bowker, Hong Zhuang, Massimiliano Petracci and David E. Gerrard 

Chickens Affected by Wooden Breast

Tamara Z. Ferreira, Liris Kindlein, Joshua J. Flees, Lauren K. Shortnacy, Sergio L. Vieira, Vladimir P. Nascimento, Kathryn J. Meloche and Jessica D. Starkey

125 Muscle Abnormalities and Meat Quality Consequences in Modern Turkey Hybrids

Marco Zampiga, Francesca Soglia, Giulia Baldi, Massimiliano Petracci, Gale M. Strasburg and Federico Sirri

133 Transcriptional Profiles of Skeletal Muscle Associated With Increasing Severity of White Striping in Commercial Broilers

Yuwares Malila, Tanaporn Uengwetwanit, Sopacha Arayamethakorn, Yanee Srimarut, Krittaporn V. Thanatsang, Francesca Soglia,

Gale M. Strasburg, Wanilada Rungrassamee and Wonnop Visessanguan

149 Physiological and Pathological Mitochondrial Clearance is Related to Pectoralis Major Muscle Pathogenesis in Broilers With Wooden Breast Syndrome

Marina Hosotani, Takeshi Kawasaki, Yasuhiro Hasegawa, Yui Wakasa, Maki Hoshino, Naoki Takahashi, Hiromi Ueda, Tomohide Takaya, Tomohito Iwasaki and Takafumi Watanabe

161 Molecular Phenotyping of White Striping and Wooden Breast Myopathies in Chicken

Christophe Praud, Justine Jimenez, Eva Pampouille, Nathalie Couroussé, Estelle Godet, Elisabeth Le Bihan-Duval and Cecile Berri

177 Application of Bioelectrical Impedance Analysis to Detect Broiler Breast Filets Affected With Woody Breast Myopathy

Amit Morey, Avery E. Smith, Laura Jewell Garner and Marlin K. Cox

184 Knockdown of Death-Associated Protein Expression Induces Global Transcriptome Changes in Proliferating and Differentiating Muscle Satellite Cells

Katherine A. Horton, Kelly R. B. Sporer, Robert J. Tempelman,

Yuwares Malila, Kent M. Reed, Sandra G. Velleman and Gale M. Strasburg

196 The Potential of Guanidino Acetic Acid to Reduce the Occurrence and Severity of Broiler Muscle Myopathies

Edgar Orlando Oviedo-Rondón and Hernan Alejandro Córdova-Noboa

209 Characterising the Influence of Genetics on Breast Muscle Myopathies in Broiler Chickens

Richard A. Bailey, Eduardo Souza and Santiago Avendano

221 The Role of Incubation Conditions in the Onset of Avian Myopathies Edgar Orlando Oviedo-Rondón, Sandra G. Velleman and Michael J. Wineland 


\section{OPEN ACCESS}

Edited and reviewed by: Colin Guy Scanes, University of Arkansas, United States

${ }^{*}$ Correspondence: Sandra G. Velleman velleman.1@osu.edu

Specialty section:

This article was submitted to Avian Physiology, a section of the journal Frontiers in Physiology

Received: 31 August 2020 Accepted: 25 September 2020 Published: 04 November 2020

Citation: Velleman SG and Petracci M (2020) Editorial: Avian Muscle Development and Growth Mechanisms: Association With Muscle Myopathies and Meat Quality. Front. Physiol. 11:601184. doi: 10.3389/fphys.2020.601184

\section{Editorial: Avian Muscle Development and Growth Mechanisms: Association With Muscle Myopathies and Meat Quality}

\author{
Sandra G. Velleman ${ }^{1 *}$ and Massimiliano Petracci ${ }^{2}$ \\ ${ }^{1}$ Department of Animal Sciences, The Ohio State University, Wooster, OH, United States, ${ }^{2}$ Department of Agricultural and \\ Food Sciences, Alma Mater Studiorum - University of Bologna, Bologna, Italy
}

Keywords: satellite cells, poultry, myopathies, meat, spaghetti meat, wooden breast

Editorial on the Research Topic

Avian Muscle Development and Growth Mechanisms: Association With Muscle Myopathies and Meat Quality

\section{INTRODUCTION}

The Research Topic Avian Muscle Development and Growth Mechanisms: Association with Muscle Myopathies and Meat Quality is the first comprehensive treatise associating the basic biology of muscle growth with the incidence of breast muscle myopathies including the influence of genetics, effect on meat quality, and the development of new detection methods with more accuracy than standard phenotypic palpation. For this special Research Topic, a total of 20 manuscripts were accepted for publication: 13 original research contributions; 4 hypothesis and theory; and 3 reviews.

A couple of review papers explore different aspects of early and late muscle development which can be related to the occurrence of breast muscle myopathies in fast-growing broilers. Velleman summarizes recent findings on how the Wooden Breast myopathy is associated with fibrosis and re-assembling of extracellular matrix proteins with special emphasis on the fibrilforming collagens (namely type I and III) and its implications on breast muscle functionality and resulting meat quality in different fast-growing commercial hybrids with varying occurrence of phenotypically detectable wooden breast condition. Halevy presents a mini-review on how satellite cell proliferation and differentiation as well as prenatal muscle development and postnatal pectoral muscle growth, are dramatically affected by environmental conditions during chicken embryonic development and initial stages after hatching. This paper further reveals the extreme importance of duration and extent of the change in environmental thermal load and its timing during these stages in determining muscle development and growth in poultry, even in the occurrence of muscle myopathies. This topic is also explored by Oviedo-Rondón et al. who showed that metabolic disorders in avian species can start very early in life, and suboptimal incubation conditions may trigger some of the key alterations on muscle metabolism and be associated with the onset of myopathies. Zampiga et al. reviewed the current understanding of the breast myopathies and possible implications on the quality of turkey meat. Overall, it was clearly stated that the relevance and practical importance of emerging growth-related breast abnormalities in the turkey is limited if compared to the broiler chickens. Therefore, the occurrence of PSE-like condition is still the major 
issue for the turkey industry. On the same species, Horton et al. explored for the first time the role of a specific group of proteins involved in cellular apoptosis in the early stages of muscle growth and development. It was demonstrated that decreased expression of death-associated proteins significantly affects the transcriptome profile of satellite cells in turkey pectoral muscles, thus impairing its proliferation and differentiation ability.

Most of the original research covers several key topics in the growth of the breast and modifications in the growth process as it relates to incidence of breast muscle myopathies in broilers. Ferreira et al. describe alterations in the satellite cell population, macrophage population, and collagen content in wooden breast affected muscle. Satellite cells are responsible for post-hatch muscle growth and the repair of muscle with damage like that which occurs in fibrotic myopathies. In Wooden breast affected muscle, the number of proliferative satellite cells increased as did macrophage and collagen content. The wooden breast myopathy has been associated with hypoxia induced by rapid growth. Hosotani et al. describe how altered mitochondrial clearance induced by chronic hypoxia and injury to mitochondria are associated with the pathological features of wooden breast. Furthermore, Baldi et al. report that the elevated ultimate $\mathrm{pH}$ in wooden breast meat involves reduced glycolytic potential and impaired ATP energy generation which would impact the degree of muscle fiber contraction.

Thus, research contributions addressing how muscle myopathies affect the structure of the breast muscle which will ultimately alter meat quality were also included in this special Research Topic. In research addressing the cellular cytoarchitecture proteins desmin and vimentin by Soglia et al. affected breast muscles from wooden breast, white striping, and spaghetti meat myopathies were compared to normal breast muscle. It was found that wooden breast and white striping affected tissue differentially undergoing severe regeneration, and vimentin may be associated with the development of the spaghetti meat phenotype. With regard to sarcomere structure, the physiological properties of single muscle fibers from wooden breast affected muscle were compared to normal muscle by Liu et al.. They found that calcium sensitivity was significantly decreased in wooden breast muscle and wooden breast muscle fibers had 50\% larger sarcomeric volume compared to normal muscle. However, the content of myosin and actin, and maximal shortening velocity were unchanged suggestive of the synthesis of new sarcomeres with unaltered kinetics.

To further a mechanistic understanding into the cause of breast muscle myopathies, cutting edge genomic and transcriptome research were included in this treatise. Bailey et al. present the first reported estimates of the genetic basis for breast muscle defects in broilers including deep pectoral myopathy, wooden breast, white striping, and spaghetti breast. They present evidence showing that breast muscle myopathies have a low genetic relationship with performance and that it is also important to understand non-genetic effects on myopathy incidence. Transcriptional profiling studies by Malila et al. on white striping severity in commercial broilers demonstrated intracellular ion imbalance, particularly calcium, oxidative stress, and impaired programmed cell death on the progression of white striping. Praud et al. report on molecular phenotyping of the white striping and wooden breast myopathies to potentially identify biomarkers for diagnostic purposes and to improve our understanding of the physiology of wooden breast and white striping. Wooden breast also has a differential occurrence between the sexes in broiler chickens. In male birds, the cranial region of the breast muscle tends to be more severely affected compared to females. Through RNA-Seq, Brothers et al. describe the increased expression of fat metabolism, oxidative stress response, antiangiogenesis, and connective tissue proliferation genes in male broilers. Thus, these data begin to demonstrate why male broilers are more susceptible to wooden breast.

A couple of the original research contributions propose advanced tools to detect wooden breast which poses significant meat quality issues and consumer complaints in the poultry industry. In the first, Morey et al. investigated the potential use of bioelectrical impedance analysis. Very promising results were obtained when normal and wooden breast samples were compared, while it was not possible to differentiate between varying severity levels of wooden breast myopathy. In addition, the accidental outcome on the interference of spaghetti breast meat opens new research areas to investigate the ability of this analytical approach to also identify this condition. On the other hand, Phillips et al. explored for the first time the application of machine learning tools for extracting critical information contained in gene expression data obtained from pectoralis major muscle and liver of chickens exhibiting wooden breast condition and those that were normal. This innovative approach allows to establish that evolution of wooden breast involves many molecules and pathways thus suggesting that the etiology of this condition is associated not only to muscle activity but altered systemic pathology. These findings were also indirectly supported by the study of Lake et al. which reveals that the influence of wooden breast is not limited to the pectoral muscles. Indeed, it was evidenced that wooden breast is coupled with blood gas disturbances characterized primarily by the accumulation of carbon dioxide and acidification of venous blood which can be likely associated with impairment of pulmonary gas exchange. Consequently, there is good evidence to suggest that birds affected by wooden breast condition can have an increased metabolic rate that may also be poorly compensated due to cardiovascular deficiencies such as insufficient venous return or respiratory deficiency. In addition, Mallmann et al. speculated that, as in mammals, deviations in bone metabolism may be associated with growth-related abnormalities occurring in modern fast-growing broilers. A clear association emerged between distinctive histological alterations (abundant infiltration of adipose tissue, muscle fibers degeneration and necrosis, infiltration of heterophils and mononuclear cells, connective tissue proliferation, and vasculitis) and abundant infiltration of adipose tissue bone marrow in broilers affected by wooden breast condition. Indeed, Lake and Abasht suggest a new hypothesis on the etiology of wooden breast that involves 
impairment of lipid and glucose metabolism in consequence of the proposed similarities between wooden breast and type 2 diabetes regardless of its phenotypic dissimilarities. They hypothesize that these dissimilarities are due to different glucose transport metabolism between avian and mammalian skeletal muscle, and that the wooden breast phenotype most closely resembles complications of diabetes in smooth and cardiac muscle of mammalian species. Finally, Oviedo-Rondón and Córdova-Noboa summarizes current knowledge on the potential of guanidino acetic acid dietary use to mitigate growth-related breast abnormalities with emphasis on the wooden breast myopathy and the possible use of plasma creatine and related blood enzymes as markers for early detection of this condition in live birds.

\section{AUTHOR CONTRIBUTIONS}

The contributions are all original to the Research Topic. All authors contributed to the article and approved the submitted version.

Conflict of Interest: The authors declare that the research was conducted in the absence of any commercial or financial relationships that could be construed as a potential conflict of interest.

Copyright (c) 2020 Velleman and Petracci. This is an open-access article distributed under the terms of the Creative Commons Attribution License (CC BY). The use, distribution or reproduction in other forums is permitted, provided the original author(s) and the copyright owner(s) are credited and that the original publication in this journal is cited, in accordance with accepted academic practice. No use, distribution or reproduction is permitted which does not comply with these terms. 


\section{OPEN ACCESS}

Edited by:

Krystyna Pierzchala-Koziec, University of Agriculture of Kraków,

Poland

Reviewed by: Michael Kogut, United States Department of Agriculture, United States

Massimiliano Petracci,

University of Bologna, Italy

*Correspondence:

Guillermo Tellez-Isaias gtellez@uark.edu

Specialty section:

This article was submitted to Avian Physiology, a section of the journal Frontiers in Physiology

Received: 05 March 2019 Accepted: 13 May 2019 Published: 29 May 2019

Citation: de Almeida Mallmann B, Martin EM, Soo Kim K, Calderon-Apodaca NL, Baxter MFA, Latorre JD, Hernandez-Velasco X, Paasch-Martinez L, Owens CM, Dridi S, Bottje WG, Greene ES and Tellez-Isaias G (2019) Evaluation of Bone Marrow Adipose Tissue and Bone Mineralization on Broiler

Chickens Affected by Wooden Breast Myopathy. Front. Physiol. 10:674. doi: 10.3389/fphys.2019.00674

\section{Evaluation of Bone Marrow Adipose Tissue and Bone Mineralization on Broiler Chickens Affected by Wooden Breast Myopathy}

\author{
Barbara de Almeida Mallmann', Elizabeth M. Martin², Kyung Soo Kim³, \\ Norma L. Calderon-Apodaca4, Mikayla F. A. Baxter', Juan D. Latorre', \\ Xochitl Hernandez-Velasco ${ }^{4}$, Leopoldo Paasch-Martinez ${ }^{4}$, Casey M. Owens ${ }^{1}$, \\ Sami Dridi ${ }^{1}$, Walter G. Bottje ${ }^{1}$, Elizabeth S. Greene ${ }^{1}$ and Guillermo Tellez-Isaias ${ }^{1 *}$ \\ ${ }^{1}$ Department of Poultry Science, University of Arkansas, Fayetteville, AR, United States, ${ }^{2}$ Institute for Nanoscience \\ and Engineering, University of Arkansas, Fayetteville, AR, United States, ${ }^{3}$ Department of Plant Pathology, University \\ of Arkansas, Fayetteville, AR, United States, ${ }^{4}$ Departamento de Medicina y Zootecnia de Aves, Facultad de Medicina \\ Veterinaria y Zootecnia, Universidad Nacional Autónoma de México, Mexico City, Mexico
}

In humans, alterations in bone metabolism have been associated with myopathies. We postulate the hypothesis that perhaps similar pathologies can also be associated in modern chickens. Hence, this study aimed to assess the fat infiltration in bone marrow and its repercussion on broiler chicken affected by Wooden Breast (WB) myopathy. Ten Cobb 500 live birds with extreme rigidity of the Pectoralis major (PM) muscle were selected as WB affected chickens by physical examination of the muscle at 49 days of age, whereas ten chickens healthy with no physical signs of hardness in the breast muscle were considered to be unaffected. Macroscopic lesions in affected chickens included areas of firm and inflamed muscle with pale appearance, hemorrhaging, and viscous exudate on the surface. Bone marrow and sections of the PM muscle were collected and analyzed for light microscopy. Additionally, transmission electron microscopy was conducted in affected or unaffected muscle. Chickens affected with WB showed significant reductions $(P<0.05)$ in femur diameter, calcium, and phosphorous percentage but increased breast weight, compression force and filet thickness when compared with non-affected chickens. Interestingly, bone marrow from WB chicken had subjectively, more abundant infiltration of adipose tissue, when compared with non-affected chickens. Histology of the Pectoralis major of birds with WB showed abundant infiltration of adipose tissue, muscle fibers degeneration with necrosis and infiltration of heterophils and mononuclear cells, connective tissue proliferation, and vasculitis. Ultrastructural changes of WB muscle revealed lack definition of bands in muscle tissue, or any normal ultrastructural anatomy such as myofibrils. The endomysium components were necrotic, and in some areas, the endomysium was notable only as a string of necrotic tissue between degraded myofibrils. The fascia 
appeared hypertrophied, with large areas of necrosis and myofiber without structural identity with degraded mitochondria adjacent to the disrupted muscle tissue. As far as we know, this is the first study that describes a subjective increase in adipose tissue in the bone marrow of chickens affected with WB when compared with non-affected chickens, and reduced bone mineralization.

Keywords: bone marrow adipose tissue, broiler chickens, wooden breast, histology, electron microscopy

\section{INTRODUCTION}

The domestic chicken has been an essential animal model and constitutes a remarkable source of high-quality protein for humans (Stern, 2005). Within the last 60 years, genetics of the domestic chicken (Gallus gallus domesticus) have been able to create a bird that reaches commercial body weight in 5 weeks (Burt, 2007). Unfortunately, with this astonishing genetic development, other disapproving conditions such as adiposity, leg, metabolic and reproduction pathologies have also increased (Wideman and Hamal, 2011; Abdalla et al., 2018). Recent studies have identified the expression of several genes in the chicken intestine that encodes sugar transporters associated with rapid growth in modern broilers (Miska and Fetterer, 2019). More intriguing are the published studies using ingenuity pathways analysis and histology, comparing the genes of modern broiler chickens suffering from a muscle myopathy called wooden breast (WB), versus non-affected chickens, resulted in 1500 genes associated with several disease and physiological disorders (Mutryn et al., 2015; Kuttappan et al., 2017; Marchesi et al., 2018). The WB condition is a myopathy affecting the pectoralis major muscle in fast-growing commercial broiler lines (Velleman and Clark, 2015).

After birth, satellite cells are the most abundant cells in skeletal muscle (Awano et al., 2015). Nevertheless, satellite cells are activated after muscle damage and undergo myogenic differentiation (Carotenuto et al., 2016). The regeneration of the tissue exhibits some resemblances to the development of muscle throughout embryogenesis. Following tissue damage, inflammation and activation of satellite cells, proliferate, differentiate and fuse to form multinucleated myofibers (Karalaki et al., 2009; Lepper et al., 2011). During this process, $5^{\prime}$ AMPactivated protein kinase (AMPK), an enzyme that plays a role in cellular energy homeostasis is also activated (Zhang et al., 2009). However, in some metabolic diseases such as obesity in humans, the reduction of AMPK activity has linked with a significant reduction in muscle regeneration (Kuang et al., 2007). Several investigators have shown that most inflammatory pathways are interconnected in the pathogenesis of diseases affecting the musculoskeletal system in broiler chickens (Mutryn et al., 2015; Wen et al., 2017) and in mammals (Adams, 2002). In chickens, under normal conditions, the skeletal muscle also can repair damage by the activation and differentiation of fiber sub-laminar satellite cells (Halevy et al., 2000). In muscle affected by WB regeneration impairment, due to reduced satellite cells number and/or functional capacity leads to fiber substitution with ectopic tissues including fat and fibrous tissue and the loss of muscle functions (Meloche et al., 2018; Velleman, 2018).
Another critical problem that the current poultry industry is facing is excessive adiposity, which has become an added issue in meat-type chicken production (Wang et al., 2007; Resnyk et al., 2017). While in mammals, lipid tissue regulates energybalance control throw the secretion of leptin, in chickens, this important adipokine is barely expressed (Matsuzawa et al., 1995). Interestingly, genome array studies have shown that modern chickens suffer from severe obesity (Wang et al., 2006).

Adiposity induces prolonged systemic inflammation, which is associated with several metabolic disorders (Collins et al., 2018). Also, in humans, there is clear evidence indicating the link between chronic inflammation caused by adiposity and myopathies (Zoico et al., 2009; Akhmedov and Berdeaux, 2013; Fu et al., 2016). Nevertheless, there are no reports in modern broiler chickens describing the severe adiposity and its relationship with myopathies.

On the other hand, a recent study has demonstrated the importance of adipose tissue proliferation in the bone marrow in metabolic disorders (Hardouin et al., 2016). Unlike mammals, in birds, fat is added to adipocyte vacuoles without an increase in adipocyte number and do not possess brown fat (King, 1967; Johnston, 1971). A recent study have shown that WB is associated with a significant increase in $\mathrm{CO}_{2}$ and a decrease in $\mathrm{O}_{2}$. Under this hypoxia conditions or damage defective mitochondria, there is an increase in fatty acids synthesis leading to fatty acid and phospholipid accumulation, responsible of the increased breast fat percentage reported in WB and WS (Ouali et al., 2013; Kozakowska et al., 2015; Livingston et al., 2018). In the bone marrow of fit humans, adipocytes represent seventy percent of total cells. However, aging and other pathological conditions can increase the percentage of adipocytes in the bone marrow (Collins et al., 2018). However, no studies in chickens have evaluated the adipose tissue in the bone marrow and its role in myopathies. Hence, we postulate the hypothesis that the adiposity of modern broiler chickens is associated with an increase fat infiltration in bone marrow, and its repercussion on broiler chicken pectoralis major muscle affected by WB myopathy.

\section{MATERIALS AND METHODS}

\section{Animal Source}

In the present study, male Cobb 500 from Cobb-Vantress (Siloam Springs, AR, United States) were utilized. Ten Cobb 500 live birds with extreme rigidity of the Pectoralis major muscle were selected as WB affected chickens by physical examination of the muscle at 49 days of age, whereas ten chickens healthy with no 
physical signs of hardness in the breast muscle were considered to be unaffected. Macroscopic lesions in affected chickens included areas of firm and inflamed muscle with pale appearance, hemorrhaging, and viscous exudate on the surface (Figure 1). After euthanasia by $\mathrm{CO}_{2}$ asphyxiation, breast muscle tissue was harvested from unaffected and affected birds. All animal handling procedures complied with Institutional Animal Care and Use Committee at the University of Arkansas, protocol \# 18067.

\section{Compression Force and Filet Thickness}

Compression test parameters were significantly modified (Mudalal et al., 2015) to accommodate for analysis on a whole filet rather than a round cut of a filet. All butterfly filets were split in half with the right filet used for compression analysis and drip loss. Filets were compressed to $20 \%$ of the filet height three times on different areas of the cranial region using a 6-mm flat probe on a TA.XT Plus Texture Analyzer (Texture Technologies Corp., Hamilton, MA/Stable Micro Systems, Godalming, Surrey, United Kingdom). No sample cutting was required. The trigger force was set at $5 \mathrm{~g}$, probe height set at $55 \mathrm{~mm}$ (higher than the thickest filet sample), pre- and post-probe speeds were both $10 \mathrm{~mm} / \mathrm{s}$, and the test speed of the probe was $5 \mathrm{~mm} / \mathrm{s}$. The pectoralis major and both tenders were weighed to give an estimate of total meat weight of the breast and the pectoralis major was dissected. Filet thickness was measured at the thickest point with a customized micrometer gauge.

\section{Bone Parameters}

Bone parameters were measured according to the methods as described by previously (Zhang and Coon, 1997). The left femur from the chicken affected or non-affected $(n=10)$ were cleaned of attached tissues. The right femur from the same chickens was used to obtain the bone marrow for histology as described below. The left femurs were dried at $100^{\circ} \mathrm{C}$ for $24 \mathrm{~h}$ and weighed again. The samples were then incinerated in a muffle furnace (Isotemp muffle furnace, Thermo Fisher Scientific, Pittsburgh, $\mathrm{PA})$ at $600^{\circ} \mathrm{C}$ for $24 \mathrm{~h}$ in crucibles. Finally, the content of calcium and phosphorus in the femur was determined using standard

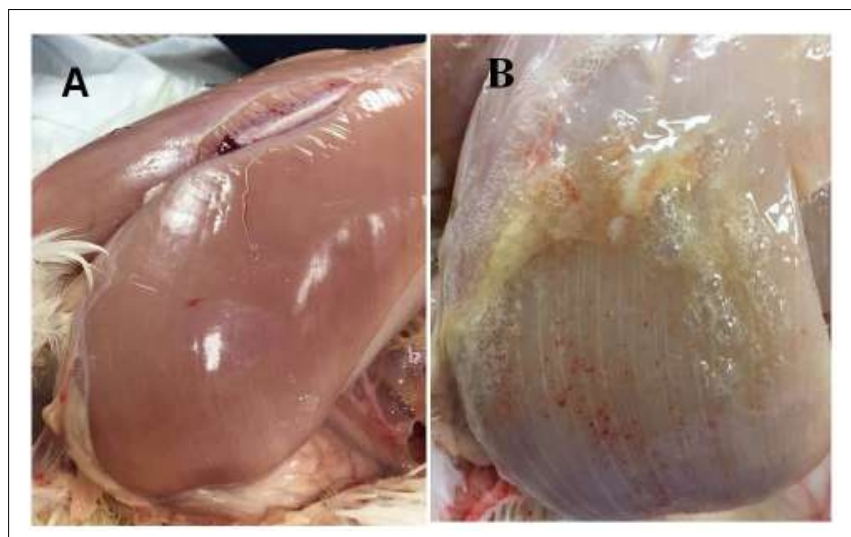

FIGURE 1 | (A) Pectoralis major non-affected chicken. (B) Pectoralis major from woody breast chicken. Muscle shows a pale color, hemorrhaging, and a viscous exudate on the surface. methods (AOAC International, 2000) and were reported as a percentage of dry matter.

\section{Muscle and Bone Marrow Histology}

Histological evaluations were performed on five samples of from affected or non-affected chickens immediately after the birds were euthanatized, the skin was removed from the breast region, and a sample of the pectoralis major muscle was excised. Muscle specimens were obtained by dissecting a $0.5-\mathrm{cm}$-wide area in the anterior portion of the muscle following the muscle fiber orientation for a length of $3 \mathrm{~cm}$. Bone marrow obtained from the right femur and muscle samples were fixed and stored in 10\% neutral buffered formalin ( $\mathrm{vol} / \mathrm{vol})$. Each of these samples was embedded in paraffin, and a $5-\mu \mathrm{m}$ section of each sample was placed on a glass slide and stained with hematoxylin and eosin for examination under a light microscope.

\section{Transmission Electron Microscope}

Pectoralis major tissue from five affected or non-affected chickens were cut at $2 \mathrm{~mm}^{2}$ and fixed with Karnovsky's fixative in a weak vacuum for $2 \mathrm{~h}$. Samples were rinsed three times with $0.05 \mathrm{M}$ cacodylate buffer $\mathrm{pH} 7.2$, post-fixed for $2 \mathrm{~h}$ in $1 \%$ osmium tetroxide, with $0.05 \mathrm{M}$ cacodylate buffer. Samples were rinsed in distilled water and stained overnight in $0.5 \%$ uranyl acetate at $4.4^{\circ} \mathrm{C}$. The tissues were dehydrated in a graded ethanol series then infiltrated with 50:50, Spurr's medium: 100\% ethanol for three changes. Samples were placed in fresh 100\% Spurr's medium, overnight with a weak vacuum. Fresh Spurr's medium was pipetted into flat embedding molds, and tissues were placed in the molds and aligned. Molds were kept overnight in a weak vacuum. The molds were placed in a $70^{\circ} \mathrm{C}$ oven overnight. The tissues, in the cured blocks, were trimmed to $1 \times 1 \mathrm{~mm}$, and sectioned at 60-90 nm, using a diamond knife with an MT2B ultra-microtome (Dupont Company, Newtown, CT). Sections were placed on 300 mesh copper grids and stained with $2 \%$ aqueous uranyl acetate, followed by lead citrate. Sections were viewed at $100 \mathrm{kV}$, with a transmission electron microscope (JEM1011, JEOL, Tokyo, Japan).

\section{Statistical Analysis}

Bone data were subjected to one-way analysis of variance as a completely randomized design using the General Linear Models procedure of SAS (SAS Institute Inc., 2002). Data are expressed as mean \pm standard error. Significant differences among the means were determined by using Duncan's multiple range test at $P<0.05$.

\section{RESULTS AND DISCUSSION}

The growth rates of modern broilers have increased by over $300 \%$ (Knowles et al., 2008). However, this intense genetic selection has been accompanied by increased body fat deposition, skeletal disorders, and greater incidence of metabolic diseases and mortality (Wideman and Hamal, 2011). In mammalian models, the molecular and cell and cellular pathways in adipose tissue development is well characterized. Nevertheless, little it is known 
about broiler chickens (Wang et al., 2017). Genotype, sex, age environmental temperature and nutrition of the broiler chicken are some of the main factors affecting fat deposition (Tưmová and Teimouri, 2010). In animal production, subcutaneous, internal, and intramuscular adipose tissue depots play economically and physiologically essential roles. However, lipid metabolism and adipogenesis in meat animals differ among species. Interestingly, the vertebrates that have a higher capacity for storing and using triglycerides as an energy reserve are found in the class Aves (Blem, 1976). Variation in fatty acid composition among species may be attributed to age, diet and physiological conditions. The amounts of body fat stored as subcutaneous, internal, and intramuscular adipose tissue depots are quantitative traits or complex phenotypes in nature, which are determined by genetic networks or molecular pathways (Hausman et al., 2009; Dodson et al., 2010). In contrast with mammals, lipid accumulation and storage occurs mainly by the addition of lipid to adipocyte vacuoles without an increase in cell number (Blem, 1976). Furthermore, avian fat bodies, unlike those of some mammals, increase in lipid content without similar changes in fat-free dry weight or relative water content of the body (King, 1967), and do not seem to possess brown fat (Johnston, 1971).

The results of the Evaluation of bone parameters and meat quality in 49-days-old broiler chickens non-affected or affected by WB myopathy are summarized in Table $\mathbf{1}$. Chickens affected with WB showed significant reductions $(P<0.05)$ in femur diameter, calcium, and phosphorous percentage when compared with nonaffected chickens (Table 1). Even though body in the present study body weight was not affected between affected or nonaffected chickens, chickens affected with WB had a significant increase in breast weight, compression force and filet thickness when compared with non-affected chickens (Table 1).

Figure 2 shows the images of the light microscopy of the bone marrow. Figure 2B shows an image of bone marrow from WB chicken with subjective more abundant infiltration of adipose tissue, when compared with non-affected chicken (Figure 2A).

Figure 3 shows the images of the light microscopy of the Pectoralis major. In comparison with non-affected chickens, birds with WB showed abundant infiltration of adipose tissue, muscle

TABLE 1 | Evaluation of bone parameters and meat quality in 49-days-old broiler chickens non-affected or affected by wooden breast myopathy.

\begin{tabular}{lcccc}
\hline & $\begin{array}{c}\text { Non- } \\
\text { affected }\end{array}$ & $\begin{array}{c}\text { Wooden Breast } \\
\text { affected }\end{array}$ & SEM & $P$-value \\
\hline Bone & & & & \\
Femur diameter (mm) & $12.34^{\mathrm{a}}$ & $11.02^{\mathrm{b}}$ & 0.15 & 0.0032 \\
Calcium (\%) & $40.48^{\mathrm{a}}$ & $38.35^{\mathrm{b}}$ & 0.025 & 0.0024 \\
Phosphorous (\%) & $21.15^{\mathrm{a}}$ & $19.05^{\mathrm{b}}$ & 1.66 & 0.0012 \\
Meat quality & & & & \\
Body Weight (kg) & 3.52 & 3.64 & 0.15 & 0.5935 \\
Breast Weight (kg) & $0.723^{\mathrm{b}}$ & $0.883^{\mathrm{a}}$ & 0.025 & 0.0019 \\
Compression force (N) & $6.26^{\mathrm{b}}$ & $12.89^{\mathrm{a}}$ & 1.44 & 0.0115 \\
Filet thickness (mm) & $42.86^{\mathrm{b}}$ & $50.19^{\mathrm{a}}$ & 1.06 & 0.0012 \\
\hline
\end{tabular}

${ }^{a, b}$ Means showing different letters between rows are significantly different. SEM $=$ standard error of the means $(n=10)$.

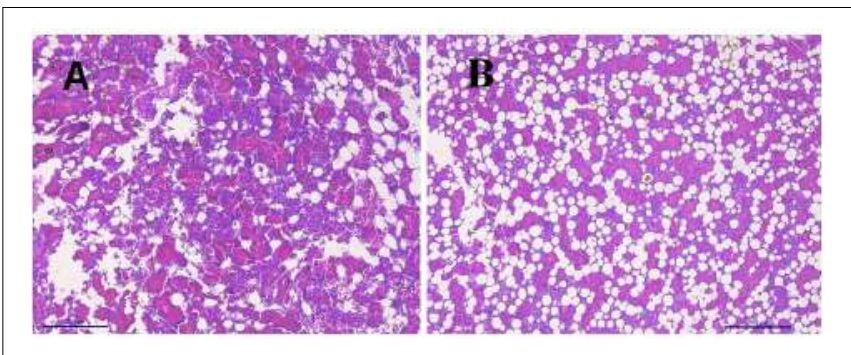

FIGURE 2 | Light microscopy bone marrow. (A) Bone marrow non-affected chicken. (B) Bone marrow from woody breast chicken with abundant infiltration of adipose tissue.

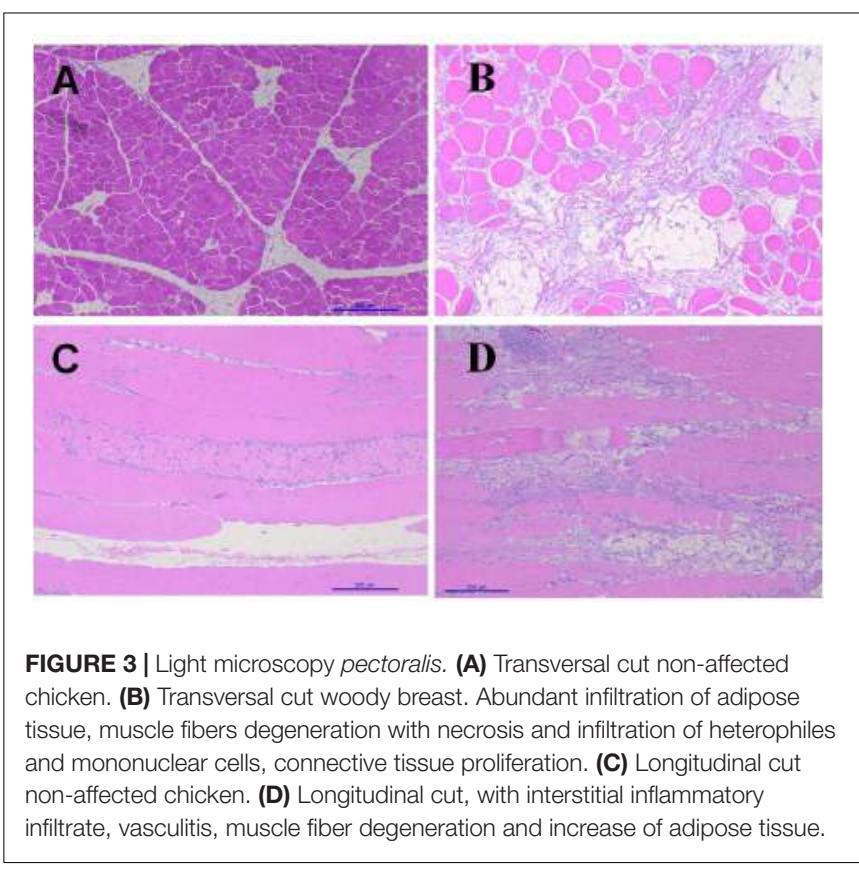

fibers degeneration with necrosis and infiltration of heterophils and mononuclear cells, connective tissue proliferation, vasculitis, and muscle fiber degeneration (Figures 3B-D). White Striping (WS) is a myopathy categorized by the manifestation of white striations parallel to muscle fibers on breast, thigh, and tender muscles of broilers (Kuttappan et al., 2016). In contrast, WB increases the tougher consistency of the breast. Usually, both these conditions occur in varying degrees on the same filet, and are associated with myodegeneration and necrosis, fibrosis, lipidosis, and regenerative changes (Velleman and Clark, 2015; Velleman et al., 2017). In the present study, non-affected chickens presented, WB eight out of the ten chickens evaluated showed severe lesions of WS when compared with six out of 10 non-affected chickens that presented mild lesions of WS (data not shown).

The images of the transmission electron microscopy of pectoralis major are presented in Figure 4. Figure 4A shows an image from a non-affected chicken. The lesions observed in the present study are similar to the lesions reported previously (Velleman et al., 1997; Velleman, 2018). The image shows 

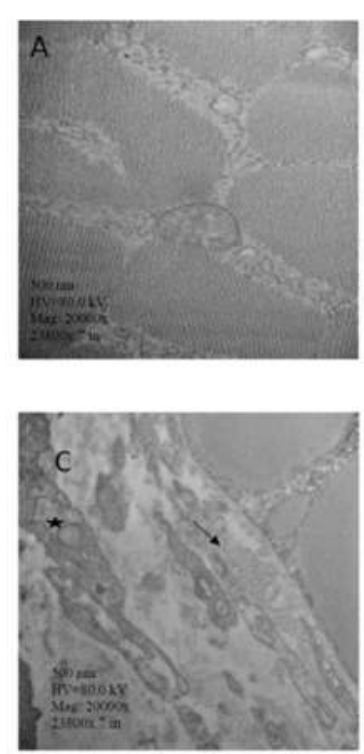
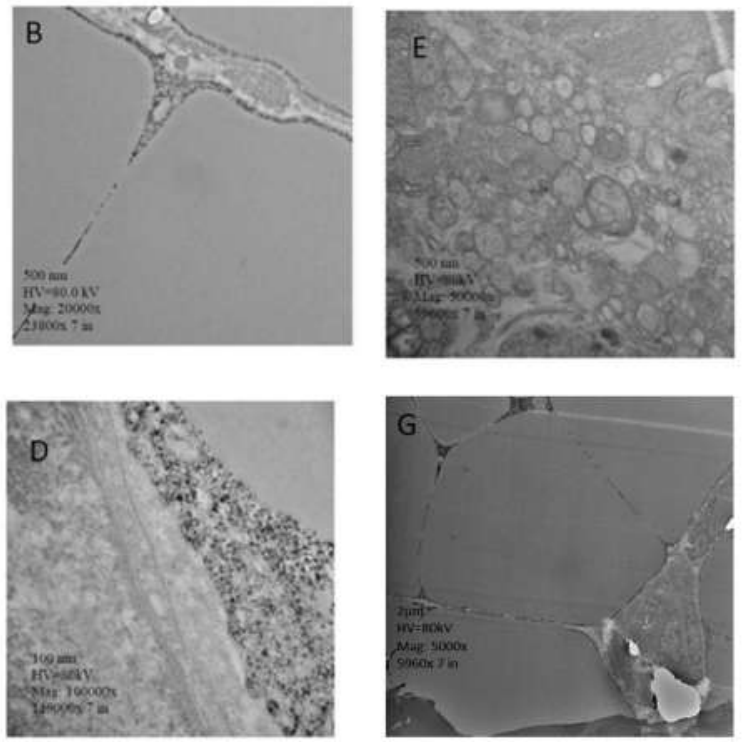
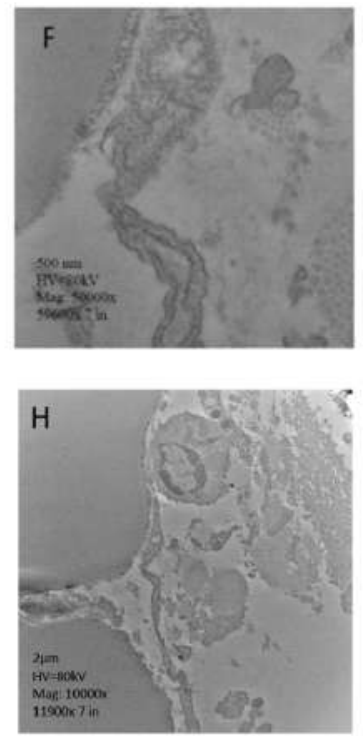

FIGURE 4 | Transmission electron microscopy, pectoralis major. (A) Muscle fibers with distinct contain myofibrils, in tangential sections. Adjacent endomysium with mitochondria and nucleus apparent are properly arranged. (B) Muscle tissue lacks definition of bands, or any characteristic ultrastructural anatomy such as myofibrils. The endomysium components are necrotic, and in some areas, the endomysium is notable only as a string of necrotic tissue between degraded myofibrils. (C) Collagen (arrow) noted in the fascia, along with mitochondria. The fascia appears hypertrophied. (D) Areas of necrotic myofiber without structural identity. Endomysium contains with degraded mitochondria adjacent to the disrupted muscle tissue. (E) Cellular components in the connective tissue are hypertrophied, with noticeable large vesicular material, and areas of separation of components with spongy appearance. (F) Cross-section of the collagen within degrading connective tissue, showing degradation of cellular components. Membranes have decomposed. Necrotic tissue is obvious in the adjoining endomysium, and muscle fibers have no noticeable myofibrils. (G) Muscle tissue is hypertrophied with lack of noticeable myofibrils. Endomysium tissue components are necrotic, showing only thread-like remains. The adjacent connective tissue appears disrupted. (H) Hypertrophied muscle tissue with no noticeable myofibrils. Adjacent collagen in cross-section with disrupted cellular debris. *Hypertrophied mitochondrion.

muscle fibers with distinct myofibrils, in tangential sections. Adjacent endomysium with mitochondria and nucleus apparent are appropriately arranged. In contrast, chickens with WB showed lack definition of bands in muscle tissue, or any normal ultrastructural anatomy such as myofibrils. The endomysium components were necrotic, and in some areas, the endomysium was notable only as a string of necrotic tissue between degraded myofibrils. The fascia appeared hypertrophied, with large areas of necrosis and myofibrils without structural identity. Endomysium contains degraded mitochondria adjacent to the disrupted muscle tissue. Furthermore, cellular components in the connective tissue are hypertrophied, with noticeable substantial vesicular material, and areas of separation of components with spongy appearance. Cross-section of the collagen within degrading connective tissue showed degradation of cellular components (Figures 4B-H).

The extraordinary muscle development of modern broilers is going together with increased adipose tissue, predominantly fat adhered to mesentery, along the intestine from the pylorus to the colon (Wang et al., 2007; Leng et al., 2016). Published studies have identified several genes connected with adiposity in chickens, which may help to regulate the excessive accumulation of fat in the gastrointestinal tract (Bourneuf et al., 2006; Bornelöv et al., 2018).

Cells of adipose tissue appear to be highly dynamic since these cells secrete or express many endocrine proteins (Saely et al., 2012; Wensveen et al., 2015). Among them, leptin, a central adipokine that influences growth, metabolism, and behavior the appetite was discovered in the visceral fat of the ob/ob mutant obese mouse (Ingalls et al., 1996). In contrast to mammals, recent studies indicate that in modern chickens, leptin plays a minor role in ruling hungriness, but has a higher endocrine role with the ovary and the testicles (Bornelöv et al., 2018). This biological effect may explain the insatiable appetite of broilers. Modern broilers suffer from severe stress and increase intestinal permeability by depriving the feed for $24 \mathrm{~h}$ (Baxter et al., 2017).

In the present study, the increased intramuscular lipid deposits observed at a histologic level in WB lesions are in agreement with several previous reports (Velleman and Clark, 2015; Soglia et al., 2015, 2017). In humans, obesity is associated with an increment of adipose tissue in the muscle, a condition that has been linked with muscle functionally, muscle integrity, and atrophy (Meyer and Ward, 2016). Hence, adipose-based inflammation links adiposity, metabolic disorders and musculoskeletal damage (Zhuo et al., 2012). In mammals, several inflammatory pathways have been linked with myopathies (De Boer et al., 2016; Collins et al., 2018). Under normal conditions, macrophages play a crucial role during early regeneration of damage muscular tissue, which is followed by a series of inflammatory courses, eventually restoring structure and function (Akhmedov and Berdeaux, 2013; Laumonier and Menetrey, 2016). However, the metabolic complications associated with adiposity induce unsuitable 
recruitment macrophages and hypoxia (Karalaki et al., 2009). Furthermore, adiposity changes the ratio of macrophage type 2 (regenerative) to macrophage type 1 (inflammatory) with increased release of inflammatory cytokines (Wensveen et al., 2015; Thomas and Apovian, 2017; Bijnen et al., 2018). The biggest limitation of the present hypothesis manuscript is that this is a single descriptive study and no mechanistic experiments were conducted to evaluate assays of macrophage function/metabolism. Until today, there are no information of the existence of macrophage type 1 and 2 in chickens.

The extreme hypertrophy in modern broiler chickens has limited space for capillaries in the perimysial connective tissues that result in a poor vascularization (Alnahhas et al., 2016). Hence, a reduction in oxygen and increase in waste products lead to severe oxidative stress and inflammation in the muscle end in myopathies such as WB, spaghetti meat, or white striping (Huang and Ahn, 2018). These changes have been confirmed by RNA-seq analysis, microscopic and biochemical studies. It is now clear that chickens with $\mathrm{WB}$ suffer localized hypoxia, increased muscle degradation, reduced glucose utilization, increased intracellular calcium and muscle fiber-type switching (Mutryn et al., 2015; Zambonelli et al., 2016). Furthermore, muscle affected by WB exhibit higher amount of free calcium and sodium as a result of a loss in the intracellular ion homeostasis and an increase of glycolytic activity leading to an increase of $\mathrm{pH}$ in affected muscles (Soglia et al., 2018).

On the other hand, mitochondria dysfunction will not only reduce precious energy required for a dynamic tissue such as the muscles but will increase the levels of oxidative stress (Schwarz et al., 2014; Espinosa-Diez et al., 2015). Hence, nutrient overload and adiposity are associated with micronutrient deficiencies, inflammation and lipid peroxidation compromising the integrity of one of the most critical organelles of cells, the cell membrane (Rambold and Pearce, 2018). To further complicate these conditions, obese humans are in a state of chronic hypoxia since adipose tissue has lower capillary vessels, which exacerbates apoptosis, inflammation, and insulin resistance (Engin A, 2017; Engin A.B, 2017).

The alterations in sarcomere and mitochondrial structure, as well as collagen organization reported in WB lesions in the present study, are in agreement with those reported previously (Velleman et al., 1997). Nevertheless, as far as we know, this is the first study that describes a subjective increase in adipose tissue in bone marrow of chickens affected with WB when compared with non-affected chickens, and reduced bone mineralization.

Compared with the other fat depots, in humans, bone marrow adipose tissue plays a crucial role in bone alterations and has been recognized as an essential biomarker of compromised bone integrity (Han et al., 2011; Riondino et al., 2014; Cawthorn and Scheller, 2017). However, analyses of bone marrow adipose tissue development in metabolic diseases and myopathies in chickens are scarce and should be scientifically evaluated to apprehend the role of bone modifications in pathophysiological contexts. It is also important to consider that adipose tissue accumulation in young and adult animals has different implications. Research with meat animals may very well lead to a new understanding of the regulation of lipid metabolism and adipocyte physiology. Because of the propensity to overeat and become obese, the broiler chicken also represents an attractive biomedical model for lipids, energy metabolism, eating disorders and obesity in humans (Wang et al., 2017).

Little is known about macrophages type 1 and types 2 in chickens. Unpublished data from our laboratory suggest that broiler chickens fed with a soy/corn diet exhibit subjectively, infiltration of inflammatory cells that resemble macrophage crowding. If confirmed, this will be a significant breakthrough in chicken immunology. Further studies to evaluate the role of visceral fat, particularly, measurements quantity of fat accumulated in the body of the birds including abdominal fat pad and under skin as well as in the muscle (lipid content of breast muscle), adipokines, and inflammasome, at different ages of broiler chickens are needed to confirm the hypothesis presented in this single and descriptive study.

\section{CONCLUSION}

Adiposity in modern broilers is a condition that deserves attention. However, there are no studies that have evaluated the relationship between adipose tissue, and the pathways and mechanisms leading to myopathies such as WB. Stress and inflammation can result from a variety of biological, chemical, environmental, or nutritional factors to mention a few. In mammals, adiposity induces chronic systemic inflammatory, a condition linked to several metabolic disorders including cardiovascular and musculoskeletal diseases. Equally important, is the relationship of bone marrow adipose tissue in bone mineralization and hematopoiesis pathologies. The interest in bone marrow adipose tissue is a hot topic because it rises in various pathophysiological diseases. The results of the present hypothesis and theory study suggest that bone marrow adipose tissue and bone mineralization may be linked in the pathogeny caused by WB myopathy in modern broiler chickens.

\section{DATA AVAILABILITY}

All data used in this study are available from the corresponding author upon reasonable request.

\section{ETHICS STATEMENT}

All animal handling procedures complied with Institutional Animal Care and Use Committee at the University of Arkansas, protocol \# 18067.

\section{AUTHOR CONTRIBUTIONS}

BdAM, EM, and GT-I designed the experiments and wrote the manuscript. BdAM and EM performed the experiments. $\mathrm{MB}$, 
$\mathrm{KSK}, \mathrm{CO}, \mathrm{NC}-\mathrm{A}, \mathrm{WB}$, and LP-M aided in the analysis and interpretation of data. GT-I, JL, and XH-V contributed to editing the manuscript. $\mathrm{CO}, \mathrm{SD}, \mathrm{WB}$, EG, and GT-I supervised the project and wrote the manuscript.

\section{REFERENCES}

Abdalla, B. A., Chen, J., Nie, Q., and Zhang, X. (2018). Genomic insights into the multiple factors controlling abdominal fat deposition in a chicken model. Front. Genet. 9:262. doi: 10.3389/fgene.2018.00262

Adams, G. R. (2002). Invited review: autocrine/paracrine IGF-I and skeletal muscle adaptation. J. Appl. Physiol. 93, 1159-1167. doi: 10.1152/japplphysiol.01264. 2001

Akhmedov, D., and Berdeaux, R. (2013). The effects of obesity on skeletal muscle regeneration. Front. Physiol. 4:371. doi: 10.3389/fphys.2013.00371

Alnahhas, N., Berri, C., Chabault, M., Chartrin, P., Boulay, M., Bourin, M. C., et al. (2016). Genetic parameters of white striping in relation to body weight, carcass composition, and meat quality traits in two broiler lines divergently selected for the ultimate $\mathrm{pH}$ of the pectoralis major muscle. BMC Genet. 17:61. doi: 10.1186/s12863-016-0369-2

Awano, H., Blaeser, A., Wu, B., Lu, P., Keramaris-Vrantsis, E., and Lu, Q. (2015). Dystroglycanopathy muscles lacking functional glycosylation of alphadystroglycan retain regeneration capacity. Neuromuscul. Disord. 25, 474-484. doi: 10.1016/j.nmd.2015.03.004

Baxter, M. F., Merino-Guzman, R., Latorre, J. D., Mahaffey, B. D., Yang, Y., Teague, K. D., et al. (2017). Optimizing fluorescein isothiocyanate dextran measurement as a biomarker in a 24 -h feed restriction model to induce gut permeability in broiler chickens. Front. Vet. Sci. 4:56. doi: 10.3389/fvets.2017.00056

Bijnen, M., Josefs, T., Cuijpers, I., Maalsen, C. J., van de Gaar, J., Vroomen, M., et al. (2018). Adipose tissue macrophages induce hepatic neutrophil recruitment and macrophage accumulation in mice. Gut 67, 1317-1327. doi: 10.1136/gutjnl2016-313654

Blem, C. R. (1976). Patterns of lipid storage and utilization in birds. Am. Zool. 16, 671-684. doi: 10.1093/icb/16.4.671

Bornelöv, S., Seroussi, E., Yosefi, S., Benjamini, S., Miyara, S., Ruzal, M., et al. (2018). Comparative omics and feeding manipulations in chicken indicate a shift of the endocrine role of visceral fat towards reproduction. BMC Genomics 19:295. doi: 10.1186/s12864-018-4675-0

Bourneuf, E., Hérault, F., Chicault, C., Carré, W., Assaf, S., Monnier, A., et al. (2006). Microarray analysis of differential gene expression in the liver of lean and fat chickens. Gene 372, 162-170. doi: 10.1016/j.gene.2005.12.028

Burt, D. (2007). Emergence of the chicken as a model organism: implications for agriculture and biology. Poult. Sci. 86, 1460-1471. doi: 10.1093/ps/86.7.1460

Carotenuto, F., Coletti, D., Di Nardo, P., and Teodori, L. (2016). $\alpha$-linolenic acid reduces TNF-induced apoptosis in $\mathrm{C} 2 \mathrm{C} 12$ myoblasts by regulating expression of apoptotic proteins. Eur. J. Transl. Myol. 26:6033. doi: 10.4081/ejtm.2016. 6033

Cawthorn, W. P., and Scheller, E. L. (2017). Bone marrow adipose tissue: formation, function, and impact on health and disease. Front. Endocrinol. 8:112. doi: 10.3389 /fendo. 2017.00112

Collins, K. H., Herzog, W., MacDonald, G. Z., Reimer, R. A., Rios, J. L., Smith, I. C., et al. (2018). Obesity, metabolic syndrome, and musculoskeletal disease: common inflammatory pathways suggest a central role for loss of muscle integrity. Front. Physiol. 9:112. doi: 10.3389/fphys.2018.00112

De Boer, A. A., Monk, J. M., Liddle, D. M., Hutchinson, A. L., Power, K. A., Ma, D. W., et al. (2016). Fish-oil-derived n-3 polyunsaturated fatty acids reduce NLRP3 inflammasome activity and obesity-related inflammatory cross-talk between adipocytes and CD11b + macrophages. J. Nutr. Biochem. 34, 61-72. doi: 10.1016/j.jnutbio.2016.04.004

Dodson, M. V., Hausman, G. J., Guan, L., Du, M., Rasmussen, T. P., Poulos, S. P., et al. (2010). Lipid metabolism, adipocyte depot physiology and utilization of meat animals as experimental models for metabolic research. Int. J. Biol. Sci. 6:691. doi: 10.7150/ijbs.6.691

Engin, A (2017). "Adipose tissue hypoxia in obesity and its impact on preadipocytes and macrophages: hypoxia hypothesis," in Obesity and Lipotoxicity, eds A. B.

\section{ACKNOWLEDGMENTS}

The authors gratefully acknowledge The Materials Characterization Facility at the University of Arkansas, Fayetteville.

Engin and A. Engin (Cham: Springer), 305-326. doi: 10.1007/978-3-31948382-5_13

Engin, A. B (2017). "Adipocyte-macrophage cross-talk in obesity," in Obesity and Lipotoxicity, eds A. B. Engin and A. Engin (Cham: Springer), 327-343. doi: 10.1007/978-3-319-48382-5_14

Espinosa-Diez, C., Miguel, V., Mennerich, D., Kietzmann, T., Sánchez-Pérez, P., Cadenas, S., et al. (2015). Antioxidant responses and cellular adjustments to oxidative stress. Redox Biol. 6, 183-197. doi: 10.1016/j.redox.2015.07.008

Fu, X., Zhu, M., Zhang, S., Foretz, M., Viollet, B., and Du, M. (2016). Obesity impairs skeletal muscle regeneration through inhibition of AMPK. Diabetes 65, 188-200. doi: $10.2337 / \mathrm{db} 15-0647$

Halevy, O., Geyra, A., Barak, M., Uni, Z., and Sklan, D. (2000). Early posthatch starvation decreases satellite cell proliferation and skeletal muscle growth in chicks. J. Nutr. 130, 858-864. doi: 10.1093/jn/130.4.858

Han, T., Tajar, A., and Lean, M. (2011). Obesity and weight management in the elderly. Br. Med. Bull. 97, 169-196. doi: 10.1093/bmb/ldr002

Hardouin, P., Rharass, T., and Lucas, S. (2016). Bone marrow adipose tissue: to be or not to be a typical adipose tissue? Front. Endocrinol. 7:85. doi: 10.3389/fendo. 2016.00085

Hausman, G., Dodson, M., Ajuwon, K., Azain, M., Barnes, K., Guan, L., et al. (2009). Board-invited review: the biology and regulation of preadipocytes and adipocytes in meat animals. J. Anim. Sci. 87, 1218-1246. doi: 10.2527/jas.20081427

Huang, X., and Ahn, D. U. (2018). The Incidence of muscle abnormalities in broiler breast meat-a review. Korean J. Food Sci. Anim. Resour. 38, 835-850. doi: 10.5851/kosfa.2018.e2

Ingalls, A. M., Dickie, M. M., and Shell, G. (1996). Obese, a new mutation in the house mouse. Obes. Res. 4:101. doi: 10.1002/j.1550-8528.1996.tb00519.x

International, A. O. A. C. (2000). "Animal feeds," in Official Methods of Analysis of AOAC International, ed. W. Horwaitz (Gaithersburg, MD: AOAC International), 1-54.

Johnston, D. W. (1971). The absence of brown adipose tissue in birds. Comp. Biochem. Physiol. 40, 1107-1108.

Karalaki, M., Fili, S., Philippou, A., and Koutsilieris, M. (2009). Muscle regeneration: cellular and molecular events. In Vivo 23, 779-796.

King, J. R. (1967). Adipose tissue composition in experimentally induced fat deposition in the White-crowned Sparrow. Comp. Biochem. Physiol. 21, $393-$ 403. doi: 10.1016/0010-406x(67)90801-8

Knowles, T. G., Kestin, S. C., Haslam, S. M., Brown, S. N., Green, L. E., Butterworth, A., et al. (2008). Leg disorders in broiler chickens: prevalence, risk factors and prevention. PLoS One 3:e1545. doi: 10.1371/journal.pone.0001545

Kozakowska, M., Pietraszek-Gremplewicz, K., Jozkowicz, A., and Dulak, J. (2015). The role of oxidative stress in skeletal muscle injury and regeneration: focus on antioxidant enzymes. J. Muscle Res. Cell. Motil. 36, 377-393. doi: 10.1007/ s10974-015-9438-9

Kuang, S., Kuroda, K., Le Grand, F., and Rudnicki, M. A. (2007). Asymmetric selfrenewal and commitment of satellite stem cells in muscle. Cell 129, 999-1010. doi: 10.1016/j.cell.2007.03.044

Kuttappan, V. A., Bottje, W., Ramnathan, R., Hartson, S. D., Coon, C. N., Kong, B.-W., et al. (2017). Proteomic analysis reveals changes in carbohydrate and protein metabolism associated with broiler breast myopathy. Poult. Sci. 96, 2992-2999. doi: 10.3382/ps/pex069

Kuttappan, V. A., Hargis, B. M., and Owens, C. M. (2016). White striping and woody breast myopathies in the modern poultry industry: a review. Poult. Sci. 95, 2724-2733. doi: $10.3382 / \mathrm{ps} /$ pew216

Laumonier, T., and Menetrey, J. (2016). Muscle injuries and strategies for improving their repair. J. Exp. Orthop. 3:15. doi: 10.1186/s40634-016-0051-7

Leng, L., Zhang, H., Dong, J. Q., Wang, Z. P., Zhang, X. Y., Wang, S. Z., et al. (2016). Selection against abdominal fat percentage may increase intramuscular fat content in broilers. J. Anim. Breed. Genet. 133, 422-428. doi: 10.1111/jbg.12204 
Lepper, C., Partridge, T. A., and Fan, C.-M. (2011). An absolute requirement for Pax7-positive satellite cells in acute injury-induced skeletal muscle regeneration. Development 138, 3639-3646. doi: 10.1242/dev.067595

Livingston, M., Ferket, P., Brake, J., and Livingston, K. (2018). Dietary amino acids under hypoxic conditions exacerbates muscle myopathies including wooden breast and white stripping. Poult. Sci. 98, 1517-1527. doi: 10.3382/ps/pey463

Marchesi, J. A. P., Ibelli, A. M. G., Peixoto, J. O., Cantão, M. E., Pandolfi, J. R. C., Marciano, C. M. M., et al. (2018). Whole transcriptome analysis of the pectoralis major muscle reveals molecular mechanisms involved with white striping in broiler chickens. Poult. Sci. 98, 590-601. doi: 10.3382/ps/pey429

Matsuzawa, Y., Shimomurn, I., Nakumura, Y., Keno, Y., Kotani, K., Tokunaga, K., et al. (1995). Pathophysiology and pathogenesis of visceral fat obesity. Obes. Res. 3, 187s-194s. doi: 10.1002/j.1550-8528.1995.tb00462.x

Meloche, K. J., Dozier, W. A. III, Brandebourg, T. D., and Starkey, J. D. (2018). Skeletal muscle growth characteristics and myogenic stem cell activity in broiler chickens affected by wooden breast. Poult. Sci. 97, 4401-4414. doi: 10.3382/ps/ pey287

Meyer, G. A., and Ward, S. R. (2016). Developmental biology and regenerative medicine: addressing the vexing problem of persistent muscle atrophy in the chronically torn human rotator cuff. Phys. Ther. 96, 722-733. doi: 10.2522/ptj. 20150029

Miska, K. B., and Fetterer, R. H. (2019). Expression of amino acid and sugar transporters, aminopeptidase, and the di-and tri-peptide transporter PepT1; differences between modern fast growing broilers and broilers not selected for rapid growth. Poult. Sci. 98, 2272-2280. doi: 10.3382/ps/pey583

Mudalal, S., Lorenzi, M., Soglia, F., Cavani, C., and Petracci, M. (2015). Implications of white striping and wooden breast abnormalities on quality traits of raw and marinated chicken meat. Animal 9, 728-734. doi: 10.1017/ S175173111400295X

Mutryn, M. F., Brannick, E. M., Fu, W., Lee, W. R., and Abasht, B. (2015). Characterization of a novel chicken muscle disorder through differential gene expression and pathway analysis using RNA-sequencing. BMC Genomics 16:399. doi: 10.1186/s12864-015-1623-0

Ouali, A., Gagaoua, M., Boudida, Y., Becila, S., Boudjellal, A., Herrera-Mendez, C. H., et al. (2013). Biomarkers of meat tenderness: present knowledge and perspectives in regards to our current understanding of the mechanisms involved. Meat Sci. 95, 854-870. doi: 10.1016/j.meatsci.2013.05.010

Rambold, A. S., and Pearce, E. L. (2018). Mitochondrial dynamics at the interface of immune cell metabolism and function. Trends Immunol. 39, 6-18. doi: 10. 1016/j.it.2017.08.006

Resnyk, C. W., Carré, W., Wang, X., Porter, T. E., Simon, J., Le Bihan-Duval, E., et al. (2017). Transcriptional analysis of abdominal fat in chickens divergently selected on bodyweight at two ages reveals novel mechanisms controlling adiposity: validating visceral adipose tissue as a dynamic endocrine and metabolic organ. BMC Genomics 18:626. doi: 10.1186/s12864-017-4035-5

Riondino, S., Roselli, M., Palmirotta, R., Della-Morte, D., Ferroni, P., Guadagni, F., et al. (2014). Obesity and colorectal cancer: role of adipokines in tumor initiation and progression. World J. Gastroenterol. 20:5177. doi: 10.3748/wjg. v20.i18.5177

Saely, C. H., Geiger, K., and Drexel, H. (2012). Brown versus white adipose tissue: a mini-review. Gerontology 58, 15-23. doi: 10.1159/000321319

SAS Institute Inc. (2002). 9.4 User's Guide. Cary, NC: SAS Documentation.

Schwarz, K., Siddiqi, N., Singh, S., Neil, C. J., Dawson, D., Frenneaux, M. P., et al. (2014). The breathing heart - mitochondrial respiratory chain dysfunction in cardiac disease. Int. J. Cardiol. 171, 134-143. doi: 10.1016/j.ijcard.2013.12.014

Soglia, F., Gao, J., Mazzoni, M., Puolanne, E., Cavani, C., Petracci, M., et al. (2017). Superficial and deep changes of histology, texture and particle size distribution in broiler wooden breast muscle during refrigerated storage. Poult. Sci. 96, 3465-3472. doi: 10.3382/ps/pex115

Soglia, F., Mudalal, S., Babini, E., Di Nunzio, M., Mazzoni, M., Sirri, F., et al. (2015). Histology, composition, and quality traits of chicken Pectoralis major muscle affected by wooden breast abnormality. Poult. Sci. 95, 651-659. doi: $10.3382 / \mathrm{ps} / \mathrm{pev} 353$

Soglia, F., Zeng, Z., Gao, J., Puolanne, E., Cavani, C., Petracci, M., et al. (2018). Evolution of proteolytic indicators during storage of broiler wooden breast meat. Poult. Sci. 97, 1448-1455. doi: 10.3382/ps/pex398

Stern, C. D. (2005). The chick: a great model system becomes even greater. Dev. Cell 8, 9-17. doi: 10.1016/s1534-5807(04)00425-3
Thomas, D., and Apovian, C. (2017). Macrophage functions in lean and obese adipose tissue. Metabolism 72, 120-143. doi: 10.1016/j.metabol.2017.04.005

Tůmová, E., and Teimouri, A. (2010). Fat deposition in the broiler chicken: a review. Sci. Agric. Biochem. 41, 121-128. doi: 10.3382/ps/pex184

Velleman, S. (2018). Association of posthatch muscle growth with the progression of breast muscle myopathies. J. Anim. Sci. 96:96. doi: 10.1093/jas/sky073.178

Velleman, S. G., and Clark, D. L. (2015). Histopathologic and myogenic gene expression changes associated with wooden breast in broiler breast muscles. Avian Dis. 59, 410-418. doi: 10.1637/11097-042015-reg.1

Velleman, S. G., Clark, D. L., and Tonniges, J. R. (2017). Fibrillar collagen organization associated with broiler wooden breast fibrotic myopathy. Avian Dis. 61, 481-490. doi: 10.1637/11738-080217-Reg.1

Velleman, S. G., McFarland, D. C., Li, Z., Ferrin, N. H., Whitmoyer, R., Dennis, J. E., et al. (1997). Alterations in sarcomere structure, collagen organization, mitochondrial activity, and protein metabolism in the avian low score normal muscle weakness. Dev. Growth Differ. 39, 563-570. doi: 10.1046/j.1440-169x. 1997.t01-4-00003.x

Wang, G., Kim, W. K., Cline, M. A., and Gilbert, E. R. (2017). Factors affecting adipose tissue development in chickens: a review. Poult. Sci. 96, 3687-3699. doi: $10.3382 / \mathrm{ps} / \mathrm{pex} 184$

Wang, H., Li, H., Wang, Q., Wang, Y., Han, H., and Shi, H. (2006). Microarray analysis of adipose tissue gene expression profiles between two chicken breeds. J. Biosci. 31, 565-573. doi: 10.1007/bf02708408

Wang, H. B., Li, H., Wang, Q. G., Zhang, X. Y., Wang, S. Z., Wang, Y. X., et al. (2007). Profiling of chicken adipose tissue gene expression by genome array. BMC Genomics 8:193. doi: 10.1186/1471-2164-8-193

Wen, C., Jiang, X., Ding, L., Wang, T., and Zhou, Y. (2017). Effects of dietary methionine on breast muscle growth, myogenic gene expression and IGFI signaling in fast-and slow-growing broilers. Sci. Rep. 7:1924. doi: 10.1038/ s41598-017-02142-z

Wensveen, F. M., Valentić, S., Šestan, M., Turk Wensveen, T., and Polić, B. (2015). The "Big Bang" in obese fat: events initiating obesity-induced adipose tissue inflammation. Eur. J. Immunol. 45, 2446-2456. doi: 10.1002/eji. 201545502

Wideman, R. F., and Hamal, K. R. (2011). Idiopathic pulmonary arterial hypertension: an avian model for plexogenic arteriopathy and serotonergic vasoconstriction. J. Pharmacol. Toxicol. Methods 63, 283-295. doi: 10.1016/j. vascn.2011.01.002

Zambonelli, P., Zappaterra, M., Soglia, F., Petracci, M., Sirri, F., Cavani, C., et al. (2016). Detection of differentially expressed genes in broiler pectoralis major muscle affected by white striping-wooden breast myopathies. Poult. Sci. 95, 2771-2785. doi: 10.3382/ps/pew268

Zhang, B., and Coon, C. N. (1997). The relationship of various tibia bone measurements in hens. Poult. Sci. 76, 1698-1701. doi: 10.1093/ps/76.12.1698

Zhang, B. B., Zhou, G., and Li, C. (2009). AMPK: an emerging drug target for diabetes and the metabolic syndrome. Cell Metab. 9, 407-416. doi: 10.1016/j. cmet.2009.03.012

Zhuo, Q., Yang, W., Chen, J., and Wang, Y. (2012). Metabolic syndrome meets osteoarthritis. Nat. Rev. Rheumatol. 8, 729-737. doi: 10.1038/nrrheum.2012. 135

Zoico, E., Rossi, A., Di Francesco, V., Sepe, A., Olioso, D., Pizzini, F., et al. (2009). Adipose tissue infiltration in skeletal muscle of healthy elderly men: relationships with body composition, insulin resistance, and inflammation at the systemic and tissue level. J. Gerontol. A Biol. Sci. Med. Sci. 65, 295-299. doi: 10.1093/gerona/glp155

Conflict of Interest Statement: The authors declare that the research was conducted in the absence of any commercial or financial relationships that could be construed as a potential conflict of interest.

Copyright (C) 2019 de Almeida Mallmann, Martin, Soo Kim, Calderon-Apodaca, Baxter, Latorre, Hernandez-Velasco, Paasch-Martinez, Owens, Dridi, Bottje, Greene and Tellez-Isaias. This is an open-access article distributed under the terms of the Creative Commons Attribution License (CC BY). The use, distribution or reproduction in other forums is permitted, provided the original author(s) and the copyright owner(s) are credited and that the original publication in this journal is cited, in accordance with accepted academic practice. No use, distribution or reproduction is permitted which does not comply with these terms. 


\section{OPEN ACCESS}

Edited by:

Walter Gay Bottje,

University of Arkansas, United States

Reviewed by:

Richard Bailey,

Aviagen, United Kingdom

Xiaofei Wang,

Tennessee State University,

United States

${ }^{*}$ Correspondence: Behnam Abash

abasht@udel.edu

Specialty section:

This article was submitted to

Avian Physiology,

a section of the journal

Frontiers in Physiology

Received: 22 January 2019

Accepted: 31 May 2019

Published: 18 June 2019

Citation:

Brothers B, Zhuo Z, Papah MB and Abasht B (2019) RNA-Seq Analysis Reveals Spatial and Sex

Differences in Pectoralis Major Muscle

of Broiler Chickens Contributing to Difference in Susceptibility

to Wooden Breast Disease.

Front. Physiol. 10:764

doi: 10.3389/fphys.2019.00764

\section{RNA-Seq Analysis Reveals Spatial and Sex Differences in Pectoralis Major Muscle of Broiler Chickens Contributing to Difference in Susceptibility to Wooden Breast Disease}

\author{
Brilynn Brothers ${ }^{1}$, Zhu Zhuo ${ }^{2}$, Michael B. Papah ${ }^{2}$ and Behnam Abasht ${ }^{2 *}$ \\ ${ }^{1}$ Department of Biological Sciences, University of Delaware, Newark, DE, United States, ${ }^{2}$ Department of Animal and Food \\ Sciences, University of Delaware, Newark, DE, United States
}

Wooden Breast Disease (WBD) is a novel myopathy affecting the pectoralis major muscle of modern broiler chickens. The etiology of WBD is not currently known, but has been linked to increased feed efficiency, growth rate, and muscle yield in broiler chickens. Differential effect of WBD has been detected between regions of the P. major and between sexes of broilers - male birds and the cranial aspect of the muscle tend to be more severely affected by the disease than females and the caudal aspect. This study aimed to characterize biological differences in the P. major between regions of the muscle and sexes of birds. Samples were taken from the cranial and caudal aspects of P. major muscles of 3-week-old, unaffected male and female birds for RNA sequencing. RNA was extracted and used for preparation of cDNA libraries, which were sequenced by the Delaware Biotechnology Institute (DBI) using HiSeq2500. Sequence reads were aligned to the chicken reference genome with HISAT, and genes were analyzed for differential expression between regions of the breast muscle and sexes of birds using CuffDiff. Functional analysis was performed on differentially expressed genes (DEGs) between sex groups using DAVID and Ingenuity Pathway Analysis (IPA). There were 12 DEGs between cranial and caudal samples, and 260 between male and female birds. Out of the 260 genes differentially expressed between sexes, 189 were upregulated in males. Of this subset, 103 genes (55\%) were located on the Z-chromosome. There was increased expression of genes involved in fat metabolism and oxidative stress responses in the cranial region of the P. major muscle, as well as increased expression of fat metabolism, oxidative stress response, antiangiogenesis, and connective tissue proliferation genes in male broilers. These results support the hypothesis that there are biological characteristics in male broilers and the cranial region of the breast muscle that may make them more susceptible to WBD, as well as raising the possibility of a metabolic switch in modern broiler chickens that may be more prominent in males.

Keywords: wooden breast, broiler, chicken, RNA sequencing, myopathy, gene expression, metabolism 


\section{INTRODUCTION}

Demand for poultry meat has greatly increased over the last 50 years. To meet the still-growing demand for poultry meat, the poultry industry has implemented methods to increase growth rate, feed efficiency, and muscle yield, especially that of the breast muscle, in turkeys and chickens. These improvements in the efficiency of the poultry industry may have inadvertently induced a higher incidence of muscle disorders and abnormalities, such as deep pectoral muscle disease and white striping (Petracci et al., 2013). Myopathies such as these have negative impacts on the poultry industry, as they decrease the quality of chicken breast meat, resulting in the breast being condemned entirely or downgraded for use in manufactured products such as pet food.

Relatively recently, a novel myopathy known as Wooden Breast Disease (WBD) has made an appearance in modern broiler chickens across the world. As described by Sihvo et al. (2014), WBD is characterized by a pale, bulging pectoralis major muscle (P. major) that is palpably firm. Recent histological studies indicate that the earliest signs of WBD appear in the first week post-hatch, when affected birds begin to display localized phlebitis with perivascular lipid infiltration (Papah et al., 2017). Later stages of WBD demonstrate diffuse myodegeneration, inflammatory cell infiltration, fibrosis, necrosis, and lipid infiltration (Papah et al., 2017). Though they may occur independently of each other, WBD is often coexistent with another muscle condition, namely, white striping on the muscle (Sihvo et al., 2014; Mudalal et al., 2015; Dalle Zotte et al., 2017; Griffin et al., 2018).

Many aspects of WBD have been characterized at histological and molecular levels, however, its exact etiology is currently unknown. To the best of our knowledge, there has not been any indication of a pathogenic association with the disease, and it is arguable whether or not there is a strong genetic determinism for WBD. However, most agree that the disease is related to the increase in muscle yield, growth rate, and feed efficiency of modern broiler chickens over the past 50 years (Kuttappan et al., 2012; Lorenzi et al., 2014; Sihvo et al., 2014; Russo et al., 2015; Zhou et al., 2015; Abasht et al., 2019), as birds that have not been selectively bred for these economically desirable traits are unaffected by WBD. Additionally, environmental factors impacting growth rate in broilers play major roles in the expression of WBD. In a recent study by Meloche et al. (2018c), reducing feed intake in broilers directly reduced growth rate, and in turn decreased the incidence and severity of WBD, as well as levels of molecular markers for broiler myopathies such as creatine kinase and lactate dehydrogenase. Likewise, reducing digestible lysine, as well as dietary energy and other amino acid densities in feed can reduce the severity of breast muscle myopathies (Meloche et al., 2018a,b). However, birds with reduced nutrient treatments in these studies typically had decreases live performance traits such as body and P. major weight (Meloche et al., 2018a,b). It has also been reported that earlier hatch times and increased temperature during incubation day 14-18 result in fewer and less severe P. major myopathies in broiler chickens (Clark et al., 2017). However, it can not be disregarded that birds undergoing the increased incubation temperature treatment also had lower body weights and P. major weights than control birds (Clark et al., 2017).

A previous study from our laboratory on differential gene expression between WBD-affected and unaffected tissue revealed altered biological pathways correlated with the disease. Mutryn et al. (2015) found over 1500 differentially expressed genes (DEGs) between birds affected and unaffected by WBD, suggesting hypoxia, oxidative stress, connective tissue disorders, and cellular repair mechanisms in WBD-affected tissues. A study by Abasht et al. (2016) on the WBD metabolic profile demonstrates evidence of decreased glucose metabolism through glycolysis. Additional studies on gene expression show evidence of myodegeneration, inflammation, lipid infiltration, and fibrosis (Zambonelli et al., 2016; Papah et al., 2018) in WBD-affected tissues.

In most WBD-affected samples, the cranial aspect of the pectoralis major muscle appears to be more severely affected than the caudal, as it tends to be firmer, thicker, and display a higher degree of white striations than the caudal aspect (Bailey et al., 2015; Clark and Velleman, 2016; Papah et al., 2017). Additionally, it has been observed that male modern broiler chickens have a higher incidence rate of WBD than females, and typically are more severely affected by the disease (Trocino et al., 2015). Considering the correlation of WBD with increased body and breast muscle mass and growth rates, it could be assumed that male birds and the cranial aspect of the pectoralis major muscle are more susceptible to WBD because they grow faster and larger than female birds and the caudal aspect of the muscle. It is a fact that male broiler chickens tend to have higher pectoralis major and overall body masses than females, as well as typically growing at a faster rate (Scheuermann et al., 2003; Tatara et al., 2012; Trocino et al., 2015). Therefore, the key to understanding increased WBD incidence and severity in male broilers and the cranial region of the muscle is understanding the biological differences between sexes of birds and regions of the pectoralis major that are associated with higher growth rates and body size.

This study aims to provide answers for a few questions: Why is the cranial aspect of the pectoralis major more affected by WBD than the caudal, and why are males more affected than females? Additionally, why does the breast muscle grow larger and faster in male birds than females, and why does the cranial aspect grow faster and become thicker than the caudal aspect? This study utilizes RNA sequencing techniques to look at differential gene expression between cranial and caudal sections of the pectoralis major, as well as between male and female birds in search of differing biological pathways that may lead to differing susceptibility to WBD.

\section{MATERIALS AND METHODS}

\section{Chickens and Sample Collection}

A total of 171 Cobb500 broiler chickens of the same egg age and parent flock were hatched at the University of Delaware poultry farm and placed on the floor with wood shavings in chicken houses with automatic ventilation and a light cycle consisting of $1 \mathrm{~h}$ of darkness followed by $23 \mathrm{~h}$ of light per day. Houses 
were kept initially at $95^{\circ} \mathrm{F}$ and lowered by $5^{\circ} \mathrm{F}$ each week until they reached $70^{\circ} \mathrm{F}$. Birds were fed standard commercial starter and grower diets and were allowed free access to feed and water. Bird houses were kept below a stocking density of $30 \mathrm{~kg} / \mathrm{m}^{2}$ throughout the entirety of the experiment in accordance with the Animal Care and Use Handbook 2014 of the College of Agriculture and Natural Resources, University of Delaware. The animal protocols were reviewed and approved by the University of Delaware's Institutional Animal Care and Use Committee.

Birds were weighed each week from the day they hatched until they were humanely euthanized by cervical dislocation at day $7,14,21$, or 56 of age. Birds euthanized at days 7, 14, and 21 had their sexes determined by identification of gonads during necropsy, as secondary sex characteristics are unnoticeable until around 4 weeks of age. For birds euthanized at day 56 , their sexes were determined by observation of secondary sex characteristics.

At 3 weeks of age, 24 birds were randomly selected and euthanized by cervical dislocation followed by necropsy and sample collection for histologic examination and RNAsequencing. Tissue samples were taken from the cranial and caudal aspects of the P. major muscle of all 24 birds. Samples were approximately $2 \mathrm{~g}$ in mass and were harvested along the longitudinal axis of the muscle fibers. To ensure that the same muscle fibers were used for microscopy and RNA-seq, each sample was split into two at the middle perpendicular to muscle fibers, with one portion immediately immersed into $10 \%$ neutral buffered formalin for histology, while the other was flash frozen in liquid nitrogen before transfer to $-80^{\circ} \mathrm{C}$ for RNA-seq. The muscle samples were taken from the same regions for all experimental chickens.

\section{Selection and Identification of Samples for RNA Sequencing}

This study aimed to use samples from unaffected chickens to examine the spatial and sex effects on the occurrence of WBD. We used samples from unaffected birds because we aim to study the innate biological differences between sexes of birds and regions of the P. major muscle without any discernible clinical, gross and histological changes attributable to WBD. There are a few important reasons we chose to study birds at 3 weeks of age. At this point in their life, broiler chickens do not typically present discernible clinical or gross changes associated with WBD. Detection of these changes frequently occur after 3 weeks of age (Papah et al., 2017). Hence, by utilizing 3-week-old birds, we are simultaneously selecting birds that do not have apparent clinical disease, but may be exhibiting molecular signatures of the developing disease that would become fulminant later in life. Indeed, a recent study in our laboratory demonstrated that molecular changes associated with WBD precede its clinical/gross presentation (Papah et al., 2018). Another important factor is that at 3 weeks of age, male and female broilers are beginning to diverge in terms of body weight and growth rates, with males growing faster and larger. Up until this point, male and female broilers grow similarly. Results from the current study supports this observation (see Figure 1). By studying birds that are at this point of divergence, we have a higher chance of seeing differences in gene expression between the sexes.

To identify and determine samples to be used in this study, all muscle tissue samples were subjected to microscopic analysis for tissues changes due to $\mathrm{WBD}$; this was in addition to gross evaluation. Processing of samples for histologic assessment followed routine $\mathrm{H} / \mathrm{E}$ protocol as used in the previous study (Papah et al., 2017) where cross and longitudinal sections of the tissues were obtained.

To classify all 24 samples on the basis of degree of tissue pathology associated with WBD, four categories were identified; namely unaffected, mild, moderate, and severe (Table 1). The degree and extent of microscopic lesions in the two sections (cross and longitudinal) per sample was used to determine the degree of tissue pathology under the four categories. Microscopic parameters used included myofiber degeneration

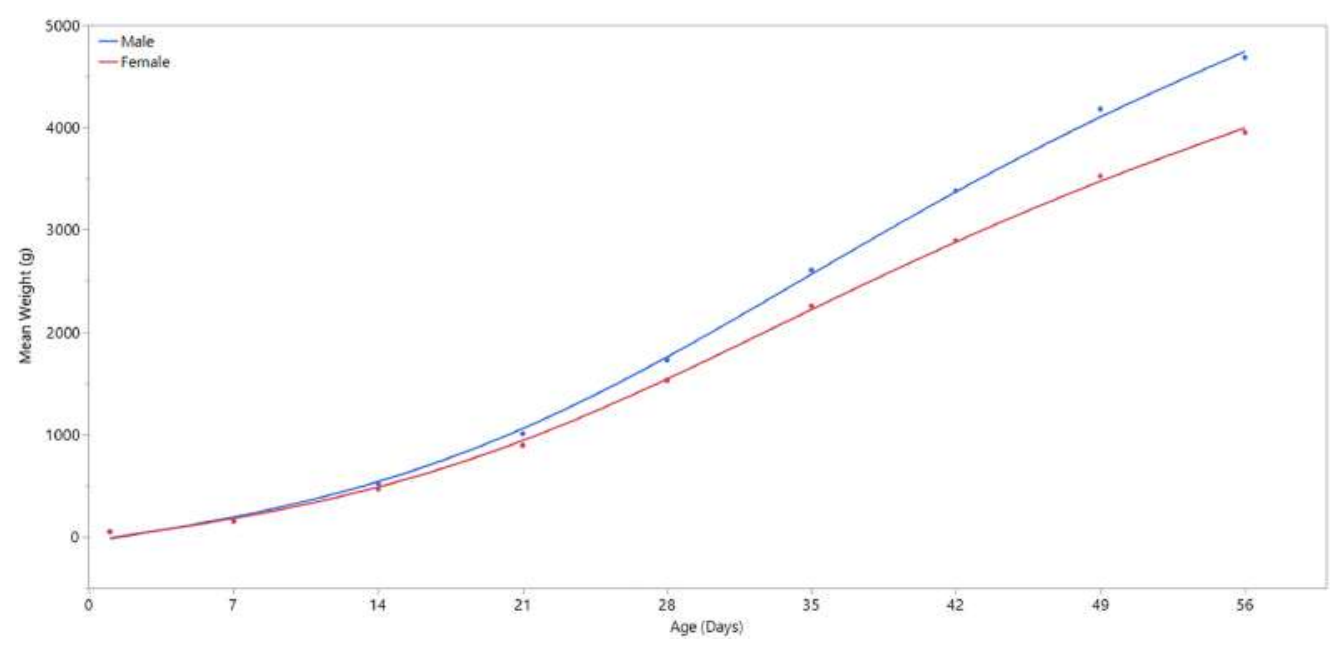

FIGURE 1 | Comparison of weekly body weight by sex. Day 1 refers to the day of hatch. Male broilers have significantly higher average body weights than females at ages $7,14,21,28,35,42,49$, and 56 days $(P \leq 0.0001)$. 
TABLE 1 | Histological presentation of pectoral muscle samples harvested from the cranial and caudal pectoral regions of broiler chickens at 3 weeks of age.

\begin{tabular}{llc}
\hline Cranial pectoral & Caudal pectoral & Number of samples \\
\hline Normal & Normal & 7 \\
Mild & Normal & 5 \\
Mild & Mild & 5 \\
Mild & Moderate & 2 \\
Moderate & Normal & 2 \\
Moderate & Mild & 1 \\
Moderate & Moderate & 1 \\
Severe & Moderate & 1 \\
Total & & 24 \\
\hline
\end{tabular}

and fragmentation, inflammatory cell infiltration, interstitial edema, necrosis, variability in myofiber sizes, lipid infiltration and fibrosis. Samples without any lesions or subtle focal degeneration (occupying $<5 \%$ of the tissue slide) were placed under the unaffected category. Samples exhibiting single-cell myodegenerative changes occupying $5-25 \%$ of the tissue slide with focal infiltration of inflammatory cells were placed under the mild category. Samples with $25-50 \%$ of the tissue slide displaying myodegenerative changes with multifocal inflammation and focal interstitial edema were considered to be moderate. Samples with multifocal to diffuse myodegeneration over $>50 \%$ of the tissue slide, myonecrosis and inflammatory cell infiltration as well as variability of fiber sizes and focal lipid infiltration were considered to be severely affected (Figure 2). It should be noted that muscle samples from the cranial and caudal regions were examined independently. Therefore, for a sample to qualify for RNA-sequencing analysis in the current study, both cranial and caudal samples had to be unaffected.

Pectoralis major muscle samples from 7 birds exhibited normal morphology in both the cranial and caudal portions, and 12 samples from 6 birds ( 3 males and 3 females) were selected for RNA-sequencing (Table 2). We performed Principal Component Analysis to ensure that our sample size was adequate for the study (Supplementary Figure 1).

\section{RNA Sequencing}

A total of 12 cranial and caudal tissue samples from male and female birds were used for RNA extraction and sequencing. RNA was isolated from the samples using the mirVana miRNA Isolation Kit (Thermo Fisher Scientific). RNA quality was tested using Nanodrop 1000 and Fragment Analyzer, which confirmed that the quality of the isolated RNA was acceptable for it to be used throughout the rest of the protocol. Complementary DNA (cDNA) libraries were synthesized using the TruSeq Stranded mRNA Sample Preparation Kit (Illumina), utilizing low sample protocol. Libraries were then sent to the Delaware Biotechnology Institute (DBI) where they were paired-end (2X101) sequenced with Illumina HiSeq 2500.
A

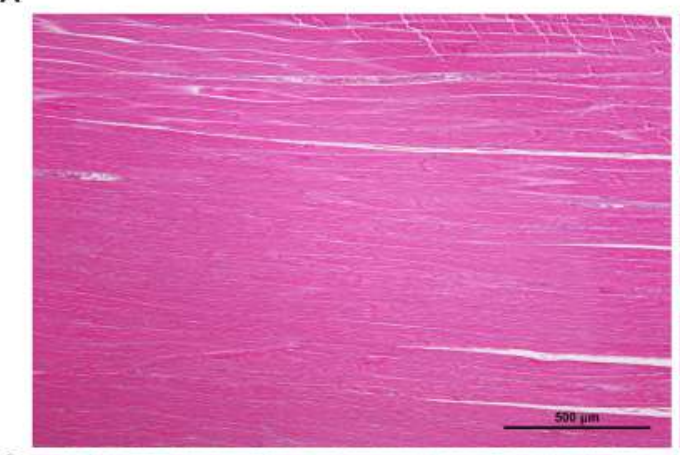

c

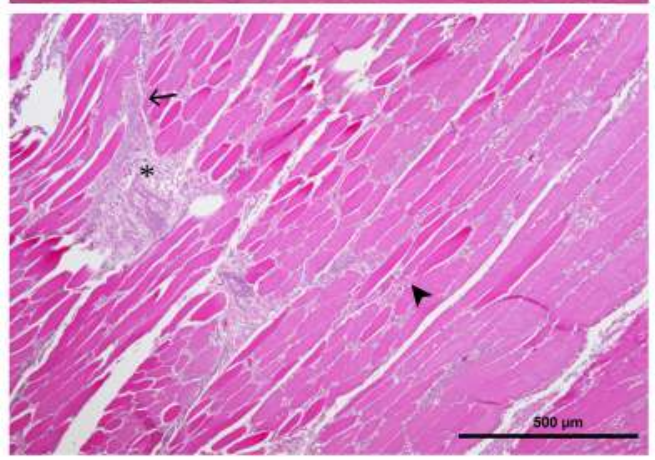

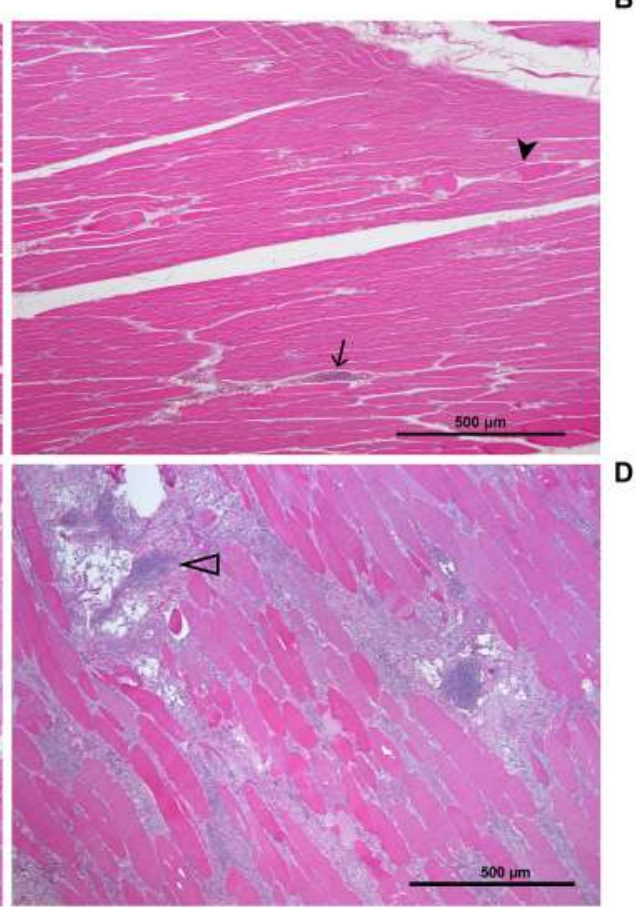

FIGURE 2 | Histological sections of P. major sample showing the various stages of pathology associated with WB: (A) unaffected muscle sample; (B) mildly affected muscle tissue exhibiting multifocal myodegeneration (arrowhead) and focal infiltration by inflammatory cells (arrow); (C) moderately affected sample as shown by diffuse myofiber degeneration multifocal inflammatory cell infiltration and fibrosis (asterisk); (D) severely/markedly affected muscle sample as indicated by diffuse myodegeneration, diffuse myofiber inflammation, lipid infiltration and phlebitis (open arrowhead). 
TABLE 2 | Total body masses of birds used in the RNA-seq study.

\begin{tabular}{llc}
\hline Bird ID\# & Sex & Total body mass (g) \\
\hline 484951 & M & 1068 \\
485058 & M & 982 \\
485061 & M & 1000 \\
484918 & F & 888 \\
485060 & F & 846 \\
485067 & F & 869 \\
\hline
\end{tabular}

\section{Data Analysis}

The quality of the resulting sequences was checked using FastQC v0.11.5. Low quality bases were trimmed with Trimmomatic v0.36, using parameters TRAILING:20 and SLIDINGWINDOW:4:15. After trimming, the reads were aligned to the chicken reference genome Gallus_gallus-5.0.89 using HISAT v2.1.0, then differential expression analysis was performed using Cuffdiff v2.2.1. Significant genes were defined as those with a false discovery rate (FDR) adjusted $p$-value of less than 0.05. Differential expression analysis of cranial vs. caudal aspects of the P. major muscle was performed separately for males and females. The analyses detected no DEGs in females and only 12 in males, indicating a nearly consistent gene expression profile between cranial and caudal aspects of the muscle in both male and female datasets. For greater statistical power, cranial and caudal data were combined for males vs. female analysis, and likewise male and female data were combined for cranial vs. caudal comparison. To identify biological processes and pathways that are significantly enriched by the differentially expressed genes, the gene list was analyzed using the Database for Annotation, Visualization and Integrated Discovery (DAVID) v6.8 (Huang et al., 2009a,b) and Ingenuity Pathways Analysis (IPA) by QIAGEN (Krämer et al., 2014).

\section{RESULTS}

\section{Body Weight Analysis}

A linear regression analysis was performed using JMP statistical software (JMP Pro ${ }^{\circledast}$ Version 13, SAS Institute Inc.) to determine statistical significance of body weight differences between male and female broilers over time, and to confirm the divergence of body weight and growth rate between male and female broilers at 3 weeks of age (Figure 1). Except for the first day of life, body weight differed significantly ( $p$ value $\leq 0.0001$ ) between males and females in all days of weight measurement (Supplementary Table 1).

\section{RNA-Seq Analysis}

\section{Cranial vs. Caudal}

There were 12 differentially expressed genes found between cranial and caudal tissue samples (Table 3). Eight of these genes were upregulated and four were downregulated in the cranial samples.

As there were only 12 significant DEGs in the cranial versus caudal comparison, IPA and DAVID analysis were not performed
TABLE 3 | Cranial vs. caudal differentially expressed genes.

\begin{tabular}{lccc}
\hline Gene & Caudal FPKM & Cranial FPKM & Log2 (fold change) \\
\hline PITX2 & 1.44 & 4.47 & 1.63 \\
HOXA11 & 1.05 & 3.20 & 1.60 \\
NRN1 & 2.81 & 7.93 & 1.50 \\
PLIN1 & 3.98 & 9.52 & 1.26 \\
FABP4 & 47.22 & 91.08 & 0.95 \\
HBAD & 37.58 & 70.28 & 0.90 \\
KY & 26.75 & 49.26 & 0.88 \\
G0S2 & 49.17 & 90.03 & 0.87 \\
FMOD & 21.15 & 8.65 & -1.29 \\
COL12A1 & 4.76 & 1.59 & -1.59 \\
COL22A1 & 1.52 & 0.45 & -1.76 \\
COL11A1 & 6.14 & 1.71 & -1.88
\end{tabular}

${ }^{1}$ A positive (or negative) log2 (fold change) signifies higher (or lower) expression of the corresponding gene in the cranial aspect of the muscle.

TABLE 4 | Top 10 upregulated and downregulated genes in males ${ }^{1}$.

\begin{tabular}{lrrc}
\hline Gene & Female FPKM & Male FPKM & Log2 (Fold Change) \\
\hline CHAC1 & 1.53 & 14.28 & 3.22 \\
ENSGALG00000046114 & 0.85 & 7.23 & 3.09 \\
C7 & 1.79 & 8.10 & 2.18 \\
PDK4 & 1.96 & 8.71 & 2.16 \\
NOV & 0.41 & 1.79 & 2.12 \\
RPL3L & 1.69 & 6.98 & 2.04 \\
CCK & 3.22 & 12.08 & 1.91 \\
FMOD & 6.57 & 21.76 & 1.73 \\
CSMD1 & 1.07 & 3.46 & 1.69 \\
MT4 & 42.37 & 133.88 & 1.66 \\
CD24 & 4.94 & 1.62 & -1.60 \\
TNN11 & 22.05 & 6.96 & -1.66 \\
CLEC19A & 4.09 & 1.29 & -1.67 \\
GCH1 & 4.95 & 1.33 & -1.90 \\
ENSGALG00000044018 & 3.19 & 0.83 & -1.94 \\
AMPH & 9.26 & 2.25 & -2.04 \\
ENSGALG00000036306 & 3.05 & 0.56 & -2.45 \\
RAPGEF4 & 3.51 & 0.46 & -2.93 \\
KCHIP2 & 2.14 & 0.27 & -2.96 \\
ENSGALG00000029783 & 4.22 & 0.11 & \\
\hline & & & \\
\hline
\end{tabular}

${ }^{1}$ Excludes 13 genes with male FPKM values of less than 0.1 , located on the $W$ chromosome or an unplaced scaffold. A positive (or negative) log2 (fold change) signifies higher (or lower) expression of the corresponding gene in male birds.

on this dataset. Rather, we explored the functions of these genes individually: Perilipin 1 (PLIN1), G0/G1 switch gene 2 (G0S2), and fatty acid-binding protein 4 (FABP4) are all involved in lipid metabolism; neuritin 1 (NRN1) is involved in nervous system development; kyphoscoliosis peptidase (KY) is involved in muscle growth, and homeobox A11 (HOXA11) and pituitary homeobox 2 (PITX2) are transcription factors. HBAD refers to the gene coding for hemoglobin subunit alpha-D. Fibromodulin (FMOD), collagen type XI alpha 1 chain (COL11A1), collagen type XII alpha 1 chain (COL12A1), and collagen type XXII alpha 1 chain (COL22A1) are all involved in collagen synthesis and interactions (Gene, 2004). 


\section{Male vs. Female}

A total of 260 genes were differentially expressed between males and females, 189 of which were upregulated in males. Of the 71 genes downregulated in males, 13 of them had expression values of or very close to zero. These genes were mapped to the $\mathrm{W}$ chromosome (3 genes) or unplaced scaffolds (9 genes), except for one gene mapped to the $\mathrm{Z}$ chromosome, which is likely a genome assembly error. Because they demonstrate an FPKM value of 1.7-43.4 in females but zero in males, these genes on unplaced scaffolds are also likely to be on the $\mathrm{W}$ chromosome, which does not exist in male birds. The top 10 upregulated and downregulated genes in males are identified in Table 4.

Of the 260 differentially expressed genes between males and females of the current study, 58 overlapped with differentially expressed genes found in the previous study by Papah et al. (2018) studying differential gene expression between WBD-affected and unaffected 3-week-old male broilers (Supplementary Figure 2). Of the 58 overlapping genes, 47 of them (81\%) shared the same directionality.

Ingenuity Pathway Analysis of the differentially expressed genes between male and female unaffected samples provided predictions of differentially regulated biological functions (Table 5) and pathways (Table 6), as well as predicting the possible activation or deactivation of upstream regulators (Table 7 ).

Additionally, by entering a list of the genes upregulated in males into DAVID we were able to find gene ontologies upregulated in male birds. DAVID analysis yielded 12 main gene

TABLE 5 | Top biological functions for males vs. females.

\begin{tabular}{|c|c|c|}
\hline Biological function & $P$-value & Number of molecules \\
\hline \multicolumn{3}{|l|}{ Diseases and disorders } \\
\hline Cancer & 7.86E-03 - 2.39E-08 & 163 \\
\hline Organismal injury and & 7.86E-03 - 2.39E-08 & 165 \\
\hline \multicolumn{3}{|l|}{ Abnormalities } \\
\hline Cardiovascular disease & 7.86E-03 - 4.77E-07 & 43 \\
\hline Neurological disease & 7.86E-03 - 4.77E-07 & 41 \\
\hline Gastrointestinal disease & 7.73E-03 - 1.19E-06 & 154 \\
\hline \multicolumn{3}{|l|}{$\begin{array}{l}\text { Molecular and cellular } \\
\text { functions }\end{array}$} \\
\hline Cell morphology & 7.86E-03 - 1.37E-06 & 37 \\
\hline Lipid metabolism & 7.86E-03 - 2.90E-06 & 31 \\
\hline Small molecule biochemistry & 7.86E-03 - 2.90E-06 & 44 \\
\hline Molecular transport & 7.73E-03 - 2.94E-06 & 26 \\
\hline Cell death and survival & 7.86E-03 - 4.40E-06 & 64 \\
\hline \multicolumn{3}{|l|}{$\begin{array}{l}\text { Physiological system } \\
\text { development and function }\end{array}$} \\
\hline Cardiovascular system & 7.86E-03 - 1.58E-07 & 43 \\
\hline \multicolumn{3}{|l|}{ Development and function } \\
\hline Organ development & 7.66E-03 - 1.58E-07 & 32 \\
\hline Organ morphology & 7.86E-03 - 1.35E-06 & 43 \\
\hline Skeletal and muscular system & 7.86E-03 - 1.37E-06 & 43 \\
\hline \multicolumn{3}{|l|}{ Development and function } \\
\hline Tissue morphology & 7.73E-03 - 1.37E-06 & 54 \\
\hline
\end{tabular}

ontologies upregulated in male broilers (Table 8). "Gene Count" refers to the number of genes associated with a given ontology, out of the 88 gene IDs DAVID accepted from the list of 189 genes upregulated in male broilers. Entering a separate list of the 71 downregulated genes in males did not yield significant results from DAVID.

\section{DISCUSSION}

\section{Fat Metabolism}

Analysis of differentially expressed genes between regions of the pectoralis major muscle and sexes of birds strongly suggests increased fat metabolism and deposition in male birds and the cranial aspect of the breast muscle. This correlates with the increased fat content found in the P. major muscle of birds affected with Wooden Breast and/or white striping (Mudalal et al., 2014; Soglia et al., 2016) as well as evidence of fatty infiltration observed in histological studies even before wooden breast is clinically and grossly detectable (Papah et al., 2017). This suggests that the breast muscle in male broilers, as well as the cranial aspect of the breast muscle, have a higher tendency to accumulate lipids, which may make them more susceptible to WBD.

For example, fatty acid-binding protein 4 (FABP4), which was upregulated in males, plays a major role in fat accumulation by acting as a fatty acid carrier and segregating fatty acids for triglyceride synthesis to develop adipose tissue (Smathers and Petersen, 2011). This protein is also a biomarker for adipocyte differentiation (Zhang et al., 2015). Similarly, lipoprotein lipase (LPL), upregulated in male birds, is an effective biomarker for adipogenesis. LPL, whose active form is found on the endothelium of capillaries and small-caliber blood vessels, acts by hydrolyzing triglycerides in blood into free fatty acids for oxidative phosphorylation by other cells. Additionally, the free fatty acids can be re-esterified into triglycerides for storage in

TABLE 6 | Top ten canonical pathways for males vs. females.

\begin{tabular}{lcc}
\hline Canonical pathway & $\boldsymbol{P}$-value & Molecules \\
\hline $\begin{array}{l}\text { D-myo-inositol }(1,4,5,6)- \\
\text { tetrakisphosphate biosynthesis }\end{array}$ & $9.85 \mathrm{E}-04$ & 6 \\
$\begin{array}{l}\text { D-myo-inositol }(3,4,5,6)- \\
\text { tetrakisphosphate biosynthesis }\end{array}$ & $9.85 \mathrm{E}-04$ & 6 \\
$\begin{array}{l}\text { 3-Phosphoinositide } \\
\text { Biosynthesis }\end{array}$ & $1.05 \mathrm{E}-03$ & 7 \\
$\begin{array}{l}\text { 3-Phosphoinositide } \\
\text { Degradation }\end{array}$ & $1.59 \mathrm{E}-03$ & 6 \\
$\begin{array}{l}\text { D-myo-inositol-5-phosphate } \\
\text { Metabolism }\end{array}$ & $1.80 \mathrm{E}-03$ & 6 \\
$\begin{array}{l}\text { Fatty acid } \beta \text {-oxidation I } \\
\text { superpathway of inositol } \\
\text { phosphate compounds } \\
\text { complement system }\end{array}$ & $2.00 \mathrm{E}-03$ & 3 \\
retinol biosynthesis & $2.63 \mathrm{E}-03$ & 7 \\
IGF-1 signaling & & 3 \\
& $3.05 \mathrm{E}-03$ & 3 \\
\hline
\end{tabular}


TABLE 7 | Significant activated and inhibited upstream regulators.

\begin{tabular}{|c|c|c|c|}
\hline Upstream regulator & Molecule type & Activation Z-score & Overlapping $\boldsymbol{P}$-value \\
\hline $\mathrm{ACOX} 1$ & Enzyme & -2.45 & $1.21 \mathrm{E}-03$ \\
\hline MYOD1 & Transcription regulator & -2.41 & 4.70E-03 \\
\hline N-cor & Group & -2.22 & $9.72 \mathrm{E}-05$ \\
\hline CD44 & Other & 2.00 & $6.62 \mathrm{E}-03$ \\
\hline MAPK9 & Kinase & 2.00 & $3.72 \mathrm{E}-02$ \\
\hline CTNNB1 & Transcription regulator & 2.00 & 2.32E-02 \\
\hline IL6 & Cytokine & 2.07 & $3.72 \mathrm{E}-02$ \\
\hline ERK & Group & 2.20 & 3.61E-03 \\
\hline NCOA2 & Transcription regulator & 2.22 & 3.77E-04 \\
\hline TGFB3 & Growth factor & 2.24 & $1.96 \mathrm{E}-03$ \\
\hline CEBPA & Transcription regulator & 2.35 & $3.58 \mathrm{E}-03$ \\
\hline FOXO1 & Transcription regulator & 2.42 & $5.54 \mathrm{E}-05$ \\
\hline PPARA & Ligand-dependent nuclear receptor & 2.54 & $2.41 \mathrm{E}-05$ \\
\hline NFE2L2 & Transcription regulator & 2.58 & 2.32E-03 \\
\hline
\end{tabular}

adipocytes (Zhang et al., 2015). This finding is in line with a recent study in our laboratory that showed upregulation of FABP4 in the P. major muscles of WBD-affected chickens at week 3 of age (Papah et al., 2018). Taken together, these studies suggest that active intracellular mobilization of lipids accompanies the early phase of WBD in chickens.

Fatty acid translocase (CD36), upregulated in males, is a receptor for multiple types of ligands, including long-chain fatty acids. It behaves as a fatty acid translocase in adipose tissue, as well as cardiac and skeletal muscle (Febbraio et al., 2001), and therefore may play a role in fat accumulation. In agreement, palmitic acid was predicted by IPA as an activated upstream regulator in males, suggesting higher levels of this long chain fatty acid in the breast muscle of males compared with females. CD36 has been found to play a role in other pathways that are significant to WBD, included antiangiogenesis and immune functions (Febbraio et al., 2001).

Ingenuity pathway analysis suggests activation of an upstream regulator that stimulates genes involved in fat metabolism. Peroxisome proliferator-activated receptor alpha (PPARA) is a ligand-dependent nuclear receptor that regulates expression

TABLE 8 | DAVID ontologies for genes expressed higher in male.

\begin{tabular}{lcc}
\hline Ontology & Gene count & $P$-value \\
\hline Coagulation & 7 & $4.41 \mathrm{E}-04$ \\
Cytokine production & 8 & $6.99 \mathrm{E}-04$ \\
Response to wounding & 9 & $1.76 \mathrm{E}-03$ \\
Cell adhesion & 10 & $2.37 \mathrm{E}-03$ \\
Foam cell differentiation & 3 & $3.19 \mathrm{E}-03$ \\
Lipid metabolism & 4 & $3.23 \mathrm{E}-03$ \\
Protein metabolism & 4 & $3.89 \mathrm{E}-03$ \\
Antiangiogenesis & 4 & $7.65 \mathrm{E}-03$ \\
Extracellular matrix organization & 6 & $8.66 \mathrm{E}-03$ \\
Tissue development & 17 & $1.34 \mathrm{E}-02$ \\
Immune response & 12 & $1.35 \mathrm{E}-02$ \\
Oxidative stress & 6 & $2.27 \mathrm{E}-02$
\end{tabular}

of multiple genes involved in lipid metabolism, including adiponectin (ADIPOQ), FABP4, CD36, perilipin 1 and 2 (PLIN1/PLIN2), and LPL. Because these genes and a few other PPARA target genes are upregulated in the male vs. female dataset, IPA predicted that PPARA is activated in male broilers. PPARA also plays key roles in regulating multiple steps of the fatty acid $\beta$-oxidation (Lemberger et al., 1996), supporting a hypothesis of increased mitochondrial fatty acid $\beta$-oxidation in male modern broilers that will be discussed further in the next section. IPA activation networks for PPARA and palmitic acid are shown in Figure 3.

Additional significant genes involved in fat deposition and metabolism include patatin-like phospholipase domaincontaining protein 2 (PNPLA2), pyruvate dehydrogenase kinase 4 (PDK4), retinol binding protein 7 (RBP7), solute carrier family 44 member 1 (SLC44A1), and acetyl-CoA acyltransferase 2 (ACAA2), all upregulated in the P. major muscle of males. ACAA2 is a gene with key involvement in mitochondrial fatty acid $\beta$-oxidation. It catalyzes the final step in the $\beta$-oxidation pathway, in which $\beta$-ketoacyl-CoA reacts with a free molecule of coenzyme A to produce acetyl-CoA (Nelson and Cox, 2008).

It has been observed that abdominal fat is the most variable body component of broiler chickens, and that female broilers tend to have more abdominal cavity fat deposition than males (Leenstra, 1986), which is consistent with the lower feed efficiency of females. It is well known that increased abdominal fat deposition is associated with the lower feed efficiency in chickens (Zhuo et al., 2015). These findings from prior studies agree with our results that suggests male broilers have higher uptake and oxidation of lipids by the P. major muscle. It can be hypothesized that female broilers catabolize lipids at a lower rate than male broilers due to lower expression of fat metabolism genes in the muscle, and as a result they have more accumulation of fat in the abdominal cavity.

\section{Metabolic Shift}

Evidence of increased fat metabolism in the pectoralis major muscle of male birds is especially significant because it opposes 

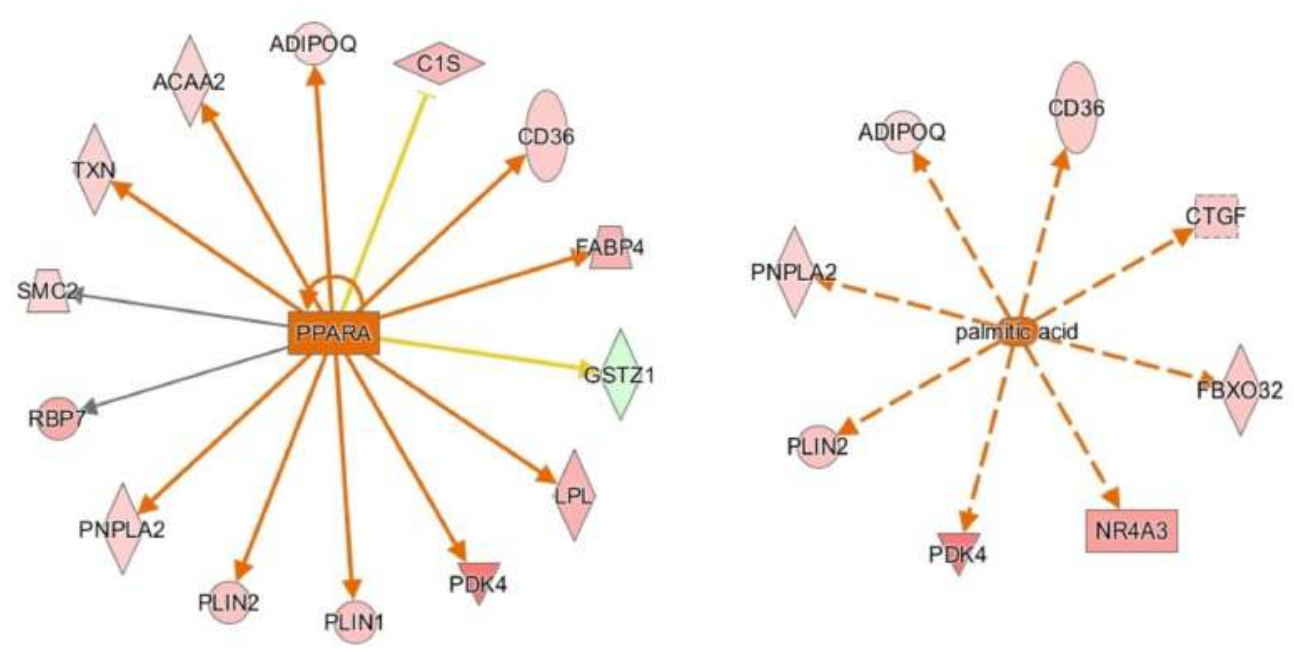

FIGURE 3 | Activation network diagrams for PPARA (left) and palmitic acid (right). Pink and green shapes represent genes upregulated or downregulated in males, respectively. Orange arrows represent IPA's prediction that the upstream regulator activates the corresponding genes. Yellow arrows represent disagreement between the direction of the gene expression (i.e., up or downregulation) and IPA's prediction of the relationship between the upstream regulator and the corresponding gene. Gray arrows mean that IPA predicts a relationship between the upstream regulator and the corresponding gene but cannot predict the exact effect. Image produced using IPA.

the normal metabolism of the muscle. The pectoralis major in chickens is a "white" muscle, meaning it consists almost entirely of type II fast-twitch fibers (Ono et al., 1993; Verdiglione and Cassandro, 2013), contains little myoglobin (hence the "white" color) (Kranen et al., 1999), operates primarily on glycolytic metabolism, and performs little to no oxidative phosphorylation of fats (Remignon et al., 1994). Remignon et al. (1994) found that two key enzymes involved in fatty acid oxidation, citrate synthase and 3-hydroxyl-CoA dehydrogenase, had significantly lower activity than lactate dehydrogenase, a key enzyme in glycolysis, in the P. major of broiler chickens. These observations held true for both fast- and slow-growing broiler lines. Because oxidative metabolism normally contributes little energy to the chicken breast muscle compared to glycolytic metabolism, it is unusual that we observed increased expression of fat metabolismrelated genes in the muscle.

This data suggests a metabolic shift from primarily glycolytic metabolism to increasing proportions of fatty acid oxidative metabolism. An important gene supporting this hypothesis is LPL, which was found to decrease glucose metabolism when overexpressed in mice (Voshol et al., 2001). Evidence of increased fatty acid oxidation and redirection of carbohydrates from glycolysis to other metabolic pathways such as the pentose phosphate, glucuronic and hexosamine biosynthetic pathways have been noted in birds affected by WBD (Abasht et al., 2016; Papah et al., 2018) and also in high feed-efficiency broilers which are more susceptible to WBD (Abasht et al., 2019). Therefore decreased glycolytic activities and increased uptake and oxidation of fatty acids appear to be a key feature of WBD.

Palamiuc et al. (2015) described inhibition of glucose metabolism and a shift of fuel preference to lipids during a metabolic shift event early in the development of amyotrophic lateral sclerosis (ALS) in mice. Affected mice experienced increased expression of LPL, CD36, PDK4, forkhead box O1 (FOXO1), and PPAR $\beta / \gamma$ during this metabolic shift. LPL, CD36, and PDK4 are upregulated in males in our data set. FOXO1 and $\mathrm{PPAR} \beta / \gamma$ are not upregulated in males in our dataset, but FOXO1 was predicted to be an activated upstream regulator by IPA. Whether increased expression of one of these genes, increased fatty acid concentration in the muscle, or another factor entirely outside of this pathway is the initial cause of the hypothesized metabolic shift in WBD has yet to be deciphered.

It is likely that the shift from glycolytic to oxidative metabolism in the muscle is a change that is energetically beneficial for faster growth and higher feed efficiency, but has had detrimental effects on the breast muscles of modern broilers. Type II muscle fibers are poor at scavenging $\mathrm{H}_{2} \mathrm{O}_{2}$, allowing accumulation of reactive oxygen species (ROS) in the muscle when lipids are utilized for extended periods of time, which is known to lead to mitochondrial dysfunction and muscular damage (Anderson and Neufer, 2006). This hypothesis is in agreement with a study by Papah et al. (2018) in which WBD-affected broilers demonstrated increased mitochondrial dysfunction. Affected mice that underwent the metabolic shift in Palamiuc et al., 2015 study displayed evidence of ROS accumulation. Mechanisms of oxidative stress and its effect on the chicken breast muscle are further discussed in Section "Oxidative Stress."

\section{Oxidative Stress}

Oxidative stress is the accumulation of ROS generated through metabolic reactions, and the resulting imbalance between ROS and antioxidant substances, which can lead to damage of DNA (Cooke et al., 2003) and injury of tissues due to reactions involving ROS, such as lipid peroxidation and protein damage (Betteridge, 2000). IPA and DAVID analysis predict 
that biological reactions to oxidative stress are upregulated in male birds. The reason for this could be that modern male broilers grow faster and larger than their female counterparts, and are therefore generating ROS at a higher rate, but do not have sufficient mechanisms to counteract ROS accumulation. Male broilers' increased susceptibility to oxidative stress may play a large part in their increased susceptibility to WBD, and supports a hypothesis that oxidative stress plays a role in the development of WBD.

The aforementioned RNA-sequencing study by Mutryn et al. (2015) identified oxidative stress as a potential factor in the development of WBD when they found many DEGs associated with an increased ROS in WBD-affected birds. Additionally, Abasht et al. (2016) found that WBD-affected birds exhibited increased biomarkers related to oxidative stress, as well as increased levels of molecules that could affect redox homeostasis, including xanthine, hypoxanthine, and urate. In the current study, upregulated genes involved in oxidative stress responses include aprataxin (APTX), NR4A3, DNA damage recognition and repair factor (XPA), TXN, ADIPOQ, CD36, POSTN, oxidative stress-induced growth inhibitor 1 (OSGIN1), G protein subunit alpha Q (GNAQ), RET, cytokine inducible $\mathrm{SH}$-containing protein (CISH), ChaC glutathione specific gamma-glutamylcyclotransferase 1 (CHAC1), and cholecystokinin (CCK). Some of these genes are involved in attempt to relieve and protect against the damage of oxidative stress. For example, it has been discovered that adiponectin, coded for by ADIPOQ, protects cells against cytotoxicity when under oxidative stress by activating adenosine monophosphate-activated protein kinase (AMPK), which in turn increases metabolism of glucose and fatty acids (Chan et al., 2012). Additionally, $\mathrm{TXN}$ is a regulator of cellular responses to oxidative stress, and overexpression of TXN protects against cytotoxicity resulting from oxidative stress (Nishinaka et al., 2013).

However, the higher expression of CHAC1, which was the top upregulated gene in male broilers, may have a detrimental effect to the muscle. A prior study found that CHAC1 expression is increased by stress in the endoplasmic reticulum, and its protein degrades glutathione in the cell (Crawford et al., 2015). This is important because glutathione is a major antioxidant in cells, and its depletion results in oxidative stress (Crawford et al., 2015). The higher expression of CHAC1 in male broilers suggests that glutathione is being degraded at a higher rate than normal, which may be playing a big role in oxidative stress in male broilers. CHAC1 is also a member of the unfolded protein response (UPR) pathway and promotes apoptosis (Mungrue et al., 2009). However, because our data doesn't show differential expression of other key genes in the UPR pathway, such as CHOP, PERK, XBP, and ATF1, we can assume that the UPR pathway isn't regulated differently between sexes of birds.

CCAAT enhancer binding protein $\alpha$ (CEBPA) is a transcription factor involved in stimulating oxidative stress responses from other genes downstream, and it was predicted to be activated in males by IPA. CEBPA and other CEBP-family proteins undergo increased expression under stress, and in turn

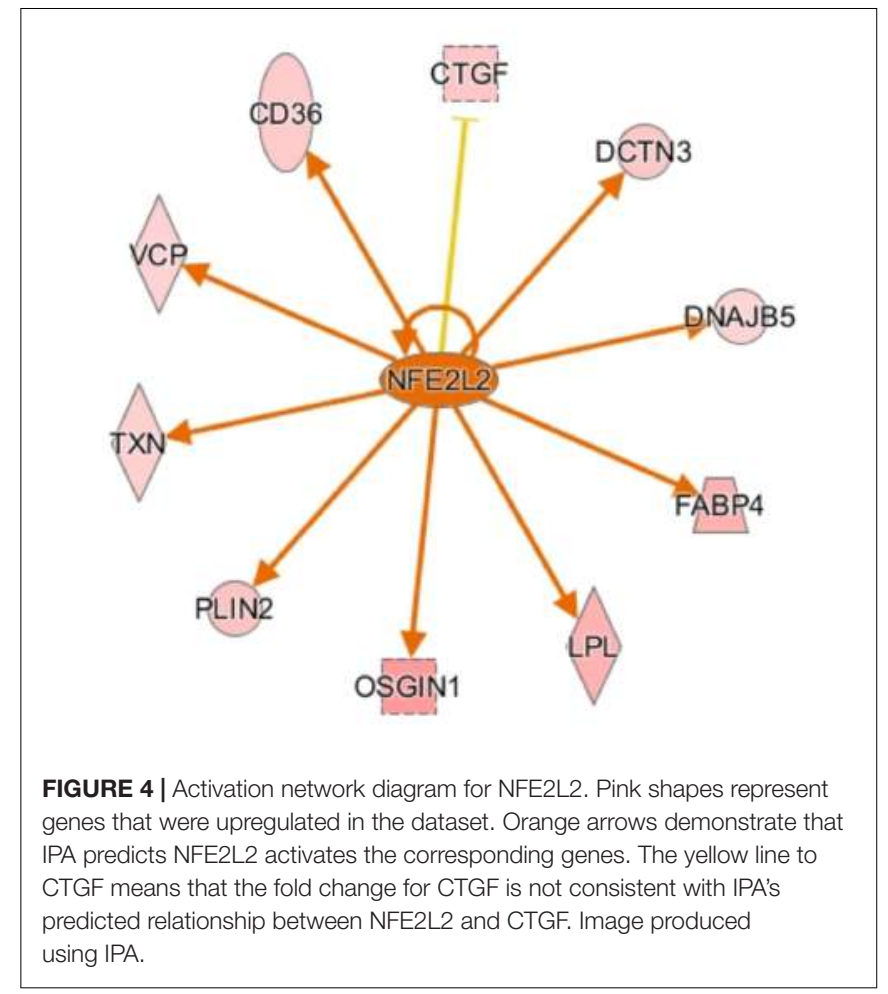

they increase expression of other oxidative stress response genes (Huggins et al., 2016), including ADIPOQ and TXN. Another activated transcription regulator, NFE2L2, acts similarly by mediating stress and inflammatory responses through regulation of CD36, OSGIN1, DNAJ heat shock protein family member B5 (DNAJB5), and ANXA1 (Figure 4). Upregulation of these stress response genes suggests the existence of increased oxidative stress in male birds, which could be a key factor in their susceptibility to WBD.

\section{Vascular Damage}

DAVID and IPA suggest that blood clotting and antiangiogenic functions are upregulated in male broilers. Additionally, IPA predicts increased cardiovascular damage in males, with significant related diseases and disorders including atherosclerosis, vascular lesions, and vaso-occlusion. These results are in agreement with a study by Papah et al. (2018). The results suggest that compared with females, male birds are more susceptible to vascular damage and increased antiangiogenic activity, even without being affected by any degree of WBD. This correlates with the observed presence of inflammation and lipogranulomas in the vasculature of WBD-affected birds (Papah et al., 2017), as well as a hypothesis that localized hypoxia caused by increased oxygen demand and compromised vasculature may be a factor in the development of WBD (Mutryn et al., 2015).

Like vascular damage, inhibition of vasculogenesis can also lead to inadequate delivery of oxygen and nutrients to skeletal muscle, and could be a factor in the development of WBD (Sihvo et al., 2018). Male broilers, which grow more quickly 
than females, would require a concomitant increase in angiogenic activity to ensure that their rapidly-growing muscle tissue has proper vasculature. Though there were some angiogenic genes upregulated in males, such as annexin A-1 (ANXA1) and endoplasmic reticulum aminopeptidase 1 (ERAP1), they appear to be overtaken by multiple upregulated antiangiogenic genes. For example, thrombospondin 1 and 2 (THBS1 and THBS2) are known antiangiogenic genes upregulated in male broilers. They inhibit angiogenesis by interference with endothelial cell migration, as well as competing with growth factors to bind to proteoglycans on endothelial cell surfaces (Tolsma et al., 1993; Lawler, 2000).

THBS1, as well as CD36, ADIPOQ, LPL, serpine family E member 2 (SERPINE2), coagulation factor XIII A chain (F13A1), and G protein subunit Alpha Q (GNAQ) are all upregulated and involved in blood coagulation and clotting, both of which were found to be upregulated functions in male broilers in DAVID and IPA. This makes sense, as it is known that the activation of coagulation is largely mediated by inflammatory cytokines (Esmon, 2005). Interestingly, a study by Mutryn et al. (2015) showed that coagulation pathways were downregulated in WBD-affected birds, despite inflammatory pathways being upregulated. Perhaps this is due to natural anticoagulant mechanisms kicking in to compensate for persisting coagulation and inflammation in later stages of WBD to prevent further damage to the body. The involvement of coagulation mechanisms in broiler growth and WBD require further investigation.

\section{Connective Tissue Proliferation}

DAVID and IPA both predict that proliferation of connective tissue is being significantly upregulated in male birds. A male predisposition for connective tissue growth correlates with findings of fibrosis and increased collagen levels in histological studies (Papah et al., 2017) as well as gene expression of WBDaffected tissues (Mutryn et al., 2015; Papah et al., 2018), which may be a factor of the stiffness of WBD-affected muscle (Mudalal et al., 2014; Soglia et al., 2016). Studies confirm increased collagen content throughout the bodies of male chickens, including the P. major muscle (Granot et al., 1991; Sakakibara et al., 2010). Males' increased propensity for connective tissue proliferation may be an important factor in their increased susceptibility to WBD.

As an example, connective tissue growth factor (CTGF), upregulated in males, is highly fibrogenic. It stimulates connective tissue proliferation and extracellular matrix (ECM) development, and is overexpressed in many fibrotic lesions (Rachfal and Brigstock, 2003). Additionally, CTGF has been identified as being transcriptionally regulated by transforming growth factor-beta (TGF- $\beta$ ) (Rachfal and Brigstock, 2003), which was also predicted by IPA to be an activated upstream regulator in male broilers. Transforming growth factor betainduced (TGFBI) is upregulated in males as well, and plays key roles in ECM development and ECM-muscle cytoskeleton interactions in mice and humans, as well as myofiber growth and myofibril bundling in zebrafish embryos (Kim and Ingham, 2009).
Additionally, periostin (POSTN) was upregulated in males. POSTN is most well-known for its involvement in osteogenesis but is also expressed in collagen-rich connective tissues throughout the body-mostly those that are subjected to constant mechanical stress (Norris et al., 2007). Considering the physical stress put on the P. major during its rapid growth in modern broilers, it is reasonable to hypothesize that the increased expression of POSTN in males could contribute to higher collagen content in male broiler breast muscles, as well as increasing their susceptibility to WBD. This hypothesis may be supported by upregulation of small muscle protein $\mathrm{x}$-linked (SMPX) and nuclear receptor subfamily 4 group A member 3 (NR4A3) in male broilers. SMPX is a gene regulated downstream of NR4A3 and is involved in muscle regulation. The exact mechanism of SMPX isn't currently clear, as there are conflicting studies arguing whether or not it is involved in muscle growth and differentiation (Eftestøl et al., 2014; Ferrán et al., 2016). However, there are studies suggesting that SMPX may be related to mechanical stress (Eftestøl et al., 2014), supporting the idea that increased biomechanical stress in the breast muscle may be a contributing factor to differential expression of genes between regions of the muscle and sexes of birds, as well as contributing to the development of WBD.

Additional DEGs (males vs. females) indicated as being involved in connective tissue proliferation by IPA include tenomodulin (TNMD), ret proto-oncogene (RET), histidine triad nucleotide binding protein 1 (HINT1), x-ray repair cross-complementing 4 (XRCC4), RAS P21 protein activator 1 (RASA1), thioredoxin (TXN), cyclin D3 (CCND3), nephroblastoma-overexpressed gene (NOV), ADIPOQ, fibulin 1 (FBLN1), Dickkopf WNT signaling pathway inhibitor 3 (DKK3), and klotho (KL).

\section{Muscle Development}

As previously explained, a key predisposing factor of WBD is increased muscle growth rates and yields in modern broiler chickens. By examining genes involved in muscle growth we seek to find differences in expression that could explain the cause of increased growth rates and muscle yields in male broilers and the cranial aspect of the breast muscle.

Unexpectedly, F-box 32 (FBXO32) was upregulated in male broilers. This is unusual because FBXO32 is involved in muscle atrophy by behaving as a ubiquitin protein ligase and enhancing proteolysis (Gomes et al., 2001). Increased expression of FBXO32 could be a response to muscle damage caused by oxidative stress, or by hypoxia as hypothesized by Mutryn et al. (2015).

While there are some DEGs involved in muscle growth in cranial vs. caudal and male vs. female analysis, there are many genes with key roles in growth that don't exhibit differential expression in either analysis, such as insulin-like growth factors 1 and 2 (IGF-1 and IGF-2), fibroblast growth factor (FGF), hepatocyte growth factor (HGF), platelet-derive growth factor (PDGF), myostatin (MSTN), myogenin (MYOG), and myoferlin (MYOF) (McFarland, 1999). This makes it difficult to draw any conclusions on the involvement of differential gene expression at week 3 post-hatch in increased growth in male broilers and the cranial aspect of the breast muscle. 
Rather than differential gene expression at 3 weeks of age mediating increased growth in male birds and the cranial region of the muscle, it is possible that differences in muscle growth are caused by the morphology of the muscle fibers themselves, decided before 3 weeks of age. Smith and Fletcher (1987) found that myofibers in the cranial region of the pectoralis major muscle had significantly smaller cross-sectional areas than those in the caudal region, suggesting a higher myofiber density in the cranial region. Additionally, studies also report a higher myofiber density and number in the muscles of male chickens both pre- and post-hatch, as well as reporting as smaller average cross-sectional area in male myofibers than in females (Henry and Burke, 1998; Scheuermann et al., 2003). The larger number of myofibers in modern male broiler chickens and the cranial aspect of the breast muscle could contribute to their increased growth rate and mass.

\section{Z Chromosome}

Out of the 189 genes upregulated in the pectoralis major of male broilers, 103 are located on the $\mathrm{Z}$ chromosome. Because we aimed to uncover the biological basis of increased growth rates in male broiler chickens, differential expression of genes on the sex chromosomes is of particular interest to us. In birds, the sex chromosomes are "W" and "Z," with females being heterogametic (ZW) and males homogametic (ZZ).

Dosage compensation is a mechanism that balances gene expression on sex chromosomes between males and females to compensate for differences in expression caused by differences in copy numbers of the sex chromosomes (Nguyen and Disteche, 2006). However, it has been found that the dosage compensation mechanism in birds is not as effective as it is in mammals. While the male-to-female ratio for expression of X-linked genes in mammals is typically close to 1.00 (Nguyen and Disteche, 2006), the male-to-female ratio for expression of $Z$-linked genes in birds has been reported to be between 1.4 and 1.6 by Ellegren et al. (2007), and between 1.33 and 1.58 by Itoh et al. (2007).
In agreement to these findings, a recent study from our laboratory reported that genes on the $\mathrm{Z}$ chromosome in male broiler chickens are expressed 1.35 times higher than that in females (Zhuo et al., 2017). As shown in Figure 5, DEGs on the Z chromosome show more uniform log 2 fold-change values than DEGs on autosomes. In addition, except one gene (not shown in Figure 5), all DEGs on Z chromosome are expressed higher in males with a mean fold-change value of 1.84 ( $\log 2$ foldchange $=0.88$ ). Collectively, these findings suggest that the copy number of the $\mathrm{Z}$ chromosome is likely to be responsible for higher expression of these genes in males.

The inherent upregulation of Z-linked genes in male birds may provide some insight on the cause of increased WBD susceptibility in male broilers. IPA provided a network of differentially expressed genes between males and females related to growth. However, very few of these genes could be related to body size or muscle growth. As an example, heat shock protein family B member 3 (HSPB3) is upregulated in males in our dataset. It is known to be highly expressed during myogenic differentiation and plays a role as a "quality control" factor (Sugiyama et al., 2000). Higher HSPB3 expression may reflect increased myogenic activity in male broilers, but it does not explain it.

There weren't many upregulated Z-linked genes involved in muscle and body growth, but we found that there were several upregulated Z-linked genes in males that were related to fat metabolism and deposition, including PLIN2, LPL, ACAA2, ALC44A1, RPP25L, HINT1, and AP3B1. This suggests a possibility that male broilers innately perform more lipid metabolizing activities, which corresponds with the hypothesis that a metabolic shift toward increased lipid metabolism may play a role in WBD development.

It should be noted that this innate difference in fat metabolism between the sexes is most likely unrelated to hormonal differences. Rather, it is likely driven by cell-autonomous sex differences in gene expression. In birds, somatic cells have

10

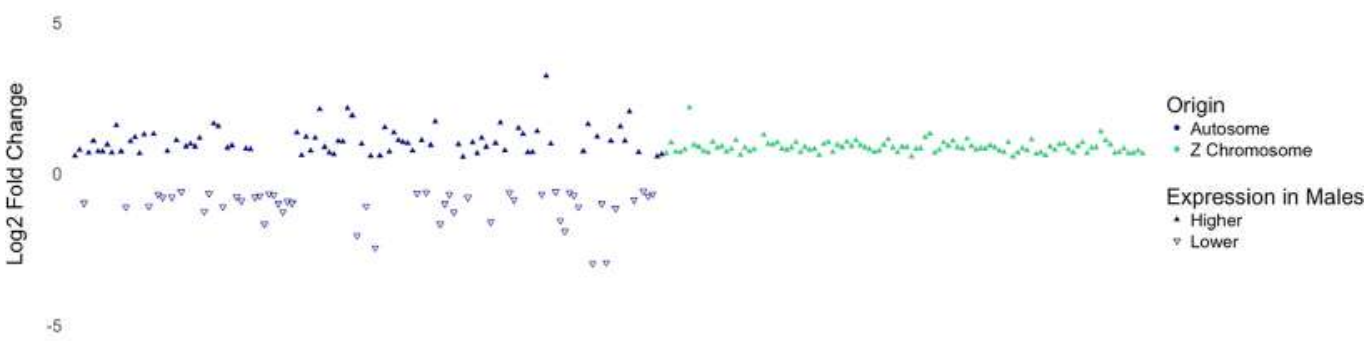

$-10$

FIGURE 5 | Log2 fold-change of differentially expressed genes separated by whether they are located on an autosome or the Z chromosome. Notice the consistency in log2-fold-change values of genes on the $Z$ chromosome. 
inherent sex identities, and different "sexes" of cells can respond differently to the same profile of gonadal hormones (Zhao et al., 2010). In addition to the avian cell-autonomous sex identity phenomenon, it has been implicated that sex hormone levels in birds may not contribute to juvenile growth or body weight differences between the sexes. A study by Burke and Edwards (1994) found that male turkeys castrated early in life did not demonstrate significantly different body weights at 3, 6, 9, 12, or 15 weeks of age from their uncastrated counterparts, suggesting that testosterone levels do not have an effect on the growth rate or body mass of poultry.

\section{CONCLUSION}

Through RNA-sequencing and analysis using DAVID and IPA, we were able to identify differences in gene expression between male and female broiler chickens, as well as the cranial and caudal aspects of the P. major muscle, that lead us to identify potential factors involved in different susceptibility to WBD between sexes of birds and regions of the breast muscle. Upregulation of genes involved in fat metabolism and deposition, vascular lesions and antiangiogenesis, and oxidative stress responses in males give us insight into the predisposing factors that may make male broilers more susceptible to WBD. Predicted changes in the activation states of transcription regulators, upstream enzymes and kinases, etc., suggest that gene expression changes observed in the current study could be linked to alteration in the activity of a few upstream regulators. Importantly, differential expression of genes on the Z-chromosome may be due to a lack of complete dosage compensation.

Though there were few differentially expressed genes identified in the cranial versus caudal analysis, there is some upregulation of genes involved in fat metabolism and muscle development in the cranial aspect of the muscle. However, we are reluctant to conclude that this reflects the mechanism of increased growth in the cranial aspect, because many key genes involved in regulating muscle growth are not differentially expressed in cranial vs. caudal or male vs. female analysis.

Unaffected birds for the current study were selected through gross and histological evaluation; however, there is a possibility that the development of WBD has started in these samples at a molecular level, and hence, some of the findings in the current study may be relevant to the onset of molecular perturbations ultimately leading to cellular and tissue damage in WBD. In agreement with this conclusion, the data collected in this project correlates with data from previous analyses of WBD-affected birds (Mutryn et al., 2015), including early developmental stages of WBD (Papah et al., 2018). However, as suggested above, some of the findings in the current study, such as higher expression of genes on $\mathrm{Z}$ chromosome in males, may be relevant to fundamental biological sex differences in modern broilers. Future studies with a heritage line of broilers in which WBD is not known to occur may be of interest to verify whether such differences are also present in slow-growing lines of chickens.

\section{DATA AVAILABILITY}

The RNA-seq data generated and analyzed for this study is deposited in NCBI's Sequence Read Archive (Study Accession No. SRP198866 and Bioproject Accession No. PRJNA543674).

\section{ETHICS STATEMENT}

Animal protocols for this study were approved by the University of Delaware Institutional Animal Care and Use Committee.

\section{AUTHOR CONTRIBUTIONS}

BA conceptualized and oversaw the study, contributed to the scientific discussion, and revised the manuscript. BB performed RNA isolation, contributed to preparation of cDNA libraries, conducted IPA and DAVID analysis, and wrote the original draft of the manuscript. MP conducted histopathological examination of muscle samples and drafted its relevant literature in the Section "Selection and Identification of Samples for RNA Sequencing." ZZ contributed to preparation of the cDNA libraries and conducted bioinformatic (RNA-seq) analysis.

\section{FUNDING}

This project was supported by the Agriculture and Food Research Initiative Competitive Grant No. 2016-67015-25027 from the USDA National Institute of Food and Agriculture. Funding for the support of BIOMIX was received from the Delaware INBRE (NIGMS P20GM103446), the State of Delaware, and the Delaware Biotechnology Institute.

\section{ACKNOWLEDGMENTS}

We gratefully acknowledge the in-kind support by CobbVantress Inc., in providing hatching eggs. We appreciate RNA sequencing services provided by the University of Delaware DNA Sequencing and Genotyping Center at the Delaware Biotechnology Institute (DBI). RNA-seq data was analyzed on DBI's shared computing cluster-BIOMIX. We also thank the members in Dr. Abasht's laboratory for assistance with animal experiment, samples and data collection, and data visualization.

\section{SUPPLEMENTARY MATERIAL}

The Supplementary Material for this article can be found online at: https://www.frontiersin.org/articles/ 10.3389/fphys.2019.00764/full\#supplementary-material 


\section{REFERENCES}

Abasht, B., Mutryn, M. F., Michalek, R. D., and Lee, W. R. (2016). Oxidative stress and metabolic perturbations in wooden breast disorder in chickens. PLoS One 11:e0153750. doi: 10.1371/journal.pone.0153750

Abasht, B., Zhou, N., Lee, W. R., Zhuo, Z., and Peripolli, E. (2019). The metabolic characteristics of susceptibility to wooden breast disease in chickens with high feed efficiency. Poul. Sci. doi: 10.3382/ps/pez183

Anderson, E. J., and Neufer, P. D. (2006). Type II skeletal myofibers possess unique properties that potentiate mitochondrial $\mathrm{H} 2 \mathrm{O} 2$ generation. Am. J. Physiol. Cell Physiol. 290, C844-C851.

Bailey, R. A., Watson, K. A., Bilgili, S. F., and Avendano, S. (2015). The genetic basis of pectoralis major myopathies in modern broiler chicken lines. Poult. Sci. 94, 2870-2879. doi: 10.3382/ps/pev304

Betteridge, D. J. (2000). What is oxidative stress? Metabolism 49, 3-8. doi: 10.1016/ s0026-0495(00)80077-3

Burke, W. H., and Edwards, H. M. (1994). Effect of castration on body weight, muscle growth, and bone characteristics of male Nicholas strain turkeys. Poult. Sci. 73, 457-463. doi: 10.3382/ps.0730457

Chan, K., Lam, K. S., Cheng, O., Kwan, J. S., Ho, P. W., Cheng, K. K., et al. (2012). Adiponectin is protective against oxidative stress induced cytotoxicity in amyloid-beta neurotoxicity. PLoS One 7:e52354. doi: 10.1371/journal.pone. 0052354

Clark, D. L., and Velleman, S. G. (2016). Spatial influence on breast muscle morphological structure, myofiber size, and gene expression associated with wooden breast myopathy in broilers. Poult. Sci. 95, 2930-2945. doi: 10.3382/ ps/pew243

Clark, D. L., Walter, K. G., and Velleman, S. G. (2017). Incubation temperature and time of hatch impact broiler muscle growth and morphology. Poult. Sci. 96, 4085-4095. doi: 10.3382/ps/pex202

Cooke, M. S., Evans, M. D., Dizdaroglu, M., and Lunec, J. (2003). Oxidative DNA damage: mechanisms, mutation and disease. FASEB J. 17, 1195-1214. doi: 10.1096/fj.02-0752rev

Crawford, R. R., Prescott, E. T., Sylvester, C. F., Higdon, A. N., Shan, J., Kilberg, M. S., et al. (2015). Human CHAC1 protein degrades glutathione, and mRNA induction is regulated by the transcription factors ATF4 and ATF3 and a bipartite ATF/CRE regulatory element. J. Biol. Chem. 290, 15878-15891. doi: 10.1074/jbc.M114.635144

Dalle Zotte, A., Tasoniero, G., Puolanne, E., Remignon, H., Cecchinato, M., Catelli, E., et al. (2017). Effect of wooden breast appearance on poultry meat quality, histological traits, and lesions characterization. Czech J. Anim. Sci. 62, 51-57. doi: 10.17221/54/2016-cjas

Eftestøl, E., Alver, T. N., Gundersen, K., and Bruusgaard, J. C. (2014). Overexpression of SMPX in adult skeletal muscle does not change skeletal muscle fiber type or size. PLoS One 9:e99232. doi: 10.1371/journal.pone. 0099232

Ellegren, H., Hultin-Rosenberg, L., Brunström, B., Dencker, L., Kultima, K., and Scholz, B. (2007). Faced with inequality: chicken do not have a general dosage compensation of sex-linked genes. BMC Biol. 5:40. doi: 10.1186/17417007-5-40

Esmon, C. T. (2005). The interactions between inflammation and coagulation. Br. J. Haematol. 131, 417-430. doi: 10.1111/j.1365-2141.2005. 05753.x

Febbraio, M., Hajjar, D. P., and Silverstein, R. L. (2001). CD36: a class B scavenger receptor involved in angiogenesis, atherosclerosis, inflammation, and lipid metabolism. J. Clin. Invest. 108, 785-791. doi: 10.1172/jci 14006

Ferrán, B., Martí-Pàmies, I., Alonso, J., Rodríguez-Calvo, R., Aguiló, S., Vidal, F., et al. (2016). The nuclear receptor NOR-1 regulates the small muscle protein, X-linked (SMPX) and myotube differentiation. Sci. Rep. 6:25944. doi: 10.1038/ srep 25944

Gene (2004). National Center for Biotechnology Information. Bethesda, MD: National Library of Medicine (US). Available at: https://www.ncbi.nlm.nih.gov/ gene/

Gomes, M. D., Lecker, S. H., Jagoe, R. T., Navon, A., and Goldberg, A. L. (2001). Atrogin-1, a muscle-specific F-box protein highly expressed during muscle atrophy. Proc. Natl. Acad. Sci. U.S.A. 98, 14440-14445. doi: 10.1073/pnas. 251541198
Granot, I., Pines, M., Plavnik, I., Wax, E., Hurwitz, S., and Bartov, I. (1991). Skin tearing in broilers in relation to skin collagen: effect of sex, strain, and diet. Poult. Sci. 70, 1928-1935. doi: 10.3382/ps.0701928

Griffin, J. R., Moraes, L., Wick, M., and Lilburn, M. S. (2018). Onset of white striping and progression into wooden breast as defined by myopathic changes underlying Pectoralis major growth. Estimations of growth parameters as predictors for stage of myopathy progression. Avian Pathol. 47, 2-13. doi: 10.1080/03079457.2017.1356908

Henry, M. H., and Burke, W. H. (1998). Sexual dimorphism in broiler chick embryos and embryonic muscle development in late incubation. Poult. Sci. 77, 728-736. doi: $10.1093 / \mathrm{ps} / 77.5 .728$

Huang, D. W., Sherman, B. T., and Lempicki, R. A. (2009a). Bioinformatics enrichment tools: paths toward the comprehensive functional analysis of large gene lists. Nucleic Acids Res. 1, 1-13. doi: 10.1093/nar/gkn923

Huang, D. W., Sherman, B. T., and Lempicki, R. A. (2009b). Systemic and integrative analysis of large gene lists using DAVID bioinformatic resources. Nat. Protoc. 4, 44-57. doi: 10.1038/nprot.2008.211

Huggins, C. J., Mayekar, M. K., Martin, N., Saylor, K. L., Gonit, M., Jailwala, P., et al. (2016). C/EBP $\gamma$ is a critical regulator of cellular stress response networks through heterodimerization with ATF4. Mol. Cell. Biol. 36, 693-713. doi: 10. 1128/MCB.00911-15

Itoh, Y., Melamed, E., Yang, X., Kampf, K., Wang, S., Yehya, N., et al. (2007). Dosage compensation is less effective in birds than in mammals. J. Biol. 6:2. doi: 10.1186/jbiol53

Kim, H. R., and Ingham, P. W. (2009). The extracellular matrix protein TGFBI promotes myofibril bundling and muscle fibre growth in the zebrafish embryo. Dev. Dyn. 238, 56-65. doi: 10.1002/dvdy.21812

Krämer, A., Green, J., Pollard, J., and Tugendreich, S. (2014). Casual analysis approaches in ingenuity pathway analysis. Bioinformatics 30, 523-530. doi: 10.1093/bioinformatics/btt703

Kranen, R. W., Van Kuppevelt, T. H., Goedhart, H. A., Veerkamp, C. H., Lambooy, E., and Verkamp, J. H. (1999). Hemoglobin and myoglobin content in muscles of broiler chickens. Poult. Sci. 78, 467-476. doi: 10.1093/ps/78.3.467

Kuttappan, V. A., Brewer, V. B., Apple, J. K., Waldroup, P. W., and Owens, C. M. (2012). Influence of growth rate on the occurrence of white striping in broiler breast fillets. Poult. Sci. 91, 2677-2685. doi: 10.3382/ps.2012-02259

Lawler, J. (2000). The functions of thrombospondin-1 and -2. Curr. Opin. Cell Biol. 12, 634-640. doi: 10.1016/s0955-0674(00)00143-5

Leenstra, F. R. (1986). Effect of age, sex, genotype and environment on fat deposition in broiler chickens-a review. World Poul. Sci. J. 42, 12-25. doi: 10.1079/wps19860002

Lemberger, T., Desvergne, B., and Wahli, W. (1996). Peroxisome proliferatoractivated receptors: a nuclear receptor signaling pathway in lipid physiology. Annu. Rev. Cell Dev. Biol. 12, 335-363. doi: 10.1146/annurev.cellbio.12.1.335

Lorenzi, M., Mudalal, S., Cavani, C., and Petracci, M. (2014). Incidence of white striping under commercial conditions in medium and heavy broiler chickens in Italy. J. Appl. Poul. Res. 23, 754-758. doi: 10.3382/japr.2014-00968

McFarland, D. C. (1999). Influence of growth factors on poultry myogenic satellite cells. Poult. Sci. 78, 747-758. doi: 10.1093/ps/78.5.747

Meloche, K. J., Fancher, B. I., Emmerson, D. A., Bilgili, S. F., and Dozier, W. A. (2018a). Effects of reduced digestible lysine density on myopathies of the pectoralis major muscles in broiler chickens at 48 and 62 days of age. Poult. Sci. 97, 3311-3324. doi: 10.3382/ps/pey171

Meloche, K. J., Fancher, B. I., Emmerson, D. A., Bilgili, S. F., and Dozier, W. A. (2018b). Effects of reduced dietary energy and amino acid density on pectoralis major myopathies in broiler chickens at 36 and 49 days of age 1. Poult. Sci. 97, 1794-1807. doi: 10.3382/ps/pex454

Meloche, K. J., Fancher, B. I., Emmerson, D. A., Bilgili, S. F., and Dozier, W. A. (2018c). Effects of quantitative nutrient allocation on myopathies of the Pectoralis major muscles in broiler chickens at 32,43, and 50 days of age. Poult. Sci. 97, 1786-1793. doi: 10.3382/ps/pex453

Mudalal, S., Babini, E., Cavani, C., and Petracci, M. (2014). Quantity and functionality of protein fractions in chicken breast fillets affected by white striping. Poult. Sci. 93, 2108-2116. doi: 10.3382/ps.2014-03911

Mudalal, S., Lorenzi, M., Soglia, F., Cavani, C., and Petracci, M. (2015). Implications of white striping and wooden breast abnormalities on quality traits of raw and marinated chicken meat. Animal 9, 728-734. doi: 10.1017/ S175173111400295X 
Mungrue, I. N., Pagnon, J., Kohannim, O., Gargalovic, P. S., and Lusis, A. J. (2009). CHAC1/MGC4504 is a novel proapoptotic component of the unfolded protein response, downstream of the ATF4-ATF3-CHOP cascade. J. Immunol. 182, 466-476. doi: 10.4049/jimmunol.182.1.466

Mutryn, M., Brannick, E., Fu, W., Lee, W., and Abasht, B. (2015). Characterization of a novel chicken muscle disorder through differential gene expression and pathway analysis using RNA-sequencing. BMC Genomics 16:399. doi: 10.1186/ s12864-015-1623-0

Nelson, D. L., and Cox, M. M. (2008). Oxidation of Fatty Acids. Lehninger Principles of Biochemistry, 5th Edn. New Yorkm, NY: W.H. Freeman and Company, 652-655.

Nguyen, D. K., and Disteche, C. M. (2006). Dosage compensation of the active X chromosome in mammals. Nat. Genet. 38, 47-53. doi: 10.1038/ng1705

Nishinaka, Y., Masutani, H., Nakamura, H., and Yodoi, J. (2013). Regulatory roles of thioredoxin in oxidative stress-induced cellular responses. Redox Rep. 6, 289-295. doi: 10.1179/135100001101536427

Norris, R. A., Damon, B., Mironov, V., Kasyanov, V., Ramamurthi, A., MorenoRodriguez, R., et al. (2007). Periostin regulates collagen fibrillogenesis and the biomechanical properties of connective tissues. J. Cell Biochem. 101, 695-711. doi: $10.1002 / j c b .21224$

Ono, Y., Iwamoto, H., and Takahara, H. (1993). The relationship between muscle growth and the growth of different fiber types in the chicken. Poult. Sci. 72, 568-576. doi: 10.3382/ps.0720568

Palamiuc, L., Schlagowski, A., Ngo, S. T., Vernay, A., Dirrig-Grosch, S., Henriques, A., et al. (2015). A metabolic switch toward lipid use in glycolytic muscle is an early pathologic event in a mouse model of amyotrophic lateral sclerosis. $E M B O$ Mol. Med. 7, 526-546. doi: 10.15252/emmm.201404433

Papah, M. B., Brannick, E. M., Schmidt, C. J., and Abasht, B. (2017). Evidence and role of phlebitis and lipid infiltration in the onset and pathogenesis of wooden breast disease in modern broiler chickens. Avian Pathol. 46, 623-643. doi: 10.1080/03079457.2017.1339346

Papah, M. B., Brannick, E. M., Schmidt, C. J., and Abasht, B. (2018). Gene expression profiling of the early pathogenesis of wooden breast disease in commercial broiler chickens usin g RNA-sequencing. PLoS One 13:e0207346. doi: 10.1371/journal.pone.0207346

Petracci, M., Mudalal, S., Bonfiglio, A., and Cavani, C. (2013). Occurrence of white striping under commercial conditions and its impact on breast meat quality in broiler chickens. Poult. Sci. 92, 1670-1675. doi: 10.3382/ps.2012-03001

Rachfal, A. W., and Brigstock, D. R. (2003). Connective tissue growth factor (CTGF/CCN2) in hepatic fibrosis. Hepatol. Res. 26, 1-9. doi: 10.1016/s13866346(03)00115-3

Remignon, H., Gardahaut, M. F., Marche, G., and Ricard, F. H. (1994). Selection for rapid growth increases the number and the size of muscle fibres without changing their typing in chickens. J. Muscle Res. Cell Motil. 16, 95-102. doi: $10.1007 / \mathrm{bf} 00122527$

Russo, E., Drigo, M., Longoni, C., Pezzotti, R., Fasoli, P., and Recordati, C. (2015). Evaluation of white striping prevalence and predisposing factors in broilers at slaughter. Poult. Sci. 94, 1843-1848. doi: 10.3382/ps/pev172

Sakakibara, K., Tabata, S., Shiba, N., Gotoh, T., Nishimura, S., and Iwamoto, H. (2010). Myofibre composition and total collagen content in M. iliotibialis lateralis and M. pectoralis of Silkie and White Leghorn chickens. Br. Poult. Sci. 41, 570-574. doi: $10.1080 / 713654983$

Scheuermann, G. N., Bilgili, S. F., Hess, J. B., and Mulvaney, D. R. (2003). Breast muscle development in commercial broiler chickens. Poult. Sci. 82, 1648-1658. doi: 10.1093/ps/82.10.1648.

Sihvo, H. K., Airas, N., Lindén, J., and Puolanne, E. (2018). Pectoral vessel density and early ultrastructural changes in broiler chicken wooden breast myopathy. J. Comp. Pathol. 161, 1-10. doi: 10.1016/j.jcpa.2018.04.002

Sihvo, H. K., Immonen, K., and Puolanne, E. (2014). Myodegeneration with fibrosis and regeneration in the pectoralis major muscle of broilers. Vet. Pathol. 51, 619-623. doi: 10.1177/0300985813497488

Smathers, R. L., and Petersen, D. R. (2011). The human fatty acid-binding protein family: evolutionary divergences and functions. Hum. Genomics 5, 170-191.
Smith, D. P., and Fletcher, D. L. (1987). Chicken breast muscle fiber type and diameter as influenced by age and intramuscular location. Poult. Sci. 67, 908-913. doi: 10.3382/ps.0670908

Soglia, F., Laghi, L., Canonico, L., Cavani, C., and Petracci, M. (2016). Function property issues in broiler breast meat related to emerging muscle abnormalities. Food Res. Int. 89, 1071-1076. doi: 10.1016/j.foodres.2016.04.042

Sugiyama, Y., Suzuki, A., Kishikawa, M., Akutsu, R., Hirose, T., Waye, M. M. Y., et al. (2000). Muscle develops a specific form of small heat shock protein complex composed of MKBP/HSPB2 and HSPB3 during myogenic differentiation. J. Biol. Chem. 275, 1095-1104. doi: 10.1074/jbc.275.2.1095

Tatara, M. R., Brodzki, A., Pyz-Lukasik, R., Pasternak, K., and Szpetnar, M. (2012). Sex-related differences in skeletal muscle amino acid concentrations in 20 week old turkeys. Jpn. Poul. Sci. Assoc. 49, 219-223. doi: 10.2141/jpsa.011099

Tolsma, S. S., Volvert, O. V., Good, D. J., Frazier, W. A., Polverini, P. J., and Bouck, N. (1993). Peptides derived from two separate domains of the matrix protein thrombospondin-1 have anti-angiogenic activity. J. Cell Biol. 122, 497-511. doi: $10.1083 /$ jcb.122.2.497

Trocino, A., Piccirillo, A., Birolo, M., Radaelli, G., Bertotto, D., Filiou, E., et al. (2015). Effect of genotype, gender, and feed restriction on growth, meat quality, and the occurrence of white striping and wooden breast in broiler chickens. Poult. Sci. 94, 2996-3004. doi: 10.3382/ps/pev296

Verdiglione, R., and Cassandro, M. (2013). Characterization of muscle fiber type in the pectoralis major muscle of slow-growing local and commercial chicken strains. Poult. Sci. 92, 2433-2437. doi: 10.3382/ps.201303013

Voshol, P. J., Jong, M. C., Dahlmans, V. E. H., Kratky, D., Levak-Frank, S., Zechner, R., et al. (2001). In muscle-specific lipoprotein lipase-overexpressing mice, muscle triglyceride content is increased without inhibition of insulinstimulated whole-body and muscle-specific glucose uptake. Diabetes 50, 25852590. doi: $10.2337 /$ diabetes.50.11.2585

Zambonelli, P., Zappaterra, M., Soglia, F., Petracci, M., Sirri, F., Cavani, C., et al. (2016). Detection of differentially expressed genes in broiler pectoralis major muscle affected by white striping - wooden breast myopathies. Poult. Sci. 95, 2771-2785. doi: 10.3382/ps/ pew268

Zhang, W., Bai, S., Liu, D., Cline, M. A., and Gilbert, E. R. (2015). Neuropeptide Y promotes adipogenesis in chicken adipose cells in vitro. Comp. Biochem. Physiol. A Mol. Integr. Physiol. 181, 62-70. doi: 10.1016/j.cbpa.2014.11.012

Zhao, D., McBride, D., Nandi, S., McQueen, H. A., McGrew, M. J., Hocking, P. M., et al. (2010). Somatic sex identity is cell autonomous in the chicken. Nature 464, 237-242. doi: 10.1038/nature 08852

Zhou, N., Lee, W. R., and Abasht, B. (2015). Messenger RNA sequencing and pathway analysis provide novel insights into the biological basis of chickens' feed efficiency. BMC Genomics 16:195. doi: 10.1186/s12864-015-1364-0

Zhuo, Z., Lamont, J., and Abasht, B. (2017). RNA-seq analyses identify frequent allele specific expression and no evidence of genomic imprinting in specific embryonic tissues of chicken. Sci. Rep. 7:11944. doi: 10.1038/s41598-01712179-9

Zhuo, Z., Lamont, J., Lee, W. R., and Abasht, B. (2015). RNA-seq analysis of abdominal fat reveals differences between modern commercial broiler chickens with high and low feed efficiencies. PLoS One 10:e0135810. doi: 10.1371/journal. pone. 0135810

Conflict of Interest Statement: The authors declare that the research was conducted in the absence of any commercial or financial relationships that could be construed as a potential conflict of interest.

Copyright ( 2019 Brothers, Zhuo, Papah and Abasht. This is an open-access article distributed under the terms of the Creative Commons Attribution License (CC BY). The use, distribution or reproduction in other forums is permitted, provided the original author(s) and the copyright owner(s) are credited and that the original publication in this journal is cited, in accordance with accepted academic practice. No use, distribution or reproduction is permitted which does not comply with these terms. 


\section{Distribution and Expression of Vimentin and Desmin in Broiler Pectoralis major Affected by the Growth-Related Muscular Abnormalities}

Francesca Soglia ${ }^{1}$, Maurizio Mazzoni ${ }^{2}$, Martina Zappaterra ${ }^{1}$, Mattia Di Nunzio ${ }^{1}$ Elena Babini ${ }^{1}$, Martina Bordini ${ }^{1}$, Federico Sirri ${ }^{1}$, Paolo Clavenzani ${ }^{2}$, Roberta Davoli ${ }^{*}$ and Massimiliano Petracci ${ }^{1 *}$

\section{OPEN ACCESS}

Edited by:

Colin Guy Scanes,

University of Arkansas, United States

Reviewed by:

Hong Zhuang,

US National Poultry Research Centre (USDA-ARS), United States Wes Schilling, Mississippi State University, United States Macdonald Wick

The Ohio State University, United States

*Correspondence: Roberta Davoli roberta.davoli@unibo.it Massimiliano Petracci m.petracci@unibo.it

Specialty section: This article was submitted to Avian Physiology,

a section of the journal

Frontiers in Physiology

Received: 17 April 2019 Accepted: 17 December 2019

Published: 17 January 2020

Citation:

Soglia F, Mazzoni M, Zappaterra M, Di Nunzio M, Babini E Bordini M, Sirri F, Clavenzani P. Davoli R and Petracci M (2020) Distribution and Expression of Vimentin and Desmin in Broiler Pectoralis major Affected by the Growth-Related Muscular Abnormalities.

Front. Physiol. 10:1581. doi: 10.3389/fphys.2019.01581
'Department of Agricultural and Food Sciences, Alma Mater Studiorum - University of Bologna, Bologna, Italy, ${ }^{2}$ Department of Veterinary Medical Sciences, Alma Mater Studiorum - University of Bologna, Bologna, Italy

Desmin (DES) and Vimentin (VIM) exert an essential role in maintaining muscle cytoarchitecture and since are considered reliable markers for muscle regeneration, their expression has been extensively investigated in dystrophic muscles. Thus, exhibiting features similar to those of human dystrophic muscles, the present study aimed at assessing the distribution of VIM and DES proteins and the expression of the corresponding genes in Pectoralis major muscles affected by white striping (WS), wooden breast (WB), and spaghetti meat (SM) abnormalities as well as in those having macroscopically normal appearance (NORM). For this purpose, 20 Pectoralis major muscles (5/group) were collected from the same flock of fast-growing broilers to perform immunohistochemistry, immunoblotting and gene expression. Immunohistochemical analyses showed an increased number of fibers immunoreactive to both VIM and DES in WS and WB, while only a few immunoreactive fibers were observed in NORM. Concerning the protein level, if compared with NORM, a 55\% increase in VIM content was found in WB affected cases $(P<0.05)$ thus suggesting the development of intense regenerative processes in an early-stage within these muscles. The significantly higher amount of DES (+53\%) found in WS might be attributed to a progression of the regenerative processes that require its synthesis to preserve the structural organization of the developing fibers. On the other hand, significantly lower VIM and DES contents were found in SM. About gene expression, VIM mRNA levels gradually increased from the NORM to the SM group, with significantly higher gene expressions in WB and SM samples compared to the NORM group ( $P=0.009$ for WB vs. NORM and $P=0.004$ for SM vs. NORM). Similarly, the expression of DES gene showed an increase from the NORM to WB group ( $P=0.05)$. Overall, the findings of the present study suggest that intense regenerative processes take place in both WB and WS muscles although a different progression of regeneration might be hypothesized. On the other hand, the lack of correspondence between VIM gene expression and its protein product observed in SM suggests that VIM may also exert a role in the development of the SM phenotype.

Keywords: vimentin, desmin, white striping, wooden breast, spaghetti meat 


\section{INTRODUCTION}

In the past few years, several studies have been carried out in order to investigate the underpinning factors involved in the development of the muscular growth-related abnormalities [White Striping (WS), Wooden Breast (WB), and Spaghetti Meat (SM)] that are currently affecting the Pectoralis major (P. major) muscles in modern fast-growing broilers (Petracci et al., 2019). Although previous studies deeply investigated the role of genetics (Mutryn et al., 2015; Alnahhas et al., 2016; Zambonelli et al., 2016; Pampouille et al., 2018; Papah et al., 2018), the exact etiology and the chronology of events leading to the development of these conditions are not fully understood. The microscopic examinations of the $P$. major affected by the aforementioned abnormalities evidence a profoundly altered muscular architecture with fiber degeneration up to necrosis, and concomitant occasional regenerative processes (Velleman, 2019). Besides, the skeletal muscle shows a proliferation of poorly organized connective tissue (fibrosis) and increased fat deposition (lipidosis) (Soglia et al., 2019).

Ultrastructural studies (Granger and Lazarides, 1979; Tokuyasu et al., 1985) demonstrated that, by constituting a three-dimensional scaffold around the Z-disk, vimentin (VIM) and desmin (DES) play a relevant role in maintaining sarcomere cytoarchitecture. DES is an important component of the cytoskeleton of striated muscles (Lazarides, 1980) located at the periphery of Z-disks where it is arranged in a honeycomblike structure within the $\mathrm{Z}$ plane of the myofibers and is involved in the connection of neighboring Z-disks (Granger and Lazarides, 1979). On the other hand, VIM is mainly located in cells of mesenchymal origin including myoblasts (Price and Sanger, 1982) and co-localize with desmin (Granger and Lazarides, 1979). Although DES and VIM coexist during early myogenesis, the last is gradually reduced as the development of myotubes proceeds (Tokuyasu et al., 1985). In detail, in early myogenesis and immature myotubes, VIM and DES are found as longitudinally arranged filaments and randomly distributed cytoplasmic strands, respectively (Gard and Lazarides, 1980). Their peculiar arrangements in muscle tissue might play an essential role in maintaining the cylindrical form of developing myotubes and allowing to parallelly align the myofibers (Tokuyasu et al., 1985). In particular, it was demonstrated that as the newly synthesized DES filaments converge and replace the pre-existing VIM-based network, DES distribution within the fibers reflects that of VIM (Bennett et al., 1979; Granger and Lazarides, 1979; Cary and Klymkowsky, 1994). Subsequently, a substantial re-organization of the intermediate filaments scaffolding takes place and involves an evolution from a longitudinally to a transversely arranged system. Concurrently, the composition of the intermediate filaments is profoundly changed: VIM synthesis is reduced whereas DES arises as the main component of mature muscle cells (Bennett et al., 1979; Granger and Lazarides, 1979).

In this context, because of their importance for maintaining muscle cytoarchitecture, the expression and distribution of VIM and DES have been previously investigated in humans affected by different neuromuscular and myopathic disorders including polymyositis, central core myopathy, nemaline myopathy and Duchenne muscular dystrophy (Gallanti et al., 1992; Banwell, 2001), but also in mdx mice (Holland et al., 2015), pigs used as a model for studying Duchenne muscular dystrophy (Fröhlich et al., 2016) and Golden retrievers affected by muscular dystrophy (Cooper et al., 1988). As a consequence, an increased abundance of VIM and DES is undoubtedly considered as an evidence of muscular dystrophy and can be considered a reliable marker for the regenerative processes taking place within the muscle tissue (Bornemann and Schmalbruch, 1992; Gallanti et al., 1992; Fröhlich et al., 2016). Within this context, since the microscopic examinations carried out on $P$. major muscles of broiler chickens affected by WS, WB, and SM abnormalities evidenced the occurrence of occasional regenerative processes (Baldi et al., 2018; Velleman, 2019), it seems reasonable to hypothesize that the expression and distribution of the intermediate filaments VIM and DES might be involved in the occurrence of these conditions. Furthermore, evident signs of regeneration are found not only within the WS, WB, and SM affected muscles but even within those cases exhibiting a macroscopically normal appearance (Soglia et al., 2019). Thus, the present study aimed at assessing the distribution of VIM and DES as well as the expression of the corresponding genes within the $P$. major muscle samples exhibiting either a macroscopically normal appearance or showing WS, WB, and SM phenotypes in order to evaluate the possible implication of these two intermediate filaments components in the occurrence of the aforementioned conditions in fast-growing chickens.

\section{MATERIALS AND METHODS}

Twenty $P$. major muscles (5 muscles/group) were collected at $3 \mathrm{~h}$ post-mortem from the same flock of fast-growing broilers farmed and slaughtered under commercial conditions (45 dayold Ross 308 males slaughtered at $3.0 \mathrm{~kg}$ live weight). All samples, $6 \times 3 \times 1 \mathrm{~cm}$ in size, were excised from the ventral surface of the $P$. major muscle (facing the skin), excluding the most superficial $3 \mathrm{~mm}$. According to the procedure described in our previous study (Soglia et al., 2017), samples collected for immunohistochemistry and protein extraction were excised from the superficial section of the cranial portion of the P. major, quickly frozen in isopentane (cooled with liquid nitrogen) and stored at $-80^{\circ} \mathrm{C}$ until processing. In detail, to assess the distribution and expression of the intermediate filament proteins DES and VIM within the muscles, immunohistochemical analyses SDS-PAGE followed by immunoblotting were carried out. Further samples of the same P. major were excised from the same position, immediately frozen in liquid nitrogen and then stored in a deep freezer until RNA extraction. RNA was extracted individually from the 22 gathered samples (2 P. major muscles belonging to the slow-growing genotype and the 20 fastgrowing ones) and the expression of the genes encoding for VIM and DES was assessed by RT-qPCR. According to their macroscopic features the $P$. major were classified as normal (NORM), WS, WB, and SM following the criteria adopted in 
our previous studies (Soglia et al., 2016; Baldi et al., 2018). Only severe cases were sampled and, to avoid eventual interference, the muscles concurrently affected by more than one defect (i.e., combined WS/WB and WS/SM) were discarded. In addition, two P. major samples were collected from slow-growing chickens farmed and slaughtered under commercial conditions (160 day-old Leghorn cocks slaughtered at $2.5 \mathrm{~kg}$ of live weight). The findings obtained for these two slow-growing birds were compared with those found for the NORM muscles belonging to the fast-growing group. In this way, we can obtain both a comparison with a control group belonging to the fast-growing genotype (NORM vs. the P. major with abnormalities) and a further comparison between the NORM fast-growing group with its slow-growing and not selected for meat production purpose counterpart. All the farming and slaughter procedures performed in this study were in accomplishment with the Italian legislation, Legislative decree of the $4^{\text {th }}$ of March 2014 No. 26, article 2 point $\mathrm{F}$, and did not require further specific authorization. Moreover, all farming procedures followed the Council Directive 98/58/EC concerning the protection of animals kept for farming purposes, Council Directive 2008/120/EC and Council Directive 43/2007/EC laying down minimum rules for the protection of chickens kept for meat production. Animal transport was performed according to Council Regulation (EC) No. 1/2005, slaughter was performed in accomplishment with the Council Regulation (EC) No. 1099/2009 on the protection of animals at the time of killing and under the control of the Veterinary Service from the Italian Ministry of Health, as indicated in the Regulation (EU) 2017/625 of the European Parliament. The samples used for the present study were gathered from carcasses intended for meat consumption. The research did not involve any experiment on animals and for this reason, no ethics approval was necessary.

\section{Immunohistochemistry}

The specimens were oriented for the transverse fibers sectioning. For each P. major muscle, 10 serial cross-sections $(10 \mu \mathrm{m}$ thick) were cut on a cryostat microtome at $-20^{\circ} \mathrm{C}$ and mounted on poly-L-lysine coated glass slides (Sigma-Aldrich, St. Louis, MO, United States). For immunohistochemistry, the avidin-biotin-peroxidase complex $(\mathrm{ABC})$ method was used. Briefly, the sections were rinsed in phosphate buffer saline and incubated in 5\% normal goat serum (for $30 \mathrm{~min}$ at room temperature) to reduce the non-specific binding of the secondary antibodies. The sections were then incubated at $4{ }^{\circ} \mathrm{C}$ in a humid chamber for $24 \mathrm{~h}$ with the monoclonal mouse antiserum antiVIM and the polyclonal rabbit antiserum anti-DES (61013 and 10570, Progen Biotechnik GmbH, Heidelberg, Germany, respectively) both diluted 1:1000. After washing, the sections were incubated at room temperature for $1 \mathrm{~h}$ with the biotinconjugated goat anti-mouse IgG and biotin-conjugated goat anti-rabbit IgG secondary antibodies, both diluted 1:200 (Vector Laboratories, Burlingame, CA, United States), and then treated with ABC (Vector elite kit, Vector Laboratories, Burlingame, CA, United States). The immune reactions were visualized through a 3,3'-diaminobenzidine (DAB) chromogen solution (Vector DAB kit, Vector Laboratories, Burlingame, CA, United States).

\section{SDS-PAGE Analysis}

The myofibrillar and sarcoplasmic proteins were extracted following the procedure described by Liu et al. (2014) with slight modifications. Briefly, one gram (in duplicate) of frozen P. major muscle was homogenized by Ultra-Turrax (IKA, Germany) (30 s at $13,000 \mathrm{rpm}$, in ice) in $20 \mathrm{~mL}$ of cold Rigor Buffer $(75 \mathrm{mM}$ $\mathrm{KCl}, 10 \mathrm{mM} \mathrm{KH} \mathrm{PO}_{4}, 2 \mathrm{mM} \mathrm{MgCl}$, 2 mM EGTA; pH 7.0) (Sigma-Aldrich, St. Louis, MO, United States). The homogenate was centrifuged for $10 \mathrm{~min}$ at $10,000 \times g$ at $4^{\circ} \mathrm{C}$ and the supernatant collected (sarcoplasmic protein). The procedure was repeated and the resultant pellet, identified as the myofibrillar protein fraction, was re-suspended by homogenization in $20 \mathrm{~mL}$ of cold Rigor Buffer. After being quantified (Bradford, 1976) the protein concentration (both myofibrillar and sarcoplasmic proteins) of each extract was adjusted to $1.0 \mathrm{mg} / \mathrm{mL}$ and each sample was mixed 1:1 (v/v) with Sample Buffer (50 mM Tris$\mathrm{HCl}, 8 \mathrm{M}$ Urea, 2M Thiourea, 75 mM DTT, 3\% (v/v) SDS; pH 6.8) (Sigma-Aldrich, St. Louis, MO, United States) (Fritz et al., 1989). SDS-PAGE analysis was run, in duplicates, on $5 \mu \mathrm{g}$ of proteins according to the procedure described by Laemmli (1970) by using 7.5\% polyacrylamide hand-cast gels. A molecular weight marker (Precision Plus Standard Proteins, All Blue Prestained, Bio-Rad Laboratories, Hercules, CA, United States) was loaded into each gel to assess the molecular weight of the protein bands. Gels were run on a Bio-Rad Mini Protean II electrophoresis apparatus at constant voltage $(110 \mathrm{~V})$ for about $1 \mathrm{~h}$. Gels were stained with Coomassie Brilliant Blue R-250 (1 g/L) containing 40\% (v/v) methanol and $10 \%(\mathrm{v} / \mathrm{v})$ acetic acid (Sigma-Aldrich, St. Louis, MO, United States) in distilled water and de-stained in distilled water. Gel images were acquired by using a GS- $800^{\mathrm{TM}}$ Calibrated Densitometer (Bio-Rad Laboratories).

\section{Western Blot}

Myofibrillar proteins $(10 \mu \mathrm{g})$ were loaded in $10 \%$ MiniPROTEAN TGX Stain-Fee ${ }^{\mathrm{TM}}$ Gels (Bio-Rad Laboratories), which are able to produce, after UV-induction, a stable, quantitative, and western blotting compatible protein fluorescent signal due to the reaction of the trihalocompound incorporated into gel formulations with the tryptophan residues contained in proteins. After electrophoresis (carried out in the same conditions described earlier), gels were activated by UV exposure for $5 \mathrm{~min}$ and protein fluorescence was acquired using a ChemiDoc $^{\text {TM }}$ MP Imaging System (Bio-Rad Laboratories) with the Image Lab software (version 5.2.1). Proteins were then transferred onto a nitrocellulose membrane using a trans-blot turbo system (Bio-Rad Laboratories) and probed at room temperature for $60 \mathrm{~min}$ with the specific monoclonal mouse antiserum anti-VIM and the polyclonal rabbit antiserum anti-DES (61013 and 10570, Progen Biotechnik GmbH, Heidelberg, Germany, respectively) both diluted 1:10,000. After washing, the membranes were incubated with secondary anti-mouse and anti-rabbit antibodies for $60 \mathrm{~min}(1: 15,000)$ (Merk Millipore, Burlington, MA, United States). Membranes were subsequently incubated at room temperature with HRPconjugated streptavidin (Merk Millipore, Burlington, MA, United States) for $20 \mathrm{~min}$. Final detection was performed 
with enhanced chemiluminescence $\left(\right.$ Clarity $^{\mathrm{TM}}$ Western ECL Substrate) Western Blotting detection kit (Bio-Rad Laboratories) and the images were acquired using the ChemiDoc ${ }^{\mathrm{TM}} \mathrm{MP}$ Imaging System (Bio-Rad Laboratories). Densitometry differences were analyzed with the Image Lab software and normalized for the total fluorescent protein signal intensity (Valli et al., 2018).

\section{Gene Expression}

Total RNA was extracted using TRIzol ${ }^{\circledR}$ reagent (15596026, Invitrogen $^{\mathrm{TM}}$, Thermo Fisher Scientific, Waltham, MA, United States), the quality and integrity of the RNA were both checked and RNA was retrotranscribed to complementary DNA (cDNA) using iScript ${ }^{\mathrm{TM}}$ cDNA Synthesis Kit (1708891, Bio-Rad Laboratories) following manufacturer's instructions. Primers were designed using Primer3Plus web software ${ }^{1}$ and the complete information of the sequences are reported in Supplementary Table 1. Since Ensembl on-line genome database reported two different transcripts for the Gallus gallus VIM gene (last accessed on $30^{\text {th }}$ of January 2019), two primer couples have been designed to quantify them. In particular, a couple of primers was designed on the nucleotide sequence common to both VIM isoforms, while another one was conceived to amplify a sequence specific to the longer VIM transcript. Gene expression was analyzed by quantitative Real-Time PCR (RT-qPCR) using the standard curve method (Pfaffl, 2004) on Rotor-Gene ${ }^{\mathrm{TM}}$ 6000 (Corbett Life Science, Concorde, NSW, Australia). The samples were first used to assess the expression level of three normalizing genes already tested also in previous studies (Bagés et al., 2015; Zambonelli et al., 2016): glyceraldehyde-3-phosphate dehydrogenase $(G A P D H)$, ribosomal protein L32 (RPL32) and tyrosine 3-monooxygenase/tryptophan 5-monooxygenase activation protein, zeta (YWHAZ). Primers and the used RT-qPCR conditions are reported in Supplementary Table 1. The expression levels of these 3 genes were evaluated using NormFinder (Andersen et al., 2004) and RPL32 and YWHAZ, the 2 most stably expressed normalizing genes, were used as reference genes to normalize the expression of DES and VIM genes. For each gene, a couple of primers has been designed and used to obtain the amplicon for the standard curve and to quantify the gene expression. Threshold cycles obtained for the samples were converted by Rotor-Gene ${ }^{\mathrm{TM}}$ 6000 in mRNA molecules/ $\mu$ l using for each gene the relative standard curve (Pfaffl, 2004). Samples were assayed at least in triplicate to obtain, among the repetitions, coefficients of variation below 0.2 .

\section{Statistical Analysis}

Data concerning SDS-PAGE and Western Blot analyses were analyzed by using the one-way ANOVA option of Statistica 10 (StatSoft Inc., 2014) considering the type of abnormality (NORM, WS, WB, and SM) as the main effect. The same model was furthermore applied to analyze the normalized gene expressions of the DES gene and the two VIM transcripts. Mean values were subsequently separated through the parametric Tukey-HSD

\footnotetext{
${ }^{1}$ http://www.bioinformatics.nl/cgi-bin/primer3plus/primer3plus.cgi
}

test. Also, Student's $t$-test was applied to test the differences in protein and gene expression levels between the NORM muscles belonging to the fast-growing and their slow-growing counterpart. All statistical differences were considered significant at a level of $P \leq 0.05$. In addition, Spearman's correlations among the gene expressions of DES and VIM transcripts were calculated on the whole dataset of $20 P$. major samples and inside each group (NORM, WS, WB, and SM) to find gene expression patterns (Zappaterra et al., 2015). Correlations were performed using rcorr function of Hmisc package in $\mathrm{R}$ environment ( $\mathrm{R}$ Core Team, 2018) and nominal $P \leq 0.05$ was considered statistically significant.

\section{RESULTS}

\section{Immunohistochemistry}

The results for immunohistochemical analyses are reported in Figure 1. Only a few fibers immunoreactive to VIM and DES were found in NORM muscles (Figures 1A1,B1). On the other hand, an increased number of fibers immunoreactive to both

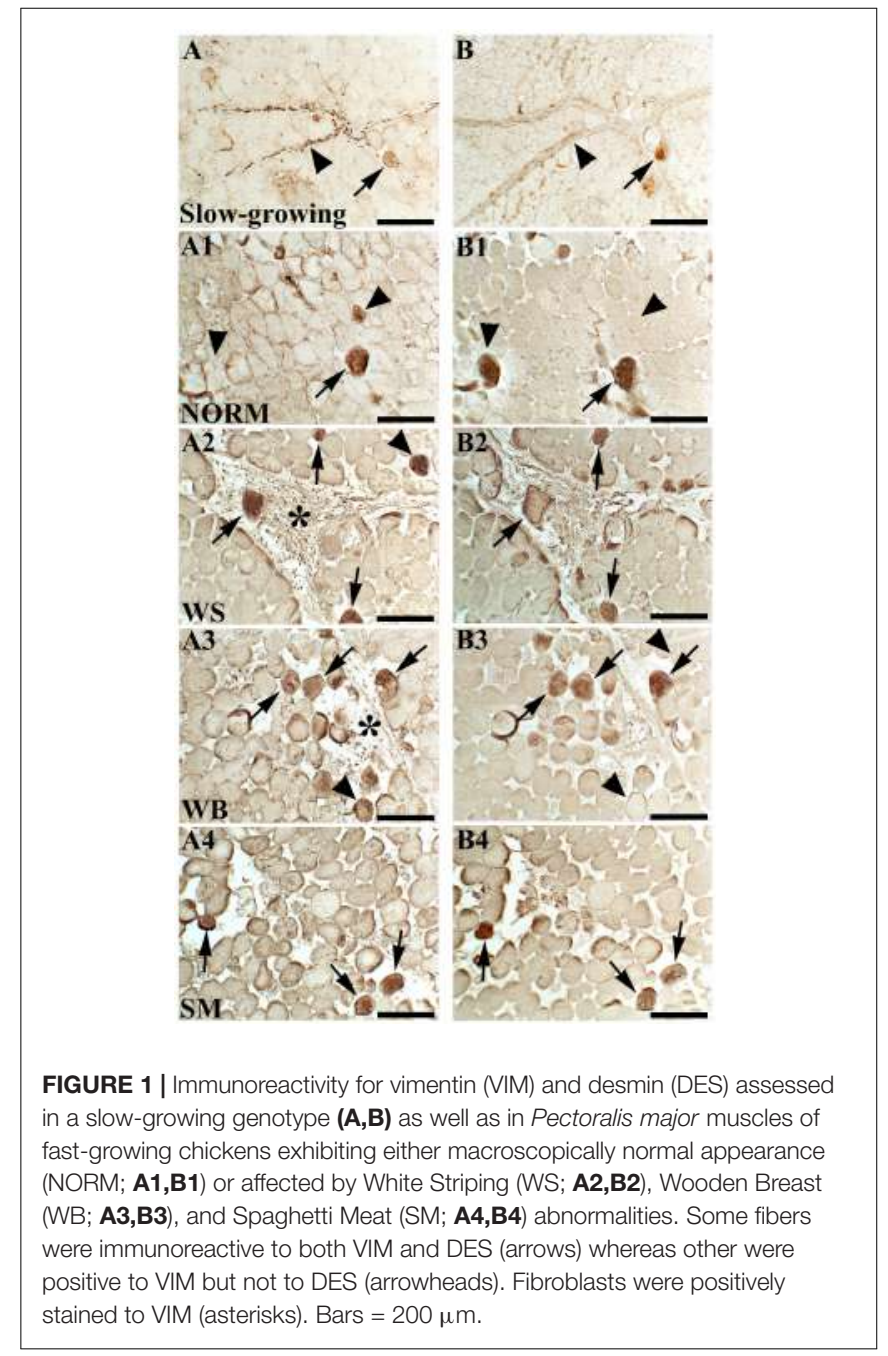


VIM and DES were observed in P. major affected by muscular abnormalities (Figures 1A2,A3,B2,B3). In detail, many positively stained fibers were found in WS and WB muscles, in which several fibroblasts exhibited a strong VIM immunoreactivity (Figures 1A2,A3,B2,B3). Similarly, some fibers showed diffuse immunoreactivity to both VIM and DES in SM affected muscles (Figures 1A4,B4). Overall, in WS, WB and SM affected muscles, most of the fibers positively stained to VIM and DES exhibited a strong immunoreactivity at the sarcolemmal and sub-sarcolemmal levels, whereas a weak or lacking reaction was found in the area corresponding to the core. Besides, some fibers were immunoreactive to VIM but not to DES (Figures 1A1A3,B1-B3). Astonishing differences were found by comparing the results obtained for the NORM muscles belonging to fastgrowing with those found for the slow-growing genotype, shown in Figures 1A,B. In detail, VIM immunoreactivity was observed within the intermyofibrillar network and, although only a few fibers were positively stained in sub-sarcolemmal position, a strong immunoreaction was found in fibroblasts (Figure 1A). Only a few fibers were positively stained to both VIM and DES and a partial reactivity to DES was observed in correspondence to the perimysial compartment (Figures 1A,B).

\section{SDS-PAGE and Western Blot Analyses}

The electrophoretic profiles of the myofibrillar and the sarcoplasmic proteins assessed on $P$. major belonging to the fast-growing genotype (NORM, WS, WB, and SM) as well as in those muscles excised from the slow-growing strain are displayed in Figures $\mathbf{2 A} \mathbf{A}$, respectively. Although the electrophoretic patterns did not differ among the samples belonging to fast-growing groups, relevant variations can be observed by comparing the intensity of the bands ascribed to the myofibrillar protein fraction of the fast-growing and of the slow-growing genotype. Indeed, if compared with NORM, a significant increase $(P \leq 0.001)$ in the optical densities of the bands having a molecular weight of 35, 85 and $125 \mathrm{kDa}$ were found in slow-growing chickens: $+57,+54$, and $+26 \%$, respectively.

Figure 3 shows the findings concerning the expression level of VIM and DES assessed through Western Blot analyses. Concerning VIM (Figure 3A), if compared with NORM, a 55\% increase in the expression level of the protein was found in WB affected muscles (100 vs. $155 \% ; P \leq 0.05$ ) whereas WS exhibited intermediate values (113\%). As for DES (Figure 3B), a $53 \%$ increased expression of this protein was observed in WS muscles whereas the content measured in WB did not differ $(P>0.05)$ from that of the NORM group. In addition, a significant reduction $(P \leq 0.05)$ in the expression level of both VIM and DES ( -24 and $-54 \%$, respectively) was observed in SM.

Remarkable differences were found by comparing the findings concerning the expression level of VIM and DES in NORM fast-growing muscles with those excised from the slowgrowing genotype. Indeed, if compared with their fast-growing counterpart, a significant reduction $(P \leq 0.001)$ in the content of both VIM and DES was found in slow-growing samples $(-71$ and $-51 \%$, respectively).

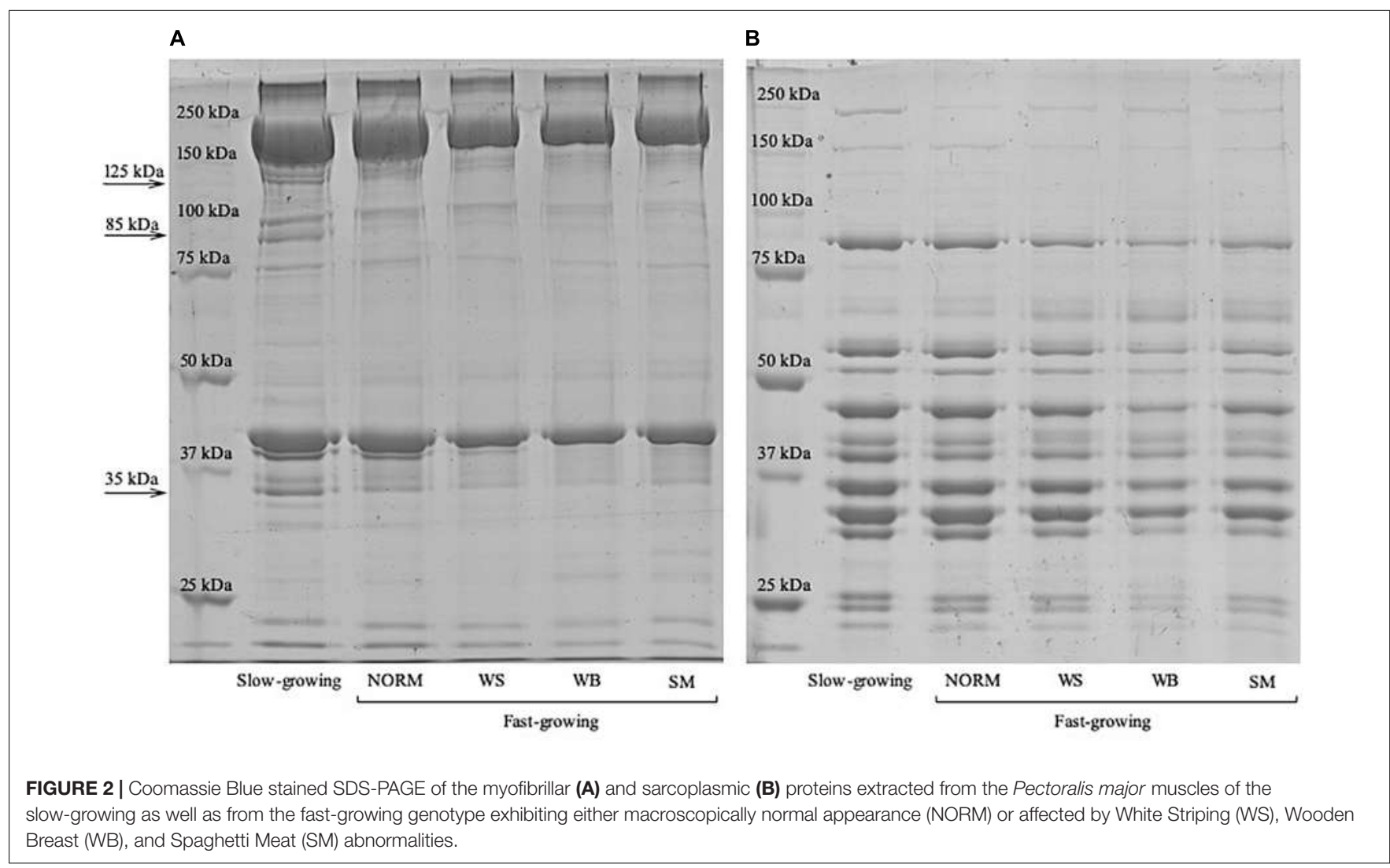




\section{Gene Expression}

The normalized gene expression levels of the two VIM sequences and DES gene are reported in Table 1 and Figure 4. Both $V I M$ gene sequences showed differences in their expressions between groups ( $P \leq 0.05$ for the group effect, in Table 1) with transcription levels progressively increasing when passing from the NORM to the SM (Figures 4A,B). In particular, the normalized expression values obtained with the VIM long isoform-specific primers showed to be significantly higher in WB $(2.45 \pm 0.35)$ and SM samples $(2.58 \pm 0.33)$ compared to NORM group $(1.60 \pm 0.44 ; P \leq 0.01$ for WB vs. NORM and $P \leq 0.01$ for SM vs. NORM comparison; Figure 4A). Group effect was also found significant for the transcription levels of the VIM sequence common to both VIM isoforms $(P \leq 0.05$; Table 1) but with higher intra-group variability. Therefore, although the SM mean for VIM common sequence $(5.48 \pm 5.58)$ was four times higher than that of NORM group $(1.31 \pm 0.86)$, due to the high intra-group standard deviations, no significant $(P>0.05)$ differences were found and only a trend toward significance between NORM (1.31 \pm 0.86$)$ and WB groups (4.37 $\pm 3.36 ; P=0.08$; Figure 4B) was observed. The $D E S$ gene expression showed a significant increase when passing from NORM to WB group $(0.37 \pm 0.23$ and $1.01 \pm 0.59$ in Table $\mathbf{1}$, respectively) and a decrease in the DES normalized mean for SM samples $(0.85 \pm 0.40$, Table 1). Both WB and SM groups significantly differed from NORM samples $(P=0.05$ for both WB vs. NORM and SM vs. NORM comparisons, Figure 4C), showing anyway a consistent intragroup variability. The normalized transcription levels of the DES gene resulted to be positively correlated with VIM common sequence expression both when correlations are performed considering all the studied samples together or each group separately (Table 2). Conversely, a positive correlation $(r=0.90$;
$P \leq 0.05)$ was noticed between DES and VIM long-isoform in NORM samples, but the same genes yielded an opposite correlation in the WS group $(r=-0.99 ; P \leq 0.001)$. Interestingly, also the two studied VIM sequences followed a similar trend, with a positive correlation linking them in NORM individuals ( $r=0.900 ; P \leq 0.05)$ that became negative in WS ones $(r=-0.99 ; P \leq 0.001$; Table 2$)$. Similarly to what was observed for proteins, the slow-growing genotype showed considerably lower normalized expressions for both VIM sequences and DES compared to the NORM group. For VIM long isoformspecific sequence, individuals belonging to the slow-growing breed showed a normalized transcription level 15-times lower than the NORM group $(0.10 \pm 0.02$ in slow-growing genotype; $P \leq 0.01$ for the difference). Notably lower expressions were also noticed for VIM common sequence $(0.07 \pm 0.01)$ and DES $(0.10 \pm 0.04)$.

\section{DISCUSSION}

In the present study, the distribution and expression of DES and VIM have been assessed on fast-growing having either macroscopically normal appearance (NORM) or affected by muscular abnormalities, namely WS, WB, and SM. Overall, the results obtained through immunohistochemistry evidenced the presence of fibers immunoreactive to VIM as well as positively stained to both VIM and DES in all the experimental groups. Since VIM and DES are normally expressed during muscle regeneration and their localization was proposed as a reliable marker of muscle fiber regeneration (Bornemann and Schmalbruch, 1992; Gallanti et al., 1992; Yang and Makita, 1996), it might be assumed that those fibers immunoreactive to VIM and/or positive for both VIM and DES were

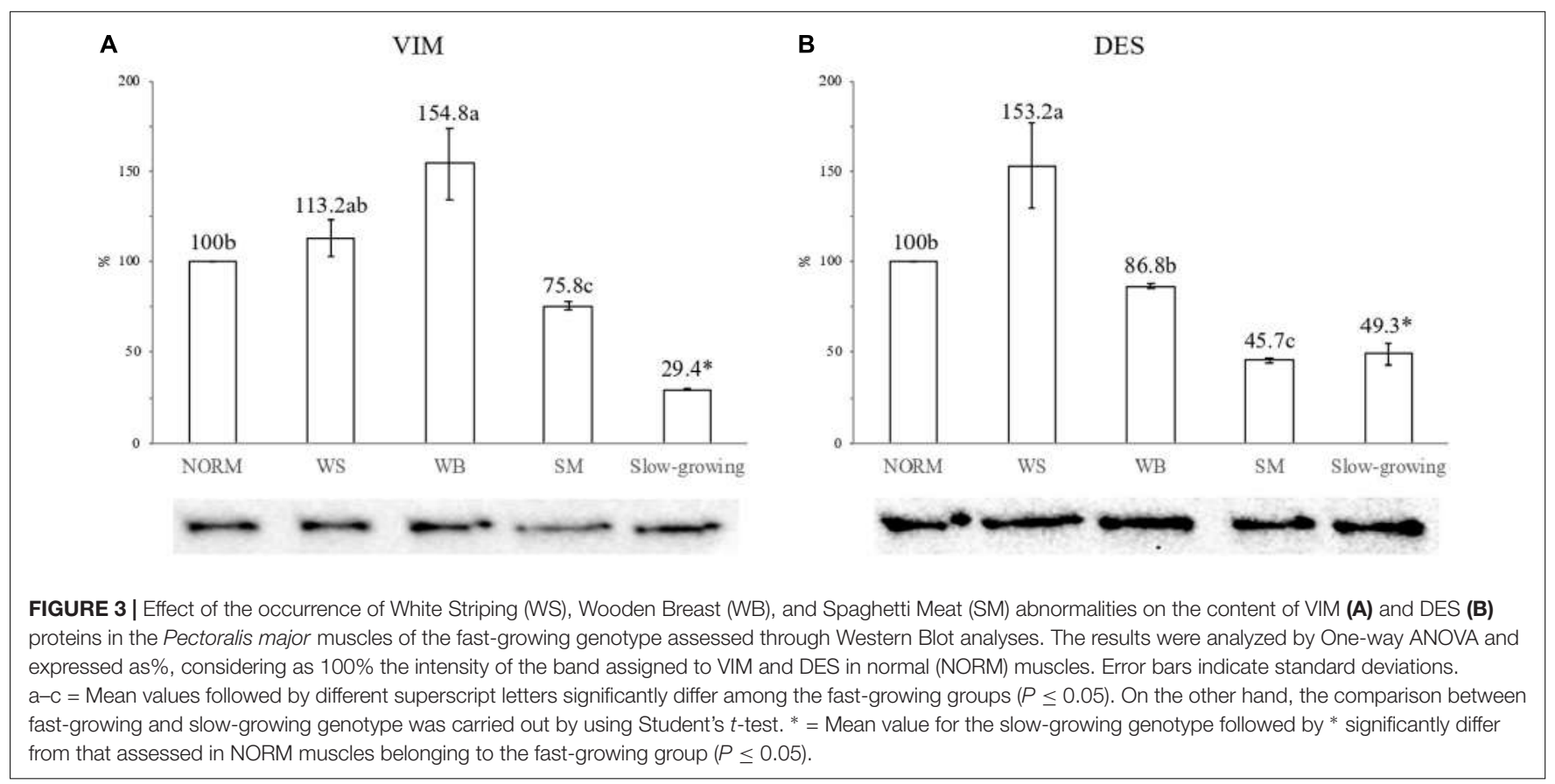


TABLE 1 | One-way ANOVA results for the type of abnormality effect.

\begin{tabular}{|c|c|c|c|c|c|}
\hline \multirow[t]{2}{*}{ Gene } & \multirow[t]{2}{*}{$\mathbf{N}$} & \multirow[t]{2}{*}{ Mean } & \multirow[t]{2}{*}{$S D$} & \multicolumn{2}{|c|}{ Group effect } \\
\hline & & & & $F$-value & $P(>F)$ \\
\hline VIM (long transcript) & & & & 14.89 & 0.001 \\
\hline NORM & 5 & 1.60 & 0.44 & & \\
\hline WS & 5 & 1.99 & 0.64 & & \\
\hline WB & 5 & 2.45 & 0.35 & & \\
\hline SM & 5 & 2.58 & 0.33 & & \\
\hline All & 20 & 2.16 & 0.58 & & \\
\hline VIM (common sequence) & & & & 4.58 & 0.046 \\
\hline NORM & 5 & 1.31 & 0.86 & & \\
\hline WS & 5 & 2.51 & 2.71 & & \\
\hline WB & 5 & 4.37 & 3.36 & & \\
\hline SM & 5 & 5.48 & 5.58 & & \\
\hline All & 20 & 3.42 & 3.66 & & \\
\hline$D E S$ & & & & 3.45 & 0.079 \\
\hline NORM & 5 & 0.37 & 0.23 & & \\
\hline WS & 5 & 0.77 & 0.51 & & \\
\hline WB & 5 & 1.01 & 0.59 & & \\
\hline SM & 5 & 0.85 & 0.40 & & \\
\hline All & 20 & 0.75 & 0.48 & & \\
\hline
\end{tabular}

For each group, mean and standard deviation (SD) of the normalized gene expressions are reported.

undergoing regenerative processes. However, some fibers showed immunoreactivity to VIM but not to DES. It can thus be likely hypothesized that different consecutive phases of regeneration were taking place within these muscles as also evidenced by the small diameter of these fibers themselves. Indeed, VIM is mainly expressed in immature myotubes and myoblasts (Price and Sanger, 1982) and its expression gradually decreases as their development proceeds (Tokuyasu et al., 1985). In this context, the fibers immunoreactive to VIM might be considered at the beginning of the regenerative process in which this protein is transiently expressed (Gallanti et al., 1992). On the other hand, it might be hypothesized that in those fibers positively stained to both VIM and DES an advanced phase of muscle regeneration is in progress thus leading to a strong immunoreactivity to DES (Bornemann and Schmalbruch, 1992; Gallanti et al., 1992). In detail, the presence of fibers immunoreactive to both the intermediate filaments is particularly evident in those muscles affected by abnormalities (WS, WB, and SM). These results are in agreement with the findings of previous studies carried out on dystrophic muscles in which the expression and distribution of DES and VIM have been assessed (Cooper et al., 1988; Gallanti et al., 1992; Holland et al., 2015; Fröhlich et al., 2016). Indeed, an increased expression of VIM and DES was considered as a reliable marker of muscle regeneration (Bornemann and Schmalbruch, 1992; Gallanti et al., 1992; Fröhlich et al., 2016) and hypothesized to be a compensatory mechanism supporting the structural organization of the sarcomere (Gallanti et al., 1992). Besides, if compared with NORM muscles belonging to the fast-growing genotype, the few fibers immunoreactive to VIM and DES observed in the slow-growing genotype, suggest that in these animals, not selected for growth performances, only mild regeneration processes, ascribable to a physiological turnover, occur.

The results of Western Blot analyses corroborate those obtained through immunohistochemistry and provide elements to further characterize the muscles exhibiting WS, WB, and SM. In detail, the significantly higher amount of VIM observed in WB suggests the development of intense regenerative processes in an early-stage within these muscles. On the other hand, the 1.5fold increase in DES content found in WS might be attributed to a progression of the regenerative processes that require the synthesis of this intermediate filament protein to preserve the structural organization of the developing fibers. Therefore, overall, it might be speculated that regenerative processes were taking place in both WB and WS muscles even though a different progression step in the development of regenerative fibers was observed. This hypothesis could be further tested in follow-up investigation, by measuring the expression of VIM and DES at different stages of the animals' growth. Also, the significantly lower content of VIM and DES observed in the muscles belonging to the slow-growing genotype (29.4 and $49.3 \%$, respectively), in comparison with their NORM fast-growing counterpart, confirmed the occurrence of a less intense regeneration that might be barely related to a physiological turnover of the muscle fibers. It might be pointed out that our immunoblots for VIM and DES revealed the presence of a single band that, according to the marker, has a molecular weight of approximately $110 \mathrm{kDa}$. These results are in agreement with the findings obtained in previous studies (Traub et al., 1993; Henderson et al., 2017). Thus, considering the high specificity of the antibodies used for Western Blotting (the same adopted for immunohistochemistry), we hypothesized that this band might be the result of the formation of dimers, either homodimers (only VIM or DES) or heterodimers (VIM-DES) at the Z-disk (Henderson et al., 2017). Indeed, in various developmental and pathological conditions, VIM and DES are co-expressed (Stewart, 1990; Traub et al., 1993) and were observed to form heterodimers (Traub et al., 1993; Henderson et al., 2017).

About gene expression results, both couples of primers designed on the VIM gene successfully amplified all samples and the two isoforms reported in the Ensembl database for VIM gene were quantified. Therefore, these results suggest that also a longer VIM isoform is commonly expressed in the P. major muscle of fast-growing. This longer VIM isoform (Ensembl ID = ENSGALT00000014123.6) is reported to have a longer promoter and exon 1 sequences compared to the shorter VIM transcript (Ensembl ID = ENSGALT00000083582.2; last accessed on $30^{\text {th }}$ of January 2019). Similar alternative usage of the gene promoter and first exon has been also observed for human VIM gene in breast cancer and adrenal carcinoma cell lines (Zhou et al., 2010), but the functional properties of these transcripts are still unclear. In the present study, both VIM common sequence and VIM long transcript-specific sequence showed a concurrent expression in the samples, in agreement with the results in human cells (Zhou et al., 2010), but different patterns of correlations were noticed between the two VIM sequences as well as between them and DES gene expression. In particular, despite both VIM sequences followed a similar increasing trend 


\section{A}

\section{$V I M$ long transcript normalized gene expression}

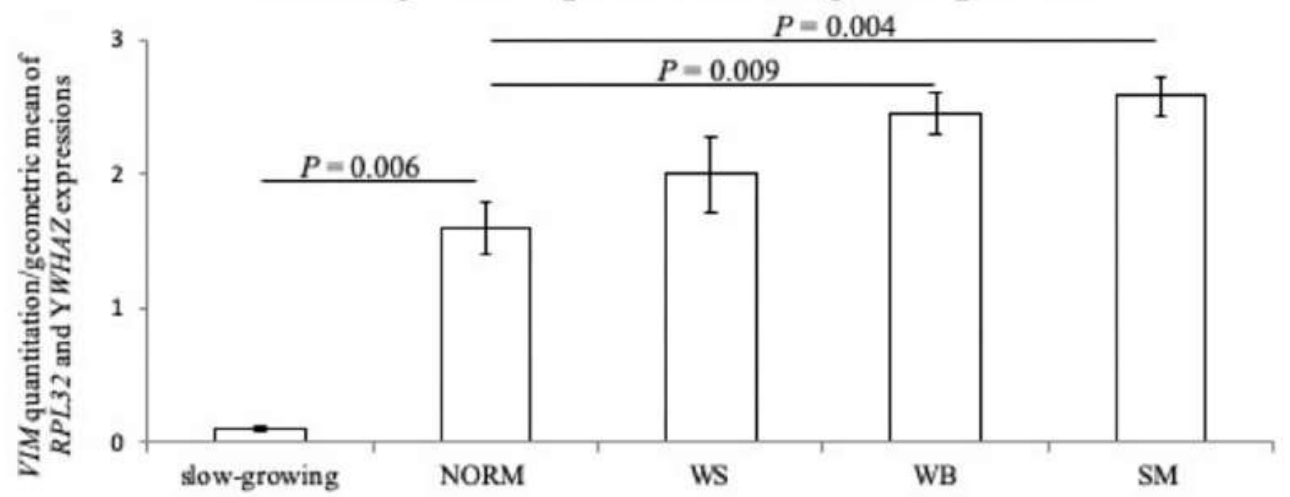

B VIM common sequence normalized gene expression

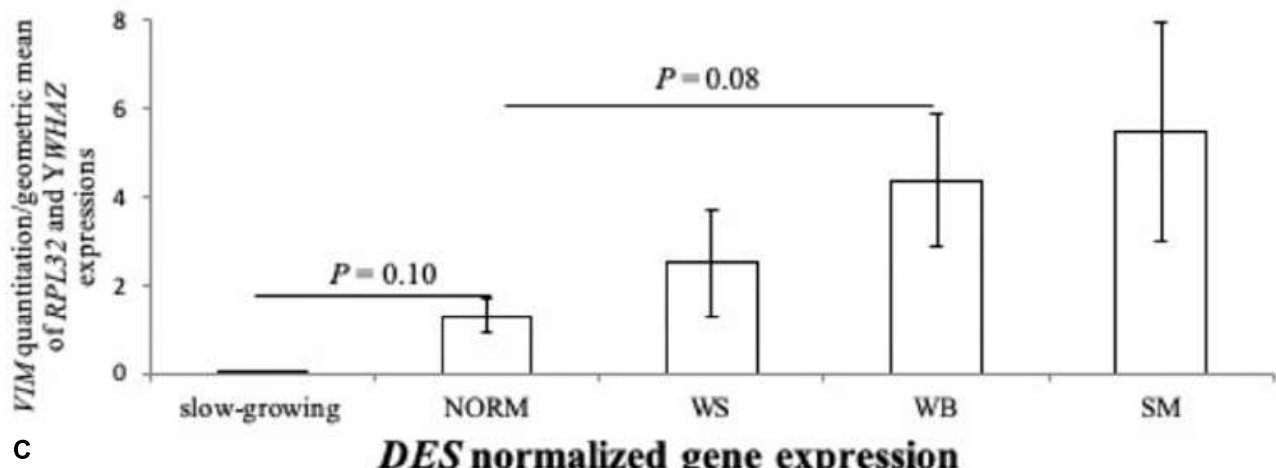

C

\section{DES normalized gene expression}

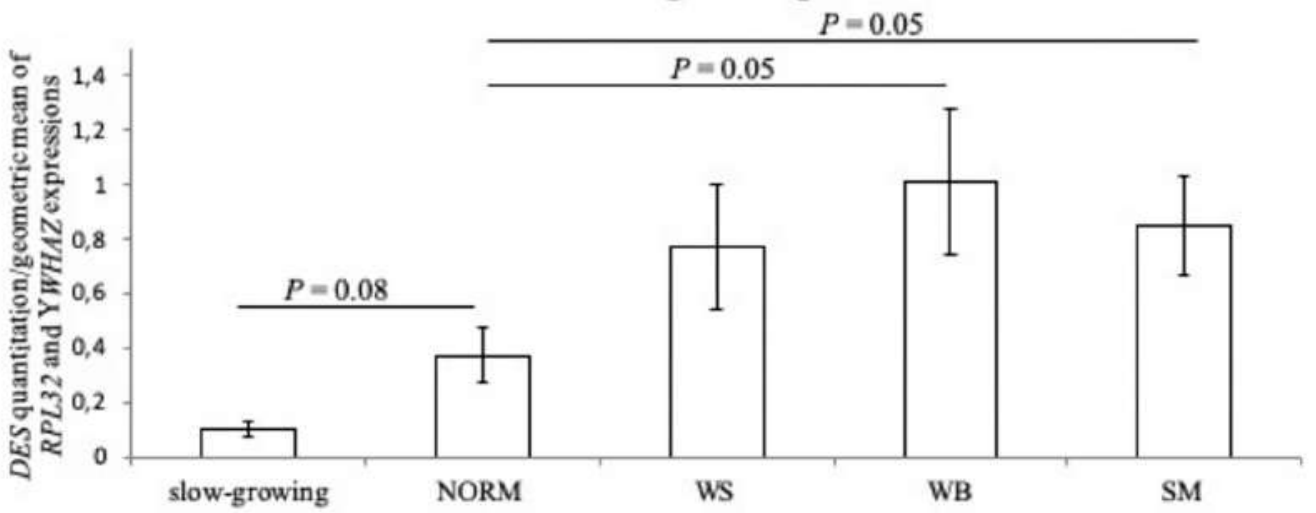

FIGURE 4 | The normalized expression values observed for the tested groups: normal (NORM), White-Striping (WS), Wooden Breast (WB), and Spaghetti Meat (SM) samples. Furthermore, the normalized gene expression levels were also reported for the slow-growing samples, and their values were used to compare the gene expressions measured in NORM group. Panel (A) shows the normalized expression levels obtained with the primer couple specific for Vimentin (VIM) long-isoform; (B) reports the normalized expression levels obtained with the primers designed on Vimentin (VIM) sequence common to both gene isoform; (C) shows the normalized expression levels of Desmin (DES) gene. The measured standard errors are graphically represented by error bars, and only the significant $P$-values $(P \leq 0.05)$ and trends toward significance $(P \leq 0.10)$ are reported for the comparisons between group gene expressions.

when passing from NORM to SM groups, the two isoforms were positively correlated in broilers exhibiting a macroscopically normal appearance, while the same were negatively correlated in the P. major muscles affected by WS condition. This twist in the correlations can suggest that the splicing of the alternative VIM transcripts may have a role in the occurrence of WS abnormality and that, more in general, a co-regulation pattern may exist between the two VIM transcripts. This hypothesis would need anyway to be proven with further dedicated studies, to elucidate the possible effects of the different VIM transcripts in macroscopically normal and in abnormal P. major muscles. Moreover, the two VIM sequences showed opposite correlations with DES gene expression in the WS group, suggesting that the two VIM isoforms may also play distinct functional roles in the 
TABLE 2 | Spearman's correlations between the expression levels of VIM and DES genes considering all the samples and each type of abnormality.

\begin{tabular}{lccc}
\hline All & $\begin{array}{c}\text { VIM } \\
\text { (long transcript) }\end{array}$ & $\begin{array}{c}\text { VIM } \\
\text { (common sequence) }\end{array}$ & DES \\
\hline VIM(long transcript) & 1 & n.s. & n.s. \\
VIM(common sequence) & n.s. & 1 & $\mathbf{0 . 9 0 ^ { * * * }}$ \\
DES & n.s. & $\mathbf{0 . 9 0 ^ { * * * }}$ & 1 \\
\hline & & NORM
\end{tabular}

\begin{tabular}{lccc}
\cline { 2 - 3 } SM & & \\
VIM(long transcript) & 1 & $\mathbf{0 . 9 0 *}$ & n.s. \\
VIM(common sequence) & n.s. & 1 & $\mathbf{0 . 9 0 ^ { * }}$ \\
DES & n.s. & $\mathbf{0 . 9 0 ^ { * }}$ & 1 \\
\hline & & WS
\end{tabular}

\begin{tabular}{lccc}
\cline { 2 - 3 } WB & & & \\
VIM(long transcript) & 1 & $-\mathbf{0 . 9 9 * * *}$ & $\mathbf{- 0 . 9 9 * * *}$ \\
VIM(common sequence) & n.s. & 1 & $\mathbf{0 . 9 9 * * *}$ \\
DES & n.s. & $\mathbf{0 . 9 0 *}$ & 1 \\
\hline
\end{tabular}

Significant correlations are reported in bold. ${ }^{*} P \leq 0.05$; ***P $\leq 0.0001$. n.s., not significant.

regenerative process taking place in WS affected muscles. On the whole, these results seem to confirm that WS muscles may be in a more advanced stage of muscle regeneration, since the negative correlation occurring between DES and VIM long transcriptspecific sequence reflects the negative relation linking VIM and $D E S$ during the development of immature myotubes, where DES expression gradually increases while VIM is down-regulated (Sejersen and Lendahl, 1993).

The comparison between the expression of the VIM and DES genes and the protein levels further evidenced that, although the highest level of expression of DES gene was found in WB, this is not associated with a higher amount of DES protein, whose content was found to be significantly higher in WS. We hypothesize that this might be attributed to a potential delay in the DES protein translation as a consequence of the remarkably higher VIM content observed in WB muscles. Indeed, a higher VIM content was found in WB and suggested that the higher VIM gene expression results in a successfully translated protein. These results seem to support the hypothesis that a more advanced regeneration can be observed in WS in comparison with $\mathrm{WB}$ in which regenerative processes in an early-stage associated with still high levels of translated VIM protein are found. Intriguingly, no linear correspondence between VIM gene expression and its protein product was observed in SM affected muscles. Indeed, the highest VIM mRNA level in these muscles was associated with a significantly lower content of the relative protein. This unexpected result together with the strong VIM immunoreactivity observed in fibroblasts, in agreement with previous studies (Bornemann and Schmalbruch, 1992; Gallanti et al., 1992; Chang et al., 2002), led us to consider in deeper the mechanisms underlying VIM transcription and its translation processes. In detail, this evidence observed also in our samples, may reflect the regulative action exerted by VIM on fibroblast proliferation and differentiation (Cheng et al., 2016). VIM has been proven to coordinate fibroblast proliferation in regenerative processes as a response to wound healing (Cheng et al., 2016). Thus, this suggests that VIM protein plays a major role in the regulation of pathological fibrosis in which differentiated fibroblasts are responsible for inducing fibrosis via increased extracellular matrix synthesis and impedes normal function of the organ (reviewed in Darby and Hewitson, 2007). This role of VIM in regulating fibroblasts proliferation (Cheng et al., 2016) seems to be consistent with the results obtained in the present research in which the highest VIM content is observed in WB affected muscles that concurrently exhibit proliferation of connective tissue. Within this context, the increased expression of VIM together with the higher content of the related protein in WB may have induced the fibro-adipogenic precursors to differentiate into fibroblasts. On the other hand, the lack of translation of VIM mRNA into VIM protein noticed in SM samples may have resulted in an impaired differentiation of fibro-adipogenic precursors to fibroblasts (as evidenced by the findings of immunohistochemical analyses). It can be likely speculated that this may lead to an altered extracellular matrix synthesis that inducing a reduction in the number of fibroblasts within the perimysial compartment, ultimately, results in the progressive rarefaction of the perimysial connective tissue that is distinctively observed in SM affected muscles. However, this hypothesis needs to be tested in future studies. Interestingly, the amount of VIM protein has been already reported to depend mainly on post-transcriptional regulation of VIM gene expression (Beggs et al., 2015), thus suggesting that a similar regulative process may have occurred also in SM samples. Therefore, we hypothesize that the different phenotypes associated with WS, WB, and SM in P. major muscles may be at least in part, a direct consequence of VIM protein synthesis. Moreover, a deeper knowledge of the molecular processes influencing both the VIM protein synthesis from mRNA and the post-transcriptional regulation of gene expression is needed to assist the identification of the factors involved in the occurrence of the muscular growth-related abnormalities in broilers.

\section{CONCLUSION}

The distribution and expression of the intermediate filament VIM and DES proteins can be considered a reliable marker of the regenerative processes taking place within the $P$. major in modern broilers affected by WS, WB, and SM abnormalities but also in those having a macroscopically normal appearance. Overall, the patterns of immunoreactivity together with the results obtained through immunoblotting and gene expression suggested that intense regenerative processes were taking place in both WB and WS muscles even though a different progression in the development of regenerative-fibers can be likely hypothesized, with the first being in an early stage of regeneration if compared with WS once. This could be further tested in follow-up investigation, by measuring the expression of VIM and DES at different stages of the animals' growth. 
On the other hand, the lack of correspondence between VIM gene expression and its protein product observed in SM suggested that VIM may also have a major role in the occurrence of SM phenotype. Anyway, further research will be needed to understand if post-transcriptional mechanisms affecting the translation of VIM mRNA to protein can exert an important effect in the development of the WB and SM abnormalities.

\section{DATA AVAILABILITY STATEMENT}

The raw data supporting the conclusions of this article will be made available by the authors, without undue reservation, to any qualified researcher.

\section{ETHICS STATEMENT}

All the aspects related to farming, handling, transportation, and slaughtering of the birds strictly accomplished with the European Union legislation.

\section{REFERENCES}

Alnahhas, N., Berri, C., Chabault, M., Chartrin, P., Boulay, M., Bourin, M. C. et al. (2016). Genetic parameters of white striping in relation to body weight, carcass composition, and meat quality traits in two broiler lines divergently selected for the ultimate $\mathrm{pH}$ of the pectoralis major muscle. BMC Genet. 17:61. doi: 10.1186/s12863-016-0369-2

Andersen, C. L., Jensen, J. L., and Ørntoft, T. F. (2004). Normalization of real-time quantitative reverse transcription-PCR data: a model-based variance estimation approach to identify genes suited for normalization, applied to bladder and colon cancer data sets. Cancer Res. 64, 5245-5250. doi: 10.1158/0008-5472. CAN-04-0496

Bagés, S., Estany, J., Tor, M., and Pena, R. N. (2015). Investigating reference genes for quantitative real-time PCR analysis across four chicken tissues. Gene 561, 82-87. doi: 10.1016/j.gene.2015.02.016

Baldi, G., Soglia, F., Mazzoni, M., Sirri, F., Canonico, L., Babini, E., et al. (2018). Implications of white striping and spaghetti meat abnormalities on meat quality and histological features in broilers. Animal 12, 164-173. doi: 10.1017/ S1751731117001069

Banwell, B. L. (2001). Intermediate filament-related myopathies. Pediatr. Neurol. 24, 257-263. doi: 10.1016/s0887-8994(00)00248-4

Beggs, J. E., Tian, S., Jones, G. G., Xie, J., Iadevaia, V., Jenei, V., et al. (2015). The MAP kinase-interacting kinases regulate cell migration, vimentin expression and eIF4E/CYFIP1 binding. Biochem. J. 467, 63-76. doi: 10.1042/BJ20141066

Bennett, G. S., Fellini, S. A., Toyama, Y., and Holtzer, H. (1979). Redistribution of intermediate filament subunits during skeletal myogenesis and maturation in vitro. J. Cell Biol. 82, 577-584. doi: 10.1083/jcb.82.2.577

Bornemann, A., and Schmalbruch, H. (1992). Desmin and vimentin in regenerating muscles. Muscle Nerve 15, 14-20. doi: 10.1002/mus.880150104

Bradford, M. M. (1976). A rapid and sensitive method for the quantitation of microgram quantities of protein utilizing the principle of protein-dye binding. Anal. Biochem. 72, 248-254. doi: 10.1006/abio.1976.9999

Cary, R. B., and Klymkowsky, M. W. (1994). Differential organization of desmin and vimentin in muscle is due to differences in their head domains. J. Cell Biol. 126, 445-456. doi: 10.1083/jcb.126.2.445

Chang, H. Y., Chi, J. T., Dudoit, S., Bondre, C., van de Rijn, M., Botstein, D., et al. (2002). Diversity, topographic differentiation, and positional memory in human fibroblasts. Proc. Natl. Acad. Sci. U.S.A. 99, 12877-12882. doi: 10.1073/pnas. 162488599

Cheng, F., Shen, Y., Mohanasundaram, P., Lindström, M., Ivaska, J., Ny, T., et al. (2016). Vimentin coordinates fibroblast proliferation and keratinocyte

\section{AUTHOR CONTRIBUTIONS}

All authors listed have made a substantial, direct and intellectual contribution to the work, and approved it for publication. MP, FSi, and FSo planned the experiment. MP, FSo, MM, FSi, and RD designed the study. FSo, MM, MZ, MD, EB, and MB performed the laboratory analyses. FSo, MD, and EB performed Western blot and SDS page analysis and interpreted the results. MM and PC performed the immunohistochemical analysis. MZ and MB performed the gene expression quantitation. FSo, MZ, and MB organized the databases and performed the statistical analysis. FSo, MZ, MP, RD, and MM wrote the first draft of the manuscript. All authors contributed to manuscript revision.

\section{SUPPLEMENTARY MATERIAL}

The Supplementary Material for this article can be found online at: https://www.frontiersin.org/articles/10.3389/fphys. 2019.01581/full\#supplementary-material

differentiation in wound healing via TGF- $\beta$-Slug signaling. Proc. Natl. Acad. Sci. U.S.A. 113, E4320-E4327. doi: 10.1073/pnas.1519197113

Cooper, B. J., Winand, N. J., Stedman, H., Valentine, B. A., Hoffman, E. P., Kunkel, L. M., et al. (1988). The homologue of the Duchenne locus is defective in X-linked muscular dystrophy of dogs. Nature 334, 154-156. doi: 10.1038/ $334154 \mathrm{a} 0$

Darby, I. A., and Hewitson, T. D. (2007). Fibroblast differentiation in wound healing and fibrosis. Int. Rev. Cytol. 257, 143-179. doi: 10.1016/S0074-7696(07) 57004-X

Fritz, J. D., Swartz, D. R., and Greaser, M. L. (1989). Factors affecting polyacrylamide gel electrophoresis and electroblotting of high-molecularweight myofibrillar proteins myofibrillar proteins. Anal. Biochem. 180, 205-210. doi: 10.1016/0003-2697(89)90116-4

Fröhlich, T., Kemter, E., Flenkenthaler, F., Klymiuk, N., Otte, K. A., Blutke, A., et al. (2016). Progressive muscle proteome changes in a clinically relevant pig model of Duchenne muscular dystrophy. Sci. Rep. 6:33362. doi: 10.1038/srep33362

Gallanti, A., Prelle, A., Moggio, M., Ciscato, P., Checcarelli, N., Sciacco, M., et al. (1992). Desmin and vimentina s markers of regeneration on muscle diseases. Acta Neuropathol. 85, 88-92. doi: 10.1007/bf00304637

Gard, D. L., and Lazarides, E. (1980). The synthesis and distribution of desmin and vimentin during myogenesis in vitro. Cell 19, 263-275. doi: 10.1016/00928674(80)90408-0

Granger, B. L., and Lazarides, E. (1979). Desmin and vimentin coexist at the periphery of the myofibril Z disk. Cell 18, 1053-1063. doi: 10.1016/00928674(79)90218-6

Henderson, C. A., Gomez, C. G., Novak, S. M., Mi-Mi, L., and Gregorio, C. C. (2017). Overview of the muscle cytoskeleton. Compr. Physiol. 7, 891-944. doi: 10.1002/cphy.c160033

Holland, A., Henry, M., Meleady, P., Winkler, C. K., Krautwald, M., Brinkmeier, H., et al. (2015). Comparative label-free mass spectrometric analysis of mildly versus severely affected mdx mouse skeletal muscles identifies annexin, lamin, and vimentin as universal dystrophic markers. Molecules 20, 11317-11344. doi: 10.3390/molecules200611317

Laemmli, U. K. (1970). Cleavage of structural proteins during the assembly of the head of bacteriophage T4. Nature 227, 680-685. doi: 10.1038/227680a0

Lazarides, E. (1980). Intermediate filaments as mechanical integrators of cellular space. Nature 283, 249-256.

Liu, J., Ruusunen, M., Puolanne, E., and Ertbjerg, P. (2014). Effect of pre-rigor temperature incubation on sarcoplasmic protein solubility, calpain activity and meat properties in porcine muscle. LWT Food Sci. Technol. 55, 483-489. doi: 10.1016/j.lwt.2013.10.001 
Mutryn, M. F., Brannick, E. M., Fu, W., Lee, W. R., and Abasht, B. (2015). Characterization of a novel chicken muscle disorder through differential gene expression and pathway analysis using RNA-sequencing. BMC Genom. 16:399. doi: 10.1186/s12864-015-1623-0

Pampouille, E., Berri, C., Boitard, S., Hennequet-Antier, C., Beauclercq, S. A., Godet, E., et al. (2018). Mapping QTL for white striping in relation to breast muscle yield and meat quality traits in broiler chickens. BMC Genom. 19:202. doi: 10.1186/s12864-018-4598-9

Papah, M. B., Brannick, E. M., Schmidt, C. J., and Abasht, B. (2018). Gene expression profiling of the early pathogenesis of wooden breast disease in commercial broiler chickens using RNA-sequencing. PLoS One 13:e0207346. doi: 10.1371/journal.pone.0207346

Petracci, M., Soglia, F., Madruga, M., Carvalho, L., Ida, E., and Estevez, M. (2019). Wooden-Breast, white striping, and spaghetti meat: causes, consequences and consumer perception of emerging broiler meat abnormalities. Compr. Rev. Food Sci. Food Saf. 18, 565-583. doi: 10.1111/1541-4337. 12431

Pfaffl, M. W. (2004). “Quantification strategies in real-time PCR," in $A-Z$ of Quantitative PCR, ed. S. A. Bustin, (La Jolla, CA: International University Line IUL), 87-112.

Price, M. G., and Sanger, J. W. (1982). "Intermediate filaments in striated muscles. A review of structural studies in embryonic and adult skeletal and cardiac muscles," in Cell and Muscle Motility, eds R. M. Dowben, and J. W. Shay, (New York, NY: Plenum), 1-40.

R Core Team (2018). R: A Language and Environment for Statistical Computing. Vienna, Austria: R Foundation for Statistical Computing. Available at: https: //www.R-project.org/

Sejersen, T., and Lendahl, U. (1993). Transient expression of the intermediate filament nestin during skeletal muscle development. J. Cell Sci. 106, 1291-1300.

Soglia, F., Gao, J., Mazzoni, M., Puolanne, E., Cavani, C., Petracci, M., et al. (2017). Superficial and deep changes of histology, texture and particle size distribution in broiler wooden breast muscle during refrigerated storage. Poult. Sci. 96, 3456-3472. doi: 10.3382/ps/pex115

Soglia, F., Mazzoni, M., and Petracci, M. (2019). Spotlight on avian pathology: current growth-related breast muscle abnormalities in broilers. Avian. Pathol. 48, 1-3. doi: 10.1080/03079457.2018.1508821

Soglia, F., Mudalal, S., Babini, E., Di Nunzio, M., Mazzoni, M., Sirri, F., et al. (2016). Histology, composition, and quality traits of chicken Pectoralis major muscle affected by wooden breast abnormality. Poult. Sci. 95, 651-659. doi: $10.3382 / \mathrm{ps} /$ pev353

StatSoft Inc. (2014). STATISTICA (Data Analysis Software System), Version 10. Available at: http://www.statsoft.com
Stewart, M. (1990). Intermediate filaments: structure assembly and molecular interactions. Curr. Opin. Cell Biol. 2, 91-100. doi: 10.1016/s0955-0674(05) 80037-7

Tokuyasu, K. T., Maher, P. A., and Singer, S. J. (1985). Distribution of vimentin and desmin in developing chick myotubes in vivo. II. Immunoelectron microscopic study. J. Cell Biol. 100, 1157-1166. doi: 10.1083/jcb.100.4.1157

Traub, P., Kuhn, S., and Grub, S. (1993). Separation and characterization of homo and hereto-oligomers of the intermediate filament proteins desmin and vimentin. J. Mol. Biol. 230, 837-856. doi: 10.1006/jmbi.1993.1205

Valli, V., Taccari, A., Di Nunzio, M., Danesi, F., and Bordoni, A. (2018). Health benefits of ancient grains. Comparison among bread made with ancient, heritage and modern grain flours in human cultured cells. Food Res. Int. 107, 206-215. doi: 10.1016/j.foodres.2018.02.032

Velleman, S. (2019). Recent developments in breast muscle myopathies associated with growth in poultry. Annu. Rev. Anim. Biosci. 7, 289-308. doi: 10.1146/ annurev-animal-020518-115311

Yang, Y., and Makita, T. (1996). Immunocytochemical colocalization of desmin and vimentin in human fetal skeletal muscle cells. Anat. Rec. 246, 64-70. doi: 10.1002/(SICI)1097-0185

Zambonelli, P., Zappaterra, M., Soglia, F., Petracci, M., Sirri, F., Cavani, C., et al. (2016). Detection of differentially expressed genes in broiler pectoralis major muscle affected by white striping - wooden breast myopathies. Poult. Sci. 95, 2771-2785. doi: 10.3382/ps/pew268

Zappaterra, M., Braglia, S., Bigi, M., Zambonelli, P., and Davoli, R. (2015). Comparison of expression levels of fourteen genes involved in the lipid and energy metabolism in two pig breeds. Livest. Sci. 181, 156-162. doi: 10.1016/j. livsci.2015.09.007

Zhou, Z., Kahns, S., and Nielsen, A. L. (2010). Identification of a novel vimentin promoter and mRNA isoform. Mol. Biol. Rep. 37, 2407-2413. doi: 10.1007/ s11033-009-9751-8

Conflict of Interest: The authors declare that the research was conducted in the absence of any commercial or financial relationships that could be construed as a potential conflict of interest.

Copyright (c) 2020 Soglia, Mazzoni, Zappaterra, Di Nunzio, Babini, Bordini, Sirri, Clavenzani, Davoli and Petracci. This is an open-access article distributed under the terms of the Creative Commons Attribution License (CC BY). The use, distribution or reproduction in other forums is permitted, provided the original author(s) and the copyright owner(s) are credited and that the original publication in this journal is cited, in accordance with accepted academic practice. No use, distribution or reproduction is permitted which does not comply with these terms. 


\section{OPEN ACCESS}

Edited by:

Sandra G. Velleman, The Ohio State University,

United States

Reviewed by:

Casey M. Owens,

University of Arkansas, United States

Massimiliano Petracci,

University of Bologna, Italy

Sami Dridi,

University of Arkansas, United States

${ }^{*}$ Correspondence:

Chris M. Ashwell

cmashwe/@ncsu.edu

Specialty section:

This article was submitted to

Avian Physiology,

a section of the journal

Frontiers in Physiology

Received: 04 September 2019 Accepted: 27 January 2020

Published: 25 February 2020

Citation:

Phillips CA, Reading BJ, Livingston $M$, Livingston $K$ and Ashwell CM (2020) Evaluation via

Supervised Machine Learning of the Broiler Pectoralis Major and Liver Transcriptome in Association With the Muscle Myopathy Wooden Breast. Front. Physiol. 11:101 doi: 10.3389/fphys.2020.00101

\section{Evaluation via Supervised Machine Learning of the Broiler Pectoralis Major and Liver Transcriptome in Association With the Muscle Myopathy Wooden Breast}

\author{
Chelsea A. Phillips' ${ }^{1}$ Benjamin J. Reading'2, Matthew Livingston'1, Kimberly Livingston' \\ and Chris M. Ashwell ${ }^{*}$.
}

${ }^{1}$ Prestage Department of Poultry Science, North Carolina State University, Raleigh, NC, United States, ${ }^{2}$ Department of Applied Ecology, North Carolina State University, Raleigh, NC, United States

The muscle myopathy wooden breast (WB) has recently appeared in broiler production and has a negative impact on meat quality. WB is described as hard/firm consistency found within the pectoralis major (PM). In the present study, we use machine learning from our PM and liver transcriptome dataset to capture the complex relationships that are not typically revealed by traditional statistical methods. Gene expression data was evaluated between the PM and liver of birds with WB and those that were normal. Two separate machine learning algorithms were performed to analyze the data set including the sequential minimal optimization (SMO) of support vector machines (SVMs) and Multilayer Perceptron (MLP) Artificial Neural Network (ANN). Machine learning algorithms were compared to identify genes within a gene expression data set of approximately 16,000 genes for both liver and PM, which can be correctly classified from birds with or without WB. The performance of both machine learning algorithms SMO and MLP was determined using percent correct classification during the cross-validations. By evaluating the WB transcriptome datasets by $5 \times$ cross-validation using ANNs, the expression of nine genes ranked based on Shannon Entropy (Information Gain) from PM were able to correctly classify if the individual bird was normal or exhibited WB 100\% of the time. These top nine genes were all protein coding and potential biomarkers. When PM gene expression data were evaluated between normal birds and those with WB using SVMs they were correctly classified $95 \%$ of the time using 450 of the top genes sorted ranked based on Shannon Entropy (Information Gain) as a preprocessing step. When evaluating the 450 attributes that were 95\% correctly classified using SVMs through Ingenuity Pathway Analysis (IPA) there was an overlap in top genes identified through MLP. This analysis allowed the identification of critical transcriptional responses for the first time in both liver and muscle during the onset of WB. The information provided has revealed many molecules and pathways making up a complex molecular mechanism involved with the progression of wooden breast and suggests that the etiology of the myopathy is not limited to activity in the muscle alone, but is an altered systemic pathology.

Keywords: wooden breast, machine learning, poultry transcriptomics, support vector machines, artificial neural networks, transforming growth factor 


\section{INTRODUCTION}

The occurrence of wooden breast (WB) in commercial poultry production is rising, leaving producers with an inferior product and, ultimately, unsatisfied consumers (Mudalal et al., 2014; Petracci et al., 2015). Much of the incidence is thought to be attributed to artificial selection that has led to the development of broilers with greater muscle yield, better feed conversion rates, and faster growth. Frequent detection of muscle myopathies has been thought to be associated with increased growth rates and breast muscle yields (Sihvo et al., 2014; Trocino et al., 2015; Kuttappan et al., 2016; Abasht et al., 2019). WB is described as a hard or firm consistency deep within the pectoralis major (PM) muscle (Sihvo et al., 2014). Alterations of the meat composition have been observed including increased moisture, collagen, sodium, calcium and fat content (Zambonelli et al., 2014). When compared to PM without the myopathy, the PM meat quality with WB is lower due to greater cooking losses and greater shear force when compared to PM without the myopathy (Zambonelli et al., 2014; Trocino et al., 2015). Consequently, the meat texture of the breast is tougher and less desirable to the consumer. Extensive histological evaluation of WB has been characterized by necrosis, chronic fibrosis, infiltration of fat and connective tissue, and the presence of inflammatory cells and macrophages (Sihvo et al., 2014; Trocino et al., 2015; Kuttappan et al., 2016; Papah et al., 2017).

The severity of WB is often categorized on a scale ranging from 0 to 3 ( 0 , normal; 1 , mild; 2 , moderate; 3 , severe) (Trocino et al., 2015). WB lesions can be detected through manual palpation of the PM as early as 3 weeks of age (Mutryn et al., 2015). Research covering muscle myopathies in broilers reveals that myopathies have increased in recent years and have been correlated with the selection of larger breast muscle (Petracci et al., 2015). The cause of WB is currently unknown, but it is more prevalent in older, heavier male broilers than young birds (Kuttappan et al., 2017; Brothers et al., 2019). Larger breast muscle has been associated with hypertrophied muscle fibers which is thought to impact blood supply and number of satellite cells that are needed in muscle regeneration (Daughtry et al., 2017; Malila et al., 2019).

The increase in prevalence and severity of $\mathrm{WB}$ has the potential to result in excessive economic loses. It has been projected that these losses could exceed more than \$200 million USD/yr (Kuttappan et al., 2016). Often, the PM muscle of birds with WB can appear pale, bulging, and covered in a clear viscous fluid (Sihvo et al., 2014). Considering consumers frequently purchase chicken breasts based on visual appearance of the meat, this unpleasant appearance will undoubtedly have a negative impact on sales (Petracci et al., 2015). Even if WB is undetectable by the outward appearance prior to purchase, the hardness of the inner muscle will become evident once the meat is handled or consumed. Ultimately, this is bad for the consumer, the company, and poultry production as a whole by breaking consumer trust and potentially initiating the desire for an alternative product.

Currently, researchers are investigating the nutritional, physiological, and genetic factors that surround this myopathy through the use of many common techniques that span from the inclusion of feed additives to molecular approaches using gene expression analysis and histology (Velleman and Clark, 2015; Kuttappan et al., 2016; Papah et al., 2018; Petracci et al., 2019). In the present study, we use machine learning analysis of PM and liver transcriptome datasets to capture the complex relationships that are not typically revealed by traditional statistical methods. This was achieved through the use of algorithms to identify genes within an extensive RNA sequencing dataset whose expression can be used to correctly distinguish normal tissue apart from severe/moderate WB. Previous gene expression datasets have characterized many molecular relationships present in birds with WB (Mutryn et al., 2015; Papah et al., 2018; Brothers et al., 2019). This study uses a different mechanism to evaluate gene expression data in the hopes for a more concise evaluation of the WB myopathy.

\section{MATERIALS AND METHODS}

\section{Facilities and Rearing}

This experiment was conducted at the North Carolina State University Chicken Education Unit between the months of March and April, 2019. All procedures used in this study were reviewed and approved by the Institutional Animal Care and Use Committee. Eggs were collected from a resident 25-weekold broiler breeder flock of known, similar genetic background and stored for no more than 7 days at $15^{\circ} \mathrm{C}$. Incubation was performed based on the methods described by Livingston (Livingston et al., 2018a). Day of hatch chicks were sex-sorted and a total of 128 male chicks were individually neck tagged and placed into eight replicate pens $\left(1.2 \mathrm{~m} \times 4.0 \mathrm{~m} ; 4.8 \mathrm{~m}^{2}\right)$ with 16 chicks per pen (blocked by location within the house). Each pen was supplied with one bell water drinker, two tube feeders, and bedded with fresh pine shavings $(15 \mathrm{~cm}$ deep). Broilers were provided ad libitum access to a common commercial starter diet (1-14 days) and a common grower diet (14-45 days) manufactured at the NC State University Feed Mill (Table 1). Eight broilers from each pen, for a total of 64, were selected (based on experiment-wise mean BW) for processing at 45 days. Birds were evaluated for $\mathrm{WB}$ and samples of PM and liver tissue were collected for analysis of gene expression and stored at $-80^{\circ} \mathrm{C}$. PM tissue was sampled from the deep medial region of the birds left breast and liver tissue was sampled from caudal region of the left lobe.

\section{Processing}

At 45 days, selected broilers were collected and transported to the North Carolina State University Chicken Education Unit's broiler processing facility followed by shackling and stunning in a salt saturated saline head stun cabinet. Birds were head stunned with a $110 \mathrm{v} / 60 \mathrm{hz}$ CF2000 poultry stun knife set to $150 \mathrm{~mA}$ for $10 \mathrm{~s}$. Broilers were exsanguinated for $120 \mathrm{~s}$ by opening of the jugular vein and carotid artery with a single knife cut by a trained technician followed by scalding in hot water $\left(60^{\circ} \mathrm{C}\right)$ for $120 \mathrm{~s}$. This was followed by feather picking for $30 \mathrm{~s}$ (Meyn Food Processing Technology B.V., Westeinde Amsterdam, Netherlands). Head and feet were removed, vent opened (VC Poultry Vent Cutter, Jarvis Product 
TABLE 1 | Composition of basal starter and grower $\operatorname{diets}^{1}$.

\begin{tabular}{|c|c|c|}
\hline Ingredients & Starter & Grower \\
\hline Corn & 55.22 & 57.45 \\
\hline Soybean meal (48\% CP) & 36.9 & 31.87 \\
\hline Poultry fat & 2.36 & 5 \\
\hline Dicalcium phosphate (18.5\% P) & 2.02 & 2.39 \\
\hline Glycine & 1.25 & 1.25 \\
\hline Limestone & 0.77 & 0.6 \\
\hline Salt & 0.5 & 0.5 \\
\hline DL-Methionine & 0.28 & 0.24 \\
\hline Choline chloride (60\%) & 0.2 & 0.2 \\
\hline L-Threonine & 0.1 & 0.09 \\
\hline L-Lysine & 0.05 & 0.07 \\
\hline Selenium premix ${ }^{2}$ & 0.05 & 0.05 \\
\hline Vitamin premix ${ }^{3}$ & 0.05 & 0.05 \\
\hline Mineral premix ${ }^{4}$ & 0.2 & 0.2 \\
\hline Coccidiostat $^{5}$ & $\underline{0} . \underline{05}$ & $\underline{0} . \underline{05}$ \\
\hline Total & 100 & 100 \\
\hline \multicolumn{3}{|l|}{$\underline{\text { Calculated nutrient content }}$} \\
\hline Crude protein & 22.5 & 20.2 \\
\hline Calcium & 0.9 & 0.9 \\
\hline Available phosphorus & 0.45 & 0.5 \\
\hline Potassium & 0.89 & 0.82 \\
\hline Total lysine & 1.27 & 1.14 \\
\hline Total methionine & 0.62 & 0.55 \\
\hline Total threonine & 0.85 & 0.76 \\
\hline Total methionine + cysteine & 0.97 & 0.87 \\
\hline Sodium & 0.21 & 0.2 \\
\hline Metabolizable energy (kcal/g) & 2.85 & 3.03 \\
\hline
\end{tabular}

${ }^{1}$ Starter diet was fed to approximately 14 days of age, $1820 \mathrm{~g}$ per bird. ${ }^{2}$ Selenium premix provided $0.2 \mathrm{mg} \mathrm{Se}\left(\mathrm{SS} \mathrm{Na}_{2} \mathrm{SeO}_{3}\right.$ ) per $\mathrm{kg}$ of diet. ${ }^{3}$ Vitamin premix supplied the following per $\mathrm{kg}$ of diet: 13,200 IU vitamin A, 4,000 IU vitamin $D_{3}, 33 \mathrm{IU}$ vitamin E, $0.02 \mathrm{mg}$ vitamin $B_{12}, 0.13 \mathrm{mg}$ biotin, $2 \mathrm{mg}$ menadione $\left(K_{3}\right), 2 \mathrm{mg}$ thiamine, $6.6 \mathrm{mg}$ riboflavin, $11 \mathrm{mg}$ d-pantothenic acid, $4 \mathrm{mg}$ vitamin $B_{6}, 55 \mathrm{mg}$ niacin, and $1.1 \mathrm{mg}$ folic acid. ${ }^{4}$ Mineral premix supplied the following per $\mathrm{kg}$ of diet: manganese, $120 \mathrm{mg}$; zinc, $120 \mathrm{mg}$; iron, $80 \mathrm{mg}$; copper, $10 \mathrm{mg}$; iodine, $2.5 \mathrm{mg}$; and cobalt,

$1 \mathrm{mg} .{ }^{5}$ Coccidiostat supplied monensin sodium at $90 \mathrm{mg} / \mathrm{kg}$ of food.

Corp., Middleton, CT, United States), and viscera and giblets removed manually. Liver and PM tissue were removed and snap frozen on liquid nitrogen for RNA sequencing analysis. Hot carcass weights (HCW) were collected prior to carcasses being air chilled at $3.0^{\circ} \mathrm{C}$ for approximately $24 \mathrm{~h}$. At $24 \mathrm{~h}$ postmortem examination of the PM muscle was evaluated after breast muscle was removed from carcass and bones by a trained and experienced technician for WB using a one to four-point ordinal scale of measurement in accordance with the methods described previously by Livingston with a score of one being normal and four being most severe (Livingston et al., 2018b).

\section{RNA Sequencing Analysis}

Pectoralis major and liver tissue samples from $45 \mathrm{~d}$ broilers with varying severities of WB scores were obtained and preserved in RNALater. RNA extracted from the PM of 15 birds with moderate to severe WB was compared to RNA extracted from five normal PM. For liver RNA evaluation, 10 samples from birds with moderate to severe WB and five livers from birds with normal
PM were used. RNA was extracted from the tissues using Qiagen RNeasy Mini protocol (Qiagen, Valencia, CA, United States) following the manufacturer's instructions. The RNA quality was assessed by Nanodrop 2000 spectrophotometer (Thermo, United States). Two micrograms of RNA from each sample were taken to the North Carolina State University Genomics Sciences Laboratory for library preparation and sequencing on the Illumina HiSeq 2500 sequencer. RNA sequencing was analyzed using CLC Genomics Workbench (Qiagen, Valencia, CA, United States; licensed to NCSU) version 11 following the software manual ${ }^{1}$. RNA sequencing reads and annotations were mapped to the chicken genome (galgal5) from NCBI. Raw reads were processed by the default settings of reads' quality control and adapter trimming. The false discovery rate p-value (FDRp) was calculated to correct for multiple testings' and an FDR adjusted $p \leq 0.05$ was considered statistically significant. Fold change and $\log _{2}$ fold change differences in gene expression between WB scores moderate to severe and normal were also calculated.

\section{Machine Learning Analysis}

Gene expression data were analyzed from the PM and liver using the Waikato Environment for Knowledge Analysis (WEKA) version 3.8.3. Two different pattern recognition machine learning algorithms were performed to analyze the data set: sequential minimal optimization support vector machines (SVMs) and artificial neural network multilayer perceptron (MLP) (Cortes and Vapnik, 1995; Eibe et al., 2016). The machine learning algorithms were compared to identify gene expression patterns within the data set of 15,569 genes, which could be used to correctly classify birds as either exhibiting moderate to severe WB or normal (dichotomous class assignment). Briefly, the 15,569 genes from the gene expression dataset were ranked based on Shannon Entropy (Information Gain) in dichotomous classification assignment by SVMs (Keerthi et al., 2001; Eibe et al., 2016). Information gain ranking was then used to identify those gene expression patterns most relevant to assignment of each bird as having WB or normal by either MLP or SVMs. Reduction of data dimensionality for each machine learning algorithm was then performed by sequential exclusion of those gene expression patterns least relevant to the class assignment (50-2,000 per sequence). This step eliminates overfitting of the machine learning classifiers. To identify the minimum number of gene expression patterns required for classification, 1-50 genes were sequentially excluded from the dataset until only the top 2-7 remained. This step identifies underfitting of the machine learning classifiers and the point of optimal classification for the MLP and SVMs was determined to be the intersection between the underfitting and overfitting curve plots.

Class assignment of all machine learning algorithms was evaluated vis-à-vis by two cross-validation strategies (classification as either WB or normal). The first being a percentage split, where $66 \%$ of the total data were randomly

\footnotetext{
${ }^{1}$ http://resources.qiagenbioinformatics.com/manuals/clcgenomicsworkbench/ current/User_Manual.pdf
} 
used for training and the remaining $34 \%$ of the data were used in testing. The second cross-validation was a stratified hold-out ( $n$-fold) method with 5-fold, where 4 -fold of the randomized gene expression data were used for the training and 1-fold was used for testing. This was repeated five times, such that all normal replicate samples were used at least once in testing and the average model performance was recorded.

The performance of the two machine learning algorithms SVMs and MLP was determined using percent correct classification during the cross-validations, which indicated the likelihood that each individual biological replicate could accurately be assigned into the classes of WB or normal based on the gene expression data provided. Kappa statistic and ROC score also were recorded. Any kappa statistic greater than 0 indicated that the machine learning classifier is performing better than random chance along with a ROC score of greater than 0.500 . The random probability of chance for dichotomous assignment was assumed to be $50 \%$ based on the Law of Probability.

A negative control of machine learning was created through 10 separate randomizations of the individual birds within the dataset. The SVMs and MLP were unable to predict WB or normal, indicating the machine learning herein is true.

\section{Pathway Analysis}

Ingenuity Pathway Analysis (IPA; Qiagen, Valencia, CA, United States $)^{2}$ software was used for canonical pathway analysis, upstream regulatory analysis, and gene network discovery. SVM analysis of the top 450 performing differentially expressed genes from the PM of birds with moderate/severe to normal WB were used in IPA and the top 150 were used from the liver dataset. IPA calculation of $\mathrm{z}$-scores using the gene expression fold change values measures the state of activation or inhibition of the molecules involved in the molecular networks. The analysis of biological mechanisms occurring in the differentially expressed genes of the chicken in IPA are based on mammalian systems for human, rat, and mouse.

\section{RESULTS}

\section{Multilayer Perceptron}

By using the novel approach of evaluating the WB PM transcriptome dataset by 5 -fold cross-validation using MLP, the expression of nine genes (NUP43, KPNA7, DEAF1, NUD19, CCDC85A, SLC25A30, ENSGAL00000015075, PACSIN3, and $R P L 19)$ were able to correctly classify if the PM tissue from an individual bird was normal or exhibited $\mathrm{WB}$ in $100 \%$ of the individual genomes when using the top nine genes ranked based on Shannon Entropy (Information Gain) (Figure 1). MLP is an artificial neural network, which can distinguish data that are not linearly separable, but instead is a feed forward mechanism that maps a dataset into suitable outputs. The top nine genes in the PM were further analyzed for their individual biological roles (Table 2). When the liver transcriptome dataset was evaluated by $66 \%$ split using MLP, the expression of

${ }^{2} \mathrm{http} / / /$ www.ingenuity.com
75 genes were capable of correctly classifying the PM tissue of an individual bird as exhibiting WB or normal in 100\% of the individual genomes (Figure 2). The kappa statistic and ROC score were optimal at 1 for both PM and liver. The individual expression of transcripts CARD19 and ITCH predicted WB or normal using MLP 5-fold cross validation 93.333\% of the time whereas BUD13 and ENSGALG00000039590 individually predicted WB or normal $100 \%$ of the time using $66 \%$ split (Table 3).

\section{Sequential Minimal Optimization}

When the PM gene expression data set was evaluated between birds with WB and those that were normal using WEKA SMO function of SVMs by a 5-fold cross-validation method they were correctly classified $95 \%$ of the time using 450 of the top genes ranked based on Shannon Entropy (Information Gain) as a preprocessing step (Figure 3). The kappa statistic and ROC score were optimal at 0.8571 and 0.9000 , consecutively. The liver gene expression data set was evaluated using the SMO function of SVMs by both a 5 -fold cross-validation method and the $66 \%$ split method was capable of predicting WB or normal $100 \%$ of the time with 100 to 200 of the top genes ranked based on Shannon Entropy (Information Gain) (Figure 4). Optimal kappa statistic and ROC scores of 1 were achieved for both methods. In both machine learning algorithms, the stratified hold-out method appeared to accurately estimate the machine learning classifier correctly more often than the percentage split method.

\section{Ingenuity Pathway Analysis}

Lastly, Ingenuity Pathway Analysis (IPA) was used to evaluate the top 450 genes ranked based on Shannon Entropy (Information Gain) from the PM transcriptome using SMO and 150 of the top genes ranked based on Shannon Entropy (Information Gain) for the liver transcriptome using SMO. Interestingly, many of the top analysis ready molecules detected in IPA were the same molecules detected using the completely separate machine learning approach of MLP. The repeatability in identification of these genes leads to greater confidence in the role they are having in the WB myopathy. These included BUD13 from the

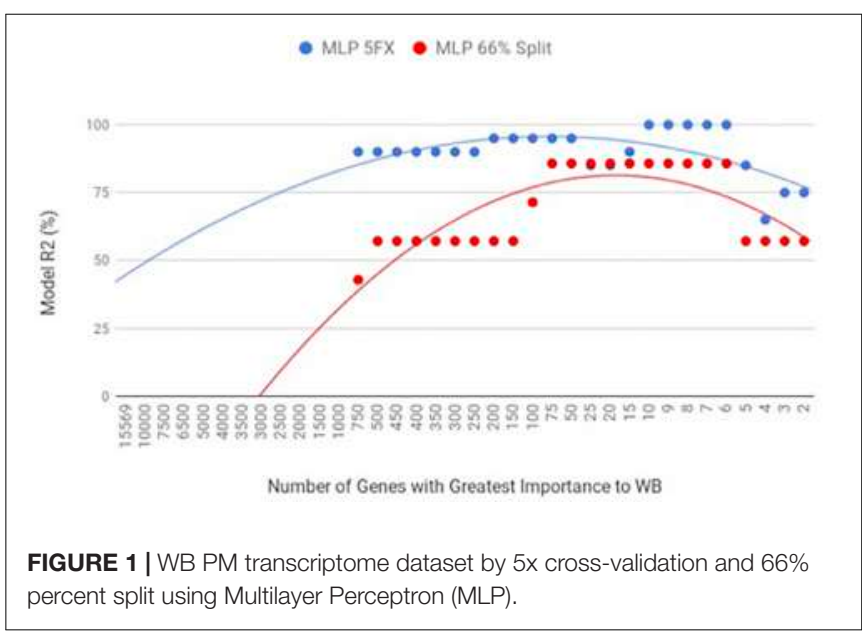




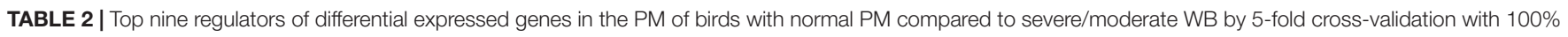
correct classification and percent split with $85.71 \%$ correct classification using MLP.

\begin{tabular}{|c|c|c|c|c|}
\hline Gene & Gene type & Short description & Log-fold change & $P$-value \\
\hline NUP43 & Protein Coding & Forms Nuclear Pore Complex (NPC) & 0.2005 & 0.4900 \\
\hline KPNA7 & Protein Coding & Forms Nuclear Pore Complex (NPC) & -3.4539 & $2.62 \mathrm{E}-06$ \\
\hline$D E A F 1$ & Protein Coding & Zinc Finger Domain Transcription Regulator; Inhibits Cell Proliferation & 0.3406 & 0.2972 \\
\hline NUDT19 & Protein Coding & Enzyme involved in Peroxisomal Lipid Metabolism & 0.9608 & $1.28 \mathrm{E}-04$ \\
\hline CCDC85A & Protein Coding & Unknown & 2.0774 & 0.0009 \\
\hline SLC25A30 & Protein Coding & Renal Mitochondrial Carrier & 0.1223 & 0.9574 \\
\hline ENSGALG00000015075 & Protein Coding & Beta-1,3-glucuronyltransferase 1 & -0.3370 & 0.5767 \\
\hline PACSIN3 & Protein Coding & Links Actin Cytoskeleton with Vesicle Formation & -0.9920 & $1.58 \mathrm{E}-04$ \\
\hline RPL19 & Protein Coding & Ribosomal Protein Component of the 60S Subunit & -0.8323 & 0.0066 \\
\hline
\end{tabular}

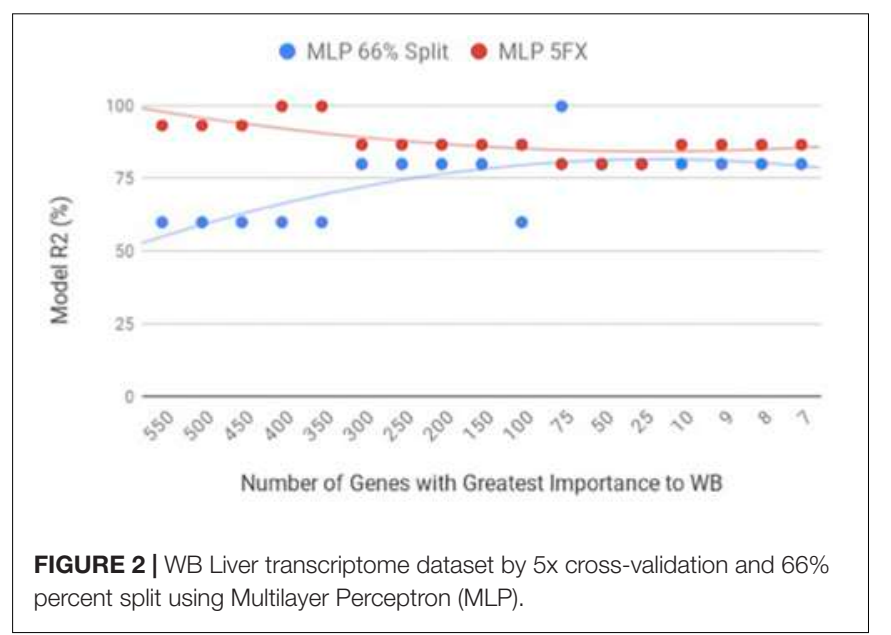

liver transcriptome dataset and CCDC85A and KPNA7 from the PM dataset (Tables 2, 3).

The top associated network function in IPA for the PM transcriptome dataset was for RNA Damage and Repair, Protein Synthesis. This pathway involves RPL19 which is a component of the 60S ribosomal subunit detected through MLP of WEKA (Figure 5). When evaluating the liver transcriptome dataset three top network associations identified were Skeletal and Muscular
System Development and Function, Developmental Disorder, Hereditary Disorder (Figure 6), Connective Tissue Disorders, Hematological Disease, Hereditary Disorder (Figure 7), and Cell Cycle, Embryonic Development, Cellular Movement (Figure 8). Figure 6 depicts CARD19 which was also detected using MLP of WEKA. This network is an association of the relationship this group of molecules has in association with the skeletal and muscular system development and function. Figure 8 represents molecules in the cell cycle such as TGF- $\beta, M A P 2 K 1 / 2$, and calcineurin proteins which are important in skeletal muscle myoblast regulation and differentiation.

When comparing the performance of the top ranked genes between MLP and SVM, many genes were identified as top performing in both ML models. In the PM data set, evaluation of the top nine molecules with increased expression, KPNA7 was observed in both MLP (100\% classification) and the top analysis ready molecules in IPA from the best performing data set using SVM (Table 2). RPL19 was observed as a top regulator in MLP with the ability to correctly classify WB or normal $100 \%$ of the time and was an affected molecule in the IPA analysis of the top associated network (Figure 5). In the same data set CCDC85A was observed as a molecule with decreased expression in both ML models and able to correctly classify WB $100 \%$ of the time using MLP (Table 2).

Assessing the liver dataset there were many overlaps using the two ML models. BUD13 was a top regulator in

TABLE 3 | Top regulators of differential expressed genes in the liver of birds with normal PM compared to severe/moderate WB tissues using MLP.

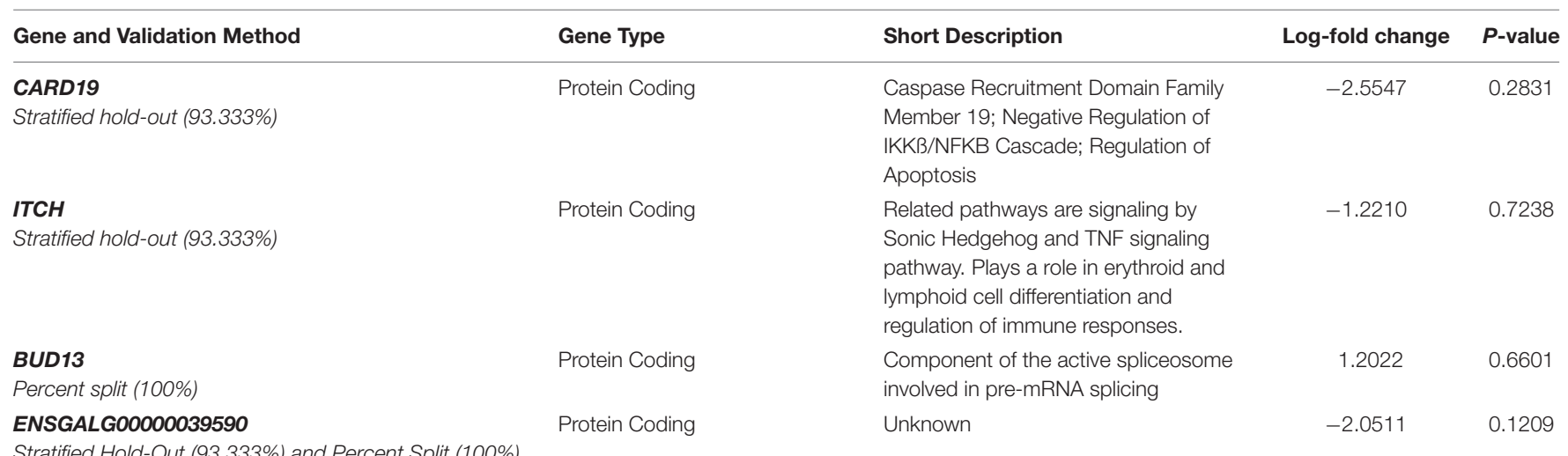



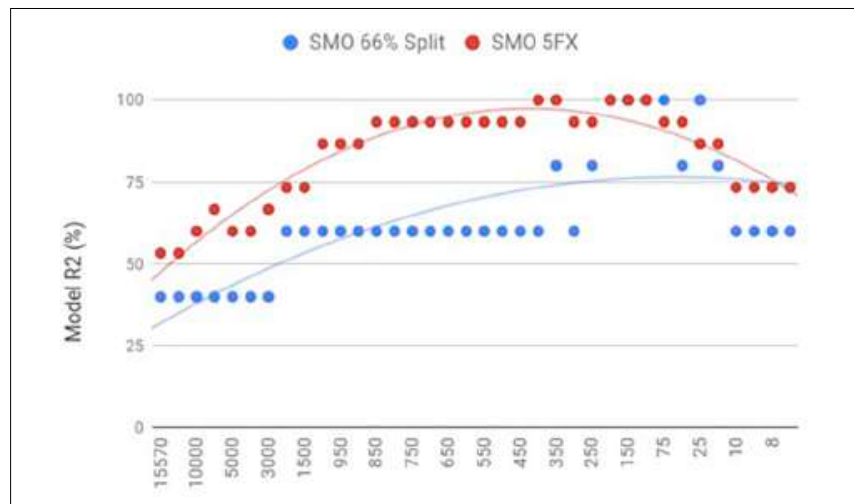

Number of Genes with Greatest Importance to WB

FIGURE 3 | WB PM transcriptome dataset by 5x cross-validation and 66\% percent split using Sequential Minimal Optimization (SMO).

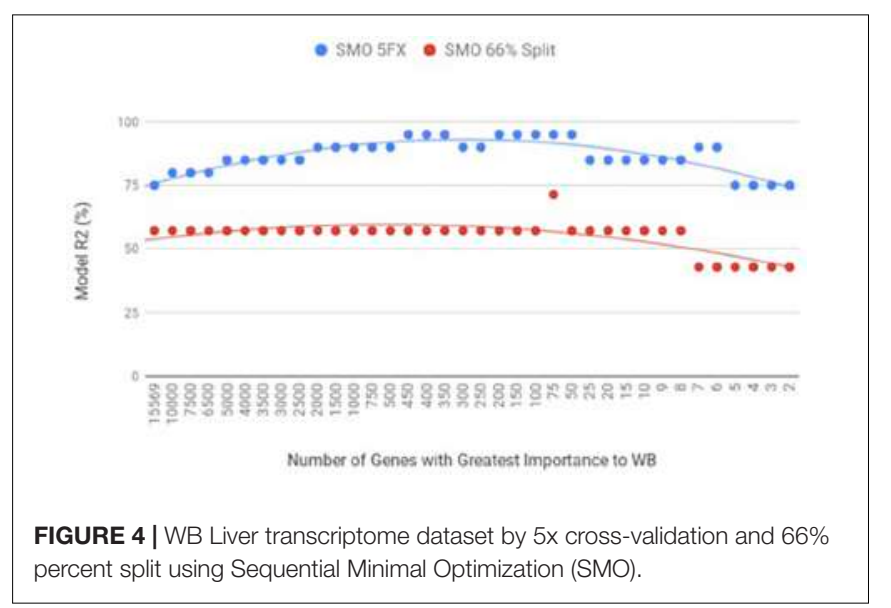

MLP and a top analysis ready molecule with increased expression in IPA (Table 3). The associated network related to skeletal and muscular system development and function from IPA had CARD19 as a downregulated molecule in IPA and a top regulator in MLP that was able to predict $\mathrm{WB}$ or normal $93.33 \%$ of the time (Figure 6). Lastly, the IPA network associated with cell cycle, embryonic development, and cellular movement included the down regulation of the ITCH molecule which was also detected in MLP as being able to correctly classify $93.33 \%$ of the time (Figure 8).

\section{DISCUSSION}

Prior systematic studies of the abnormal muscle phenotype referred to as wooden breast using gene expression measurements has identified multiple processes that may contribute to the development of the myopathy. Processes including muscle fiber regeneration, inflammatory response, myodegeneration, hypoxia, fibrosis, lipidosis, and altered energy metabolism are likely involved in the manifestation of wooden breast (Mutryn et al., 2015; Zambonelli et al., 2016;
Papah et al., 2018; Malila et al., 2019; Petracci et al., 2019). This study attempts to connect organismal level physiology and metabolism with the activity in the breast muscle by including the evaluation of liver gene expression by a novel analysis method.

\section{Machine Learning Approach}

Traditional statistics is not well designed to handle datasets which have more variables than observations therefore this is an alternative method to analyze and make interpretations of datasets. Using the traditional statistical approach on this dataset resulted in almost 1000 genes being identified as differentially expressed (FDR corrected $p<0.05$ ) across the etiology of WB in the PM and only one gene in the liver. This large number of targets (or too few) when subjected to pathway analysis in IPA results in more pathways (or none) than can be interpreted, therefore an alternative approach was warranted. The use of machine learning on the transcriptome datasets allows for the recognition of consistent patterns or systematic relationships within the datasets and therefore can be used to make predictions. Through this process, the machine learns by building a model from example inputs and then makes predictions on new data by the learned pattern recognition. This is the first report using machine learning to identify gene expression patterns associated with WB in muscle as well as peripheral tissues that may be influencing the myopathy development.

\section{PM Fibrosis}

Evaluating the top nine genes ranked based on Shannon Entropy (Information Gain), all the genes are protein coding genes (Table 2). The top two genes NUP43 and KPNA7 are two essential Nuclear Pore Complexes (NPC). KPNA7 was also observed as one of the top nine upregulated genes with a fold-change for birds with WB (Mutryn et al., 2015). NPC's are macromolecular proteins found within the nuclear envelope in eukaryotic cells. These complexes are surrounded by decondensed chromatin and are responsible for the exchange of large molecules such as proteins and RNA between the nucleus and cytoplasm (Kelley et al., 2010). Prior to cell division there is an increase in expression of NPC found in the nuclear envelope (NE). Once mitosis begins the NE is broken down and NPCs form subcomplexes, which are essential for later reassembly of the NPC. This process of reconstruction is unknown, however, it has been shown when the KPNA7 NPC is depleted, mitotic defects and deformation of the nucleus occur (Vuorinen et al., 2018). In the mouse, KPNA7 is mostly expressed in oocytes and zygotes and is responsible for epigenetic reprogramming which occurs during fertilization and zygotic gene activation (Hu et al., 2010). Recently, NPCs have been observed in regulation of gene expression and have been associated with both gene silencing and activation (Galy et al., 2000; Casolari et al., 2004). The expression of nucleoporins, which make up the NPCs, vary depending on cell types and changes in the structure of NPCs are used in the regulation of cellular differentiation (D’Angelo, 2018). In mammals, the tissue specific NPC, Nup210 has an effect on the regulation muscle development and maintenance. Nup210 regulates myofiber maturation, growth and even survival through the use of a 


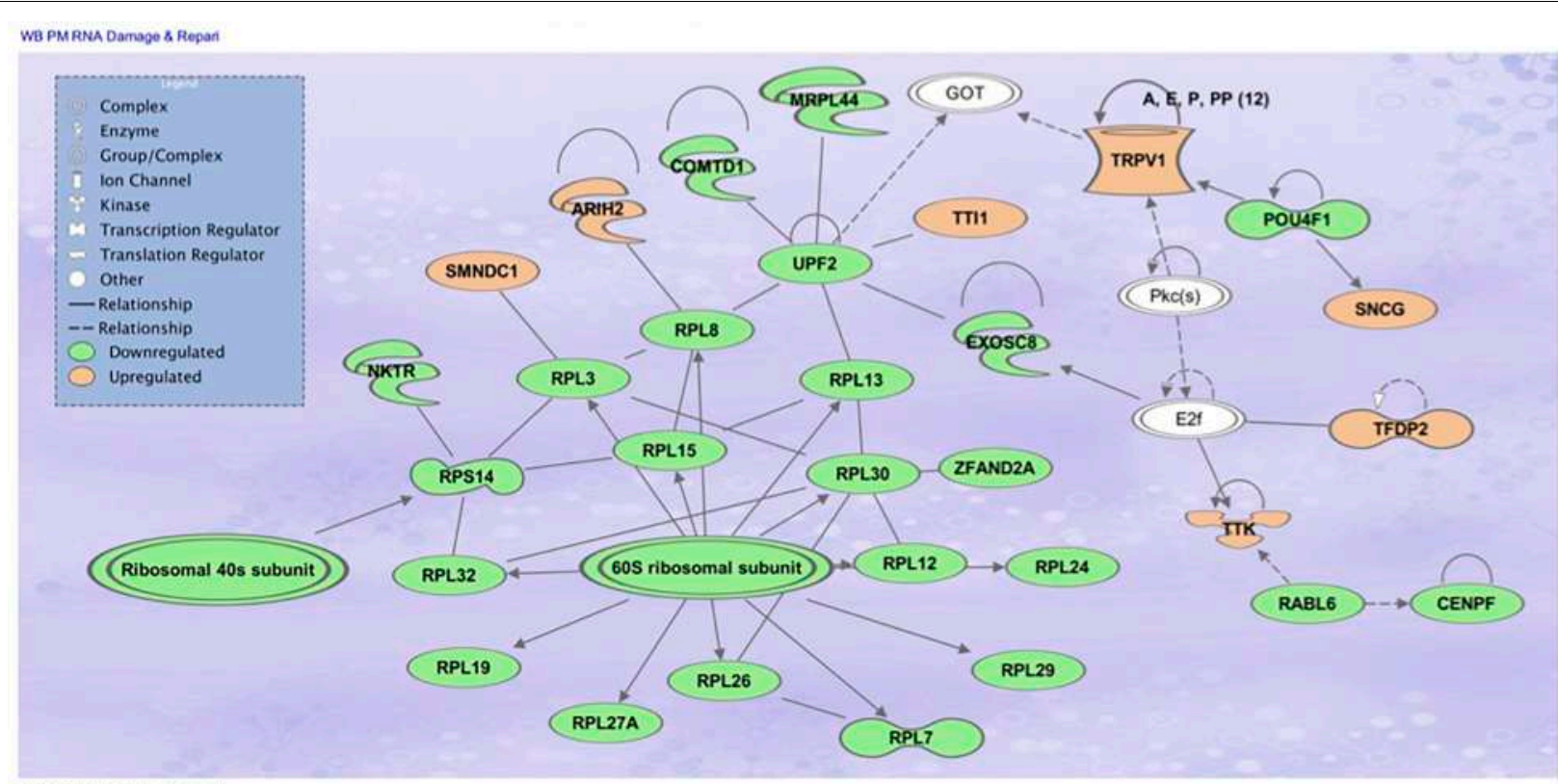

FIGURE 5 | Top associated network function in IPA for the PM transcriptome dataset of birds with normal PM compared to severe/moderate WB: RNA Damage and Repair and Protein Synthesis.

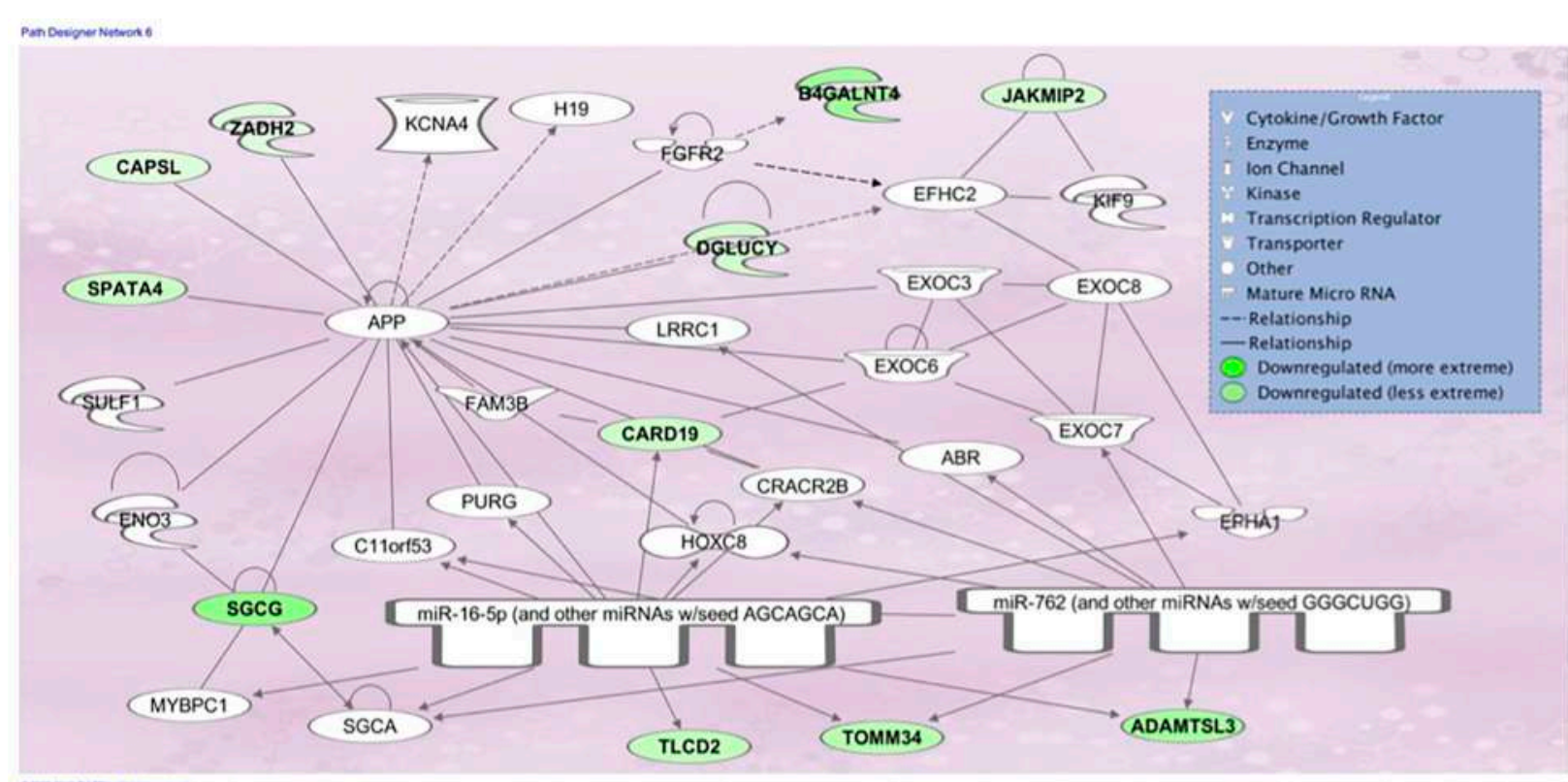

FIGURE 6 | Associated network function in IPA for the liver transcriptome dataset of birds with normal PM compared to severe/moderate WB: Skeletal and Muscular System Development and Function, Developmental Disorder, Hereditary Disorder.

dependent transcription factor $\mathrm{Mef} 2 \mathrm{C}$ in the regulation of structural and maturation related muscle genes (D'Angelo et al., 2012; Raices et al., 2017). In the absences of Nup210 the initial formation of muscle fibers occurs during development, however in older animals abnormal muscle structure develops and muscle degeneration can even occur (Raices et al., 2017).

\section{PM Regeneration}

Another top gene, DEAF1, is a part of the SAND domain in the molecule Ski. The activity of Ski was originally identified in the chicken as a transduced retroviral oncogene, however, research has indicated homologs which are not associated with endogenous viral loci ( $\mathrm{Li}$ et al., 1986). The c-Ski residue is 


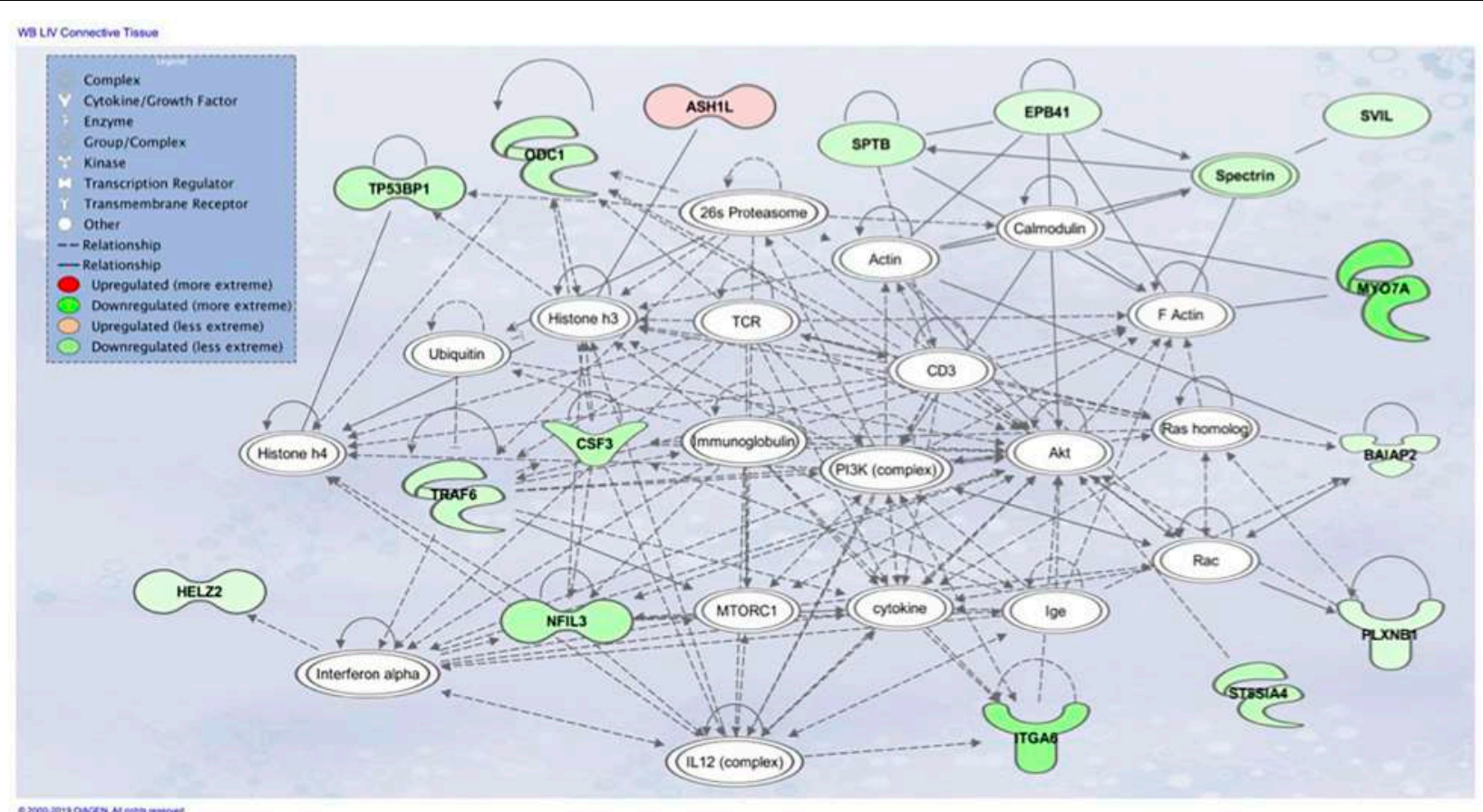

FIGURE 7| Associated network function in IPA for the liver transcriptome dataset of birds with normal PM compared to severe/moderate WB: Connective Tissue Disorders, Hematological Disease, Hereditary Disorder.

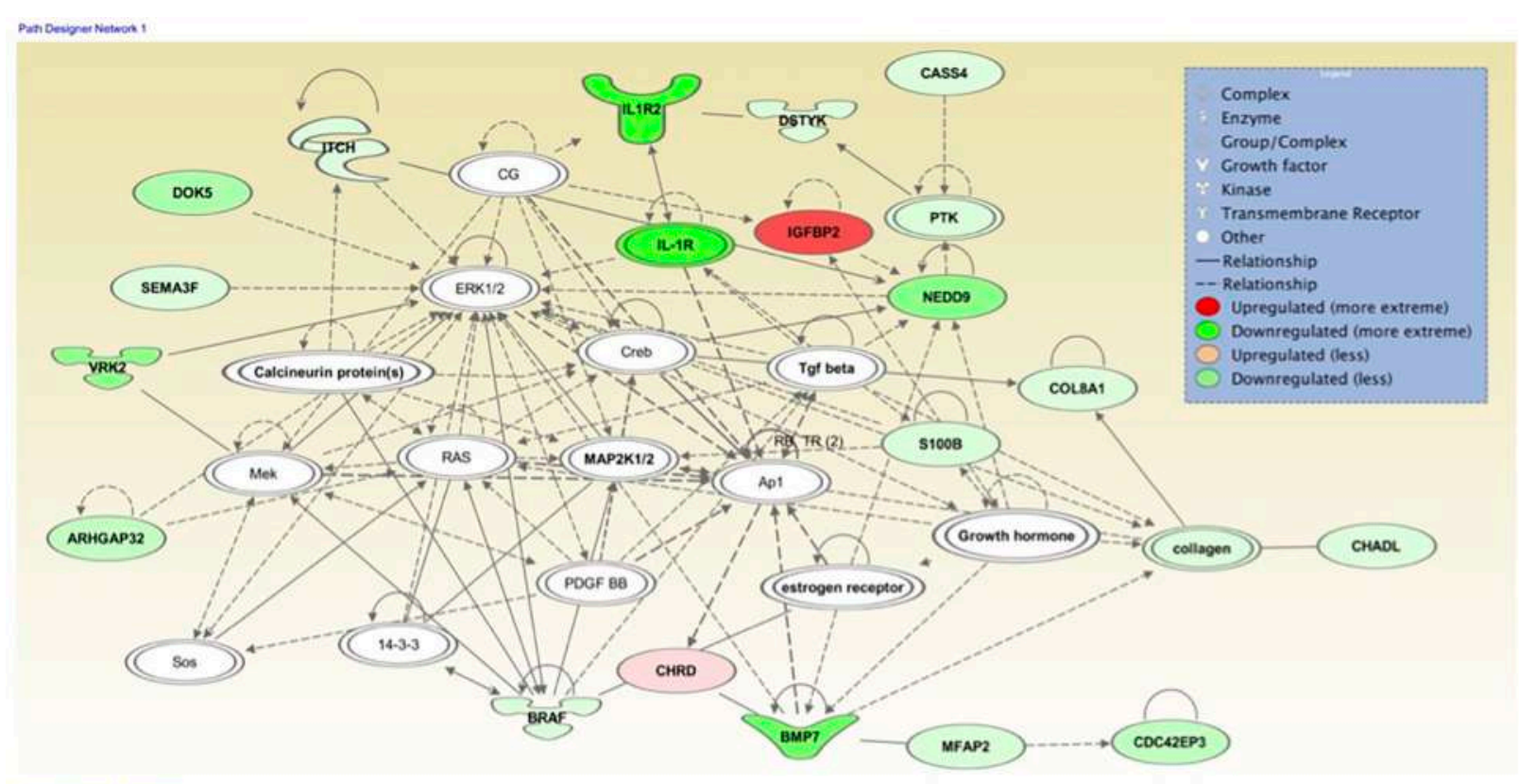

FIGURE 8| Associated network function in IPA for the liver transcriptome dataset of birds with normal PM compared to severe/moderate WB: Cell Cycle, Embryonic Development, Cellular Movement.

primarily found in the nucleus and is highly conserved in many species. The SAND domain is involved in protein-protein interactions and is responsible for the interactions of SKI with
Smad4, FHL2, and MeCP2 (Engle et al., 2008). Ski can act as an activator or repressor to gene transcription depending on the transcription factor it interacts with. C-Ski has been 
shown to bind with Smad4 and block activation of transforming growth factor (TGF- $\beta$ ). TGF- $\beta$ leads to an increase in $\beta$-catenin within the cytoplasm and $\beta$-catenin is an activator of canonical Wnt signaling (Staal, 2016). TGF- $\beta$, TNF- $\alpha$, and IGF-2 are growth factors that regulate myoblast differentiation rather than activation (Rosenthal et al., 1991; Li et al., 2005; Carter et al., 2009). The key role of TGF- $\beta$ in muscle repair is to regulate the balance between fibrosis and regeneration. In Japanese quail, myogenic differentiation is induced through the activation of myogenic regulatory factors (MRFs), MyoD, and myogenin as well as inhibiting HDAC activity (Colmenares and Stavnezer, 1989; Kobayashi et al., 2007). Satellite cells are activated by expression of early myogenic regulatory factors, MyoD and Myf5. Later, myogenin and MRF4 are expressed. The Ski response element to myogenin is located upstream of the promoter region.

The gene RPL19 was discovered as one of the top nine regulators in the MLP model able to $100 \%$ correctly classify PM samples as WB or normal sample. This protein coding gene encodes a ribosomal protein that makes up the $60 \mathrm{~S}$ ribosomal subunit (Davies and Fried, 1995). Like many of the genes observed, RPL19 also plays a role in genetic regulation. In mammals, this protein contains a $\mathrm{CpG}$ island at the $5^{\prime}$ transcriptional start site, which would indicate an area for modifications to the expression of this gene (Davies and Fried, 1995). RPL19 was also involved in the top molecular network associated with RNA damage and repair protein synthesis when evaluating the 450 attributes that were 95\% correctly classified using SMO through Ingenuity Pathway Analysis (IPA) (Figure 5). Transcripts related to spliceosomes were also detected in IPA analysis in Figure 6, involving the previously mentioned gene BUD13 as a component of the spliceosome. Similar to this finding previous research evaluating gene expression data in the PM has indicated differential expression of small nucleolar RNAs including snoRNAs and miRNAs, which are often involved in ribosomal and protein synthesis (Zambonelli et al., 2014).

CCDC85A, another top regulator identified with MLP, was able to correctly classify as a single attribute WB and normal PM birds $95 \%$ of the time using stratified cross-validation. CCDC85A was also a top analysis molecule when evaluating the 450 attributes that were 95\% correctly classified using SMO through Ingenuity Pathway Analysis (IPA). CCDC85A is a protein coding gene for coiled coil domain containing 85 a (Iwai et al., 2008). In humans, CCDC85A is regulated by p53 and results in the degradation of $\beta$-catenin. This protein suppresses $\beta$-catenin activity through interaction with T-cell factors to result in Wnt signaling (Iwai et al., 2008). $\beta$-catenin is an activator of canonical Wnt signaling (Staal, 2016). Wnt signaling is responsible for the activation of satellite cells in adult skeletal muscle and perturbations of this pathway can result in muscle fibrosis (Cisternas et al., 2014). If Wnt signaling occurs too often, the satellite cells become exhausted and eventually lose the ability to renew (Ryall et al., 2008). This has been characterized by increased extracellular matrix molecules such as fibronectin, collagen, and macrophages leading to the inability of muscles to regenerate and ultimately the loss of activity, leading to similar traits associated with WB (Cisternas et al., 2014). The addition of $\mathrm{Wnt} 3 \mathrm{~A}$ protein in mice has been shown to increase the rate at which progenitor cells are converted from a myogenic to a fibrogenic state resulting in increased deposition of connective tissue (Brack et al., 2007). The expression of myogenic regulatory factors $(\mathrm{MRF})$ responsible for normal formation of new myotubes, such as MyoD, Myf5, myogenin, and Pax3/7, are activated by Wnt (Yokoyama and Asahara, 2011). However, in the event that these progenitor cells lose the ability to mediate repair, the muscle tissue is replaced by adipose and fibrotic tissue, which also appears to be a phenotype associated with WB (Laumonier and Menetrey, 2016). Our findings were not the first to detect changes in Wnt signaling due to WB. Others have shown that WB results in statistically significant differential expression of WNT7A (Zambonelli et al., 2014). Wnt7a is responsible for stimulating skeletal muscle growth and repair through the induction of satellite cells via the mTOR pathway (Bentzinger et al., 2014). In contrast, others have found that there is an increase in gene expression of the MRF's however, they tend to vary depending on the lineage of the bird used (Velleman and Clark, 2015). This has led to a different understanding of the disease state which may not be entirely genetic or environmental but rather both, which is commonly referred to as epigenetic. It could be that environmental conditions stimulate pathways leading to genomic modifications, potentially resulting in phenotypic alterations.

Our working hypothesis, based on the ML analysis, is that the underlying mechanism resulting in fibrosis and hence, $\mathrm{WB}$, is related to genetic regulation, possibly through NPCs, CCDC85A, and $\beta$-catenin. These activate the Wnt signaling pathway via TGF- $\beta$, mTOR and IGF-II pathways, potentially resulting in WB pathology. It is possible that a pattern of Mendelian inheritance does not result in direct causation of WB, but rather modifications that result in changes in the expression of genes such as histone modifications and DNA methylation.

Following damage or rapid growth, skeletal muscle satellite cells are failing to regenerate myoblasts and results in fibrotic scar tissue; overall, because stem cells are restricted to a limited number of divisions, we hypothesize satellite cells are being exhausted and eventually resulting in WB (Sacco et al., 2010). This is similar to the hypothesis presented by Daughtry et al. (2017), who thought that a disruption in satellite cell homeostasis was involved in muscle myopathies. Throughout the life of an organism, the number of satellite cells available for regeneration of cells decreases. For satellite cells, aging has been characterized by delayed activation and the inability to proliferate and differentiate. A decrease in the efficiency of Wnt, TGF and IGF signaling pathways has been shown to limit satellite cell proliferation and myoblast differentiation (Barton-Davis et al., 1998; Carlson et al., 2009). It is known that fast twitch muscle is the leading muscle type found in the PM of broilers. Fast twitch muscle has fewer satellite cells than those of slow twitch resulting in differences in the course of muscle regeneration (Collins and Partridge, 2005). Differences depicted in fast twitch fibers include the TGF- $\beta$ expression pattern, early activation of the myogenic regulatory factors, and better regeneration efficiency (Zimowska et al., 2009, 2017). After injury, satellite cells are activated by expression of early myogenic regulatory factors, MyoD and Myf5. Next, late myogenic regulatory factors are expressed, 
which consist of myogenin and MRF4. Pax 3/7 are paired box transcription factors that directly and indirectly regulate myogeneic regulatory factors as skeletal muscle progenitor cells. Together these altered pathways are likely contributing to the development of the PM myopathy.

\section{Organismal Metabolic Influence - Liver Transcriptome}

The liver was considered as a tissue of importance related to WB due to the vast array of metabolic functions including the responsibility to synthesize, metabolize and excrete many molecules (Zaefarian et al., 2019). In the bird, $11 \%$ of all protein synthesis occurs in the liver which are then transported via systemic circulation to other tissues (Denbow, 2000). Evaluation of the liver transcriptome dataset using IPA resulted in the identification of Skeletal and Muscular System Development and Function, Developmental Disorder, Hereditary Disorder (Figure 6), Connective Tissue Disorders, Hematological Disease, Hereditary Disorder (Figure 7) and Cell Cycle, Embryonic Development, Cellular Movement (Figure 8) network associations. Similarly when evaluating the PM through IPA analysis of differential gene expression data, connective tissue disorders, embryonic development and cell cycle pathways have previously been detected (Mutryn et al., 2015).

The association network Skeletal and Muscular System Development and Function, Developmental Disorder, Hereditary Disorder (Figure 6) depicts molecules involved in muscle function and development such as SGCG which protects and maintains the structure of muscle cells through the sarcoglycan protein. In mammals, mutations of this gene result in the loss of $y$-sarcoglycan protein and ultimatley muscle dystrophy and fibrosis (Heydemann et al., 2009). Disfunction in SGCG has been shown to result in enhanced TGF- $\beta$ availability and therefore increased SMAD signaling leading to fibrosis (Heydemann et al., 2009). It is thought that proteins from this gene could be mediating their effect by regulating myostatin activity. Myostatin (MSTN), a family member of TGF- $\beta$, inhibits myoblast differentiation by repressing myogenic regulatory factors (Langley et al., 2002). MSTN prevents differentiation via the transcription factor SMAD3, which can be activated by both TGF- $\beta$ and MSTN. Other molecules identified in IPA with changes in expression due to WB were JAKMIP2, which is invovlved in microtubule binding and CAPSL which is involved in calcium ion binding. This IPA pathway has also been detected when evaluating the PM of differential gene experssion between male and females birds with WB (Brothers et al., 2019).

CARD19 in this molecular network was also detected in MLP of WEKA as being able to predict normal or WB $93.333 \%$ using the cross-validation method (Figure 8 and Table 3). CARD proteins (caspase recruitment domain) are a domain of proteins which regulate apoptosis and inflammation (Jang et al., 2015). Studies evaluating CARD19 and its role in the IKKB and NF-kB pathway have been contradictory. Early data suggests CARD19 is a negative regulator of $\mathrm{NF}-\mathrm{kB}$, which is a transcription factors that signals IKK, however, a more recent study in mice suggests in the absence of CARD19 there was an increase in TNF- $\alpha$ which would subsequently increase IKKß and NF-kB (Rios et al., 2018).
IKKß has been shown to decrease $\beta$-catenin activation which as previously mentioned is an activator canonical Wnt signaling (Lamberti et al., 2001; Staal, 2016).

Downstream TNF- $\alpha$ kinases IKKß and NF-kB play many roles in regulating physiological reactions including regulators of tuberous sclerosis complex (TSC), which repress the mechanistic Target of Rapamycin (mTOR) pathway (Lee et al., 2007). IKKß in association with TSC allows for the activation of mTOR. MTOR coordinates cell growth and is the major regulator of metabolic processes (Figure 7). Many factors are responsible for the activation of mTORC1 including Wnt signaling, growth factors, and TNF- $\alpha$ through interaction with TSC (Laplante and Sabatini, 2009). Activation of mTORC1 positively results in cell growth and proliferation through the activation of protein and lipid synthesis pathways. Disruptions in these pathways is associated with tumor development and fibrogenesis, and macrophage regulation. Ribosome biogenesis has been observed to be promoted through activation of mTORC1 by transcription of ribosomal RNA which can be observed as being downregulated in Figure 5 and Table 3 of the PM dataset (Mayer et al., 2004). Much of the IPA network association Connective Tissue Disorders, Hematological Disease, Hereditary Disorder invovles moleculues related to cell adhesion and cytoskeleton (Figure 7). Intersestingly, mTORC2 regulates cytoskeleton organization (Jacinto et al., 2004).

Cell Cycle, Embryonic Development, Cellular Movement (Figure 8) network association represents molecules in the cell cycle such as: ITCH, NEDD9, DOK5, and IGFBP2. ITCH in this molecular network was also detected in MLP of WEKA as being able to predict normal or WB $93.333 \%$ using the cross-validation method. The Itch protein encodes a member of the Nedd4 family ubiquitin ligases that targets specific proteins for lysosomal degradation. Itch plays a role in lymphoid cell differentiation and the regulation of immune response and pathways related to this protein include the TNF- $\alpha$ signaling pathway. NEDD9 plays a role in the TGF- $\beta$ pathway and growth signals initiating cellular proliferation and has been identified as upregulated in hepatic fibrosis (Dooley et al., 2008). DOK5 in humans is strongly expressed in muscle and is involved in the positive activation of MAPK and possibly insulin activation (Grimm et al., 2001; Cai et al., 2003). DOK5 has been identified as a membrane associated protein triggered by insulin-like growth factor binding protein-5 (IGFBP-5) for intracellular signaling resulting in pro-fibrotic effects and is thought to promote fibrosis (Yasuoka et al., 2014). IGFBP2 is an insulin-like growth factor binding protein involved in cellular signaling. While muscle is the primarily a site for glucose disposal, the avian liver does function to control muscle growth through the allocation of resources by regulating the birds nutritional balance. The muscle has a paracrine effect, whereas the liver has an endocrine role in circulating IGFs and IGFBPs. Signaling pathways in the liver play an important role in regulating many aspects of energy metabolism and cell cycle processes (Nguyen et al., 2014). Circulating molecules have been identified that play a role of signaling between the liver and muscle (Liu et al., 2013). Some signaling pathways are only responsive within the liver while others are only responsive in the muscle (proliferation and differentiation). Most of the IPA pathways identified resulted in an effect which would be detected within skeletal muscle. 
Liver and muscle are important tissues in understanding regulation of metabolic homeostasis, genes involved in glycogenesis, glycolysis, and lipogenesis are responsive in both liver and muscle even though the expression patterns are very different between the two tissues. It is not unexpected to observe effects of the liver on muscle development since the liver is the major location of protein, lipid, and carbohydrate metabolism supporting the rapid growth of broiler chickens.

Histological evaluation of WB has revealed multifocal degeneration and necrosis of fibers and accumulation of immune related cells such as macrophages, heterophils and lymphocytes (Sihvo et al., 2014; Trocino et al., 2015; Kuttappan et al., 2016). Affected areas have characteristics of fibrosis separating muscle fibers and thickening of the interstitium. Fibrosis has been characterized as hardening or scaring of tissue as a result of the accumulation of the extra cellular matrix proteins, including collagen and fibronectin eventually leading to loss of activity to the tissue (Wynn, 2008). Fibrosis has been detected in various tissues lung, liver, kidney and skeletal muscle, however, the mechanism resulting in fibrosis has been similar in tissues (Cisternas et al., 2014). Pathways detected through IPA of the liver and PM have indicated many molecules which directly and indirectly lead to tissue fibrosis. The fibrosis associated with WB may be driven by signal(s) originating in the liver or other tissues.

\section{CONCLUSION}

In conclusion, using a machine learning approach, we were able to identify predictors that were able to accurately differentiate normal tissue from WB tissue using liver and PM transcriptomes from individual birds. Through the use of IPA, predictors from both PM and liver tissue identified gene networks associated with skeletal muscle disorders and other networks that could be associated with the development of WB. Given that gene expression data from both PM and liver transcriptomes were able to predict $\mathrm{WB}$ or normal tissue using select genes, with some redundancy between tissues, suggests that WB is the result of systematic disruptions in one or more regulatory pathways involving abnormal muscle development, deposition, or maintenance. The data herein suggests that WB phenotype could potentially be mediated through genes which ultimately result in the up- or down- regulation of pathways that are

\section{REFERENCES}

Abasht, B., Zhou, N., Lee, W. R., Zhuo, Z., and Peripolli, E. (2019). The metabolic characteristics of susceptibility to wooden breast disease in chickens with high feed efficiency. Poult. Sci. 98, 3246-3256. doi: 10.3382/ps/ pez183

Barton-Davis, E. R., Shoturma, D. I., Musaro, A., Rosenthal, N., and Sweeney, H. L. (1998). Viral mediated expression of insulin-like growth factor I blocks the aging-related loss of skeletal muscle function. Proc. Natl. Acad. Sci. U.S.A. 95, 15603-15607. doi: 10.1073/pnas.95.26.15603

Bentzinger, C. F., Maltzahn, J., von Dumont, N. A., Stark, D. A., Wang, Y. X., Nhan, K., et al. (2014). Wnt7a stimulates myogenic stem cell motility and engraftment resulting in improved muscle strength. J. Cell Biol. 205, 97-111. doi: $10.1083 /$ JCB.201310035 largely involved with metabolic regulation and basic cellular maintenance, such as Wnt and mTOR, respectively. In mammals, dysregulation in either of these canonical pathways has been shown to result in similar characteristics identified in WB and further investigation of these pathways in chickens exhibiting WB is warranted.

\section{DATA AVAILABILITY STATEMENT}

The datasets generated and analyzed for this study can be found in the NCBI-Gene Expression Omnibus database under Accession Series GSE144000: https://www.ncbi.nlm.nih.gov/geo/ query/acc.cgi? acc $=$ GSE144000.

\section{ETHICS STATEMENT}

The animal study was reviewed and approved by the NC State Institutional Animal Care and Use Committee, 15-061-A, approved on $07 / 06 / 2015$.

\section{AUTHOR CONTRIBUTIONS}

ML, KL, and CA conceived and designed the experiment. ML and KL performed the animal experiment. CA managed the RNA sequencing data collection and analysis. CP and BR analyzed the data. CP drafted the manuscript and it was revised and reviewed by BR, ML, KL, and CA.

\section{FUNDING}

This project was supported by a grant from United States Poultry and Egg Association (2016-0126).

\section{ACKNOWLEDGMENTS}

The authors would like to thank those who contributed to this project including Shelly Nolin, Allysa Stern, and Coltin Caraway for their support and guidance.

Brack, A. S., Conboy, M. J., Roy, S., Lee, M., Kuo, C. J., Keller, C., et al. (2007). Increased wnt signaling during aging alters muscle stem cell fate. Science 129:2865. doi: 10.1126/science. 1144090

Brothers, B., Zhuo, Z., Papah, M. B., and Abasht, B. (2019). RNA-Seq analysis reveals spatial and sex differences in pectoralis major muscle of broiler chickens contributing to difference in susceptibility to wooden breast disease. Front. Physiol. 10:764. doi: 10.3389/fphys.2019.00764

Cai, D., Dhe-Paganon, S., Melendez, P. A., Lee, J., and Shoelson, S. E. (2003). Two new substrates in insulin signaling, IRS5/DOK4 and IRS6/DOK5. J. Biol. Chem. 278, 25323-25330. doi: 10.1074/jbc.M212430200

Carlson, M. E., Conboy, M. J., Hsu, M., Barchas, L., Jeong, J., Agrawal, A., et al. (2009). Relative roles of TGF- $\beta 1$ and Wnt in the systemic regulation and aging of satellite cell responses. Aging Cell 8, 676-689. doi: 10.1111/j.1474-9726.2009. 00517.x 
Carter, E. J., Cosgrove, R. A., Gonzalez, I., Eisemann, J. H., Lovett, F. A., Cobb, L. J., et al. (2009). MEK5 and ERK5 are mediators of the pro-myogenic actions of IGF-2. J. Cell Sci. 122, 3104-3112. doi: 10.1242/jcs.045757

Casolari, J. M., Brown, C. R., Komili, S., West, J., Hieronymus, H., and Silver, P. A. (2004). Genome-wide localization of the nuclear transport machinery couples transcriptional status and nuclear organization. Cell 117, 427-439. doi: 10.1016/S0092-8674(04)00448-9

Cisternas, P., Henriquez, J. P., Brandan, E., and Inestrosa, N. C. (2014). Wnt signaling in skeletal muscle dynamics: myogenesis, neuromuscular synapse and fibrosis. Mol. Neurobiol. 49, 574-589. doi: 10.1007/s12035-013-8540-5

Collins, C. A., and Partridge, T. A. (2005). Self-renewal of the adult skeletal muscle satellite cell. Cell Cycle 4, 1338-1341. doi: 10.4161/cc.4.10.2114

Colmenares, C., and Stavnezer, E. (1989). The ski oncogene induces muscle differentiation in quail embryo cells. Cell 59, 293-303. doi: 10.1016/00928674(89)90291-2

Cortes, C., and Vapnik, V. (1995). Support-vector networks. Mach. Learn. 20, 273-297.

D’Angelo, M. A. (2018). Nuclear pore complexes as hubs for gene regulation. Nucleus 9, 142-148. doi: 10.1080/19491034.2017.1395542

D’Angelo, M. A., Gomez-Cavazos, J. S., Mei, A., Lackner, D. H., and Hetzer, M. W. (2012). A change in nuclear pore complex composition regulates cell differentiation. Dev. Cell 22, 446-458. doi: 10.1016/J.DEVCEL.2011.11.021

Daughtry, M. R., Berio, E., Shen, Z., Suess, E. J. R., Shah, N., Geiger, A. E., et al. (2017). Satellite cell-mediated breast muscle regeneration decreases with broiler size. Poult. Sci. 96, 3457-3464. doi: 10.3382/ps/pex068

Davies, B., and Fried, M. (1995). The L19 ribosomal protein gene (RPL19): gene organization, chromosomal mapping, and novel promoter region. Genomics 25, 372-380. doi: 10.1016/0888-7543(95)80036-L

Denbow, D. M. (2000). "Gastrointestinal anatomy and physiology," in Sturkie's Avian Physiology, 5th Edn, ed. E. Whittow, (New York, NY: Academic Press).

Dooley, S., Hamzavi, J., Ciuclan, L., Godoy, P., Ilkavets, I., Ehnert, S., et al. (2008). Hepatocyte-specific Smad7 expression attenuates TGF- $\beta$-mediated fibrogenesis and protects against liver damage. Gastroenterology 135, 642-659. doi: 10.1053/j.gastro.2008.04.038

Eibe, F., Hall, M. A., and Witten, I. H. (2016). The WEKA Workbench. Online Appendix for Data Mining: Practical Machine Learning Tools and Techniques. San Francisco, CA: Morgan Kaufmann Publishers Inc.

Engle, K. M., Mei, T.-S., Wasa, M., and Yu, J.-Q. (2008). NIH public access. Acc. Chem. Res. 45, 788-802. doi: 10.1038/jid.2014.371

Galy, V., Olivo-Marin, J.-C., Scherthan, H., Doye, V., Rascalou, N., and Nehrbass, U. (2000). Nuclear pore complexes in the organization of silent telomeric chromatin. Nature 403, 108-112. doi: 10.1038/47528

Grimm, J., Sachs, M., Britsch, S., Di Cesare, S., Schwarz-Romond, T., Alitalo, K., et al. (2001). Novel p62dok family members, dok-4 and dok-5, are substrates of the c-Ret receptor tyrosine kinase and mediate neuronal differentiation. J. Cell Biol. 154, 345-354. doi: 10.1083/jcb.200102032

Heydemann, A., Ceco, E., Lim, J. E., Hadhazy, M., Ryder, P., Moran, J. L., et al. (2009). Latent TGF-beta-binding protein 4 modifies muscular dystrophy in mice. J. Clin. Invest. 119, 3703-3712. doi: 10.1172/JCI39845

Hu, J., Wang, F., Yuan, Y., Zhu, X., Wang, Y., Zhang, Y., et al. (2010). Novel importin- $\alpha$ family member Kpna7 is required for normal fertility and fecundity in the mouse. J. Biol. Chem. 285, 33113-33122. doi: 10.1074/jbc.M110.117044

Iwai, A., Hijikata, M., Hishiki, T., Isono, O., Chiba, T., and Shimotohno, K. (2008). Coiled-coil domain containing $85 \mathrm{~B}$ suppresses the $\beta$-catenin activity in a p53-dependent manner. Oncogene 27, 1520-1526. doi: 10.1038/sj.onc.1210801

Jacinto, E., Loewith, R., Schmidt, A., Lin, S., Rüegg, M. A., Hall, A., et al. (2004). Mammalian TOR complex 2 controls the actin cytoskeleton and is rapamycin insensitive. Nat. Cell Biol. 6, 1122-1128. doi: 10.1038/ncb1183

Jang, T. H., Kim, S. H., Jeong, J. H., Kim, S., Kim, Y. G., and Park, H. H. (2015). Crystal structure of caspase recruiting domain (CARD) of apoptosis repressor with CARD (ARC) and its implication in inhibition of apoptosis. Sci. Rep. 5:9847. doi: 10.1038/srep09847

Keerthi, S. S., Shevade, S. K., Bhattacharyya, C., and Murthy, K. R. K. (2001). "Improvements to platt's SMO algorithm for SVM classifier design," in Proceedings of the Neural Computation (Germany: MITP), 637-649.

Kelley, J. B., Talley, A. M., Spencer, A., Gioeli, D., and Paschal, B. M. (2010). Karyopherin alpha7 (KPNA7), a divergent member of the importin alpha family of nuclear import receptors. BMC Cell Biol. 11:63. doi: 10.1186/14712121-11-63

Kobayashi, N., Goto, K., Horiguchi, K., Nagata, M., Kawata, M., Miyazawa, K., et al. (2007). c-Ski activates MyoD in the nucleus of myoblastic cells through suppression of histone deacetylases. Genes Cell 12, 375-385. doi: 10.1111/j. 1365-2443.2007.01052.x

Kuttappan, V. A., Hargis, B. M., and Owens, C. M. (2016). White striping and woody breast myopathies in the modern poultry industry: a review. Poult. Sci. 95, 2724-2733. doi: 10.3382/ps/pew216

Kuttappan, V. A., Owens, C. M., Coon, C., Hargis, B. M., and Vazquez-A Non, M. (2017). Research Note Incidence of broiler breast myopathies at 2 different ages and its impact on selected raw meat quality parameters. Poult. Sci. 96, 3005-3009. doi: 10.3382/ps/pex072

Lamberti, C., Lin, K.-M., Yamamoto, Y., Verma, U., Verma, I. M., Byers, S., et al. (2001). Regulation of-catenin function by the IB Kinases*. J. Biol. Chem. 276, 42276-42286. doi: 10.1074/jbc.M104227200

Langley, B., Thomas, M., Bishop, A., Sharma, M., Gilmour, S., and Kambadur, R. (2002). Myostatin inhibits myoblast differentiation by down-regulating MyoD expression. J. Biol. Chem. 277, 49831-49840. doi: 10.1074/JBC.M204291200

Laplante, M., and Sabatini, D. M. (2009). mTOR signaling at a glance. J. Cell Sci. 122, 3589-3594. doi: 10.1242/jcs.051011

Laumonier, T., and Menetrey, J. (2016). Muscle injuries and strategies for improving their repair. J. Exp. Orthop. 3:15. doi: 10.1186/s40634-016-0051-7

Lee, D.-F., Kuo, H.-P., Chen, C.-T., Hsu, J.-M., Chou, C.-K., Wei, Y., et al. (2007). IKK $\beta$ suppression of TSC1 links inflammation and tumor angiogenesis via the mTOR pathway. Cell 130, 440-455. doi: 10.1016/J.CELL.2007.05.058

Li, Y., Turck, C. M., Teumer, J. K., and Stavnezert, E. (1986). Unique sequence, ski, in sloan-kettering avian retroviruses with properties of a new cellderived oncogene. J. Virol. 57, 1065-1072. doi: 10.1128/jvi.57.3.1065-1072. 1986

Li, Y.-P., Chen, Y., John, J., Moylan, J., Jin, B., Mann, D. L., et al. (2005). TNF- $\alpha$ acts via 38 MAPK to stimulate expression of the ubiquitin ligase atrogin1/MAFbx in skeletal muscle. FASEB J. 19, 362-370. doi: 10.1096/fj.04-2364com

Liu, S., Brown, J. D., Stanya, K. J., Homan, E., Leidl, M., Inouye, K., et al. (2013). A diurnal serum lipid integrates hepatic lipogenesis and peripheral fatty acid use. Nature 502, 550-554. doi: 10.1038/nature12710

Livingston, M. L., Ferket, P. R., Brake, J., and Livingston, K. A. (2018a). Dietary amino acids under hypoxic conditions exacerbates muscle myopathies including wooden breast and white stripping. Poult. Sci. 98, 1517-1527. doi: $10.3382 / \mathrm{ps} /$ pey463

Livingston, M. L., Landon, C., Barnes, H. J., and Brake, J. (2018b). White striping and wooden breast myopathies of broiler breast muscle is affected by timelimited feeding, genetic background, and egg storage. Poult. Sci. 98, 217-226. doi: $10.3382 / \mathrm{ps} /$ pey333

Malila, Y., Thanatsang, K., Arayamethakorn, S., Uengwetwanit, T., Srimarut, Y., Petracci, M., et al. (2019). Absolute expressions of hypoxia-inducible factor-1 alpha (HIF1A) transcript and the associated genes in chicken skeletal muscle with white striping and wooden breast myopathies. PLoS One 14:e0220904. doi: 10.1371/journal.pone.0220904

Mayer, C., Zhao, J., Yuan, X., and Grummt, I. (2004). mTOR-dependent activation of the transcription factor TIF-IA links rRNA synthesis to nutrient availability. Genes Dev. 18, 423-434. doi: 10.1101/gad.285504

Mudalal, S., Babini, E., Cavani, C., and Petracci, M. (2014). Quantity and functionality of protein fractions in chicken breast fillets affected by white striping. Poult. Sci. 93, 2108-2116. doi: 10.3382/ps.2014-03911

Mutryn, M. F., Brannick, E. M., Fu, W., Lee, W. R., and Abasht, B. (2015). Characterization of a novel chicken muscle disorder through differential gene expression and pathway analysis using RNA-sequencing. BMC Genom. 16:399. doi: 10.1186/s12864-015-1623-0

Nguyen, T. T., Almon, R. R., Dubois, D. C., Sukumaran, S., Jusko, W. J., and Androulakis, I. P. (2014). Tissue-specific gene expression and regulation in liver and muscle following chronic corticosteroid administration. Gene Regul. Syst. Biol. 8, 75-87. doi: 10.4137/GRSB.S13134

Papah, M. B., Brannick, E. M., Schmidt, C. J., and Abasht, B. (2017). Evidence and role of phlebitis and lipid infiltration in the onset and pathogenesis of wooden breast disease in modern broiler chickens. Avian Pathol. 46, 623-643. doi: 10.1080/03079457.2017.1339346 
Papah, M. B., Brannick, E. M., Schmidt, C. J., and Abasht, B. (2018). Gene expression profiling of the early pathogenesis of wooden breast disease in commercial broiler chickens using RNA-sequencing. PLoS One 13:e0207346. doi: 10.1371/journal.pone.0207346

Petracci, M., Mudalal, S., Soglia, F., and Cavani, C. (2015). Meat quality in fastgrowing broiler chickens. Worlds Poult. Sci. J. 71, 363-374. doi: 10.1017/ S0043933915000367

Petracci, M., Soglia, F., Madruga, M., Carvalho, L., Ida, E., and Estévez, M. (2019). Wooden-breast, white striping, and spaghetti meat: causes, consequences and consumer perception of emerging broiler meat abnormalities. Compr. Rev. Food Sci. Food Saf. 18, 565-583. doi: 10.1111/1541-4337.12431

Raices, M., Bukata, L., Sakuma, S., Borlido, J., Hernandez, L. S., Hart, D. O., et al. (2017). Nuclear pores regulate muscle development and maintenance by assembling a localized Mef2C complex. Dev. Cell 41, 540-554. doi: 10.1016/j. devcel.2017.05.007

Rios, K. E., Liem, A., Kashyap, A., Maynard, S., Paul, S., and Schaefer, B. C. (2018). CARD19: a mitochondrial caspase recruitment domain protein with a role in regulating pro-inflammatory innate immune responses. J. Immunol. 200:169. doi: 10.1007/3-540-30683-8_249

Rosenthal, S. M., Brunetti, A., Brown, E. J., Mamula, P. W., and Goldfine, I. D. (1991). Regulation of insulin-like growth factor (IGF) I receptor expression during muscle cell differentiation. Potential autocrine role of IGF-II. J. Clin. Invest. 87, 1212-1219. doi: 10.1172/JCI115121

Ryall, J. G., Schertzer, J. D., and Lynch, G. S. (2008). Cellular and molecular mechanisms underlying age-related skeletal muscle wasting and weakness. Biogerontology 9, 213-228. doi: 10.1007/s10522-008-9131-0

Sacco, A., Mourkioti, F., Tran, R., Choi, J., Llewellyn, M., Kraft, P., et al. (2010). Short telomeres and stem cell exhaustion model duchenne muscular dystrophy in mdx/mTR mice. Cell 143, 1059-1071. doi: 10.1016/j.cell.2010. 11.039

Sihvo, H. K., Immonen, K., and Puolanne, E. (2014). Myodegeneration with fibrosis and regeneration in the pectoralis major muscle of broilers. Vet. Pathol. 51, 619-623. doi: 10.1177/0300985813497488

Staal, F. J. T. (2016). Wnt signalling meets epigenetics. Stem Cell Invest. 3, 38-38. doi: $10.21037 /$ sci.2016.08.01

Trocino, A., Piccirillo, A., Birolo, M., Radaelli, G., Bertotto, D., Filiou, E., et al. (2015). Effect of genotype, gender and feed restriction on growth, meat quality and the occurrence of white striping and wooden breast in broiler chickens. Poult. Sci. 94, 2996-3004. doi: 10.3382/ps/pev296

Velleman, S. G., and Clark, D. L. (2015). Histopathologic and myogenic gene expression changes associated with wooden breast in broiler breast muscles. Avian Dis. 59, 410-418. doi: 10.1637/11097-042015-Reg.1
Vuorinen, E. M., Rajala, N. K., Ihalainen, T. O., and Kallioniemi, A. (2018) Depletion of nuclear import protein karyopherin alpha 7 (KPNA7) induces mitotic defects and deformation of nuclei in cancer cells. BMC Cancer 18:325. doi: 10.1186/s12885-018-4261-5

Wynn, T. (2008). Cellular and molecular mechanisms of fibrosis. J. Pathol. 214, 199-210. doi: 10.1002/path.2277

Yasuoka, H., Yamaguchi, Y., and Feghali-Bostwick, C. A. (2014). The membraneassociated adaptor protein DOK5 is upregulated in systemic sclerosis and associated with IGFBP-5-induced fibrosis. PLoS One 9:e87754. doi: 10.1371/ journal.pone.0087754

Yokoyama, S., and Asahara, H. (2011). The myogenic transcriptional network. Cell. Mol. Life Sci. 68, 1843-1849. doi: 10.1007/s00018-011-0629-2

Zaefarian, F., Abdollahi, M. R., Cowieson, A., and Ravindran, V. (2019). Avian liver: the forgotten organ. Animal 9:E63. doi: 10.3390/ANI9020063

Zambonelli, P., Zappaterra, M., Soglia, F., Petracci, M., Sirri, F., Cavani, C., et al. (2014). Genetics and genomics detection of differentially expressed genes in broiler pectoralis major muscle affected by white striping-wooden breast myopathies. Poult. Sci. 95, 2771-2785. doi: 10.3382/ps/pew268

Zambonelli, P., Zappaterra, M., Soglia, F., Petracci, M., Sirri, F., Cavani, C., et al. (2016). Detection of differentially expressed genes in broiler pectoralis major muscle affected by white striping - wooden breast myopathies. Poult. Sci. 95, 2771-2785. doi: 10.3382/ps/pew268

Zimowska, M., Duchesnay, A., Dragun, P., Oberbek, A., Moraczewski, J., and Martelly, I. (2009). Immunoneutralization of TGF $\beta 1$ improves skeletal muscle regeneration: effects on myoblast differentiation and glycosaminoglycan content. Int. J. Cell Biol. 2009, 1-16. doi: 10.1155/2009/659372

Zimowska, M., Kasprzycka, P., Bocian, K., Delaney, K., Jung, P., Kuchcinska, K., et al. (2017). Inflammatory response during slow- and fast-twitch muscle regeneration. Muscle Nerve 55, 400-409. doi: 10.1002/mus. 25246

Conflict of Interest: The authors declare that the research was conducted in the absence of any commercial or financial relationships that could be construed as a potential conflict of interest.

Copyright (c) 2020 Phillips, Reading, Livingston, Livingston and Ashwell. This is an open-access article distributed under the terms of the Creative Commons Attribution License (CC BY). The use, distribution or reproduction in other forums is permitted, provided the original author(s) and the copyright owner(s) are credited and that the original publication in this journal is cited, in accordance with accepted academic practice. No use, distribution or reproduction is permitted which does not comply with these terms. 


\title{
Glucolipotoxicity: A Proposed Etiology for Wooden Breast and Related Myopathies in Commercial Broiler Chickens
}

\author{
Juniper A. Lake ${ }^{1,2}$ and Behnam Abasht ${ }^{1,2 *}$ \\ ${ }^{1}$ Center for Bioinformatics and Computational Biology, University of Delaware, Newark, DE, United States, ${ }^{2}$ Department \\ of Animal and Food Sciences, University of Delaware, Newark, DE, United States
}

\section{OPEN ACCESS}

Edited by:

Massimiliano Petracci,

University of Bologna, Italy

Reviewed by:

Giulia Baldi,

University of Bologna, Italy

Vincenzo Tufarelli,

University of Bari Aldo Moro, Italy

Yuwares Malila,

National Center for Genetic Engineering and Biotechnology

(BIOTEC), Thailand

*Correspondence: Behnam Abasht abasht@udel.edu

Specialty section:

This article was submitted to Avian Physiology,

a section of the journal

Frontiers in Physiology

Received: 20 January 2020 Accepted: 13 February 2020

Published: 13 March 2020

Citation:

Lake JA and Abasht B (2020) Glucolipotoxicity: A Proposed Etiology for Wooden Breast and Related Myopathies in Commercial Broiler Chickens. Front. Physiol. 11:169. doi: 10.3389/fphys.2020.00169
Wooden breast is one of several myopathies of fast-growing commercial broilers that has emerged as a consequence of intensive selection practices in the poultry breeding industry. Despite the substantial economic burden presented to broiler producers worldwide by wooden breast and related muscle disorders such as white striping, the genetic and etiological underpinnings of these diseases are still poorly understood. Here we propose a new hypothesis on the primary causes of wooden breast that implicates dysregulation of lipid and glucose metabolism. Our hypothesis addresses recent findings that have suggested etiologic similarities between wooden breast and type 2 diabetes despite their phenotypic disparities. Unlike in mammals, dysregulation of lipid and glucose metabolism is not accompanied by an increase in plasma glucose levels but generates a unique skeletal muscle phenotype, i.e., wooden breast, in chickens. We hypothesize that these phenotypic disparities result from a major difference in skeletal muscle glucose transport between birds and mammals, and that the wooden breast phenotype most closely resembles complications of diabetes in smooth and cardiac muscle of mammals. Additional basic research on wooden breast and related muscle disorders in commercial broiler chickens is necessary and can be informative for poultry breeding and production as well as for human health and disease. To inform future studies, this paper reviews the current biological knowledge of wooden breast, outlines the major steps in its proposed pathogenesis, and examines how selection for production traits may have contributed to its prevalence.

Keywords: wooden breast, white striping, broiler, myopathy, pectoralis major, diabetes, spaghetti meat, dorsal cranial myopathy

\section{INTRODUCTION}

The last half century has witnessed remarkable gains in commercial broiler production characterized primarily by rapid growth, high feed efficiency, and high breast muscle yield (Havenstein et al., 2003a,b). Alongside improvements to production traits, intense breeding programs and enhanced management practices may have elicited the emergence of several 
muscle disorders among fast-growing broiler strains. Myopathies such as wooden breast, white striping, spaghetti meat, and dorsal cranial myopathy significantly impact meat quality, causing substantial economic losses in the poultry industry (Zimermann et al., 2012; Kuttappan et al., 2016; Baldi et al., 2018; Zanetti et al., 2018). Speculation on the causes of these muscle disorders has focused largely on impaired oxygen supply, buildup of metabolic waste, and overstretching or compartmentalization of the muscle due to sustained rapid growth of skeletal muscle and consequent vascular marginalization (Kuttappan et al., 2013; Mudalal et al., 2015; Dalle Zotte et al., 2017; Lilburn et al., 2018). While each of these may well be a contributing factor, a critical analysis of the literature and findings in our laboratory has prompted us to submit a new hypothesis. Here we propose that dysregulation of lipid and glucose metabolism is an important underlying cause of wooden breast and related muscle disorders in commercial broiler chickens (Figure 1). Moreover, we suggest that there are substantial similarities in the mechanistic underpinnings of wooden breast in broiler chickens and type 2 diabetes in mammals.

\section{LIPID ACCUMULATION IN WOODEN BREAST PHENOTYPE}

Evidence of lipid accumulation (Papah et al., 2017) and altered lipid metabolism (Papah et al., 2018; Lake et al., 2019) early in the onset of wooden breast suggest that dysregulation of lipid metabolism is a key etiological feature of the myopathy. Lipid accumulation in the pectoralis major of wooden breast affected chickens is evident at the molecular, microscopic, and macroscopic levels. Affected breast muscle has an overall higher percent composition of fat (Soglia et al., 2016), an altered fatty acid profile (Gratta et al., 2019), and is substantially more likely than unaffected filets to exhibit signs of white striping, characterized by fatty white striations running parallel to the muscle fiber that are visible to the naked eye (Tasoniero et al., 2016; Dalle Zotte et al., 2017). Microscopic evidence of lipid accumulation and its associated pathological changes includes the presence of lipid droplets, lipogranulomas, and lipid-laden foam cells in the pectoralis major as early as the first week of age (Papah et al., 2017). At the molecular level, metabolomic profiling of 7-week-old birds shows that affected tissues have higher levels of multiple long chain fatty acids, including palmitate, palmitoleate, stearate, and oleate, as well as accumulation of various phospholipid and triglycerides catabolites (Abasht et al., 2016). Lipid accumulation is also supported by the upregulation of lipid metabolism genes in the pectoralis major of 2- and 3 -week-old birds that were later diagnosed with wooden breast at 7 weeks of age (Papah et al., 2018; Lake et al., 2019). These genes include fatty acid translocase (CD36), fatty acid binding protein 4 (FABP4), lipoprotein lipase ( $L P L)$, and peroxisome proliferator-activated receptor gamma (PPARG) among others (Lake et al., 2019). Such studies provide overwhelming evidence of altered lipid metabolism in the pectoralis major of wooden breast affected broilers starting in early phases of the disease.
Of the genes mentioned above, $L P L$ stands out due to its encoded protein's key role as a metabolic gatekeeper in terms of partitioning circulating lipids among tissues (Wang and Eckel, 2009). The $L P L$ gene encodes lipoprotein lipase, an enzyme that attaches to the surface of vascular endothelial cells and serves as the rate-limiting catalyst for hydrolysis of triglycerides in two types of circulating lipoproteins - portomicrons/chylomicrons and very-low-density lipoproteins (VLDL) - providing nonesterified fatty acids and monoglycerides for use by surrounding tissues (Mead et al., 2002; Wang and Eckel, 2009). Regulation of LPL expression occurs in a tissue-specific manner and alterations to LPL levels in one tissue can affect systemic nutrient partitioning by reducing substrate availability to other tissues (Wang and Eckel, 2009). Increased expression of LPL is a consistent and early signal of the overprovision of lipids to the pectoralis major in affected birds. LPL is upregulated in 2- and 3-week-old broilers that later develop wooden breast (Papah et al., 2018; Lake et al., 2019), it has been proposed as a contributor to sex-linked differences in wooden breast incidence rate and severity (Brothers et al., 2019), and its increased expression in affected birds has been localized to the site where disease is first microscopically apparent - the endothelium of veins undergoing phlebitis (Papah and Abasht, 2019).

Lipid accumulation in the pectoralis major has been consistently linked to wooden breast, but the mechanism by which it may be involved in the myopathy has not been sufficiently explored. Papah et al. (2017) pointed out the resemblance of the lipid infiltration and phlebitis of wooden breast to atherosclerosis, although symptoms are restricted to the veins of affected broilers. It has also been suggested that increased expression of lipid metabolism genes in the early stages of wooden breast may signify a pathogenetic relationship with metabolic syndrome and type 2 diabetes in humans (Lake et al., 2019). Ectopic fat deposition and the resulting lipotoxicity, specifically in skeletal muscle, are known to be major metabolic risk factors for type 2 diabetes and related conditions (Rasouli et al., 2007), and we believe they play an important etiological role in wooden breast and similar muscle disorders. However, an important missing link between lipid accumulation and other aspects of the wooden breast phenotype is the fate of glucose in the pectoralis major.

\section{LIPOTOXIC INHIBITION OF GLYCOLYSIS AND GLYCOGENESIS}

The pectoralis major of wooden breast affected birds exhibits signs of severely altered glucose metabolism, specifically inhibition of glycolysis. This is strongly supported by lower levels of glycolytic intermediates, such as glucose-6-phosphate and fructose-6-phosphate, and glycolytic end products, such as pyruvate and lactate, in affected birds (Abasht et al., 2016). Glycogen levels are also significantly lower (Abasht et al., 2016), which discounts the idea that increased synthesis of glycogen (glycogenesis) from glucose-6-phosphate or decreased degradation (such as glycogen storage disease) cause the reduction in glycolytic intermediates and end products. 


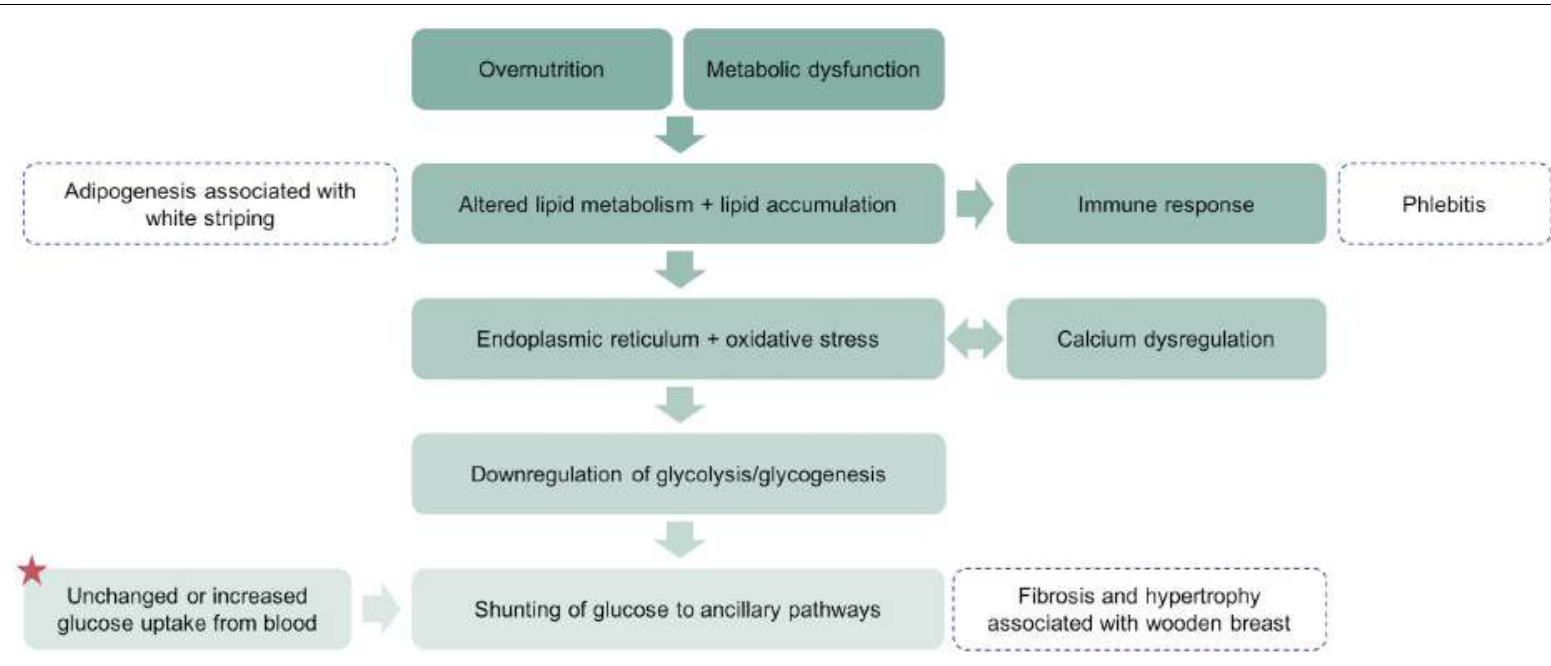

FIGURE 1 | A simplified schematic representation of the proposed pathogenesis of wooden breast and white striping in the pectoralis major muscle. A key difference between wooden breast in broilers and type 2 diabetes in mammals is the dependence on insulin-independent glucose transport in skeletal muscle of chickens. This results in unchanged or increased uptake of glucose from blood (star) even when glycolysis and glycogenesis are substantially downregulated and shunting of that glucose into alternative pathways that contribute to the wooden breast phenotype.

Inhibition of glycolysis is also supported by downregulation of the glycolytic enzyme 6-phosphofructo-2-kinase (PFKFB3) (Mutryn et al., 2015) as well as the isoform primarily expressed in skeletal muscle, PFKFB1 (unpublished data), in 7-week-old affected birds. In that study, PFKFB3 and PFKFB1 were the only detected HIF1-dependent gene that were downregulated rather than upregulated (Mutryn et al., 2015). The suppression of glycolysis in affected birds is actually at odds with a common hypothesis that hypoxia resulting from breast muscle growth and vascular deficiency is the primary cause of wooden breast and other breast muscle disorders in modern broilers (Boerboom et al., 2018; Sihvo et al., 2018; Livingston et al., 2019a; Petracci et al., 2019). Hypoxia is widely known to stimulate glycolysis in lieu of more oxygen-demanding means of ATP production (Semenza et al., 1994). What must be determined, then, is the trigger for altered glucose metabolism as well as the fate of glucose that doesn't undergo glycolysis in the pectoralis major.

Reduced glucose uptake from plasma is an unlikely cause of reduced glycolysis as there is no difference in plasma glucose levels between affected and unaffected birds (Livingston et al., 2019a,b) and expression of various glucose transporter genes is actually upregulated in the pectoralis major of affected birds (unpublished data). The liver has a major role in maintaining blood glucose levels, compensating for increased plasma glucose by synthesizing more hepatic glycogen (Hers, 1976). However, the livers of wooden breast affected birds also have lower glycogen content than livers of unaffected birds (Kawasaki et al., 2018). Although additional research on glucose uptake in wooden breast is required, this evidence suggests that glucose is taken up by the breast muscle, but not used for energy production or storage as fully as it is in unaffected birds.

We believe that the observed decrease in glycolytic flux is primarily a result of cellular stress responses to lipid accumulation in the pectoralis major. When lipids accumulate in cells and tissues that are not adequately equipped to metabolize or store them, such as liver and muscle, they can activate a broad range of cellular stress and immune responses such as toll-like receptor (TLR) signaling (Lee and Hwang, 2006), increased production of reactive oxygen species (ROS) (Goglia and Skulachev, 2003), endoplasmic reticulum stress, and the unfolded protein response (Volmer and Ron, 2015). These responses create deleterious effects, collectively referred to as lipotoxicity, and can destabilize metabolic functions such as glycolysis. In wooden breast, we believe oxidative stress is the key inhibitor of glycolysis based on downregulation of the glycolytic gene PFKFB3 in affected broilers (Mutryn et al., 2015). PFKFB3 encodes the enzyme that catalyzes the conversion of fructose-6-phosphate to fructose 2,6-bisphosphate, which itself is a potent activator of glycolysis via allosteric regulation of phosphofructokinase 1. PFKFB3 is also considered a regulator of oxidative stress because of its ability to sense and respond to redox homeostasis, shunting glucose to the pentose phosphate pathway in response to elevated levels of ROS (Seo and Lee, 2014; Yamamoto et al., 2014).

In mammals, lipotoxic inhibition of glucose oxidation in skeletal muscle and adipose tissue is accompanied by downregulation of insulin-sensitive glucose transporter 4 (GLUT4), resulting in the insulin resistance and high plasma glucose levels that characterize type 2 diabetes (Randle et al., 1965; Groop and Ferrannini, 1993). Reduced GLUT4-mediated glucose transport is especially impactful in skeletal muscle, which is the major site of glucose uptake in the postprandial state (DeFronzo and Tripathy, 2009). This process has been demonstrated in a study of muscle-specific overexpression of LPL in transgenic mice (Kim et al., 2001). In that experiment, overexpression of muscle-specific LPL caused major metabolic changes in skeletal muscle, including a 3 -fold increase in triglyceride content, an increased number of lipid droplets 
around the mitochondrial region, a 52\% decrease in insulinstimulated glucose uptake, a $48 \%$ decrease in glycolysis, and an $88 \%$ decrease in glycogen synthesis (Kim et al., 2001). The key difference between this mouse model of type 2 diabetes and the wooden breast phenotype is that wooden breast is not associated with a significant change in plasma glucose levels (Livingston et al., 2019a,b). However, this disparity is likely a result of differences between avian and mammalian glucose transport and insulin signaling in skeletal muscle.

\section{INSULIN-INDEPENDENT GLUCOSE TRANSPORT IN CHICKEN SKELETAL MUSCLE}

The polarity and size of glucose molecules prevent their transport across lipid membranes by simple diffusion (Navale and Paranjape, 2016). Two families of glucose transporters, sodiumglucose linked transporters (SGLTs) and facilitated glucose transporters (GLUTs), control the movement of glucose into and out of cells (Navale and Paranjape, 2016) and thus play an important role in glucose homeostasis and regulation of blood glucose levels. In mammals, glucose transport in skeletal muscle relies primarily on the activity of GLUT1 and GLUT4 (Klip et al., 1996). GLUT1 is preferentially restricted to the cell surface and provides insulin-independent basal levels of glucose while GLUT4 is sequestered in intracellular vesicles and rapidly translocated to the cell surface in response to insulin, exercise, or hypoxia (Klip et al., 1996; Shepherd and Kahn, 1999). Impairment of the insulin signaling cascade in skeletal muscle of mammals, which can occur in response to chronic high-fat conditions (Zierath et al., 1997), reduces the insulin-stimulated translocation of GLUT4 to the plasma membrane. Pancreatic $\beta$-cells compensate for a reduced insulin response by increasing secretion of insulin, but eventually this becomes insufficient and reduced GLUT4 translocation causes accumulation of glucose in the blood.

Avian glucose transport and insulin signaling are not as well characterized as those of mammals (Braun and Sweazea, 2008), but it is well known that chickens are naturally hyperglycemic and insulin resistant, possessing circulating insulin concentrations approximately equal to those of mammals but extremely high plasma glucose levels (Akiba et al., 1999; Tokushima et al., 2005). Insulin resistance in chickens has been partly attributed to hyperactivity of insulin receptor signaling in skeletal muscle, where phosphatidylinositol 3-kinase (PI3K) activity, a key component of the insulin signaling pathway, was found to be 30 times higher in chickens than in rats (Dupont et al., 2004). In fact, insulin privation via immuno-neutralization was found to have no effect on PI3K activity in chicken skeletal muscle, although it resulted in altered expression of major metabolic transcription factors in both the liver and skeletal muscle (Godet et al., 2008). Another major contributor is perhaps the intrinsic lack of any GLUT4 homologs in the chicken genome and resulting predominance of insulin-independent glucose transport. An attempt to detect GLUT4 homologs in various tissues of broiler chickens found that GLUT4 cDNA was completely undetectable in any of the 19 chicken tissues that were tested, which included the pectoralis major (Seki et al., 2003). The intrinsic lack of GLUT4 and low expression levels of other common glucose transporters (GLUT1, GLUT2, GLUT3, and GLUT8) in skeletal muscle of broilers (Kono et al., 2005) suggests that glucose transport is regulated differently in chickens than it is in mammals and may primarily be insulin-independent.

The reliance on insulin-independent glucose transporters in chicken skeletal muscle may explain why there are no mammalian skeletal muscle disorders that are equivalent to wooden breast. Rather, the wooden breast phenotype shares striking similarities with various complications of type 2 diabetes in smooth muscle and cardiac muscle, such as atherosclerosis, diabetic cardiomyopathy and myocardial fibrosis, diabetic nephropathy, pulmonary fibrosis, diabetic retinopathy, and non-alcoholic fatty liver disease. These diseases frequently involve lipid accumulation, inflammation, oxidative stress, calcium dysregulation, endoplasmic reticulum stress, hypoxia, hypertrophy, and fibrosis - features that have also been welldocumented in wooden breast. The greater role of insulinindependent glucose transport in cardiac and smooth muscle allows glucose uptake even when glycolysis and glycogenesis are impaired. The importance of insulin-insensitive glucose transport in development of diabetic retinopathy (Kumagai, 1999), atherosclerosis (Wall et al., 2018), diabetic nephropathy (Brosius and Heilig, 2005), pulmonary fibrosis (Cho et al., 2017), diabetic myocardial fibrosis (Asbun and Villarreal, 2006; Gorski et al., 2019) and non-alcoholic fatty liver disease (Nanji et al., 1995) has been demonstrated and is in support of our hypothesis that wooden breast shares substantial etiological factors with type 2 diabetes in mammals.

\section{PATHOLOGICAL SHUNTING OF GLUCOSE TO ANCILLARY PATHWAYS}

The suppression of glycolysis and glycogenesis concurrent with unchanged or increased uptake of glucose from blood results in increased flux of glucose through alternative metabolic pathways (Fantus et al., 2006), including the pentose phosphate, glucuronic acid, hexosamine biosynthesis, and aldose reductase (polyol) pathways. There is evidence that all of these pathways are upregulated in affected birds (Abasht et al., 2016; Papah et al., 2018).

Glucose that is phosphorylated to glucose-6-phosphate can directly enter both the pentose phosphate pathway and the glucuronic acid pathway. In addition to generating carbon skeletons and ribose 5-phosphate, a precursor to nucleotide synthesis, the pentose phosphate pathway is a major source of $\mathrm{NADPH}$, the main reductant that drives free radical detoxification and anabolic growth (Kruger and Von Schaewen, 2003). Accumulation of pentose phosphate pathway intermediates 6-phosphogluconate and sedoheptulose 7-phosphate support the upregulation of this pathway in 7-week-old affected birds (Abasht et al., 2016). Glucose-6phosphate is also utilized to produce glucuronic acid, a precursor to ascorbic acid and a building block of proteoglycans, 
glycosaminoglycans, and glycolipids. Elevated levels of ascorbate and UDP-glucuronate in affected birds suggests that this pathway is upregulated as well (Abasht et al., 2016).

Glucose-6-phosphate can also be converted to fructose6-phosphate and consumed in the hexosamine biosynthesis pathway. The hexosamine biosynthesis pathway is responsible for production of uridine diphosphate $\mathrm{N}$-acetylglucosamine (UDP-GlcNAc), a nucleotide sugar and coenzyme used for protein glycosylation and the synthesis of glycosaminoglycans, proteoglycans, and glycolipids (Fantus et al., 2006). The gene encoding this pathway's rate-limiting enzyme, glutaminefructose-6-phosphate transaminase 2 (GFPT2), was found to be upregulated in 3-week-old broilers affected by wooden breast (Papah et al., 2018). In fact, three of the four genes involved in the hexosamine biosynthesis pathway - GFPT2, phosphoglucomutase 3 (PGM3), and UDP-N-acteylglucosamine pyrophosphorylase 1 (UAP1) - show increased expression in the pectoralis major of high-feed-efficiency broilers, which are more susceptible to wooden breast than those with low feed efficiency (Abasht et al., 2019). In agreement, affected birds have higher levels of hexosamine biosynthesis pathway intermediates isobar UDPacetylglucosamine and UDP-acetylgalactosamine at 7 weeks of age (Abasht et al., 2016). Increased production of proteoglycans is further supported by Clark and Velleman (2017), who found greater RNA expression of decorin in the pectoralis major of affected birds. The protein encoded by this gene is a small leucinerich proteoglycan that regulates collagen cross-linking, although it is unclear if increased collagen cross-linking is a universal feature of the wooden breast phenotype (Velleman et al., 2017; Baldi et al., 2019). Proteoglycans and glycosaminoglycans are important components of the extracellular environment and increased production of them is associated with extensive remodeling of the extracellular matrix.

Excess glucose is also consumed in the polyol pathway, a two-step process that converts glucose first to sorbitol and then to fructose (Yan, 2018). Upregulation of the polyol pathway, specifically its first step, in wooden breast and white striping is supported by an accumulation of sorbitol in the pectoralis major (Abasht et al., 2016; Boerboom et al., 2018). One notable effect of polyol pathway stimulation is the induction of collagen synthesis (Bleyer et al., 1994; Ha et al., 1997) due at least in part to transcriptional activation of transforming growth factor- $\beta$ (TGF$\beta$ ) (Ishii et al., 1998; Han et al., 1999; Tokudome et al., 2004). All three isoforms of TGF- $\beta$ are considered important regulators of inflammation, extracellular matrix protein deposition, and fibrosis, and altered activity of TGF- $\beta$ proteins is known to contribute to various fibroproliferative disorders in humans (Pohlers et al., 2009). Increased expression of transforming growth factor $\beta 3$ (TGFB3) in the pectoralis major muscle of affected birds at 7 weeks (Mutryn et al., 2015) may represent a link between altered glucose metabolism and increased collagen production in wooden breast, the latter of which is widely considered to be an important feature of the myopathy contributing to the characteristic firmness of the pectoralis major (Sihvo et al., 2014; Soglia et al., 2016; Papah et al., 2017).

Finally, glucose can be used in the production of advanced glycation end products (Fantus et al., 2006). Advanced glycation end products are proteins or lipids that become glycated as a result of exposure to sugars and can have numerous pathological effects, such as induction of cytokine production, increased vascular permeability and inflammation, inhibition of vascular dilation, and enhanced oxidative stress (Basta et al., 2004). These effects are consistent with current knowledge of wooden breast, although altered production of advanced glycation end products has not yet been reported.

Increased flux of glucose through these alternative pathways (Figure 2) is a pathological manifestation of altered glucose utilization, likely resulting from reduced glycolysis and glycogenesis alongside unchanged or increased import of glucose from the blood. This process is often referred to as glucose toxicity and is considered a key component of pancreatic $\beta$-cell dysfunction, insulin resistance, and chronic complications of diabetes such as diabetic neuropathy, retinopathy, and nephropathy in mammals.

\section{DISRUPTION OF REDOX HOMEOSTASIS AND OXIDATIVE STRESS}

Oxidative stress occurs when the production and accumulation of ROS become unbalanced with an organism's ability to detoxify reactive intermediates and can lead to cell and tissue damage. Broiler chickens show signs of greater oxidative stress in the pectoralis major than layers (Zahoor et al., 2017) and broilers with high feed efficiency have greater oxidative stress response in the breast muscle compared to those with low feed efficiency (Zhou et al., 2015). In wooden breast, affected broilers have metabolite profiles indicative of altered redox homeostasis involving higher free radical exposure than unaffected birds (Abasht et al., 2016). Similarly, various genes involved in oxidative stress response are upregulated in wooden breast birds at both 3 and 7 weeks of age (Abasht et al., 2015; Mutryn et al., 2015; Papah et al., 2018).

As previously discussed, lipotoxicity is likely a major contributor to oxidative stress in the early stages of wooden breast. Fatty acids are particularly prone to ROS-induced oxidative damage via a process called lipid peroxidation, which proceeds in an aggressively self-propagating chain reaction that can induce damage to proteins and DNA (Goglia and Skulachev, 2003; Schrauwen, 2007). When the supply of fatty acids overwhelms the storage and oxidative capacity of cells, which is more likely in mitochondria-scarce, type IIB pectoralis major muscle fibers, they accumulate in the cell and around the mitochondria where they can have extremely damaging effects (Schrauwen and Hesselink, 2004; Anderson and Neufer, 2006). Fatty acid transport into the mitochondria is generally regulated by the enzyme carnitine palmitoyltransferase 1 (Schrauwen, 2007). Lower levels of free carnitine in the pectoralis major of affected birds (Abasht et al., 2016) may reflect increased transport of long-chain fatty acids into the mitochondria by carnitine palmitoyltransferase 1. Fatty acids and fatty acid derivatives inside the mitochondrial matrix are extremely vulnerable to ROS-induced lipid peroxidation and can cause substantial mitochondrial damage due to the complex nature of the 


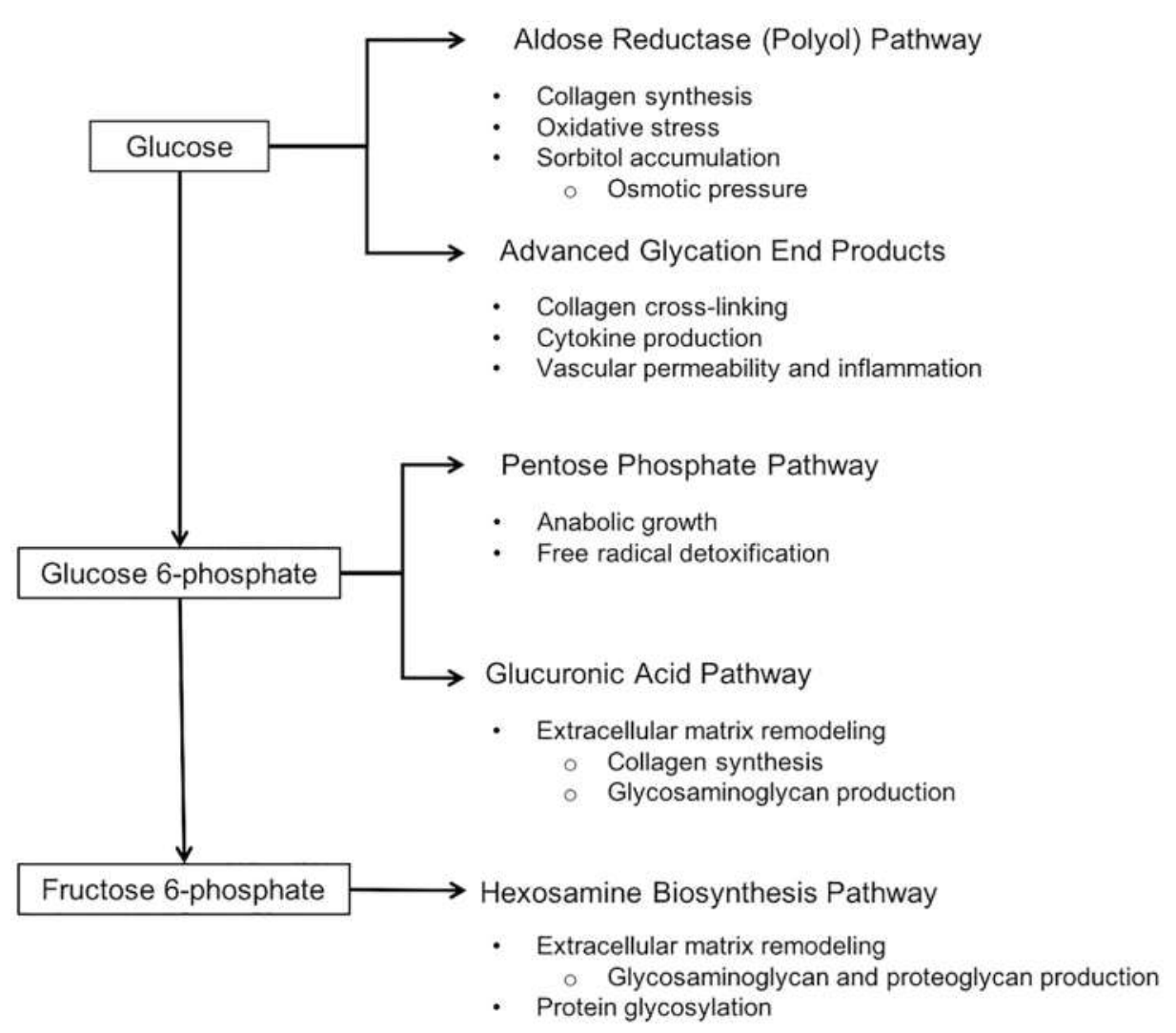

FIGURE 2 | Altered carbohydrate metabolism in wooden breast and relevant effects of each pathway. Wooden breast is associated with a reduced flux of glucose through glycolytic and glycogenic pathways and shunting of glucose into ancillary pathways, including the aldose reductase, pentose phosphate, glucuronic acid, and hexosamine biosynthesis pathways. It has not yet been demonstrated whether or not wooden breast also involves increased synthesis of advanced glycation end products.

matrix space (Goglia and Skulachev, 2003). Oxidative damage to the mitochondria due to fatty acid accumulation may be attenuated by mitochondrial energy uncoupling, mediated largely by uncoupling protein 3 (UCP3), an enzyme activated by lipid peroxides (Goglia and Skulachev, 2003; Rousset et al., 2004; Schrauwen and Hesselink, 2004). However, UCP3 was found to be downregulated in wooden breast affected birds at 3 weeks of age (Papah et al., 2018), suggesting an impaired uncoupling response. High coupling efficiency in an environment with severe lipid accumulation would directly contribute to ROS production and lipid peroxidation and also allow for increased mitochondrial damage by ROS inside the mitochondrial matrix.

After initial derangement of redox homeostasis, multiple factors can contribute to a positive feedback loop of oxidative stress. Most ROS are generated as normal by-products during mitochondrial electron transport, specifically at respiratory complexes I and III of the oxidative phosphorylation pathway (Rousset et al., 2004), and are prevented from reaching damaging levels by various cellular defenses such as superoxide dismutase and glutathione peroxidase (Anderson and Neufer, 2006). Potential genetic variation in antioxidant response may be a key contributor to wooden breast susceptibility in broilers, as altered redox homeostasis can inhibit the activity of anti-oxidant enzymes and increase ROS production at mitochondrial respiratory complex I (Yabe-Nishimura, 1998; Chung, 2004; Yan, 2018). In diabetes, the polyol pathway is believed to play a critical role in oxidative stress and vascular damage due to its derangement of redox homeostasis (Yabe-Nishimura, 1998; Chung, 2004; Yan, 2018). The first of two reactions in the pathway is conversion of glucose and $\mathrm{NADPH}$ to sorbitol and NADP+ by aldose reductase and is suggested to be upregulated in the pectoralis major of affected birds based on the accumulation of sorbitol (Abasht et al., 2016; Boerboom et al., 2018). Mutryn et al. (2015) proposed NADPH-oxidase activity as another contributor to altered $\mathrm{NADP}+/ \mathrm{NADPH}$ homeostasis related to inflammatory and immune responses. The consumption of NADPH by aldose reductase and NADPH-oxidase reduces the activity of other NADPH-dependent enzymes such as glutathione reductase, an important anti-oxidative enzyme, and nitric oxide synthase, which produces nitric oxide from L-arginine (YabeNishimura, 1998). Nitric oxide is a soluble gas produced by endothelial cells that has many functions, including regulation of vascular homeostasis, vasodilation, angiogenesis, endothelial cell growth, and protection of vessels from injury (Tousoulis et al., 2012). 
Disruption of NAD+/NADH homeostasis can also increase ROS production, wherein overproduction of the electron donor $\mathrm{NADH}$ increases activity of NADH-dependent mitochondrial respiratory complex I (Hirst et al., 2008). The conversion of sorbitol and NAD+ to fructose and NADH by sorbitol dehydrogenase (SORD) in the second reaction of the polyol pathway is one potential contributor to this imbalance; however, this is unlikely in wooden breast as decreased SORD expression (unpublished data) and greater accumulation of sorbitol (Abasht et al., 2016) in affected birds provide testimony for reduced SORD activity. Rather, potentially higher levels of $\mathrm{NADH}$ in wooden breast may be partly attributed to lower activity of the glycerol3-phosphate shuttle (Abasht et al., 2019) or lower activity of lactate dehydrogenase. An imbalance of cytosolic NAD+/NADH would not only increase ROS production but could also limit NAD+ supply to key metabolic enzymes required for sustaining glycolysis and the citric acid cycle. Free NAD+ is a cofactor for glyceraldehyde 3-phosphate dehydrogenase and oxoglutarate dehydrogenase, both of which are downregulated in affected birds according to differential expression analysis (Abasht et al., 2015; Papah et al., 2018). Free NAD+ is also a cofactor for the pyruvate dehydrogenase complex which serves as a link between glycolysis and the citric acid cycle (Yan, 2018).

\section{CALCIUM CYCLING ABNORMALITIES}

Maintenance of intracellular $\mathrm{Ca}^{2+}$ pools is fundamental to generating the $\mathrm{Ca}^{2+}$ signals required for numerous cellular processes (Berridge et al., 2003). The pectoralis major of wooden breast affected birds exhibits upregulation of genes encoding both parvalbumin and sarcoplasmic/endoplasmic reticulum calcium ATPase (SERCA) 2 (Mutryn et al., 2015) as well as increased abundance of SERCA protein (Soglia et al., 2016). Both proteins are involved in sequestering calcium and their upregulation constitutes evidence of a compensatory response to increased intracellular calcium in muscle cells (Mutryn et al., 2015). Parvalbumin is a $\mathrm{Ca}^{2+}$-binding protein that functions as a calcium buffer and SERCAs are intracellular pumps located in the sarcoplasmic/endoplasmic reticulum (SR) membranes that use ATP to translocate $\mathrm{Ca}^{2+}$ from the cytoplasm to the SR lumen (Berridge et al., 2003). Evidence of dysregulated calcium homeostasis and impaired excitation-contraction coupling is also present in 2- and 3-week-old broilers, prior to manifestation of wooden breast phenotype at market age (Papah et al., 2018; Lake et al., 2019). Increased levels of calcium in the pectoralis major of birds affected by white striping, wooden breast, or both (Sandercock et al., 2009; Tasoniero et al., 2016; Zambonelli et al., 2016) suggests that myocellular uptake of $\mathrm{Ca}^{2+}$ from extracellular spaces is affected in addition to dysregulation of intracellular calcium pools.

It is unclear what provides the primary stimulus for calcium dysregulation in wooden breast and, unfortunately, there is no consensus regarding a single cause of altered calcium cycling in diabetes despite extensive research. However, one popular hypothesis contends that chronic exposure to excessive nutrients, specifically glucose and lipids, in tissues unequipped to fully metabolize, store, or dispose of them can initiate SR and mitochondrial stress that result in disruption of calcium homeostasis (Arruda and Hotamisligil, 2015). At least three mechanisms can aid in understanding this process: (1) fatty acids and ROS stimulate release of $\mathrm{Ca}^{2+}$ from the SR and inhibit removal of $\mathrm{Ca}^{2+}$ from the cytosol, (2) formation of the mitochondrial permeability transition pore and mitochondrial depolarization cause release of $\mathrm{Ca}^{2+}$ from the mitochondria, and (3) depolarization of the plasma membrane causes an influx of $\mathrm{Ca}^{2+}$ from extracellular spaces. The SR lumen serves as the most important $\mathrm{Ca}^{2+}$ store in the cell (Arruda and Hotamisligil, 2015), but an excess of fatty acids, fatty acid derivatives, and ROS can cause release of $\mathrm{Ca}^{2+}$ from the SR lumen by activating ryanodine and inositol trisphosphate $\left(\mathrm{IP}_{3}\right)$-sensitive calcium channels (Cheah, 1981; Ursini et al., 2004; Berezhnov et al., 2008; Wei et al., 2009). Certain types of fatty acids can also inhibit SERCA activity, preventing calcium removal from the cytosol (Berezhnov et al., 2008; Fu et al., 2011). Fluxes in intracellular calcium can be buffered by increased calcium uptake in the mitochondria, where $\mathrm{Ca}^{2+}$ can elevate ATP production (Berridge et al., 2003). However, excess mitochondrial $\mathrm{Ca}^{2+}$ can sensitize the mitochondrial permeability transition pore, leading to membrane depolarization and the rapid release of $\mathrm{Ca}^{2+}$ into the cytosol (Gunter et al., 1994; Berezhnov et al., 2008). High concentrations of long-chain fatty acids could also stimulate formation of the mitochondrial permeability transition pore (Penzo et al., 2002).

Calcium released from internal stores in the SR and mitochondria cannot explain why the pectoralis major of wooden breast birds has an increased percent composition of $\mathrm{Ca}^{2+}$. Dysregulation of cation homeostasis that extends beyond $\mathrm{Ca}^{2+}$ in affected birds provides evidence that an influx of $\mathrm{Ca}^{2+}$ into the cytoplasm from extracellular spaces involves cell membrane depolarization. Specifically, affected birds show higher sodium and lower magnesium and phosphorus in the pectoralis major as well as higher blood potassium levels (Zambonelli et al., 2016; Livingston et al., 2019b). At resting condition, the concentration of $\mathrm{K}^{+}$is higher inside the cell while the concentration of $\mathrm{Na}^{+}$ and $\mathrm{Ca}^{2+}$ is higher outside the cell (Bahar et al., 2016), but the opening of voltage-gated calcium and sodium channels during membrane depolarization results in the rapid influx of $\mathrm{Ca}^{2+}$ and $\mathrm{Na}^{+}$from extracellular spaces (Bahar et al., 2016). The subsequent efflux of $\mathrm{K}^{+}$through voltage-dependent and voltageindependent channels allows repolarization of the membrane (Bahar et al., 2016). Calcium influx in wooden breast might be induced by certain types of fatty acids (Huang et al., 2006), by oxidative damage to the cell membrane (Ursini et al., 2004), or by the gradient-dependent activity of $\mathrm{Na}^{+} / \mathrm{Ca}^{2+}$ exchangers that exchange three moles of $\mathrm{Na}^{+}$for one mole of $\mathrm{Ca}^{2+}$ (Berridge et al., 2003). Opening of voltage gated calcium channels can exacerbate the release of calcium from endoplasmic reticulum stores in a process called calcium-induced calcium release (Endo, 2009).

One notable effect of altered calcium cycling in skeletal muscle, specifically of increased ATP-dependent SERCA activity, is a substantial increase in oxygen consumption and concurrent elevation of resting metabolic rate (Smith et al., 2013). Disruption of calcium homeostasis is potentially a major contributor to the venous hypercapnia, venous hypoxemia, and muscular 
hypoxia of the pectoralis major that have been documented in wooden breast affected broilers (Mutryn et al., 2015; Livingston et al., 2019a,b). Other effects of calcium dysregulation include mitochondrial damage (Orrenius et al., 2015), aberrant excitation/contraction signaling (Berridge et al., 2003), chronic SR stress (Fu et al., 2011), stimulation of skeletal muscle growth (Ermak and Davies, 2001), calcium-dependent proteolysis (Yin et al., 2013), and ultimately cell death (Hajnóczky et al., 2006). As a major site of post-translational protein modification, the SR is crucial to producing functional proteins. Calciumdependent proteolysis and SR stress could contribute to the lower protein content and lower water holding capacity of wooden breast filets due to their effects on protein degradation and protein production.

Dysregulation of calcium signaling can also induce expression of genes encoding myoglobin and slow muscle fiber type proteins, a feature of wooden breast discovered through differential expression analysis (Mutryn et al., 2015) and confirmed with RNA in situ hybridization (RNA ISH) (Papah and Abasht, 2019). Expression of myoglobin and other muscle-specific genes is regulated by a synergistic interaction between transcription factors in the nuclear factor of activated $t$ cells (NFAT) and myocyte enhancer factor-2 (MEF-2) families (Chin et al., 1998). Transcriptional activation of NFAT and MEF-2 is mediated by calcineurin, a calcium-activated phosphatase, such that calcium fluxes and intracellular concentrations will ultimately determine the fiber type composition within a specific skeletal muscle (Chin et al., 1998). Lipid supplementation and hypoxic conditions have also been demonstrated to stimulate myoglobin expression, possibly through calcium-independent pathways (De Miranda et al., 2012).

\section{VENOUS INFLAMMATION AND VASCULAR PERMEABILITY}

Venous and perivascular inflammation and lipid accumulation are the first microscopic signs of wooden breast, potentially contributing to the edema, petechial hemorrhages, and tissue damage that are macroscopically apparent in late stages of the disease (Papah et al., 2017). It has been noted that these symptoms resemble atherosclerosis in humans (Papah et al., 2017), a condition closely linked to diabetes mellitus that consists of chronic inflammation induced by excessive lipid accumulation (Li et al., 2014). The upregulation of genes associated with vascular disease in 3-week-old broilers that later develop wooden breast (Papah et al., 2018) corroborates this hypothesis, but fails to explain why vascular inflammation is limited to veins. Recent work using RNA ISH to localize expression of a selection of genes in affected pectoralis major muscle provides an important clue. In that study, higher LPL expression was found in the veins of affected breast muscle and not the arteries (Papah and Abasht, 2019), suggesting a causal link between venous and perivascular lipid accumulation and the inflammatory response. This connection between increased LPL activity and inflammation has been thoroughly studied in the context of atherosclerosis.
The participation of LPL in the pathogenesis of atherosclerosis is two-fold: it mediates both the increased hydrolysis of lipoprotein triglycerides as well as the retention of lipoprotein remnants. High LPL activity increases local hydrolysis of triglycerides in portomicrons and VLDL, which produces free fatty acids, portomicron remnants, intermediate-density lipoproteins, and low-density lipoproteins (Alvarenga et al., 2011). Elevated levels of fatty acids and lipoprotein remnants cause damage to vessels as they trigger an inflammatory response, induce endothelial cell apoptosis, and increase endothelial permeability (Toborek et al., 2002; Eiselein et al., 2007; Rocha et al., 2016). A critical element of the inflammatory response caused by fatty acids is the activation of TLRs in the presence of high glucose (Lee and Hwang, 2006; Dasu and Jialal, 2010). TLR activation initiates an inflammatory cascade that includes the release of pro-inflammatory cytokines and cell adhesion molecules as well as $\mathrm{T}$ cell activation and the rapid differentiation of monocytes into macrophages (Krutzik et al., 2005). In wooden breast affected birds, toll-like receptor 2 type 2 precursor (TLR2-2) was one of the top 30 genes identified as a biomarker of wooden breast severity, with significant elevation in moderately affected birds compared to unaffected or severely affected birds (Abasht et al., 2015). The author suggested that this expression pattern may be indicative of the progression of the disease involving regulation by some negative feedback mechanism. If disease severity and disease progression are considered more or less equivalent, a similar pattern can be seen with lipid metabolism genes, which are generally upregulated in 2- and 3-week-old birds but relatively unchanged or downregulated in 7-weekold birds (Mutryn et al., 2015; Papah et al., 2018; Papah and Abasht, 2019). Due to the apparent coupling of lipid metabolism and venous inflammation, negative feedback regulation of lipid metabolism could be associated with a reduction in the innate immune response.

Endothelial cell damage and apoptosis due to increased lipoprotein metabolism increases the permeability of the endothelium and triggers leukocyte adhesion and transmigration into the vessel wall (Aghajanian et al., 2008). Lipoprotein remnants, which normally enter back into circulation to be cleared by the liver (Alvarenga et al., 2011), can then diffuse into the tunica intima where LPL mediates binding between remnant particles and proteoglycans in the sub-endothelial extracellular matrix (Olin et al., 1999). The subsequent activation of extracellular matrix proteins such as matrix metalloproteinases can elicit remodeling of the extracellular matrix (Amin et al., 2017), a process that is a well-substantiated in the wooden breast pathogenesis (Mutryn et al., 2015; Papah et al., 2018). LPL-mediated bridging normally occurs between lipoprotein remnants and heparan sulfate proteoglycans on cell surfaces before lipoproteins undergo hydrolysis (Olin et al., 1999). Increased retention of lipoprotein remnants in the extracellular matrix and on cell surfaces increases the likelihood that they will be modified (e.g., oxidized) or taken up by scavenging macrophages to form foam cells, triggering further inflammatory reactions (Botham et al., 2007). The proatherosclerotic role of both active and inactive LPL has been demonstrated in mice (Wang et al., 2007), suggesting that both the hydrolysis and bridging functions of LPL are important. 


\section{BROILER SELECTION AND WOODEN BREAST}

Consolidation of poultry breeding and intense selection for production traits have undoubtedly played an oversized role in the rise of muscle disorders such as wooden breast, white striping, and spaghetti meat among commercial broilers. Even without completely understanding the genetic architectures underlying these traits, it is possible to speculate how selection for specific performance metrics might increase the predisposition of meattype chickens to these myopathies. Much of this speculation can be framed in the context of supply and demand of nutrients in the pectoralis major muscle. Juvenile growth rate, which has increased substantially since the 1950s independent of improvements to feed formulation (Havenstein et al., 2003b) and which is correlated with wooden breast severity, represents a major component of the supply side of this equation. The rapid growth rate of modern broilers reflects increased delivery of nutrients to the whole body, stemming from some combination of greater appetite, suppressed satiety (Barbato, 1994), as well as improved digestion and absorption in the digestive system (Smith et al., 1990).

Selection for feed efficiency has also raised digestive and absorptive capacity but may not increase the overall supply of nutrients to the body as increased energy absorption from feed is at least partly offset by reduced feed consumption. A larger effect of using feed efficiency as a major selection criterion relates to its correlation with fat distribution and blood lipid levels (Griffin et al., 1989; Zhuo et al., 2015). Unlike selection for juvenile growth rate, which is accompanied by increased fat deposition in adipose tissue depots, selection for increased feed efficiency is accompanied by reduced fat deposition in adipose tissue depots (Abasht et al., 2020). This reflects altered nutrient partitioning in high feed efficiency birds which is likely multifaceted, involving changes to metabolic processes in several organs such as reduced adipogenesis in abdominal fat, reduced lipogenesis in the liver, and increased lipoprotein triglyceride hydrolysis in skeletal muscle. Regardless of the specific biological mechanisms, there is evidence that improvements to such performance traits are associated with a shifting of fat metabolism away from the liver and normal adipose depots toward skeletal muscle (Griffin et al., 1989; Larkina et al., 2010; Zhuo et al., 2015). In commercial broiler chickens, selection for large breast muscle partitions more nutrients toward the pectoralis major, which may be particularly susceptible to metabolic perturbations due to its primary composition of type IIB glycolytic muscle fibers (Anderson and Neufer, 2006).

It should be noted, though, that wooden breast does not correlate perfectly with performance traits like growth rate, feed efficiency, abdominal fat percentage, or breast muscle yield, suggesting the existence of etiological factors that may be unrelated to nutrient partitioning. For example, a mutation that affects coupling of lipid or glucose uptake and utilization in the pectoralis major might constitute the spark on the proverbial fire of nutrient accumulation and toxicity. Lake et al. (2019) proposed a potential mechanism by which this type of mutation, seemingly unrelated to performance traits, might inadvertently become the target of extreme selection pressure. Organ hypertrophy is a common symptom of chronic diabetes complications, including diabetic cardiomyopathy, nephropathy, and non-alcoholic fatty liver disease. Assuming a similar pathogenesis in wooden breast, it is possible that selection for high breast muscle yield would directly select for pathological hypertrophy symptomatic of the wooden breast phenotype. This inherent conflation of desired performance traits with muscle disorder symptoms suggests that the only long-term solution to myopathies like wooden breast and white striping involves selection against their causal variants.

\section{CONCLUDING REMARKS}

We believe that the wooden breast phenotype in commercial broilers is a manifestation of lipotoxicity and glucotoxicity resulting from the chronic oversupply of both lipids and carbohydrates to the pectoralis major and also the disruption of normal lipid and glucose metabolism. Dependence on insulin-independent glucose transport in the skeletal muscle of chickens causes lipid accumulation in the pectoralis major to be accompanied by unchanged or increased uptake of glucose, causing metabolic and structural alterations that closely resemble complications of diabetes in smooth and cardiac muscle of mammals. In addition to improving our understanding of the etiology and pathogenesis of wooden breast and related myopathies, this hypothesis supports the use of these muscle disorders as models of human metabolic diseases.

\section{DATA AVAILABILITY STATEMENT}

The datasets generated for this study are available on request to the corresponding author.

\section{AUTHOR CONTRIBUTIONS}

JL wrote the original draft of the manuscript. BA provided indispensable guidance, expertise, and unpublished data from previous research. All authors contributed to critical feedback and helped to shape the research, analysis, and manuscript.

\section{FUNDING}

This work was supported by the University of Delaware and by Agriculture and Food Research Initiative Competitive Grant No. 2016-67015-25027 from the USDA National Institute of Food and Agriculture.

\section{ACKNOWLEDGMENTS}

The authors wish to thank Dr. Erin Seifert and Dr. Nishanth E. Sunny for their comments and support regarding the manuscript. 


\section{REFERENCES}

Abasht, B., Fu, W., Mutryn, M. F., Brannick, E. M., and Lee, W. R. (2015). "Identification of gene expression biomarkers associated with severity of Wooden Breast disease in broiler chickens," in Proceeding of XXII European Symposium on the Quality of Poultry Meat, Nantes.

Abasht, B., Mignon-Grasteau, S., Bottje, W., and Lake, J. (2020). "Genetics and genomics of feed utilization efficiency traits in poultry species," in Advances in Poultry Genetics and Genomics, eds S. E. Aggrey, H. Zhou, M. Tixier-Boichard, and D. D. Rhoads (Cambridge: Burleigh Dodds Science Publishing. Limited).

Abasht, B., Mutryn, M. F., Michalek, R. D., and Lee, W. R. (2016). Oxidative stress and metabolic perturbations in wooden breast disorder in chickens. PLoS One 11:e0153750. doi: 10.1371/journal.pone.0153750

Abasht, B., Zhou, N., Lee, W. R., Zhuo, Z., and Peripolli, E. (2019). The metabolic characteristics of susceptibility to wooden breast disease in chickens with high feed efficiency. Poult. Sci. 98, 3246-3256. doi: 10.3382/ps/pez183

Aghajanian, A., Wittchen, E. S., Allingham, M. J., Garrett, T. A., and Burridge, K. (2008). Endothelial cell junctions and the regulation of vascular permeability and leukocyte transmigration. J. Thromb. Haemost. 6, 1453-1460. doi: 10.1111/ j.1538-7836.2008.03087.x

Akiba, Y., Chida, Y., Takahashi, T., Ohtomo, Y., Sato, K., and Takahashi, K. (1999). Persistent hypoglycemia induced by continuous insulin infusion in broiler chickens. Br. Poult. Sci. 40, 701-705. doi: 10.1080/00071669987124

Alvarenga, R. R., Zangeronimo, M. G., Pereira, L. J., Rodrigues, P. B., and Gomide, E. M. (2011). Lipoprotein metabolism in poultry. Worlds Poult. Sci. J. 67, 431-440. doi: 10.1017/s0043933911000481

Amin, M., Pushpakumar, S., Muradashvili, N., Kundu, S., Tyagi, S. C., and Sen, U. (2017). Regulation and involvement of matrix metalloproteinases in vascular diseases. Front. Biosci. 21:89-118. doi: 10.1002/jmri.25711.PET/MRI

Anderson, E. J., and Neufer, P. D. (2006). Type II skeletal myofibers possess unique properties that potentiate mitochondrial H2O2 generation. Am. J. Physiol. Cell Physiol. 290, C844-C851. doi: 10.1152/ajpcell.00402.2005

Arruda, A. P., and Hotamisligil, G. S. (2015). Calcium homeostasis and organelle function in the pathogenesis of obesity and diabetes. Cell Metab. 22, 381-397. doi: 10.1016/j.cmet.2015.06.010

Asbun, J., and Villarreal, F. J. (2006). The pathogenesis of myocardial fibrosis in the setting of diabetic cardiomyopathy. J. Am. Coll. Cardiol. 47, 693-700. doi: 10.1016/j.jacc.2005.09.050

Bahar, E., Kim, H., and Yoon, H. (2016). ER stress-mediated signaling: action potential and $\mathrm{Ca} 2+$ as key players. Int. J. Mol. Sci. 17:1558. doi: 10.3390/ ijms 17091558

Baldi, G., Soglia, F., Laghi, L., Tappi, S., Rocculi, P., Tavaniello, S., et al. (2019). Comparison of quality traits among breast meat affected by current muscle abnormalities. Food Res. Int. 115, 369-376. doi: 10.1016/j.foodres.2018.1 1.020

Baldi, G., Soglia, F., Mazzoni, M., Sirri, F., Canonico, L., Babini, E., et al. (2018). Implications of white striping and spaghetti meat abnormalities on meat quality and histological features in broilers. Animal 12, 164-173. doi: 10.1017/ S1751731117001069

Barbato, G. F. (1994). Genetic control of food intake in chickens. J. Nutr. 124, 1341S-1348S. doi: 10.1093/jn/124.suppl_8.1340s

Basta, G., Schmidt, A. M., and De Caterina, R. (2004). Advanced glycation end products and vascular inflammation: implications for accelerated atherosclerosis in diabetes. Cardiovasc. Res. 63, 582-592. doi: 10.1016/j. cardiores.2004.05.001

Berezhnov, A. V., Fedotova, E. I., Nenov, M. N., Kokoz, Y. M., Zinchenko, V. P., and Dynnik, V. V. (2008). Destabilization of the cytosolic calcium level and the death of cardiomyocytes in the presence of derivatives of long-chain fatty acids. Biophysics (Oxf.) 53, 564-570. doi: 10.1134/s0006350908060183

Berridge, M. J., Bootman, M. D., and Roderick, H. L. (2003). Calcium signalling: dynamics, homeostasis and remodelling. Nat. Rev. Mol. Cell Biol. 4, 517-529. doi: $10.1038 / \mathrm{nrm} 1155$

Bleyer, A. J., Fumo, P., Snipes, E. R., Goldfarb, S., Simmons, D. A., and Ziyadeh, F. N. (1994). Polyol pathway mediates high glucose-induced collagen synthesis in proximal tubule. Kidney Int. 45, 659-666. doi: 10.1038/ki.1994.88

Boerboom, G., van Kempen, T., Navarro-villa, A., and Perez-Bonilla, A. (2018). Unraveling the cause of white striping in broilers using metabolomics. Poult. Sci. 97, 3977-3986. doi: 10.3382/ps/pey266
Botham, K. M., Moore, E. H., De Pascale, C., and Bejta, F. (2007). The induction of macrophage foam cell formation by chylomicron remnants. Biochem. Soc. Trans. 35, 454-458. doi: 10.1042/bst0350454

Braun, E. J., and Sweazea, K. L. (2008). Glucose regulation in birds. Comp. Biochem. Physiol. B Biochem. Mol. Biol. 151, 1-9. doi: 10.1016/j.cbpb.2008.05.007

Brosius, F. C., and Heilig, C. W. (2005). Glucose transporters in diabetic nephropathy. Pediatr. Nephrol. 20, 447-451. doi: 10.1007/s00467-004-1748-x

Brothers, B., Zhuo, Z., Papah, M. B., and Abasht, B. (2019). RNA-seq analysis reveals spatial and sex differences in pectoralis major muscle of broiler chickens contributing to difference in susceptibility to wooden breast disease. Front. Physiol. 10:764. doi: 10.3389/fphys.2019.00764

Cheah, A. M. (1981). Effect of long chain unsaturated fatty acids on the calcium transport of sarcoplasmic reticulum. Biochim. Biophys. Acta 648, 113-119. doi: 10.1016/0005-2736(81)90025-0

Chin, E. R., Olson, E. N., Richardson, J. A., Yang, Q., Humphries, C., Shelton, J. M., et al. (1998). A calcineurin-dependent transcriptional pathway controls skeletal muscle fiber type. Genes Dev. 12, 2499-2509. doi: 10.1101/gad.12.16.2499

Cho, S. J., Moon, J. S., Lee, C. M., Choi, A. M. K., and Stout-Delgado, H. W. (2017). Glucose transporter 1-dependent glycolysis is increased during aging-related lung fibrosis, and phloretin inhibits lung fibrosis. Am. J. Respir. Cell Mol. Biol. 56, 521-531. doi: 10.1165/rcmb.2016-0225OC

Chung, S. S. M. (2004). Contribution of polyol pathway to diabetes-induced oxidative stress. J. Am. Soc. Nephrol. 14(8 Suppl. 3), 233S-236S. doi: 10.1097/ 01.asn.0000077408.15865.06

Clark, D. L., and Velleman, S. G. (2017). Spatial influence on breast muscle morphological structure, myofiber size, and gene expression associated with the wooden breast myopathy in broilers. Poult. Sci. 95, 2930-2945. doi: 10.3382/ps/ pew243

Dalle Zotte, A., Tasoniero, G., Puolanne, E., Remignon, H., Cecchinato, M., Catelli, E., et al. (2017). Effect of "Wooden Breast" appearance on poultry meat quality, histological traits, and lesions characterization. Czech J. Anim. Sci. 62, 51-57. doi: 10.17221/54/2016-CJAS

Dasu, M. R., and Jialal, I. (2010). Free fatty acids in the presence of high glucose amplify monocyte inflammation via Toll-like receptors. Am. J. Physiol. Endocrinol. Metab. 300, E145-E154. doi: 10.1152/ajpendo.00490.2010

De Miranda, M. A., Schlater, A. E., Green, T. L., and Kanatous, S. B. (2012). In the face of hypoxia: myoglobin increases in response to hypoxic conditions and lipid supplementation in cultured Weddell seal skeletal muscle cells. J. Exp. Biol. 215(Pt 5), 806-813. doi: 10.1242/jeb.060681

DeFronzo, R. A., and Tripathy, D. (2009). Skeletal muscle insulin resistance is the primary defect in type 2 diabetes. Diabetes Care 32(Suppl. 2), S157-S163. doi: $10.2337 / \mathrm{dc} 09-\mathrm{s} 302$

Dupont, J., Dagou, C., Derouet, M., Simon, J., and Taouis, M. (2004). Early steps of insulin receptor signaling in chicken and rat: apparent refractoriness in chicken muscle. Domest. Anim. Endocrinol. 26, 127-142. doi: 10.1016/j.domaniend. 2003.09.004

Eiselein, L., Wilson, D. W., Lamé, M. W., and Rutledge, J. C. (2007). Lipolysis products from triglyceride-rich lipoproteins increase endothelial permeability, perturb zonula occludens-1 and F-actin, and induce apoptosis. Am. J. Physiol. Heart Circ. Physiol. 292, H2745-H2753. doi: 10.1152/ajpheart.00686.2006

Endo, M. (2009). Calcium-induced calcium release in skeletal muscle. Physiol. Rev. 89, 1153-1176. doi: 10.1152/physrev.00040.2008

Ermak, G., and Davies, K. J. A. (2001). Calcium and oxidative stress: from cell signaling to cell death. Mol. Immunol. 38, 713-721. doi: 10.1016/s01615890(01)00108-0

Fantus, I. G., Goldberg, H. J., Whiteside, C. I., and Topic, D. (2006). "The hexosamine biosynthesis pathway," in The Diabetic Kidney. Contemporary Diabetes, eds P. Cortes and C. E. Mogensen (Totowa, NJ: Humana Press).

Fu, S., Li, P., Watkins, S. M., Ivanov, A. R., Hofmann, O., Hotamisligil, G. S., et al. (2011). Aberrant lipid metabolism disrupts calcium homeostasis causing liver endoplasmic reticulum stress in obesity. Nature 473, 528-531. doi: 10.1038/ nature 09968

Godet, E., Porter, T. E., Tesseraud, S., Simon, J., Duclos, M. J., Métayer-Coustard, S., et al. (2008). Insulin immuno-neutralization in chicken: effects on insulin signaling and gene expression in liver and muscle. J. Endocrinol. 197, 531-542. doi: 10.1677/JOE-08-0055

Goglia, F., and Skulachev, V. P. (2003). A function for novel uncoupling proteins: antioxidant defense of mitochondrial matrix by translocating fatty 
acid peroxides from the inner to the outer membrane leaflet. FASEB J. 17, 1585-1591. doi: 10.1096/fj.03-0159hyp

Gorski, D. J., Petz, A., Reichert, C., Twarock, S., Grandoch, M., and Fischer, J. W. (2019). Cardiac fibroblast activation and hyaluronan synthesis in response to hyperglycemia and diet-induced insulin resistance. Sci. Rep. 9:1827. doi: 10. 1038/s41598-018-36140-6

Gratta, F., Fasolato, L., Birolo, M., Zomeño, C., Novelli, E., Petracci, M., et al. (2019). Effect of breast myopathies on quality and microbial shelf life of broiler meat. Poult. Sci. 98, 2641-2651. doi: 10.3382/ps/pez001

Griffin, H., Acamovic, F., Guo, K., and Peddie, J. (1989). Plasma lipoprotein metabolism in lean and in fat chickens produced by divergent selection for plasma very low density lipoprotein concentration. J. Lipid Res. 30, 1243-1250.

Groop, L. C., and Ferrannini, E. (1993). Insulin action and substrate competition. Baillieres Clin. Endocrinol. Metab. 7, 1007-1032. doi: 10.1016/s0950-351x(05) 80243-5

Gunter, T. E., Gunter, K. K., Sheu, S.-S., and Gavin, C. E. (1994). Mitochondrial calcium transport: physiological and pathological relevance. Am. J. Physiol. 267(2 Pt 1), C313-C339.

Ha, S. W., Seo, Y. K., Kim, J. G., Kim, I. S., Sohn, K. Y., Lee, B. H., et al. (1997). Effect of high glucose on synthesis and gene expression of collagen and fibronectin in cultured vascular smooth muscle. Exp. Mol. Med. 29, 59-64. doi: 10.1038/emm.1997.9

Hajnóczky, G., Csordás, G., Das, S., Garcia-Perez, C., Saotome, M., Sinha Roy, S., et al. (2006). Mitochondrial calcium signalling and cell death: approaches for assessing the role of mitochondrial Ca2+ uptake in apoptosis. Cell Calcium 40, 553-560. doi: 10.1016/j.ceca.2006.08.016

Han, D. C., Isono, M., Hoffman, B. B., and Ziyadeh, F. N. (1999). High glucose stimulates proliferation and collagen type I synthesis in renal cortical fibroblasts: mediation by autocrine activation of TGF-beta. J. Am. Soc. Nephrol. 10, 1891-1899.

Havenstein, G. B., Ferket, P. R., and Qureshi, M. A. (2003a). Carcass composition and yield of 1957 versus 2001 broilers when fed representative 1957 and 2001 broiler diets. Poult. Sci. 82, 1509-1518. doi: 10.1093/ps/82.10.1509

Havenstein, G. B., Ferket, P. R., and Qureshi, M. A. (2003b). Growth, livability, and feed conversion of 1957 versus 2001 broilers when fed representative 1957 an 2001 broiler diets. Poult. Sci. 82, 1500-1508. doi: 10.1093/ps/82.10.1500

Hers, H. G. (1976). The control of glycogen metabolism in the liver. Annu. Rev. Biochem. 45, 167-190. doi: 10.1007/978-3-642-66461-8_14

Hirst, J., King, M. S., and Pryde, K. R. (2008). The production of reactive oxygen species by complex I. Biochem. Soc. Trans. 36, 976-980. doi: 10.1042/ BST0360976

Huang, J. M., Xian, H., and Bacaner, M. (2006). Long-chain fatty acids activate calcium channels in ventricular myocytes. Proc. Natl. Acad. Sci. U.S.A. 89, 6452-6456. doi: 10.1073/pnas.89.14.6452

Ishii, H., Tada, H., and Isogai, S. (1998). An aldose reductase inhibitor prevents glucose-induced increase in transforming growth factor- $\beta$ and protein kinase C activity in cultured human mesangial cells. Diabetologia 41, 362-364. doi: $10.1007 / \mathrm{s} 001250050916$

Kawasaki, T., Iwasaki, T., Yamada, M., Yoshida, T., and Watanabe, T. (2018). Rapid growth rate results in remarkably hardened breast in broilers during the middle stage of rearing: a biochemical and histopathological study. PLoS One 13:e0193307. doi: 10.1371/journal.pone.0193307

Kim, J. K., Fillmore, J. J., Chen, Y., Yu, C., Moore, I. K., Pypaert, M., et al. (2001). Tissue-specific overexpression of lipoprotein lipase causes tissuespecific insulin resistance. Proc. Natl. Acad. Sci. U.S.A. 98, 7522-7527. doi: 10.1073/pnas.121164498

Klip, A., Volchuk, A., He, L., and Tsakiridis, T. (1996). The glucose transporters of skeletal muscle. Semin. Cell Dev. Biol. 7, 229-237. doi: 10.1006/scdb.1996.0031

Kono, T., Nishida, M., Nishiki, Y., Seki, Y., Sato, K., and Akiba, Y. (2005). Characterisation of glucose transporter (GLUT) gene expression in broiler chickens. Br. Poult. Sci. 46, 510-515. doi: 10.1080/00071660500181289

Kruger, N. J., and Von Schaewen, A. (2003). The oxidative pentose phosphate pathway: structure and organisation. Curr. Opin. Plant Biol. 6, 236-246. doi: 10.1016/s1369-5266(03)00039-6

Krutzik, S. R., Tan, B., Li, H., Ochoa, M. T., Liu, P. T., Sharfstein, S. E., et al. (2005). TLR activation triggers the rapid differentiation of monocytes into macrophages and dendritic cells. Nat. Med. 11, 653-660. doi: 10.1016/j.jaci. 2011.08.037.Evidence
Kumagai, A. K. (1999). Glucose transport in brain and retina: implications in the management and complications of diabetes. Diabetes Metab. Res. Rev. 15, 261-273. doi: 10.1002/(SICI)1520-7560(199907/08)15:4<261:AIDDMRR43<3.0.CO;2-Z

Kuttappan, V. A., Hargis, B. M., and Owens, C. M. (2016). White striping and woody breast myopathies in the modern poultry industry: a review. Poult. Sci. 95, 2724-2733. doi: $10.3382 /$ ps/pew216

Kuttappan, V. A., Shivaprasad, H. L., Shaw, D. P., Valentine, B. A., Hargis, B. M., Clark, F. D., et al. (2013). Pathological changes associated with white striping in broiler breast muscles. Poult. Sci. 92, 331-338. doi: 10.3382/ps.2012-02646

Lake, J. A., Papah, M. B., and Abasht, B. (2019). Increased expression of lipid metabolism genes in early stages of wooden breast links myopathy of broilers to metabolic syndrome in humans. Genes (Basel) 10:746. doi: 10.3390/ genes10100746

Larkina, T. A., Sazanova, A. L., Fomichev, K. A., Barkova, O. Y., Malewski, T., Jaszczak, K., et al. (2010). HMG1A and PPARG are differently expressed in the liver of fat and lean broilers. J. Appl. Genet. 52, 225-228. doi: 10.1007/s13353010-0023-Z

Lee, J. Y., and Hwang, D. H. (2006). The modulation of inflammatory gene expression by lipids: mediation through Toll-like receptors. Mol. Cells 21, 174-185.

Li, Y., He, P. P., Zhang, D. W., Zheng, X. L., Cayabyab, F. S., Yin, W. D., et al. (2014). Lipoprotein lipase: from gene to atherosclerosis. Atherosclerosis 237, 597-608. doi: 10.1016/j.atherosclerosis.2014.10.016

Lilburn, M. S., Griffin, J. R., and Wick, M. (2018). From muscle to food: oxidative challenges and developmental anomalies in poultry breast muscle. Poult. Sci. 98, 4255-4260. doi: 10.3382/ps/pey409

Livingston, M. L., Ferket, P. R., Brake, J., and Livingston, K. A. (2019a). Dietary amino acids under hypoxic conditions exacerbates muscle myopathies including wooden breast and white stripping. Poult. Sci. 98, 1517-1527. doi: $10.3382 / \mathrm{ps} / \mathrm{pey} 463$

Livingston, M. L., Landon, C. D., Barnes, H. J., Brake, J., and Livingston, K. A. (2019b). Dietary potassium and available phosphorous on broiler growth performance, carcass characteristics, and wooden breast. Poult. Sci. 98, 28132822. doi: $10.3382 / \mathrm{ps} / \mathrm{pez} 015$

Mead, J. R., Irvine, S. A., and Ramji, D. P. (2002). Lipoprotein lipase: structure, function, regulation, and role in disease. J. Mol. Med. 80, 753-769. doi: 10.1007/ s00109-002-0384-9

Mudalal, S., Lorenzi, M., Soglia, F., Cavani, C., and Petracci, M. (2015). Implications of white striping and wooden breast abnormalities on quality traits of raw and marinated chicken meat. Animal 9, 728-734. doi: 10.1017/ S175173111400295X

Mutryn, M. F., Brannick, E. M., Fu, W., Lee, W. R., and Abasht, B. (2015). Characterization of a novel chicken muscle disorder through differential gene expression and pathway analysis using RNA-sequencing. BMC Genomics 16:399. doi: 10.1186/s12864-015-1623-0

Nanji, A. A., Fogt, F., and Griniuviene, B. (1995). Alterations in glucose transporter proteins in alcoholic liver disease in the rat. Am. J. Pathol. 146, 329-334.

Navale, A. M., and Paranjape, A. N. (2016). Glucose transporters: physiological and pathological roles. Biophys. Rev. 8, 5-9. doi: 10.1007/s12551-015-0186-2

Olin, K. L., Potter-Perigo, S., Barrett, P. H. R., Wight, T. N., and Chait, A. (1999). Lipoprotein lipase enhances the binding of native and oxidized low density lipoproteins to versican and biglycan synthesized by cultured arterial smooth muscle cells. J. Biol. Chem. 274, 34629-34636. doi: 10.1074/jbc.274.49. 34629

Orrenius, S., Gogvadze, V., and Zhivotovsky, B. (2015). Calcium and mitochondria in the regulation of cell death. Biochem. Biophys. Res. Commun. 460, 72-81. doi: 10.1016/j.bbrc.2015.01.137

Papah, M. B., and Abasht, B. (2019). Dysregulation of lipid metabolism and appearance of slow myofiber- specific isoforms accompany the development of Wooden Breast myopathy in modern broiler chickens. Sci. Rep 9:17170. doi: 10.1038/s41598-019-53728-8

Papah, M. B., Brannick, E. M., Schmidt, C. J., and Abasht, B. (2017). Evidence and role of phlebitis and lipid infiltration in the onset and pathogenesis of Wooden Breast disease in modern broiler chickens. Avian Pathol. 46, 623-643. doi: 10.1080/03079457.2017.1339346

Papah, M. B., Brannick, E. M., Schmidt, C. J., and Abasht, B. (2018). Gene expression profiling of the early pathogenesis of wooden breast disease in 
commercial broiler chickens using RNA-sequencing. PLoS One 13:e0207346. doi: 10.1371/journal.pone.0207346

Penzo, D., Tagliapietra, C., Colonna, R., Petronilli, V., and Bernardi, P. (2002). Effects of fatty acids on mitochondria: implications for cell death. Biochim. Biophys. Acta 1555, 160-165. doi: 10.1016/s0005-2728(02)00272-4

Petracci, M., Soglia, F., Madruga, M., Carvalho, L., Ida, E., and Estevez, M. (2019). Wooden-Breast, white striping, and spaghetti meat: causes, consequences and consumer perception of emerging broiler meat abnormalities. Compr. Rev. Food Sci. Food Saf. 18, 565-583. doi: 10.1111/1541-4337.12431

Pohlers, D., Brenmoehl, J., Löffler, I., Müller, C. K., Leipner, C., Schultze-Mosgau, S., et al. (2009). TGF- $\beta$ and fibrosis in different organs - molecular pathway imprints. Biochim. Biophys. Acta - Mol. Basis Dis. 1792, 746-756. doi: 10.1016/ j.bbadis.2009.06.004

Randle, P. J., Garland, P. B., Newsholme, E. A., and Hales, C. N. (1965). The glucose fatty acid cycle in obesity and maturity onset diabetes mellitus. Ann. N. Y. Acad. Sci. 131, 324-333. doi: 10.1111/j.1749-6632.1965.tb34800.x

Rasouli, N., Molavi, B., Elbein, S. C., and Kern, P. A. (2007). Ectopic fat accumulation and metabolic syndrome. Diabetes Obes. Metab. 9, 1-10. doi: 10.1111/j.1463-1326.2006.00590.x

Rocha, D. M., Caldas, A. P., Oliveira, L. L., Bressan, J., and Hermsdorff, H. H. (2016). Saturated fatty acids trigger TLR4-mediated inflammatory response. Atherosclerosis 244, 211-215. doi: 10.1016/j.atherosclerosis.2015.11.015

Rousset, S., Alves-Guerra, M.-C., Mozo, J., Miroux, B., Cassard-Doulcier, A.-M., Bouillaud, F., et al. (2004). The biology of mitochondrial uncoupling. Diabetes 53, S130-S135. doi: 10.2337/diabetes.53.2007.s130

Sandercock, D. A., Barker, Z. E., Mitchell, M. A., and Hocking, P. M. (2009). Changes in muscle cell cation regulation and meat quality traits are associated with genetic selection for high body weight and meat yield in broiler chickens. Genet. Sel. Evol. 41:8. doi: 10.1186/1297-9686-41-8

Schrauwen, P. (2007). High-fat diet, muscular lipotoxicity and insulin resistance. Proc. Nutr. Soc. 66, 33-41. doi: 10.1017/S0029665107005277

Schrauwen, P., and Hesselink, M. K. C. (2004). Oxidative capacity, lipotoxicity, and mitochondrial damage in type 2 diabetes. Diabetes 53, 1412-1417. doi: 10.2337/diabetes.53.6.1412

Seki, Y., Sato, K., Kono, T., Abe, H., and Akiba, Y. (2003). Broiler chickens (Ross strain) lack insulin-responsive glucose transporter GLUT4 and have GLUT8 cDNA. Gen. Comp. Endocrinol. 133, 80-87. doi: 10.1016/S0016-6480(03)001 $45-\mathrm{X}$

Semenza, G. L., Roth, P. H., Fang, H. M., and Wang, G. L. (1994). Transcriptional regulation of genes encoding glycolytic enzymes by hypoxia-inducible factor 1 . J. Biol. Chem. 269, 23757-23763.

Seo, M., and Lee, Y. H. (2014). PFKFB3 regulates oxidative stress homeostasis via its S-glutathionylation in cancer. J. Mol. Biol. 426, 830-842. doi: 10.1016/j.jmb. 2013.11.021

Shepherd, P. R., and Kahn, B. B. (1999). Glucose transporters and insulin action: implications for insulin resistance and diabetes mellitus. N. Engl. J. Med. 341, 248-257. doi: 10.1056/nejm199907223410406

Sihvo, H. K., Airas, N., Lindén, J., and Puolanne, E. (2018). Pectoral vessel density and early ultrastructural changes in broiler chicken wooden breast myopathy. J. Comp. Pathol. 161, 1-10. doi: 10.1016/j.jcpa.2018.04.002

Sihvo, H. K., Immonen, K., and Puolanne, E. (2014). Myodegeneration with fibrosis and regeneration in the pectoralis major muscle of broilers. Vet. Pathol. 51, 619-623. doi: 10.1177/0300985813497488

Smith, I. C., Bombardier, E., Vigna, C., and Tupling, A. R. (2013). ATP consumption by sarcoplasmic reticulum Ca2+ pumps accounts for $40-50 \%$ of resting metabolic rate in mouse fast and slow twitch skeletal muscle. PLoS One 8:e68924. doi: 10.1371/journal.pone.0068924

Smith, M. W., Mitchell, M. A., and Peacock, M. A. (1990). Effects of genetic selection on growth rate and intestinal structure in the domestic fowl (Gallus domesticus). Comp. Biochem. Physiol. A Comp. Physiol. 97, 57-63. doi: 10.1016/ 0300-9629(90)90722-5

Soglia, F., Mudalal, S., Babini, E., Di Nunzio, M., Mazzoni, M., Sirri, F., et al. (2016). Histology, composition, and quality traits of chicken Pectoralis major muscle affected by wooden breast abnormality. Poult. Sci. 95, 651-659. doi: $10.3382 / \mathrm{ps} / \mathrm{pev} 353$

Tasoniero, G., Cullere, M., Cecchinato, M., Puolanne, E., and Dalle Zotte, A. (2016). Technological quality, mineral profile, and sensory attributes of broiler chicken breasts affected by White Striping and Wooden Breast myopathies. Poult. Sci. 95, 2707-2714. doi: 10.3382/ps/pew215

Toborek, M., Lee, Y. W., Garrido, R., Kaiser, S., and Hennig, B. (2002). Unsaturated fatty acids selectively induce an inflammatory environment in human endothelial cells. Am. J. Clin. Nutr. 75, 119-125. doi: 10.1093/ajcn/75. 1.119

Tokudome, T., Horio, T., Yoshihara, F., Suga, S. I., Kawano, Y., Kohno, M., et al. (2004). Direct effects of high glucose and insulin on protein synthesis in cultured cardiac myocytes and DNA and collagen synthesis in cardiac fibroblasts. Metabolism 53, 710-715. doi: 10.1016/j.metabol.2004.01.006

Tokushima, Y., Takahashi, K., Sato, K., and Akiba, Y. (2005). Glucose uptake in vivo in skeletal muscles of insulin-injected chicks. Comp. Biochem. Physiol. B Biochem. Mol. Biol. 141, 43-48. doi: 10.1016/j.cbpc.2005.01.008

Tousoulis, D., Kampoli, A. M., Tentolouris, C., Papageorgiou, N., and Stefanadis, C. (2012). The role of nitric oxide on endothelial function. Curr. Vasc. Pharmacol. 10, 4-18. doi: 10.2174/157016112798829760

Ursini, F., Roveri, A., van Amsterdam, F. T. M., Ratti, E., Maiorino, M., Zamburlini, A., et al. (2004). Effect of hydrogen peroxide on calcium homeostasis in smooth muscle cells. Arch. Biochem. Biophys. 297, 265-270. doi: 10.1016/0003-9861(92) 90671-i

Velleman, S. G., Clark, D. L., and Tonniges, J. R. (2017). Fibrillar collagen organization associated with broiler wooden breast fibrotic myopathy. Avian Dis. 61, 481-490. doi: 10.1637/11738-080217-Reg.1

Volmer, R., and Ron, D. (2015). Lipid-dependent regulation of the unfolded protein response. Curr. Opin. Cell Biol. 33, 67-73. doi: 10.1016/j.ceb.2014.1 2.002

Wall, V. Z., Barnhart, S., Kanter, J. E., Kramer, F., Shimizu-Albergine, M., Adhikari, N., et al. (2018). Smooth muscle glucose metabolism promotes monocyte recruitment and atherosclerosis in a mouse model of metabolic syndrome. JCI Insight 3:e96544. doi: 10.1172/jci.insight.96544

Wang, H., and Eckel, R. H. (2009). Lipoprotein lipase: from gene to obesity. Am. J. Physiol. Metab. 297, E271-E288. doi: 10.1152/ajpendo.90920.2008

Wang, J., Xian, X., Huang, W., Chen, L., Wu, L., Zhu, Y., et al. (2007). Expression of LPL in endothelial-intact artery results in lipid deposition and vascular cell adhesion molecule-1 upregulation in both LPL and ApoE-deficient mice. Arterioscler. Thromb. Vasc. Biol. 27, 197-203. doi: 10.1161/01.ATV.0000249683. 80414.d9

Wei, Y., Wang, D., Gentile, C. L., and Pagliassotti, M. J. (2009). Reduced endoplasmic reticulum luminal calcium links saturated fatty acid-mediated endoplasmic reticulum stress and cell death in liver cells. Mol. Cell. Biochem. 331, 31-40. doi: 10.1007/s11010-009-0142-1

Yabe-Nishimura, C. (1998). Aldose reductase in glucose toxicity: a potential target for the prevention of diabetic complications. Pharmacol. Rev. 50, 21-33.

Yamamoto, T., Takano, N., Ishiwata, K., Ohmura, M., Nagahata, Y., Matsuura, T., et al. (2014). Reduced methylation of PFKFB3 in cancer cells shunts glucose towards the pentose phosphate pathway. Nat. Commun. 5:3480. doi: 10.1038/ ncomms 4480

Yan, L. (2018). Redox imbalance stress in diabetes mellitus: role of the polyol pathway. Anim. Model. Exp. Med. 1, 7-13. doi: 10.1002/ame2.12001

Yin, H., Price, F., and Rudnicki, M. A. (2013). Satellite cells and the muscle stem cell niche. Physiol. Rev. 93, 23-67. doi: 10.1152/physrev.00043.2011

Zahoor, I., De Koning, D. J., and Hocking, P. M. (2017). Transcriptional profile of breast muscle in heat stressed layers is similar to that of broiler chickens at control temperature. Genet. Sel. Evol. 49:69. doi: 10.1186/s12711-017-0346-x

Zambonelli, P., Zappaterra, M., Soglia, F., Petracci, M., Sirri, F., Cavani, C., et al. (2016). Detection of differentially expressed genes in broiler pectoralis major muscle affected by White Striping - Wooden Breast myopathies. Poult. Sci. 95, 2771-2785. doi: 10.3382/ps/pew268

Zanetti, M. A., Tedesco, D. C., Schneider, T., Teixeira, S. T. F., Daroit, L., Pilotto, F., et al. (2018). Economic losses associated with Wooden Breast and White Striping in broilers. Semin. Cienc. Agrar. 39, 887-892. doi: 10.5433/1679-0359. 2018v39n2p887

Zhou, N., Lee, W. R., and Abasht, B. (2015). Messenger RNA sequencing and pathway analysis provide novel insights into the biological basis of chickens' feed efficiency. BMC Genomics 16:195. doi: 10.1186/s12864-015-1364-0

Zhuo, Z., Lamont, S. J., Lee, W. R., and Abasht, B. (2015). RNA-seq analysis of abdominal fat reveals differences between modern commercial broiler chickens 
with high and low feed efficiencies. PLoS One 10:e0135810. doi: 10.1371/journal. pone. 0135810

Zierath, J. R., Houseknecht, K. L., Gnudi, L., and Kahn, B. B. (1997). High-fat feeding impairs insulin-stimulated GLUT4 recruitment via an early insulinsignaling defect. Diabetes 46, 215-223. doi: 10.2337/diab.46.2.215

Zimermann, F. C., Fallavena, L. C. B., Salle, C. T. P., Moraes, H. L. S., Soncini, R. A., Barreta, M. H., et al. (2012). Downgrading of heavy broiler chicken carcasses due to myodegeneration of the anterior latissimus dorsi: pathologic and epidemiologic studies. Avian Dis. 56, 418-421. doi: 10.1637/9860-072111case.1
Conflict of Interest: The authors declare that the research was conducted in the absence of any commercial or financial relationships that could be construed as a potential conflict of interest.

Copyright (c) 2020 Lake and Abasht. This is an open-access article distributed under the terms of the Creative Commons Attribution License (CC BY). The use, distribution or reproduction in other forums is permitted, provided the original author(s) and the copyright owner(s) are credited and that the original publication in this journal is cited, in accordance with accepted academic practice. No use, distribution or reproduction is permitted which does not comply with these terms. 
OPEN ACCESS

Edited by:

Massimiliano Petracci,

University of Bologna, Italy

Reviewed by:

Kent M. Reed,

University of Minnesota Twin Cities,

United States

Martina Bordini,

University of Bologna, Italy

*Correspondence:

Orna Halevy

orna.halevy@mail.huji.ac.il;

halevyo@gmail.com

Specialty section:

This article was submitted to

Avian Physiology,

a section of the journal

Frontiers in Physiology

Received: 05 January 2020

Accepted: 02 March 2020

Published: 31 March 2020

Citation:

Halevy O (2020) Timing Is

Everything-The High Sensitivity

of Avian Satellite Cells to Thermal

Conditions During Embryonic

and Posthatch Periods.

Front. Physiol. 11:235.

doi: 10.3389/fphys.2020.00235

\section{Timing Is Everything - The High Sensitivity of Avian Satellite Cells to Thermal Conditions During Embryonic and Posthatch Periods}

\author{
Orna Halevy* \\ Department of Animal Sciences, The Hebrew University of Jerusalem, Rehovot, Israel
}

Myofiber formation is essentially complete at hatch, but myofiber hypertrophy increases posthatch through the assimilation of satellite cell nuclei into myofibers. Satellite cell proliferation and differentiation occur during the early growth phase, which in meattype poultry terminates at around 8 days posthatch. Thus, any factor that affects the accumulation of satellite cells during late-term embryogenesis or early posthatch will dictate long-term muscle growth. This review will focus on the intimate relationship between thermal conditions during chick embryogenesis and the early posthatch period, and satellite cell myogenesis and pectoralis growth and development. Satellite cells are highly sensitive to temperature changes, particularly when those changes occur during crucial periods of their myogenic activity. Therefore, timing, temperature, and duration of thermal treatments have a great impact on satellite cell activity and fate, affecting muscle development and growth in the long run. Short and mild thermal manipulations during embryogenesis or thermal conditioning in the early posthatch period promote myogenic cell proliferation and differentiation, and have long-term promotive effects on muscle growth. However, chronic heat stress during the first 2 weeks of life has adverse effects on these parameters and may lead to muscle myopathies.

Keywords: skeletal muscle, broiler, satellite cell, myofiber, thermal stress, myopathies

\section{INTRODUCTION}

Vertebrates are born or hatch with a defined number of myofibers in the muscle. Muscle growth will then involve mainly growth in myofiber size (i.e., hypertrophy), where there is a large increase in the synthesis of contractile and regulatory proteins. In meat-type poultry (i.e., broilers), this process occurs largely from the second week of age onward and involves the accretion of nuclei. The source for these additional nuclei, at least during the early growth period, is a population of muscle progenitor cells called satellite cells (SCs; Moss and Leblond, 1971), also termed "adult myoblasts" (Hartley et al., 1992). This review will focus on the implications of environmental conditions, specifically thermal conditions, during embryogenesis and early posthatch periods, for

Abbreviations: BW, body weight; SC, satellite cell; Ta, ambient temperature; Tb, body temperature; TC, thermal conditioning; TM, thermal manipulation. 
the proliferation and differentiation of SCs, as well as for pectoralis muscle development and growth in broilers. Possible mechanisms underlying these effects will be discussed.

\section{SATELLITE CELLS AND THEIR INVOLVEMENT IN POSTNATAL MUSCLE GROWTH AND REGENERATION}

Satellite cells were initially discovered by Mauro (1961) based on their location in the myofiber, in a niche between the basal lamina and the sarcolemma. Although originating from the somites at early stages of embryonic development (Gros et al., 2005; Relaix et al., 2005), SCs can only be distinguished at later stages; in chickens, they are present at low numbers on embryonic day 14 (E14) and become the majority at E18 (Hartley et al., 1992; Feldman and Stockdale, 1992; Yablonka-Reuveni, 1995). In contrast to the embryonic and fetal myoblasts, which undergo proliferation and terminal differentiation during the first and second waves of myogenesis in the embryo, the SCs proliferate and do not differentiate (Stockdale, 1992; reviewed in Chal and Pourquié, 2017). After birth or hatch, upon the third wave of myogenesis, SCs largely proliferate and then undergo terminal differentiation. This third wave is short; in broilers, it lasts around 8 days (Halevy et al., 2000, 2001, 2004, 2006a,b; Allouh et al., 2008). Thereafter, SC activity rapidly declines, and their numbers drop to $1-5 \%$ of total myonuclei. Satellite cells then become largely quiescent and remain in their niche in that state. They will only become active again during muscle regeneration: upon damage (e.g., injury, toxins), stress (e.g., heat or cold stress), or diseases (myopathies), SCs will be reactivated into the myogenic program, entering the cell cycle and undergoing terminal differentiation to form new myofibers (reviewed in Hawke and Garry, 2001; Zammit et al., 2006; Yablonka-Reuveni, 2011).

The muscle-specific basic helix-loop-helix family of transcription factors regulates the myogenic program of embryonic and fetal myoblasts, as well as of SCs, where they are expressed in a sequential pattern (reviewed in Naya and Olson, 1999). The paired-box containing transcription factor Pax7 plays an essential role in the formation of adult skeletal muscle (Seale et al., 2000); it is expressed by quiescent SCs and becomes highly expressed upon their activation and proliferation, then declines when the SCs undergo terminal differentiation (Halevy et al., 2004; Zammit et al., 2006).

Muscle growth and regeneration are tightly regulated by autocrine and paracrine factors, some of which are involved in SC activation and proliferation [e.g., hepatocyte growth factor (HGF), fibroblast growth factor (FGF)], whereas others are more involved in terminal differentiation and hypertrophy [e.g., insulin-like growth factor (IGF), sonic hedgehog] or inhibit these processes [e.g., transforming growth factor $\beta$ (TGF $\beta$ ), myostatin] (reviewed in Hawke and Garry, 2001; Halevy et al., 2006b; Yablonka-Reuveni, 2011).

Satellite cells are considered to be adult muscle stem cells due to their ability to divide in an asymmetric manner into either daughter progenitor cells that will undergo myogenic differentiation or daughter stems cells that will replenish the muscle cell reservoir (Collins et al., 2005; Shinin et al., 2006; Kuang et al., 2007; reviewed in Brack and Rando, 2012; Tierney and Sacco, 2016). Moreover, SCs, under specific environmental cues, possess the ability to transdifferentiate into other mesenchymal lineages, such as chondrogenic, adipogenic, or fibrogenic tissues (Asakura et al., 2001; Shefer et al., 2004; Brack et al., 2007; Yin et al., 2013).

\section{THERMOREGULATION IN BIRDS}

Birds are endotherms; hence, they are able to maintain their body temperature $(\mathrm{Tb})$ within a narrow range. However, due to genetic selection for growth and meat production over the last decades (Havenstein et al., 2003a,b), meat-type poultry have difficulty coping with extreme environmental temperatures, especially heat stress. The consequences are adverse effects on food consumption and performance, morbidity and mortality, and inferior meat quality (Cooper and Washburn, 1998; Sandercock et al., 2001; Yahav et al., 2005; Cahaner, 2008). Among the different approaches used to avoid the harmful consequences of thermal stress and to improve the acquisition of thermotolerance are acclimation by thermal conditioning (TC) during the early postnatal period and thermal manipulations (TMs) during the incubation period (Nichelmann and Tzschentke, 2002; Tzschentke et al., 2004; Yahav, 2009). These approaches are based on the presumed occurrence of epigenetic adaptation with a lifelong impact due to changes in factors such as ambient temperature $(\mathrm{Ta})$ at "critical developmental phases" of the thermoregulation system during embryogenesis and early posthatch periods (Tzschentke et al., 2004). This is reflected in a reduction in $\mathrm{Tb}$, decreased thyroid and corticosterone hormone levels, and changes in the vasomotor response (Yahav, 2015). Therefore, the ability to define these "critical phases" is crucial for improving thermotolerance acquisition. Mid and late embryogenesis are good periods for TMs (Yahav, 2009), as these are the periods during which the hypothalamo-pituitary-thyroid axis, associated with thermoregulation, and the hypothalamo-pituitary-adrenal axis, associated with stress, are developing (Thommes et al., 1984). The development of the chicken brain and thermoregulation is completed during the first 10 days posthatch (Arad and Itsaki-Glucklish, 1991), making this period ideal for TC in posthatch chicks.

\section{THERMAL CONDITIONING IN THE EARLY POSTHATCH PERIOD PROMOTES SC PROLIFERATION AND MUSCLE GROWTH}

The first evidence that mild $\mathrm{TC}$ at $37^{\circ} \mathrm{C}$ for $24 \mathrm{~h}$ promotes body weight $(\mathrm{BW})$ gain in broilers emerged from experiments aimed at enhancing acclimation of broilers to hot temperatures before their marketing. Interestingly, only chicks that underwent TC on day 5 of age, but not later, had higher BW, while 
lower rates of morbidity and mortality were observed in all treated chicks (Yahav and Hurwitz, 1996; Yahav and Plavnik, 1999). The higher BW was accompanied by higher growth of the pectoralis muscle (De Basilio et al., 2001). Fine-tuning experiments showed that TC on day 3 posthatch is optimal for chicken acclimation to high temperatures, as well as for gains in BW and performance at marketing (Yahav and McMurtry, 2001). Satellite cell proliferative activity in chickens is at its peak on day 3 posthatch (Halevy et al., 2004, 2006b), suggesting this day to be the best time for SC activity induction by TC. Indeed, in a follow-up study, it was demonstrated that mild TC on day 3 boosts the number of SCs in the pectoralis muscle and accelerates their differentiation on later days, resulting in higher pectoral muscle growth until marketing (Halevy et al., 2001). The dramatic response of SCs to the TC was most likely due to an indirect, systemic effect rather than a direct heat effect. Analysis of different factors that might be affected by the TC revealed induction of HGF and a muscle IGF-I isotype (Halevy et al., 2001) - growth factors that are upregulated following muscle stress (Tatsumi et al., 1998; Goldspink et al., 2008). It also seemed that a reduction in triiodothyronine $\left(\mathrm{T}_{3}\right)$ in response to the TC (Yahav and Hurwitz, 1996; Yahav, 2009) may also have an effect on the accelerated rate of SC differentiation (Ambrosio et al., 2017). Despite the promising results of TC on day 3 of age for both thermal acquisition and pectoralis muscle growth, this procedure proved to be somewhat demanding for the farmer and is therefore not widely used in the commercial rearing of broilers. Conducting TMs on chicken embryos during sensitive periods of the thyroid and adrenal axes' development and myogenesis could provide long-lasting promotive epigenetic effects on both thermoregulation acquisition and muscle growth.

\section{THERMAL MANIPULATIONS DURING EMBRYOGENESIS AND SKELETAL MUSCLE GROWTH}

The development of the thyroid and adrenal axes in the chick embryo between E8 and E12 (Thommes et al., 1984) largely overlaps with the second wave of myogenesis, when the major part of the myofiber mass is being generated. Proliferative SC activity occurs later on, between E15 and E19, with a peak on E17 (Halevy et al., 2006a; Piestun et al., 2009b); the hypothalamopituitary-adrenal axis is activated during this period (Wise and Frye, 1975; Epple et al., 1997). These two periods could be ideal for TMs, to both increase thermotolerance and promote muscle growth posthatch. However, because egg incubation in the hatchery must be kept under tight temperature and humidity control, only mild changes in either timing, temperature, or duration of the TMs can be made to avoid any major stress response (Epple et al., 1997; Yahav et al., 2004a,b; Renaudeau et al., 2012). Different timings and durations of the TMs seem to have different impacts on long-term thermotolerance and pectoralis muscle growth. For example, among the wide range of temperatures and durations for TMs performed from E16 to E18, only an increase in temperature to $39.5^{\circ} \mathrm{C}$ for $3 \mathrm{~h}$ daily was optimal for the promotion of thermotolerance as well as performance (Yahav et al., 2004b; Tzschentke and Halle, 2009) and pectoralis muscle growth (Halevy et al., 2006a). However, this regime had to be reexamined for TMs on earlier embryonic days. While TMs between E8 and E10 for $3 \mathrm{~h}$ daily did not have any promotive effect on these parameters (Collin et al., 2007), intermittent TM for 12 h/day between E8 and E16 had a long-lasting effect on thermotolerance, BW and pectoralis muscle growth (Loyau et al., 2014) up to 70 days posthatch (Piestun et al., 2013). In another experiment, it was demonstrated that intermittent, but not continuous TM between E8 and E16, increases the embryos' relative BW; yet, in all embryos, $\mathrm{Tb}$ decreased, as did thyroid hormone levels (Piestun et al., 2009a). Owing to the intermittent TM between E8 and E16, the increase in pectoralis muscle growth during embryogenesis was later reflected in increased myofiber hypertrophy (Piestun et al., 2011) and pectoral muscle growth (Piestun et al., 2013; Loyau et al., 2014) up to 35 days posthatch. The phenomenon of a long-term effect of embryonic TM on muscle growth could have several explanations. First, the immediate response to the TM was an increase in the number of myoblasts, a trend that continued posthatch, suggesting an increase in the muscle progenitor reservoir during the second and third waves of myogenesis (Piestun et al., 2015). Second, under a similar TM regime, a gene-array assay on pectoralis major muscle taken from control vs. treated embryos revealed different patterns of expression for genes involved in, among others, cell proliferation, energy metabolism, and mitochondrial function, vascularization, and muscle growth (Loyau et al., 2016). Taken together, it is concluded that TMs during periods in embryogenesis corresponding to the development of the thyroid and adrenal axes and myogenic waves result in changes in epigenetic processes, which underlie the changes in specific genes' expression leading to long-lasting effects on thermotolerance and muscle growth.

\section{NEGATIVE EFFECTS OF THERMAL STRESS ON SC MYOGENESIS IN POSTHATCH CHICKS}

The early posthatch period is critical for muscle growth; during this period, SCs complete their proliferation and undergo terminal differentiation and fusion to myofibers. Therefore, changes in nutrition (Halevy et al., 2000; Mozdziak et al., 2002; Bigot et al., 2003; Kornasio et al., 2011; Velleman et al., 2014; Powell et al., 2014, 2016) or environmental conditions (Halevy et al., 1998, 2001; Liu et al., 2010; Hadad et al., 2014a) during this period largely affect these processes, resulting in long-term effects on muscle growth. As already noted, TC on day 3 of age is beneficial for SC proliferation and differentiation (Halevy et al., 2001). However, prolonged thermal stress during the first 2 weeks posthatch had the opposite effect on these cells. Cell proliferation and numbers declined along with myofiber hypertrophy (Hadad et al., 2014a; Piestun et al., 2017; Patael et al., 2019), all of which had shortand long-term adverse effects on BW and pectoralis muscle weight (Hadad et al., 2014a,b; Patael et al., 2019). Interestingly, 
these adverse effects were minimal or non-existent under mild heat stress, where the temperature was only $2^{\circ} \mathrm{C}$ higher than commercial production temperatures. Moreover, in chicks grown under mild cold conditions, the proliferation of SCs exceeded that in the other groups (Patael et al., 2019). Taken together, it can be concluded that the Ta under which chicks are reared early posthatch is a critical factor influencing the immediate activity of the muscle progenitor cells and muscle hypertrophy at later ages.

Detailed studies on cultured SCs from broilers (Harding et al., 2015, 2016) and turkeys (Clark et al., 2016, 2017) revealed that the pectoralis muscle, in particular, is highly sensitive to temperature changes. Yet, a decline in SCs did not result from increased apoptosis in either cultured cells (Harding et al., 2015) or the treated chicks (Patael et al., 2019). A surprising finding was that the heat stress caused dramatic changes in SC fate toward adipogenesis and in muscle structure toward myopathy. Accumulation of lipid droplets was noticed shortly upon heat stress of cultured SCs (Harding et al., 2015; Clark et al., 2017), in SCs derived from heat-treated chicks (Piestun et al., 2017), and later, as the cells underwent differentiation, in myotubes and myofibers. The lipid accumulation was due to the induction of adipogenic gene and protein expression in the SCs (Harding et al., 2015; Clark et al., 2017; Piestun et al., 2017; Patael et al., 2019), suggesting transdifferentiation of these cells to the adipogenic lineage. Interestingly, these SCs did not seem to lose their myogenic characteristics completely, and along with the fat deposition, they underwent terminal differentiation to myotubes and fusion to myofibers. It may well be that, although the myogenic phenotype remains, these specific myofibers lose their full ability to contract, thus rendering the muscle less functional and contributing to its myopathic appearance.

\section{THERMAL STRESS IN THE EARLY POSTHATCH PERIOD AND PECTORAL MUSCLE MYOPATHIES}

Chronic heat stress, even when mild, during the first 2 weeks posthatch causes long-term adverse effects on the pectoralis muscle. The pectoralis muscle of the heat-stressed chicken presents large amounts of collagen deposition (i.e., fibrotic tissue) between the myofibers and large areas of fat droplets (Figure 1; Patael et al., 2019), all of which are typical features of myopathies (Kuttappan et al., 2016; Sihvo et al., 2014, 2017; Velleman et al., 2018). The various myopathies in poultry, such as white striping, wooden breast, and spaghetti meat, are thought to result from genetic selection for BW and, in particular, breast meat production, and inaccurate or suboptimal nutrition and/or management (Branciari et al., 2009; Kuttappan et al., 2012, 2016; Petracci et al., 2013; Velleman and Clark, 2015; Tonniges et al., 2019). These myopathies have a severe economic impact on the poultry industry due to a sheer loss in meat quantity and quality (Alnahhas et al., 2016; Kuttappan et al., 2016; Baldi et al., 2018). The direct metabolic and physiological causes

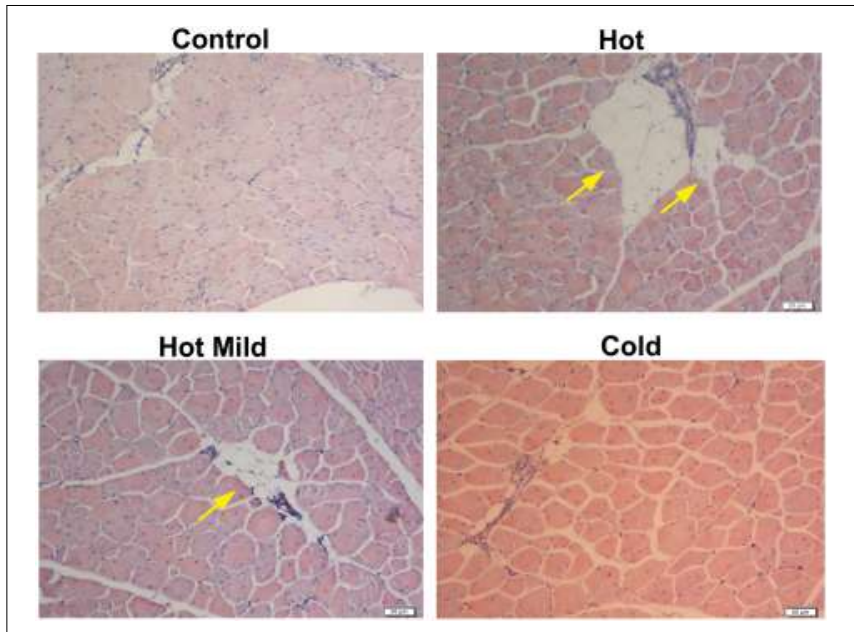

FIGURE 1 | Morphological structure of pectoralis muscle in cross-sections derived from control and treated chicks on day 35 of age and stained with hematoxylin-eosin. Meat-type chick (Cobb strain) groups were reared in temperature-controlled rooms under various Ta regimes beginning on day 1 and gradually reduced until day 13 , as follows: control, commercial temperature, $33-27^{\circ} \mathrm{C}$; hot, $39-33^{\circ} \mathrm{C}$; mild hot, $35-30^{\circ} \mathrm{C}$; cold, $29-27^{\circ} \mathrm{C}$. From day 14 onward, Ta was similar to all groups. Note the relatively smaller myofiber diameters in the hot group as compared to the other groups, and the large areas of fat deposition in the hot and hot mild groups (yellow arrows). Bar, $50 \mu \mathrm{m}$. Adapted from Patael et al. (2019), used with permission.

underlying these myopathies are not fully understood. They could be due, at least in part, to extensive genetic selection for BW or pectoral muscle growth, but not for supportive tissues (e.g., cardiovascular system), leading to lack of vascularization mainly in the pectoralis muscle and, hence, lack of oxygen supply and energy storage (Alnahhas et al., 2016; Malila et al., 2019). A very recent study reported a direct association between dysregulation of lipid metabolism and the development of wooden breast (Papah and Abasht, 2019). Thermal stress, especially during the early growth period, probably further aggravates this situation, leading to myodegeneration, expansion of the muscle's interstitial fibro-adipogenic progenitors (Contreras et al., 2016), and/or recruitment of myofibroblasts and adipogenic cells from nearby tissues, resulting in increased fibrotic tissue and fat deposition between the myofibers (Bailey et al., 2015). This is in parallel to the reduction in the number of SCs and their changes in fate, which may lead to ineffective myofiber function (Harding et al., 2015; Piestun et al., 2017; Patael et al., 2019). In contrast, featherless broilers subjected to cold stress during the first week posthatch developed a better vascular system and had a higher number of muscle cell progenitors and better muscle hypertrophy than feathered broilers (Hadad et al., 2014a,b). In addition, broilers that were kept under colder temperatures during the first 2 weeks posthatch had hardly any signs of collagen or fat deposition in the pectoralis muscle (Figure 1; Patael et al., 2019). Taken together, the evidence suggests that thermal stress should be minimized in modern broilers that have high rates of metabolism and 
heat production, on the one hand, and a shortage of blood supply on the other.

\section{CONCLUSION}

Changes in Ta have a pronounced impact on muscle growth, as well as on thermal acclimation in birds in general and in fast growing poultry in particular. These changes are observed already during embryonic development and into the posthatch period. During periods when they are highly active, SCs are very sensitive to temperature changes; these changes affect their proliferation and differentiation activities, as well as their fate, with longterm effects on muscle growth and structure. While short and mild heat stress promotes SC activity and muscle growth, more chronic heat stress has severe consequences, even resulting in muscle myopathies. Therefore, the duration and degree of the change in $\mathrm{Ta}$, as well as its timing during the embryonic and posthatch periods, are of the utmost importance in determining the development and growth of muscle in poultry.

\section{REFERENCES}

Allouh, M. Z., Yablonka-Reuveni, Z., and Rosser, B. W. C. (2008). Pax7 reveals a greater frequency and concentration of satellite cells at the ends of growing skeletal muscle fibers. J. Histochem. Cytochem. 56, 77-87. doi: 10.1369/jhc. 7a7301.2007

Alnahhas, N., Berri, C., Chabault, M., Chartrin, P., Boulay, M., and Bourin, M. C. (2016). Genetic parameters of white striping in relation to body weight, carcass composition, and meat quality traits in two broiler lines divergently selected for the ultimate $\mathrm{pH}$ of the pectoralis major muscle. BMC Genetics 17, 61-70. doi: 10.1186/s12863-016-0369-2

Ambrosio, R., De Stefano, M. A., Di Girolamo, D., and Salvatore, D. (2017). Thyroid hormone signaling and deiodinase actions in muscle stem/progenitor cells. Mol. Cell. Endocrinol. 459, 79-83. doi: 10.1016/j.mce.2017.06.014

Arad, Z., and Itsaki-Glucklish, S. (1991). Ontogeny of brain temperature in quail chicks (Coturnix japonica). Physiol. Zool. 64, 1356-1370. doi: 10.1086/physzool. 64.5.30156250

Asakura, A., Komaki, M., and Rudnicki, M. (2001). Muscle satellite cells are multipotential stem cells that exhibit myogenic, osteogenic and adipogenic differentiation. Differentiation 68, 245-253. doi: 10.1046/j.1432-0436.2001. 680412.x

Bailey, R. A., Watson, K. A., Bilgili, S. F., and Avendano, S. (2015). The genetic basis of pectoralis major myopathies in modern broiler chicken lines. Poult. Sci. 94, 2870-2879. doi: 10.3382/ps/pev304

Baldi, G., Soglia, F., Mazzoni, M., Sirri, F., Canonico, L., Babini, E., et al. (2018). Implications of white striping and spaghetti meat abnormalities on meat quality and histological features in broilers. Animal 12, 164-173. doi: 10.1017/ s1751731117001069

Bigot, K., Mignon-Grasteau, S., Picard, M., and Tesseraud, S. (2003). Effects of delayed feed intake on body, intestine, and muscle development in neonate broilers. Poult. Sci. 82, 781-788. doi: 10.1093/ps/82.5.781

Brack, A. S., Conboy, M. J., Roy, S., Lee, M., Kuo, C. J., Keller, C., et al. (2007). Increased Wnt signaling during aging alters muscle stem cell fate and increases fibrosis. Science 317, 807-810. doi: 10.1126/science.1144090

Brack, A. S., and Rando, T. A. (2012). Tissue specific stem cells: lessons from skeletal muscle satellite cells. Cell Stem Cell 10, 504-514. doi: 10.1016/j.stem. 2012.04.001

Branciari, R., Mugnai, C., Mammoli, R., Miraglia, D., Ranucci, D., Dal Bosco, A., et al. (2009). Effect of genotype and rearing system on chicken behavior and muscle fiber characteristics. J. Anim. Sci. 87, 4109-4117. doi: 10.2527/jas.20092090

\section{AUTHOR CONTRIBUTIONS}

The author confirms being the sole contributor to this manuscript and approved it for publication. $\mathrm{OH}$ conceptualized the manuscript, reviewed the literature, and wrote the manuscript in its entirety.

\section{FUNDING}

OH's studies were supported in part by the Binational Agricultural Research and Development (BARD) fund, grant nos. IS-4592-13 and IS-3836-06R.

\section{ACKNOWLEDGMENTS}

The author thanks Prof. Shlomo Yahav for careful reading of the manuscript. $\mathrm{OH}$ holds the Charles Charkowsky Chair in Poultry Science and Animal Hygiene.

Cahaner, A. (2008). "Breeding fast-growing, high-yield broilers for hot conditions," in Poultry Production in Hot Climates, 2nd Edn, ed. N. J. Daghir (Oxfordshire: CAB Int.), 30-47. doi: 10.1079/9781845932589.0030

Chal, J., and Pourquié, O. (2017). Making muscle: skeletal myogenesis in vivo and in vitro. Development 144, 2104-2122. doi: 10.1242/dev.151035

Clark, D. L., Coy, C. S., Strasburg, G. M., Reed, K. M., and Velleman, S. G. (2016). Temperature effect on proliferation and differentiation of satellite cells from turkeys with different growth rates. Poult. Sci. 95, 934-947. doi: 10.3382/ps/ pev437

Clark, D. L., Strasburg, G. M., Reed, K. M., and Velleman, S. G. (2017). Influence of temperature and growth selection on turkey pectoralis major muscle satellite cell adipogenic gene expression and lipid accumulation. Poult. Sci. 96, 10151027. doi: 10.3382/ps/pew374

Collin, A., Berri, C., Tesseraud, S., Rodón, F. E., Skiba-Cassy, S., Crochet, S., et al. (2007). Effects of thermal manipulation during early and late embryogenesis on thermotolerance and breast muscle characteristics in broiler chickens. Poult. Sci. 86, 795-800. doi: 10.1093/ps/86.5.795

Collins, C. A., Olsen, I., Zammit, P. S., Heslop, L., Petrie, A., Partridge, T. A., et al. (2005). Stem cell function, self-renewal, and behavioral heterogeneity of cells from the adult muscle satellite cell niche. Cell 122, 289-301. doi: 10.1016/j.cell. 2005.05.010

Contreras, O., Rebolledo, D. L., Oyarzún, J. E., Olguín, H. C., and Brandan, E. (2016). Connective tissue cells expressing fibro/adipogenic progenitor markers increase under chronic damage: relevance in fibroblast-myofibroblast differentiation and skeletal muscle fibrosis. Cell Tissue Res. 364, 647-660. doi: 10.1007/s00441-015-2343-0

Cooper, M. A., and Washburn, K. W. (1998). The relationships of body temperature to weight gain, feed consumption, and feed utilization in broilers under heat stress. Poult. Sci. 77, 237-242. doi: 10.1093/ps/77.2.237

De Basilio, V., Vilariño, M., Yahav, S., and Picard, M. (2001). Early age thermal conditioning and a dual feeding program for male broilers challenged by heat stress. Poult. Sci. 80, 29-36. doi: 10.1093/ps/80.1.29

Epple, A., Gower, B., Busch, M. T., Gill, T., Milakofsky, L., Piechotta, R., et al. (1997). Stress responses in avian embryos. Am. Zool. 37, 536-545. doi: 10.1093/ icb/37.6.536

Feldman, J. L., and Stockdale, F. E. (1992). Temporal appearance of satellite cells during myogenesis. Dev. Biol. 153, 217-226. doi: 10.1016/0012-1606(92)90 $107-\mathrm{r}$

Goldspink, G., Wessner, B., and Bachl, N. (2008). Growth factors, muscle function and doping. Curr. Opin. Pharmacol. 8, 352-357. doi: 10.1016/j.coph.2008.02. 002 
Gros, J., Manceau, M., Thomas, V., and Marcelle, C. (2005). A common somitic origin for embryonic muscle progenitors and satellite cells. Nature 435, 654958.

Hadad, Y., Cahaner, A., and Halevy, O. (2014a). Featherless and feathered broilers under control versus hot conditions. 2. Breast muscle development and growth in pre- and post-hatch periods. Poult. Sci. 93, 1076-1087.

Hadad, Y., Halevy, O., and Cahaner, A. (2014b). Featherless and feathered broilers under control versus hot conditions. 1. Breast meat yield and quality. Poult. Sci. 93, 1067-1075. doi: 10.3382/ps.2013-03591

Halevy, O., Biran, I., and Rozenboim, I. (1998). Various light source treatments affect body and skeletal muscle growth by affecting skeletal muscle satellite cell proliferation in broilers. Compar. Biochem. Physiol. 120, 317-323. doi: 10.1016/ s1095-6433(98)10032-6

Halevy, O., Geyra, A., Barak, M., Uni, Z., and Sklan, D. (2000). Early posthatch starvation decreases satellite cell proliferation and skeletal muscle growth in chicks. J. Nutr. 130, 858-864.

Halevy, O., Krispin, A., Leshem, Y., McMurtry, J. P., and Yahav, S. (2001). Early-age heat exposure affects skeletal muscle satellite cell proliferation and differentiation in chicks. Am. J. Physiol. Regul. Integr. Comp. Physiol. 281, R302-R309.

Halevy, O., Piestun, Y., Allouh, M. Z., Rosser, B. W., Rinkevich, Y., Reshef, R., et al. (2004). Pattern of Pax7 expression during myogenesis in the posthatch chicken establishes a model for satellite cell differentiation and renewal. Dev. Dyn. 231, 489-502.

Halevy, O., Piestun, Y., Rozenboim, I., and Yablonka-Reuveni, Z. (2006a). Inovo exposure to monochromatic green light promotes skeletal muscle cell proliferation and synchronizes myofiber development in posthatch chicks. Am. J. Physiol. Regul. Integr. Comp. Physiol. 290, R1062-R1070.

Halevy, O., Rozenboim, I., and Yahav, S. (2006b). Enhancement of meat production by environmental manipulation in embryo and young broilers. World Poult. Sci. J. 62, 485-497.

Harding, R. L., Clark, D. L., Halevy, O., Coy, C. S., Yahav, S., and Velleman, S. G. (2015). The effect of temperature on apoptosis and adipogenesis on skeletal muscle satellite cells derived from different muscle types. Physiol. Rep. 3:e12539. doi: 10.14814/phy2.12539

Harding, R. L., Halevy, O., Yahav, Y., and Velleman, S. G. (2016). The effect of temperature on proliferation and differentiation of chicken skeletal muscle satellite cells isolated from different muscle types. Physiol. Rep. 4:e12770. doi: 10.14814 phy2.12770

Hartley, R. S., Bandman, E., and Yablonka-Reuveni, Z. (1992). Skeletal muscle satellite cells appear during late chicken embryogenesis. Dev. Biol. 153, 206-216.

Havenstein, G. B., Ferket, P. R., and Qureshi, M. A. (2003a). Carcass composition and yield of 1957 vs. 2001 broilers fed representative 1957 and 2001 broiler diets. Poult. Sci. 82, 1509-1518.

Havenstein, G. B., Ferket, P. R., and Qureshi, M. A. (2003b). Growth, livability, and feed conversion of 1957 vs. 2001 broilers when fed representative 1957 and 2001 broiler diets. Poult. Sci. 82, 1500-1508.

Hawke, T. J., and Garry, D. J. (2001). Myogenic satellite cells: physiology to molecular biology. J. Appl. Physiol. 91, 534-551.

Kornasio, R., Kedar, O., Halevy, O., and Uni, Z. (2011). Effect of in ovo feeding and its interaction with timing of first feed on glycogen reserves, muscle growth, and body weight. Poult. Sci. 90, 1467-1477.

Kuang, S., Kuroda, K., Le Grand, F., and Rudnicki, M. A. (2007). Asymmetric self-renewal and commitment of satellite stem cells in muscle. Cell 129, 999-1010

Kuttappan, V. A., Brewer, V. B., Apple, J. K., Waldroup, P. W., and Owens, C. M. (2012). Influence of growth rate on the occurrence of white striping in broiler breast fillets. Poult. Sci. 91, 2677-2685.

Kuttappan, V. A., Hargis, B. M., and Owens, C. M. (2016). White striping and woody breast myopathies in the modern poultry industry: a review. Poult Sci. 95, 2724-2733.

Liu, W., Wang, Z., and Chen, Y. (2010). Effects of monochromatic light on developmental changes in satellite cell population of pectoral muscle in broilers during early posthatch period. Anat. Rec. (Hoboken) 293, 1315-1324. doi: 10 . 1002/ar.21174

Loyau, T., Hennequet-Antier, C., Coustham, V., Berri, C., Leduc, M., Crochet, S., et al. (2016). Thermal manipulation of the chicken embryo triggers differential gene expression in response to a later heat challenge. BMC Genomics 17:329. doi: 10.1186/s12864-016-2661-y

Loyau, T., Métayer-Coustard, S., Berri, C., Crochet, S., Cailleau-Audouin, E., Sannier, M., et al. (2014). Thermal manipulation during embryogenesis has long-term effects on muscle and liver metabolism in fast-growing chickens. PLoS One 9:e105339. doi: 10.1371/journal.pone.0105339

Malila, Y., Thanatsang, K., Arayamethakorn, S., Uengwetwanit, T., Srimarut, Y., Petracci, M., et al. (2019). Absolute expressions of hypoxia-inducible factor-1 alpha (HIF1A) transcript and the associated genes in chicken skeletal muscle with white striping and wooden breast myopathies. PLoS One 14:e0220904. doi: 10.1371/journal.pone.0220904

Mauro, A. (1961). Satellite cell of skeletal muscle fibers. J. Biophys. Biochem. Cytol. 9, 493-495.

Moss, F. P., and Leblond, C. P. (1971). Satellite cells as the source of nuclei in muscles of growing rats. Anat. Rec. 170, 421-435.

Mozdziak, P. E., Walsh, T. J., and McCoy, D. W. (2002). The effect of early posthatch nutrition on satellite cell mitotic activity. Poult. Sci. 81, 1703-1708.

Naya, F. J., and Olson, E. (1999). MEF2: a transcriptional target for signaling pathways controlling skeletal muscle growth and differentiation. Curr. Opin. Cell Biol. 6, 683-688.

Nichelmann, M., and Tzschentke, B. (2002). Ontogeny of thermoregulation in precocial birds. Comp. Biochem. Physiol. 131, 751-763.

Papah, M. B., and Abasht, B. (2019). Dysregulation of lipid metabolism and appearance of slow myofiber-specific isoforms accompany the development of Wooden Breast myopathy in modern broiler chickens. Sci. Rep. 9:17170. doi: 10.1038/s41598-019-53728-8

Patael, T., Piestun, Y., Soffer, A., Mordechay, S., Yahav, S., Velleman, S. G., et al. (2019). Early posthatch thermal stress causes long-term adverse effects on pectoralis muscle development in broilers. Poult. Sci. 98, 3268-3277. doi: 10. 3382/ps/pez123

Petracci, M., Mudalal, S., Bonfiglio, A., and Cavani, C. (2013). Occurrence of white striping under commercial conditions and its impact on breast meat quality in broiler chickens. Poult. Sci. 92, 1670-1675. doi: 10.3382/ps.2012-03001

Piestun, Y., Druyan, S., Brake, J., and Yahav, S. (2013). Thermal manipulations during broiler incubation alter performance of broilers to 70 days of age. Poult. Sci. 5, 1155-1163. doi: 10.3382/ps.2012-02609

Piestun, Y., Halevy, O., Shinder, D., Ruzal, M., Druyan, S., and Yahav, S. (2011). Thermal manipulations during broiler embryogenesis improves post-hatch performance under hot conditions. J. Thermal Biol. 36, 469-474.

Piestun, Y., Halevy, O., and Yahav, S. (2009a). Thermal manipulations of broiler embryos-the effect on thermoregulation and development during embryogenesis. Poult. Sci. 88, 2677-2688.

Piestun, Y., Harel, M., Barak, M., Yahav, S., and Halevy, O. (2009b). Thermal manipulations in late-term chick embryos affect skeletal muscle development and promote myoblast proliferation and muscle hypertrophy. J. Appl. Physiol. $106,233-240$.

Piestun, Y., Patael, T., Yahav, S., Velleman, S. D., Harding, R. L., Coy, C. S., et al. (2017). Early post-hatch thermal stress affects broiler muscle development and satellite cell fate. Poult. Sci. 96, 2877-2888.

Piestun, Y., Yahav, S., and Halevy, O. (2015). Thermal manipulation during embryogenesis affects myoblast proliferation and skeletal muscle growth in meat-type chickens. Poult. Sci. 94, 2528-2536.

Powell, D. J., McFarland, D. C., Cowieson, A. J., Muir, W. I., and Velleman, S. G. (2014). The effect of nutritional status and muscle fiber type on myogenic satellite cell fate and apoptosis. Poult. Sci. 93, 163-173.

Powell, D. J., Velleman, S. G., Cowieson, A. J., Singh, M., and Muir, W. I. (2016). Influence of hatch time and access to feed on intramuscular fat deposition in the broiler. Poult. Sci. 95, 1449-1456.

Relaix, F., Rocancourt, D., Mansouri, A., and Buckingham, M. (2005). A Pax3/Pax7-dependent population of skeletal muscle progenitor cells. Nature 435, 948-953.

Renaudeau, D., Collin, A., Yahav, S., de Basilio, V., Gourdine, J. L., and Collier, R. J. (2012). Adaptation to hot climate and strategies to alleviate heat stress in livestock production. Animal 6, 707-728. doi: 10.1017/S1751731111002448

Sandercock, D. A., Hunter, R. R., Nute, G. R., Mitchel, M. A., and Hocking, P. M. (2001). Acute heat stress-induced alterations in blood acid-base status and skeletal muscle membrane integrity in broilers chickens at 2 ages: implications for meat quality. Poult. Sci. 80, 418-425. 
Seale, P., Sabourin, L. A., Girgis-Gabardo, A., Mansouri, A., Gruss, P., and Rudnicki, M. A. (2000). Pax7 is required for the specification of myogenic satellite cells. Cell 102, 777-786.

Shefer, G., Wieklinski-Lee, M., and Yablonka-Reuveni, Z. (2004). Skeletal muscle satellite cells can spontaneously enter an alternative mesenchymal pathway. J. Cell Sci. 117, 5393-5404.

Shinin, V., Gayraud-Morel, B., Gomes, D., and Tajbakhsh, S. (2006). Asymmetric division and cosegregation of template DNA strands in adult muscle satellite cells. Nat. Cell Biol. 8, 677-687.

Sihvo, H.-K., Immonen, K., and Puolanne, E. (2014). Myodegeneration with fibrosis and regeneration in the pectoralis major muscle of broilers. Vet. Pathol. 51, 619-623.

Sihvo, H.-K., Immonen, K., and Puolanne, E. (2017). Myodegeneration with fibrosis and regeneration in the pectoralis major muscle of broilers. Vet. Pathol. $54,119-128$

Stockdale, F. E. (1992). Myogenic cell lineages. Dev. Biol. 154, 284-298.

Tatsumi, R., Anderson, J. E., Neveret, C. J., Halevy, O., and Allen, R. E. (1998). $\mathrm{HGF} / \mathrm{SF}$ is present in normal adult skeletal muscle and is capable of activating satellite cells. Dev. Biol. 194, 114-128.

Thommes, R. C., Clark, N. B., Mok, L. L. S., and Malone, S. (1984). Hypothalmoadenohypophyseal-thyroid interrelationships in the chick embryo. V. The effects of thyroidectomy on T4 levels in blood plasma. Gen. Comp. Endocrinol. 54, 324-327.

Tierney, M. T., and Sacco, A. (2016). Satellite cell heterogeneity in skeletal muscle homeostasis. Trends Cell Biol. 26, 434-444. doi: 10.1016/j.tcb.2016. 02.004

Tonniges, J. R., Clark, D. L., and Velleman, S. G. (2019). The effect of the wooden breast fibrotic myopathy in broilers on fibrillar collagen organization and decorin-collagen binding. Avian Dis. 63, 48-60. doi: 10.1637/11985-102218Reg. 1

Tzschentke, B., Basta, D., Janke, O., and Maier, I. (2004). Characteristics of early development of body functions and epigenetic adaptation to the environment in poultry: focus on development of central nervous mechanisms. Avian Poult. Biol. Rev. 15, 107-118.

Tzschentke, B., and Halle, I. (2009). Influence of temperature stimulation during the last 4 days of incubation on secondary sex ratio and later performance male and female broiler chickens. Br. Poult. Sci. 50, 634-640.

Velleman, S. G., and Clark, D. L. (2015). Histopathologic and myogenic gene expression changes associated with wooden breast in broiler breast muscles. Avian Dis. 59, 410-418.

Velleman, S. G., Clark, D. L., and Tonniges, J. R. (2018). The effect of the wooden breast myopathy on sarcomere structure and organization. Avian Dis. 62, 28-35.

Velleman, S. G., Coy, C. S., and Emmerson, D. A. (2014). Effect of the timing of posthatch feed restrictions on the deposition of fat during broiler breast muscle development. Poult. Sci. 93, 2622-2627.
Wise, P. M., and Frye, B. E. (1975). Functional development of the hypothalamohypophyseal-adrenal cortex axis in chick embryo, Gallus domesticus. J. Exp. Zool. 185, 277-292.

Yablonka-Reuveni, Z. (1995). Development and postnatal regulation of adult myoblasts. Microsc. Res. Techol. 30, 366-380.

Yablonka-Reuveni, Z. (2011). The skeletal muscle satellite cell: still young and fascinating at 50. J. Histochem. Cytochem. 59, 1041-1059.

Yahav, S. (2009). Alleviating heat stress in domestic fowl - different strategies. World Poult. Sci. J. 65, 719-732.

Yahav, S. (2015). "Regulation of body temperature: strategies and mechanisms," in Sturkie's Avian Physiology, 6th Edn, ed. C. G. Scanes (Cambridge: Academic Press), 869-905.

Yahav, S., Collin, A., Shinder, D., and Picard, M. (2004a). Thermal manipulations during broiler chick embryogenesis: effects of timing and temperature. Poult. Sci. 83, 1959-1963.

Yahav, S., Sasson-Rath, R., and Shinder, D. (2004b). The effect of thermal manipulations during embryogenesis of broiler chicks (Gallus domesticus) on hatchability, body weight and thermoregulation after hatch. J. Thermal Biol. 29, 245-250.

Yahav, S., and Hurwitz, S. (1996). Induction of thermotolerance in male broiler chickens by temperature conditioning and early age. Poult. Sci. 75, 402-406.

Yahav, S., and McMurtry, J. P. (2001). Thermotolerance acquisition in broiler chickens by temperature conditioning early in life - the effect of timing and ambient temperature. Poult. Sci. 80, 1662-1666.

Yahav, S., and Plavnik, Y. (1999). Effect of early-stage thermal conditioning and food restriction on performance and thermotolerance of male broiler chickens. Br. Poult. Sci. 40, 120-126.

Yahav, S., Shinder, D., Tanny, J., and Cohen, S. (2005). Sensible heat loss: the broiler's paradox. World Poult. Sci. J. 61, 419-434.

Yin, H., Pasut, A., Soleimani, V. D., Bentzinger, C. F., Antoun, G., Thorn, S., et al. (2013). MicroRNA-133 controls brown adipose determination in skeletal muscle satellite cells by targeting Prdm16. Cell Metab. 17, 210-224. doi: 10.1016/ j.cmet.2013.01.004

Zammit, P. S., Partridge, T. A., and Yablonka-Reuveni, Z. (2006). The skeletal muscle satellite cell: the stem cell that came in from the cold. J. Histochem. Cytochem. 54, 1177-1191.

Conflict of Interest: The author declares that the research was conducted in the absence of any commercial or financial relationships that could be construed as a potential conflict of interest.

Copyright (c) 2020 Halevy. This is an open-access article distributed under the terms of the Creative Commons Attribution License (CC BY). The use, distribution or reproduction in other forums is permitted, provided the original author(s) and the copyright owner(s) are credited and that the original publication in this journal is cited, in accordance with accepted academic practice. No use, distribution or reproduction is permitted which does not comply with these terms. 


\title{
Blood Gas Disturbances and
} Disproportionate Body Weight Distribution in Broilers With Wooden Breast

\author{
Juniper A. Lake1,2, Erin M. Brannick², Michael B. Papah², Cory Lousenberg², \\ Sandra G. Velleman ${ }^{3}$ and Behnam Abasht ${ }^{1,2 *}$
}

' Center for Bioinformatics and Computational Biology, University of Delaware, Newark, DE, United States, ${ }^{2}$ Department of Animal and Food Sciences, University of Delaware, Newark, DE, United States, ${ }^{3}$ Department of Animal Sciences, Ohio Agricultural Research and Development Center, The Ohio State University, Wooster, OH, United States

\section{OPEN ACCESS}

Edited by:

Krystyna Pierzchala-Koziec, University of Agriculture in Krakow,

Poland

Reviewed by: Francesca Soglia,

University of Bologna, Italy

Elizabeth Ruth Gilbert,

Virginia Polytechnic Institute and State

University, United States

*Correspondence: Behnam Abasht abasht@udel.edu

Specialty section:

This article was submitted to

Avian Physiology,

a section of the journal

Frontiers in Physiology

Received: 09 January 2020 Accepted: 19 March 2020

Published: 07 April 2020

Citation:

Lake JA, Brannick EM, Papah MB, Lousenberg C, Velleman SG and Abasht B (2020) Blood Gas Disturbances and Disproportionate Body Weight Distribution in Broilers

With Wooden Breast.

Front. Physiol. 11:304.

doi: 10.3389/fphys.2020.00304
Wooden breast syndrome is a widespread and economically important myopathy and vasculopathy of fast growing, commercial broiler chickens, primarily affecting birds with high feed efficiency and large breast muscle yield. To investigate potential systemic physiological differences between birds affected and unaffected by wooden breast, a total of 103 market-age Cobb 500 broilers were sampled for 13 blood parameters and the relative weights of the pectoralis major muscle, pectoralis minor muscle, external oblique muscle, wing, heart, lungs, liver, and spleen. Blood analysis was performed on samples taken from the brachial vein of live birds and revealed significant differences in venous blood gases between affected and unaffected chickens. Chickens with wooden breast exhibited significantly higher potassium $\left(\mathrm{K}^{+}\right)$and lower partial pressure of oxygen $\left(\mathrm{pO}_{2}\right)$, oxygen saturation (sO2), and $\mathrm{pH}$. Additionally, affected males had significantly higher partial pressure of carbon dioxide $\left(\mathrm{pCO}_{2}\right)$ and total carbon dioxide $\left(\mathrm{TCO}_{2}\right)$ than unaffected males. Wooden breast affected broilers also possessed a significantly heavier pectoralis major muscle and whole feathered wing compared to unaffected broilers. Blood gas disturbances characterized by high $\mathrm{pCO}_{2}$ and low $\mathrm{pH}$ are indicative of insufficient respiratory gas exchange, suggesting that wooden breast affected broilers have an elevated metabolic rate that may also be inadequately compensated due to cardiovascular deficiencies such as poor venous return or respiratory insufficiency. Lung tissues from 12 birds with extreme $\mathrm{sO}_{2}$ values were subsequently examined to assess whether lung pathology contributed to the observed blood gas disturbance. Comparison of lung morphology between affected and unaffected birds revealed no apparent differences that could contribute to decreased parabronchial gas exchange. However, an interesting finding was the detection of pulmonary phlebitis in one of the wooden breast-affected samples consistent with vascular changes observed in pectoralis major muscle exhibiting the wooden breast phenotype. Our results suggest that the effects of wooden breast are not limited to the pectoralis major muscle and further indicate the importance of research into metabolic changes associated with the myopathy.

Keywords: wooden breast, white striping, broiler, pectoralis major muscle, myopathy, pulmonary phlebitis 


\section{INTRODUCTION}

Modern commercial broiler chickens have undergone intensive selection for production traits such as high muscle yield, rapid growth, and high feed efficiency to meet consumer demand for low-cost lean chicken meat, specifically chicken breast meat. Such breeding strategies have produced remarkable results, nearly halving the time for birds to reach market weight while simultaneously increasing the breast muscle weight by about two-thirds since the 1950s (Petracci et al., 2015). Unfortunately, modern commercial broilers also experience increased prevalence of breast muscle myopathies, some of which cause substantial economic losses due to negative effects on meat quality (Kuttappan et al., 2016).

One such myopathy, commonly called wooden breast (WB), causes the pectoralis major muscle to become grossly pale, enlarged, and palpably firm, with visible signs of inflammation such as petechial hemorrhages and tissue edema (Sihvo et al., 2014). These macroscopic manifestations of the disorder are accompanied by considerable degradation of meat quality (Mudalal et al., 2015; Chatterjee et al., 2016) such that moderately or severely affected breast muscle cannot be sold as prime breast muscle filets and is instead condemned or sold for lower revenue products. In addition, increased locomotor difficulties, decreased wing mobility, and higher rates of dorsal recumbency among affected birds (Papah et al., 2017; Norring et al., 2018; Gall et al., 2019) suggest that WB may also be detrimental to bird welfare.

Research on WB and related myopathies is focused largely on the pectoralis major muscle, with only minor attention paid to potential systemic disparities accompanying the condition. However, factors that predispose broilers to WB - growth rate, feed efficiency, and breast muscle yield - can broadly be categorized as relating to general metabolism and body form. To date, there has been no comprehensive comparison of muscle and organ weights beyond the pectoralis major muscle weight and abdominal fat percentage between WB affected and unaffected broilers. An analysis of body weight distribution in WB birds may aid in identifying systemic physiological predisposition to or pathophysiologic effects of the myopathy. Intensive selection for commercially valuable traits has been shown to underpin biological imbalances in meat-type chickens, such as insufficient cardiopulmonary capacity to accommodate sustained rapid growth, resulting in pulmonary hypertension syndrome, or ascites (Wideman and French, 2000). It is known that WB-affected broilers possess larger breast muscles relative to body weight (Mutryn et al., 2015b) with higher crosssectional areas (Dalle Zotte et al., 2017), and lower abdominal fat as a percentage of body weight (Mutryn et al., 2015b), but other potential differences in body weight distribution have not been examined.

Microscopic characterizations of WB have also largely been limited to the pectoralis major muscle (Sihvo et al., 2014, 2017, 2018; Papah et al., 2017), although evidence of altered blood gas values (Livingston et al., 2019a,b) in affected birds may indicate systemic disturbances or inadequate respiratory gas exchange. Two studies have provided initial insight into differences in blood parameters between WB-affected and unaffected broilers (Livingston et al., 2019a,b). Livingston et al. (2019a) evaluated the venous blood of male broilers at 35 days of age and found that WB severity was significantly associated with reduced partial pressure of oxygen $\left(\mathrm{pO}_{2}\right)$ and increased total carbon dioxide $\left(\mathrm{TCO}_{2}\right)$, bicarbonate $\left(\mathrm{HCO}_{3}{ }^{-}\right)$, and base excess (BE). The same blood analysis conducted at 42 days of age produced similar results with regard to blood gas changes in WB affected birds, with the additional finding that packed cell volume (PCV, hematocrit; $\mathrm{Hct}$ ) was significantly associated with WB severity (Livingston et al., 2019a). Livingston et al. (2019b) subsequently reported higher potassium $\left(\mathrm{K}^{+}\right)$levels in affected birds in a study evaluating the venous blood of male broilers at 42 days of age.

Thus, the objective of the current study was to further the systemic characterization of WB myopathy by comparing blood parameters, body weight distribution, and lung histology between affected and unaffected broilers.

\section{MATERIALS AND METHODS}

\section{Ethics Statement}

The University of Delaware Institutional Animal Care and Use Committee approved the animal protocol (48R-2015-0) followed for this scientific study. Euthanasia was performed by means of cervical dislocation, and all efforts were made to maximize bird welfare.

\section{Experimental Animals and Wooden Breast Disease Scoring}

This experiment was conducted in chicken houses located at the University of Delaware under environmental conditions simulating a commercial setting. As part of a genome-wide association study, a total of 542 Cobb 500 broilers from the same breeding population were raised in 4 chicken houses and provided free access to feed and water. At 47 days of age, 103 birds were selected for blood analysis based on manual palpation of the breast muscle. These birds were selected to achieve an approximately equal number exhibiting no palpable breast muscle firmness (48 total; 26 male and 22 female) and severe palpable firmness ( 55 total; 39 male and 16 female). Blood analysis using i-STAT requires sampling of blood from live birds. Therefore, bird selection was performed using breast muscle palpation of live birds while all statistical analyses utilized more accurate scoring of WB based on gross evaluation of the pectoralis major muscle at necropsy, as described below.

Birds were euthanized by cervical dislocation at 52 or 53 days of age. During necropsy, the pectoralis major muscles were evaluated for gross lesions and palpable firmness associated with WB and each bird was assigned a WB score using a $0-4$ scale; 0-Normal indicates the bird had no macroscopic signs of the myopathy, 1-Very Mild indicates $1 \%$ or less of the breast muscle was affected, 2-Mild indicates between $1 \%$ and $10 \%$ of the breast muscle was affected, 3-Moderate indicates between 10 and $50 \%$ of the breast muscle was affected, and a score of 4-Severe indicates that more than $50 \%$ was affected. This scoring system is the same one implemented by Lake et al. (2019) and 
separates unaffected and mildly and moderately affected chickens with higher resolution.

\section{Blood Analysis}

At 47 days of age, $1 \mathrm{ml}$ of blood was drawn from the brachial wing vein of each bird using a $3 \mathrm{ml}$ syringe with 23-gauge needle that had been prepared by aspirating and expelling a small volume of liquid heparin prior to blood collection. The blood was deposited immediately into a new i-STAT CG8 + cartridge inserted in the i-STAT 1 Analyzer (model 300A, Abbott Point of Care Inc., Princeton, NJ, United States) to perform rapid blood analysis. While designed for clinical use in humans, the i-STAT system's performance in Gallus gallus has been demonstrated in previous studies (Steinmetz et al., 2007; Livingston et al., 2019a). CG8 + test cartridges were used to test blood chemistry parameters including sodium $\left(\mathrm{Na}^{+}\right), \mathrm{K}^{+}$, ionized calcium (iCa), and glucose (Glu); hematologic parameters Hct and hemoglobin $(\mathrm{Hb})$; and blood gas parameters including $\mathrm{pH}$, partial pressure of carbon dioxide $\left(\mathrm{pCO}_{2}\right), \mathrm{TCO}_{2}, \mathrm{HCO}_{3}{ }^{-}, \mathrm{BE}$, oxygen saturation $\left(\mathrm{sO}_{2}\right)$, and $\mathrm{pO}_{2}$. After all measurements were completed, data was downloaded from the analyzer and consolidated for statistical analysis. Body weight at 47 days was also measured at this time.

\section{Body Weight Distribution}

The same 103 birds used for blood analyses were also used to evaluate body weight distribution. After euthanasia, the left pectoralis major muscle, left pectoralis minor muscle, left external oblique muscle, heart, lungs, liver, spleen, and whole feathered left wing, disarticulated at the shoulder, were dissected from each bird. The weight of each dissected body part was recorded, along with the body weight of the bird before necropsy.

\section{Statistical Analysis}

Statistical analyses were performed using JMP software (SAS Institute, Cary, NC, United States). To improve statistical power, birds were grouped according to WB status: unaffected birds included those assigned a WB score of 0 -Normal or 1-Very Mild and affected birds included those assigned a score of 2-Mild, 3-Moderate, or 4-Severe. All i-STAT measurements were analyzed using a mixed linear model with WB status, sex, WBsex interaction, and body weight at 47 days as fixed effects and poultry house as a random effect. Body part weights were analyzed using a mixed linear model with WB status, sex, WBsex interaction, and body weight at necropsy as fixed effects and poultry house as a random effect. Effects with $P \leq 0.05$ were considered significant for all tests.

\section{Histological Evaluation of Lungs}

Of the 103 broilers used in this study, six birds ( 3 males and 3 females) with the lowest $\mathrm{sO}_{2}$ values and six birds with the highest $\mathrm{sO}_{2}$ values ( 3 males and 3 females) were selected for microscopic examination of lung tissue based on blood gas values measured at 47 days of age. In the low $\mathrm{sO}_{2}$ group, 5 birds were classified as affected, with WB scores of 3-Moderate or 4-Severe, and 1 bird was classified as unaffected, with a WB score of 0-Normal. In the high $\mathrm{sO}_{2}$ group, all 6 birds were classified as unaffected, with a WB score of 0-Normal. From each of the selected birds, lung tissue from the cranial and caudal aspects from either the left or right lung were harvested and fixed by immersion in $10 \%$ neutral buffered formalin. Samples were processed routinely for staining with hematoxylin and eosin as described by Papah et al. (2017) before histologic evaluation with a light microscope and morphometric analysis with the Aperio LV1 digital microscope (Leica Biosystems, Buffalo Grove, IL, United States).

Lung tissue was examined microscopically by a veterinarian (Papah) and a certified veterinary anatomic pathologist (Brannick) for histopathologic lesions or other tissue changes which could affect systemic blood parameters, such as the presence of inflammation, fibrosis, lymphoid follicular hyperplasia (lymphocytic nodules), cartilaginous nodules, edema or hemorrhage in the gas exchange areas, thickening of parabronchial walls, and obstruction of parabronchi. Assessment of all slides was performed in a blinded fashion and later microscopic lesions were assessed for an association with $\mathrm{sO}_{2}$ status (high or low), sex, or WB status.

\section{RESULTS AND DISCUSSION}

\section{Body Weight Distribution}

The effects of WB, sex, WB-sex interaction, and body weight on the weights of the dissected left pectoralis major muscle, left pectoralis minor muscle, left external oblique muscle, whole feathered left wing, heart, lungs, liver, and spleen are provided in Table 1. Wooden breast had a significant association with the pectoralis major muscle and whole feathered left wing, which were both larger in affected birds compared to unaffected birds. Previous studies have found similar results with regard to pectoralis major yield (Mutryn et al., 2015b; Livingston et al., 2019b), providing fodder for speculation that high breast muscle yield is responsible for development of the WB and WS phenotypes due to overstretching of the myofibers and a reduction in capillary density (Kuttappan et al., 2013; Dalle Zotte et al., 2017). However, microscopic lesions of WB can be detected as early as 1 week of age in the pectoralis major muscle (Papah et al., 2017). Thus, hypotheses suggesting that WB arises from overstretching and ischemia may be inadequate or incomplete. Without discounting the potential contribution of pectoralis major growth rate to WB development, it is important to consider alternative interpretations of these results. For example, it has been proposed that muscle hypertrophy may be symptomatic of WB rather than causal, similar to the pathological hypertrophy of organs in chronic complications of diabetes mellitus in mammals (Lake et al., 2019; Lake and Abasht, 2020).

The external oblique muscle showed an interesting effect of the WB-sex interaction, with affected males and unaffected females having the highest average weights of those muscles and unaffected males having the lowest weights. Similarly, the weight of the pectoralis minor muscle was highest in unaffected females and lowest in unaffected males. One potential explanation for the observed group means of the external oblique muscle is as an adaptive response to the size of the combined pectoralis major and pectoralis minor muscle. Avian respiration relies on 
TABLE 1 | Effects of wooden breast (WB) status, sex, the interaction of WB and sex, and body weight on the weight of the left pectoralis major, left pectoralis minor, left whole feathered wing, left external oblique, heart, lungs, liver, and spleen of broiler chickens.

\begin{tabular}{|c|c|c|c|c|c|c|c|c|c|c|c|c|c|}
\hline & & \multicolumn{3}{|c|}{ WB } & \multicolumn{3}{|c|}{ Sex } & \multicolumn{5}{|c|}{ WB $\times$ Sex } & \multirow{2}{*}{$\begin{array}{c}\text { Body Weigh } \\
\text { P-value }\end{array}$} \\
\hline & & Affected & Unaffected & $P$-value & Male & Female & $P$-value & $\begin{array}{l}\text { Affected } \\
\text { Male }\end{array}$ & $\begin{array}{l}\text { Affected } \\
\text { Female }\end{array}$ & $\begin{array}{c}\text { Unaffected } \\
\text { Male }\end{array}$ & $\begin{array}{c}\text { Unaffected } \\
\text { Female }\end{array}$ & $P$-value & \\
\hline Broilers (n) & & 55 & 48 & & 65 & 38 & & 39 & 16 & 26 & 22 & & \\
\hline \multirow[t]{2}{*}{ P.major (g) } & Mean & 425.51 & 400.59 & 0.002 & 402.70 & 423.41 & 0.079 & 417.84 & 433.19 & 387.56 & 413.63 & 0.505 & $<0.001$ \\
\hline & SE & 4.67 & 4.68 & & 4.54 & 7.71 & & 6.66 & 10.42 & 6.78 & 9.41 & & \\
\hline \multirow[t]{2}{*}{ P.minor (g) } & Mean & 81.66 & 83.58 & 0.305 & 80.26 & 84.97 & 0.074 & $81.14^{a, b}$ & $82.17^{a, b}$ & $79.38^{a}$ & $87.78^{b}$ & 0.047 & $<0.001$ \\
\hline & SE & 1.55 & 1.61 & & 1.54 & 2.06 & & 1.89 & 2.61 & 1.98 & 2.41 & & \\
\hline \multirow[t]{2}{*}{ Wing (g) } & Mean & 169.62 & 160.31 & $<0.001$ & 169.07 & 160.87 & 0.008 & 174.72 & 164.53 & 163.42 & 157.20 & 0.350 & $<0.001$ \\
\hline & SE & 1.78 & 1.85 & & 1.77 & 2.38 & & 2.18 & 3.01 & 2.28 & 2.78 & & \\
\hline \multirow[t]{2}{*}{ Ext. obl. (g) } & Mean & 7.53 & 7.03 & 0.213 & 6.88 & 7.68 & 0.153 & $7.60^{\mathrm{a}}$ & $7.46^{a, b}$ & $6.15^{\mathrm{b}}$ & $7.90^{\mathrm{a}}$ & 0.018 & 0.006 \\
\hline & SE & 0.26 & 0.28 & & 0.26 & 0.40 & & 0.35 & 0.52 & 0.37 & 0.48 & & \\
\hline \multirow[t]{2}{*}{ Heart (g) } & Mean & 17.74 & 17.83 & 0.867 & 17.84 & 17.74 & 0.893 & 17.86 & 17.62 & 17.81 & 17.85 & 0.786 & $<0.001$ \\
\hline & SE & 0.32 & 0.33 & & 0.31 & 0.50 & & 0.44 & 0.67 & 0.46 & 0.61 & & \\
\hline \multirow[t]{2}{*}{ Lungs (g) } & Mean & 15.51 & 16.35 & 0.209 & 17.23 & 14.64 & 0.006 & 16.58 & 14.45 & 17.88 & 14.83 & 0.483 & 0.158 \\
\hline & SE & 0.52 & 0.54 & & 0.52 & 0.71 & & 0.65 & 0.91 & 0.68 & 0.84 & & \\
\hline \multirow[t]{2}{*}{ Liver (g) } & Mean & 62.43 & 64.19 & 0.334 & 60.09 & 66.53 & 0.013 & 58.17 & 66.70 & 62.02 & 66.35 & 0.241 & $<0.001$ \\
\hline & SE & 1.30 & 1.36 & & 1.29 & 1.86 & & 1.68 & 2.42 & 1.76 & 2.22 & & \\
\hline \multirow[t]{2}{*}{ Spleen (g) } & Mean & 4.20 & 4.46 & 0.223 & 4.03 & 4.62 & 0.056 & 3.83 & 4.57 & 4.24 & 4.67 & 0.460 & $<0.001$ \\
\hline & SE & 0.13 & 0.14 & & 0.14 & 0.21 & & 0.19 & 0.27 & 0.20 & 0.25 & & \\
\hline
\end{tabular}

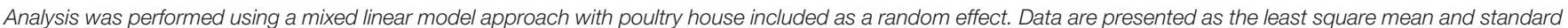
error. Means not sharing a common superscript letter within the interaction effect are significantly different $(P<0.05$, Tukey's HSD test).

movement of the sternum to allow expansion of the bellowslike air sacs during inhalation (Schmidt-Nielsen, 1971). The external obliques are ventilatory muscles that insert onto the base of the uncinate processes of the ribs, extensions of bone that project caudally from the vertical segment of each rib, and move the sternum dorsally during expiration (Codd, 2005). Because inhalation and exhalation are active processes driven by musculoskeletal movements, additional weight, especially on the sternum increases the metabolic demand of respiration, reduces the overall effectiveness of respiratory movements (Tickle et al., 2014), and may result in strengthening of respiratory muscles. This is in accordance with unaffected males having the smallest pectoralis major muscle, pectoralis minor muscle, and external oblique muscle in the present model.

Compared to females in the present study, males possessed larger wings and lungs, but a smaller liver after accounting for WB and body weight. There was no significant difference in the size of the pectoralis major muscle between males and females in our model. Body weight had a significant effect on the sizes of all body parts except the lungs, with larger birds possessing generally heavier body parts but relatively smaller lungs.

\section{Blood Analysis}

The effects of WB, sex, WB-sex interaction, and body weight on 13 blood parameters are shown in Table 2. Compared to unaffected birds, $\mathrm{WB}$ affected birds exhibited significantly higher venous $\mathrm{K}^{+}$and significantly lower $\mathrm{pH}, \mathrm{sO}_{2}$, and $\mathrm{pO}_{2}$. Affected male birds also possessed significantly higher $\mathrm{pCO}_{2}$ and $\mathrm{TCO}_{2}$ values compared to unaffected male birds. Although affected female birds had $\mathrm{pCO}_{2}$ and $\mathrm{TCO}_{2}$ values higher than those of unaffected female birds, the effect was not significant potentially due to the smaller number of female birds sampled. These results are largely in accordance with previously published data of blood parameters measured at 42 days of age (Livingston et al., 2019a,b), although no significant effect of WB on Hct or BE was detected in the present study. The present model also detected a significant sex effect for BE and a WB-sex interaction effect for Glu, with males possessing higher $\mathrm{BE}$ than females and affected males possessing higher Glu compared to affected females. It is unclear what might be causing these specific sex effects.

Elevated $\mathrm{pCO}_{2}$ in conjunction with a decline in $\mathrm{pH}$ in $\mathrm{WB}$ affected birds is possibly indicative of an acid-base disorder called respiratory acidosis. While respiratory acidosis is classically defined in terms of arterial blood gas measurements, the high correlation of arterial and venous $\mathrm{pCO}_{2}$ and $\mathrm{pH}$ is wellestablished in humans, dogs, and chickens, as is the use of venous measurements for investigation of acid-base disturbances (Forster et al., 1972; Ilkiw et al., 1991; Wideman et al., 2003; Yildizdaş et al., 2004). Respiratory acidosis occurs when the body produces more $\mathrm{CO}_{2}$ than can be removed by the lungs (D’Addesio, 1992). As carbon dioxide accumulates in the blood, it causes blood $\mathrm{pH}$ to decrease, triggering compensatory mechanisms in the kidneys, such as $\mathrm{HCO}_{3}{ }^{-}$retention, to mitigate the rising acidity. Elevated $\mathrm{HCO}_{3}{ }^{-}$levels are suggestive of renal compensation and possible chronic respiratory acidosis, as the kidneys increase excretion of acid and hydrogen ions and increase reabsorption of $\mathrm{HCO}_{3}{ }^{-}$(Carter et al., 1959). Livingston et al. (2019a) previously found increased $\mathrm{HCO}_{3}{ }^{-}$levels in WB-affected broilers. In the present study, $\mathrm{HCO}_{3}{ }^{-}$values are higher in affected males compared to unaffected males, though 
TABLE 2 | Effects of wooden breast (WB) status, sex, the interaction of WB and sex, and body weight on blood sodium (Na $\left.{ }^{+}\right)$, potassium ( $\mathrm{K}^{+}$), ionized calcium (iCa), glucose (Glu), hematocrit $(\mathrm{Hct})$, hemoglobin $(\mathrm{Hb}), \mathrm{pH}$, partial pressure of carbon dioxide $\left(\mathrm{pCO}_{2}\right)$, total carbon dioxide $\left(\mathrm{TCO}_{2}\right)$, partial pressure of oxygen $\left(\mathrm{pO}_{2}\right)$, oxygen saturation $\left(\mathrm{SO}_{2}\right)$, bicarbonate $\left(\mathrm{HCO}_{3}{ }^{-}\right)$, and base excess (BE).

\begin{tabular}{|c|c|c|c|c|c|c|c|c|c|c|c|c|c|}
\hline & & \multicolumn{3}{|c|}{ WB } & \multicolumn{3}{|c|}{ Sex } & \multicolumn{5}{|c|}{ WB x Sex } & \multirow{2}{*}{$\begin{array}{c}\text { Body Weight } \\
\text { P-value }\end{array}$} \\
\hline & & Affected & Unaffected & $P$-value & Male & Female & $P$-value & $\begin{array}{l}\text { Affected } \\
\text { Male }\end{array}$ & $\begin{array}{l}\text { Affected } \\
\text { Female }\end{array}$ & $\begin{array}{c}\text { Unaffected } \\
\text { Male }\end{array}$ & $\begin{array}{l}\text { Unaffected } \\
\text { Female }\end{array}$ & $P$-value & \\
\hline Broilers (n) & & 55 & 48 & & 65 & 38 & & 39 & 16 & 26 & 22 & & \\
\hline \multirow[t]{2}{*}{$\mathrm{Na}^{+}(\mathrm{mmol} / \mathrm{L})$} & Mean & 150.17 & 149.24 & 0.123 & 149.91 & 149.50 & 0.611 & 150.05 & 150.30 & 149.78 & 148.71 & 0.240 & 0.320 \\
\hline & SE & 0.42 & 0.47 & & 0.42 & 0.60 & & 0.58 & 0.75 & 0.56 & 0.74 & & \\
\hline \multirow[t]{2}{*}{$\mathrm{K}^{+}(\mathrm{mmol} / \mathrm{L})$} & Mean & 5.04 & 4.86 & 0.045 & 4.98 & 4.91 & 0.569 & 5.11 & 4.97 & 4.85 & 4.86 & 0.372 & 0.127 \\
\hline & SE & 0.05 & 0.06 & & 0.05 & 0.09 & & 0.08 & 0.11 & 0.08 & 0.11 & & \\
\hline \multirow[t]{2}{*}{ iCa (mmol/L) } & Mean & 1.41 & 1.39 & 0.416 & 1.40 & 1.40 & 0.983 & 1.41 & 1.41 & 1.40 & 1.39 & 0.932 & 0.266 \\
\hline & SE & 0.02 & 0.02 & & 0.02 & 0.02 & & 0.02 & 0.02 & 0.02 & 0.02 & & \\
\hline \multirow[t]{2}{*}{ Glu (mmol/L) } & Mean & 220.06 & 222.58 & 0.366 & 224.29 & 218.36 & 0.119 & $226.71^{a}$ & $213.42^{b}$ & $221.87^{a, b}$ & $223.30^{a, b}$ & 0.006 & 0.310 \\
\hline & SE & 1.85 & 2.08 & & 1.87 & 2.75 & & 2.61 & 3.43 & 2.52 & 3.39 & & \\
\hline \multirow[t]{2}{*}{ Hct (\%PCV) } & Mean & 23.79 & 22.84 & 0.151 & 23.31 & 23.32 & 0.994 & 23.82 & 23.76 & 22.80 & 22.87 & 0.919 & 0.216 \\
\hline & SE & 0.50 & 0.55 & & 0.50 & 0.69 & & 0.67 & 0.85 & 0.64 & 0.83 & & \\
\hline \multirow[t]{2}{*}{$\mathrm{Hb}(\mathrm{g} / \mathrm{dL})$} & Mean & 8.08 & 7.76 & 0.161 & 7.93 & 7.92 & 0.989 & 8.10 & 8.07 & 7.75 & 7.78 & 0.903 & 0.211 \\
\hline & SE & 0.17 & 0.19 & & 0.17 & 0.24 & & 0.23 & 0.29 & 0.22 & 0.29 & & \\
\hline \multirow[t]{2}{*}{$\mathrm{pH}$} & Mean & 7.360 & 7.389 & 0.007 & 7.382 & 7.367 & 0.268 & 7.364 & 7.356 & 7.401 & 7.377 & 0.396 & 0.600 \\
\hline & SE & 0.008 & 0.009 & & 0.008 & 0.011 & & 0.011 & 0.014 & 0.010 & 0.013 & & \\
\hline \multirow[t]{2}{*}{$\mathrm{pCO}_{2}(\mathrm{mmHg})$} & Mean & 47.26 & 42.16 & 0.002 & 44.69 & 44.73 & 0.984 & $48.82^{a}$ & $45.70^{a, b}$ & $40.55^{b}$ & $43.77^{a, b}$ & 0.041 & 0.825 \\
\hline & SE & 0.88 & 1.02 & & 0.86 & 1.55 & & 1.42 & 1.95 & 1.26 & 1.96 & & \\
\hline \multirow[t]{2}{*}{$\mathrm{TCO}_{2}(\mathrm{mmol} / \mathrm{L})$} & Mean & 27.81 & 26.87 & 0.125 & 28.01 & 26.67 & 0.111 & $29.06^{a}$ & $26.57^{a, b}$ & $26.96^{b}$ & $26.78^{a, b}$ & 0.045 & 0.333 \\
\hline & SE & 0.38 & 0.43 & & 0.38 & 0.58 & & 0.55 & 0.74 & 0.53 & 0.73 & & \\
\hline \multirow[t]{2}{*}{$\mathrm{pO}_{2}(\mathrm{mmHg})$} & Mean & 39.85 & 44.75 & $<0.001$ & 42.03 & 42.57 & 0.751 & 39.52 & 40.18 & 44.54 & 44.97 & 0.923 & 0.352 \\
\hline & SE & 1.16 & 1.22 & & 1.15 & 1.48 & & 1.42 & 1.79 & 1.38 & 1.71 & & \\
\hline \multirow[t]{2}{*}{$\mathrm{sO}_{2}(\%)$} & Mean & 70.57 & 79.07 & $<0.001$ & 74.86 & 74.78 & 0.974 & 70.20 & 70.94 & 79.52 & 78.62 & 0.647 & 0.452 \\
\hline & SE & 1.37 & 1.49 & & 1.35 & 1.95 & & 1.83 & 2.46 & 1.76 & 2.34 & & \\
\hline \multirow[t]{2}{*}{$\mathrm{HCO}_{3}-(\mathrm{mmol} / \mathrm{L})$} & Mean & 26.42 & 25.55 & 0.133 & 26.66 & 25.31 & 0.087 & 27.62 & 25.22 & 25.70 & 25.40 & 0.052 & 0.371 \\
\hline & $\mathrm{SE}$ & 0.36 & 0.41 & & 0.36 & 0.55 & & 0.53 & 0.70 & 0.50 & 0.69 & & \\
\hline \multirow[t]{2}{*}{$\mathrm{BE}(\mathrm{mmol} / \mathrm{L})$} & Mean & 1.00 & 0.46 & 0.375 & 1.60 & -0.13 & 0.036 & 2.32 & -0.31 & 0.88 & 0.05 & 0.113 & 0.443 \\
\hline & SE & 0.42 & 0.47 & & 1.60 & 0.61 & & 0.58 & 0.75 & 0.56 & 0.74 & & \\
\hline
\end{tabular}

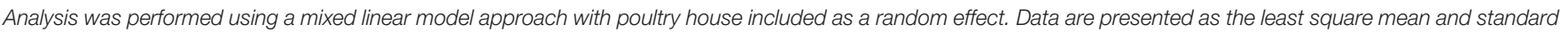
error. Means not sharing a common superscript letter within the interaction effect are significantly different $(P<0.05$, Tukey's HSD test).

the WB-sex interaction effect is not quite significant in our model $(P$-value $=0.052)$.

Respiratory acidosis is caused either by an increase in $\mathrm{CO}_{2}$ production (increased metabolism), a relative decrease in respiratory gas exchange (cardiopulmonary insufficiency), or both (Epstein and Singh, 2001). Previous studies comparing feed-restricted vs. non-feed-restricted broilers and slow-growing vs. fast-growing broilers (Julian and Mirsalimi, 1992; Olkowski et al., 1999) have demonstrated how an increase in metabolism can cause changes to blood gas values similar to those seen in the present study. Similarly, respiratory acidosis caused by cardiopulmonary insufficiency has been demonstrated by comparing ascitic versus non-ascitic chickens (Malan et al., 2003). In the present study, body weight was investigated for a potential association with blood parameter values but did not show any significant effects (Table 2). The weight of the whole feathered left wing was also tested as a main effect for blood gas values as the brachial vein, from which blood samples were taken, drains the peripheral wing tissue. However, it also showed no significant effects for blood gas values and so was excluded (data not shown). The fact that WB has a significant effect on blood gas values beyond what can be explained by body weight or wing weight suggests that a higher metabolic rate due to faster growth is not the cause of the apparent blood gas disturbance. It is, therefore, important to explore other potential metabolic, respiratory, or cardiovascular differences between WB affected and unaffected birds that might explain the results seen in this study and in previous studies (Livingston et al., 2019a,b).

Metabolically, WB involves substantial alterations in the pectoralis major muscle, including an apparent increase in lipid metabolism and decrease in glycolysis (Mutryn et al., 2015a; Abasht et al., 2016; Papah et al., 2018). Increased reliance on lipids for energy production rather than glucose can raise oxygen consumption due to the lower phosphate/oxygen ratio of fatty acids, but would not be expected to raise $\mathrm{CO}_{2}$ production (Brand, 2005). Other features of WB, such as hypercontraction of muscle fibers (Velleman et al., 2018) or increased activity of ATP-powered calcium pumps, could raise the body's energy 
requirements and total metabolic rate without contributing to growth rate. Sarcoplasmic/endoplasmic reticulum $\mathrm{Ca}^{2+}$-ATPase (SERCA) pumps have been found to account for $40-50 \%$ of the resting metabolic rate in mouse skeletal muscle, or $12-15 \%$ of whole body resting oxygen consumption (Smith et al., 2013). Upregulation of SERCAs in the pectoralis major muscle of affected birds at market age is supported by transcriptional and proteomic evidence (Mutryn et al., 2015a; Soglia et al., 2016), and the disruption of intracellular calcium homeostasis has been identified as a key feature of the early pathogenesis of WB (Papah et al., 2018; Lake et al., 2019). The contribution of sarcoplasmic reticulum calcium cycling to increased resting energy expenditure in the WB phenotype has been proposed (Lake and Abasht, 2020) but remains unexplored experimentally.

While numerous potential pulmonary causes of insufficient gas exchange exist, none are particularly well-supported by existing knowledge of WB or the results of the present study. Our data indicate WB is not associated with reduced lung size, altered lung morphology (see following section), or reduced respiratory musculature (i.e., the external oblique muscle).

However, the circulatory system is also intimately involved in respiratory exchange and can reduce gas exchange at the bloodgas barrier by decreasing the rate at which blood passes by the gas exchange surface in the lungs (Ludders, 2015). Evaluations of microscopic lesions associated with WB have cataloged extensive damage to the veins in the p. major muscle of affected birds as well as evidence of hemodynamic perturbations resulting from such damage (Papah et al., 2017; Sihvo et al., 2017). In many cases, venous inflammation progresses to circumferential transmural and valvular infiltration of veins by inflammatory cells (Papah et al., 2017). Subsequent congestion, edema, valvular damage, and stenosis (narrowing) or obstruction of the venous lumen (Papah et al., 2017; Sihvo et al., 2017) all indicate significant impairment of venous return and, implicitly, a reduction of cardiac output. Poor venous return in affected birds could lead to increased accumulation of metabolic waste including $\mathrm{CO}_{2}$, as indicated by blood gas analysis in this study, and negatively affect gas exchange by slowing the flow of blood back to the gas exchange surfaces of the lungs. Impaired venous return from phlebitis may exacerbate what some characterize as existing vascular insufficiency in the pectoralis major of commercial broilers. At the cellular level, large breast muscles are produced by increasing the number, diameter, and length of muscle fibers (Scheuermann et al., 2004; Roy et al., 2006), causing a reduction of capillary density among other effects (Hoving-Bolink et al., 2000; Joiner et al., 2014).

Apart from blood gases, the only other analyte measurement that was significantly altered in affected birds in the present study was potassium. Hyperkalemia, increased $\mathrm{K}^{+}$, is a known effect of high blood $\mathrm{CO}_{2}$ and therefore a common symptom of respiratory acidosis (Scribner et al., 1955; Ladé and Brown, 1963; Kilburn, 1966). Extracellular $\mathrm{K}^{+}$concentration is tightly regulated in the body to maintain it within the necessary range for cellular functions such as electrical excitability of cardiac and skeletal muscle (Aronson and Giebisch, 2011). The majority of the body's $\mathrm{K}^{+}$is located in the intracellular fluid of skeletal muscle and is shifted between muscle cells and extracellular space by the activity of various ion transport pathways (Youn and McDonough, 2009). A net loss of $\mathrm{K}+$ from cells during respiratory acidosis is mediated primarily by $\mathrm{Na}^{+}-\mathrm{H}^{+}$exchange and $\mathrm{Na}^{+} / \mathrm{K}^{+}$-ATPase activity, although extracellular elevation of bicarbonate enhances $\mathrm{Na}^{+}-\mathrm{HCO}_{3}{ }^{-}$ cotransport and prevents the severity of hyperkalemia seen in metabolic acidosis where bicarbonate levels are reduced (Aronson and Giebisch, 2011).

\section{Histological Evaluation of the Lungs}

Histologic analysis demonstrated that there was no clinically significant lung disease (pneumonia, etc.) observed in tissues from either high $\mathrm{sO}_{2}$ or low $\mathrm{sO}_{2}$ birds to explain detectable differences in blood oxygen saturation. A single lung specimen from the low $\mathrm{sO}_{2}$ group exhibited moderate localized multifocal lymphoplasmacytic phlebitis consistent with vascular changes observed in WB musculature (Figure 1a) and was confirmed to have been collected from a low $\mathrm{sO}_{2} \mathrm{WB}$ affected bird following analysis. All pulmonary tissues examined from both high $\mathrm{sO}_{2}$ and low $\mathrm{sO}_{2}$ birds exhibited one or more foci of chondroosseous metaplasia, characterized by focal to multifocal islands of well-differentiated non-neoplastic cartilage or bone tissue within otherwise healthy pulmonary tissue. Chondro-osseous metaplasia is thought to arise from within pulmonary connective tissue over time in response to low oxygenation in tissues (chronic hypoxia) and has been previously reported in broiler chickens in association with ascites syndrome (Maxwell, 1988). In the present study, there was no gross evidence of fulminant ascites in any of the birds examined. While present in both WB affected and WB unaffected birds, the metaplastic change in pulmonary tissues was more extensive resulting in larger, more numerous metaplastic foci in WB affected birds (Figures $\mathbf{1 b}, \mathbf{c}$ ). This finding may indicate that while lung tissues are susceptible to hypoxic injury even in clinically normal broilers, the WB condition may exacerbate tissue changes due to regionalized or systemic hypoxia.

Other minor histopathologic findings included bronchusassociated lymphoid tissue (BALT) hyperplasia, occasionally with discrete nodular follicle formation, and extramedullary hematopoiesis (EMH). Generalized and nodular lymphoid hyperplasia likely indicate immune response to inhaled antigens or irritants and are not suspected to be related to WB. The airway associated lymphoid tissues did not appear to impinge upon or obstruct airway lumens. The physiologic process of $\mathrm{EMH}$ is common in tissues of young animals, but can sometimes occur to produce additional red blood cells in response to low tissue oxygenation. However, the limited extent and level of EMH in the lung tissues in the present study indicate that $\mathrm{EMH}$ is likely an incidental rather than clinically significant finding. Mild, limited EMH is a common finding in avian tissues, including lung, even in clinically normal birds. Artifactual changes from specimen collection and processing, such as acute hemorrhage with no tissue reaction and collapse of air spaces with no indication of true airway obstruction (atelectasis) were observed but disregarded for purposes of analysis.

Altogether, WB status, sex, or $\mathrm{sO}_{2}$ status were not associated with histopathologic changes in the lungs, suggesting that structural abnormalities or disease in the lungs are not likely 


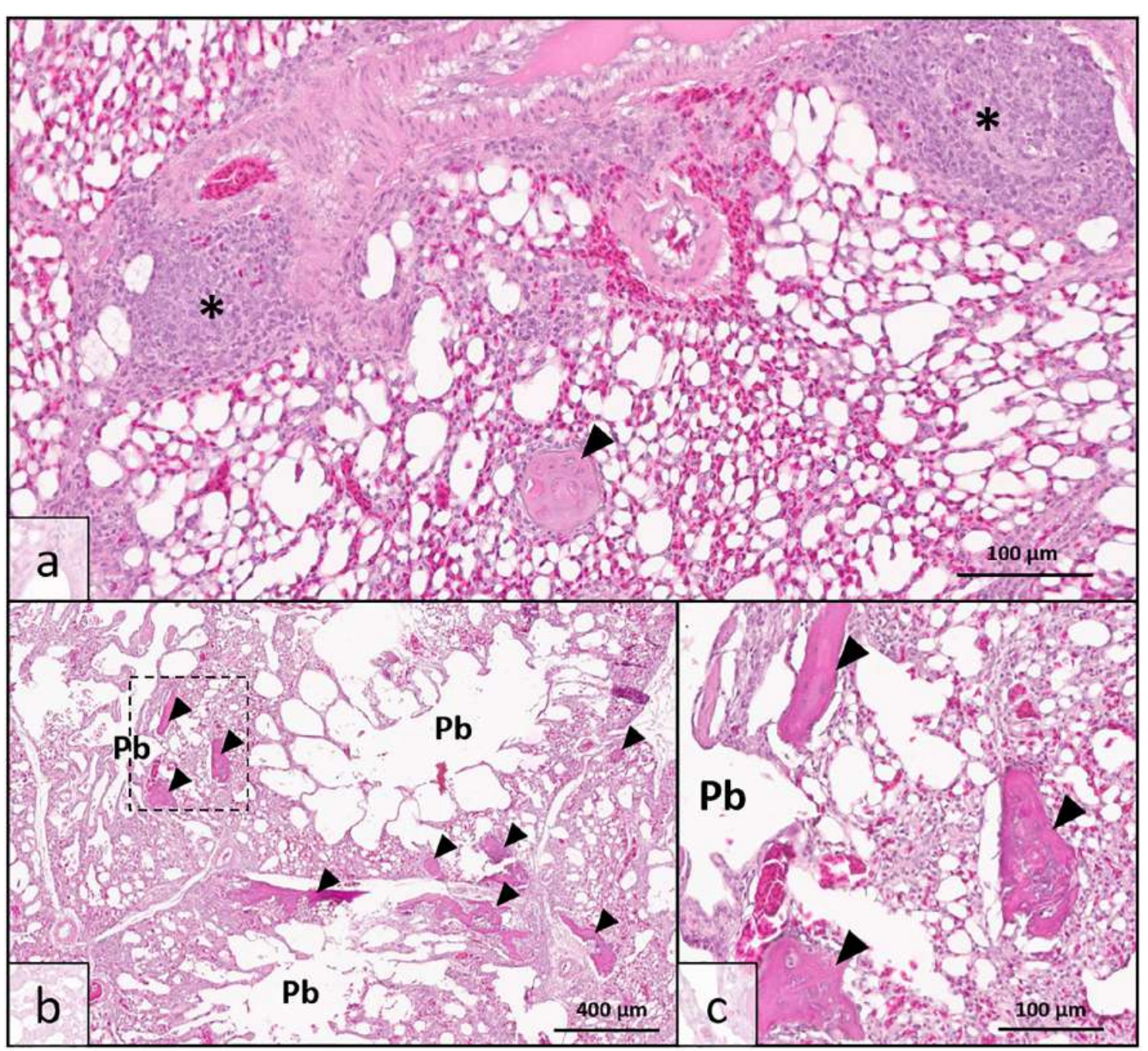

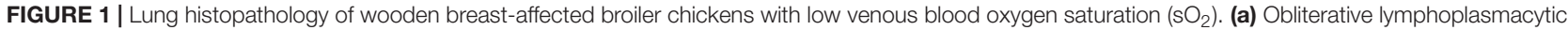
venous inflammation, interpreted as phlebitis (*), was observed in one bird with sparing of adjacent arterial vessel (top) consistent with the wooden breast phenotype in muscle tissues. Chondro-osseous metaplasia (arrowhead) may be occurring as a sequela of low oxygenation in pulmonary tissues. (b) Multiple parabronchi (Pb) exhibit chondro-osseous metaplasia (arrowheads) in wooden breast-affected birds with low $\mathrm{sO}_{2}$ values. The number and size of metaplastic foci were greater than either wooden breast-affected birds with high $\mathrm{sO}_{2}$ values or unaffected birds. (c) Higher magnification of region demarcated by dotted line in $1 \mathrm{~b}$ showing metaplastic foci in greater detail. [Hematoxylin and Eosin stain, scale bar indicates $100 \mu \mathrm{m}$ in (a,c) and $400 \mu \mathrm{m}$ in (b)].

contributors to the WB phenotype. The chondro-osseous metaplasia lesions observed in this study are likely sequelae of the WB syndrome rather than precipitating factors in the development of the disease. The finding of pulmonary phlebitis in one WB bird suggests that the phlebitis associated with WB is not restricted to the musculature but may be systemic in nature.

Previously, our laboratory reported altered gene expression in lung tissue of WB affected broilers (Wong, 2018) which may be connected with the present finding of pulmonary phlebitis. In that study, the top significant canonical pathways identified from the list of differentially expressed genes included atherosclerosis signaling, adipogenesis, and LXR/RXR activation, a pathway involved in the regulation of lipid metabolism and inflammation. Increased expression of genes involved in lipid metabolism and uptake, such as lipoprotein lipase (LPL), fatty acid binding protein 4 (FABP4), and adiponectin c1q and collagen domain containing (ADIPOQ), in the lung tissue of WB affected broilers (Wong, 2018) is reminiscent of the transcriptomic changes associated with $\mathrm{WB}$ in the pectoralis major muscle (Papah et al., 2018; Lake et al., 2019) and may reflect a similar mechanism. Papah and Abasht (2019) recently found increased $L P L$ expression in veins undergoing phlebitis in the pectoralis major muscle of WB affected birds, further substantiating the link between increased lipid metabolism and venous inflammation. In light of this knowledge, it is possible that phlebitis in the lungs is more widespread and not fully represented by the limited number of sections and samples examined in the present study. 


\section{CONCLUSION}

The findings of the present study indicate that WB is associated with blood gas disturbances characterized primarily by increased venous $\mathrm{K}^{+}$and $\mathrm{pCO}_{2}$ and decreased $\mathrm{pH}, \mathrm{sO}_{2}$ and $\mathrm{pO}_{2}$. The accumulation of carbon dioxide and acidification of venous blood occurs when the metabolic demands of the tissue exceed the capacity of the respiratory or circulatory system. Factors that may contribute to increased metabolic demand in WB affected birds include hypercontraction of muscle fibers and, importantly, the dysregulation of calcium homeostasis. Cardiovascular and pulmonary deficiencies, specifically venous damage caused by phlebitis and disproportionate growth of the pectoralis major compared to respiratory muscles, potentially also contribute to inadequate respiratory gas exchange in affected birds. Blood gas disturbances, musculature differences, and pulmonary chondro-osseous metaplasia and phlebitis demonstrated herein may further indicate broader systemic implications of the metabolic dysfunction and circulatory insufficiency already described in existing literature regarding WB. Since these results were obtained in 7-week-old birds, findings are more informative of the WB myopathy's effects (sequelae) rather than its etiology and further studies are required to ascertain whether a blood gas disturbance is present during early stages of the disease.

\section{DATA AVAILABILITY STATEMENT}

The datasets generated for this study are available on request to the corresponding author.

\section{REFERENCES}

Abasht, B., Mutryn, M. F., Michalek, R. D., and Lee, W. R. (2016). Oxidative stress and metabolic perturbations in wooden breast disorder in chickens. PLoS One 11:e0153750. doi: 10.1371/journal.pone.0153750

Aronson, P. S., and Giebisch, G. (2011). Effects of $\mathrm{pH}$ on potassium: new explanations for old observations. J. Am. Soc. Nephrol. 22, 1981-1989. doi: 10.1681/asn.2011040414

Brand, M. D. (2005). The efficiency and plasticity of mitochondrial energy transduction. Biochem. Soc. Trans. 33, 897-904. doi: 10.1042/bst20050897

Carter, N., Seldin, D., and Teng, H. (1959). Tissue and renal response to chronic respiratory acidosis. J. Clin. Invest. 38, 949-960. doi: 10.1172/jci103878

Chatterjee, D., Zhuang, H., Bowker, B. C., Rincon, A. M., and Sanchez-Brambila, G. (2016). Instrumental texture characteristics of broiler pectoralis major with the wooden breast condition. Poult. Sci. 95, 2449-2454. doi: 10.3382/ps/pew204

Codd, J. R. (2005). Activity of three muscles associated with the uncinate processes of the giant Canada goose Branta canadensis maximus. J. Exp. Biol. 208, 849-857. doi: 10.1242/jeb.01489

D’Addesio, J. (1992). Metabolic and respiratory acidosis. Top. Emerg. Med. 14, 51-55.

Dalle Zotte, A., Tasoniero, G., Puolanne, E., Remignon, H., Cecchinato, M., Catelli, E., et al. (2017). Effect of "Wooden Breast" appearance on poultry meat quality, histological traits, and lesions characterization. Czech J. Anim. Sci. 62, 51-57. doi: 10.17221/54/2016-CJAS

Epstein, S. K., and Singh, N. (2001). Respiratory acidosis. Respir. Care 46, 366-383.

Forster, H. V., Dempsey, J. A., Thomson, J., Vidruk, E., and DoPico, G. A. (1972). Estimation of arterial $\mathrm{PO} 2, \mathrm{PCO} 2, \mathrm{pH}$, and lactate from arterilazed venous blood. J. Appl. Physiol. 32, 134-137. doi: 10.1152/jappl.1972.32.1.134

\section{ETHICS STATEMENT}

The animal study was reviewed and approved by the University of Delaware Institutional Animal Care and Use Committee.

\section{AUTHOR CONTRIBUTIONS}

BA conceptualized, designed, and supervised the study. JL performed the statistical analysis and wrote the original draft of the manuscript. EB, MP, and CL conducted histopathological examination of lung samples and $\mathrm{EB}$ wrote sections of the manuscript relevant to that examination. BA, JL, SV, EB, and MP contributed to manuscript revision. All authors read and approved the submitted version.

\section{FUNDING}

This project was supported by the Agriculture and Food Research Initiative Competitive Grant No. 2016-67015-25027 from the United States Department of Agriculture.

\section{ACKNOWLEDGMENTS}

The authors gratefully acknowledge the in-kind support by Cobb-Vantress, Inc., in providing chicks and chicken feed. We also greatly appreciate assistance of many graduate and undergraduate students at the University of Delaware Department of Animal and Food Sciences with sample and data collection.

Gall, S., Suyemoto, M. M., Sather, H. M. L., Sharpton, A. R., Barnes, H. J., and Borst, L. B. (2019). Wooden breast in commercial broilers associated with mortality, dorsal recumbency, and pulmonary disease. Avian Dis. 63, 514-519. doi: 10.1637/11995-111218-case.1

Hoving-Bolink, A. H., Kranen, R. W., Klont, R. E., Gerritsen, C. L. M., and De Greef, K. H. (2000). Fibre area and capillary supply in broiler breast muscle in relation to productivity and ascites. Meat Sci. 56, 397-402. doi: 10.1016/S03091740(00)00071-1

Ilkiw, J. E., Rose, R. J., and Martin, I. C. A. (1991). A comparison of simultaneously collected arterial, mixed venous, jugular venous and cephalic venous blood samples in the assessment of blood-gas and acid-base status in the dog. J. Vet. Intern. Med. 5, 294-298. doi: 10.1111/j.1939-1676.1991.tb03136.x

Joiner, K., Hamlin, G., Lien, R., and Bilgili, S. (2014). Evaluation of capillary and myofiber density in the pectoralis major muscles of rapidly growing, highyield broiler chickens during increased heat stress. Avian Dis. 58, 377-382. doi: 10.1637/10733-112513-reg.1

Julian, A. R. J., and Mirsalimi, S. M. (1992). Blood oxygen concentration of fastgrowing and slow-growing broiler chickens, and chickens with ascites from right ventricular failure. Avian Dis. 36, 730-732.

Kilburn, K. H. (1966). Movements of potassium during acute respiratory acidosis and recovery. J. Appl. Physiol. 21, 679-684. doi: 10.1152/jappl.1966.21.2.679

Kuttappan, V. A., Hargis, B. M., and Owens, C. M. (2016). White striping and woody breast myopathies in the modern poultry industry: a review. Poult. Sci. 95, 2724-2733. doi: 10.3382/ps/pew216

Kuttappan, V. A., Shivaprasad, H. L., Shaw, D. P., Valentine, B. A., Hargis, B. M., Clark, F. D., et al. (2013). Pathological changes associated with white striping in broiler breast muscles. Poult. Sci. 92, 331-338. doi: 10.3382/ps.201202646 
Ladé, R. I., and Brown, E. B. (1963). Movement of potassium between muscle and blood in response to respiratory acidosis. Am. J. Physiol. Content 204, 761-764. doi: 10.1152/ajplegacy.1963.204.5.761

Lake, J. A., and Abasht, B. (2020). Glucolipotoxicity: a proposed etiology for wooden breast and related myopathies in commercial broiler chickens. Front. Physiol. 11:169. doi: 10.3389/fphys.2020.00169

Lake, J. A., Papah, M. B., and Abasht, B. (2019). Increased expression of lipid metabolism genes in early stages of wooden breast links myopathy of broilers to metabolic syndrome in humans. Genes 10:E746. doi: 10.20944/preprints201906. 0194.v1

Livingston, M. L., Ferket, P. R., Brake, J., and Livingston, K. A. (2019a). Dietary amino acids under hypoxic conditions exacerbates muscle myopathies including wooden breast and white stripping. Poult. Sci. 98, 1517-1527. doi: $10.3382 / \mathrm{ps} /$ pey463

Livingston, M. L., Landon, C. D., Barnes, H. J., Brake, J., and Livingston, K. A. (2019b). Dietary potassium and available phosphorous on broiler growth performance, carcass characteristics, and wooden breast. Poult. Sci. 98, 28132822. doi: $10.3382 / \mathrm{ps} / \mathrm{pez} 015$

Ludders, J. W. (2015). "Respiration in birds," in Duke's Physiology of Domestic Animals, eds W. O. Reece, H. E. Howard, J. P. Goff, and E. E. Uemura (Ames, IA: Wiley), 245-258.

Malan, D. D., Scheele, C. W., Buyse, J., Kwakernaak, C., Siebrits, F. K., Van Der Klis, J. D., et al. (2003). Metabolic rate and its relationship with ascites in chicken genotypes. Br. Poult. Sci. 44, 309-315. doi: 10.1080/000716603100024603

Maxwell, M. H. (1988). The histology and ultrastructure of ectopic cartilaginous and osseous nodules in the lungs of young broilers with an ascitic syndrome. Avian Pathol. 17, 201-219. doi: 10.1080/03079458808436439

Mudalal, S., Lorenzi, M., Soglia, F., Cavani, C., and Petracci, M. (2015). Implications of white striping and wooden breast abnormalities on quality traits of raw and marinated chicken meat. Animal 9, 728-734. doi: 10.1017/ S175173111400295X

Mutryn, M. F., Brannick, E. M., Fu, W., Lee, W. R., and Abasht, B. (2015a). Characterization of a novel chicken muscle disorder through differential gene expression and pathway analysis using RNA-sequencing. BMC Genomics 16:399. doi: 10.1186/s12864-015-1623-0

Mutryn, M. F., Fu, W., and Abasht, B. (2015b). "Incidence of wooden breast disease and its correlation with broiler performance and ultimate $\mathrm{pH}$ of breast muscle," in Proceedings of XXII European Symposium on Poultry Meat Quality, Nantes.

Norring, M., Valros, A., Valaja, J., Sihvo, H., Immonen, K., and Puolanne, E. (2018). Wooden breast myopathy links with poorer gait in broiler chickens. Animal 17, 1-6. doi: 10.1017/S1751731118003270

Olkowski, A. A., Korver, D., Rathgeber, B., and Classen, H. L. (1999). Cardiac index, oxygen delivery, and tissue oxygen extraction in slow and fast growing chickens, and in chickens with heart failure and ascites: a comparative study. Avian Pathol. 28, 137-146. doi: 10.1080/03079459994867

Papah, M. B., Brannick, E. M., Schmidt, C. J., and Abasht, B. (2017). Evidence and role of phlebitis and lipid infiltration in the onset and pathogenesis of wooden breast disease in modern broiler chickens. Avian Pathol. 46, 623-643. doi: 10.1080/03079457.2017.1339346

Papah, M. B., Brannick, E. M., Schmidt, C. J., and Abasht, B. (2018). Gene expression profiling of the early pathogenesis of wooden breast disease in commercial broiler chickens using RNA-sequencing. PLoS One 13:e0207346. doi: 10.1371/journal.pone.0207346

Papah, M. B., and Abasht, B. (2019). Dysregulation of lipid metabolism and appearance of slow myofiber- specific isoforms accompany the development of Wooden Breast myopathy in modern broiler chickens. Sci. Rep. 9:17170. doi: 10.1038/s41598-019-53728-8

Petracci, M., Mudalal, S., Soglia, F., and Cavani, C. (2015). Meat quality in fastgrowing broiler chickens. Worlds. Poult. Sci. J. 71, 363-374. doi: 10.1017/ S0043933915000367

Roy, B. C., Oshima, I., Miyachi, H., Shiba, N., Nishimura, S., Tabata, S., et al. (2006). Effects of nutritional level on muscle development, histochemical properties of myofibre and collagen architecture in the pectoralis muscle of male broilers. $\mathrm{Br}$. Poult. Sci. 47, 433-442. doi: 10.1080/00071660600828334
Scheuermann, G. N., Bilgili, S. F., Tuzun, S., and Mulvaney, D. R. (2004). Comparison of chicken genotypes: myofiber number in pectoralis muscle and myostatin ontogeny. Poult. Sci. 83, 1404-1412. doi: 10.1093/ps/83.8.1404

Schmidt-Nielsen, K. (1971). How birds breathe. Sci. Am. 225, 72-79. doi: 10.1038/ scientificamerican1271-72

Scribner, B. B. H., Fremont-smith, K., and Burnell, J. M. (1955). The effect of acute respiratory acidosis on the internal equilibrium of potassium. J. Clin. Invest. 34, 1276-1285. doi: 10.1172/jci103174

Sihvo, H. K., Airas, N., Lindén, J., and Puolanne, E. (2018). Pectoral vessel density and early ultrastructural changes in broiler chicken wooden breast myopathy. J. Comp. Pathol. 161, 1-10. doi: 10.1016/j.jcpa.2018.04.002

Sihvo, H. K., Immonen, K., and Puolanne, E. (2014). Myodegeneration with fibrosis and regeneration in the pectoralis major muscle of broilers. Vet. Pathol. 51, 619-623. doi: 10.1177/0300985813497488

Sihvo, H. K., Lindén, J., Airas, N., Immonen, K., Valaja, J., and Puolanne, E. (2017). Wooden breast myodegeneration of pectoralis major muscle over the growth period in broilers. Vet. Pathol. 54, 119-128. doi: 10.1177/0300985816658099

Smith, I. C., Bombardier, E., Vigna, C., and Tupling, A. R. (2013). ATP consumption by sarcoplasmic reticulum $\mathrm{Ca}^{2+}$ pumps accounts for $40-50 \%$ of resting metabolic rate in mouse fast and slow twitch skeletal muscle. PLoS One 8:e068924. doi: 10.1371/journal.pone.0068924

Soglia, F., Mudalal, S., Babini, E., Di Nunzio, M., Mazzoni, M., Sirri, F., et al. (2016). Histology, composition, and quality traits of chicken Pectoralis major muscle affected by wooden breast abnormality. Poult. Sci. 95, 651-659. doi: $10.3382 / \mathrm{ps} / \mathrm{pev} 353$

Steinmetz, H. W., Vogt, R., Kästner, S., Riond, B., and Hatt, J.-M. (2007). Evaluation of the i-STAT portable clinical analyzer in chickens (Gallus gallus). J. Vet. Diagnostic Investig. 19, 382-388. doi: 10.1177/104063870701900407

Tickle, P. G., Paxton, H., Rankin, J. W., Hutchinson, J. R., and Codd, J. R. (2014). Anatomical and biomechanical traits of broiler chickens across ontogeny. Part I. Anatomy of the musculoskeletal respiratory apparatus and changes in organ size. PeerJ 2:e432. doi: $10.7717 /$ peerj.432

Velleman, S. G., Clark, D. L., and Tonniges, J. R. (2018). The effect of the Wooden Breast myopathy on sarcomere structure and organization. Avian Dis. 62, 28-35. doi: 10.1637/11766-110217-Reg.1

Wideman, R. F., and French, H. (2000). Ascites resistance of progeny from broiler breeders selected for two generations using chronic unilateral pulmonary artery occlusion. Poult. Sci. 79, 396-401. doi: 10.1093/ps/79.3.396

Wideman, R. F., Hooge, D. M., and Cummings, K. R. (2003). Dietary sodium bicarbonate, cool temperatures, and feed withdrawal: impact on arterial and venous blood-gas values in broilers. Poult. Sci. 82, 560-570. doi: 10.1093/ps/82. 4.560

Wong, E. M. (2018). Differential gene expression in lung tissue of wooden breast syndrome affected and unaffected commercial broiler chickens. Undergraduate Thesis, University of Delaware, Newark, DE.

Yildizdaş, D., Yapicioğlu, H., Yilmaz, H. L., and Sertdemir, Y. (2004). ). Correlation of simultaneously obtained capillary, venous, and arterial blood gases of patients in a paediatric intensive care unit. Arch. Dis. Child. 89, 176-180. doi: 10.1136/adc.2002.016261

Youn, J. H., and McDonough, A. A. (2009). Recent advances in understanding integrative control of potassium homeostasis. Annu. Rev. Physiol. 71, 381-401. doi: 10.1146/annurev.physiol.010908.163241

Conflict of Interest: The authors declare that the research was conducted in the absence of any commercial or financial relationships that could be construed as a potential conflict of interest.

Copyright (c) 2020 Lake, Brannick, Papah, Lousenberg, Velleman and Abasht. This is an open-access article distributed under the terms of the Creative Commons Attribution License (CC BY). The use, distribution or reproduction in other forums is permitted, provided the original author(s) and the copyright owner(s) are credited and that the original publication in this journal is cited, in accordance with accepted academic practice. No use, distribution or reproduction is permitted which does not comply with these terms. 
OPEN ACCESS

Edited by:

Massimiliano Petracci, University of Bologna, Italy

Reviewed by: Maurizio Mazzoni, University of Bologna, Italy Martina Zappaterra, University of Bologna, Italy Nabeel Alnahhas, Laval University, Canada

*Correspondence: Jiao Liu

jiao.liu@scuec.edu.cn

Specialty section: This article was submitted to Avian Physiology, a section of the journal Frontiers in Physiology

Received: 29 January 2020 Accepted: 16 March 2020

Published: 09 April 2020

Citation:

Liu J, Puolanne E,

Schwartzkopf M and Arner A (2020) Altered Sarcomeric Structure and Function in Woody Breast Myopathy of Avian Pectoralis Major Muscle. Front. Physiol. 11:287. doi: 10.3389/fphys.2020.00287

\section{Altered Sarcomeric Structure and Function in Woody Breast Myopathy of Avian Pectoralis Major Muscle}

\author{
Jiao Liu1,2*, Eero Puolanne ${ }^{3}$, Matthias Schwartzkopf ${ }^{4}$ and Anders Arner ${ }^{2}$ \\ ${ }^{1}$ College of Life Sciences, South-Central University for Nationalities, Wuhan, China, ${ }^{2}$ Thoracic Surgery, Department \\ of Clinical Sciences, Lund, Faculty of Medicine, Lund University, Lund, Sweden, ${ }^{3}$ Department of Food and Nutrition, \\ University of Helsinki, Helsinki, Finland, ${ }^{4}$ Deutsches Elektronen-Synchrotron, Hamburg, Germany
}

The "Woody" or "Wooden" breast disease is a severe myopathy of pectoralis major muscle recently identified within rapidly growing broiler lines all around the world with a prevalence rate around $20 \%$, or even higher. Although of significant ethical and economic impact, little is known regarding the structural and functional aspects of the contractile apparatus in the woody breast muscle. The aim of the present study was to determine physiological properties of the contractile system in the morphologically intact muscle fibers of focally damaged woody breast in comparison with normal muscle fibers to gain insight into the muscle function of the animal and possibly mechanisms involved in the disease development. Muscle samples were taken from woody breast (non-lesioned areas) and normal breast muscles from broilers. Length-tension curves, maximal active stress, maximal shortening velocity, calcium sensitivity, rate of tension development, lattice spacing and muscle biochemical composition were investigated on single skinned fibers. Sarcomeres of woody breast fibers were more compliant, which is very likely related to the wider spacing (18\% wider compared to controls) between thick and thin filament. No differences were found in optimal sarcomere length $(2.68 \pm 0.04$ vs. $2.65 \pm 0.05 \mu \mathrm{m})$ nor in maximal active stress $(116 \pm 17$ vs. $125 \pm 19 \mathrm{mN} \mathrm{mm}^{-2}$ ). However, woody breast fibers had less steep descending arm as shown in length-tension curve. Woody breast muscle fibers had $40 \%$ bigger sarcomeric volume compared to controls. Content of contractile proteins (myosin and actin), and maximal shortening velocity were unchanged indicating that the growth in woody breast muscle fiber was associated with synthesis of new contractile units with unaltered kinetics. Calcium sensitivity was decreased in woody breast muscle fibers significantly. In conclusion, the results show that the rapid growth of muscle in woody breast disease is associated with significant structural and functional changes in the pectoralis major musculature, associated with alterations in the mechanical anchoring of contractile filaments.

Keywords: avian muscle, hypertrophy, muscular dystrophy, muscle disease, sarcomere 


\section{INTRODUCTION}

Consumption of poultry meat has increased dramatically worldwide during recent years, due to its comparatively low environmental impact as well as a healthy nutritional profile, with low fat and high protein contents. In order to satisfy the high and increasing demands, breeders have been genetically selecting broilers toward a fast growth rate, and high breast muscle (pectoralis major) yield over the past few decades. As a consequence, the growth rate of broilers has increased during the last 50 years almost 3 -fold, resulting in weights of more than $2 \mathrm{~kg}$ in less than 40 days, with breast weights of about $20 \%$ of the whole carcass weight (Petracci et al., 2015).

Unfortunately, the improvement of growth rate is accompanied by increasing prevalence rates of several muscular abnormalities in the breast muscle, such as pale, soft and exudative (PSE) like meat (Chen et al., 2018), white striping (Kuttappan et al., 2012) and the woody (also denoted wooden) breast condition (Sihvo et al., 2014). Compared to the other two abnormalities, the woody breast is a more recent problem and has already been reported by several big chicken meat producing countries worldwide. The prevalence rate is around 10-20\% (Trocino et al., 2015) and has been shown even higher in some reports (Mutryn et al., 2015; Petracci et al., 2019). Woody breast muscles are easily identified by hand palpation having a hard consistency, with pale appearance, and in more severe cases, hemorrhages in lesion areas. Histological examination has revealed that the affected areas in woody breast muscle usually have moderate to severe myodegeneration and regeneration, such as muscle fiber necrosis, infiltration of inflammatory cells, fibrosis, fat accumulation and centralized nuclei (Sihvo et al., 2014). Besides the economic loss, woody breast myopathy gives rise to serious concerns regarding animal welfare (Norring et al., 2019). In view of the $10-20 \%$ or higher prevalence and the large number of slaughtered broilers worldwide (more than 45 billion per year worldwide, AVEC, 2011 report) woody breast would by far be the most common muscle disease in the world today.

Ever since woody breast was first reported as a broiler meat quality deterioration problem several years ago, studies have been done (see review by Petracci et al., 2019) in aspects including pathological description of the lesions (Sihvo et al., 2014), post mortem physical and chemical characteristics of affected breast muscle (Soglia et al., 2015), and the properties of the muscle during further food manufacturing (Mudalal et al., 2015). Based on the currently available literature, it is likely that the occurrence of the woody breast is related to the heavier breast muscles or hypoxia (Soglia et al., 2015; Baldi et al., 2017; Dalle Zotte et al., 2017; Papah et al., 2017; Petracci et al., 2019) suggesting that rapid growth is involved in the pathogenesis. On the muscle fiber level, woody breast affected muscle tends to have higher abnormal fiber prevalence, but to our knowledge, little is known regarding on the structural and functional aspects of the contractile apparatus within woody breast muscle fibers under physiological conditions. The proper assembly and organization of the basic contractile units in each myofibril have critical roles in the morphology and, most importantly, the function of muscle fibers. It is also important to note that the structure and mechanical function of the sarcomere can feedback on the growth and adaptation of the muscle structure/function, with significant contacts between the cytoskeleton and signaling pathways involved in the maintenance of the cellular structure (e.g., titin in the sarcomere, Lange et al., 2005; vimentin and desmin in the cytoskeleton, Paulin and Li, 2004; Soglia et al., 2020); dystrophin in the dystroglycan complex (Rahimov and Kunkel, 2013). It is therefore relevant to gain more knowledge regarding the structural characteristics and function of the contractile apparatus in the woody breast muscle, since it will help us to understand how this myopathy influences muscle function in vivo, and cast light on mechanisms underlying the muscle dystrophy disease development in general.

In the current study, we examined woody breast muscle from a muscle physiology perspective. Muscle samples were prepared from normal breast and non-lesioned areas of woody breast. Sarcolemma was permeabilized (skinned preparations) preserving the intracellular contractile machinery enabling analysis length-tension curves, maximal active stress and maximal shortening velocity. Sarcomeric structure was measured using small angle $\mathrm{x}$-ray scattering. We report alterations in the mechanical properties, including altered sarcomere mechanics and structure, which would influence mechanical function in vivo and possibly be related to the induction of hypertrophic growth.

\section{MATERIALS AND METHODS}

\section{Sample Selection}

Eight normal and eight woody breast broiler pectoralis major muscles were dissected from sixteen Ross 308 chickens at 32 days old of both genders from the regular slaughter line in a local commercial slaughterhouse (average animal slaughter weight $\sim 2.2 \mathrm{~kg}$ as given by the slaughterhouse). Both normal and woody breast muscles were obtained from the same cohort of slaughtered animals.

Woody breast affected carcasses were identified by palpation on the slaughter line at $15 \mathrm{~min}$ post mortem together with normal carcasses. After being excised, the status of the breast muscles was confirmed visually and by palpating according to the criteria described by Sihvo et al. (2016) based on the appearance of white and hard areas vs. normal color and texture. Since the current study focused on identifying early changes in the woody breast condition development, we included woody breast affected muscles with only focal lesions in the cranial area and less affected medial area. Comparisons were made with normal breast muscle obtained from non-affected animals. Samples for analysis were taken from the medial part of normal controls and woody breast affected muscles. The samples were immediately fixed for histology or treated with chemical skinning as described below.

The study was fully based on slaughterhouse material obtained from the normal food production slaughterhouse line, after the animals were slaughtered. The study does not involve any study of live animals. No interventions or examinations were done prior to the slaughter. 


\section{Histological Analysis}

Muscle bundles (10 mm long $\times 2 \mathrm{~mm}$ thick) were slightly stretched, pinned to cork plates and fixed in buffered $4 \%$ paraformaldehyde for $24 \mathrm{~h}$ at $4^{\circ} \mathrm{C}$. After transfer to a $70 \%$ of ethanol solution, samples were trimmed to obtain both longitudinal and transverse profiles of the muscle fibers, embedded in paraffin, sectioned at 4 micrometers, stained with hematoxylin and eosin, for visualization of tissue and muscle structure, and examined by light microscopy.

\section{Skinned Fiber Preparations}

Fiber bundles ( $10 \mathrm{~mm}$ long $\times 2 \mathrm{~mm}$ thick) were tied at both ends with silk thread, stretched to the approximate in situ length on plastic holders and chemically skinned (sarcolemma permeabilized) essentially as described by in Iorga et al. (2012). The skinned preparations were kept relaxed in ATP-containing solution with composition (in mmol L-1): $\mathrm{K}_{2}$ EGTA $10, \mathrm{Na}_{2}$ ATP 7, MgAcetate 2, MOPS 10, and K-propionate 122, $\mathrm{pH} 7.0$, with $50 \%$ glycerol at $-20^{\circ} \mathrm{C}$ for further analysis.

The woody breast affected samples displayed a mixture of degenerating muscle and morphologically "normal" muscle areas. Since we aimed at examining early stages of the myopathy, we isolated single muscle fibers from the morphologically intact areas and mounted for subsequent analysis. Fibers with clear sarcomere patterns and homogeneous width along fiber axis were included in the experiments.

\section{Sarcomere Length Measurements}

Each isolated single fiber was attached using cellulose acetate glue (Sigma-Aldrich) between a force transducer (AE801, Kronex) and a micromanipulator enabling length changes. Experiments were performed in standard relaxation solution which contained (in mmol L-1) $\mathrm{K}_{2}$ EGTA 6, MgATP 5, free $\mathrm{Mg}^{2+}$ 0.5, phosphocreatine 15, MOPS buffer 20, DTE 2 and $\mathrm{K}$-propionate to an ionic strength of 200 , with creatine kinase $0.5-1 \mathrm{mg} \mathrm{ml}-1$ at $\mathrm{pH}$ 7.0. Free $\left[\mathrm{Ca}^{2+}\right]$ was approximately $1 \mathrm{nmol}$ L-1 (pCa 9) (All chemicals were purchased in Sigma-Aldrich). For each animal, 4-5 fibers were analyzed.

\section{Length-Tension Relationship}

To determine the relationship between fiber length and passive/active force, single fibers were mounted at slack length between a force transducer and a micro manipulator as described above, and repeatedly activated at room temperature using a solution with high $\mathrm{Ca} 2+\left(\mathrm{pCa}\left(-\log \left[\mathrm{Ca}^{2+}\right]\right)=4.7\right)$, same composition as the relaxing solution, except that $\mathrm{K}_{2}$ EGTA is replaced by $\mathrm{K}_{2}$ CaEGTA) and relaxed in between at room temperature. Muscles were stretched between contraction steps, and passive and active forces were recorded at each length step. Fiber length (relative to slack) was determined using light microscopy. Optimal stretch for active force was 1.3 of the slack length, and fiber width was determined at that length and used to calculate the maximal active stress (i.e., force per cross sectional area). Two to three single fibers from each animal were measured. For each fiber the force response at each L/Ls step was first normalized by its maximal force. The data from the fibers of each animal was averaged, and used as representative in the statistical analysis.

\section{Fiber Width Determination}

Fiber diameter at optimal stretch (1.3 relative to slack length, $\mathrm{L} / \mathrm{Ls}$ ) was determined from different single fibers ( $>50$ fibers for both woody and normal beast group) during sarcomere length measurements, length-tension experiments, force velocity experiment and $\mathrm{k}_{\mathrm{TR}}$ determinations using light microscopy and an ocular scale (Zeiss Axiovert 35, inverted microscope, $20 \times$ objective with $2.5 \times$ magnification and a $10 \times$ ocular). Three measurements at different positions along the long axis were taken from each fiber and averaged. In total, 6-7 measurements of fiber diameter from each animal were obtained and the average of these measurements for each animal were calculated and compared.

\section{Determination of Force-Velocity Relationship, Stiffness and Rate of Tension Development}

To obtain information on the kinetics of the actin-myosin interaction we determined the force-velocity relationship, i.e., the dependence of shortening velocity on force and the maximal shortening velocity $\left(\mathrm{V}_{\max }\right)$ at room temperature. Single fibers were mounted as described above between the force transducer and a motor, enabling rapid changes in muscle length and force via a computer-controlled feedback system (Aurora Scientific 802B, ON, Canada). A series of 14 force steps was applied at the plateau of a maximal contraction. Stable after-loaded force levels were obtained in about $15 \mathrm{~ms}$. For each step, length and force signals were recorded during $300 \mathrm{~ms}$. The afterload $(\mathrm{Pa})$ and the isometric force $(\mathrm{Po})$, as well as the mean shortening velocity ( $\mathrm{V}$, between 20 and $70 \mathrm{~ms}$ after the force step in muscle length $\mathrm{ML} \mathrm{s}^{-1}$ ) were analyzed for each step. Force velocity curves were constructed from the relationship between $\mathrm{V}$ and relative force $(\mathrm{Pa} / \mathrm{Po})$. For each animal, 1 fiber was analyzed.

For each force step, the linear regression for the time period $20-70 \mathrm{~ms}$ (used to calculate velocity) was extrapolated to the time of release and the intercept of this line was used to determine the series elastic recoil.

In separate experiments the rate of tension development $\left(\mathrm{k}_{\mathrm{TR}}\right)$ was estimated using a protocol described by Brenner and Eisenberg (1986). The fibers were shortened by $28 \%$ and restretched to initial length after $30 \mathrm{~ms}$. The apparent rate constant for the force development was estimated from half-time of the force increase.

\section{Calcium Sensitivity Measurement}

In another series of measurements, two bundles around $0.2 \mathrm{~mm}$ in diameter from each animal sample were analyzed. The averaged result of the two bundles represented one animal. Preparations were attached to a force transducer (Kronex) and stretched to 1.3 times of slack length in relaxing solution. After an initial activation at $\mathrm{pCa} 4.7$, a series of solutions with intermediate free $\left[\mathrm{Ca}^{2+}\right]$, pCa: $9,6.0,5.7,5.5,5.2$, and 4.7 , calculated as described by Fabiato (1988), were used to activate the muscle. 
The maximal force obtained at pCa 4.7 was used to normalize each contraction in response at lower $\mathrm{pCa}$ values. A hyperbolic equation $\left[\mathrm{F}=\mathrm{c}^{\mathrm{h}} /\left(\mathrm{EC}_{50}^{\mathrm{h}}+\mathrm{c}^{\mathrm{h}}\right)\right]$ was fitted to the normalized force responses $(\mathrm{F})$ at different free $\left[\mathrm{Ca}^{2+}\right](\mathrm{c})$, to determine the $\mathrm{EC}_{50}$ (concentration giving half maximal force) and the Hill coefficient (h, describing the steepness of the curve).

\section{SDS-PAGE Analysis}

A muscle bundle (around $2 \mathrm{mg}$ wet weight, including several muscle fibers) was dissected from the skinned preparations, rinsed and homogenized using glass homogenizers in $100 \times$ volume of $1 \times$ Laemmli sample buffer (Bio-Rad) containing $1 \%$ SDS and $50 \mathrm{mmol} \mathrm{L}^{-1}$ DTT and heated to $95^{\circ} \mathrm{C}$ for $10 \mathrm{~min}$ until all the tissue were completed dissolved. Samples were separated on $4-15 \%$ gradient gels (Mini-PROTEAN TGX Precast Gels, Bio-Rad). Three different volumes of the homogenates, including one woody and normal breast muscle sample, together with three different known amounts of albumin standard and a molecular weight standard were loaded on each gel. Gels were stained using PAGEBLUE Protein Staining Solution (Thermo Fisher) and the quantification of stained protein bands were done using a CHEMIDOC XRS and the QUANTITY ONE program (Bio-Rad, RRID: SCR_014280). For each lane, the intensity of the bands corresponding to myosin heavy chain (MHC, $220 \mathrm{kDa})$ and actin $(42 \mathrm{kDa})$ were determined. For each homogenate, a linear regression curve was fitted to the relation between loaded volume, and the MHC and actin band intensity respectively. The ratio between this slope and the slope of the albumin standard was then used to calculate the protein content $\left(\mu \mathrm{g} \mathrm{mg}^{-1}\right.$ wet muscle weight) of the sample.

\section{Small Angle X-Ray Scattering}

Small angle $\mathrm{x}$-ray scattering (SAXS) was used to determine the lattice spacing of the normal and woody breast muscle samples. Skinned muscle preparations were mounted in relaxation solution in a cuvette with Kapton window and stretched to different relative length (sarcomere length). The SAXS experiments were performed at the beamline P03, at Petra III, DESY, Hamburg (Buffet et al., 2012). A beam with a wavelength of $0.95 \AA$ and a size of $16 \times 22 \mu \mathrm{m}^{2}$ was used. The equatorial diffraction patterns were recorded with 0.1 to $1 \mathrm{~s}$ exposures using a PILATUS 300K detector (DECTRIS Ltd.) in $5.1 \mathrm{~m}$ distance as described previously (Liu et al., 2018). The signal was calibrated using rat tail collagen. A polynomial function was fitted to the equatorial intensity signal for background subtraction and the center of mass for the reflections was determined. The spacing of the equatorial 1.0 and 1.1 reflections were measured. Based on collagen calibration, $d_{10}$ and $d_{11}$ from the muscle samples were calculated which are related to the spacing between two adjacent thick filaments mathematically. Lattice volume (Vol) was determined as described by Millman (1998) by multiplying lattice area with sarcomere length (SL):

$$
\mathrm{Vol}=2 / \sqrt{3} \times \mathrm{d} 10^{2} \times \mathrm{SL}
$$

Vol was estimated from the slope of the relationship between sarcomere length and $1 /(\mathrm{d} 10)^{2}$. The $1.1 / 1.0$ intensity ratio was determined as an indicator of mass transfer from thick to thin filament, e.g., when cross-bridge attachment is initiated, an increase of 1.1/1.0 will be observed according to Millman (1998).

\section{Statistical Analysis}

All data are given as mean \pm SEM with number of animals indicated. Several muscle fibers were analyzed for each animal, the average was taken as a representative value for that animal. Statistical analyses were made using SigmaPlot 14 (RRID: SCR_003210) for Windows (Systat Software Inc.). Twoway ANOVA, and the Holm-Sidak method were used for comparisons of normal and woody breast fibers at stretches, $\mathrm{L} / \mathrm{Ls} \geq 1.8$. Student's $t$-test was applied after confirmation of normality (Shapiro-Wilk) and equal variance (Brown-Forsythe), and Mann-Whitney Rank Sum Test used for active tension comparison at L/Ls 1.6.

\section{RESULTS}

Figure 1 shows longitudinal- and cross-sections of samples from normal and woody breast muscles. The control muscle displays mainly well-organized muscle fibers with equal staining, with small areas of inflammatory infiltrates centered on larger blood vessels. In woody breast samples, discontinuities were found among the fibers in longitudinal sections accompanied by extensive fibrosis and inflammation. Transverse sectioning of muscle fibers clearly shows varying fiber diameters, presence of degrading fibers and a significant fibrosis. These morphological data thus show a clear dystrophy in the woody breast group and essentially normal structure in the control group. In the mechanical analysis, we examined morphologically intact fibers from woody breast affected muscle; possibly displaying early changes associated with disease development, and did not include

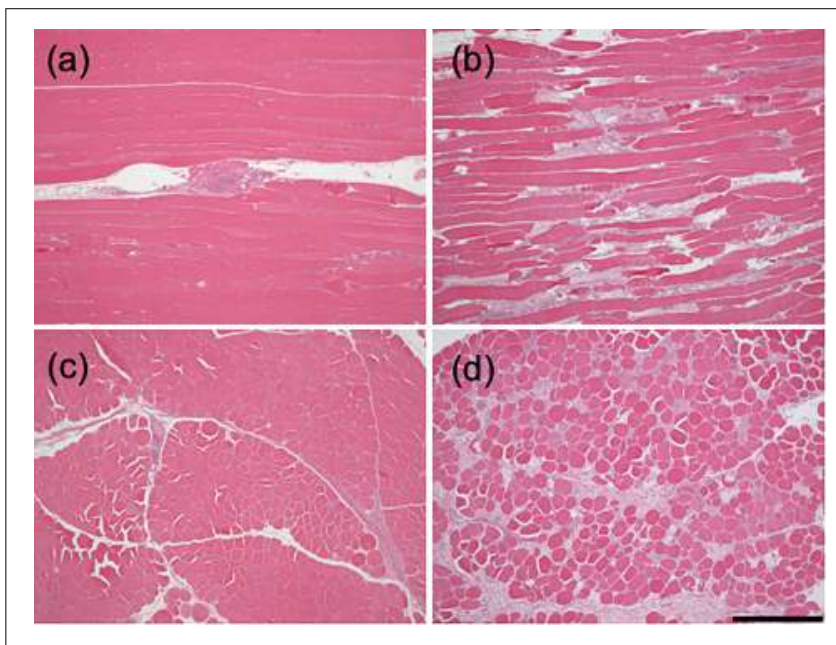

FIGURE 1 | Histology of woody and normal breast muscle. Longitudinal (a,b) and cross-sections $\mathbf{( c , d )}$ of clinically normal pectoralis major muscle $\mathbf{( a , c )}$ woody breast (b,d) muscles (bar $=0.5 \mathrm{~mm}$ ). 


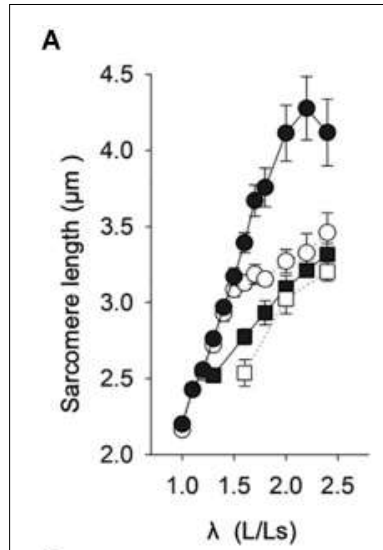

C

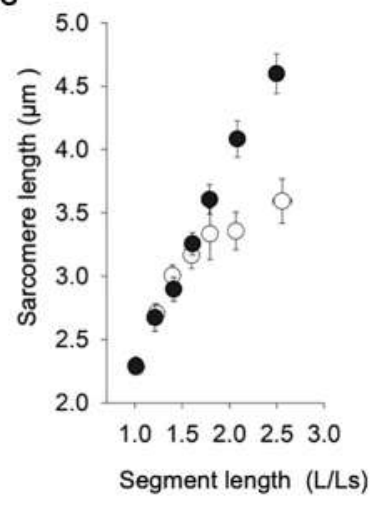

B

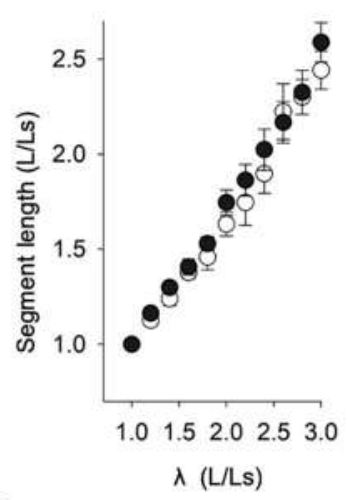

D

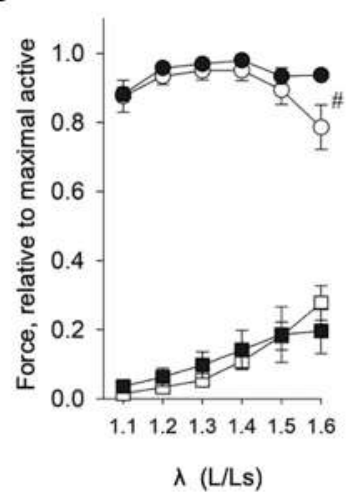

FIGURE 2 | Length-force analysis of woody and normal breast muscle. (A) Relation between muscle length (related to slack length, Ls) and sarcomere length in normal breast (open circles) and woody breast (filled circles) muscles (data from eight animals, 4-5 fibers each, grouped according to L/Ls, each L/Ls: $n=6-30$ ). Filled squares show the relation of woody breast fibers compressed by $20 \%$ (to the same fiber diameter as that of normal breast fibers) using 4\% dextran T-500, and open squares show the relation of normal breast fibers compressed by $20 \%$ using $4 \%$ dextran T-500. For $L / L s$ values $\geq 1.8$, the sarcomere length of the woody breast group was significantly longer compared to normal muscle fibers $(P<0.05$, Two-way ANOVA). (B) Relation between segment length, i.e., the distance between two carbon dots adhered to the fiber surface (relative to slack length, Ls, $\sim 60 \mu \mathrm{m}$ ), and muscle fiber length (relative to slack fiber length). (C) Relation between sarcomere length and segment length (relative to Ls) in normal (open circles) and woody (filled circles) muscle fibers (data from six animals, 2-3 fibers each, grouped according to segment length/Ls, $n=6-13)$. (D) Active (circles) and passive (squares) length-force relationships of normal (open symbols) and woody breast (filled symbols) fibers (average data from eight animals, 2-3 fibers each, grouped according to L/Ls, each L/Ls: $n=6-8$ ). \# Indicates significant difference in active force at $L / L s=1.6(P<0.05)$.

severely damaged fibers. Therefore, only skinned fibers with clearly visible sarcomere patterns were included.

The first step was to investigate the relationship between sarcomere length and stretch of the fiber (given as muscle length/slack length, $\lambda, L / L s$ ) in relaxed fibers from both groups. Figure 2A (including data from 6-30 fibers from 8 samples, grouped according to $\mathrm{L} / \mathrm{Ls}$ ) showed that for $\mathrm{L} / \mathrm{Ls}$ values $\leq 1.7$, no difference in sarcomere lengths between normal breast (open circles) and woody breast groups (filled circles) was observed. At $\mathrm{L} / \mathrm{Ls}$ values $\geq 1.8$, sarcomeres were longer in the woody

breast groups $(P<0.05$, two-way ANOVA) compared to the normal breast sarcomere lengths. In the control normal breast muscle, sarcomere length increased with stretch up to $\sim 1.5 \mathrm{~L} / \mathrm{Ls}$, reaching about $3.2 \mu \mathrm{m}$, and then increased less steeply with fiber length increase to about $3.4 \mu \mathrm{m}$ at $2.5 \mathrm{~L} / \mathrm{Ls}$. In contrast, the sarcomere length of woody breast fibers increased with stretch and reached $\sim 4.2 \mu \mathrm{m}$ at $2.4-2.5 \mathrm{~L} / \mathrm{Ls}$. These data suggest that passive structures limiting sarcomere extension during muscle fiber stretch are lacking or weaker in the woody breast group. We observed that upon extreme extension of fiber length, $70 \%$ of normal breast single fibers broke when L/Ls was above 2.6, whereas most of the woody breast fibers were able to be extended to 2.8-3.0 L/Ls. We therefore performed the stretch experiments up to $2.5 \mathrm{~L} / \mathrm{Ls}$. Since the stretch experiments of Figure 2A were done with length normalized to whole fiber length, we also examined if inhomogeneity along the fiber length or endcompliance, e.g., in the mounting, could affect the relationship between stretch and sarcomere length. Figure 2B shows that the extension of segments (about $60 \mu \mathrm{m}$, corresponding to about 25 sarcomeres) followed the extension of the whole fiber and Figure 2C shows that the difference in sarcomere extension between normal and woody breast fibers was observed also using this method.

As shown in Table 1, we found that woody breast fibers were about $20 \%$ wider in diameter compared to normal breast fibers. To study if the wider fiber diameter was related to the increased sarcomere extensibility when fibers were stretched

TABLE 1 | Mechanical data and contractile protein expression.

\begin{tabular}{|c|c|c|c|}
\hline & Normal breast & Woody breast & $P$ \\
\hline Optimal L/Ls & $1.34 \pm 0.05(8)$ & $1.34 \pm 0.03(8)$ & n.s. \\
\hline $\begin{array}{l}\text { Optimal sarcomere length, } \\
\mu \mathrm{m}\end{array}$ & $2.68 \pm 0.04(5)$ & $2.65 \pm 0.05(6)$ & n.s. \\
\hline Fiber diameter, $\mu \mathrm{m}$ & $73.0 \pm 1.3(8)$ & $88.0 \pm 2.5(8)$ & 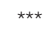 \\
\hline Maximal active tension, $\mathrm{mN}$ & $0.50 \pm 0.08(8)$ & $0.73 \pm 0.08(8)$ & n.s. \\
\hline $\begin{array}{l}\text { Maximal active stress, } \mathrm{mN} \\
\mathrm{mm}^{-2}\end{array}$ & $116 \pm 17(8)$ & $125 \pm 19(8)$ & n.s. \\
\hline Vmax, ML s ${ }^{-1}$ & $1.51 \pm 0.08(4)$ & $1.68 \pm 0.16(5)$ & n.s. \\
\hline Stiffness, $\mathrm{ML}(\mathrm{Pa} / \mathrm{Po})^{-1}$ & $0.036 \pm 0.003(6)$ & $0.043 \pm 0.004(6)$ & n.s. \\
\hline $\mathrm{k}_{\mathrm{TR}}, \mathrm{s}^{-1}$ & $19 \pm 2(6)$ & $10 \pm 1(5)$ & $\star \star$ \\
\hline $\mathrm{EC}_{50}, \mathrm{pCa}$ units & $5.81 \pm 0.02(6)$ & $5.71 \pm 0.03(6)$ & * \\
\hline $\mathrm{MHC}, \mu \mathrm{g} \mathrm{mg}^{-1}$ wet weight & $11.0 \pm 1.4(5)$ & $12.6 \pm 1.4(6)$ & n.s. \\
\hline Actin, $\mu \mathrm{g} \mathrm{mg}^{-1}$ wet weight & $9.0 \pm 1.3(5)$ & $8.8 \pm 1.1(6)$ & n.s. \\
\hline MHC/actin, molar ratio & $0.27 \pm 0.01(5)$ & $0.31 \pm 0.01(6)$ & * \\
\hline
\end{tabular}

Fiber diameter, sarcomere length and active force were determined at optimal stretch (relative to slack length, L/Ls) for active force. The maximal shortening velocity $(\mathrm{Vmax})$ is given in muscle length/s $\left(M L \mathrm{~s}^{-1}\right)$. The series elastic stiffness is given as in muscle length/relative force [ML (Pa/Po)-1] where $\mathrm{Pa}$ and $\mathrm{PO}$ are afterload and isometric force, respectively. The rate of tension development $\left(k_{T R}\right)$ is given in $\mathrm{s}^{-1}$. The $\mathrm{Ca}^{2+}$ concentration where half of maximal force was developed $\left(E C_{50}\right)$ is given in $\mathrm{pCa}$ units. Number of animals is given in parenthesis. Myosin heavy chain (MHC) and actin were determined using quantitative SDS-PAGE. For fiber diameter: 6-7 fibers were analyzed per animal; for optimal lengths, maximal tension and stress: 2-3 fibers per animal; for Vmax and stiffness 1 fiber per animal; for MHC and actin: a sample containing several fibers per animal, loaded in different concentrations on the same gel; for $k_{T R}, 2$ fibers per animal; for $E C_{50}, 2$ fiber bundles per animal. ${ }^{\star} P<0.05,{ }^{* *} P<0.01,{ }^{\star * *} P<0.001$, n.s., non-significant (Student's t-test). 


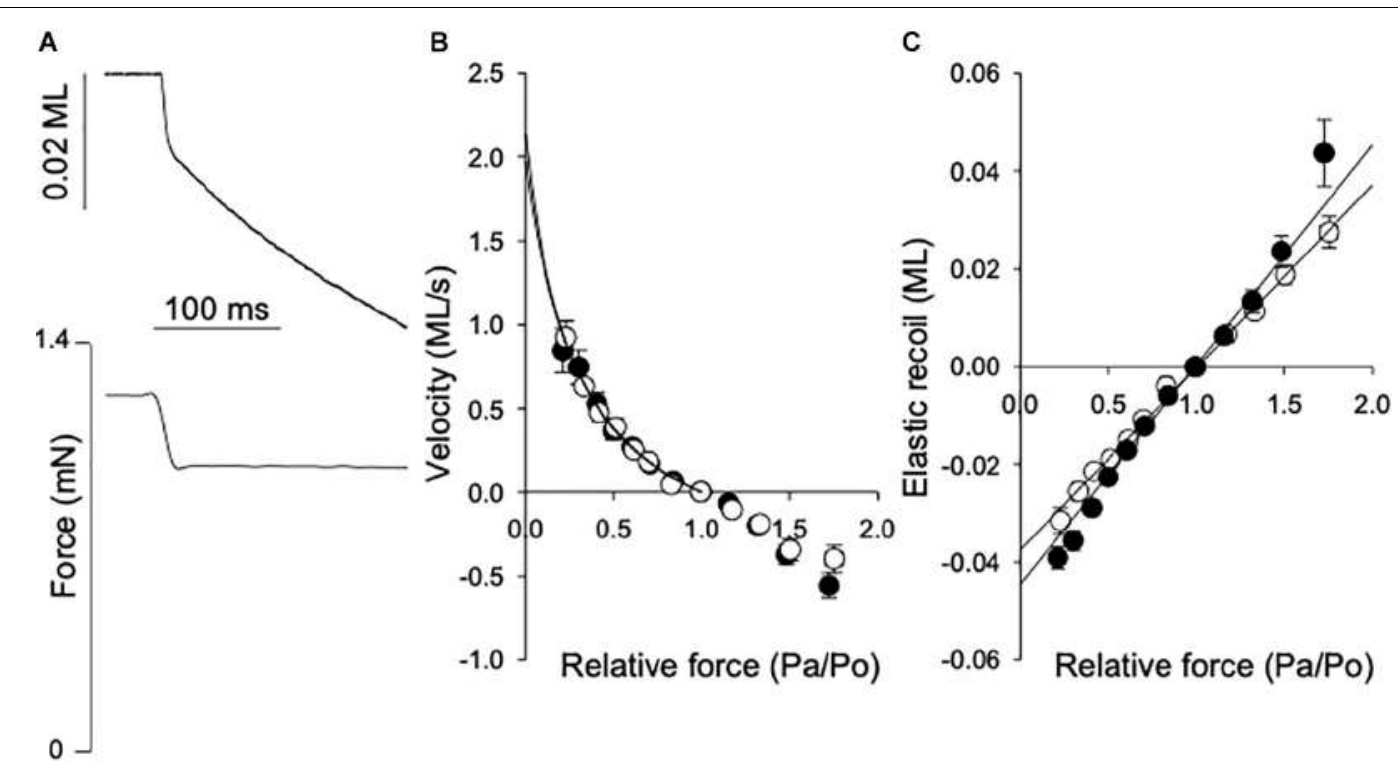

FIGURE 3 | Force-velocity relationship and series elasticity. (A) Shows original traces of the fiber length shortening response (lower) to a rapid change in contraction force (top), from a normal breast muscle. The force step and length response are indicated in the figure. Shortening velocity = reduction in muscle length $(\mathrm{ML})$ /shortening time (s). The afterload force (Pa) and reduction in muscle length were determined between 20 and $70 \mathrm{~ms}$ after the force step. (B) Shows the velocity (ML s-1) at different after loads related to the isometric force (Po). Data from 6 experiments were grouped according to Pa/Po and the data for shortening (i.e., velocity $\geq 0$ ) were fitted by the hyperbolic Hill equation (Eq. 1). The equation was extrapolated to Pa/Po $=0$ to determine the maximal shortening velocity, $\mathrm{Vmax}$ (fit to data in figure: normal breast, open symbols: 2.1; woody breast, filled symbols: $2.0 \mathrm{ML} \mathrm{s} \mathrm{s}^{-1}$ ). (C) Depicts the initial elastic recoil for different force steps. The data between 0.5 and 1.5 Pa/Po were fitted by a straight line [Normal breast, open symbols: 0.037; woody breast, filled symbols: 0.045 ML (Pa/Po)-1].

(Figure 2A), we used osmotic compression (4\% dextran added) to compress the woody breast fiber diameter to the same size as that of the control group (filled squares, Figure $\mathbf{2 A}$, diameter $71 \pm 5 \mu \mathrm{m}, n=6)$. Under these conditions, the sarcomere length response to stretch in the woody breast fibers became similar to that of the normal control fibers, suggesting that the altered coupling between fiber length and sarcomere length was related to the increase in fiber diameter. For comparison, the compression of normal breast fibers by $20 \%$ (diameter $63 \pm 4 \mu \mathrm{m}, n=4$ ) had minor effects on the relationship between stretch and sarcomere length (open squares, Figure 2A).

Our next step was to examine the length-force relationships, i.e., the passive and active force at different stretch (sarcomere length). As seen in Figure 2D, the relationship between stretch and active force was bell shaped with an optimal stretch of $\sim 1.3 \mathrm{~L} / \mathrm{Ls}$ in both groups. The mechanical data are summarized in Table 1. The woody breast fibers had unchanged optimal stretch and optimal sarcomere length, but significantly increased fiber diameter at optimal stretch, compared to the normal controls. Both the maximal active force and calculated maximal active stress tended to be higher in the woody breast group, although not significantly different from the controls. We observed that at $1.6 \mathrm{~L} / \mathrm{Ls}$, the woody breast fibers had significantly $(P<0.05)$ higher active force compared to normal breast fibers in the descending limb of the active length-force curve (rightmost circles in Figure 2D). Passive force, in the relaxed muscle, reflecting properties of elastic components in parallel with contractile components, increased during extension of the fibers in a similar manner in the two groups (squares in Figure 2D).

The maximal shortening velocity $\left(\mathrm{V}_{\max }\right)$ reflects the turnover of myosin-actin cross-bridges under unloaded conditions, mainly determined by the cross-bridge dissociation rate (Huxley, 1957). To determine this parameter, we performed isotonic quick release experiments, where the muscle was maximally activated to a stable isometric force (Po) and then exposed to a series of after loads $(\mathrm{Pa})$, as illustrated in Figure 3A. The velocity (V) determined during the time period 20-70 ms after each release is plotted against relative after load $(\mathrm{Pa} / \mathrm{Po})$. In Figure $3 \mathbf{B}$, data from 4-5 experiments were grouped according to after load and fitted with the Hill force-velocity equation in the following form:

$$
V=\frac{b \times\left(1-\frac{P a}{P o}\right)}{a+\frac{P a}{P o}}
$$

$\mathrm{V}_{\max }$ was calculated from the fitted parameters $\mathrm{a}$ and $\mathrm{b}$ as $\mathrm{b} / \mathrm{a}$. The mean values of $\mathrm{V}_{\max }$ determined in individual muscle fibers are shown in Table 1. The mean values for parameter a were $0.38 \pm 0.06$ and $0.32 \pm 0.08$ and $\mathrm{b} 0.58 \pm 0.11$ and $0.52 \pm 0.08$, $\mathrm{ML} \mathrm{s}{ }^{-1}$, in normal and woody breast respectively, $n=4$ and 5. No difference was found between normal and woody breast muscle fibers suggesting that although we have observed changes in woody breast muscle fibers, e.g., compliance, fiber width and lattice spacing (Figure 2 and Table 1), these differences are not associated with altered kinetics in the actin-myosin interaction when muscle is doing concentric work (i.e., shortening under load). It should be noted that the woody breast fibers appeared 
to be somewhat more compliant in the negative velocity region $(\mathrm{Pa} / \mathrm{Po}>1)$ of the force-velocity relationship, which might suggest altered behavior when muscle is doing eccentric work (i.e., stretched while contracting).

Results of stiffness and $\mathrm{k}_{\mathrm{TR}}$ determination showed that woody breast fibers have more complaint sarcomeres compared to the controls. The isotonic quick release experiments enabled determination of the properties of the series elastic components using the initial length change after the force step. It should be noted that our setup enabled force steps complete within about $15 \mathrm{~ms}$, but would reflect the series elastic components in the muscles. In Figure 3C the elastic recoil (SE) is plotted against afterload $(\mathrm{Pa} / \mathrm{Po})$. The data between 0.5 and $1.5 \mathrm{~Pa} / \mathrm{Po}$ were fitted by a straight line, where the slope would be the inverse of the spring constant of a linear spring. The values in Table 1 show a slightly more compliant SE in the woody breast group compared to that of the normal breast muscle, although the mean values were not significantly different. In a separate series of experiments the rate of tension development $\left(\mathrm{k}_{\mathrm{TR}}\right)$ was estimated using a shortening, re-stretch protocol. A significantly lower rate was observed in the woody breast group (Table 1), since the serieselastic components are stretched during the force redevelopment, the data are consistent with a higher compliance associated with minor changes in cross-bridge kinetics in the woody breast group.

Contractile protein content was determined using quantitative SDS-PAGE (Figure 4) with Coomassie staining and different amounts loaded on each gel. We did not perform a detailed analysis of the protein composition, but no obvious differences in band appearance were observed on the gels between woody and normal breast samples, as shown in Figure 4. Using an albumin standard, we determined myosin heavy chain (Mw. $\sim 220 \mathrm{kD})$ and actin (Mw. $\sim 42 \mathrm{kD})$ contents, and related the contents to skinned fiber wet weight. No significant differences in myosin or actin were found between normal and woody breast samples (Table 1), although myosin content was slightly higher

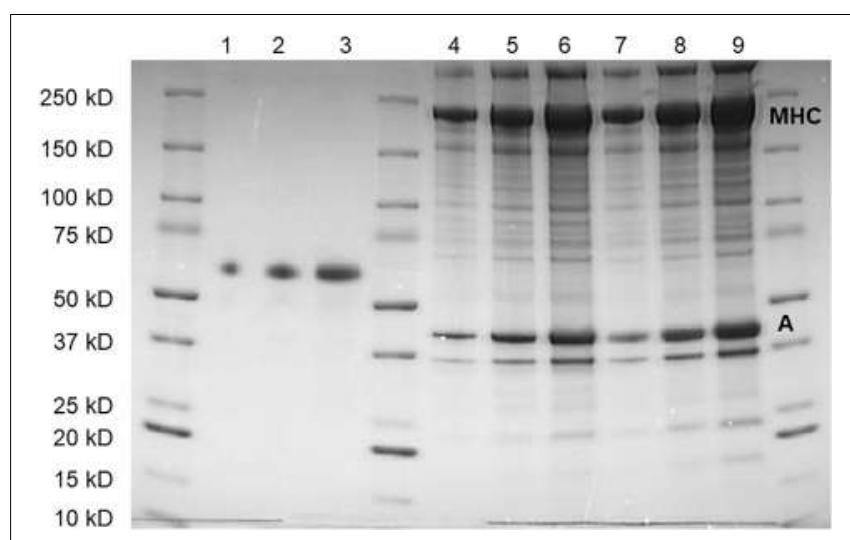

FIGURE 4 | SDS-poly acrylamide gel separation of protein extracts from normal (Lanes 4-6) and woody breast (Lanes 7-9) together with albumin standard (Lanes 1-3). Three different volumes of samples and amount of albumin were loaded on each gel. Myosin heavy chain (MHC) and actin ( $\mathrm{A})$ were identified on the basis on their apparent molecular weights. and the ratio of myosin to actin was significantly higher in the woody breast group.

Since fiber width was significantly increased in the woody breast group, we explored the possibility that also the volume of the contractile unit (sarcomere volume) was changed. We applied small angle $\mathrm{x}$-ray scattering (SAXS) using synchrotron light. In principle, the regular lateral arrangement between contractile filaments results in interference patterns reflecting the filament distances. A wider spacing result in a smaller angle of the scattering. The equatorial reflections, the outer 1.1 and the inner 1.0, were recorded from both groups (Figures 5A,B) with a wider spacing in the woody breast group (i.e., inward movement of the reflections). Panel $\mathrm{C}$ shows a decrease in the lattice spacing with stretch of sarcomeres in the normal breast group, reflecting constant volume behavior. With increased sarcomere length, lattice spacing also declined in the woody breast samples but was generally wider. As seen also in Figures $2 \mathbf{A}, \mathbf{C}$, the woody breast samples could be extended to higher sarcomere lengths. Assuming a constant volume behavior the sarcomere volume could be calculated from data in the sarcomere range 2.2$3.0 \mu \mathrm{m}$, as shown in Figure 5C. The estimated sarcomere volume was about $40 \%$ bigger in the woody breast group compared to controls, as shown in Figure 5D. The 1.1/1.0 intensity ratio, reflecting mass transfer between filaments, determined in the relaxed fibers at optimal stretch was low and not different between the two groups (normal breast $0.12 \pm 0.02$; woody breast $0.13 \pm 0.03, n=4$ and 3 ). These data show that the muscles were fully relaxed and that major differences in the cross-bridge positioning in relaxed fibers can be excluded.

The force determination at different $\left[\mathrm{Ca}^{2+}\right]$ determined at optimal stretch showed a significantly lower $\mathrm{Ca}^{2+}$ sensitivity in the woody breast group. Curve fit to the hyperbolic equation revealed no difference in the $\mathrm{h}$ parameters (normal breast: $3.1 \pm 0.3$; woody breast: $3.5 \pm 0.2, n=6$ and 6 ) but significantly higher $\mathrm{EC}_{50}$ value (Table 1) reflecting the decreased $\mathrm{Ca}^{2+}$ sensitivity.

\section{DISCUSSION}

Woody breast muscle exhibits a pronounced myopathy with a loss of muscle fibers, which would significantly affect the contractile function of the breast muscle in the affected animals. The morphological changes have been reported previously (Sihvo et al., 2014, 2016, 2018; Velleman and Clark, 2015) and also our histological analysis shows that the affected animals were identified to have a clear muscle disease. To obtain information on early stages in the disease development prior to severe cellular degeneration, the present study focused on the structurally intact fibers from the woody breast muscle and reported alterations in sarcomeric structure and compliance.

The histological analysis of woody breast muscle revealed signs of degeneration and regeneration of muscle cells, including fiber splitting and fragmentation, increased number of rounded cellular cross-sections accompanied by fibrosis, which are consistent with previous observations (Sihvo et al., 2014, 2016, 2018; Velleman and Clark, 2015; Papah et al., 2017). 

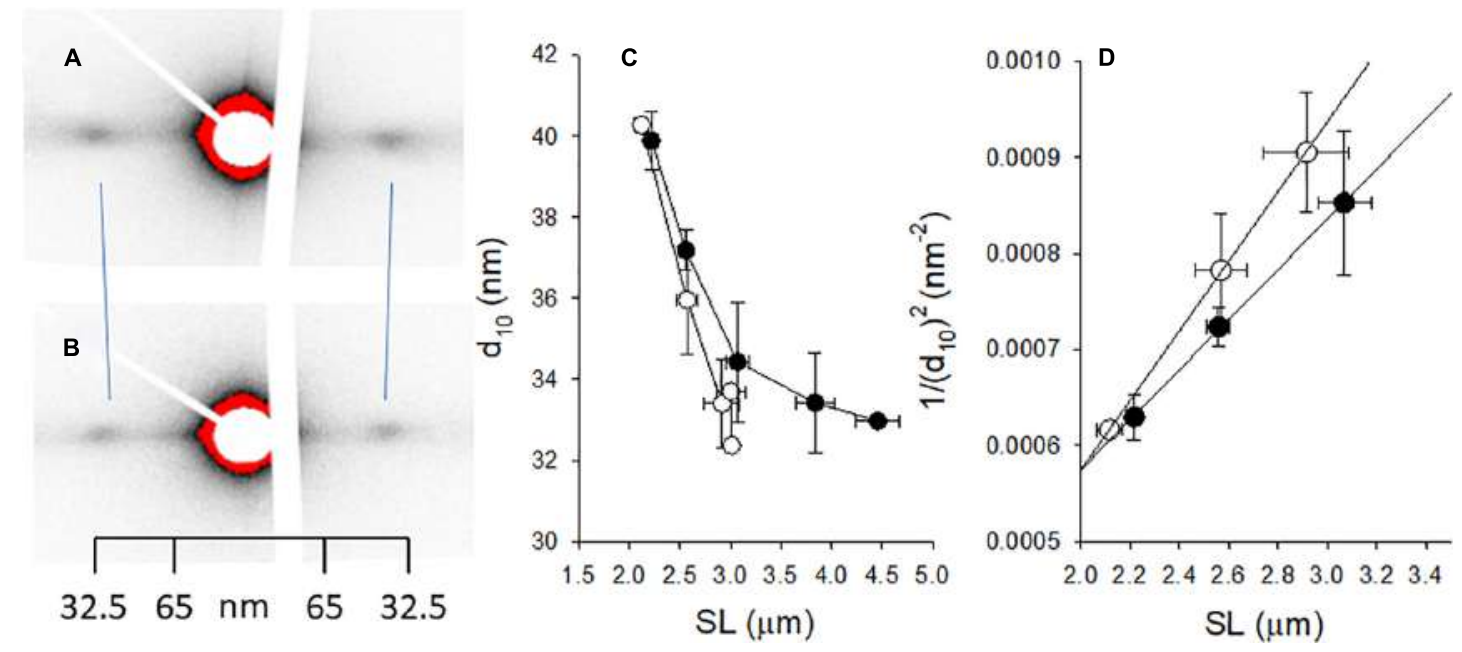

FIGURE $\mathbf{5}$ | Small angle $\mathrm{x}$-ray scattering of muscle fibers from normal control [(A) open symbols in (C,D) and woody breast] [(B) filled symbols in (C,D)]. (A,B) Show original recordings of $\mathrm{X}$-ray patterns where the 1.0 equatorial reflections are indicated. Note inward movement of reflections in the woody breast group, showing wider lattice spacing. Calibration from collagen pattern is indicated. (C) Depicts the spacing of the 10 reflection $\left(d_{10}\right)$ and in (D) the $1 /\left(d_{10}\right)^{2}$ is plotted against the sarcomere length (SL), with straight lines fitted to the data (the slope is inversely related to lattice volume, ref: Millman, 1998). The estimated lattice volumes were $3.18 \times 10^{-3}$ and $4.42 \times 10^{-3} \mu \mathrm{m}^{3}$ in normal and woody breast groups, respectively. $n=3-4$.

Velleman et al. (2017) showed altered sarcomere organization in woody breast and Papah et al. (2017) observed irregular, disconnected and altered Z-disks by electron microscopy in woody breast affected muscle. Most likely these structural changes reflect an end stage of the myopathy which would be a consequence of earlier events in the disease process. In view of the tight links between the contractile system, the cytoskeleton and the membrane attachment sites, which are all involved in the maintenance of cellular function, it is possible that early changes in these structures can be involved in the induction of hypertrophy. The changes in sarcomeric structure, observed in the present study, could affect these cellular mechanical contacts. It might cause altered responses to external mechanical perturbations and body movement, and eventually initiate the dystrophy process via membrane injury and/or activation of cellular signaling for growth and degeneration.

In order to understand the development of the woody breast condition at a cellular level, it is worthwhile to analyze morphologically intact fibers rather than degenerated ones. Therefore, we set up criteria for fibers selected for analysis from woody breast samples, including only fibers with homogenous width along the fiber axis and clear sarcomere patterns. These fibers had significantly increased diameter at defined sarcomere lengths that are optimal for active force, which suggests that cellular hypertrophy is an important component in the woody breast condition, which is in agreement with previous reports on samples fixed for histology (Clark and Velleman, 2016; Dalle Zotte et al., 2017). It should be noted that our analysis is based on single fibers, whereas the behavior of the whole muscle will also be influenced by the loss of fibers and development of fibrosis in the later stage of the condition.

To know whether the increase in muscle cell size involved the addition of contractile components, we determined the contents of actin and myosin in woody breast muscle fibers. The amount of myosin and actin per milligram of muscle (i.e., concentration) were the same between woody breast and normal breast muscles. This shows that a net synthesis of sarcomeric proteins occurs during the hypertrophy in the woody breast, thereby maintaining the myosin and actin concentrations. To examine if the increase in contractile proteins was associated with an increase in parallel-coupled functional myofibrils, we determined active force development at optimal sarcomere length. The active stress (i.e., force/cross-sectional area) observed in our study of normal, control breast muscle (pectoralis major) is similar to values previous reported in the literature (Reiser et al., 1992, 1996). In woody breast fibers, the active stress was maintained, or even slightly increased, which shows that the increase in cell size is associated with a compensatory increase in the contractile components. It also shows that a loss of contractile function, as has been observed in some other forms of hypertrophic myopathy (e.g., myostatin deficiency, Amthor et al., 2007), is not a primary component in the degenerative process in woody breast disease.

Growth and re-arrangement of the sarcomeric units can affect the contractile performance in different ways. Our measurements of optimal sarcomere length and active stress suggest that the length of the sarcomere and the number of parallel-coupled actinmyosin cross-bridges per cross-section are maintained in the woody breast muscle fibers. Active force and contractile function can also be affected by the kinetics of the actin-myosin turn-over; a faster rate can lower active force, by decreasing the time a crossbridge spends in force bearing states which would be associated with high ATPase and higher maximal shortening velocity (e.g., Rome et al., 1999). Myosin heavy chain (MHC) and myosin light chain (MLC) isoforms greatly influence the maximal shortening velocity in avian muscle (Lowey et al., 1993). The adult chicken 
pectoralis major muscle comprises mainly fast-twitch type IIB fibers with a fast myosin heavy chain type, with the addition of some slow and red-strip fibers located deeper in the tissue (Reiser et al., 1988, 1996; Remignon et al., 1995). We sampled from the breast muscle surface region in the 32-day-old broilers and report a maximal shortening velocity of about 1.6 muscle length $/ \mathrm{s}$ $\left(\mathrm{ML} \mathrm{s}^{-1}\right)$ at $22^{\circ} \mathrm{C}$. These $\mathrm{V}_{\max }$ values for the normal chicken pectoralis major muscle are lower than the values for unloaded shortening of fast white muscle fibers previously reported by Reiser et al. (1996). The difference between the reports might reflect differences between techniques (isotonic quick release experiments vs. slack test) or possibly in the age or strain of chicken studied (ROS 308 vs. White Leghorn/New Hampshire). Importantly, no difference could be noted in our measurements between normal and woody breast muscles, suggesting that the contractile kinetics and the series coupling of sarcomeres are preserved. The rate of tension development was slower as predicted by the more compliant series elasticity located in the fiber or within the sarcomere, although the data do not exclude a slightly decreased rate of tension development. Modern selection for rapid growth of broiler is often accompanied by a shift to fast twitch fiber type that has bigger fiber width. However, our results of $\mathrm{V}_{\max }$ and $\mathrm{k}_{\mathrm{TR}}$ did not prove that woody breast muscle fibers are faster. Therefore, the observed increase in cross-sectional area in woody breast muscle cannot be explained by a shifting of fiber types to the faster ones.

Although the optimal sarcomere length and active contraction were similar in the normal and woody breast muscle, we observed significant changes in the structure and compliance of sarcomeres in the latter group. In the normal muscle, sarcomeres could not be extended in the relaxed state beyond about $3.2 \mu \mathrm{m}$ when the fibers were stretched, suggesting the presence of structures limiting extreme sarcomere extension. In contrast, the woody breast fiber sarcomeres were significantly more extensible and could be extended almost linearly with stretch up to around $4.3 \mu \mathrm{m}$. This suggests that a factor limiting sarcomere extension is lacking or less prominent in the woody breast muscle fibers. The loss or conformational changes in cytoskeletal proteins acting as connecting components along sarcomere axis can be one possible reason for the compliance, but no obvious changes were seen in the present SDS-PAGE result. Previous protein analysis of woody breast muscle has not identified changes in myofibrillar proteins (Soglia et al., 2015). Therefore, to explain the altered sarcomere structure and compliance, a more detailed study on the changes within structural components and signaling factors is needed in the future.

The present study revealed enlarged sarcomeric volume in woody breast fibers compared to the controls by about $39 \%$, which could influence the distance between adjacent thick and thin filaments at each defined sarcomere length. Muscle and sarcomeres act as constant volume systems, where elongation in sarcomere length results in a decreased crosssectional area. Compression of lattice spacing reduces active force production but increases force during stretch of active muscles in frog fibers (Edman, 1999). In mammalian muscle, lattice compression has relatively fewer effects (Godt and Maughan, 1981; Zhao and Kawai, 1993; Liu et al., 2018), and only larger compression reduces active force (Maughan and Godt, 1981; Zhao and Kawai, 1993). It is thus unlikely that the difference in lattice spacing between normal and woody breast muscle fibers affected the active force generation at each stretch step. Nevertheless, the lattice spacing can influence the passive stress and extensibility of sarcomeres upon longer fiber length. Resistance might develop when adjacent filament surfaces touch each other during fiber elongation. As shown in Figure 5C, the minimum lattice spacing value $\left(\mathrm{d}_{10}\right)$ is $\sim$ $33 \mathrm{~nm}$ in both woody and normal muscle fibers. This might reflect the minimal distance between filaments in the sarcomere where the myosin heads can be accommodated. The current study showed wider lattice spacing in woody breast sarcomeres, which could thus allow more extension before the minimal lattice spacing was reached. We compressed woody breast fibers laterally by osmotic force to the diameter of normal fibers and found that the length coupling between fiber and sarcomere was similar to that in the controls, reflecting a role of wider spacing. Since normal fibers were less affected by osmotic compression, the presented results demonstrated that the effect of lateral distance on sarcomeric compliance is operating in swollen sarcomeres, as in the woody breast muscle. We observed decreased calcium sensitivity in the woody breast group and a change in the lattice spacing might be a factor contributing to this phenomenon (Godt and Maughan, 1981). Avian muscles operate mainly in the sarcomere length range covering the ascending arm of the length-force relationship (Burkholder and Lieber, 2001), which did not differ between woody and normal breast muscle according to our measurements. However, a less steep descending arm was observed in the woody breast group where significantly higher force was observed at longer sarcomere length. This could be a consequence of the wider filament spacing in the woody breast group as discussed above (Williams et al., 2013).

Modern genetic selection for fast growth and high breast yield broilers is achieved by selecting animals with more profound muscle hypertrophy (Dransfield and Sosnicki, 1999), with average larger fiber cross-sectional areas (Berri et al., 2007) and/or increased fiber number (Scheuermann et al., 2004). In our study woody and normal breast samples were taken from a flock of the same hybrid and were thus genetically very homogeneous. This suggests that a genetic predisposition plays a role in the susceptibility to develop the syndrome and some environmental factors then trigger the onset of the syndrome in some animals. It has been shown that animals that grow even faster with more pronounced fiber enlargement are more prone to develop woody breast condition (Papah et al., 2017), which suggests that food intake or animal size are important triggering factors. It has been suggested that the woody breast condition is a consequence of hypoxia, increased oxidative stress and intracellular calcium alterations, which might be due to insufficient blood supply or impaired metabolism in the fastgrowing animals (Mutryn et al., 2015; Petracci et al., 2019). It is difficult to ascertain whether these changes are primary or only consequence of the abnormal hypertrophic growth. The present study demonstrates the changes in the mechanical anchoring of contractile filaments in the woody breast muscles. This could 
affect the contractile properties and possibly activating signaling pathways associated with hypertrophic growth and ultimate muscle degeneration.

\section{DATA AVAILABILITY STATEMENT}

All data used in this study are available from the corresponding author upon reasonable request.

\section{ETHICS STATEMENT}

The study was fully based on slaughterhouse material obtained from the normal food production slaughterhouse line, after the animals were slaughtered. The study does not involve any study of live animals. No interventions or examinations were done prior to the slaughter. The slaughterhouse providing the material (in Denmark) is operating according to the local legislation and animals are bread in normal food production.

\section{REFERENCES}

Amthor, H., Macharia, R., Navarrete, R., Schuelke, M., Brown, S. C., Otto, A., et al. (2007). Lack of myostatin results in excessive muscle growth but impaired force generation. Proc. Natl. Acad. Sci. U.S.A. 104, 1835-1840. doi: 10.1073/pnas. 0604893104

AVEC (2011). Associationof Poultry Processors and Poultry Trade in the EU Countries-Annual Report 2011. Available online at: http://www.avec-poultry. eu/wp-content/uploads/2018/04/2011.pdf

Baldi, G., Soglia, F., Mazzoni, M., Sirri, F., and Petracci, M. (2017). Implications of white striping and spaghetti meat abnormalities on meat quality and histological features in broilers. Animal 12, 164-173. doi: 10.1017/ S1751731117001069

Berri, C., Le Bihan-Duval, E., Debut, M. S., Sante-Lhoutellier, V., Baeza, E., Gigaud, V., et al. (2007). Consequence of muscle hypertrophy on characteristics of Pectoralis major muscle and breast meat quality of broiler chickens. J. Anim. Sci. 85, 2005-2011. doi: 10.2527/jas.2006-398

Brenner, B., and Eisenberg, E. (1986). Rate of force generation in muscle: correlation with actomyosin ATPase activity in solution. Proc. Natl. Acad. Sci. U.S.A. 83, 3542-3546. doi: 10.2307/27542

Buffet, A., Rothkirch, A., Döhrmann, R., Körstgens, V., Abul Kashem, M. M., Perlich, J., et al. (2012). P03, the microfocus and nanofocus x-ray scattering (minaxs) beamline of the petra III storage ring: the microfocus endstation. J. Synchrotron. Radiat. 19(Pt 4), 647-653. doi: 10.1107/S09090495120 16895

Burkholder, T. J., and Lieber, R. L. (2001). Sarcomere length operating range of vertebrate muscles during movement. J. Exp. Biol. 204, 1529-1536.

Chen, H., Wang, H., Qi, J., Wang, M., Xu, X., and Zhou, G. (2018). Chicken breast quality-normal, pale, soft and exudative (PSE) and woody-influences the functional properties of meat batters. Int. J. Food Sci. Tech. 53, 654-664. doi: $10.1111 /$ ijfs. 13640

Clark, D. L., and Velleman, S. G. (2016). Spatial influence on breast muscle morphological structure, myofiber size, and gene expression associated with the wooden breast myopathy in broilers. Poult. Sci. 95, 2930-2945. doi: 10.3382/ps/ pew243

Dalle Zotte, A., Tasoniero, G., Puolanne, E., Hervé Rémignon, Cecchinato, M. Catelli, E., et al. (2017). Effect of "Wooden Breast" appearance on poultry meat quality, histological traits, and lesions characterization. Czech J. Anim. Sci. 62, 51-57. doi: 10.17221/54/2016-CJAS

Dransfield, E., and Sosnicki, A. A. (1999). Relationship between muscle growth and poultry meat quality. Poult. Sci. 78, 743-746. doi: 10.1093/ps/78.5.743

\section{AUTHOR CONTRIBUTIONS}

JL and AA designed the study, performed most of the experiments, and conducted data analysis. JL, EP, and AA did sampling in slaughterhouse. JL, AA, and MS accomplished x-ray scattering experiment. JL and AA wrote the manuscript. All the authors participated during result discussion and manuscript revision before the submission.

\section{ACKNOWLEDGMENTS}

Preparation of histological sections was performed and analyzed the KI core facility for Morphological Phenotype Analysis. We are grateful for the Igelösa Life Science AB, Lund for providing laboratory space and scientific environment. Parts of this research were carried out at the light source PETRA III at DESY, a member of the Helmholtz Association (HGF). The study was supported by a grant from Alice and Hans-Gabriel and Trolle-Wachtmeisters foundation (PI: AA 2017-2019).

Edman, K. A. P. (1999). The force bearing capacity of frog muscle fibers during stretch: its relation to sarcomere length and fiber width. J. Physiol. 519, 515-526. doi: 10.1111/j.1469-7793.1999.0515m.x

Fabiato, A. (1988). Computer programs for calculating total from specified free or free from specified total ionic concentrations in aqueous solutions containing multiple metals and ligands. Methods Enzymol. 157, 378-417. doi: 10.1016/ 0076-6879(88)57093-3

Godt, R. E., and Maughan, D. W. (1981). Influence of osmotic compression on calcium activation and tension in skinned muscle fibers of the rabbit. Pflügers. Arch. 391, 334-337. doi: 10.1007/bf00581519

Huxley, A. F. (1957). Muscle structure and theories of contraction. Prog. Biophys. Biophys. Chem. 7, 255-318. doi: 10.1016/s0096-4174(18)30128-8

Iorga, B., Wang, L., Stehle, R., Pfitzer, G., and Kawai, M. (2012). ATP binding and cross-bridge detachment steps during full $\mathrm{Ca} 2+$ activation: comparison of myofibril and muscle fiber mechanics by sinusoidal analysis. J. Physiol. 590, 3361-3373. doi: 10.2307/27542

Kuttappan, V. A., Brewer, V. B., Apple, J. K., Waldroup, P. W., and Owens, C. M. (2012). Influence of growth rate on the occurrence of white striping in broiler breast fillets. Poult. Sci. 91, 2677-2685. doi: 10.3382/ps.2012-02259

Lange, S., Xiang, F., Yakovenko, A., Vihola, A., Hackman, P., Rostkova, E., et al. (2005). The kinase domain of titin controls muscle gene expression and protein turnover. Science 308, 1599-1603. doi: 10.1126/science.1110463

Liu, J., Schwartzkopf, M., and Arner, A. (2018). Rigor bonds cause reduced sarcomeric volume in skinned porcine skeletal muscle under PSE-like conditions. Meat Sci. 139, 91-96. doi: 10.1016/j.meatsci.2018.01.014

Lowey, S., Waller, G. S., and Trybus, K. M. (1993). Function of skeletal muscle myosin heavy and light chain isoforms by an in vitro motility assay. J. Biol. Chem. 268, 20414-20418.

Maughan, D. W., and Godt, R. E. (1981). Inhibition of force production in compressed skinned muscle fibers of the frog. Pflügers Arch. 390, 161-163. doi: 10.1007/BF00590200

Millman, B. M. (1998). The filament lattice of striated muscle. Physiol. Rev. 78, 359-391. doi: 10.1152/physrev.1998.78.2.359

Mudalal, S., Lorenzi, M., Soglia, F., Cavani, C., and Petracci, M. (2015). Implications of white striping and wooden breast abnormalities on quality traits of raw and marinated chicken meat. Animal 9, 728-734. doi: 10.1017/ s175173111400295x

Mutryn, M. F., Brannick, E. M., Fu, W., Lee, W. R., and Abasht, B. (2015). Characterization of a novel chicken muscle disorder through differential gene expression and pathway analysis using RNA-sequencing. BMC Genomics 16:399. doi: 10.1186/s12864-015-1623-0 
Norring, M., Valros, A., Valaja, J., Sihvo, H.-K., Immonen, K., and Puolanne, E. (2019). Wooden breast myopathy links with poorer gait in broiler chickens. Animal 13, 1690-1695. doi: 10.1017/S1751731118003270

Papah, M. B., Brannick, E. M., Schmidt, C. J., and Abasht, B. (2017). Evidence and role of phlebitis and lipid infiltration in the onset and pathogenesis of wooden breast disease in modern broiler chickens. Avian Pathol. 46, 623-643. doi: 10.1080/03079457.2017.1339346

Paulin, D., and Li, Z. (2004). Desmin: a major intermediate filament protein essential for the structural integrity and function of muscle. Exp. Cell. Res. 301, 1-7. doi: 10.1016/j.yexcr.2004.08.004

Petracci, M., Mudalal, S., Soglia, F., and Cavani, C. (2015). Meat quality in fast-growing broiler chickens. World's Poul. Sci. J. 71, 363-374. doi: 10.1017/ S0043933915000367

Petracci, M., Soglia, F., Madruga, M., Carvalho, L., Ida, E., and Estévez, M. (2019). Wooden-breast, white striping, and spaghetti meat: causes, consequences and consumer perception of emerging broiler meat abnormalities. Compr. Rev. Food. Sci. F 18, 565-583. doi: 10.1111/1541-4337.12431

Rahimov, F., and Kunkel, L. M. (2013). The cell biology of disease: cellular and molecular mechanisms underlying muscular dystrophy. J. Cell Biol. 201, 499-510. doi: 10.1083/jcb.201212142

Reiser, P. J., Greaser, M. L., and Moss, R. L. (1988). Myosin heavy chain composition of single cells from avian slow skeletal muscle is strongly correlated with velocity of shortening during development. Dev. Biol. 129, 400-407. doi: 10.1016/0012-1606(88)90387-9

Reiser, P. J., Greaser, M. L., and Moss, R. L. (1992). Developmental changes in troponin $\mathrm{T}$ isoform expression and tension production in chicken single skeletal muscle fibers. J. Physiol. 449, 573-588. doi: 10.1113/jphysiol.1992.sp019102

Reiser, P. J., Greaser, M. L., and Moss, R. L. (1996). Contractile properties and protein isoforms of single fibers from the chicken pectoralis red strip muscle. J. Physiol. 493, 553-562. doi: 10.1113/jphysiol.1996.sp021403

Remignon, H., Gardahaut, M. F., Marche, G., and Ricard, F. H. (1995). Selection for rapid growth increases the number and the size of muscle fibers without changing their typing in chickens. J. Muscle. Res. Cell. Motil. 16, 95-102. doi: $10.1007 / \mathrm{bf} 00122527$

Rome, L. C., Cook, C., Syme, D. A., Connaughton, M. A., Ashley-Ross, M., Klimov, A., et al. (1999). Trading force for speed: why superfast crossbridge kinetics leads to superlow forces. Proc. Natl. Acad. Sci. U.S.A. 96, 5826-5831. doi: 10.1073/pnas.96.10.5826

Scheuermann, G. N., Bilgili, S. F., Tuzun, S., and Mulvaney, D. R. (2004). Comparison of chicken genotypes: myofiber number in pectoralis muscle and myostatin ontogeny. Poult. Sci. 83, 1404-1412. doi: 10.1093/ps/83.8. 1404

Sihvo, H. K., Airas, N., Lindén, J., and Puolanne, E. (2018). Pectoral vessel density and early ultrastructural changes in broiler chicken wooden breast myopathy. J. Comp. Pathol. 161, 1-10. doi: 10.1016/j.jcpa.2018. 04.002

Sihvo, H. K., Immonen, K., and Puolanne, E. (2014). Myodegeneration with fibrosis and regeneration in the pectoralis major muscle of broilers. Vet. Pathol. 51, 619-623. doi: 10.1177/0300985813497488

Sihvo, H. K., Lindén, J., Airas, N., Immonen, K., Valaja, J., and Puolanne, E. (2016). Wooden breast myodegeneration of pectoralis major muscle over the growth period in broilers. Vet Pathol. 54, 119-128. doi: 10.1177/0300985816658099

Soglia, F., Mazzoni, M., Zappaterra, M., Di Nunzio, M., Babini, E., Bordini, M., et al. (2020). Distribution and expression of vimentin and desmin in broiler pectoralis major affected by the growth-related muscular abnormalities. Front. Physiol. 10:1581. doi: 10.3389/fphys.2019.01581

Soglia, F., Mudalal, S., Babini, E., Di Nunzio, M., Mazzoni, M., Sirri, F., et al. (2015). Histology, composition, and quality traits of chicken Pectoralis major muscle affected by wooden breast abnormality. Poult. Sci. 95, 651-659. doi: $10.3382 / \mathrm{ps} / \mathrm{pev} 353$

Trocino, A., Piccirillo, A., Birolo, M., Radaelli, G., Bertotto, D., Filiou, E., et al. (2015). Effect of genotype, gender and feed restriction on growth, meat quality and the occurrence of white striping and wooden breast in broiler chickens. Poult. Sci. 94, 2996-3004. doi: 10.3382/ps/pev296

Velleman, S. G., and Clark, D. L. (2015). Histopathologic and myogenic gene expression changes associated with wooden breast in broiler breast muscles. Avian Dis. 59, 410-418. doi: 10.1637/11097-042015-Reg.1

Velleman, S. G., Clark, D. L., and Tonniges, J. R. (2017). The effect of the wooden breast myopathy on sarcomere structure and organization. Avian Dis. 62, 28-35. doi: 10.1637/11766-110217-Reg.1

Williams, C. D., Salcedo, M. K., Irving, T. C., Regnier, M., and Daniel, T. L. (2013). The length-tension curve in muscle depends on lattice spacing. Proc. Biol. Sci. 280:20130697. doi: 10.1098/rspb.2013.0697

Zhao, Y., and Kawai, M. (1993). The effect of the lattice spacing change on crossbridge kinetics in chemically skinned rabbit psoas muscle fibers. II. Elementary steps affected by the spacing change. Biophys. J. 64, 197-210. doi: 10.1016/ S0006-3495(93)81357-2

Conflict of Interest: The authors declare that the research was conducted in the absence of any commercial or financial relationships that could be construed as a potential conflict of interest.

Copyright (c) 2020 Liu, Puolanne, Schwartzkopf and Arner. This is an open-access article distributed under the terms of the Creative Commons Attribution License (CC BY). The use, distribution or reproduction in other forums is permitted, provided the original author(s) and the copyright owner(s) are credited and that the original publication in this journal is cited, in accordance with accepted academic practice. No use, distribution or reproduction is permitted which does not comply with these terms. 
OPEN ACCESS

Edited by:

Yajun Wang,

Sichuan University, China

Reviewed by:

Francesca Soglia,

University of Bologna, Italy

Xiquan Zhang,

South China Agricultural University,

China

${ }^{*}$ Correspondence: Sandra G. Velleman

Velleman.1@osu.edu

Specialty section:

This article was submitted to

Avian Physiology,

a section of the journal

Frontiers in Physiology

Received: 31 December 2019

Accepted: 16 April 2020

Published: 07 May 2020

Citation:

Velleman SG (2020) Pectoralis

Major (Breast) Muscle Extracellular Matrix Fibrillar Collagen Modifications Associated With the Wooden Breast

Fibrotic Myopathy in Broilers.

Front. Physiol. 11:461

doi: 10.3389/fphys.2020.00461

\section{Pectoralis Major (Breast) Muscle Extracellular Matrix Fibrillar Collagen Modifications Associated With the Wooden Breast Fibrotic Myopathy in Broilers}

\author{
Sandra G. Velleman* \\ Department of Animal Sciences, The Ohio State University, Wooster, OH, United States
}

The poultry industry has placed significant emphasis on the selection of meat-type broilers for increased body weight, increased meat yield especially the pectoralis major (breast) muscle, decreased time to processing, and improved feed conversion. Although significant improvements have occurred in fast-growing meat-type broilers, myopathies affecting meat quality especially in the pectoralis major muscle have occurred. Many of the broiler breast muscle myopathies are caused by inflammation leading to the necrosis of existing muscle fibers and resulting in replacement of the muscle fibers with extracellular matrix proteins especially fibrillar collagens, fibrosis. This review explores how the fibrotic deposition and organization of extracellular matrix proteins especially the fibrillar collagens, Types I and III, affects the phenotype of the Wooden Breast myopathy, functional properties of the pectoralis major muscle, and meat quality.

Keywords: collagen, fibrosis, muscle, necrosis, wooden breast

\section{INTRODUCTION}

Broilers have been selected for increased growth, carcass weight, pectoralis major (breast) muscle yield, decreased time to reach processing weight, and improved feed conversion (Havenstein et al., 1994a,b, 2003; Collins et al., 2014). Despite the overall growth improvements and increased feed efficiency, the presence of novel necrotic/fibrotic myopathies has been identified. These myopathies negatively impact breast meat quality and affect the well-being and overall function of the pectoralis major muscle. Typical named necrotic/fibrotic myopathies include, but are not limited to, Wooden Breast (WB: Sihvo et al., 2014), White Stripping (WS: Kuttappan et al., 2013), and Spaghetti Meat (SM: Baldi et al., 2018, 2019). All three of these myopathies affect the visual appearance of the pectoralis major muscle with increased collagen deposition, and altered fat and protein levels. Wooden Breast and SM have the most detrimental effects on breast meat quality with WB affected meat being hard whereas SM meat is soft after cooking. In modern heavy weight fastgrowing broilers, the pectoralis major muscle is eight times larger than broilers marketed in 1955 (Collins et al., 2014). Selection favoring greater pectoralis major muscle growth is due to consumer preference for a low-fat protein choice that is reasonable in cost. The increase in muscle mass is correlated with larger myofiber diameters from hypertrophic growth (Dransfield and Sosnicki, 1999), and a decrease in connective tissue spacing between muscle fiber bundles (perimysium) and 
individual myofibers (endomysium) (Yost et al., 2002; Velleman et al., 2003). The presence of ample connective tissue spacing is necessary for structure and function of the muscle and vascularization. Since the pectoralis major is an anaerobic muscle, the removal of lactic acid the by-product of anaerobic respiration requires vascular supply.

The fibrotic process of the replacement of muscle fibers with connective tissue is induced by chronic muscle fiber damage, necrosis, with associated tissue inflammation. In normal muscle, the damage will be repaired without changes in the myofiber structure or fibrosis. However, fibrosis results in muscles with chronic necrosis leading to the excessive deposition of fibrillar collagen as is observed with myopathies like WB. It is not the concentration of fibrillar collagen synthesized during fibrosis, but the organization of the fibrillar collagen will determine the tissue flexibility and the tenderness of the meat. In recent studies, it has been shown that there may be multiple necrotic/fibrotic myopathies associated with the broiler pectoralis major muscle (Velleman, 2015, 2019; Velleman and Clark, 2015; Clark and Velleman, 2017; Velleman et al., 2017). Many of these myopathies, largely go phenotypically undetected by palpation due to differences in the organization of fibrillar collagens. This review focuses on the organization of fibrotic fibrillar collagen deposited in the extracellular connective tissue spaces in the broiler pectoralis major muscle in 3 heavy weight fast-growing commercial broiler with differing incidence of phenotypically detectable WB.

\section{What Is the Extracellular Matrix and Why It Is a Key Element Determining Muscle Function and Meat Quality}

The extracellular matrix is defined as all the secreted molecules extrinsic to the cell composed of collagens, proteoglycans, and non-collagenous glycoproteins. The composition and structure of the extracellular matrix is not random as it is tissue-type and age specific. The matrix is thus dynamically expressed and directly impacts muscle cell proliferation, adhesion, migration, and the repair of damaged muscle fibers. In skeletal muscle there are three layers of connective tissue containing extracellular matrix macromolecules. They are the endomysium, perimysium, and epimysium. The epimysium forms a sheath around the entire muscle, the perimysium encompasses muscle fiber bundles, and the endomysium surrounds individual muscle fibers. The predominant extracellular matrix proteins in these layers are the fibrillar collagens especially Types I and III.

The fibrillar collagens are characterized by a single triplehelical domain containing three peptide chains forming an alpha helix. After the triple helical structure is formed, the collagen is secreted into the extracellular matrix space where it is aligned into a quarter stagger array leading to the formation of collagen fibrils that are stabilized by crosslinking between the collagens. Crosslinking of the fibrillar collagens is necessary for both its structural stability and functional properties and is a major determinant of meat textural properties. The covalent hydroxylsylpyridinoline (HP) crosslink is a mature, nonreducible, trivalent crosslink that forms from the condensation of two divalent ketoimine crosslinks (Reiser et al., 1992). The formation of HP crosslinks is progressive with age and the toughening of meat is directly attributable to crosslink concentration. With tissue injury, the repair process results in collagen fibrils that have higher levels of HP crosslinking (Zimmerman et al., 2001). Since collagen HP crosslinking is progressive with age and increases with tissue injury, fibrotic myopathies will likely result in increased collagen deposition and crosslinking like what is observed in the WB myopathy. Despite the likely increase in collagen crosslinking in severely affected WB muscle, Baldi et al. (2019) showed that hydroxylysylpyridinoline concentration, the principle non-reducible crosslink in fibrillar collagen is not affected in the Ross 308 strain. The muscle necrotic and fibrotic process is characterized by both changes in tissue structure and composition of the extracellular matrix. In fibrotic tissue, there is an excessive deposition of fibrillar collagen (Alexakis et al., 2007; Serrano and Muňoz-Cánoves, 2010). Thus, necrotic and fibrotic conditions in skeletal muscle will result in altered structural architecture and function with reduced elasticity. Furthermore, there is a general replacement of skeletal muscle fibers with connective tissue. Meat derived from pectoralis major muscles with necrotic and fibrotic disorders like WB will have reduced myofibrillar protein content, reduced water holding capacity, increased fat, and be tough and texturally unappealing (Mazzoni et al., 2015).

\section{Overview of Skeletal Muscle Development and Regeneration}

The predisposition to the development of degenerative breast muscle myopathies has its origins in the formation of muscle and its continued growth as well as how selection for fastgrowing heavy weights lines has altered the development and growth of the pectoralis major muscle including the extracellular matrix environment. The development and growth of skeletal muscle is a precisely regulated process with specific phases. Embryonic myoblasts are derived from the somites and once they migrate to the areas of muscle formation will further proliferate, align to form multinucleated myotubes, and ultimately muscle fibers. After the myoblasts form multinucleated myotubes they withdraw from the cell cycle. During the formation of skeletal muscle, the muscle fibers will form bundles with the bundles being separated by perimysial connective tissue spacing and the individual muscle fibers by endomysial connective tissue. At the time of hatch, muscle fiber formation is complete (Smith, 1963).

The continued posthatch growth is from the enlargement or hypertrophy of existing muscle fibers. Hypertrophy is solely dependent upon a mesodermally derived stem cells population of adult myoblasts, satellite cells. With myofiber injury, the satellite cells are responsible for the repair and regeneration of the myofiber back to its original state.

Having enough perimysial and endomysial connective tissue spacing is necessary for the livability of the avian pectoralis major muscle myofibers (Wilson et al., 1990). The perimysium is a connective layer composed of groups of collagen fibrils tightly packed that surround the entire muscle fiber bundle. In comparison, the endomysium contains a thin layer of 
collagen encompassing only individual myofibers. In addition to providing space between the muscle fiber bundles and myofibers, the intramuscular areas of connective tissue spacing provide structural support for the tissue, defines the elasticity or stretch of the muscle, and contains capillaries necessary for adult myoblast activity, satellite cells, and the removal of respiration by-products like lactic acid. Selection for increased breast muscling based on myofiber hypertrophy and not muscle fiber number through hyperplasia will result in myofibers and muscle fiber bundles that occupy the endomysial and perimysial spaces, respectively. As the connective spaces are diminished in size and the fibers and fiber bundles begin to touch, fiber degeneration ensues (Wilson et al., 1990; Velleman et al., 2003). Once the muscle fibers are damaged, satellite cell-mediated repair mechanisms are invoked. When myofiber degeneration occurs the sarcolemma (myofiber plasma membrane) is disrupted which initiates necrosis from the influx of calcium from the sarcoplasmic reticulum. The necrosis of the muscle fibers leads to an immune response (Orimo et al., 1991) with the infiltration of immune cells including neutrophils and macrophages to phagocytize the cellular debris. In necrotic/fibrotic disorders like WB the pectoralis major muscle tissue has observable lysis of existing muscle fibers with immune cell infiltration.

The degeneration or necrosis of the muscle fibers will initiate satellite cell repair mechanisms. Satellite cells are, in general, quiescent and must be activated to reenter the cell cycle to proliferate and differentiate. Satellite cells require the appropriate niche environment for activation. For satellite cell activity to occur, the muscle stem cell niche must contain vascularization within $21 \mu \mathrm{m}$ of the satellite cells (Christov et al., 2007). However, in meat-type broilers affected with $\mathrm{WB}$ the muscle is under oxidative stress (Abasht et al., 2016). One of the characterizing features of $\mathrm{WB}$ muscle is a reduction in circulatory supply. The reduction in circulatory supply in the pectoralis major muscle is further augmented by the pectoralis major muscle being an anaerobic muscle. Anaerobic glycolytic metabolism does not require oxygen. Thus, the pectoralis major muscle being a fast twitch Type II muscle does not require by nature an extensive circulatory network. Further reduction in circulatory supply in WB affected muscle will suppress satellite cell-mediated myofiber regeneration. Regeneration is a process of constructing parallel arrays of microfibrils. Alterations in the reconstruction of the myofibrillar structure will negatively impact the contractile properties of the muscle (Velleman et al., 2018) and meat quality by reducing protein content. Velleman et al. (2018) showed that the WB condition resulted in a deposition of smaller diameter myofibrils with a lack of normal sarcomere structure ranging from moderate to severe (Figure 1). In contrast, during normal posthatch muscle growth, myofiber diameter should continue to increase with age through satellite cell-mediated hypertrophic growth (Moss and LeBlond, 1971) while maintaining normal sarcomere structure in the myofibrils.

The degeneration of muscle fibers results in inflammation of the pectoralis major muscle tissue leading to an increased deposition of extracellular matrix proteins like collagen and proteoglycans. The extracellular matrix proteins form an architectural network outside the cell which determines, in part,
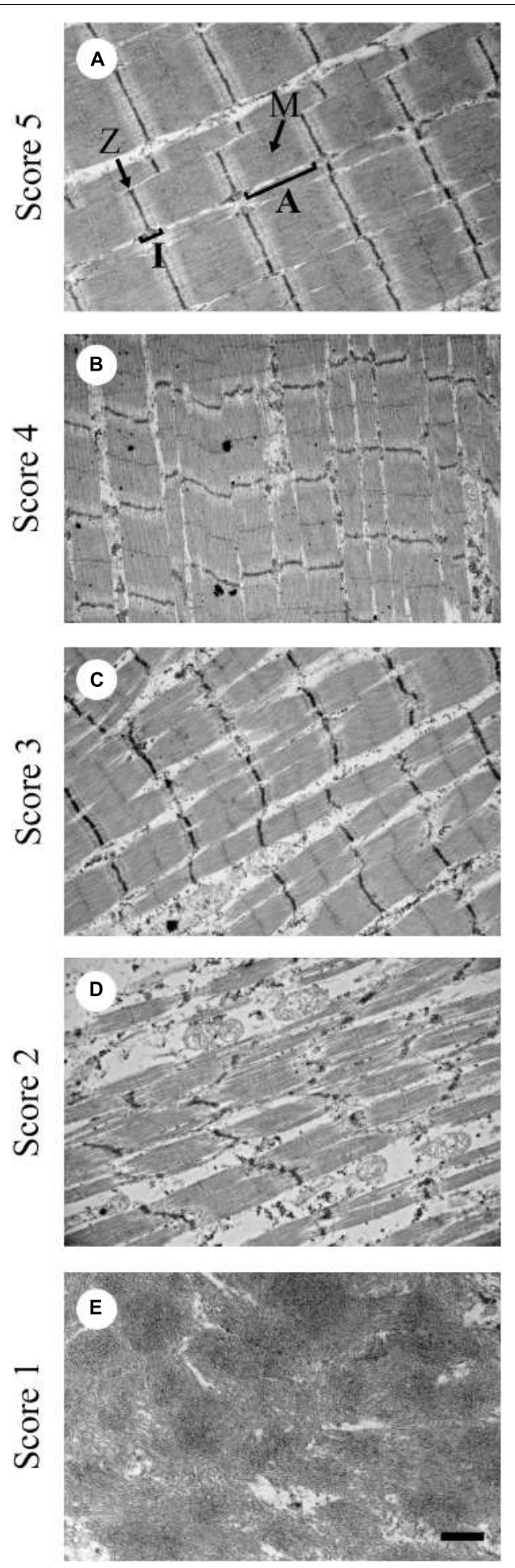

FIGURE 1 | Representative transmission electron microscopy images for sarcomere organization scoring from 1 to 5 . (A) Score of 5 shows sarcomeres with normal structure; (B) score of 4 means mildly altered; (C) score of 3 means further disorganized from a score of 4 but still moderate; (D) score of 2 means severely altered sarcomere structure; and $(\mathbf{E})$ score of 1 means complete absence of sarcomere structure. $\mathrm{A}=\mathrm{A}$ zone; $\mathrm{I}$ = I zone; $\mathrm{M}=\mathrm{M}$-line; $Z=Z$-line. Bar $=1 \mu \mathrm{m}$. (Figure reproduced from Velleman et al., 2018. Avian Dis. 62, 28-35). 
the structural stability of the tissue, stretch, and water-holding capacity. Measures of extracellular matrix protein concentration alone will not determine the functional attributes of the tissue. For example, the primary phenotypic feature of WB is a hard pectoralis major muscle detected by palpation (Sihvo et al., 2014). As shown by Velleman and Clark (2015), the phenotypic incidence of WB does not align with microscopic assessment for necrosis and fibrosis with the incidence being significantly higher when evaluated microscopically.

\section{Formation and Organization of Intramuscular Collagen Fibrils: Relationship to the Phenotype of Wooden Breast}

The process of fibrosis is a self-perpetuating response to muscle necrosis resulting in the progressive overproduction of fibrillar collagens Types I and III in the perimysial and endomysial connective tissue spaces. The phenotypic characteristics of necrotic/fibrotic myopathies is not a direct relationship to the concentration of these collagens. There are numerous factors determining the tissue phenotype resulting from fibrosis. These include, but are not limited to fibril diameter, degree of crosslinking, proteoglycan localization and type, fibril alignment, and morphometric organization of the collagen fibrils.

All collagens are composed of three polypeptide chains with the amino acid repeat Glycine-X-Y where $\mathrm{X}$ and $\mathrm{Y}$ are any amino acid but are frequently proline or lysine. The polypeptide chains will wrap around each other intracellularly to form a right-handed triple helix. At this point, the collagen molecule is exocytosed into the extracellular space where fibril and fiber formation take place. The collagen fiber is the functional form impacting tissue structure, elasticity, and ultimately meat quality. After secretion, the collagen molecules will align in parallel to
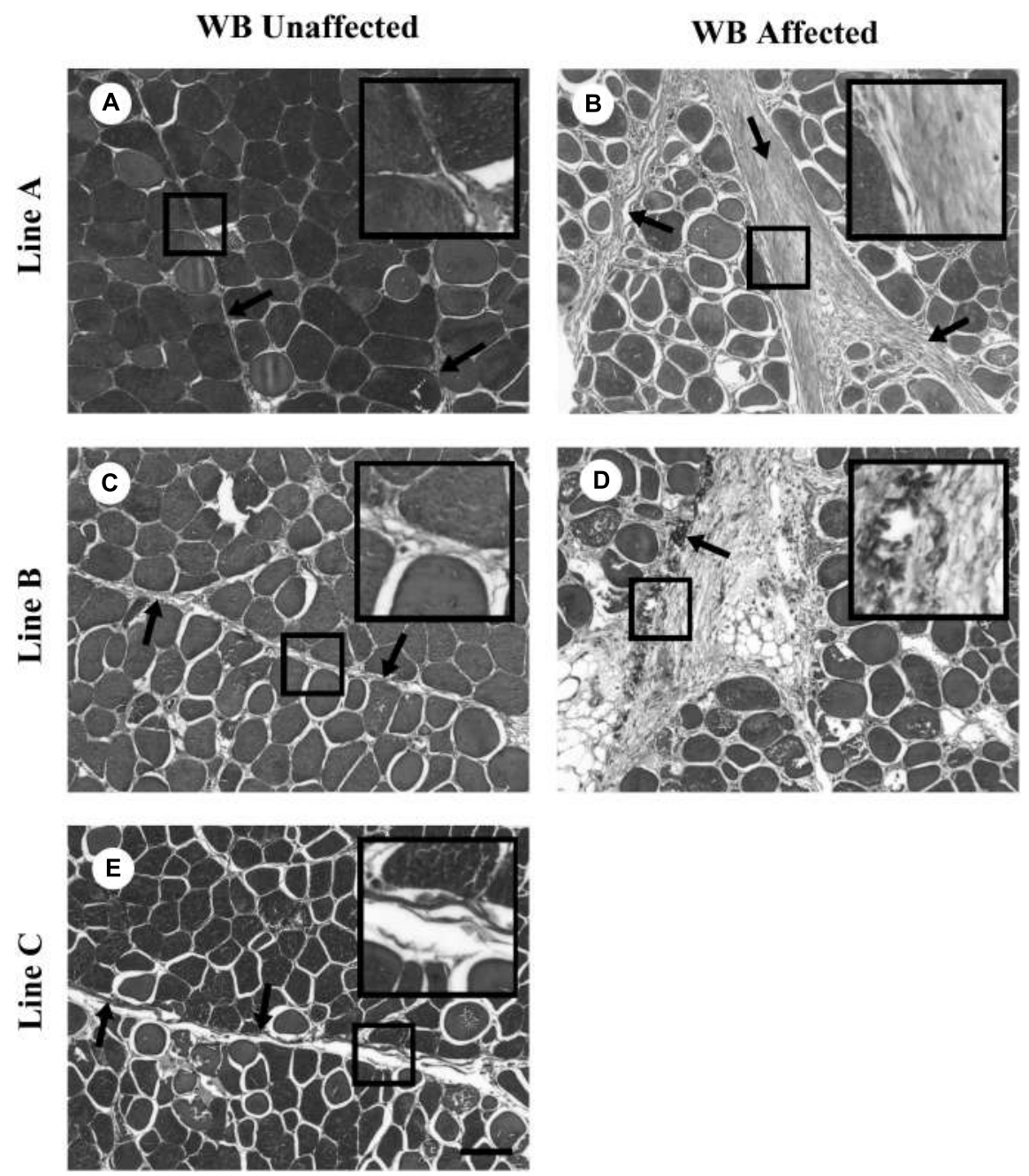

FIGURE 2 | Masson trichrome staining of collagen organization in wooden breast (WB)-unaffected and -affected pectoralis major muscle. (A,C,E) are representative images of WB-unaffected pectoralis major muscle from Lines A, B, and C, respectively. (B,D) are representative images of WB-affected pectoralis major muscle from Lines A and B, respectively. The arrows highlight fibrillar collagen and the boxes contain enlargements of the fibrillar collagen. Scale bar $=100 \mu \mathrm{m}$. (Figure reproduced from Velleman et al., 2017. Avian Dis. 61, 481-490). 
forming a quarter staggered array which is a necessary step leading to the assembly of collagen fibrils. The collagen fibrils are stabilized by the formation of reversible divalent crosslinks. The alignment of the collagen molecules is not a random process and after alignment there are gap and overlap areas within the quarter staggered array. The length of one overlap zone is $67 \mathrm{~nm}$ and this is termed a $\mathrm{D}$ banding-period. If the alignment of the collagen molecules is altered, the length of the D-period will be modified affecting collagen fibril function. After collagen fibril formation, the collagen fibrils will come together forming collagen fibers. With maturation, divalent ketoimine crosslinks are replaced with trivalent non-reversible HP crosslinks. The HP crosslink is a critical factor in tissue stiffening. After three collagen triple helices are linked together, additional helices are linked together increasing collagen fibril diameter and crosslinking. The formation of HP crosslinks is a progressive process with age and is likely a major factor in the phenotypic detection of WB by palpation and the reduction in meat quality.

Since the WB myopathy is characterized by excessive collagen fibril deposition, it is of importance to understand the ultrastructural intramuscular organization of the fibrillar collagens in fast growing meat-type broiler lines of differing parental lineage. To comprehensively study collagen fibril structure, a series of studies was conducted examining collagen fibril structure in three fast growing commercial broiler lines with distinctly different levels of phenotypically detectable WB (Velleman and Clark, 2015; Velleman et al., 2017; Tonniges et al., 2019). These lines will be referred to as A, B, and C. Line $\mathrm{C}$ does not exhibit any WB whereas Line $\mathrm{A}$ has a high degree of affected birds, and Line B has an intermediate level of phenotypic detection of WB affected birds. Velleman and Clark (2015) and Velleman et al. (2017) using light microscopy observed that Line A with a high degree of phenotypically WB affected birds, had perimysial collagen fibers characterized by parallel packing (Figure 2). In contrast, Line B by palpation had a low percentage of birds categorized as WB affected. Light microscopic examination revealed that the perimysial collagen was not packed but diffuse in structure. Furthermore, histological examination of the pectoralis major muscle showed that $70 \%$ of the Line B birds had microscopically observable necrosis and fibrosis. Therefore, phenotypic palpation for breast muscle hardness, a standard approach used by the broiler industry, is an insufficient measure for pectoralis major muscle fibrosis.

Transmission electron microscopy (TEM) analysis was used to further investigate histological differences, measure collagen D-periodicity, and collagen fiber diameter in Lines A, B, and $\mathrm{C}$ affected and unaffected WB birds (Velleman et al., 2017). Velleman et al. (2017) showed that WB affected Line A muscle had overall smaller average collagen fibril diameter and longer average collagen D-period compared to unaffected Line A muscle. Line B showed no such differences. Fibrotic collagen of WB-affected muscle of Line A exhibited D-period length and fibril diameter more like endomysial collagen of unaffected muscle of Line A. However, the fibrillar structure of fibrotic collagen in Line B affected with WB was similar to perimysial collagen of Line B-unaffected muscle. The endomysial collagen of Line A was changed with the WB myopathy while the endomysial collagen of Line B was not. Endomysial collagen of Line A exhibited decreased fibril diameter and increased D-period length with WB. Changes in D-period length and collagen fibril diameter are a direct consequence of the arrangement of collagen within the collagen fibril. Modifications of the collagen fibril are suggestive of changes in the molecular packing of collagen monomers, which will affect collagen function by altering protein binding sites or the flexibility of the collagen fibril.

More importantly the TEM study by Velleman et al. (2017) demonstrated that there are different fibrotic collagen organizations in the pectoralis major muscle of fast-growing meat type broilers. These differences in fibrosis are likely unique myopathies. Myopathies like WB have increased stiffness or hardness of the pectoralis major muscle likely due to high levels of collagen crosslinking which results in tightly packed collagen fibrils. Fibrotic myopathies undetected by palpation is due to the collagen fibrils being diffuse with lower levels of crosslinking, and the pectoralis major muscle remains texturally soft and in extreme case SM may be found. Despite not being phenotypically
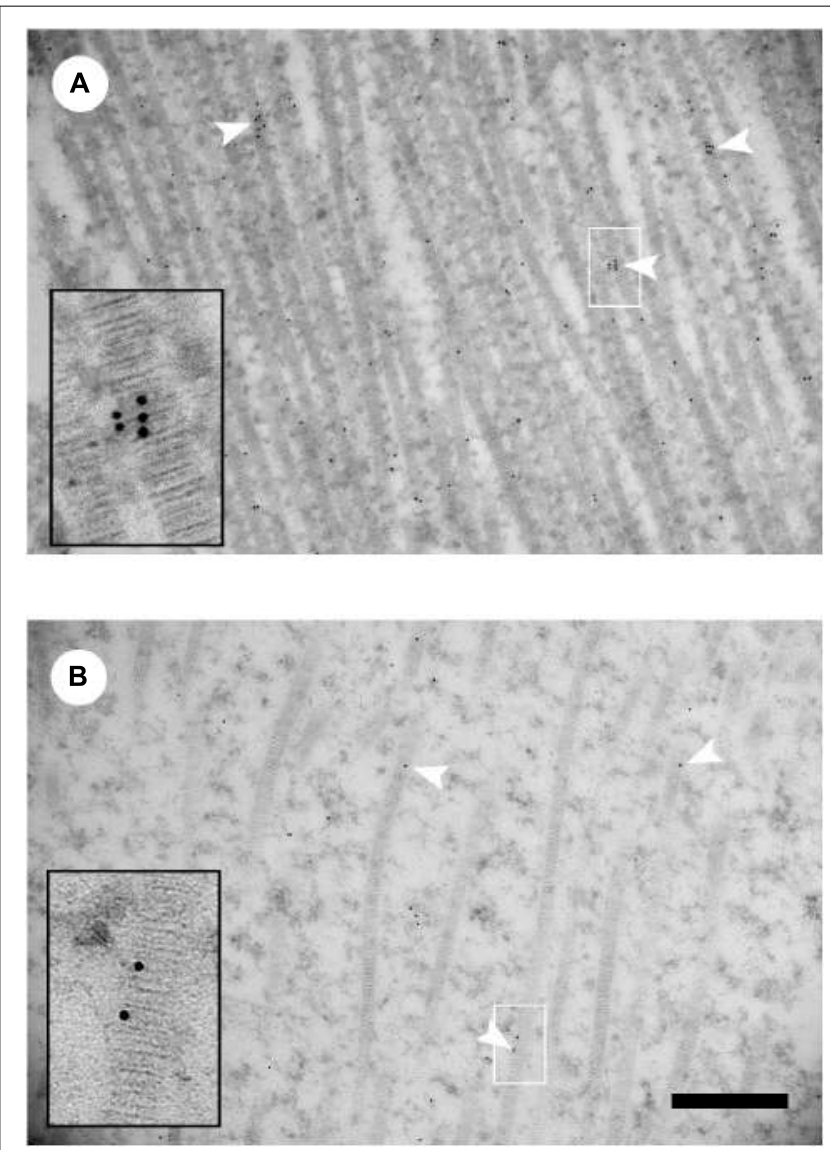

FIGURE 3 | Decorin immunogold transmission electron microscopy images of collagen fibrils from wooden breast-affected muscle of Line A (A) and Line B (B). White arrowheads indicate gold particles labeling collagen fibrils. Insets show enlarged images of gold labeling individual collagen fibrils in the region indicated by the white box. Scale bar $=500 \mathrm{~nm}$. (Figure reproduced from Tonniges et al. (2019). Avian Dis. 63, 48-60). 
detectable, necrosis and fibrosis will still alter the nutritional value of the breast meat by reducing protein levels and increasing fat concentration.

Since the organization of collagen fibrils is altered by the fibrotic process, it is necessary to determine mechanistically how this organizational change occurs. A primary candidate for altering collagen fibrillar structure is the chondroitin and dermatan sulfate proteoglycan decorin. Decorin plays a critical role in the formation of the quarter stagger array aligning the collagen helices (Weber et al., 1996) and subsequent HP crosslinking (Reiser et al., 1992) by binding to the $\mathrm{D}$ banding period. Extracellular matrix organization has been implicated in other fibrotic conditions including but not limited to lung fibrosis (Blaauboer et al., 2014) and human renal fibrosis disease (Stokes et al., 2000). Velleman and Clark (2015) reported a high correlation of decorin expression in WB affected muscle in Line $\mathrm{A}$ but not in Line B.

Tonniges et al. (2019) investigated the association of decorin binding and collagen organization in Lines A, B, and C using TEM and immunogold labeling decorin. The WB myopathy caused larger diameter collagen fibril bundles. However, Line A with the high phenotypic detection of WB had larger collagen fibril bundles compared to WB affected pectoralis major muscles in Line B. Line A had collagen fibril bundles upto $8.4 \mu \mathrm{m}$ and Line B had a maximum diameter of $5.1 \mu \mathrm{m}$. It was further found that Line A WB affected muscle had smaller diameter collagen fibril bundles with more decorin collagen binding than Line B. Figure 3 shows a representative TEM decorin immunogold image for Lines $A$ and $B$. In addition to the observable differences in decorin-collagen binding, the images also illustrate the distinct differences in collagen organization.

To summarize this review provides novel insight into the fibrillar organization of collagen in the broiler pectoralis major muscle and its impact on the phenotypic detection of $\mathrm{WB}$ affected birds. Despite Tonniges et al. (2019) reporting that smaller diameter collagen fibril bundles in WB affected muscle having more decorin-collagen binding, it is unlikely for decorin to play a major role in the alignment and organization fibrotic fibrillar WB collagen. In contrast decorin knockout studies in mice demonstrated irregular collagen fiber organization was

\section{REFERENCES}

Abasht, B., Mutryn, M. F., Michalek, R. D., and Lee, W. R. (2016). Oxidative stress and metabolic perturbations in wooden breast disorder in chickens. PLoS One 11:e0153750. doi: 10.1371/journal.pone.015 3750

Alexakis, C., Partridge, T., and Bou-Gharios, G. (2007). Implication of the satellite cell in dystrophic muscle fibrosis: a self-perpetuating mechanism of collagen overproduction. Am. J. Physiol. 293, C661-C669. doi: 10.1152/ajpcell.00061. 2007

Asgari, M., Latifi, N., Heris, H. K., Vali, H., and Mongeau, L. (2017). In vitro fibrillogenesis of tropocollagen type III in collagen type I affects its relative fibrillar topology and mechanics. Sci. Rep. 7:1392. doi: 10.1038/s41598-01701476-y directly associated with fragility of tissues like the skin tearing with force (Danielson et al., 1997). The presence of multiple fibrotic myopathies in the broiler pectoralis major muscle is likely associated with the expression and organization of Types I and III collagen.

Skeletal muscle contains fibrillar collagens Types I and III. The expression of collagen is age dependent. During early development, Type III collagen is the predominant collagen but with increased age there is a shift toward Type I collagen (Bornstein and Sage, 1980; Light and Champion, 1984; Kovanen and Suominen, 1989). With injury and inflammation as occurs in myopathies like WB, the expression of collagen will shift back to more of an embryonic-like tissue with increased levels of Type III collagen. As the tissue is repaired Type I collagen expression will then increase and Type III collagen will decrease (Bailey et al., 1975; Barnes et al., 1976; Weber et al., 1978; Merkel et al., 1988). Collagen fibrils can be homotypic composed of only a single collagen type or heterotypic containing a mixture of collagen types. Although the functional impact of collagen fibril composition is not well understood, it has been postulated that collagen fibril composition can influence muscle stiffness and meat toughness (McCormick, 1994). Studies investigating differential collagen fibril composition and its impacts has not been reported to date for broiler skeletal muscle. In other tissue systems, heterotypic collagen fibrils containing both Types I and III collagen have reduced stiffness and collagen fibril diameter (Romanic et al., 1991; Notbohm et al., 1993; Asgari et al., 2017). Interestingly, when collagen Type III expression is increased as observed with skeletal muscle injury (Hurme et al., 1991; Gibertini et al., 2014), Type III collagen is localized in highly aligned and tightly packed collagen fibrils (Brisson et al., 2015; McConnell et al., 2016) as observed in the phenotypically detectable WB. Thus, collagen Type III expression may be associated with the fibrotic organization of collagen fibrils. Future studies will need to address collagen fibril composition and its association with broiler pectoralis major muscle fibrosis.

\section{AUTHOR CONTRIBUTIONS}

The author confirms being the sole contributor of this work and has approved it for publication.

Bailey, A., Bazin, S., Sims, T., Le Lous, M., Nicoletis, C., and Delaunay, A. (1975). Characterization of the collagen of human hypertrophic and normal scars. Biochim. Biophys. Acta 405, 412-421. doi: 10.1016/0005-2795(75)90106-3

Baldi, G., Soglia, F., Laghi, L., Tappi, S., Rocculi, P., Tavaniello, S., et al. (2019). Comparison of quality traits among breast meat by current muscle abnormalities. Food Res. Internatl. 115, 369-376. doi: 10.1016/j.foodres.2018. 11.020

Baldi, G., Soglia, F., Mazzoni, M., Sirri, F., Canonico, L., Babini, E., et al. (2018). Implications of white striping and spaghetti meat abnormalities on meat quality and histological features in broilers. Animal 12, 164-173. doi: 10.1017/ S1751731117001069

Barnes, M., Morton, L., Bennett, R., Bailey, A., and Sims, T. (1976). Presence of type III collagen in guinea-pig dermal scar. Biochem. J. 157, 263-266. doi: $10.1042 /$ bj 1570263 
Blaauboer, M. E., Boeijen, F. R., Emson, C. L., Turner, S. M., Zandieh-Doulabi, B., Hanemaaijer, R., et al. (2014). Extracellular matrix proteins: a positive feedback loop in lung fibrosis? Matrix Biol. 34, 170-178. doi: 10.1016/j.matbio.2013. 11.002

Bornstein, P., and Sage, H. (1980). Structurally distinct collagen types. Annu. Rev. Biochem. 49, 957-1003.

Brisson, B. K., Mauldin, E. A., Lei, W., Vogel, L. K., Power, A., Lo, D., et al. (2015) Type III collagen directs stromal organization and limits metastasis in a murine model of breast cancer. Am. J. Pathol. 185, 1471-1486. doi: 10.1016/j.ajpath. 2015.01.029

Christov, C., Chrétien, F., Abou-Khalil, R., Bassez, G., Vallet, G., Authier, F.-J., et al. (2007). Muscle satellite cells and endothelial cells: close neighbors and privileged partners. Mol. Biol. Cell 18, 1397-1409. doi: 10.1091/mbc.e06-080693

Clark, D. L., and Velleman, S. G. (2017). Spatial influence on breast muscle morphological structure, myofiber size, and gene expression associated with the wooden breast myopathy in broilers. Poult. Sci. 95, 2930-2945. doi: 10.3382/ps/ pew243

Collins, K. E., Kiepper, B. H., Ritz, C. W., McLendon, B. L., and Wilson, J. L. (2014). Growth, livability, feed consumption, and carcass composition of the Athens Canadian random bred 1955 meat-type chicken versus the 2012 high-yielding Cobb 500 broiler. Poult. Sci. 93, 2953-2962. doi: 10.3382/ps.2014-04224

Danielson, K. G., Baribault, H., Holmes, D. F., Graham, H., Kadler, K. V., and Iozzo, R. V. (1997). Targeted disruption of decorin leads to abnormal collagen fibril morphology and skin fragility. J. Cell Biol. 136, 729-743. doi: 10.1083/jcb.136. 3.729

Dransfield, E., and Sosnicki, A. A. (1999). Relationship between muscle growth and poultry meat quality. Poult. Sci. 78, 743-746. doi: 10.1093/ps/78.5.743

Gibertini, S. G., Zanotti, S., Savadori, P., Curcio, M., Saredi, S., Saerno, F., et al. (2014). Fibrosis and inflammation are greater in muscles of beta-sarcoglycannull mouse than mdx mouse. Cell Tissue Res. 356, 427-443. doi: 10.1007/ s00441-014-1854-4

Havenstein, G. B., Ferket, P. R., and Qureshi, M. A. (2003). Carcass composition and yield of 1957 versus 2001 broilers when fed representative 1957 and 2001 broiler diets. Poult. Sci. 82, 1509-1518. doi: 10.1093/ps/82.10.1509

Havenstein, G. B., Ferket, P. R., Scheideler, S. E., and Larson, B. T. (1994a). Growth, livability, and feed conversion of 1957 vs. 1991 broilers when fed "typical" 1957 and 1991 broiler diets. Poult. Sci. 73, 1785-1794. doi: 10.3382/ps.0731785

Havenstein, G. B., Ferket, P. R., Schedidler, S. E., and Rives, D. V. (1994b). Carcass composition and yield of 1991 vs. 1957 broilers when fed "typical" 1957 and 1991 broiler diets. Poult. Sci. 73, 1795-1804. doi: 10.3382/ps.0731795

Hurme, T., Kalimo, H., Sandberg, M., Lehto, M., and Vuorio, E. (1991). Localization of type I and III collagen and fibronectin production in injured gastrocnemius muscle. Lab. Investig. 64, 76-81.

Kovanen, V., and Suominen, H. (1989). Age- and training-related changes in collagen metabolism of rat skeletal muscle. Eur. J. Appl. Physiol. 58, 765-771. doi: 10.1007/bf00637389

Kuttappan, V. A., Shivaprasad, H. I, Shaw, B. A., Valentine, B. A., Hargis, B. M., Clark, F. D., et al. (2013). Pathological changes associated with white striping in broiler breast muscles. Poult. Sci. 92, 331-338. doi: 10.3382/ps.2012-02646

Light, N., and Champion, A. E. (1984). Characterization of muscle epimysium, perimysium, and endomysium collagens. Biochem. J. 219, 1017-1026. doi: 10.1042/bj2191017

Mazzoni, M., Petracci, M., Meluzzi, A., Cavani, C., Clavenzani, P., and Sirri, F. (2015). Relationship between pectoralis major muscle histology and quality traits of chicken meat. Poult. Sci. 94, 123-130. doi: 10.3382/ps/peu043

McConnell, J. C., O’Connell, O. V., Brennan, K., Weiping, L., Howe, M., Joseph, L., et al. (2016). Increased peri-ductal collagen micro-organization may contribute to raised mammographic density. Breast Cancer Res. 18:5. doi: 10.1186/s13058015-0664-218

McCormick, R. J. (1994). The flexibility of the collagen compartment of muscle. Meat Sci. 36, 79-91. doi: 10.1016/0309-1740(94)90 035-3

Merkel, J., DiPaolo, B., Hallock, G., and Rice, D. (1988). Type I and type III collagen content of healing wounds in fetal and adult rats. Proc. Soc. Exp. Biol. Med. 187, 493-497. doi: 10.3181/00379727-187-42694

Moss, F. P., and LeBlond, C. P. (1971). Satellite cells are the source of nuclei in muscles of growing rats. Anat. Rec. 170, 421-435. doi: 10.1002/ar.1091700405

Notbohm, H., Mosler, S., Müller, P. K., and Brinckmann, J. (1993). In vitro formation and aggregation of heterotypic collagen I and III fibrils.
Int. J. Biol. Macromol. 15, 299-304. doi: 10.1016/0141-8130(93)90 030-p

Orimo, S., Hiyamuta, E., Arahata, K., and Sugita, H. (1991). Analysis of inflammatory cells and complement C3 in bupivacaine-induced myonecrosis. Muscle Nerve 14, 515-520. doi: 10.1002/mus.880140605

Reiser, J. M., McCormick, R. J., and Rucker, R. B. (1992). The enzymatic and non-enzymatic crosslinking of collagen and elastin. FASEB J. 6, 2439-2449.

Romanic, A. M., Adachi, E., Kadler, K. E., Hojima, Y., and Prockop, D. J. (1991). Copolymerization of pNcollagen III and collagen I. pNcollagen III decreases the rate of incorporation of collagen I into fibrils, the amount of collagen I incorporate, and the diameter of the fibrils formed. J. Biol. Chem. 266, 12703-12709.

Serrano, A. L., and Muňoz-Cánoves, P. (2010). Regulation and dysregulation of fibrosis in skeletal muscle. Exp. Cell Res. 316, 3050-3058. doi: 10.1016/j.yexcr. 2010.05.035

Sihvo, H.-K., Immonen, K., and Puolanne, E. (2014). Myodegeneration with fibrosis and regeneration in the pectoralis major muscle of broilers. Vet. Pathol. 51, 619-623. doi: 10.1177/0300985813497488

Smith, J. H. (1963). Relation to body size to muscle cell size and number in the chicken. Poult. Sci. 42, 619-623.

Stokes, M. B., Holler, S., Cui, Y., Hudkins, K. L., Eitner, F., Fogo, A., et al. (2000). Expression of decorin, biglycan, and collagen type I in human renal fibrosing disease. Kidney Int. 57, 487-498. doi: 10.1046/j.1523-1755.2000.00868.x

Tonniges, J. R., Clark, D. L., and Velleman, S. G. (2019). The effect of the wooden breast fibrotic myopathy in broilers on fibrillar collagen organization and decorin-collagen binding. Avian. Dis. 63, 48-60. doi: 10.1637/11985-102218Reg.1

Velleman, S. G. (2015). Relationship of skeletal muscle development and growth to breast muscle myopathies: a review. Avian. Dis. 59, 525-531. doi: 10.1637/ 11223-063015-Review.1

Velleman, S. G. (2019). Recent developments in breast muscle myopathies associated with growth in poultry. Ann. Rev. Anim. Biosci. 7, 289-308. doi: 10.1146/annurev-animal-020518-115311

Velleman, S. G., Anderson, J. W., Coy, C. S., and Nestor, K. E. (2003). Effect of selection for growth rate on muscle damage during turkey breast muscle development. Poult. Sci. 82, 1069-1074. doi: 10.1093/ps/82.7.1069

Velleman, S. G., and Clark, D. L. (2015). Histopathologic and myogenic gene expression changes associated with wooden breast in broiler breast muscles. Avian. Dis. 59, 410-418. doi: 10.1637/11097-042015-Reg.1

Velleman, S. G., Clark, D. L., and Tonniges, J. R. (2017). Fibrillar collagen organization associated with the broiler wooden breast fibrotic myopathy Avian. Dis. 61, 481-490. doi: 10.1637/11738-080217-Reg.1

Velleman, S. G., Clark, D. L., and Tonniges, J. R. (2018). The effect of the wooden breast myopathy on sarcomere structure and organization. Avian. Dis. 62, 28-35. doi: 10.1637/11766-110217-Reg.1

Weber, I. T., Harrison, R. W., and Iozzo, R. V. (1996). Model structure of decorin and implication for collagen fibrillogenesis. J. Biol. Chem. 271, 31767-31770.

Weber, L., Meigel, W., and Spier, W. (1978). Collagen polymorphism in pathologic human scars. Arch. Dermatol. Res. 261, 63-71.

Wilson, B. W., Nieberg, P. S., and Buhr, R. J. (1990). Turkey muscle growth and focal myopathy. Poult. Sci. 69, 1553-1562. doi: 10.3382/ps.0691553

Yost, J. K., Kenney, P. B., Slider, S. D., Russell, R. W., and Killefer, J. (2002). Influence of selection for breast muscle mass on myosin isoform composition and metabolism of deep pectoralis muscles of male and female turkeys. Poult. Sci. 81, 911-917. doi: 10.1093/ps/81.6.911

Zimmerman, S. D., Thomas, D. P., Velleman, S. G., Li, X., Hansen, T. R., and McCormick, R. J. (2001). Time course of collagen and decorin changes in rat cardiac and skeletal muscle post-MI. Am. J. Physiol. Heart Circ. Physiol. 281, H1816-H1822. doi: 10.1152/ajpheart.2001.281.4.H1816

Conflict of Interest: The author declares that the research was conducted in the absence of any commercial or financial relationships that could be construed as a potential conflict of interest.

Copyright (c) 2020 Velleman. This is an open-access article distributed under the terms of the Creative Commons Attribution License (CC BY). The use, distribution or reproduction in other forums is permitted, provided the original author(s) and the copyright owner(s) are credited and that the original publication in this journal is cited, in accordance with accepted academic practice. No use, distribution or reproduction is permitted which does not comply with these terms. 


\section{OPEN ACCESS}

Edited by:

Krystyna Pierzchala-Koziec, University of Agriculture in Krakow,

Poland

Reviewed by:

Vivek A. Kuttappan, Novus International, Brazil

Macdonald Wick

The Ohio State University,

United States

*Correspondence:

Massimiliano Petracci

m.petracci@unibo.it

David E. Gerrard

dgerrard@vt.edu

Specialty section:

This article was submitted to

Avian Physiology,

a section of the journal

Frontiers in Physiology

Received: 21 January 2020

Accepted: 26 March 2020

Published: 08 May 2020

Citation:

Baldi G, Yen C-N, Daughtry MR, Bodmer J, Bowker BC, Zhuang $H$, Petracci M and Gerrard DE (2020) Exploring the Factors Contributing to the High Ultimate $\mathrm{pH}$ of Broiler Pectoralis Major Muscles Affected by

Wooden Breast Condition

Front. Physiol. 11:343.

doi: 10.3389/fphys.2020.00343

\section{Exploring the Factors Contributing to the High Ultimate $\mathrm{pH}$ of Broiler Pectoralis Major Muscles Affected by Wooden Breast Condition}

\author{
Giulia Baldi', Con-Ning Yen², Morgan R. Daughtry², Jocelyn Bodmer², Brian C. Bowker ${ }^{3}$, \\ Hong Zhuang ${ }^{3}$, Massimiliano Petracci ${ }^{1 *}$ and David E. Gerrard ${ }^{2 *}$
}

${ }^{1}$ Department of Agricultural and Food Sciences, University of Bologna, Bologna, Italy, ${ }^{2}$ Department of Animal and Poultry Sciences, Virginia Polytechnic Institute and State University, Blacksburg, VA, United States, ${ }^{3}$ US National Poultry Research Center, Quality \& Safety Assessment Research Unit, Athens, GA, United States

The elevated ultimate $\mathrm{pH}\left(\mathrm{pH}_{u}\right)$ found in wooden breast (WB) meat suggests an altered muscular energetic status in WB but also could be related to a prematurely terminated post-mortem $\mathrm{pH}$ decline. The aims of this study were to explore the factors contributing to the elevated $\mathrm{pH}_{u}$ and establish whether the occurrence of WB defect alters muscle post-mortem carbohydrate metabolism and determine if the contractile apparatus reflects such changes. A total of 24 carcasses from Ross 308 male chickens were obtained from a commercial producer and harvested using commercial processing procedures. Carcasses were categorized into unaffected (NORM) and WB groups ( $n=12$ each), and samples were collected from cranial bone-in pectoralis major (PM) muscles at $15 \mathrm{~min}$ and $24 \mathrm{~h}$ post-mortem for the determination of $\mathrm{pH}$, glycolytic metabolites, adenonucleotides, buffering capacity, phosphofructokinase (PFK) activity, and in vitro $\mathrm{pH}$ decline. Twenty-four additional deboned PM samples (12 NORM and 12 WB) were collected from the same processing plant to assess muscle histology and sarcomere length at four different locations throughout the PM muscle. Data show that the reduced glycolytic potential of WB muscles only partially explains the higher $(P<0.001) \mathrm{pH}_{u}$ of WB meat, as residual glycogen along with unaltered PFK activity suggests that neither glycogen nor a deficiency of PFK is responsible for arresting glycolysis prematurely. The dramatic reduction in ATP concentrations in the early postmortem period suggests a defective ATP-generating pathway that might be responsible for the reduced $\mathrm{pH}$ decline in WB samples. Further, the addition of excess of ATPase extended post-mortem glycolysis of WB meat in an in vitro glycolytic system. WBaffected samples have longer $(P<0.001)$ sarcomeres compared to NORM, indicating the existence of compromised energy-generating pathways in myopathic muscles that may have had consequences on the muscle contraction and tension development, as in vivo, also during the post-mortem period. Considering the overall reduced 
glycolytic potential and the myodegenerative processes associated with WB condition, we speculate that the higher $\mathrm{pH}_{u}$ of $\mathrm{WB}$ meat might be the outcome of a drastically impaired energy-generating pathway combined with a deficiency and/or a dysfunction of muscle ATPases, having consequences also on muscle fiber contraction degree.

Keywords: wooden breast, post-mortem metabolism, pH, sarcomere length, glycolysis

\section{INTRODUCTION}

In an attempt to address the ever-growing demand for poultry meat, selection pressures on chicken genetics have resulted in huge gains in animal growth rates, feed conversion, and enhanced growth of the pectoralis major (PM) muscle chickens, which represents the most profitable portion in the broiler industry, at least in Western countries (Petracci et al., 2019). As a result, however, the incidence of metabolic and growthrelated disorders affecting PM muscles of broiler chickens has increased, suggesting genetic improvements have given rise to the occurrence of muscular abnormalities (Griffin et al., 2018; Velleman, 2019). Among these, wooden breast (WB) has been reported in several plants all over the world with an occurrence up to $50 \%$ of affected individuals within the same flock (Griffin et al., 2018; Livingston et al., 2018), resulting in costly economic deficits (Kuttappan et al., 2017b). This global widespread muscular abnormality appears as either a focally or diffusely palpably firm consistency in the breast muscle, which is undesirable, pale, out-bulging, and often displays superficial exudate and petechiae (Sihvo et al., 2014). Microscopically, WB-affected muscles exhibit an altered muscular architecture, showing a diffuse thickening of the endomysial and perimysial connective tissue associated with several degrees of fiber necrosis, inflammatory cell infiltrations, as well as extreme collagen and fat deposition (Sihvo et al., 2014; Soglia et al., 2016; Clark and Velleman, 2017). Moreover, selection for enhancing breast muscle mass has increased muscle fiber size through greater post-hatch hypertrophic growth (Clark and Velleman, 2017; Velleman, 2019), thus reducing capillary-tofiber ratio and resulting in severe circulatory insufficiency (i.e., hypoxia), oxidative stress mechanisms, as well as remarkable alterations in muscle metabolism (Sihvo et al., 2018). Indeed, metabolomic studies provide evidence of dysregulated lipid and carbohydrate metabolism in affected birds (Mutryn et al., 2015; Abasht et al., 2016; Papah et al., 2017) to such a degree that the elevated ultimate $\mathrm{pH}\left(\mathrm{pH}_{u}\right)$ of $\mathrm{WB}$ meat is usually considered a hallmark of this muscular abnormality. However, even though many aspects of WB abnormality have been investigated at the molecular level, to the best of our knowledge, there has not been any indication concerning postmortem metabolism of WB-affected muscles. Considering that glycogen is not usually a limiting factor in chicken PM muscle (Chauhan and England, 2018), the high $\mathrm{pH}_{u}$ of WB meat may be related to a prematurely terminated post-mortem $\mathrm{pH}$ decline. Thus, having information about the enzymes and metabolites involved in post-mortem glycolysis might be crucial to understand the reasons behind the altered energetic status of myopathic muscles and provide new insights into this condition.
Moreover, since the rate and the extent of post-mortem muscular acidification influence the degree of myofibrillar contraction (Ertbjerg and Puolanne, 2017), it could be speculated that the extreme stiffness of WB muscles might be partially due to a hypercontraction of sarcomeres caused by an abnormal acidification process or premature rigor formation. Within this context, the present study aimed at widening the knowledge about WB condition by exploring the factors contributing to the elevated $\mathrm{pH}_{u}$ and establishing whether its occurrence exerts an effect on muscle post-mortem glycolysis and muscle fiber contraction.

\section{MATERIALS AND METHODS}

\section{Muscle Sampling}

A total of 24 carcasses were obtained from the same flock of broiler chickens (Ross 308 strain, males, 48 days of age, 3.0$3.5 \mathrm{~kg}$ live weight), reared and harvested using standard US commercial procedures. Carcasses were selected immediately after evisceration ( $\sim 15 \mathrm{~min}$ post-mortem) from the line of a commercial broiler processing plant and categorized by experienced personnel in unaffected (NORM) and WB ( $n=12$ /group) based on the criteria proposed by Sihvo et al. (2014). More specifically, WB carcasses were selected based on palpable hardness and muscle rigidity throughout the whole PM muscle, picking the most severe cases. Carcasses were stored in cold storage room at $4^{\circ} \mathrm{C}$, and samples of about $1 \mathrm{~cm}^{3}$ were collected from the cranial position of bonein PM muscles of each carcass at both 15 and 1,440 min $(24 \mathrm{~h})$ post-mortem, snap frozen in liquid nitrogen, and stored at $-80^{\circ} \mathrm{C}$ until analyses. Samples were then used for the assessment of $\mathrm{pH}$, glycolytic metabolites, adenine nucleotides, buffering capacity, phosphofructokinase (PFK) activity, and in vitro $\mathrm{pH}$ decline. Further, 24 additional deboned PM muscles were collected from the deboning line of the same commercial broiler processing plant at $3 \mathrm{~h}$ post-mortem and categorized into NORM and WB ( $n=12$ /group) following the same criteria described before. Fillets were stored at $4^{\circ} \mathrm{C}$ until $24 \mathrm{~h}$ post-mortem (i.e., until rigor mortis resolution), and samples from four different locations throughout the PM muscle were obtained in order to evaluate the spatial effects of WB condition on muscle histology and sarcomere length. Specifically, samples were collected from anteroventral, anterodorsal, posteroventral, and posterodorsal regions of WBaffected PM muscles, while samples from NORM breast muscles were acquired from anteroventral section (Figure 1). Samples belonging to anterodorsal and posterodorsal positions were 


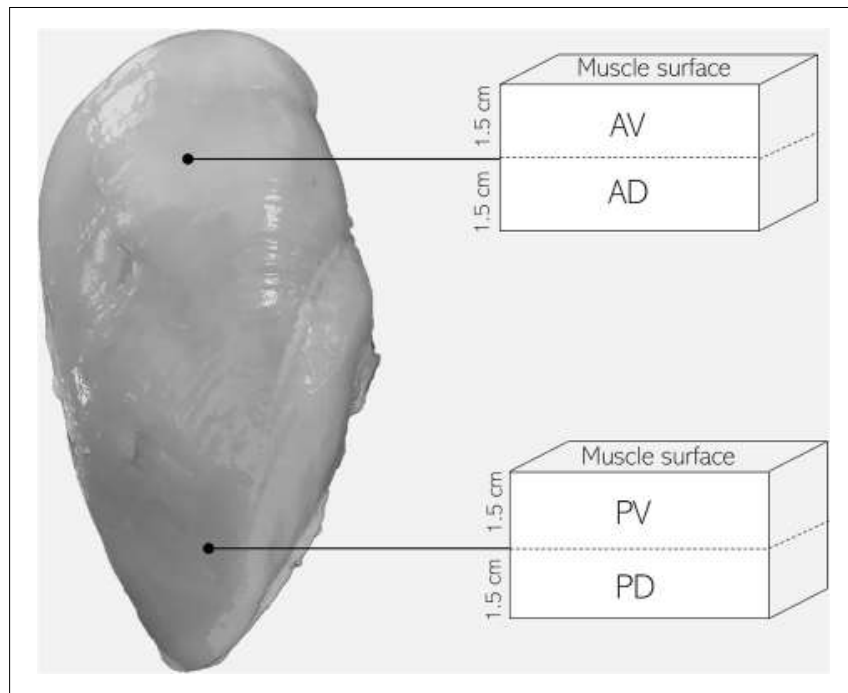

FIGURE 1 | Schematic diagram of pectoralis major muscle sampling location for the evaluation of muscle histology and sarcomere length ( $\mathrm{AV}$, anteroventral; AD, anterodorsal; PV, posteroventral; PD, posterodorsal).

collected by making an incision in the breast muscle in order to obtain samples located $1.5 \mathrm{~cm}$ underneath the anteroventral and posteroventral samples, respectively. All the aspects of rearing, handling, transportation, and harvest of birds were accomplished under US laws.

\section{pH and Glycolytic Metabolites}

Samples were processed as described by Matarneh et al. (2018) with slight modifications. Briefly, frozen 15- and 1,440 -min PM muscle samples ( $n=12$ /group) were powdered under liquid nitrogen using a mortar and pestle, and three aliquots of approximately $0.1 \mathrm{~g}$ were collected. Powdered samples were lysed using a Tissue Lyser II system (Qiagen, Boston, MA, United States) in $0.8 \mathrm{ml}$ of ice-cold $5 \mathrm{mM}$ sodium iodoacetate and $150 \mathrm{mM} \mathrm{KCl}$ solution $(\mathrm{pH}=7.0)$. Following centrifugation at $17,000 \times g$ for $5 \mathrm{~min}$ and equilibration to $25^{\circ} \mathrm{C}, \mathrm{pH}$ of supernatants was directly measured using an Orion Ross Ultra $\mathrm{pH}$ glass electrode (Thermo Scientific, Pittsburgh, PA, United States). Samples designated for glucose, glucose-6-phosphate (G6P), lactate, and adenine nucleotides (ATP, ADP, AMP, and IMP) were lysed in $1 \mathrm{ml}$ of ice-cold $0.5 \mathrm{M}$ perchloric acid and incubated on ice for $20 \mathrm{~min}$. Homogenates were centrifuged at $17,000 \times g$ for $5 \mathrm{~min}$, then supernatants were transferred into new tubes and neutralized with $2 \mathrm{M} \mathrm{KOH}$. As for muscle glycogen determination, another sample was lysed in $1 \mathrm{ml}$ of $1.25 \mathrm{M} \mathrm{HCl}$, heated at $90^{\circ} \mathrm{C}$ for $2 \mathrm{~h}$, and centrifuged at $17,000 \times g$ for $5 \mathrm{~min}$. Following, supernatants were transferred into new tubes and neutralized with $1.25 \mathrm{M}$ $\mathrm{KOH}$. Glycogen, glucose, G6P, and lactate were determined using enzymatic methods modified for a 96-well plate as described by Hammelman et al. (2003), and glycolytic potential (GP) was calculated as suggested by Scheffler et al. (2013) following the equation: GP $(\mu \mathrm{mol} / \mathrm{g})=2 *$ (glucose $+\mathrm{G} 6 \mathrm{P}+$ glycogen $)+$ lactate. Adenine nucleotide concentrations were quantified using HP Agilent 1100 HPLC system (Agilent Technologies, Santa Clara, CA) and external standards.

\section{In vitro Glycolysis Model}

Frozen 15-min NORM and WB samples ( $n=6 /$ group) were powdered in liquid nitrogen and homogenized at 1:10 (wt/vol) in a glycolysis buffer containing $5 \mathrm{mM} \mathrm{MgCl}_{2}, 10 \mathrm{mM} \mathrm{Na}_{2} \mathrm{HPO}_{4}$, $60 \mathrm{mM} \mathrm{KCl}, 5 \mathrm{mM} \mathrm{Na}$-ATP, $0.5 \mathrm{mM}$ ADP, $0.5 \mathrm{mM} \mathrm{NAD}^{+}$, $25 \mathrm{mM}$ carnosine, $30 \mathrm{mM}$ creatine, $40 \mathrm{mM}$ glycogen, and $10 \mathrm{mM}$ sodium acetate ( $\mathrm{pH}$ 7.4) (England et al., 2014). In order to test the effect of ATPase on muscle acidification, either 0 or $2 \mathrm{U} / \mathrm{ml}$ of ATPase were incorporated into the in vitro model. Reaction vessels were incubated at $25^{\circ} \mathrm{C}$ for the duration of the trial. Aliquots for $\mathrm{pH}$ determination were removed from reaction vessels at $0,15,30,120,240$, and $1,440 \mathrm{~min}$ and homogenized at $1: 4(\mathrm{vol} / \mathrm{vol})$ ratio with a $25 \mathrm{mM}$ sodium iodoacetate and $750 \mathrm{mM} \mathrm{KCl}$ solution $(\mathrm{pH}=7.0)$. Samples were centrifuged at $17,000 \times g$ for $5 \mathrm{~min}$, equilibrated to $25^{\circ} \mathrm{C}$, and $\mathrm{pH}$ was measured directly using an Orion Ross Ultra $\mathrm{pH}$ glass electrode (Thermo Scientific, Pittsburgh, PA, United States).

\section{Phosphofructokinase Activity Assay}

PFK activity of both NORM and WB PM muscles ( $n=6$ /group) was determined according to the procedures described by England et al. (2014). Briefly, $\sim 0.1 \mathrm{~g}$ of the 15-min samples was homogenized at 1:10 (wt/vol) in ice-cold $100 \mathrm{mM} \mathrm{K}_{2} \mathrm{HPO}_{4}$ solution ( $\mathrm{pH}=7.4)$. Subsequently, aliquots of tissue homogenate were added to a reaction buffer containing $120 \mathrm{mM}$ MES, $3.2 \mathrm{mM}$ $\mathrm{MgSO}_{4}, 2 \mathrm{mM}$ ATP, $1 \mathrm{mM}$ NADH, 3 mM fructose-6-phosphate, $2 \mathrm{U} / \mathrm{ml}$ triosephosphate isomerase, $1 \mathrm{U} / \mathrm{ml}$ glycerol-3-phosphate dehydrogenase, and $1 \mathrm{U} / \mathrm{ml}$ aldolase $(\mathrm{pH}=7.0)$. Enzymatic activity was measured spectrophotometrically at $340 \mathrm{~nm}$ and reported as $\mathrm{nmol} \mathrm{NADH} \times \min ^{-1} \times \mathrm{g}^{-1}$.

\section{Buffering Capacity}

Buffering capacity of NORM and WB samples ( $n=12$ /group) was determined according to Matarneh et al. (2015). Briefly, $\sim 1$ g of the 1,440 min meat was homogenized in $5 \mathrm{mM}$ sodium iodoacetate and $150 \mathrm{mM} \mathrm{KCl}$ solution $(\mathrm{pH}=7.0)$ at $1: 10$ ratio (wt/vol). After equilibration to $25^{\circ} \mathrm{C}$, samples were titrated using $0.1 \mathrm{M} \mathrm{NaOH}$. Samples $\mathrm{pH}$ was measured using an Orion Ross Ultra pH glass electrode (Thermo Scientific, Pittsburgh, PA, United States) and buffering capacity was calculated as follows: buffering capacity $=\Delta \mathrm{B} / \Delta \mathrm{pH}$, where $\Delta \mathrm{B}$ is the increment of base expressed as $\mu \mathrm{mol} \mathrm{NaOH} / \mathrm{g}$ of tissue, and $\Delta \mathrm{pH}$ is the corresponding $\mathrm{pH}$ variation.

\section{Histology}

Muscle samples of approximately $1 \mathrm{~cm}^{3}$ were removed from anteroventral, anterodorsal, posteroventral, and posterodorsal regions of $\mathrm{WB}$ de-boned chicken $\mathrm{PM}$ muscles $(n=12)$ at $24 \mathrm{~h}$ post-mortem. Meat samples were placed in 10\% (vol/vol) buffered formalin fixative $(\mathrm{pH}=7)$ and stored at 
$4^{\circ} \mathrm{C}$. Subsequently, samples were dehydrated in a graded series of ethanol, oriented for cross-sectional fiber sectioning, and paraffin-embedded. Paraffin blocks were cut at $6 \mu \mathrm{m}$ with a microtome, mounted on saline-coated microscope slides, and hematoxylin and eosin stained. In more detail, muscle sections were stained with hematoxylin for $6 \mathrm{~min}$, rinsed with running deionized water, submerged in eosin for $2 \mathrm{~s}$, and rinsed again with deionized water. Slides were then rinsed in 50 and $70 \%$ ethanol 10 times, then in $95 \%$ ethanol for $30 \mathrm{~s}$, then in $100 \%$ ethanol for $60 \mathrm{~s}$. Muscle sections were then rinsed in xylene seven times, dried with a Kimwipe, and mounted. Digital photomicrographs were taken using a Nikon ECLIPSE microscope (Nikon Instruments, Inc., United States) equipped with a $40 \times$ objective.

\section{Sarcomere Length}

Muscle samples of approximately $1 \mathrm{~cm}^{3}$ and oriented along the muscle fibers were collected at $24 \mathrm{~h}$ post-mortem from the anteroventral zone of NORM PM $(n=6)$ and anteroventral, anterodorsal, posteroventral, and posterodorsal locations of WB PM de-boned muscles ( $n=6 /$ location) used for histology evaluation, placed in $10 \%$ (vol/vol) buffered formalin fixative $(\mathrm{pH}=7.0)$ and stored at $4^{\circ} \mathrm{C}$. Subsequently, samples were dehydrated in a graded series of ethanol, oriented for longitudinal fiber sectioning, and paraffin-embedded. Paraffin blocks were cut at $3 \mu \mathrm{m}$ with a microtome, mounted on saline-coated microscope slides, and hematoxylin and eosin stained. Each slide contained a minimum of two sections. Digital photomicrographs were taken with Nikon ECLIPSE microscope (Nikon Instruments, Inc., United States) equipped with an oil immersion $100 \times$ objective and processed using ImageJ software NIH Image. For each section, 15 myofibrils at least 5 sarcomeres long were selected for the assessment of sarcomere length, which was evaluated as the ratio between the total length of the myofibril (i.e., the distance between A bands) and the number of sarcomeres.

\section{Statistical Analysis}

Overall data from the experiment were analyzed using the oneway ANOVA option of the GLM procedure of SAS software (SAS Institute, Inc., United States). Data concerning $\mathrm{pH}$, glycolytic metabolites, and adenine nucleotides were analyzed using the occurrence of WB defect as the main effect within each sampling time (15 and 1,440 $\mathrm{min}$ ). As for the in vitro study, the statistical model included the addition of ATPase to the meat as the main effect for each sampling time ( 0 , $15,30,120,240$, and $1,440 \mathrm{~min}$ ), while results concerning buffering capacity between NORM and WB groups were compared within each $\mathrm{pH}$ value. Data regarding sarcomere length detected from AV sampling position were analyzed by comparing unaffected and affected samples, while the spatial influence of WB through affected PM muscles was evaluated using sampling location (anteroventral, anterodorsal, posteroventral, and posterodorsal) as the main effect. Data regarding the effect of $\mathrm{WB}$ condition on muscle glycolytic potential and PFK activity were analyzed by comparing NORM and WB experimental groups. Means were then evaluated using Tukey's multiple range test of the GLM procedure and

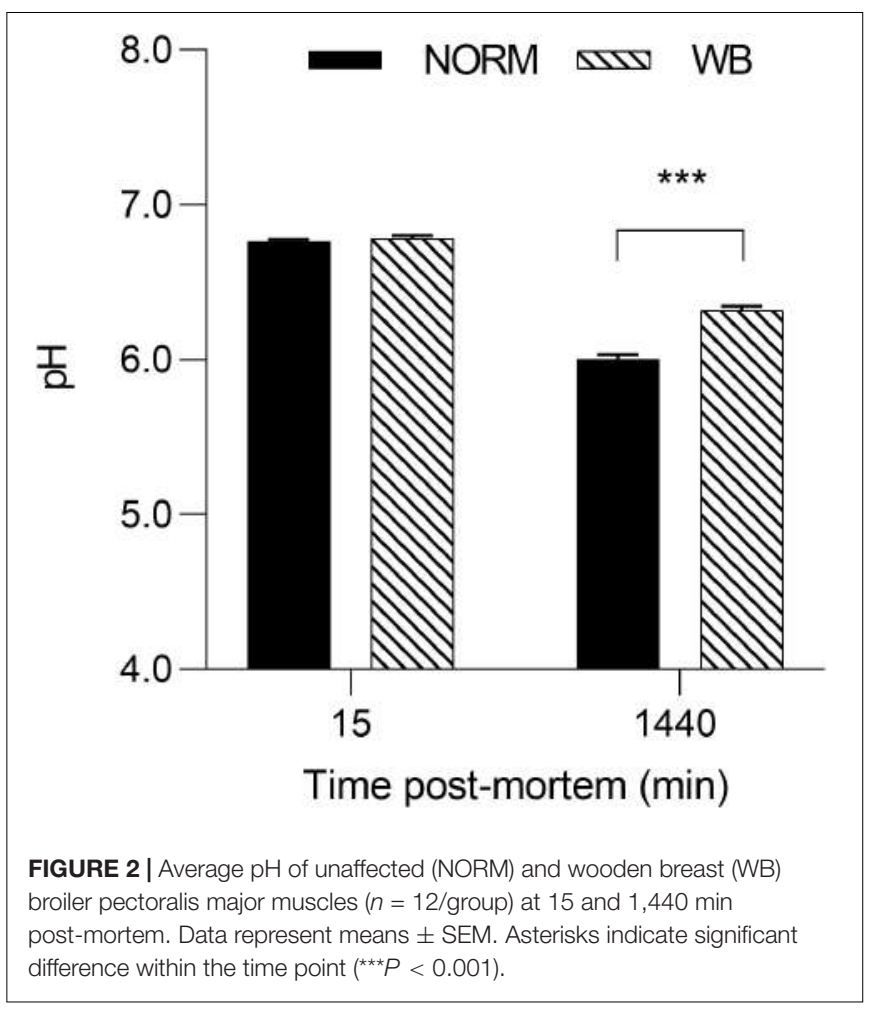

considered significant at $P<0.05$. All data are expressed as means \pm SEM.

\section{RESULTS AND DISCUSSION}

\section{pH and Glycolytic Metabolites}

Given that selection programs have exerted significant consequences on muscle metabolism (Petracci et al., 2017), understanding post-mortem glycolysis in WB-affected birds may be useful in providing greater understanding into this muscular abnormality. Results concerning the effect of WB condition on muscle $\mathrm{pH}$ and glycolytic metabolites are displayed in Figures 2, 3 , respectively. While no differences were detected at $15 \mathrm{~min}$ post-mortem, a significantly higher $\mathrm{pH}_{u}$ of $6.32(P<0.001)$ was observed in muscles affected by WB. The higher $\mathrm{pH}_{u}$ of WB meat found within this experiment and confirmed by previous studies (Mudalal et al., 2015; Kuttappan et al., 2017b; Baldi et al., 2019; Tasoniero et al., 2019) is commonly considered a hallmark of this myopathy, whereas the regular $\mathrm{pH}_{u}$ values of chicken pectoral muscles range typically from 5.8 to 5.9 (Petracci et al., 2017).

Accordingly, lactate contents followed $\mathrm{pH}$ decline, with WB muscles accumulating less lactate by 1,440 min post-mortem $(P<0.001$; Figure 3A). Reduced lactate formation was also found in severely affected WB chickens by Malila et al. (2019) and might be explained with the loss of lactate dehydrogenase (LDH) enzyme from abnormal muscle fibers (Abasht et al., 2016). Moreover, it has been recently suggested that decreased lactate concentrations found in WB meat might be linked to 


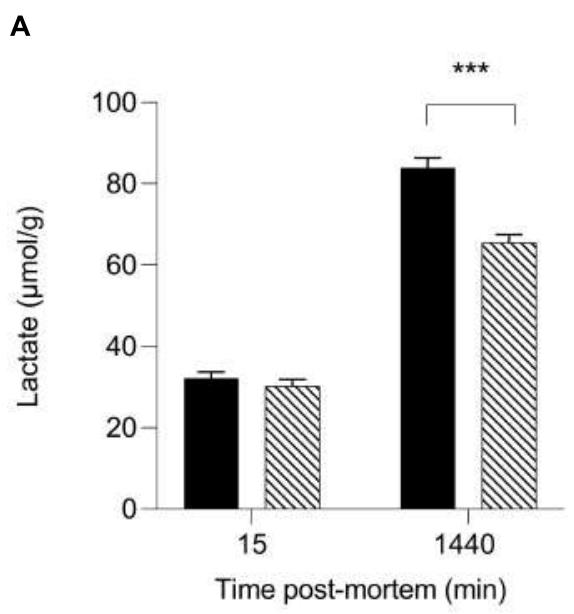

C

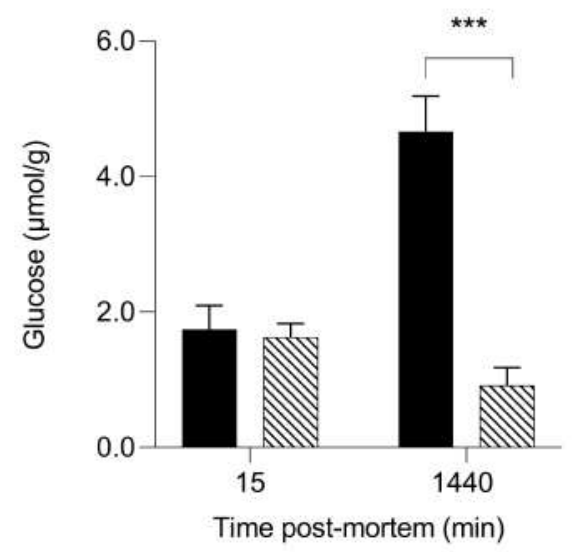

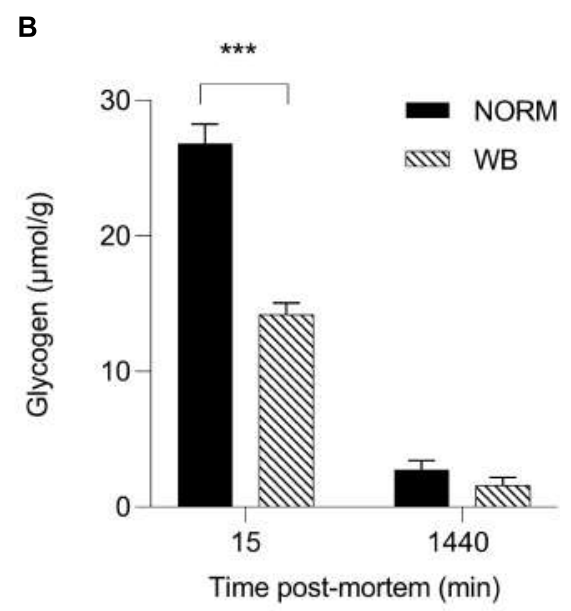

D

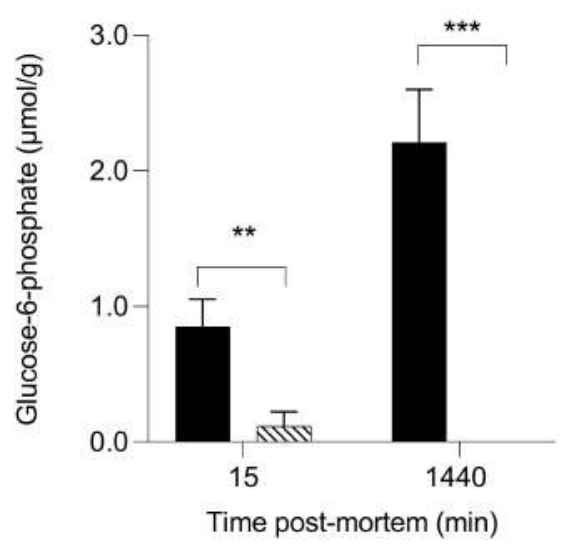

FIGURE 3 | Average lactate (A, $\mu \mathrm{mol} / \mathrm{g})$, glycogen (B, $\mu \mathrm{mol} / \mathrm{g})$, glucose (C, $\mu \mathrm{mol} / \mathrm{g})$, and glucose-6-phosphate (D, $\mu \mathrm{mol} / \mathrm{g})$ of unaffected (NORM) and wooden breast (WB) broiler pectoralis major muscles ( $n=12$ /group) at 15 and 1,440 min post-mortem. Data represent means \pm SEM. Asterisks indicate a significant difference within the time point $\left({ }^{\star \star \star} P<0.001\right.$; $\left.{ }^{\star \star} P<0.01\right)$.

an alteration of genes involved in lactate metabolism due to chronic hypoxic conditions responsible for exportation of lactate out of muscle cells (Zhao et al., 2019). However, lower lactate levels at 1,440 min along with the higher $\mathrm{pH}_{u}$ of $\mathrm{WB}$ samples suggest premature cessation of post-mortem metabolism, which is often thought to be associated with the depletion of muscular glycogen and/or an indication of a reduced amount prior to harvest. Curiously, glycogen content was found to be lower in WB-affected muscles at $15 \mathrm{~min}$ post-mortem $(P<0.001$; Figure 3B), corroborating the findings of Abasht et al. (2019) and Malila et al. (2019), but no differences were detected at 1,440 min. It has been reported that glycogen storage in breast muscle decreases with the increase in muscle fiber size (Berri et al., 2007), suggesting that selection for hybrids with high growth rate and muscle yield (mainly achieved through fiber hypertrophy) might lead to reduced muscular glycogen concentrations in modern broiler breast muscles. On the other hand, G6P concentrations at both 15 and 1,440 min were likewise reduced in WB meat $(P<0.01$ and $P<0.001$, respectively; Figure 3D), while glucose concentration was found to be higher $(P<0.001$; Figure 3C) in NORM samples at $1,440 \mathrm{~min}$. As a direct consequence of the diminished content of glycolytic metabolites, WB-affected muscles exhibit lower glycolytic potentials $(P<0.001)$, reduced by $30 \%$ when compared to NORM samples (Figure 4). Lower glycolytic potential in WB muscles indicates reduced substrate flux through glycolysis and, therefore, lower production of both $\mathrm{H}^{+}$and lactate, causing a higher $\mathrm{pH}_{u}$ of the forthcoming meat. These results confirmed what has been extensively reported in previous genetic and proteomic studies (Abasht et al., 2016; Zambonelli et al., 2016; Kuttappan et al., 2017a; Malila et al., 2019), suggesting that muscles showing severe myopathic lesions are usually characterized by downregulated carbohydrate metabolism and, as a consequence, a reduced content of glycolytic metabolites. Indeed, overall data collected within this study corroborate altered glycogen and glucose metabolic pathways. Considering the extreme hypoxic conditions and inflammatory processes taking place in WB muscles, the reduced content of glycolytic metabolites could be explained by a rerouting of the carbohydrate flow from glycolysis to other metabolic pathways in order to 


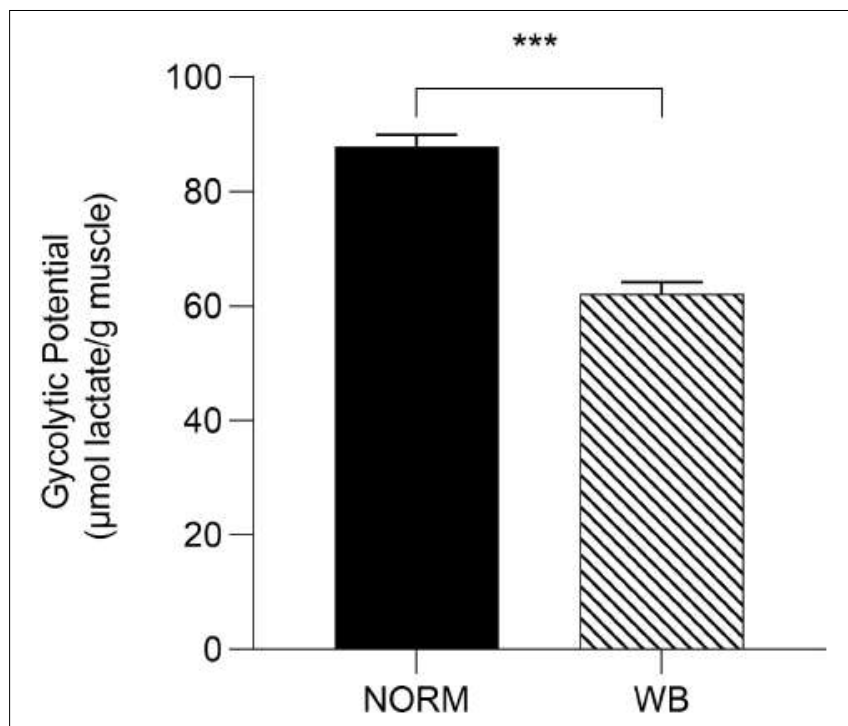

FIGURE 4 | Glycolytic potential ( $\mu$ mol lactate/g meat) of unaffected (NORM) and wooden breast (WB) broiler pectoralis major muscles ( $n=12 /$ group). Data represent means \pm SEM. Asterisks indicate a significant difference between experimental groups $\left({ }^{\star \star \star} P<0.001\right)$.

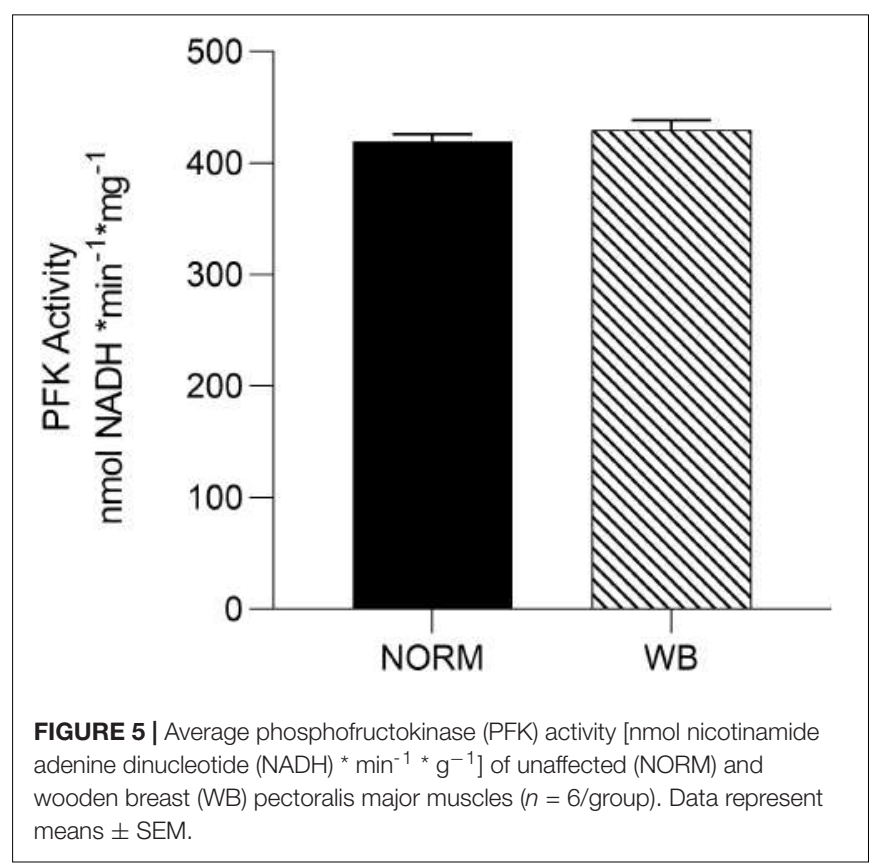

contrast muscle inflammation (Lake et al., 2019). Finally, the presence of residual glycogen in WB muscle found at $24 \mathrm{~h}$ post-mortem suggests that glycolysis did not arrest prematurely due to substrate deficiency.

\section{Phosphofructokinase Activity}

In the presence of residual glycogen, Matarneh et al. (2018) reported that $\mathrm{pH}_{u}$ is determined by the activity of PFK, a key regulatory enzyme in the glycolytic pathway that irreversibly catalyzes the conversion of fructose 6phosphate into fructose 1,6-biphosphate. Results concerning PFK activity are reported in Figure 5. Surprisingly, no differences were detected in PFK activity between affected and unaffected muscles, meaning that PFK is not responsible for arresting post-mortem glycolysis in WB-affected samples. However, Minchenko et al. (2002) reported that in hypoxic conditions, PFK plays a key role as a regulator of glucose consumption in order to maintain energetic homeostasis. With this in mind, the unaltered PFK activity found in WB muscles might represent an attempt of muscle cells to combat hypoxia by regulating glucose metabolism through different mechanisms. Further explanation for this outcome remains to be studied.

\section{Adenine Nucleotides}

In an attempt to better understand post-mortem metabolism in WB-affected muscles, adenine nucleotides levels were also measured. England et al. (2014) reported that in some cases, the depletion of adenosine nucleotides (ATP, ADP, IMP, and AMP) could arrest glycolysis while PFK is still functioning. Results concerning adenine nucleotides levels are shown in Figure 6. ATP content (Figure 6A) was 50\% lower in WBaffected muscle at 15 min post-mortem $(P<0.001)$ compared to NORM (3.10 vs. $6.51 \mu \mathrm{mol} / \mathrm{g})$. Intriguingly, WB-affected samples had higher IMP levels at 15 min post-mortem. No differences were detected in AMP concentrations (Figure 6C), while both ADP (Figure 6B) and IMP (Figure 6D) contents were lower in WB muscles at 1,440 min post-mortem $(P<0.05$ and $<0.001$, respectively). The lower IMP content found at 1,440 min within this study corroborates findings from Abasht et al. (2016) and Soglia et al. (2019), who suggest that lower levels of adenosine nucleotides along with higher levels of catabolites such as xanthine and urate might explain a greater nucleotide degradation in affected muscles. On the other hand, lower ADP concentration of $\mathrm{WB}$ muscle at 1,440 min postmortem might be the result of WB muscles attempting to combat the deficit in ATP concentrations by generating ATP from two molecules of ADP. This reaction also produces one molecule of AMP, which in turn could be deaminated to IMP and $\mathrm{NH}_{3}$. This might also explain the higher content of IMP in WB muscles at $15 \mathrm{~min}$ post-mortem, as muscle attempts to compensate for the lack of ATP in the first minutes after the death. However, the reasons behind the drastic reduction in ATP content might be different. The first hypothesis is related to creatine, a compound that plays a key role in muscular energy metabolism since it is directly involved in ATP synthesis in vivo (Mora et al., 2008). A study conducted by Sundekilde et al. (2017) revealed that dystrophic muscles present significantly lower creatine content, thus suggesting a perturbation in the energy-generating pathway. This result has also been confirmed by Soglia et al. (2019) and Wang et al. (2019) who found remarkably reduced creatine content in muscles affected by WB. Thus, being creatine present in lower concentrations in myopathic muscles, it is reasonable to speculate that the ATPgenerating pathway might be compromised, resulting in reduced ATP concentrations in the early post-mortem. The second 


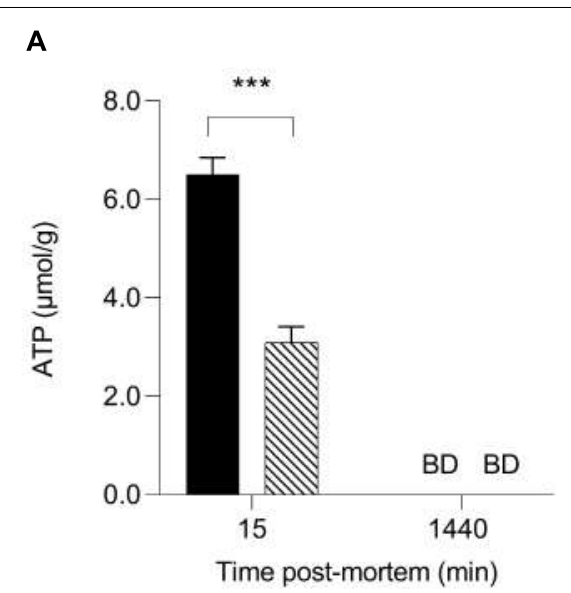

C

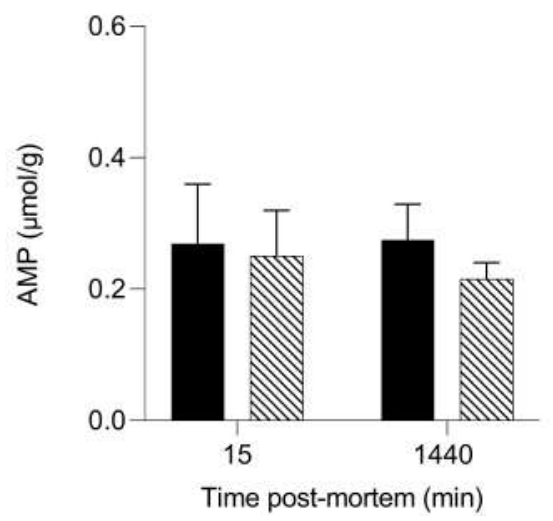

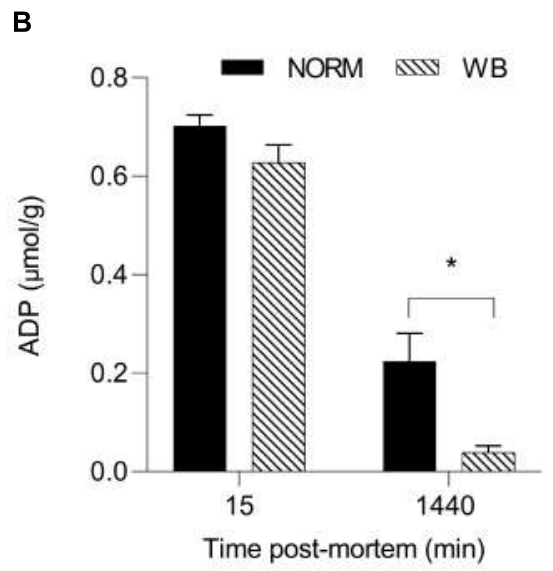

D

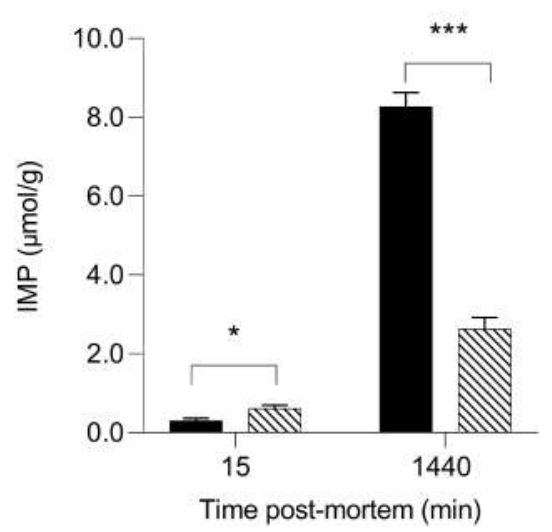

FIGURE 6 | Average ATP (A, $\mu \mathrm{mol} / \mathrm{g})$, ADP (B, $\mu \mathrm{mol} / \mathrm{g})$, AMP (C, $\mu \mathrm{mol} / \mathrm{g})$, and IMP (D, $\mu \mathrm{mol} / \mathrm{g})$ of unaffected (NORM) and wooden breast $(\mathrm{WB})$ broiler pectoralis major muscles ( $n=12$ /group) at 15 and 1,440 min post-mortem. Data represent means \pm SEM. Asterisks indicate a significant difference within the time point $\left({ }^{\star \star *} P<0.001 ;{ }^{*} P<0.05\right)$. BD, below limit of detection.

hypothesis concerns the impaired mitochondrial functionality of WB muscles found in a recent study (Sihvo et al., 2018), where the authors reported that WB-affected samples exhibit mitochondrial swelling, vacuolation, and cristae loss, clear indicators of osmotic imbalance and hypoxia. The same authors also suggest that muscles that experienced distress, such as hypoxia at any time during the lifetime of an animal, might be more prone to develop post-mortem alterations. Under these circumstances, since in vivo ATP synthesis happens through mitochondrial respiration, it is reasonable to postulate that the reduced ATP content found within this study might be partially due to the impaired mitochondria functionality found in WB-affected muscles. Aside from these theories, it is noteworthy to highlight that the severe histopathological lesions associated with WB condition (which will be discussed later in the paper) might represent the most logical explanation for the reduced nucleotide content found within affected samples, since the presence of necrotic fibers may have resulted in a lower ATP content. However, the drastic reduction in ATP concentrations in the early post-mortem period, along with the remarkably lower glycolytic potential of myopathic muscles, undoubtedly provoked a reduced extent of $\mathrm{pH}$ decline in WB samples (i.e., higher $\mathrm{pH}_{u}$ ). ADP and
AMP were not depleted by 1,440 min post-mortem, suggesting that adenine nucleotides alone were not likely responsible for arresting post-mortem glycolysis.

\section{Buffering Capacity}

The $\mathrm{pH}_{u}$ of a muscle also depends on the buffering systems of the muscle itself (Laack et al., 2001), thus, exploring muscle's buffering capacity may provide further knowledge about WB post-mortem glycolysis. Buffering capacity was significantly affected by the occurrence of WB defect (Figure 7). WB samples exhibited significantly lower buffering capacity $(P<0.001)$ for each $\mathrm{pH}$ value considered within the range of 6.4-7.0, meaning that WB-affected muscles have a lower ability to buffer $\mathrm{H}^{+}$ produced during post-mortem glycolysis. However, a reduced buffering capacity should have implied the achievement of a lower $\mathrm{pH}_{u}$, yet in WB meat, this condition is coupled with an overall reduced glycolytic potential that consequently led to decreased $\mathrm{H}^{+}$accumulation. Nevertheless, muscle's ability to buffer the acidic end-products of glycolysis is due by half to myofibrillar proteins, while phosphate compounds and histidinecontaining dipeptides (e.g., anserine and carnosine) contributed to the other half (Matarneh et al., 2017). In more detail, 


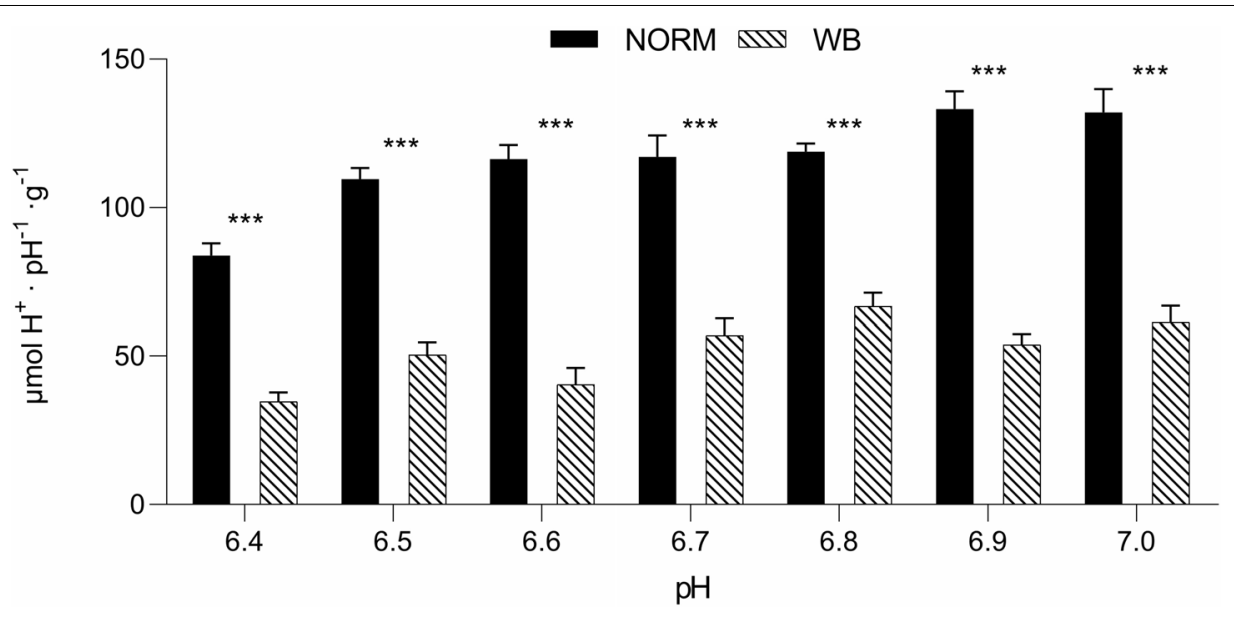

FIGURE 7 | Buffering capacity ( $\left.\mu \mathrm{mol} \mathrm{H} \mathrm{H}^{+} \cdot \mathrm{pH}^{-1} \cdot \mathrm{g}^{-1}\right)(\mathrm{pH}$ range 6.4-7.0) in unaffected (NORM) and wooden breast $(\mathrm{WB})$ pectoralis major muscles $(n=12 / g r o u p)$. Data represent means \pm SEM. Asterisks indicate a significant difference within the same $\mathrm{pH}$ value $\left({ }^{\star * \star} P<0.001\right)$.

anserine and carnosine, in light of their pKa (6.38 and 7.04, respectively), are considered the most important compounds for the maintenance of muscle homeostasis and prevention of tissue damage (Davey, 1960; Jung et al., 2013). As in vivo, also during the conversion of muscle to meat histidine dipeptides play a determinant role in counteracting rapid changes in $\mathrm{pH}$. However, a recent study conducted by Soglia et al. (2019) revealed that the concentrations of these compounds were significantly lower in WB muscles compared to unaffected ones. More specifically, anserine and carnosine levels were reduced by 37.2 and $46.1 \%$, respectively, in myopathic muscles, corroborating data from Sundekilde et al. (2017). Within this context, it is reasonable to assume that the reduced content of buffering compounds coupled with compromised muscle fibers structure and functionality (Sihvo et al., 2014; Velleman and Clark, 2015) severely affected the buffering capacity of muscles affected by WB condition.

\section{In vitro pH Decline}

Considering the overall dataset, the reduced content of glycolytic metabolites only partially explains the higher $\mathrm{pH}_{u}$ detected in WB muscle. Residual glycogen found in WB samples at 1,440 min post-mortem along with unaltered PFK activity suggests neither glycogen content nor PFK activity was responsible for arresting glycolysis. On the other hand, the absence of G6P at 1,440 min raises the possibility that glycogenolysis was somehow inhibited. Thus, since post-mortem glycolysis is regulated by ATPase activity (Scopes, 1974), an in vitro system was used to simulate muscle acidification with or without excess ATPase in order to further test the factors contributing to the higher $\mathrm{pH}_{u}$ in WB meat. Using this system, we were able to compare $\mathrm{pH}$ decline in affected and unaffected samples with or without $2 \mathrm{U}$ ATPase under the same environment (Figure 8). Because muscle samples from both groups were homogenized in the same buffer, differences in $\mathrm{pH}$ decline should be a function of the incorporated muscle tissue. The $\mathrm{pH}$ of the in vitro system was significantly affected by the addition of ATPase in the buffer. At both 0 and 15 min post-mortem, the addition of ATPase significantly raised muscular pH. NORM + ATPase and WB + ATPase groups showed significantly higher $\mathrm{pH}$ values when compared to their counterparts without ATPase. Scopes (1974) explained that the increased muscle $\mathrm{pH}$ with the addition of ATPase is due to immediate use of $\mathrm{H}^{+}$ions with $\mathrm{ADP}$ and phosphocreatine to produce creatine and ATP prior to anaerobic glycolysis. Thus, for every re-phosphorylation of ADP by phosphocreatine, a $\mathrm{H}^{+}$ion is consumed by the creatine kinase reaction, causing a slight increase of the $\mathrm{pH}$ (Scopes, 1974). However, after an initial slower rate, addition of ATPase increased the rate of $\mathrm{pH}$ decline of NORM + ATPase samples, which exhibited the lowest $\mathrm{pH}$ values for each time point. This is likely due to the addition of an excess ATPase to the system that stimulated additional glycolytic flux and consequently results in a lower $\mathrm{pH}$. After $30 \mathrm{~min}$ post-mortem, the addition of ATPase appeared to increase the rate of $\mathrm{pH}$ decline in $\mathrm{WB}$ muscle and, at 1,440 min, reaction vessels containing WB muscle with an excess of ATPase showed significantly lower values when compared to their counterparts without ATPase (5.67 vs. 6.01; $P<0.05$ ). No statistically significant differences were detected between NORM and WB + ATPase groups at 1,440 min, suggesting that an excess of ATPase can extend in vitro post-mortem glycolysis in WB muscles. Considering the presence of significant damaged/abnormal myofibers in WB tissue, it is reasonable to hypothesize that a deficiency and/or a dysfunction of muscular ATPases might be one of the factors responsible for arresting post-mortem glycolysis prematurely in WB muscles. Further, given the abundance of myosin ATPase in skeletal muscle, ATP hydrolysis by myofibrillar component likely drives post-mortem metabolism (Ferguson and Gerrard, 2014). Thus, further studies should be conducted in order to investigate if post-mortem metabolism is repressed because of a deficiency rather than a reduced functionality of myosin ATPases.

\section{Histology}

Although the effect of WB abnormality on muscle fibers and microstructure has been deeply investigated, few studies take into 


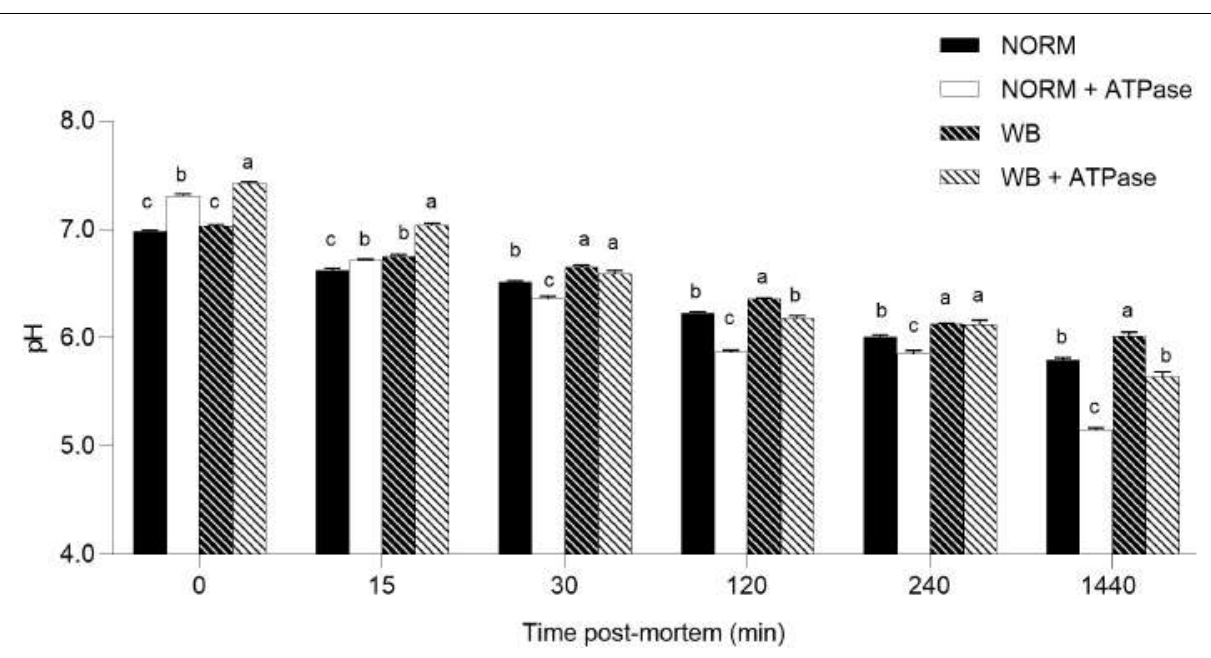

FIGURE 8 | Average $\mathrm{pH}$ of the in vitro model ( $n=6$ /group). Data represent means \pm SEM. (a-c) Means lacking a common letter significantly differ within a time point $(P<0.05)$.
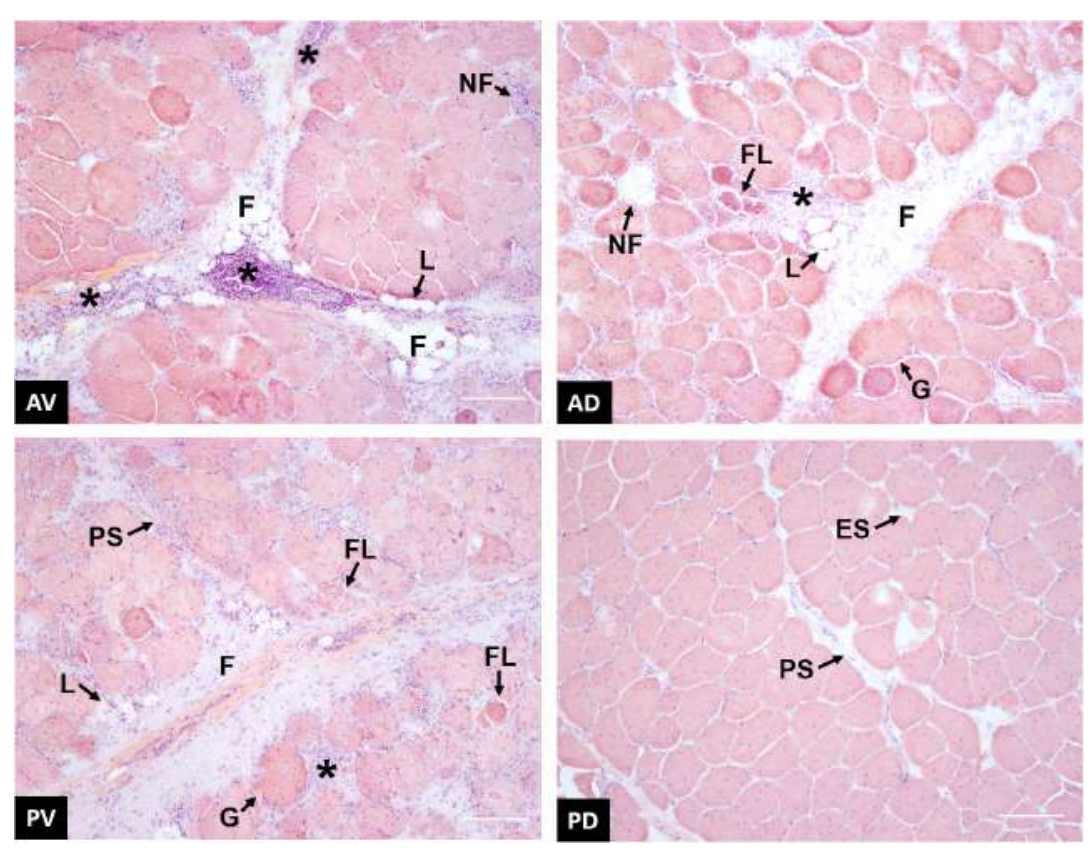

FIGURE 9 | Representative images of wooden breast (WB)-affected samples collected from anteroventral (AV), anterodorsal (AD), posteroventral (PV), and posterodorsal (PD) positions of the pectoralis major muscle. * inflammatory cells infiltrate; F, proliferation of connective tissue; L, fat tissue deposition; NF, necrotic fiber; FL, fiber lysis; PS, perimysial spacing; ES, endomysial spacing; G, giant hypertrophic fiber. Scale bar $=150 \mu \mathrm{m}$.

consideration the evaluation of fiber morphology throughout different zones of PM muscle (Clark and Velleman, 2017; Soglia et al., 2017). Figure 9 contains representative images showing the morphological structure of anteroventral, anterodorsal, posteroventral, and posterodorsal regions of broiler PM affected by WB. In agreement with previous studies (Sihvo et al., 2014; Clark and Velleman, 2017; Soglia et al., 2017), anteroventral region exhibited severe myodegenerative lesions, including several extensive macrophage infiltrations, accumulation of fat (i.e., lipidosis) and connective tissue (i.e., fibrosis), as well as the presence of necrotic fibers surrounded by inflammatory cell infiltrates. The anteroventral region appears to have the highest degree of macrophage infiltration when compared to all other locations. Affected areas are demarcated by the replacement of damaged fibers with the deposition of connective tissue, causing a complete reorganization of the skeletal muscle structure. According to Sihvo et al. (2014), a severe thickening in the interstitial tissue separating muscle fiber bundles and nuclei internalization have been widely detected in anteroventral area. The main causes associated with this altered histological profile 
have been widely attributed to the reduced microcirculation due to excessive development of the pectoral muscle, which consequently leads to impaired muscle fiber metabolism and oxygen supply (Zambonelli et al., 2016; Sihvo et al., 2017). Among published studies, there are contrasting results about the effect of WB defect on the histologic characteristics of breast muscle's inner cranial region probably due to the severity grade of the myopathy. According to Soglia et al. (2017), the anterodorsal zone exhibited several myodegenerative traits, such as fibrosis, lipidosis, necrotic fibers surrounded by macrophage infiltrations, and the presence of giant and hypercontracted fibers. Thickening of the endomysial spaces with deposition of connective tissue was observed as well. Moreover, the existence of fibers characterized by small diameters could be the result of fiber lysis, a process taking place in the muscle as a reparative and/or adaptive mechanism against fiber necrosis and degeneration. Additionally, Clark and Velleman (2017) found the presence of regenerating myofibers in the same region of PM muscles affected by WB. In the most severe cases, WB abnormality affects both the cranial and the caudal regions of superficial pectoral muscle (Kuttappan et al., 2016). From histological examinations conducted within this study, the posteroventral region showed an overall compromised muscular structure architecture, nearly comparable to what was observed for the anteroventral region. In addition to fibrosis, lipidosis, and fiber lysis, thickening of the perimysial and endomysial spaces and inflammatory cell infiltrations surrounding necrotic fibers have been observed. These findings are quite conflicting with what was observed in the same broiler PM region by Clark and Velleman (2017), who reported that the histological profile of the posteroventral area was just slightly affected by the occurrence of WB myopathy. However, the divergence detected between these studies might be ascribable to the different severity grades of WB condition and/or slaughter age of animals. Furthermore, based on the current knowledge, no studies have been conducted to investigate the histological traits of caudal deep section of PM muscle affected by WB. Contrary to other locations, the histological profile of posterodorsal region did not appear as severely compromised as other sampling locations. Neither infiltrating inflammatory cells nor fiber necrosis were found, while endomysial and perimysial spacing, as well as nuclei internalization and endomysial connective tissue proliferation, were evident. Generally, when compared to their superficial counterparts, the histological profile of the deep sections appeared to be less impacted by the occurrence of WB. The different degrees of myodegeneration between the upper and the inner sections of the pectoral muscle might be attributed to the different distance from the blood vessels responsible for oxygen translocation (Soglia et al., 2017). The greater physical proximity of the muscle fibers to capillaries and vessels might have resulted in a better oxygenation of the muscular tissue, thus limiting muscle damages related to oxidative stress.

\section{Sarcomere Length}

Because the rate and the extent of post-mortem muscular acidification influence the degree of myofibrillar contraction (Ertbjerg and Puolanne, 2017), it has been speculated if the
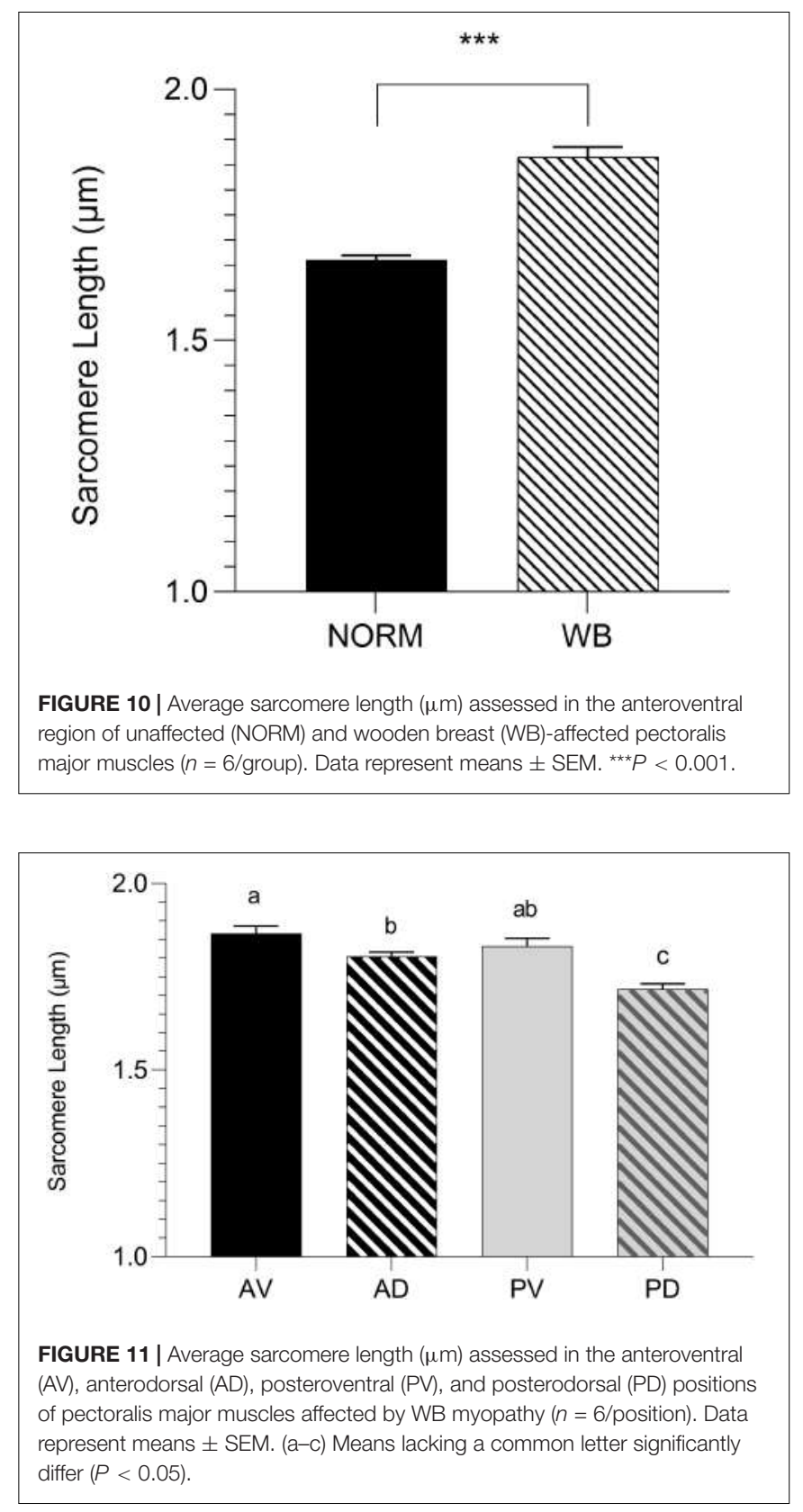

extreme stiffness of WB muscles could be partially due to a hypercontraction of sarcomeres caused by an abnormal acidification process. Contrary to what was expected, WB samples belonging to the anteroventral region (superficial, cranial) showed significantly $(P<0.001)$ longer sarcomeres when compared to samples collected from the same area of NORM PM muscles (1.86 versus $1.66 \mu \mathrm{m}$, respectively; Figure 10). This result corroborates data from Tijare et al. (2016) and Sun et al. (2018), where investigators suggested that the longer sarcomeres detected in the cranial region of WB muscle might be due to the increased collagen content and the loss of muscle fiber vitality that might prevent shortening. In light of the results collected within this study, it could be postulated 
that the overall reduced content of ATP detected among WBaffected samples might result in a defective shortening process. Specifically, because the force for shortening is ATP-driven (Ertbjerg and Puolanne, 2017), with a reduced content of ATP present in the muscle, the sarcomeres are less likely to shorten because there is not enough energy to allow muscle contraction (Owens and Sams, 1997). Moreover, a study conducted by Mutryn et al. (2015) suggested that the higher concentration of reactive oxygen species detected in WB muscles may impact calcium release from the sarcoplasmic reticulum, damaging the ability of muscle cells to contract. Thus, it is reasonable to hypothesize that the hypoxic conditions along with the compromised energy-generating pathways of myopathic muscles might have consequences on the degree of muscle contraction, as in vivo, also during post-mortem. Moreover, considering these outcomes, it can be postulated that the typical hardness of WB muscles is not due to muscle fiber hypercontraction, otherwise, it is more likely linked to the excessive collagen deposition, as suggested in previous studies (Sihvo et al., 2014; Soglia et al., 2016; Baldi et al., 2019). Further, considering that sarcomere length is not uniform across the muscle (Moo et al., 2016), the spatial effect of WB myopathy on sarcomere length was investigated in different regions of PM muscle (anterodorsal, anteroventral, posteroventral, and posterodorsal) (Figure 11). Intriguingly, samples belonging to the anteroventral region of WB fillets showed the longest sarcomere length values (1.86 $\mu \mathrm{m}$ ), while those belonging to the posterodorsal area exhibited the shortest $(1.71 \mu \mathrm{m})$ and anteroventral and posteroventral displayed intermediate values $(1.80$ and $1.83 \mu \mathrm{m}$, respectively). Further, the superficial sections of both cranial and caudal regions (anteroventral and posteroventral, respectively) showed significantly longer $(P<0.05)$ sarcomeres when compared to their deep counterparts. These divergences detected along muscle's thickness might be attributed to the different physical conformation and morphology of WB muscles, which usually show out-bulging and swollen areas in both cranial and caudal zones. In addition, considering fiber morphology results, anteroventral and posteroventral regions showed the most outstanding damages linked to $\mathrm{WB}$ disease (i.e., extreme collagen deposition, inflammatory cell infiltrations, endomysial, and perimysial spacing, etc.). Thus, it is reasonable to hypothesize that the profound injuries detected at histological level linked to a loss of muscle fiber functionality and vitality might have led to defective myofiber contraction mechanism in vivo that in turn resulted in longer sarcomeres.

\section{CONCLUSION}

This study aimed to better understand the effects of WB condition on muscle post-mortem metabolism in an attempt to provide new insights about the factors contributing to the high $\mathrm{pH}_{u}$ of affected muscles. Reduced glycolytic potential only partially explains the higher $\mathrm{pH}_{u}$ in $\mathrm{WB}$ meat, as residual glycogen and PFK activity were unaffected in WB samples, arguing against glycogen content or PFK activity being the primary culprit for arresting glycolysis in WB. Further, the dramatic reduction in ATP concentrations in the early postmortem period might suggest a defective ATP-generating pathway in vivo. It should be pointed out that the severe histopathologic damages associated to WB condition in general, and fiber necrosis in particular, might represent the logical culprits for the reduced ATP content found in WB. However, since ADP and AMP were not depleted by $24 \mathrm{~h}$ postmortem, adenine nucleotides alone were not responsible for arresting post-mortem glycolysis. It has been also tested that the addition of excess ATPase through an in vitro glycolytic system can extend post-mortem glycolysis in WB muscles. In light of all the mechanisms involved in the occurrence of WB abnormality, it might be complex to define a single factor contributing to the higher $\mathrm{pH}_{u}$ of affected muscles. Considering the overall reduced glycolytic metabolites and the extreme myodegenerative processes associated with WB condition, data suggest that the higher $\mathrm{pH}_{u}$ of $\mathrm{WB}$ meat might be the outcome of a drastically impaired energy-generating pathway combined with a deficiency and/or a dysfunction of muscle ATPases, having consequences also on muscle fiber contraction degree.

\section{DATA AVAILABILITY STATEMENT}

The datasets generated for this study are available on request to the corresponding author.

\section{ETHICS STATEMENT}

In this experiment, meat samples were directly collected postmortem from carcasses belonging to animals that were farmed and slaughtered under commercial conditions in a federal slaughterhouse in Athens, Georgia (USA). Therefore, an ethical review process was not required for this study since the authors have not personally reared, killed nor handled the animals. All the aspects of farming, handling, transportation, and slaughter of birds were under the responsibility of the commercial slaughterhouse and accomplished under U.S. laws.

\section{AUTHOR CONTRIBUTIONS}

All authors listed have made a substantial, direct and intellectual contribution to the work, and approved it for publication. GB, DG, BB, HZ, and MP planned the experiment. GB designed the study, organized the databases and performed the statistical analysis, and wrote the first draft of the manuscript. GB, C-NY, MD, and JB performed the laboratory analyses and interpreted the results. All authors contributed to manuscript revision. 


\section{REFERENCES}

Abasht, B., Mutryn, M. F., Michalek, R. D., and Lee, W. R. (2016). Oxidative stress and metabolic perturbations in wooden breast disorder in chickens. PLoS One 11:e153750. doi: 10.1371/journal.pone.0153750

Abasht, B., Zhou, N., Lee, W. R., Zhuo, Z., and Peripolli, E. (2019). The metabolic characteristics of susceptibility to wooden breast disease in chickens with high feed efficiency. Poult. Sci. 98, 3246-3256. doi: 10.3382/ps/pez183

Baldi, G., Soglia, F., Laghi, L., Tappi, S., Rocculi, P., Tavaniello, S., et al. (2019). Comparison of quality traits among breast meat affected by current muscle abnormalities. Food Res. Int. 115, 369-376. doi: 10.1016/j.foodres.2018.11.020

Berri, C., Le Bihan-Duval, E., Debut, M., Santé-Lhoutellier, V., Baeza, E., Gigaud, V., et al. (2007). Consequence of muscle hypertrophy on characteristics of Pectoralis major muscle and breast meat quality in broiler chickens. J. Anim. Sci. 85, 2005-2011. doi: 10.2527/jas.2006-398

Chauhan, S. S., and England, E. M. (2018). Postmortem glycolysis and glycogenolysis: insights from species comparisons. Meat Sci. 144, 118-126. doi: 10.1016/j.meatsci.2018.06.021

Clark, D. L., and Velleman, S. G. (2017). Spatial influence on breast muscle morphological structure, myofiber size, and gene expression associated with the wooden breast myopathy in broilers. Poult. Sci. 95, 2930-2945. doi: 10.3382/ps/ pew243

Davey, C. L. (1960). The significance of carnosine and anserine in striated skeletal muscle. Arch. Biochem. Biophys. 89, 303-308. doi: 10.1016/0003-9861(60) 90059-X

England, E. M., Matarneh, S. K., Scheffler, T. L., Wachet, C., and Gerrard, D. E. (2014). PH inactivation of phosphofructokinase arrests postmortem glycolysis. Meat Sci. 98, 850-857. doi: 10.1016/j.meatsci.2014.07.019

Ertbjerg, P., and Puolanne, E. (2017). Muscle structure, sarcomere length and influences on meat quality: a review. Meat Sci. 132, 139-152. doi: 10.1016/j. meatsci.2017.04.261

Ferguson, D. M., and Gerrard, D. E. (2014). Regulation of post-mortem glycolysis in ruminant muscle. Anim. Prod. Sci. 54:464. doi: 10.1071/an13088

Griffin, J. R., Moraes, L., Wick, M., and Lilburn, M. S. (2018). Onset of white striping and progression into wooden breast as defined by myopathic changes underlying Pectoralis major growth. Estimation of growth parameters as predictors for stage of myopathy progression. Avian Pathol. 47, 2-13. doi: 10.1080/03079457.2017.1356908

Hammelman, J. E., Bowker, B. C., Grant, A. L., Forrest, J. C., Schinckel, A. P., and Gerrard, D. E. (2003). Early postmortem electrical stimulation simulates PSE pork development. Meat Sci. 63, 69-77. doi: 10.1016/S0309-1740(02)00057-8

Jung, S., Bae, Y. S., Kim, H. J., Jayasena, D. D., Lee, J. H., Park, H. B., et al. (2013). Carnosine, anserine, creatine, and inosine 5 '-monophosphate contents in breast and thigh meats from 5 lines of korean native chicken. Poult. Sci. 92 3275-3282. doi: 10.3382/ps.2013-03441

Kuttappan, V. A., Bottje, W., Ramnathan, R., Hartson, S. D., Coon, C. N., Kong, B. W., et al. (2017a). Proteomic analysis reveals changes in carbohydrate and protein metabolism associated with broiler breast myopathy. Poult. Sci. 96, 2992-2999. doi: 10.3382/ps/pex069

Kuttappan, V. A., Owens, C. M., Coon, C., Hargis, B. M., and Vazquez-A Non, M. (2017b). Research Note Incidence of broiler breast myopathies at 2 different ages and its impact on selected raw meat quality parameters. Poult. Sci. 96, 3005-3009. doi: 10.3382/ps/pex072

Kuttappan, V. A., Hargis, B. M., and Owens, C. M. (2016). White striping and woody breast myopathies in the modern poultry industry: a review. Poult. Sci. 95, 2724-2733. doi: $10.3382 /$ ps/pew216

Laack, R. L. J. M., Kauffman, R. G., and Greaser, M. L. (2001). "Determinants of ultimate $\mathrm{pH}$ on meat," in Proceedings of the 47th International Congress of Meat Science and Technology, (Warszawa: Meat and Fat Research Institute), 22-26.

Lake, J. A., Papah, M. B., and Abasht, B. (2019). Increased expression of lipid metabolism genes in early stages of wooden breast links myopathy of broilers to metabolic syndrome in humans. Genes 10, 1-18. doi: 10.3390/genes10100746

Livingston, M. L., Ferket, P. R., Brake, J., and Livingston, K. A. (2018). Dietary amino acids under hypoxic conditions exacerbates muscle myopathies including wooden breast and white stripping. Poult. Sci. 98, 1517-1527. doi: $10.3382 /$ ps/pey463

Malila, Y., Thanatsang, K., Arayamethakorn, S., Uengwetwanit, T., Srimarut, Y., Petracci, M., et al. (2019). Absolute expressions of hypoxia-inducible factor-1 alpha (HIF1A) transcript and the associated genes in chicken skeletal muscle with white striping and wooden breast myopathies. PLoS One 14:e0220904. doi: 10.1371/journal.pone.0220904

Matarneh, S. K., England, E. M., Scheffler, T. L., Oliver, E. M., and Gerrard, D. E. (2015). Net lactate accumulation and low buffering capacity explain low ultimate $\mathrm{pH}$ in the longissimus lumborum of AMPK $\gamma 3 \mathrm{R} 200 \mathrm{Q}$ mutant pigs. Meat Sci. 110, 189-195. doi: 10.1016/j.meatsci.2015.07.023

Matarneh, S. K., England, E. M., Sheffler, T. L., and Gerrard, D. E. (2017). “The conversion of muscle to meat," in Lawrie's Meat Science, eds R. A. Lawrie and D. A. Ledward (Amsterdem: Elsevier), 159-184.

Matarneh, S. K., Yen, C.-N., Elgin, J. M., Beline, M., de Luz e Silva, S., Wicks, J. C., et al. (2018). Phosphofructokinase and mitochondria partially explain the high ultimate $\mathrm{pH}$ of broiler pectoralis major muscle. Poult. Sci. 97, 1808-1817. doi: $10.3382 / \mathrm{ps} / \mathrm{pex} 455$

Minchenko, A., Leshchinsky, I., Opentanova, I., Sang, N., Srinivas, V., Armstead, V., et al. (2002). Hypoxia-inducible factor-1-mediated expression of the 6-phosphofructo-2-kinase/fructose-2,6-bisphosphatase-3 (PFKFB3) gene: its possible role in the warburg effect. J. Biol. Chem. 277, 6183-6187. doi: 10.1074/ jbc.M110978200

Moo, E. K., Fortuna, R., Sibole, S. C., Abusara, Z., and Herzog, W. (2016). In vivo sarcomere lengths and sarcomere elongations are not uniform across an intact muscle. Front. Physiol. 7:187. doi: 10.3389/fphys.2016.00187

Mora, L., Sentandreu, M. A., and Toldrá, F. (2008). Contents of creatine, creatinine and carnosine in porcine muscles of different metabolic types. Meat Sci. 79, 709-715. doi: 10.1016/j.meatsci.2007.11.002

Mudalal, S., Lorenzi, M., Soglia, F., Cavani, C., and Petracci, M. (2015). Implications of white striping and wooden breast abnormalities on quality traits of raw and marinated chicken meat. Animal 9, 728-734. doi: 10.1017/ S175173111400295X

Mutryn, M. F., Brannick, E. M., Fu, W., Lee, W. R., and Abasht, B. (2015). Characterization of a novel chicken muscle disorder through differential gene expression and pathway analysis using RNA-sequencing. BMC Genomics 16:399. doi: 10.1186/s12864-015-1623-0

Owens, C. M., and Sams, A. R. (1997). Muscle metabolism and meat quality of pectoralis from Turkeys treated with postmortem electrical stimulation. Poult. Sci. 76, 1047-1051. doi: 10.1093/ps/76.7.1047

Papah, M. B., Brannick, E. M., Schmidt, C. J., and Abasht, B. (2017). Evidence and role of phlebitis and lipid infiltration in the onset and pathogenesis of Wooden Breast Disease in modern broiler chickens. Avian. Pathol. 46, 623-643. doi: 10.1080/03079457.2017.1339346

Petracci, M., Soglia, F., and Berri, C. (2017). "Muscle metabolism and meat quality abnormalities," in Poultry Quality Evaluation: Quality Attributes and Consumer Values, eds M. Petracci and C. Berri (Sawston: Woodhead Publishing), 51-75. doi: 10.1016/b978-0-08-100763-1.00003-9

Petracci, M., Soglia, F., Madruga, M., Carvalho, L., Ida, E., and Estévez, M. (2019). Wooden-breast, white striping, and spaghetti meat: causes, consequences and consumer perception of emerging broiler meat abnormalities. Compr. Rev. Food Sci. Food Saf. 18, 565-583. doi: 10.1111/1541-4337.12431

Scheffler, T. L., Scheffler, J. M., Kasten, S. C., Sosnicki, A. A., and Gerrard, D. E. (2013). High glycolytic potential does not predict low ultimate $\mathrm{pH}$ in pork. Meat Sci. 95, 85-91. doi: 10.1016/j.meatsci.2013.04.013

Scopes, R. K. (1974). Studies with a reconstituted muscle glycolytic system. The rate and extent of glycolysis in simulated post-mortem conditions. Biochem. J. 142, 79-86. doi: 10.1042/bj1420079

Sihvo, H. K., Airas, N., Lindén, J., and Puolanne, E. (2018). Pectoral vessel density and early ultrastructural changes in broiler chicken wooden breast myopathy. J. Comp. Pathol. 161, 1-10. doi: 10.1016/j.jcpa.2018.04.002

Sihvo, H. K., Immonen, K., and Puolanne, E. (2014). Myodegeneration With Fibrosis and Regeneration in the Pectoralis Major Muscle of Broilers. Vet. Pathol. 51, 619-623. doi: 10.1177/0300985813497488

Sihvo, H. K., Lindén, J., Airas, N., Immonen, K., Valaja, J., and Puolanne, E. (2017). Wooden breast myodegeneration of pectoralis major muscle over the growth period in broilers. Vet. Pathol. 54, 119-128. doi: 10.1177/030098581 6658099

Soglia, F., Gao, J., Mazzoni, M., Puolanne, E., Cavani, C., Petracci, M., et al. (2017). Superficial and deep changes of histology, texture and particle size distribution in broiler wooden breast muscle during refrigerated storage. Poult. Sci. 96, 3465-3472. doi: 10.3382/ps/pex115 
Soglia, F., Mudalal, S., Babini, E., Di Nunzio, M., Mazzoni, M., Sirri, F., et al. (2016). Histology, composition, and quality traits of chicken Pectoralis major muscle affected by wooden breast abnormality. Poult. Sci. 95, 651-659. doi: $10.3382 / \mathrm{ps} / \mathrm{pev} 353$

Soglia, F., Silva, A. K., Lião, L. M., Laghi, L., and Petracci, M. (2019). Effect of broiler breast abnormality and freezing on meat quality and metabolites assessed by 1 H-NMR spectroscopy. Poult. Sci. 0, 1-12. doi: 10.3382/ps/ pez514

Sun, X., Koltes, D. A., Coon, C. N., Chen, K., and Owens, C. M. (2018). Instrumental compression force and meat attribute changes in woody broiler breast fillets during short-term storage. Poult. Sci. 97, 2600-2606. doi: 10.3382/ ps/pey107

Sundekilde, U. K., Rasmussen, M. K., Young, J. F., and Bertram, H. C. (2017). High resolution magic angle spinning NMR spectroscopy reveals that pectoralis muscle dystrophy in chicken is associated with reduced muscle content of anserine and carnosine. Food Chem. 217, 151-154. doi: 10.1016/j.foodchem. 2016.08.104

Tasoniero, G., Bowker, B., Stelzleni, A., Zhuang, H., Rigdon, M., and Thippareddi, H. (2019). Use of blade tenderization to improve wooden breast meat texture2. Poult. Sci. 98, 4204-4211. doi: 10.3382/ps/pez163

Tijare, V. V., Yang, F. L., Kuttappan, V. A., Alvarado, C. Z., Coon, C. N., and Owens, C. M. (2016). Meat quality of broiler breast fillets with white striping and woody breast muscle myopathies. Poult. Sci. 95, 2167-2173. doi: 10.3382/ps/ pew129

Velleman, S. G. (2019). Recent developments in breast muscle myopathies associated with growth in poultry. Annu. Rev. Anim. Biosci. 7, 289-308. doi: 10.1146/annurev-animal-020518
Velleman, S. G., and Clark, D. L. (2015). Histopathologic and Myogenic Gene Expression Changes Associated with Wooden Breast in Broiler Breast Muscles. Avian. Dis. 59, 410-418. doi: 10.1637/11097-042015-Reg.1

Wang, Y., Yang, Y., Pan, D., He, J., Cao, J., Wang, H., et al. (2019). Metabolite profile based on 1H-NMR of broiler chicken breasts affected by wooden breast myodogeneration. Food Chem. 2019, 125852. doi: 10.1016/j.foodchem.2019. 125852

Zambonelli, P., Zappaterra, M., Soglia, F., Petracci, M., Sirri, F., Cavani, C., et al. (2016). Detection of differentially expressed genes in broiler pectoralis major muscle affected by White Striping - Wooden Breast myopathies. Poult. Sci. 95, 2771-2785. doi: 10.3382/ps/pew268

Zhao, D., Kogut, M. H., Genovese, K. J., Hsu, C.-Y., Lee, J. T., and Farnell, Y.Z. (2019). Altered expression of lactate dehydrogenase and monocarboxylate transporter involved in lactate metabolism in broiler wooden breast. Poult. Sci. doi: $10.3382 / \mathrm{ps} /$ pez572 [Online ahead of print]

Conflict of Interest: The authors declare that the research was conducted in the absence of any commercial or financial relationships that could be construed as a potential conflict of interest.

Copyright (c) 2020 Baldi, Yen, Daughtry, Bodmer, Bowker, Zhuang, Petracci and Gerrard. This is an open-access article distributed under the terms of the Creative Commons Attribution License (CC BY). The use, distribution or reproduction in other forums is permitted, provided the original author(s) and the copyright owner(s) are credited and that the original publication in this journal is cited, in accordance with accepted academic practice. No use, distribution or reproduction is permitted which does not comply with these terms. 


\section{OPEN ACCESS}

Edited by:

Sandra G. Velleman, The Ohio State University, United States

Reviewed by: Lisa Bielke,

The Ohio State University, United States Kent M. Reed, University of Minnesota Twin Cities, United States

*Correspondence: Jessica D. Starkey jessica.starkey@auburn.edu

Specialty section: This article was submitted to Avian Physiology, a section of the journal

Frontiers in Physiology

Received: 29 February 2020 Accepted: 29 April 2020

Published: 27 May 2020

Citation:

Ferreira TZ, Kindlein L, Flees JJ, Shortnacy LK, Vieira SL, Nascimento VP, Meloche $\mathrm{KJ}$ and Starkey JD (2020) Characterization of Pectoralis Major Muscle Satellite

Cell Population Heterogeneity, Macrophage Density, and Collagen Infiltration in Broiler Chickens Affected by Wooden Breast.

Front. Physiol. 11:529. doi: 10.3389/fphys.2020.00529

\section{Characterization of Pectoralis Major Muscle Satellite Cell Population Heterogeneity, Macrophage Density, and Collagen Infiltration in Broiler Chickens Affected by Wooden Breast}

Tamara Z. Ferreira'1, Liris Kindlein'1, Joshua J. Flees², Lauren K. Shortnacy², Sergio L. Vieira ${ }^{3}$, Vladimir P. Nascimento ${ }^{4}$, Kathryn J. Meloche² and Jessica D. Starkey²*

${ }^{1}$ Department of Preventative Veterinary Medicine, Federal University of Rio Grande do Sul, Porto Alegre, Brazil, ${ }^{2}$ Department of Poultry Science, Auburn University, Auburn, AL, United States, ${ }^{3}$ Department of Animal Science, Federal University of Rio Grande do Sul, Porto Alegre, Brazil, ${ }^{4}$ Department of Animal Medicine, Federal University of Rio Grande do Sul, Porto Alegre, Brazil

Muscle satellite cells (MSCs) are myogenic stem cells that play a critical role in posthatch skeletal muscle growth and regeneration. Activation of regeneration pathways to repair muscle fiber damage requires both the proliferation and differentiation of different MSC populations as well as the function of resident phagocytic cells such as anti-inflammatory and pro-inflammatory macrophages. The Wooden Breast (WB) phenotype in broiler chickens is characterized by myofiber degeneration and extensive fibrosis. Previous work indicates that the resident MSC populations expressing the myogenic regulatory factors, Myf-5 and Pax7 are larger and more proliferative in broilers severely affected with WB vs. unaffected broilers. To further characterize the cellular and molecular changes occurring in WB-affected muscles, samples from pectoralis major (PM) muscles with varying severity of WB (WB score $0=$ normal; $1=$ mildly affected; $2=$ severely affected) were collected at 25 and 43 days post-hatch $(n=8$ per score per age) and processed for cryohistological and protein expression analyses. Collagen per field and densities of macrophages and MyoD+, Myf-5+, and Pax7+ MSC populations were quantified on immunofluorescence-stained cryosections. Relative collagen protein expression was quantified by fluorescent Western Blotting. In both 25 and 43-daysold broilers, the proportion of collagen per field $(P \leq 0.021)$ and macrophage density $(P \leq 0.074)$ were greater in PM exhibiting severe WB compared with normal. At day 43, populations of MyoD+, Myf-5+:MyoD+ MSC were larger and relative collagen protein expression was greater in WB-affected vs. unaffected broilers $(P \leq 0.05)$. Pax7+ MSC relative to total cells was also increased as WB severity increased in 43-days-old broilers $(P \leq 0.05)$. Densities of Myf-5+ $(P=0.092)$, MyoD+ $(P=0.030)$, Myf5+:MyoD+ 
( $P=0.046)$, and Myf-5+:MyoD+:Pax7+ $(P=0.048)$ MSC were greater in WB score 1 birds compared with WB score 0 and 2 birds. Overall, alterations in the resident MSC and macrophage populations and collagen protein content were observed in WBaffected muscle. Further investigation will be required to determine how these changes in cell population kinetics and local autocrine and paracrine signaling are involved in the apparent dysregulation of muscle maintenance in WB-affected broilers.

Keywords: Wooden Breast, muscle satellite cell, myogenic stem cell, macrophage, collagen infiltration, broiler chicken

\section{INTRODUCTION}

Both global and domestic demand for chicken meat continue to steadily increase making it arguably the most important meat protein source in the world. In the United States (US), the demand is greatest for high quality, white (breast, pectoralis major muscle, PM) meat. To meet this demand, the commercial poultry industry has placed tremendous genetic selection pressure on breast meat yield, growth rate, and feed efficiency traits and has made remarkable improvements over the last 40 years (Zuidhof et al., 2014). Unfortunately, along with those tremendous improvements has come a severe meat quality defect, the cause of which has yet to be elucidated. The defect referred to as both Woody Breast and Wooden Breast (WB) is characterized by visible bulging and extreme palpable hardness of the PM. The WB phenotype has been characterized by histopathologists as a degenerative myopathy that manifests in fast-growing, high-meat-yielding broiler chickens and results in myofiber necrosis, excessive fibrosis, and immune cell infiltration inside the perimysium (Petracci and Cavani, 2012; Sihvo et al., 2014; Velleman and Clark, 2015). The safety and wholesomeness of the product are not negatively impacted, but the poultry industry nevertheless continues to incur large economic losses due to decreased product acceptability and functionality (Kuttappan et al., 2016; Soglia et al., 2016; Tasoniero et al., 2016; Tijare et al., 2016). From a product quality standpoint, the WB phenotype has been reasonably well-characterized. However, to date, the specific cellular and molecular mechanisms that lead to the development of WB are still unclear.

Skeletal muscle satellite cells (MSCs) play a critical role in post-hatch broiler chicken skeletal muscle fiber hypertrophic growth and are essential for normal muscle maintenance and repair (Armand et al., 1983; Yablonka-Reuveni et al., 1987). The rapid increase in the muscle fiber cross-sectional area (CSA) that occurs in broiler chickens during the normal 4 to 10 -week rearing period is mediated by extensive MSC proliferation, differentiation (accompanied by withdrawal from the cell cycle), and fusion with the existing muscle fibers (Campion, 1984; Hutton et al., 2014). Thus far, the relationship between MSC function in rapidly-growing, high-yielding broilers and the development of the WB myopathy has been largely unexplored. However, it is known that the activation of muscle repair and regeneration pathways requires both the proliferation and differentiation of different MSC populations as well as the function of resident phagocytic cells such as anti-inflammatory and pro-inflammatory macrophages, which produce cytokines known to impact MSC function (Cantini et al., 1994). The relationships among the different MSC populations and macrophages and how they relate to collagen infiltration in WB-affected muscle are unclear. Therefore, the objective of this work was to explore the changes in the heterogeneity of myogenic regulatory factor (MRF) expression in MSC populations and to quantify macrophage densities and collagen protein expression in broilers with increasing severity of WB over time.

\section{MATERIALS AND METHODS}

\section{Bird Husbandry}

The Auburn University Institutional Animal Care and Use Committee approved the use of live birds and all procedures performed in this experimental protocol (PRN 2016-2829). As previous described by Meloche et al. (2018a), day-old, male, Yield Plus $\times$ Ross 708 broiler chicks were obtained from a commercial hatchery ( $n=480$, Aviagen Group, Huntsville, AL, United States). Chicks were vaccinated for Newcastle disease, Marek's disease, and infectious bronchitis at the hatchery. From 1 to 6 days of age, chicks were housed in groups of 8 in raised floor pens $\left(0.03 \mathrm{~m}^{2} /\right.$ bird $)$ bedded with new pine shavings, containing individual feeders, 2 nipple waterers per pen located in a solidsided, temperature-controlled, dehumidified research facility. At 7 days of age, all chicks were weighed and the lower and upper $12 \%$ of the BW range were excluded. The remaining 360 chicks were identified with wing bands and allocated by weight into the individual-housing pens $\left(0.20 \mathrm{~m}^{2} /\right.$ bird $)$. Ambient temperature was set to $33^{\circ} \mathrm{C}$ on day 0 and reduced to maintain comfort until day 43. Birds were exposed to a photoperiod of $23 \mathrm{~h}$ from placement to 7 days of age, followed by a photoperiod of $18 \mathrm{~h}$ for the remainder of the experiment. Light intensity was set at 30 lux from 1 to 7 days of age, 10 lux from 8 to 14 days of age, 5 lux from 15 to 24 days of age, and 3 lux from 25 to 43 days of age. Light intensity settings were verified at bird level $(30 \mathrm{~cm})$ using a photometric sensor with National Institute of Standards and Technology-traceable calibration (Model No. 403125, Extech Instruments, Waltham, MA, United States) for each intensity adjustment. All birds consumed fresh water and feed offered in four dietary phases on an ad libitum basis. Birds whose samples were chosen for this experiment all consumed the same corn and soybean meal-based Control grower 2 diet (formulated at 100\% of primary breeder nutrient recommendations for digestible Lys) from days 15 to 25 which is described in detail in Table 1 of Meloche et al. (2018a). 
TABLE 1 | Effect of Wooden Breast on density, relative density, and heterogeneity of Pectoralis major muscle satellite cell populations in broiler chickens at 25 days of age.

\begin{tabular}{|c|c|c|c|c|c|}
\hline \multirow[t]{2}{*}{ Cell population ${ }^{2,3}$} & \multicolumn{3}{|c|}{ Wooden Breast Score ${ }^{1}$} & \multirow[t]{2}{*}{ SEM $^{4}$} & \multirow[t]{2}{*}{$P$-value } \\
\hline & Normal (0) & Mild (1) & Severe (2) & & \\
\hline \multicolumn{6}{|l|}{ Density, cells per $\mathrm{mm}^{2}$} \\
\hline Myf-5-:MyoD-:Pax7- (non-myogenic) & 412.69 & 521.44 & 547.55 & 107.92 & 0.650 \\
\hline Myf-5+ & 106.63 & 84.75 & 139.37 & 19.46 & 0.161 \\
\hline MyoD+ & 16.38 & 16.50 & 12.00 & 6.44 & 0.855 \\
\hline $\operatorname{Pax} 7+$ & 60.25 & 60.63 & 78.75 & 13.85 & 0.567 \\
\hline Myf-5+:MyoD+ & 37.38 & 39.50 & 35.00 & 13.31 & 0.972 \\
\hline Myf-5+:Pax7+ & 11.25 & 17.25 & 7.50 & 6.50 & 0.573 \\
\hline MyoD+:Pax7+ & 6.75 & 12.75 & 15.75 & 5.06 & 0.454 \\
\hline Myf-5+:MyoD+:Pax7+ & 18.00 & 21.00 & 45.50 & 9.59 & 0.109 \\
\hline \multicolumn{6}{|l|}{ Relative density, $\%$ of total DAPI+ } \\
\hline Myf-5-:MyoD-:Pax7 - (non-myogenic) & 61.69 & 67.46 & 62.14 & 0.05 & 0.644 \\
\hline Myf-5+ & 15.94 & 10.96 & 15.82 & 0.03 & 0.459 \\
\hline MyoD+ & 2.45 & 2.14 & 1.36 & 0.01 & 0.487 \\
\hline $\operatorname{Pax} 7+$ & 9.01 & 7.84 & 8.94 & 0.01 & 0.825 \\
\hline Myf-5+:MyoD & 5.58 & 5.11 & 3.97 & 0.02 & 0.825 \\
\hline Myf-5+:Pax7+ & 1.68 & 2.23 & 0.85 & 0.01 & 0.569 \\
\hline MyoD+:Pax7+ & 1.01 & 1.65 & 1.79 & 0.01 & 0.649 \\
\hline MyoD+:Myf-5+: Pax7+ & 2.69 & 2.72 & 5.16 & 0.01 & 0.173 \\
\hline \multicolumn{6}{|l|}{ Relative density, \% of total myogenic } \\
\hline Myf-5+ & 41.59 & 33.65 & 41.78 & 0.05 & 0.374 \\
\hline MyoD+ & 6.38 & 6.55 & 3.59 & 0.02 & 0.537 \\
\hline $\operatorname{Pax} 7+$ & 23.50 & 24.07 & 23.60 & 0.06 & 0.997 \\
\hline Myf-5+:MyoD+ & 14.58 & 15.68 & 10.49 & 0.04 & 0.587 \\
\hline Myf-5+:Pax7+ & 4.39 & 6.85 & 2.25 & 0.02 & 0.416 \\
\hline MyoD+:Pax7+ & 2.63 & 5.06 & 4.72 & 0.02 & 0.593 \\
\hline Myf-5+:MyoD+: Pax7+ & 7.02 & 8.34 & 13.64 & 0.03 & 0.104 \\
\hline
\end{tabular}

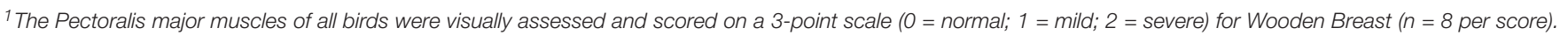

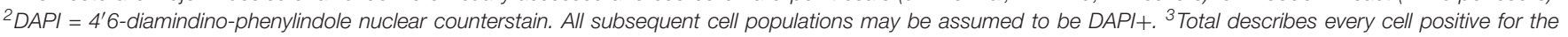

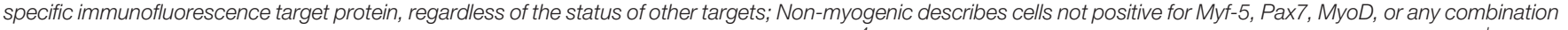

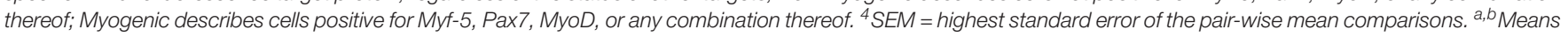
within the same row with different superscripts differ $P \leq 0.05 .{ }^{x, y}$ Means within the same row with different superscripts differ $0.0501 \leq P \leq 0.10$.

\section{Wooden Breast Scoring and Muscle Sample Collection}

At days 25 and 43 post-hatch, birds $(n=50$ per day) were euthanized by $\mathrm{CO}_{2}$ asphyxiation followed immediately by cervical dislocation and samples $(\approx 1.25 \mathrm{~cm} \times 0.635 \mathrm{~cm} \times 0.635 \mathrm{~cm})$ from the anteroventral portion of the left PM muscle were excised and processed for cryohistological immunofluorescence staining analyses according to procedures adapted from Hutton et al. (2014) and described in Meloche et al. (2018a). Muscle samples immediately adjacent those taken for cryohistology were also collected from each bird, snap frozen in liquid nitrogen, and stored at $-80^{\circ} \mathrm{C}$ prior to analysis for protein expression by quantitative, fluorescent Western Blotting as described below. Samples for this experiment ( $n=8$ per WB score per day) were obtained from birds with WB scores of 0,1 , and 2 on a 3 -point scale (WB score $0=$ normal, $1=$ mildly affected, and $2=$ severely affected) as determined by visual evaluation and physical palpation of the PM muscles at sampling. All PM muscles were scored by the same evaluator and considered "normal" if there was no palpable hardness in any of the PM, "mild" if palpable hardness was present in less than half the total PM muscle surface area, and "severe" if it exceeded this limit.

\section{Cryohistological Immunofluorescence Analysis}

Samples stored at $-80^{\circ} \mathrm{C}$ prior to analysis were warmed to $-20^{\circ} \mathrm{C}$ for at least $16 \mathrm{~h}$ and subsequently cryosectioned using a Leica CM 1950 cryomicrotome. Serial 5- $\mu$ m-thick, cross-sections were cut from each PM sample, mounted on positively charged glass slides (VWR International, Westchester, PA, United States), and stored at $4^{\circ} \mathrm{C}$ before immunofluorescence staining as described in Meloche et al. (2018a). All slides were briefly counter-stained with 4',6-diamidino-phenylindole (DAPI; $1 \mu \mathrm{g}$ per $\mathrm{mL}$; VWR International) to facilitate determination of total nuclear density. Control cryosections processed as described above, but without the addition of either primary or secondary antibodies, were used to ensure that no fluorescence signal 
beyond natural autofluorescence was observed for the selected combination of antibodies confirmed to be cross-reactive with chicken described below.

Immunofluorescence-stained cryohistological slides were imaged at 100-fold and 200-fold magnification with an inverted fluorescence microscope (Nikon Eclipse, Ti-U; Nikon Instruments, Inc., Melville, NY, United States) fitted with a UV light source (Nikon Intensilight). Images were captured and analyzed using an Evolve 512 EMCCF camera (Photometrics, Tucson, AZ, United States) and Elements imaging software (Nikon Instruments, Inc.). A representative digital image at both magnifications was captured from each slide (2 slides per bird). Slides were simultaneously immunofluorescence-stained for the nuclear MRF MSC markers, Myf-5, MyoD, and Pax7 for determination of MSC population densities and heterogeneity of MRF expression, The MSC populations (Myf-5+, MyoD+, Pax7+, Myf-5+: MyoD+, Myf-5+: Pax7+, MyoD+:Pax7+, and Myf-5+:MyoD+:Pax7+) were enumerated in the 200-fold magnification images and their densities expressed on a per $\mathrm{mm}^{2}$ basis (Tables 1, 2). All cell populations enumerated were also DAPI + in addition to their immunofluorescence profile. The total number of DAPI+ nuclei per image was determined in each image as a measure of nuclear density and to determine Total DAPI+ cells. Any cells positive for Myf-5, MyoD, Pax7 or any combination thereof were considered myogenic and those not positive for any of the target antigens were considered non-myogenic using antibodies previously validated for crossreactivity with chicken (Yablonka-Reuveni, 1995; Day et al., 2009; Tejeda et al., 2019). Additional serial slides from 43-days-old birds were immunofluorescence-stained for sarcomeric myosin, collagen, and Pax7 to facilitate the visual illustration of the extensive collagen infiltration and increases in the local MSC populations observed in PM muscles severely affected with WB compared with those receiving normal WB scores (Figures 1AC). The proportion of collagen fluorescence in each image was determined using the binary component in Elements software as previously reported by Murphy et al. (2011). Subsequently, additional slides with serial cryosections to those stained for

TABLE 2 | Effect of Wooden Breast on density, relative density, and heterogeneity of Pectoralis major muscle satellite cell populations in broiler chickens at 43 days of age.

\begin{tabular}{|c|c|c|c|c|c|}
\hline \multirow[t]{2}{*}{ Cell population ${ }^{2,3}$} & \multicolumn{3}{|c|}{ Wooden Breast Score ${ }^{1}$} & \multirow[t]{2}{*}{ SEM $^{4}$} & \multirow[t]{2}{*}{$P$-value } \\
\hline & Normal (0) & Mild (1) & Severe (2) & & \\
\hline \multicolumn{6}{|l|}{ Density, cells per $\mathrm{mm}^{2}$} \\
\hline Myf-5-:MyoD-:Pax7 - (non-myogenic) & 267.43 & 308.41 & 403.76 & 56.25 & 0.236 \\
\hline Myf-5+ & $123.62^{x}$ & $187.12^{y}$ & $121.38^{x}$ & 22.83 & 0.092 \\
\hline MyoD+ & $3.75^{a}$ & $30.63^{b}$ & $9.00^{a}$ & 7.06 & 0.032 \\
\hline $\operatorname{Pax} 7+$ & $13.50^{x}$ & $26.25^{x y}$ & $52.87^{y}$ & 11.43 & 0.067 \\
\hline Myf-5+:MyoD+ & $58.13^{a}$ & $177.13^{b}$ & $57.37^{a}$ & 36.38 & 0.046 \\
\hline Myf-5+:Pax7+ & 0.75 & 7.50 & 3.75 & 2.79 & 0.252 \\
\hline MyoD+:Pax7+ & 10.50 & 12.00 & 3.00 & 4.08 & 0.269 \\
\hline Myf-5+:MyoD+:Pax7+ & $30.00^{a}$ & $72.37^{b}$ & $41.87^{a b}$ & 11.63 & 0.048 \\
\hline \multicolumn{6}{|l|}{ Relative density, $\%$ of total DAPI+ } \\
\hline Myf-5-:MyoD-:Pax7 - (non-myogenic) & $52.71^{a b}$ & $37.57^{a}$ & $58.29^{b}$ & 0.06 & 0.050 \\
\hline Myf-5+ & 24.37 & 22.80 & 17.52 & 0.04 & 0.272 \\
\hline MyoD+ & $0.74^{a}$ & $3.73^{b}$ & $1.29^{a}$ & 0.01 & 0.033 \\
\hline $\operatorname{Pax} 7+$ & $2.67^{a}$ & $3.19^{a}$ & $7.63^{b}$ & 0.01 & 0.042 \\
\hline Myf-5+:MyoD+ & 11.46 & 21.58 & 8.28 & 0.05 & 0.115 \\
\hline Myf-5+:Pax7+ & 0.15 & 0.92 & 0.54 & 0.01 & 0.452 \\
\hline MyoD+:Pax7+ & 2.07 & 1.46 & 0.43 & 0.01 & 0.121 \\
\hline Myf-5+:MyoD+:Pax7+ & 5.91 & 8.82 & 6.05 & 0.01 & 0.243 \\
\hline \multicolumn{6}{|l|}{ Relative density, $\%$ of total myogenic } \\
\hline Myf-5+ & 51.54 & 36.51 & 42.02 & 0.06 & 0.174 \\
\hline MyoD+ & 1.56 & 5.98 & 3.12 & 0.01 & 0.105 \\
\hline $\operatorname{Pax} 7+$ & $5.63^{a b}$ & $5.12^{a}$ & $18.30^{b}$ & 0.03 & 0.029 \\
\hline Myf-5+:MyoD+ & 24.23 & 34.56 & 19.86 & 0.06 & 0.191 \\
\hline Myf-5+:Pax7+ & 0.31 & 1.46 & 1.29 & 0.01 & 0.702 \\
\hline MyoD+:Pax7+ & $4.38^{a}$ & $2.34^{a b}$ & $1.04^{b}$ & 0.01 & 0.085 \\
\hline Myf-5+:MyoD+:Pax7+ & 12.51 & 14.12 & 14.50 & 0.03 & 0.864 \\
\hline
\end{tabular}

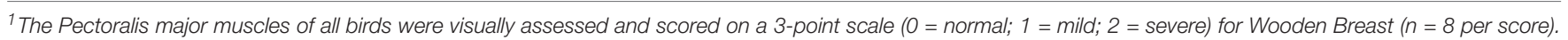

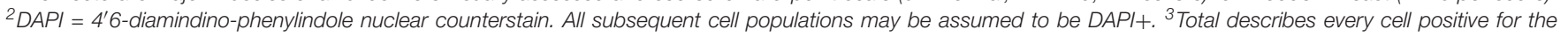

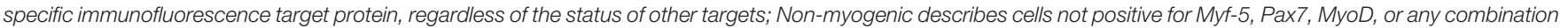

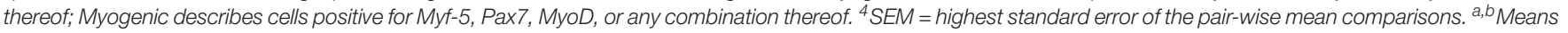
within the same row with different superscripts differ $P \leq 0.05{ }^{x, z}$ Means within the same row with different superscripts differ $0.0501 \leq P \leq 0.10$. 


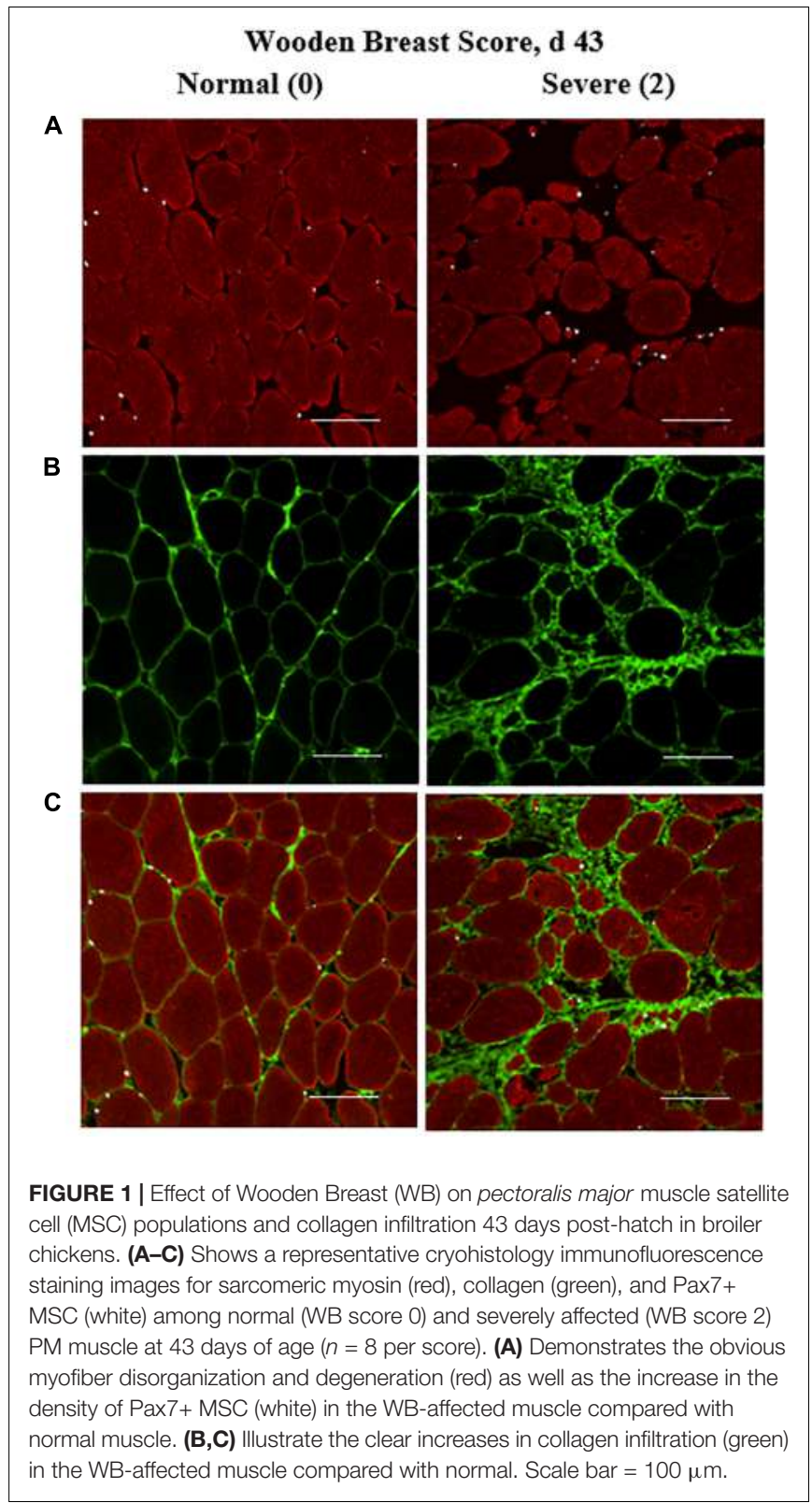

MRF heterogeneity were immunofluorescence-stained from each bird and the 200-fold digital images were used to determine the proportion of collagen per image and density $\left(\right.$ per $\mathrm{mm}^{2}$ ) of the total macrophages, including both pro and anti-inflammatory populations using a general leukocyte/macrophage marker previously validated for use in chickens Mast et al. (1998) (Figures 3A-C).

Primary antibodies utilized were as follows: rabbit IgG Type1, $\alpha 1$ Collagen [Cat. No. sc-8784-R, 1:1,500 dilution; Santa Cruz Biotechnology, Santa Cruz, CA (SCB)]; mouse IgG1 Monocyte/Macrophage (Cat. No. sc-52603, 1:750 dilution; SCB); mouse IgG2b MyoD (Cat. No. sc-377460, 1:2,000 dilution; SCB); rabbit IgG Myf-5 (Cat. No. sc-302, 1:100 dilution; SCB), mouse IgG2b sarcomeric myosin (Cat. No. MF20, 1:10 dilution, Developmental Studies Hybridoma Bank (DSHB), Iowa
A

$\mathrm{SEM} \leq \mathbf{0 . 0 1}$

$P=0.005$

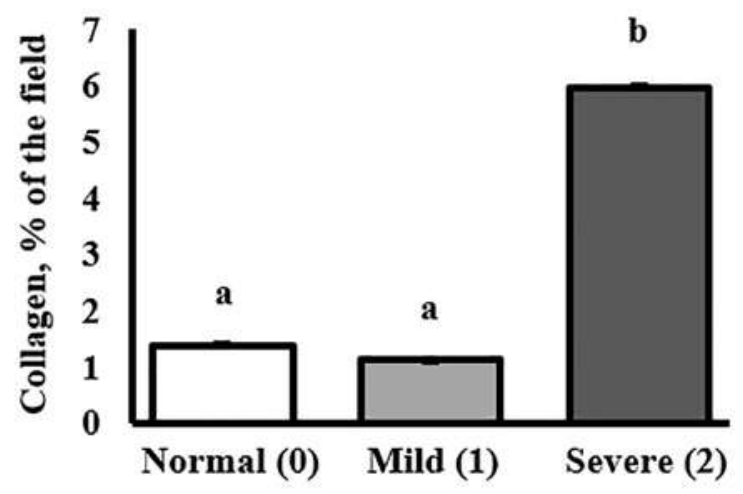

Wooden Breast Score, $\mathrm{d} 25$

B

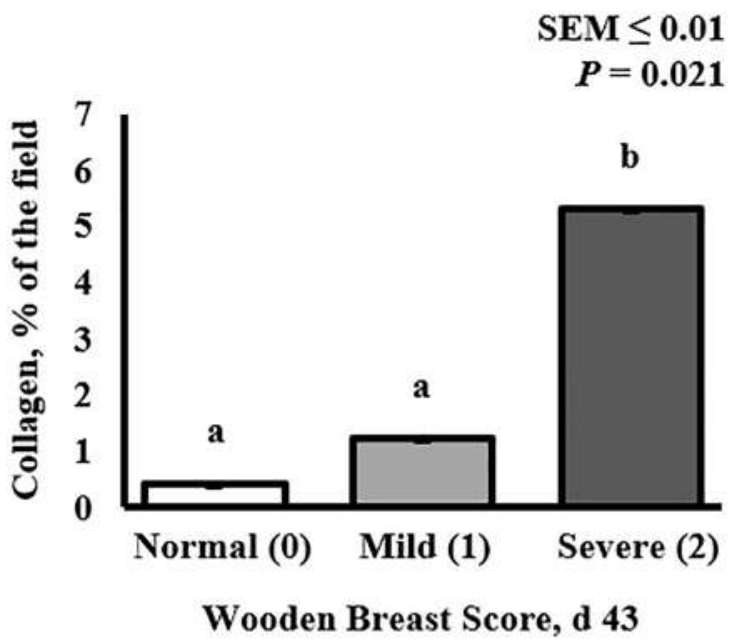

FIGURE 2 | Effect of Wooden Breast (WB) on pectoralis major muscle collagen infiltration 43 days post-hatch in broiler chickens. (A,B) Demonstrate the increase in the proportion of collagen per digital image in muscles severely affected by WB on both day $25(P=0.005)$ and $43(P=0.021)$, respectively ( $n=8$ per score). ${ }^{\text {a,b }}$ Means with different superscripts differ $P<0.05$.

City, IA, United States) and mouse IgG2b Pax7 (Cat. No. PAX7, 1:10 dilution, DSHB). Secondary antibodies (1:1,000 dilution) used for detection of the primary antibodies were as follows: AlexaFluor 488 Goat anti-rabbit IgG $(\mathrm{H}+\mathrm{L})$, AlexaFluor 546 Goat anti-mouse IgG1, AlexaFluor 633 Goat anti-mouse IgG2b, AlexaFluor 546 Goat anti-mouse IgG2b, AlexaFluor 633 Goat anti-mouse IgG1, and AlexaFluor 546 Goat antimouse IgG2b (Thermo Fisher Scientific/Invitrogen, Waltham, MA, United States).

\section{Quantitative Fluorescent Western Blot Protein Expression Analysis}

Pectoralis major muscle tissue samples $(\sim 250 \mathrm{mg})$ were placed in ice cold T-PER lysis buffer (Cat. No. 78510; Thermo 


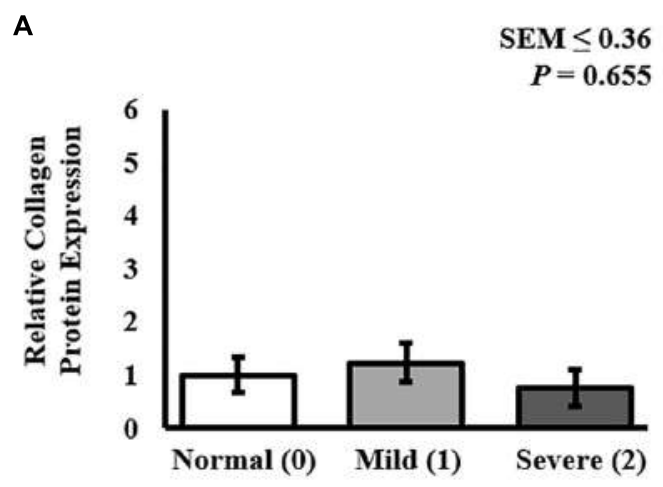

Wooden Breast Score, $d 25$
C
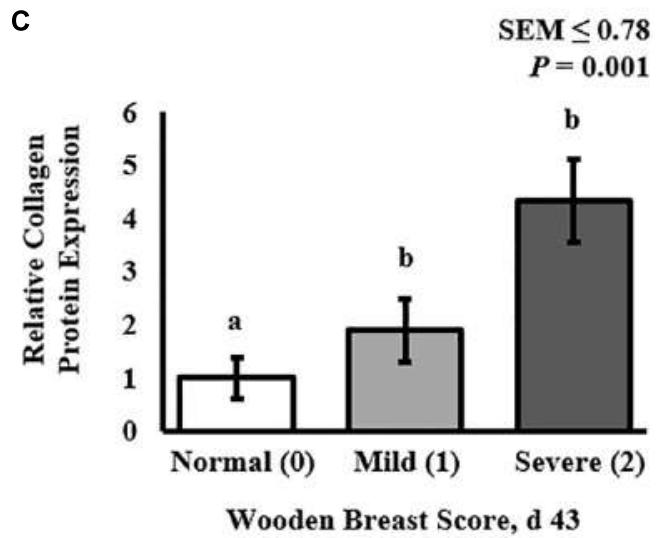

B

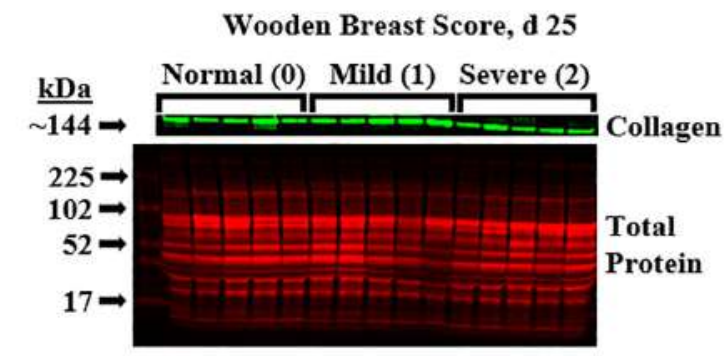

D

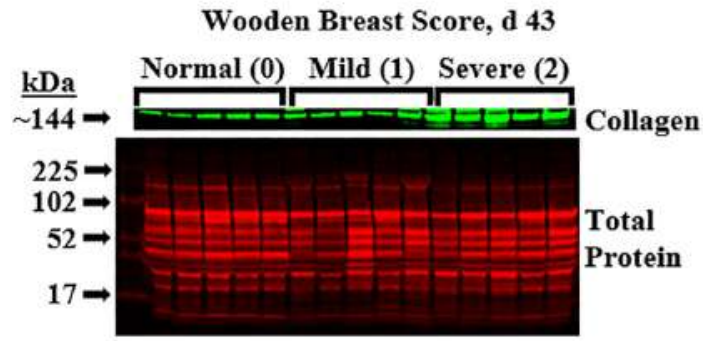

FIGURE 3 | Effect of Wooden Breast (WB) on relative collagen protein expression in the pectoralis major muscle of broiler chickens at 25 and 43 days post-hatch using fluorescent Western Blot analysis. Collagen protein expression was first normalized to total protein on a per lane (individual bird) basis and then set relative to the mean normal (WB score 0) expression ( $n=8$ per score per d). At 25 days of age $\mathbf{( A , B ) , ~ b r o i l e r ~ P M ~ m u s c l e ~ c o l l a g e n ~ p r o t e i n ~ e x p r e s s i o n ~ w a s ~ s i m i l a r ~ a m o n g ~ a l l ~ W B ~}$ scores $(P=0.655)$. At day $43(\mathbf{C}, \mathbf{D})$, collagen protein expression was increased in WB-affected PM muscles (WB scores 1 and 2$)$ compared with normal (WB score 0) muscles $(P=0.001)$. ${ }^{\text {a,b }}$ Means with different superscripts differ $P<0.05$.

Fisher Scientific) supplemented with a $2 \mathrm{X}$ final concentration of Halt protease and phosphatase inhibitor cocktail (Cat. No. 78441; Thermo Fisher Scientific). Samples were homogenized using a Qiagen TissueLyser II (Cat. No. 85300; Qiagen, Germantown, MD, United States) twice at $30 \mathrm{~Hz}$ for $2 \mathrm{~min}$ using the manufacturer's instructions for homogenization. After homogenization, samples were centrifuged at $12,000 \times g$ for $10 \mathrm{~min}$. Supernatants were carefully removed and protein concentrations were determined using a Pierce BCA Protein Assay Kit (Cat. No. 23225; Thermo Fisher Scientific) with a NanoDrop One spectrophotometer (ND-ONEC-W; Thermo Fisher Scientific). Samples at $160 \mu \mathrm{g}$ of total protein were mixed with lysis buffer to achieve a $20-\mu \mathrm{L}$ final volume. Samples were then mixed with $1 \mu \mathrm{L}$ of Cy 5 dye from the Amersham QuickStain Protein Labeling Kit (Cat. No. RPN4000; GE Healthcare, Chicago, IL, United States) to stain total protein. Samples were incubated at room temperature in the dark for 30 min per the manufacturer's instructions for the Amersham QuickStain Protein Labeling Kit. After, 4X Fluorescent Compatible Sample Buffer (Cat. No. LC2570; Invitrogen) and $\beta$-mercaptoethanol were added to each sample to achieve a final concentration of
1X sample buffer and $10 \mathrm{~m} M \beta$-mercaptoethanol. Samples were vortexed, and then heated to $95^{\circ} \mathrm{C}$ and held for $3 \mathrm{~min}$. Samples were loaded onto 4 to $20 \%$ gradient Criterion TGX precast midi gels (Cat. No. 5671094; Bio-Rad, Hercules, CA, United States) with Amersham ECL Plex Fluorescent Rainbow Markers (Cat. No. RPN851E; GE Healthcare) being added to the first and last lanes of each gel. Gels were electrophoresed at $80 \mathrm{~V}$ for $10 \mathrm{~min}$ and then $120 \mathrm{~V}$ for 60 to $65 \mathrm{~min}$ (until the dye front reached the bottom of the gel) in a Criterion Electrophoresis Midi Vertical Cell (Cat. No. 1656001; Bio-Rad). After electrophoresis, gels were transferred to low-fluorescent polyvinylidene fluoride (PVDF) membranes from a Trans-Blot Turbo RTA Midi LF PVDF Transfer Kit (Cat. No. 1704275; Bio-Rad) using a TransBlot Turbo Transfer System (Cat. No. 1704150; Bio-Rad) per the manufacturer's instructions. Membranes were then blocked for $1 \mathrm{~h}$ at room temperature using Intercept (TBS) Blocking Buffer (Cat. No. P/N: 927-60001; LI-COR Biosciences, Lincoln, NE, United States). After blocking, membranes were incubated in anti-Type 1, $\alpha 1$ Collagen (Cat. No. sc-8784-R; SCB) primary antibody diluted 1:500 in Intercept T20 (TBS) Antibody Diluent (Cat. No. P/N:927-65001; LI-COR) overnight $(\sim 16 \mathrm{~h})$ at $4^{\circ} \mathrm{C}$. 
The following morning, membranes were washed three times for 5 min each in tris-buffered saline $+0.01 \%$ Tween 20 (TBST). Membranes were incubated in AlexaFluor Plus 555 Goat antiRabbit IgG (H+L) Highly Cross-Absorbed Secondary Antibody (Cat. No. A21428; Thermo Fisher Scientific) diluted 1:5,000 in Intercept T20 (TBS) Antibody Diluent at room temperature for $1 \mathrm{~h}$. Membranes were then washed three times for 5 min each in TBST and allowed to air dry for $3 \mathrm{~h}$ in a dark room. Dried membranes were imaged using an Amersham Imager 600 (Cat. No. 29083461; GE Healthcare) using the fluorescent settings for green/Cy3 (collagen, and green fluorescent protein ladder markers), and red/Cy5 (total protein and red fluorescent protein ladder markers) channels for 5 and $4 \mathrm{~s}$, respectively. Fluorescent band intensity for collagen and total protein were quantified using Image Quant TL 8.1 software (Cat. No. 29000737; GE Healthcare). Collagen protein expression was first normalized to total protein on a per lane (individual bird) basis and then set relative to the mean WB score 0 expression (Figure 2).

\section{Statistical Analysis}

Statistical analysis was performed using the GLIMMIX procedure of SAS (PC version 9.4, SAS Inst. Inc., Cary, NC, United States). For all data analysis, WB score served as the fixed effect and the Satterthwaite adjustment was used to correct degrees of freedom with individual bird serving as the experimental unit. Bird BW and PM weight were tested as possible covariates for all independent variables and were found to be insignificant resulting in their exclusion from the model. Proportional data were analyzed using the events/experiments syntax with a binomial distribution and both continuous and proportional data were analyzed using an R-side covariance structure. All treatment means were separated using the PDIFF option and considered different when $P \leq 0.05$. Tendencies for differences among treatment means were declared when $0.0501 \leq P \leq 0.10$.

\section{RESULTS}

\section{Muscle Satellite Cell Population Heterogeneity}

Heterogeneity of MSC populations in broilers with varying WB severity were assessed and are reported in Table 1 (day 25) and 2 (day 43). Heterogeneity and densities of the Myf-5+, MyoD+, and Pax7+ MSC in the PM of broilers harvested at 25 days post-hatch were similar among WB score $(P>0.10$; Table 1). However, at day 43 post-hatch, there were considerable alterations in the Myf-5+, MyoD+, and Pax7+ MSC populations in PM of broilers with varying WB scores (Table 2). In 43days-old broilers, as WB score increased the density of the Pax7+ MSC $(P=0.067)$ and relative density of MyoD+:Pax7+ MSC as a proportion of the total MSC population tended to increase $(P=0.085)$. Score 1 or mildly WB-affected birds also had increased densities of $\mathrm{Myf}-5+(P=0.092)$, MyoD+ $(P=0.03)$, Myf5+:MyoD+ $(P=0.046)$ compared with normal and severely affected (score 2 ) birds. In addition, the density of MSC expressing all 3 MSC markers (Myf-5+:MyoD+:Pax7+) was greater in muscles of mildly affected birds compared with unaffected and severely affected with WB $(P=0.048)$. The density and relative densities of the Myf5+:Pax7+ and MyoD+:Pax7+ MSC populations were unaltered by WB $(P>0.10)$. The relative density of Pax7+ MSC as a proportion of total DAPI+ and total myogenic cells was greater in severely affected broilers compared with mildly affected birds $(P \leq 0.042$; Figure 1A). Densities of the non-myogenic Myf-5-:MyoD-:Pax7- populations were similar among WB scores $(P=0.236)$. However, the mildly affected (score 1) PM had lower proportions of total cells considered nonmyogenic than muscles from unaffected or severely affected birds 43 days post-hatch $(P=0.05$; Table 2$)$.

\section{Collagen Infiltration and Collagen Protein Expression}

Collagen infiltration into WB-affected PM muscle was assessed at both 25 and 43 days post-hatch in PM muscle cryosections by immunofluorescence staining and digital fluorescence microscopic analysis (Figures 1, 4) and quantitative results are displayed in Figure 2. At both ages, severely affected broilers (WB score 2) exhibited greater proportions of collagen per image compared with mildly affected and normal birds $(P \leq 0.021$; Figures 1, 2). Relative collagen protein expression in PM tissue was also assessed at both days 24 and 43 in the same birds sampled for the cryohistology analysis using fluorescent Western Blotting (Figure 3). On day 25 post-hatch, relative collagen expression was similar among WB score $(P=0.655)$, while on day 43 , birds affected with WB had increased collagen protein expression compared with unaffected broilers $(P=0.001)$.

\section{Muscle Macrophage Density}

The density of the total macrophage population (including both pro- and anti-inflammatory cells populations) in the PM of broilers was assessed by cryohistological and immunofluorescence analysis at both days 25 and 43 post-hatch in broilers with varying degrees of WB severity (Figure 4) and quantitative results are shown in Figure 5. The density of macrophages increased as WB score increased at both 25 $(P=0.023)$ and $43(P=0.074)$ days post-hatch (Figure 5).

\section{DISCUSSION}

The cellular and molecular mechanisms involved in the development of the broiler chicken WB myopathy are still not well-understood and the underlying cause has yet to be elucidated. Many different nutritional and management strategies have been aimed at eliminating the condition. Those strategies have included feed restrictions to slow growth rates, reductions in dietary nutrient density and specific nutrients, addition of antioxidants and chelated minerals, changes in electrolytes, changes in management such as restricting lighting and changing temperature conditions in the hatching and rearing facilities (Trocino et al., 2015; Wedekind et al., 2016; Chen et al., 2017; Kindlein et al., 2017; Livingston and Brake, 2017; Manangi et al., 2017; Meloche et al., 2018b,c,d). Yet, all these different nutritional and management strategies have failed to completely 


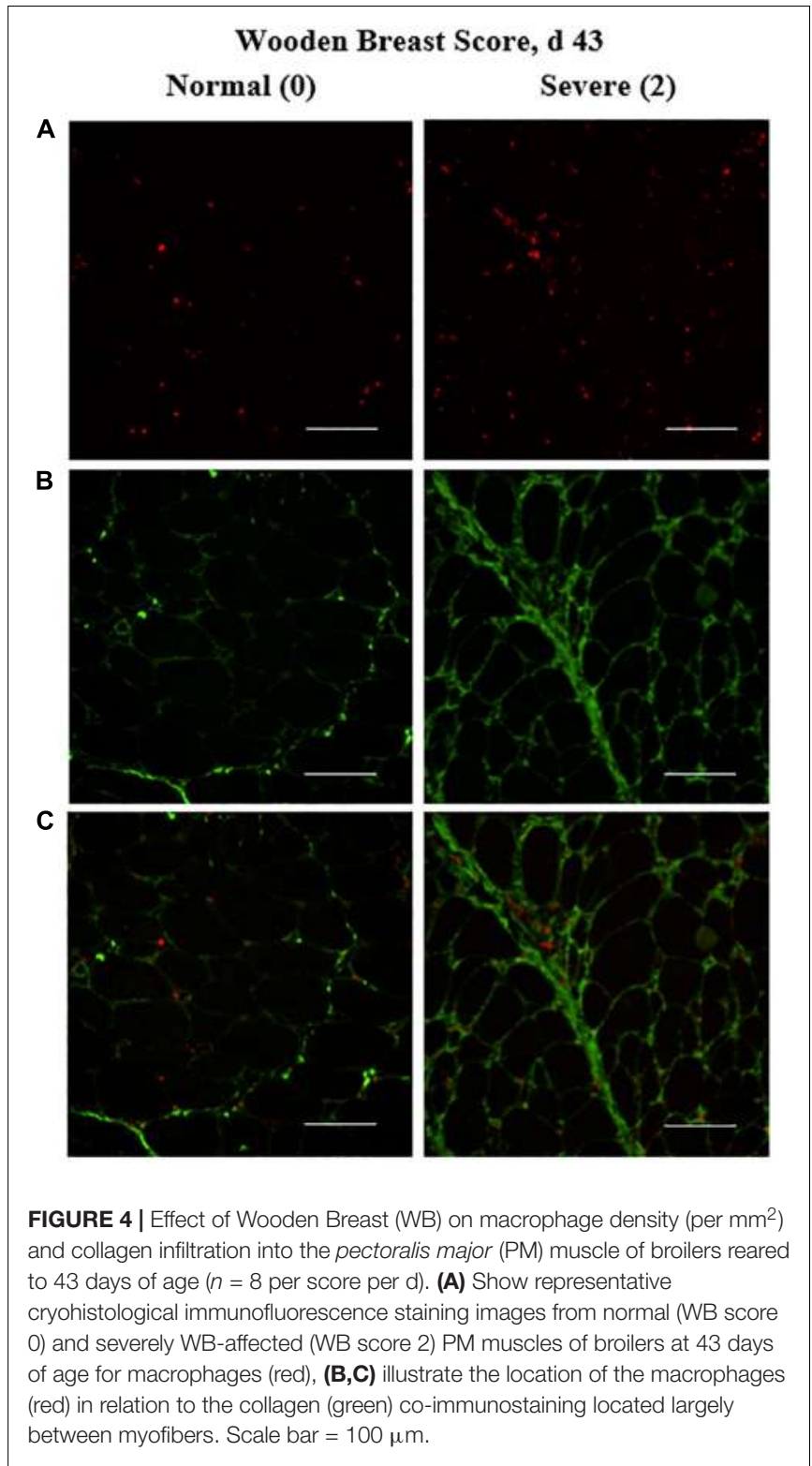

eliminate the WB myopathy in fast-growing, high-yielding commercial broilers.

The broiler industry's inability to eliminate WB through posthatch nutritional and management strategies combined with its widespread manifestation in a large proportion of the modern commercial broiler genetic lines grown globally suggests that selection of modern broilers over the last several decades for breast meat yield and feed efficiency placed inadvertent selection pressure on post-hatch hypertrophic growth of muscle fibers instead of pre-hatch muscle fiber hyperplastic growth. It is possible that this has contributed to post-hatch muscle tissue architecture with limited vascularity and oxygenation capacity creating a cellular environment that is simply incompatible with normal muscle growth and maintenance resulting in the WB myopathic phenotype. This theory is supported by recent work focused on exploring the differential transcriptomic and

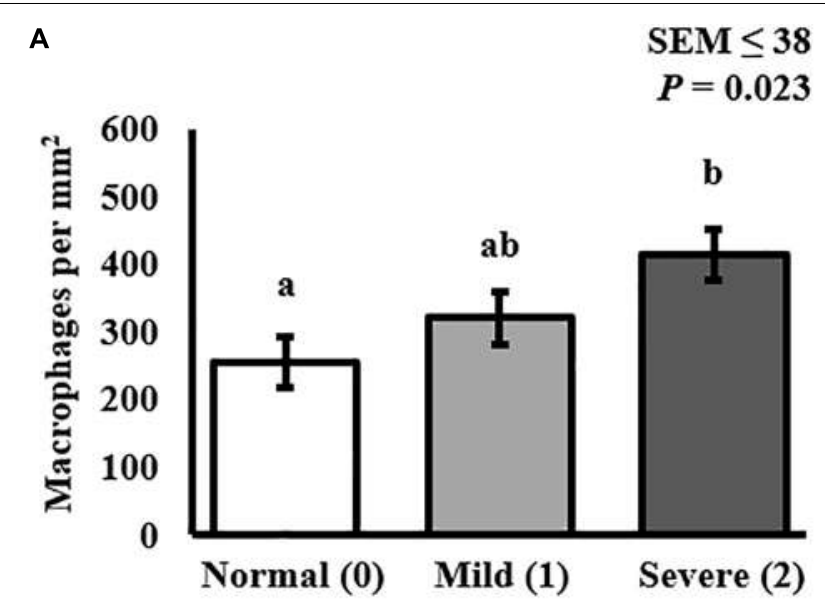

Wooden Breast Score, d 25

B

$\mathrm{SEM} \leq 50$

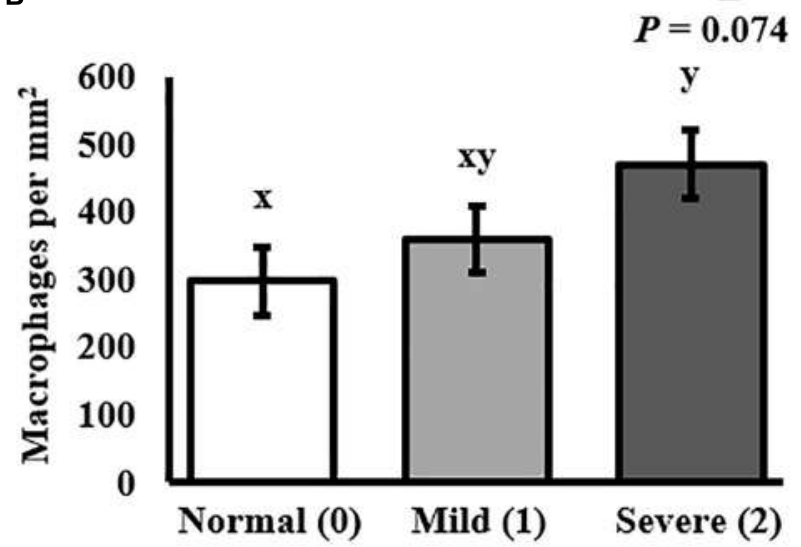

Wooden Breast Score, d 43

FIGURE 5 | Effect of Wooden Breast (WB) on macrophage density (per $\mathrm{mm}^{2}$ ) in the pectoralis major (PM) muscle of broilers reared to 43 days of age $(n=8$ per score per d). (A) Demonstrates that macrophage density increases as WB score increases in 25-days-old broilers $(P=0.023)$. (B) Demonstrates that macrophage density tended to increase as WB score increased in 43-days-old broilers $(P=0.074)$. ${ }^{\text {a,b }}$ Means with different superscripts differ $P<0.05$.

proteomic gene expression profiles in which dysregulation of various metabolic and muscle maintenance pathways have been observed (Mutryn et al., 2015; Abasht et al., 2016; Soglia et al., 2016, 2019; Kuttappan et al., 2017; Brothers et al., 2019; Greene et al., 2019).

The role of MSC function in the development of the WB phenotype is also not clear. However, the limited work conducted to date suggests that MSC function eventually becomes compromised in WB-affected broilers, leaving the rapidly growing $\mathrm{PM}$ improperly maintained, thus creating an environment in which collagen infiltration/fibrosis occurs (Velleman, 2015; Daughtry et al., 2017). The increases in mRNA 
for the MRF, MyoD and Myogenin, and the collagen crosslinking regulator, Decorin, in broilers with severe WB (Velleman and Clark, 2015) combined with the observation that MSC differentiation capacity is reduced as broilers age (Daughtry et al., 2017) support this view. Our previous findings that the mitotic activity of MSC populations and myofiber CSA distributions are significantly altered as WB scores increase further support the idea that MSC function is compromised in WB-affected broilers (Meloche et al., 2018a).

The WB myopathic phenotype in broilers has been largely characterized from a histopathological standpoint using paraffin histology, single antigen immunohistochemistry, and various traditional histological stains such as hematoxylin, eosin, and Masson's trichrome with light microscopy methods. In the current study, our objective was to use a combination of cryohistological and immunofluorescence microscopy and quantitative protein expression techniques to expand the exploration of the cellular and molecular changes that occur in WB-affected broilers over time. Here, we characterized the heterogeneity of MSC populations expressing 3 MRF (Myf5, MyoD, and Pax7) and quantified macrophage density, collagen infiltration, and collagen protein expression in normal, mildly affected, and severely affected broilers at 25 and 43 days of age. The use of experimental techniques such as cryohistology and multiplexed immunofluorescence staining as well as quantitative fluorescent Western Blotting to explore the WB myopathy at the cellular and molecular level is novel compared with current literature employing traditional paraffin histological analyses.

Here, no differences in the Myf-5, MyoD, and Pax7expressing MSC populations were observed at day 25 in broilers differentially affected with WB (Table 1). This finding is in agreement with our previous study where the size of the total Myf-5+ and Pax7+ populations were similar in PM muscles from birds with WB scores of 0,1 , and 2 at day 25 post-hatch (Meloche et al., 2018a). On day 43, however, there were alterations in the Myf-5, MyoD, and Pax7-expressing MSC populations (Table 2). Interestingly, the Score 1 or mildly WB-affected birds had increased densities of Myf-5+, MyoD+, Myf5+:MyoD+, Myf-5+:MyoD+:Pax7+ MSC populations compared with WB scores of 0 and 2 (Table 2). The changes observed in this study at 43 days in the various Myf-5 and Pax7-expressing MSC populations among various WB scores are also similar to those observed in our previous work (Meloche et al., 2018a). The shifts observed in the MSC populations expressing MyoD at day 43 are in agreement with previous reports of increased MyoD mRNA transcripts in muscle severely affected with WB (Velleman and Clark, 2015). However, the reason for these shifts in the MSC growth kinetics that occur during the development of the WB myopathy are not clear. Perhaps in a mildly affected bird, the MSC are still in the process of trying to repair the damage and in birds of the same age that have already progressed to the severe phenotype this process has already ended. Based on these results, further investigation of the proliferation and differentiation capacity of MSC from WB score 0,1 , and 2 birds both in vitro and in vivo is warranted.
The quantitative increases in collagen infiltration in immunofluorescence-stained PM cryosections within both the endomysial and perimysial layers of connective tissue in WB-affected muscles observed in this study (Figures 1, 4) align with previous literature in which traditional paraffin histopathology methods were utilized to demonstrate this striking characteristic of the WB myopathy (Sihvo et al., 2014, 2017; Velleman and Clark, 2015). In addition, the increased relative collagen protein content of WB-affected muscle observed in 43-days-old broilers is supported by others' work in which the in vivo collagen synthesis rates are upregulated in WB-affected broilers (Maharjan et al., 2019).

The increases in PM macrophage density as WB score increased are in alignment with previous qualitative work describing immune cell infiltration as a histopathological characteristic of the WB myopathy (Sihvo et al., 2014, 2017). We are unaware of other reports in which density of these resident phagocytic immune cells has been quantified in relation to WB severity over time. The major limitation of our macrophage analysis is the inability to distinguish between the pro- and anti-inflammatory macrophage populations due to the absence of commercially available antibodies reactive to these macrophage populations in chickens. Further characterization of these functionally divergent macrophage populations as well as their cell signaling secretory products is warranted.

Overall, the shifts in the MSC population MRF heterogeneity observed previously as well as in this study are novel and may indicate dysregulation of the MSC proliferation and differentiation processes in WB-affected muscles. Determining whether this apparent issue with MSC function is a symptom or cause of the WB myopathy, how local macrophages are involved, and what autocrine and paracrine cell signaling mechanisms may be driving this apparent inability to maintain rapidly growing muscles in today's high-yielding, commercial broiler chickens will require further investigation.

\section{DATA AVAILABILITY STATEMENT}

All datasets generated for this study are included in the article/supplementary material..

\section{ETHICS STATEMENT}

The animal study was reviewed and approved by the Auburn University Institutional Animal Care and Use Committee under Protocol No. 2016-2829.

\section{AUTHOR CONTRIBUTIONS}

LK, TF, SV, VN, JF, KM, and JS conceptualized the studies and contributed to the scientific discussion. TF and JS conducted the immunofluorescence analysis. TF wrote the original draft 
of the manuscript. JF, LS, and JS conducted the fluorescent Western Blot protein quantification. JS oversaw all experiments and revised the manuscript.

\section{FUNDING}

This material was based on work supported by the Brazilian Federal Agency for Support and Evaluation of Graduate Education (CAPES) under Grant 88881.131664/2016-01 awarded to TF as well as the United States Department of Agriculture National Institute of Food and Agriculture (USDA-NIFA) through Hatch Act funds to the Alabama

\section{REFERENCES}

Abasht, B., Mutryn, M. F., Michalek, R. D., and Lee, W. R. (2016). Oxidative stress and metabolic perturbations in wooden breast disorder in chickens. PLoS One 11:e0153750. doi: 10.1371/journal.pone.0153750

Armand, O., Boutineau, A. M., Mauger, A., Pautou, M. P., and Kieny, M. (1983). Origin of satellite cells in avian skeletal muscles. Arch. Anat. Microsc. Morphol. Exp. 72, 163-181.

Brothers, B., Zhuo, Z., Papah, M. B., and Abasht, B. (2019). RNA-seq analysis reveals spatial and sex differences in pectoralis major muscle of broiler chickens contributing to difference in susceptibility to wooden breast disease. Front. Physiol. 10:764. doi: 10.3389/fphys.2019.00764

Campion, D. R. (1984). The muscle satellite cell: a review. Int. Rev. Cytol. 87, 225-251.

Cantini, M., Massimino, M. L., Bruson, A., Catani, C., Dalla Libera, L., and Carraro, U. (1994). Macrophages regulate proliferation and differentiation of satellite cells. Biochem. Biophys. Res. Commun. 202, 1688-1696. doi: 10.1006/bbrc.1994. 2129

Chen, L., Borst, E., Oviedo-Rondon, E., Sarsour, A., Cordova-Noboal, A., Wineland, M., et al. (2017). Effect of age, strain, sex, and incubation temperature on severity of chicken breast myopathy ("woody breast") in broiler chickens. Poult. Sci. 96(E-Suppl. 1):256.

Daughtry, M. R., Berio, E., Shen, Z., Suess, E. J. R., Shah, N., Geiger, A. E., et al. (2017). Satellite cell-mediated breast muscle regeneration decreases with broiler size. Poult. Sci. 96, 3457-3464. doi: 10.3382/ps/ pex068

Day, K., Paterson, B., and Yablonka-Reuveni, Z. (2009). A distinct profile of myogenic regulatory factor detection within Pax7+cells at S phase supports a unique role of Myf5 during posthatch chicken myogenesis. Dev. Dyn. 238, 1001-1009. doi: 10.1002/dvdy.21903

Greene, E., Flees, J., Dadgar, S., Mallmann, B., Orlowski, S., Dhamad, A., et al. (2019). Quantum blue reduces the severity of woody breast myopathy via modulation of oxygen homeostasis-related genes in broiler chickens. Front. Physiol. 10:1251. doi: 10.3389/fphys.2019.01251

Hutton, K. C., Vaughn, M. A., Turner, B. J., Litta, G., and Starkey, J. D. (2014). Effect of vitamin D status improvement with 25-hydroxycholecalciferol on skeletal muscle growth characteristics and satellite cell activity in broiler chickens. J. Anim. Sci. 92, 3291-3299. doi: 10.2527/jas.2013-7193

Kindlein, L., Vieira, S., Steffanello, C., Ferreira, T., Valle, S., Simoes, C., et al. (2017). Wooden breast myopathy development in broilers subjected to feed restriction: growth performance, serologic profile, and meat quality. Poult. Sci. 96(E-Suppl. 1):253.

Kuttappan, V. A., Bottje, W., Ramnathan, R., Hartson, S. D., Coon, C. N., Kong, B. W., et al. (2017). Proteomic analysis reveals changes in carbohydrate and protein metabolism associated with broiler breast myopathy. Poult. Sci. 96, 2992-2999. doi: 10.3382/ps/pex069

Kuttappan, V. A., Hargis, B. M., and Owens, C. M. (2016). White striping and woody breast myopathies in the modern poultry industry: a review. Poult. Sci. 95, 2724-2733. doi: $10.3382 / \mathrm{ps} /$ pew216
Agricultural Experiment Station and a USDA-NIFA Agriculture and Food Research Initiative Competitive Grant No. 2018-670172755 awarded to JS.

\section{ACKNOWLEDGMENTS}

The sarcomeric myosin (MF20) and Pax7 (PAX7) antibody hybridoma cell lines developed by D. A. Fischman and A. Kawakami, respectively, were obtained from the Developmental Studies Hybridoma Bank, created by the NICHD of the NIH and maintained at The University of Iowa, Department of Biology, Iowa City, IA, United States.

Livingston, M., and Brake, J. (2017). Broiler live performance and carcass yield is improved by dietary potassium and available phosphorous without an increased incidence of wooden breast. Poult. Sci. 96(E-Suppl. 1), 249.

Maharjan, P., Owens, C. M., and Coon, C. (2019). In-vivo intramuscular collagen synthesis, muscle fiber growth and histomorphology of pectoralis major of a fast-growing broiler strain gallus gallus domesticus. Front. Vet. Sci. 6:470. doi: 10.3389/fvets.2019.00470

Manangi, M., Chen, J., Foran, C., Vazquez-Anon, M., Walter, K., Cerrate, S., et al. (2017). Synthetic antioxidant improves oxidative stability of breast meat and reduces incidence of Wooden breast myopathy in broilers fed diets containing oxidized fat. Poult. Sci. 96(E-Suppl. 1):294.

Mast, J., Goddeeris, B. M., Peeters, K., Vandesande, F., and Berghman, L. R. (1998). Characterisation of chicken monocytes, macrophages and interdigitating cells by the monoclonal antibody KUL01. Vet. Immunol. Immunopathol. 61, 343357. doi: 10.1016/s0165-2427(97)00152-9

Meloche, K. J., Dozier, W. A. III, and Starkey, J. D. (2018a). Skeletal muscle fiber morphometrics and in vivo myogenic stem cell mitotic activity in broiler chickens affected by Wooden Breast. Poult. Sci. 97, 4401-4414.

Meloche, K. J., Fancher, B. I., Emmerson, D. A., Bilgili, S. F., and Dozier, W. A. III (2018b). Effects of quantitative nutrient allocation on myopathies of the Pectoralis major muscles in broiler chickens at 32, 43, and 50 days of age. Poult. Sci. 97, 1786-1793. doi: 10.3382/ps/pex453

Meloche, K. J., Fancher, B. I., Emmerson, D. A., Bilgili, S. F., and Dozier, W. A. III (2018c). Effects of reduced dietary energy and amino acid density on Pectoralis major myopathies in broiler chickens at 36 and 49 days of age. Poult. Sci. 97, 1794-1807. doi: 10.3382/ps/pex454

Meloche, K. J., Fancher, B. I., Emmerson, D. A., Bilgili, S. F., and Dozier, W. A. III (2018d). Effects of reduced digestible lysine density on myopathies of the Pectoralis major muscles in broiler chickens at 48 and 62 days of age. Poult. Sci. 97, 3311-3324. doi: 10.3382/ps/pey171

Murphy, M. M., Lawson, J. A., Mathew, S. J., Hutcheson, D. A., and Kardon, G. (2011). Satellite cells, connective tissue fibroblasts and their interactions are crucial for muscle regeneration. Development 138, 3625-3637. doi: 10.1242/dev. 064162

Mutryn, M. F., Brannick, E. M., Fu, W., Lee, W. R., and Abasht, B. (2015). Characterization of a novel chicken muscle disorder through differential gene expression and pathway analysis using RNA-sequencing. BMC Genomics 16:399. doi: 10.1186/s12864-015-1623-0

Petracci, M., and Cavani, C. (2012). Muscle growth and poultry meat quality issues. Nutrients 4, 1-12. doi: 10.3390/nu4010001

Sihvo, H. K., Immonen, K., and Puolanne, E. (2014). Myodegeneration with fibrosis and regeneration in the pectoralis major muscle of broilers. Vet. Pathol. 51, 619-623. doi: 10.1177/0300985813497488

Sihvo, H. K., Linden, J., Airas, N., Immonen, K., Valaja, J., and Puolanne, E. (2017). Wooden breast myodegeneration of pectoralis major muscle over the growth period in broilers. Vet. Pathol. 54, 119-128. doi: 10.1177/0300985816658099

Soglia, F., Mazzoni, M., Zappaterra, M., Di Nunzio, M., Babini, E., Bordini, M., et al. (2019). Distribution and expression of vimentin and desmin in broiler 
pectoralis major affected by the growth-related muscular abnormalities. Front. Physiol. 10:1581. doi: 10.3389/fphys.2019.01581

Soglia, F., Mudalal, S., Babini, E., Di Nunzio, M., Mazzoni, M., Sirri, F., et al. (2016). Histology, composition, and quality traits of chicken Pectoralis major muscle affected by wooden breast abnormality. Poult. Sci. 95, 651-659. doi: $10.3382 / \mathrm{ps} / \mathrm{pev} 353$

Tasoniero, G., Cullere, M., Cecchinato, M., Puolanne, E., and Dalle Zotte, A. (2016). Technological quality, mineral profile, and sensory attributes of broiler chicken breasts affected by white striping and wooden breast myopathies. Poult. Sci. 95, 2707-2714. doi: 10.3382/ps/pew215

Tejeda, O. J., Calderon, A. J., Arana, J. A., Meloche, K. J., and Starkey, J. D. (2019). Broiler chicken myofiber morphometrics and myogenic stem cell population heterogeneity. Poult. Sci. 98, 4123-4130. doi: 10.3382/ps/pez287

Tijare, V. V., Yang, F. L., Kuttappan, V. A., Alvarado, C. Z., Coon, C. N., and Owens, C. M. (2016). Meat quality of broiler breast fillets with white striping and woody breast muscle myopathies. Poult. Sci. 95, 2167-2173. doi: 10.3382/ps/ pew129

Trocino, A., Piccirillo, A., Birolo, M., Radaelli, G., Bertotto, D., Filiou, E., et al. (2015). Effect of genotype, gender and feed restriction on growth, meat quality and the occurrence of white striping and wooden breast in broiler chickens. Poult. Sci. 94, 2996-3004. doi: 10.3382/ps/pev296

Velleman, S. G. (2015). Relationship of skeletal muscle development and growth to breast muscle myopathies: a review. Avian Dis. 59, 525-531. doi: 10.1637/ 11223-063015-Review.1

Velleman, S. G., and Clark, D. L. (2015). Histopathologic and myogenic gene expression changes associated with wooden breast in broiler breast muscles. Avian Dis. 59, 410-418. doi: 10.1637/11097-042015-Reg.1
Wedekind, K., Wineman, T., Atwell, C., Vazquez-Anon, M., and Escobar, J. (2016). Characteristics of woody breast and the effect of chelated trace minerals on woody breast in broilers. Poult. Sci. 95(E-Suppl. 1), 197.

Yablonka-Reuveni, Z. (1995). Myogenesis in the chicken: the onset of differentiation of adult myoblasts is influenced by tissue factors. Basic Appl. Myol. 5, 33-41.

Yablonka-Reuveni, Z., Quinn, L. S., and Nameroff, M. (1987). Isolation and clonal analysis of satellite cells from chicken pectoralis muscle. Dev. Biol. 119, 252-259. doi: 10.1016/0012-1606(87)90226-0

Zuidhof, M. J., Schneider, B. L., Carney, V. L., Korver, D. R., and Robinson, F. E. (2014). Growth, efficiency, and yield of commercial broilers from 1957, 1978, and 2005. Poult. Sci. 93, 2970-2982. doi: 10.3382/ps.2014-04291

Conflict of Interest: The authors declare that the research was conducted in the absence of any commercial or financial relationships that could be construed as a potential conflict of interest. Mention of trade names or commercial products in this publication is solely for the purpose of providing specific information and does not imply recommendation or endorsement by Federal University of Rio Grande do Sul or Auburn University.

Copyright (c) 2020 Ferreira, Kindlein, Flees, Shortnacy, Vieira, Nascimento, Meloche and Starkey. This is an open-access article distributed under the terms of the Creative Commons Attribution License (CC BY). The use, distribution or reproduction in other forums is permitted, provided the original author(s) and the copyright owner(s) are credited and that the original publication in this journal is cited, in accordance with accepted academic practice. No use, distribution or reproduction is permitted which does not comply with these terms. 


\section{OPEN ACCESS}

Edited by:

Krystyna Pierzchala-Koziec,

University of Agriculture in Krakow,

Poland

Reviewed by:

Elisabeth Baeza,

Institut National de Recherche pour

l'Agriculture, l'Alimentation et

l'Environnement (INRAE), France

Woo Kyun Kim,

University of Georgia, United States

*Correspondence:

Francesca Soglia

francesca.soglia2@unibo.it

Specialty section:

This article was submitted to

Avian Physiology,

a section of the journal

Frontiers in Physiology

Received: 25 March 2020

Accepted: 04 May 2020

Published: 12 June 2020

Citation:

Zampiga M, Soglia F, Baldi G,

Petracci M, Strasburg GM and Sirri F

(2020) Muscle Abnormalities and

Meat Quality Consequences

in Modern Turkey Hybrids.

Front. Physiol. 11:554.

doi: 10.3389/fphys.2020.00554

\section{Muscle Abnormalities and Meat Quality Consequences in Modern Turkey Hybrids}

\author{
Marco Zampiga1 , Francesca Soglia ${ }^{1 *}$, Giulia Baldi ${ }^{1}$, Massimiliano Petracci ${ }^{1}$, \\ Gale M. Strasburg ${ }^{2}$ and Federico Sirri ${ }^{1}$
}

${ }^{1}$ Department of Agricultural and Food Sciences, Alma Mater Studiorum - University of Bologna, Bologna, Italy, ${ }^{2}$ Department of Food Science and Human Nutrition, Michigan State University, East Lansing, MI, United States

Turkey meat is the second most consumed poultry meat worldwide and represents an economic source of high-quality protein for human consumption. To fulfill the increasing demand for turkey meat, breeding companies have been selecting genetic lines with increased growth potential and breast muscle proportion. Moreover, the progressive shift toward further processed products has emphasized the need for higher standards in poultry meat to improve its technological characteristics and functional properties (i.e., water-holding capacity). However, as observed for broiler chickens, a growing body of scientific evidence suggests that the intense selection for the aforementioned traits could be associated with a greater occurrence of growth-related myopathies and abnormalities and, consequently, to increased downgrading rates and overall reduction of meat quality characteristics. In the past, muscle abnormalities such as deep pectoral myopathy, pale-soft-and-exudative-like meat, and focal myopathy have been reported in turkey lines selected for increased growth rate. In addition, the presence of white striations in the superficial layer of pectoralis major muscle, as well as the tendency of muscle fiber bundles to separate resulting in an altered breast muscle structure, has been detected in commercial turkey abattoirs. Furthermore, past investigations revealed the presence of another quality issue depicted by an overall toughening of the breast muscle. These meat abnormalities seem to macroscopically overlap the white striping, spaghetti meat, and wooden breast conditions observed in pectoral muscle of fastgrowing, high-breast-yield chicken hybrids, respectively. Considering the high economic impact of these growth-related abnormalities in broilers, there is an increasing interest of the turkey industry in estimating the occurrence and the impact of these meat quality issues also in the modern turkey lines. Studies have been recently conducted to assess the effect of the genotype on the occurrence of these emerging growth-related defects and to evaluate how meat quality properties are affected by white-striping condition in turkeys, respectively. Therefore, this review aims to provide a critical overview of the current understanding regarding the growth-related abnormalities and their impact on meat quality in modern turkey hybrids with the hope that this information may improve the knowledge concerning their overall effect on poultry meat. 


\section{INTRODUCTION}

According to recent estimates, poultry meat consumption is increasing worldwide regardless of the country and the income level (OECD/FAO, 2016). The turkey sector represents one of the major pillars of the poultry industry (FAOSTAT, 2020), with turkey meat being the second most consumed poultry meat worldwide with an average annual consumption of 4.0 and $7.3 \mathrm{~kg} /$ per capita in the European Union (EU) and the United States, respectively (AVEC, 2019). Consumers, particularly in Western countries, are increasingly attracted toward breast meat, mainly because of its perceived healthy profile and the variety and organoleptic qualities of processed poultry meat products (Petracci and Cavani, 2012; Petracci et al., 2015). Therefore, to fulfill the increasing demand for breast meat, breeding companies have targeted the selection toward genetic lines with rapid breast muscle development and high carcass yield, achieving astonishing results as summarized by Havenstein et al. (2007). In addition, the progressive shift toward further processed products has emphasized the necessity for higher standards in poultry meat to improve its technological characteristics and functional properties (e.g., water-holding capacity) (Barbut, 2015; Petracci et al., 2015).

However, Wilson et al. (1990), finding more fragmented muscle fibers and higher plasma creatine kinase concentration in fast-growing turkey lines compared to slow-growing ones, hypothesized that the selection for growth rate and muscle development has resulted in muscles that have "outgrown their life-support systems," leading to alterations in muscle morphology, physiological state, and biochemistry. Further studies have been supporting this hypothesis, suggesting that the selection for the aforementioned traits in modern turkey lines could be associated with alterations in the musculoskeletal structure (Sosnicki and Wilson, 1991; Velleman et al., 2003; Hocking, 2014; Velleman, 2015) and a greater occurrence of growth-related abnormalities (Sosnicki and Wilson, 1991; Mahon, 1999; Owens et al., 2009), as well as impaired meat functionality and technological traits (Updike et al., 2005), which will be discussed in the following paragraph.

\section{HISTORICAL EVOLUTION OF MEAT ABNORMALITIES IN TURKEYS}

The first scientific reports regarding growth-related muscle abnormalities in turkeys were published during the 1960s and 1970s. As previously stated, the extraordinary improvements achieved in growth rate and breast muscle yield were accompanied by alterations of muscle morphology, physiology, and biochemistry with negative implications for overall meat quality traits and downgrading rates. One of the first reported growth-related meat abnormalities in turkeys was the deep pectoral myopathy (DPM). Originally described by Dickinson et al. (1968) as a "degenerative myopathy" affecting the supracoracoideus muscle (pectoralis minor or deep pectoralis muscle), DPM is characterized by necrosis, dry, stringy, woodylike texture, atypical color (i.e., "green muscle disease"), and gross edematous appearance (Sosnicki and Wilson, 1991; Jordan and Pattison, 1998; Table 1). It is widely accepted that DPM is caused by an ischemic condition induced by the swelling of the muscle during exercise (Orr and Riddell, 1977; Siller and Wight, 1978; Siller et al., 1979; Jordan and Pattison, 1998). Indeed, the compartmentalization of the deep pectoral muscle between the inelastic muscle fascia and the keel bone can determine the swelling of the supracoracoideus muscle during exercise (e.g., wing flapping), resulting in the partial occlusion of the cranial and caudal pectoral arteries leading to a limited blood circulation to the muscle, which, in turn, triggers degenerative cellular processes (Siller, 1985; Sosnicki and Wilson, 1991). Although DPM does not represent a public health concern or an animal welfare issue, affected fillets need to be trimmed and subsequently discarded as unacceptable for human consumption. However, the selection against DPM and the adoption of specific management operations have significantly reduced the incidence of this defect (Sosnicki and Wilson, 1991), which now occurs only sporadically in commercial broiler and turkey flocks.

Another very well-studied and described meat alteration in turkeys is pale, soft, and exudative (PSE)-like meat (Table 1). PSE-like meat is characterized by abnormally light or pale color, soft texture, and reduced water-holding capacity and cooking yield (Barbut, 1993, 1996; Sosnicki et al., 1998). This condition can be ascribed to antemortem stressors, such as the sudden onset of a heat wave and the transportation of turkeys to slaughter facilities, which induce an accelerated glycolytic metabolism of breast muscle fibers leading to increased muscle temperature and rapid development of rigor mortis (fast-acidifying meat, $\mathrm{pH}<6$ within $1 \mathrm{~h}$ postmortem) (Pietrzak et al., 1997). Alternatively, the breast muscle may reach a low ultimate $\mathrm{pH}$ (acidification of a greater extent) without any remarkable alterations in the acidification rate (acid meat, $\mathrm{pH}<5.8$ ) (Sosnicki et al., 1998; Barbut et al., 2008). The combination of low $\mathrm{pH}$ and high postmortem breast muscle temperature may result in a partial protein denaturation and hence loss of protein functionality (Sosnicki, 1993; Sosnicki et al., 1995). In addition, analysis of gene expression patterns of normal and PSE-like turkey meat suggests that birds yielding PSE-like meat displayed altered expression of proteins associated with calcium homeostasis, signaling pathways regulating actin cytoskeleton structure, carbohydrate metabolism, and energy production (Strasburg and Chiang, 2009; Malila et al., 2014). The presence of PSE-like meat still represents a large concern for vendors of fresh retail poultry cuts because of the moisture loss and abnormal color (Petracci et al., 2015), and also the value-added processing industry sector experiences great economic losses because of reduced protein functionality of affected meat (Owens et al., 2000; Barbut et al., 2008). For such reasons, research is encouraged to further investigate the effect of genotype, antemortem stressors, and slaughtering phases on the breast muscle acidification pattern of modern turkey hybrids to find solutions that can counteract the negative impact of PSE-like meat. A possible practical strategy could be to exploit abnormal meat for further processing through the addition of functional ingredients (i.e., phosphates, starches, hydrocolloids, vegetable proteins) to improve its technological properties (Barbut, 2009; Petracci et al., 2013). 
TABLE 1 | Description of the main breast muscle abnormalities in modern commercial turkeys and their impact on overall meat quality.

\begin{tabular}{|c|c|c|c|c|c|}
\hline $\begin{array}{l}\text { Breast muscle } \\
\text { abnormality }\end{array}$ & Macroscopic traits & Causes & $\begin{array}{l}\text { Current } \\
\text { occurrence } \\
\text { level }\end{array}$ & Impact on meat quality & Main references \\
\hline $\begin{array}{l}\text { Deep pectoral } \\
\text { myopathy }\end{array}$ & $\begin{array}{l}\text { Atypical coloration } \\
\text { (from reddish to } \\
\text { greenish) of } P \text {. minor }\end{array}$ & $\begin{array}{l}\text { Ischemic condition } \\
\text { caused by muscle } \\
\text { swelling during exercise }\end{array}$ & Sporadic & $\begin{array}{l}\text { Increased trimming and discarding rate as } \\
\text { affected fillets, presenting necrosis, dry, } \\
\text { stringy, and hardened texture, atypical } \\
\text { color, and gross edematous appearance, } \\
\text { are unacceptable for human consumption }\end{array}$ & $\begin{array}{l}\text { Dickinson et al., 1968; Orr and } \\
\text { Riddell, 1977; Siller and Wight, } \\
\text { 1978; Siller et al., 1979; Siller, } \\
\text { 1985; Sosnicki and Wilson, } \\
\text { 1991; Jordan and Pattison, } \\
\text { 1998 }\end{array}$ \\
\hline $\begin{array}{l}\text { Pale, soft, } \\
\text { exudative-like } \\
\text { condition }\end{array}$ & $\begin{array}{l}\text { Abnormal light or pale } \\
\text { color, soft texture, } \\
\text { excessive juice loss }\end{array}$ & $\begin{array}{l}\text { Rapid and/or excessive } \\
\text { postmortem muscle } \\
\text { acidification due to } \\
\text { acute preslaughter } \\
\text { stress }\end{array}$ & $\begin{array}{l}\text { High, } \\
\text { especially } \\
\text { during hot } \\
\text { seasons }\end{array}$ & $\begin{array}{l}\text { Downgrading due to muscle abnormal } \\
\text { appearance and poor functional properties } \\
\text { for further processing; loss of protein } \\
\text { functionality, which results in reduced } \\
\text { water-binding capacity and cook yield }\end{array}$ & $\begin{array}{l}\text { Barbut, 1993, 1996; Sosnicki, } \\
\text { 1993; Sosnicki et al., 1995, } \\
\text { 1998; Pietrzak et al., 1997; } \\
\text { Owens et al., 2000; Fraqueza } \\
\text { et al., 2006; Barbut et al., 2008; } \\
\text { Chiang et al., 2008; Malila et al., } \\
\text { 2014; Carvalho et al., } 2018\end{array}$ \\
\hline $\begin{array}{l}\text { Dark-firm-dry } \\
\text { condition }\end{array}$ & $\begin{array}{l}\text { Abnormal dark color } \\
\text { and dry appearance }\end{array}$ & $\begin{array}{l}\text { Reduced postmortem } \\
\text { muscle acidification } \\
\text { due to antemortem } \\
\text { chronic stress inducing } \\
\text { glycogen depletion }\end{array}$ & Moderate & $\begin{array}{l}\text { Downgrading due to muscle abnormal } \\
\text { appearance and greater potential spoilage } \\
\text { from organisms and food-borne pathogens } \\
\text { ascribable to the higher ultimate } \mathrm{pH} \text {; higher } \\
\text { water-binding capacity if used for further } \\
\text { processed products }\end{array}$ & $\begin{array}{l}\text { Mallia et al., 2000; Fraqueza } \\
\text { et al., 2006; Carvalho et al., } \\
\text { 2018; Henrikson et al., } 2018\end{array}$ \\
\hline White striping & $\begin{array}{l}\text { White striations of } \\
\text { variable thickness } \\
\text { parallel to muscle fibers } \\
\text { direction in the ventral } \\
\text { surface of pectoralis } \\
\text { major }\end{array}$ & $\begin{array}{l}\text { Alterations of regular } \\
\text { muscle architecture } \\
\text { during growth with } \\
\text { abnormal deposition of } \\
\text { adipocytes }\end{array}$ & Moderate & $\begin{array}{l}\text { Slight effect on meat proximate } \\
\text { composition (increased fat content) and } \\
\text { technological traits }\end{array}$ & $\begin{array}{l}\text { Soglia et al., 2018; Mudalal, } \\
\text { 2019; Zampiga et al., } 2019\end{array}$ \\
\hline $\begin{array}{l}\text { Spaghetti } \\
\text { meat-like }\end{array}$ & $\begin{array}{l}\text { Separation of muscle } \\
\text { fiber bundles in the } \\
\text { superficial layer of the } \\
\text { cranial region of } \\
\text { pectoralis major }\end{array}$ & $\begin{array}{l}\text { Progressive rarefaction } \\
\text { of perimysial connective } \\
\text { tissue during growth }\end{array}$ & Sporadic & $\begin{array}{l}\text { Affected parts are trimmed, whereas no } \\
\text { information is available about possible meat } \\
\text { quality implications }\end{array}$ & Zampiga et al., 2019 \\
\hline Wooden breast & $\begin{array}{l}\text { Hardening of pectoralis } \\
\text { major muscle }\end{array}$ & $\begin{array}{l}\text { Connective tissue } \\
\text { proliferation }\end{array}$ & $\begin{array}{l}\text { Not yet } \\
\text { detected }\end{array}$ & - & - \\
\hline
\end{tabular}

In addition to PSE-like condition, dark-firm-dry (DFD) abnormality is well known by many years, and almost all farmed species show a certain prevalence of this quality defect affecting color and texture with detrimental effects on consumer willingness to purchase (Mallia et al., 2000; Fraqueza et al., 2006; Carvalho et al., 2018; Table 1). Also in turkeys, DFD results from an abnormal reduced postmortem muscle acidification (ultimate $\mathrm{pH}>6.0$ ) often associated with chronic or long-term stress experienced by the birds before slaughter (Barbut et al., 2008). Indeed, prolonged feed withdrawal and transportation, as well as exposition to cold temperature, can cause exhaustion of muscle glycogen stores and therefore a reduced postmortem lactic acid production through anaerobic glycolysis (Mallia et al., 2000; Fraqueza et al., 2006; Henrikson et al., 2018). DFD meat, being characterized by a darker-than-normal color and dry appearance, has a limited consumer appeal and thus is usually downgraded and redirected for further processing in turkey plants. Moreover, according to Fraqueza et al. (2006), darker turkey breast meat with higher ultimate $\mathrm{pH}$ values exhibits a fast microbial spoilage. Avoidance of long fasting period before slaughter and exposition to cold temperatures are well-known strategies to counteract DFD condition also in turkeys (Barbut et al., 2008; Henrikson et al., 2018).
During the 1980s, some meat quality issues related to the cohesiveness and juiciness of processed turkey breast meat, as well as to an overall toughening of the pectoralis major, were associated with microscopic, degenerative alterations of skeletal muscles in market turkeys, likely induced by the selection for growth rate and muscle yield (Dutson and Carter, 1985; Grey et al., 1986; Seemann et al., 1986; Grey, 1989; Sosnicki and Wilson, 1991). In a series of studies, Sosnicki et al. (1988a,b, 1989a,b, 1991a,b) investigated the pathology of skeletal muscles in fastgrowing turkeys. The main degenerative alterations observed in the pectoralis major and iliofibularis (biceps femoris) muscle included scattered and focal necrosis, granular necrosis with phagocytosis by mononuclear cells of several adjacent muscle fibers or multifiber areas, hypercontraction of muscle fibers, as well as a strong proliferation of connective and fat tissues in the endomysium and perimysium (Sosnicki et al., 1991b). Sosnicki and Wilson (1991) defined this condition as the "focal myopathy" of the turkey. It is interesting to note that this myopathy was thought to be related with vascular defects, which could induce a localized microischemia at the terminal capillary bed level. Swatland (1990) observed a 35-fold increase in cross-sectional area of the muscle fibers of turkeys during the first 15 weeks of life, which was relatively greater than the growth of endomysial 
and perimysial connective tissue. Consequently, low values of capillary density and capillary-to-fiber ratio, as well as a greater intercapillary distance, were observed in necrotic regions of the pectoralis major and biceps femoris muscle of turkeys (Sosnicki et al., 1991a). Overall, these findings seem to indicate that the massive hypertrophic-based growth of muscle fibers was not accompanied by a concomitant growth of the connective layers, resulting in a poor vascularization of muscle fibers and then in histological defects and meat quality issues. Sosnicki and Wilson (1991) concluded that the physiological development of tissues supporting muscle as an organ (vascular, connective, adipose, nerve, etc.) has not kept pace with rapid growth and development of muscle fibers.

Intriguingly, the histological alterations observed almost 30 years ago in breast muscle of turkeys selected for high growth rate and meat yield are similar to those reported for breast muscle of fast-growing broiler chickens, which are affected by the emerging growth-related muscle abnormalities such as white striping (WS), wooden breast (WB), and spaghetti meat (SM) (Kuttappan et al., 2009, 2016; Petracci et al., 2014, 2015, 2019; Sihvo et al., 2014). Briefly, the WS condition is macroscopically characterized by the presence of white striations of variable thickness parallel to the direction of muscle fibers (Kuttappan et al., 2009), whereas WB one presents bulging and pale areas of muscle with hardened consistency (Sihvo et al., 2014). Recently, Baldi et al. (2018) described the SM condition as the tendency toward separation of muscle fiber bundles that mainly affects the superficial layer of the cranial region of pectoralis major muscle. As reviewed by Soglia et al. (2019), all these abnormalities share some common microscopic features such as the loss of normal muscle architecture, presence of abnormal fibers presenting rounded profiles, nuclear internalization, fiber degeneration, necrosis up-to-lysis associated with concurrent regenerative processes (small-caliber regenerative fibers intermingled to abnormal ones having large caliber), compromised perimysial and endomysial connective tissue, and fat and inflammatory cell infiltrations. In addition, each syndrome also exhibits distinctive histological traits such as an abnormal deposition of adipose tissue in the perimysium (lipidosis) for WS and proliferation and thickening of the perimysial network (fibrosis) in WB condition. In contrast, the SM defect is characterized by a progressive rarefaction of the endomysial and perimysial connective tissue layers (Soglia et al., 2019).

In turkeys, the presence of white striations in the superficial layer of pectoralis major muscle, as well as the tendency of muscle fiber bundles to separate, has been observed in commercial practices especially in heavy male turkey hybrids but not considered a main quality issue by producers or consumers. Past investigations (Grey et al., 1986; Seemann et al., 1986; Grey, 1989) also described a turkey meat quality issue characterized by an increased toughness of the pectoralis major muscle coupled with the presence of giant hypercontracted cells, fiber defects, and overall greater muscle cell size (Sosnicki and Wilson, 1991). In addition, the hypothetical causative mechanism of emerging muscle abnormalities in broiler chickens (i.e., limited capillary density, impaired oxygen supply, and metabolic waste product displacement from breast myofibers) seems to be analogous to that hypothesized by Sosnicki and Wilson (1991) for turkeys. However, in contrast to broiler chickens, there is limited information regarding emerging growth-related muscle defects in modern turkeys. In addition, considering the detrimental effects of these muscle abnormalities on meat quality traits in chickens, it is fundamental to understand whether this scenario could be similar for turkeys and also if the genotype could have any impact on the occurrence of such conditions.

\section{OCCURRENCE OF BREAST MEAT ABNORMALITIES IN MODERN TURKEY HYBRIDS}

In broilers, the genetic potential for breast meat yield and growth rate is considered the main factor affecting the occurrence of breast abnormalities as recently reviewed by Petracci et al. (2019). Previous studies on turkeys showed that the incidence of meat abnormalities and muscle fiber defects was greater in hybrids selected for high growth rate and breast muscle development (Wilson et al., 1990; Sosnicki and Wilson, 1991; Velleman et al., 2003). However, the effects of these promoting factors on turkey meat quality are somewhat less clear.

To examine the effects of growth selection on turkey meat quality, Updike et al. (2005) compared breast meat functionality in three lines of turkeys of differing growth traits: (1) a randombred control line (RBC2), representing commercial turkeys of the 1960s; (2) F line, which was selectively bred from RBC2 line for increased body weight at 16 weeks; and (3) a fastgrowing commercial line. The RBC2 turkey breast meat showed highest water-holding capacity and tenderness (measured by Warner-Bratzler shear force) compared to that of the other two lines. Although there were no differences in cook yield, thermally induced meat gels from the RBC2 line were strongest of the three lines. Overall, these results support the hypothesis that growth selection can negatively affect meat quality.

Environmental conditions such as heat or transportation stress may differentially affect meat quality of slow-growing and fast-growing turkeys. Chiang et al. (2008) subjected RBC2 and commercial turkeys to various periods of heat stress and examined differences in meat quality and thyroid hormone (T3 and T4) levels. They observed that the breast muscle from fast-growing commercial turkeys had higher $\mathrm{pH}$ at $15 \mathrm{~min}$ postmortem and greater cook yield compared to the slowgrowing randombred line, whereas no differences were detected in water-holding capacity. However, of particular note from a processed meat standpoint is the observation that meat from the RBC2 line had a significantly higher marinade uptake than that of commercial birds. Moreover, this parameter was very consistent over the first 3 days of heat stress, whereas that of the commercial line fluctuated significantly as a function of time. Marinade uptake is an important indicator of protein functionality, and higher ability to pick up marinade solutions is generally associated with greater quality meat products. Interestingly, the changes in marinade uptake in the commercial line were mirrored by changes in the plasma T3 levels. Both marinade uptake and T3 levels were much more consistent in the 
RBC2 line. Taken together, this study also supports the hypothesis that selection for growth performance adversely affects some meat quality parameters, particularly upon environmental stress. On the other hand, Werner et al. (2008) analyzed breast meat from two fast-growing and two slow-growing turkey lines and found no clear differences among the tested turkey strains. One of the fast-growing lines showed a significantly higher drip loss compared to the others, and muscle from both fast growing lines exhibited higher fibers diameter, although the ratio of capillaries to unit of fiber area was not different between lines. It is important to note that although the birds in this study were raised and harvested simultaneously under the same conditions, they were not subjected to environmental stress. Thus, the effects of growth selection on the PSE-like condition in turkeys may depend on environmental effects.

Whereas DPM and PSE-like conditions have been extensively studied in the past, there is a lack of scientific information about other potential emerging growth-related abnormalities in turkeys. To address these concerns, a trial was carried out by our research group to evaluate the occurrence of WS, WB, and SM-like abnormalities in two commercial turkey hybrids ( $n=486$ toms per genotype A and $\mathrm{B}$, respectively) (Zampiga et al., 2019). The genetic lines tested in the trial are currently available and used worldwide for turkey meat production. The lines belong to two major independent breeding companies, were not genetically related, and were fed the same diet and raised in the same environmental conditions. At 140 days, the toms, which showed comparable growth performance at the end of the trial, were processed in a commercial slaughterhouse following the procedure commonly adopted in the EU. The occurrence of breast meat abnormalities was assessed on 100 breasts/genotype 24 h after processing. To evaluate the severity of the WS defect, a 4-point scale evaluation system was developed: $0=$ no lesions; $1=$ mild lesions: few stripes; 2 = marked lesions: up to $50 \%$ of the breast surface covered by the white striations; $3=$ severe lesions: more than $50 \%$ of the breast surface covered by the white striation (Zampiga et al., 2019). For the WB and SM-like defect, the evaluation systems proposed by Sihvo et al. (2014) and Sirri et al. (2016) for broiler chickens were used, respectively. The percentage of breasts affected by WS was markedly higher in both the tested lines as indicated by the very low percentage $(1 \%)$ of breasts reporting no WS lesions. Moreover, a significant effect of the genotype was observed on the frequency of the different severity classes. Turkeys from group A showed a significantly lower percentage of breasts presenting severe lesions (46 vs. $60 \%$, respectively, for $\mathrm{A}$ and $\mathrm{B}$ ) and a higher incidence of those with mild lesions (17 vs. $5 \%$, respectively, for A and B). On the other hand, the overall occurrence of WB and SM-like conditions was negligible, and no significant difference emerged between the genotypes. The low incidence of the SM-like defect in breast muscle of turkeys might be partially explained by the analysis carried out to assess the intramuscular collagen properties in breasts presenting no macroscopic defects. Overall, the two turkey strains showed a similar breast muscle collagen content and, regardless of the genotype, were characterized by a good level of collagen cross-linking and maturation. These findings could support the hypothesis of a proper muscle structure, which could explain the overall low occurrence and severity of SMlike condition in both the genetic lines (Zampiga et al., 2019). Mudalal (2019), analyzing 2,300 turkey breasts from 22 flocks (20 weeks old turkeys), found that the overall occurrence of moderate and severe WS was $61.3 \%$, with moderate cases accounting for $49.4 \%$. Moreover, it has been recently reported that a visible-near infrared spectroscopy-based approach was useful to differentiate normal from severe WS turkey fillets, which could have a potential application in the turkey industry (Zaid et al., 2020).

Zampiga et al. (2019) also performed histological evaluations on pectoralis major muscles representative of each abnormality and degree of severity adopted for testing the occurrence of muscle myopathies (i.e., $\mathrm{WS}=0,1,2$, and 3 ; and $\mathrm{SM}=0$ and $1)$. Breast muscles not showing WS defect (WS $=0$ ) exhibited myofibers with a regular polygonal profile, as well as endomysial and perimysial connective tissues without remarkable alterations. In breast muscles macroscopically presenting minor WS lesions (WS $=1$ ), some necrotic fibers intermingled with apparently normal ones that have lost their polygonal profile, as well as changes of the perimysial connective layer, were observed. In severe WS (WS = 2 and 3), histological sections exhibited nuclear internalization, vacuolar and hyaline degeneration, necrosis and lysis of the fibers, inflammatory cell infiltration, variable fiber cross-sectional area (degenerating and regenerating fibers), lipidosis, and fibrosis. A similar scenario, including degenerative changes such as scattered and focal necrosis, hypercontraction of muscle fibers, and strong proliferation of connective and fat tissues in the endomysium and perimysium, as well as infiltration of the necrotic areas by mononuclear cells, has been previously reported in pectoralis major muscle of fastgrowing turkeys (Sosnicki et al., 1988a,b; Sosnicki and Wilson, 1991). Overall, these histological findings are consistent with the muscle alterations reported in previous studies conducted on commercial broiler chickens (Kuttappan et al., 2013; Radaelli et al., 2017; Baldi et al., 2018).

On the other hand, breasts affected by the SM-like defect showed a distinctive progressive rarefaction of the endomysial and perimysial connective tissue, leading to muscle fibers detaching from each other. This compromised connective tissue was generally accompanied by degenerate and necrotic (up-tolysis) fibers and fat and inflammatory cells infiltration, as well as the presence of small-caliber fibers associated with large-caliber ones showing rounded profile. To the best of our knowledge, the data reported in Zampiga et al. (2019) are the only ones available in literature regarding the SM-like condition in turkeys. Sosnicki and Wilson (1991), discussing the focal myopathies, pointed out that the excrescence of the sinus muscle fibers on the supporting connective tissue may also predispose the products to fragmentation, which could be similar to the recently observed SM-like condition. Moreover, these findings are in agreement with those previously reported by Baldi et al. (2018) regarding histological sections of broiler breast muscle affected by the SM abnormality.

Taken together, the overall occurrence of WS in commercial turkey hybrids was remarkably high, whereas that of SM-like and WB was sporadic and negligible, respectively (Table 1). Nonetheless, it should be considered that, differently from broiler 
meat, the presence of white striations in turkey breasts seems not to significantly affect consumers' perception possibly because most of the turkey meat is commercialized worldwide as readyto-eat products and meal (Remignon, 2004). Similarly, other marketing forms such as manufacturing sliced (unprocessed, raw slices) or whole carcass (e.g., for Thanksgiving and Christmas festivity meals), which are common, respectively, in the EU and US market, tend to reduce the impact of the white striations typical of the WS defect. Finally, the histological alterations found in turkey breast muscles presenting WS and SM-like conditions were similar to those reported for broilers. However, the overall degree of severity was remarkably lower in turkeys, with possible implications on overall meat quality traits that will be discussed in the following section of this review.

\section{EFFECT OF WS ON PROXIMATE COMPOSITION AND TECHNOLOGICAL TRAITS OF BREAST MEAT}

In chickens, breast muscle abnormalities have been associated with marked alterations in meat chemical composition and technological traits as recently reviewed by Petracci et al. (2019). In general, WS, WB, and SM conditions are characterized by increased amount of fat and moisture and lower protein content compared to unaffected breasts (Petracci et al., 2014, 2019; Baldi et al., 2018). In addition, WB- and WS-affected breasts displayed impairment of meat functionality resulting in poor technological traits, particularly for WB condition (Tijare et al., 2016), which could have a tremendous impact on processing attitude and hence on overall quality features of value-added processed meat products that represent an important form of commercialization for turkey meat (Remignon, 2004).

The effects of the WS abnormality on proximate composition and technological traits of turkey breast meat were recently investigated by Soglia et al. (2018). As reported by the authors, a total of 72 boneless and skinless pectoralis major muscles were collected $48 \mathrm{~h}$ postmortem and classified according to the severity of WS (i.e., thickness of the white striations in the ventral surface of the pectoral muscles) as normal (NORM), moderate (MOD), and severe (SEV). All the breasts were obtained from two separate trials on two flocks of heavy male turkeys (BUT Big 6). Rearing, feeding, and slaughtering conditions were representative of current commercial practices in the EU. Technological traits, such as $\mathrm{pH}$, drip loss, cooking loss, tenderness, marinade uptake, and nuclear magnetic resonance (NMR) relaxation properties, as well as proximate composition, were determined.

Overall, fillets presenting the WS defect were characterized by a significantly greater weight ( $20 \%$ ) compared to the normal ones, potentially confirming a growth-related origin of WS also for turkeys (Soglia et al., 2018). Similarly, previous investigations hypothesized an association between muscle degeneration and growth rate of the turkeys, with the ones showing rapid growth rate being characterized by severe degenerations (Wilson et al., 1990; Velleman et al., 2003).
However, unlike broiler chickens, the presence of WS in turkeys had a limited effect on proximate composition. Indeed, breasts presenting WS showed no significant difference in moisture and protein content, whereas total lipid was higher in SEV group compared to the NORM counterpart. Moreover, regardless of the severity of WS abnormality, affected breasts exhibited lower ash content in comparison with NORM ones. No significant differences were observed for collagen content among the groups. Mudalal (2019) reported that turkey breasts presenting severe WS were characterized by higher fat and lower protein content as well as different color indexes $\left(a^{*}\right.$ and $\left.b^{*}\right)$ compared to unaffected ones. Overall, these findings corroborate with the results of previous studies performed on broilers showing WS (Kuttappan et al., 2013; Petracci et al., 2014). Indeed, the differences in proximate composition can be explained by considering the degenerative and regenerative processes taking place within these muscles, which involve the proliferation of connective tissue and fat infiltration as microscopically observed in the skeletal muscle tissue of turkeys showing WS abnormality (Zampiga et al., 2019).

Consistent with the limited differences found in proximate composition, also the technological traits were slightly affected by the presence of WS abnormality (Soglia et al., 2018). Indeed, with the only exception of drip loss that significantly differed among the groups, no remarkable alterations were observed for overall quality traits of both raw $(\mathrm{pH}$, color, cooking losses, and shear force) and marinated (uptake, cooking losses, and shear force) meat. The lack of differences in water-holding capacity using conventional drip loss determination was confirmed by the NMR analysis aimed at assessing the water mobility and distribution within the muscle tissue. Therefore, in contrast to the studies reported for broilers, an overall absence of adverse effects of WS on breast meat quality traits of turkey was observed. The authors supposed that this difference could be explained by species-specific dissimilarity in breast muscle morphology and development among turkeys and broilers. Indeed, the increase in breast meat yield obtained by selection process in broilers was mainly accomplished by enhancing breast muscle thickness (Soglia et al., 2018). However, it was reported that the greater the pectoralis major depth and yield, the higher the likelihood to find severe myopathic alternations within the muscle tissue (Griffin et al., 2018). Therefore, it has been hypothesized that the limited impact of WS on turkey meat quality might be ascribed to a more harmonious development of breast muscle that, being not merely restricted to the depth as observed in broilers, could lead to less pronounced myopathic changes within the muscle tissue (Soglia et al., 2018).

Taken together, differently from broiler chickens, the overall impact of emerging growth-related breast muscle myopathies (WS and SM) in turkey seems rather limited. On the other hand, the presence of PSE-like meat is still a major concern for this sector (Table 1). Based on the findings obtained in previous studies carried out on broilers, several feeding strategies can be implemented with the aim to reduce the incidence of these growth-related abnormalities as extensively reviewed by Petracci et al. (2019). However, the same authors suggested that a reduction in the incidence of myopathies in response to feeding 
strategies might be ascribed to an overall limitation of muscle development and growth performance rather than to a real effect associated with the dietary treatments.

\section{CONCLUSION}

The overall occurrence of WS in commercial turkey hybrids was remarkably high, whereas that of SM-like and WB conditions could be considered sporadic and negligible, respectively. Moreover, a possible growth-related origin for WS in turkeys could be assumed. However, despite its high occurrence and the fact that histological alterations are similar to those reported for broilers, WS condition did not significantly affect meat quality traits in turkeys resulting in no negative impact either for raw or processed meat

\section{REFERENCES}

AVEC (2019). AVEC Annual Report. Available online at: https://www.avec-poultry. eu/wp-content/uploads/2019/10/05494-AVEC-annual-report-2019.pdf (accessed April 27, 2020).

Baldi, G., Soglia, F., Mazzoni, M., Sirri, F., Canonico, L., Babini, E., et al. (2018). Implications of white striping and spaghetti meat abnormalities on meat quality and histological features in broilers. Animal 12, 164-173. doi: 10.1017/ S1751731117001069

Barbut, S. (1993). Colour measurements for evaluating the pale soft exudative (PSE) occurrence in turkey meat. Food Res. Int. 26, 39-43. doi: 10.1016/09639969(93)90103-p

Barbut, S. (1996). Estimates and detection of the PSE problem in young turkey breast meat. Can. J. Anim. Sci. 76, 455-457. doi: 10.4141/cjas96-066

Barbut, S. (2009). Pale, soft, and exudative poultry meat-reviewing ways to manage at the processing plant. Poult. Sci. 88, 1506-1512. doi: 10.3382/ps.2009-00118

Barbut, S. (2015). Developments in turkey meat harvesting technologies. World Poult. Sci. J. 71, 59-70. doi: 10.1017/S0043933915000069

Barbut, S., Sosnicki, A. A., Lonergan, S. M., Knapp, T., Ciobanu, D. C., Gatcliffe, L. J., et al. (2008). Progress in reducing the pale, soft and exudative (PSE) problem in pork and poultry meat. Meat Sci. 79, 46-63. doi: 10.1016/j.meatsci. 2007.07.031

Carvalho, R. H. D., Soares, A. L., Guarnieri, P. D., Oba, A., Ida, E. I., and Shimokomaki, M. (2018). Turkey meat. Seasonal effect on meat quality and on dead on arrival index in a commercial plant. Braz. Arch. Biol. Technol. 61:106. doi: 10.1590/1678-4324-2018180106

Chiang, W., Booren, A., and Strasburg, G. (2008). The effect of heat stress on thyroid hormone response and meat quality in turkeys of two genetic lines. Meat. Sci. 80, 615-622. doi: 10.1016/j.meatsci.2008.02.012

Dickinson, E. M., Stevens, J. O., and Helfer, D. H. (1968). “A degenerative myopathy in turkeys," in Proceedings 17th Western Disease Conference, California.

Dutson, R. D., and Carter, A. (1985). Microstructure and biochemistry of avian muscle and its relevance to meat processing industries. Poult. Sci. 64, 15771590. doi: $10.3382 / \mathrm{ps} .0641577$

FAOSTAT (2020). Available online at: http://www.fao.org/faostat/en/\#data/QA (accessed March 23, 2020).

Fraqueza, M. J., Cardoso, A. S., Ferreira, M. C., and Barreto, A. S. (2006). Incidence of pectoralis major turkey muscles with light and dark color in a Portuguese slaughterhouse. Poult. Sci. 85, 1992-2000. doi: 10.1093/ps/85.11.1992

Grey, T. C. (1989). “Turkey meat texture," in Recent Advances in Turkey Science, eds C. Nixey and T. C. Grey (London: Lexis Nexis Publishing company), 289-311.

Grey, T. C., Griffiths, N. M., Jones, J. M., and Robinson, D. (1986). A study of some factors influencing the tenderness of turkey breast meat. Lebensm. Wiss. Technol. 19, 412-414.

Griffin, J. R., Moraes, L., Wick, M., and Lilburn, M. S. (2018). Onset of white striping and progression into wooden breast as defined by myopathic changes underlying pectoralis major growth. Estimation of growth parameters as products. Taken together, unlike broiler chickens, growthrelated breast muscle abnormalities are not a main concern at present for the turkey meat production chain. In this scenario, the PSE-like condition or the proneness of the meat toward oxidation, both during refrigerated and frozen storage, could be considered the main quality issues, and hence other insights are necessary to find practical solutions which could alleviate these problems.

\section{AUTHOR CONTRIBUTIONS}

MZ wrote the first draft of the manuscript. All authors contributed to the manuscript revision, and read and approved the submitted version.

predictors for stage of myopathy progression. Avian Pathol. 47, 2-13. doi: 10.1080/03079457.2017.1356908

Havenstein, G. B., Ferket, P. R., Grimes, J. L., Qureshi, M. A., and Nestor, K. E. (2007). Comparison of the performance of 1966-versus 2003-type turkeys when fed representative 1966 and 2003 turkey diets: growth rate, livability, and feed conversion. Poult. Sci. 86, 232-240. doi: 10.1093/ps/86.2.232

Henrikson, Z. A., Vermette, C. J., Schwean-Lardner, K., and Crowe, T. G. (2018). Effects of cold exposure on physiology, meat quality, and behavior of turkey hens and toms crated at transport density. Poult. Sci. 97, 347-357. doi: 10.3382/ ps/pex227

Hocking, P. M. (2014). Unexpected consequences of genetic selection in broilers and turkeys: problems and solutions. Br. Poult. Sci. 55, 1-12. doi: 10.1080/ 00071668.2014.877692

Jordan, F. T. W., and Pattison, M. (1998). "Deep pectoral myopathy of turkeys and chickens," in Poultry Diseases, eds T. W. Jordan and M. Pattison (London: Saunders), 398-399.

Kuttappan, V. A., Brewer, V. B., Clark, F. D., McKee, S. R., Meullenet, J. F., Emmert, J. L., et al. (2009). Effect of white striping on the histological and meat quality characteristics of broiler fillets. Poult. Sci. 88, 136-137.

Kuttappan, V. A., Hargis, B. M., and Owens, C. M. (2016). White striping and woody breast myopathies in the modern poultry industry: a review. Poult. Sci. 95, 2724-2733. doi: 10.3382/ps/pew216

Kuttappan, V. A., Shivaprasad, H. L., Shaw, D. P., Valentine, B. A., Hargis, B. M., Clark, F. F., et al. (2013). Pathological changes associated with white striping in broiler breast muscles. Poult. Sci. 92, 331-338. doi: 10.3382/ps.201202646

Mahon, M. (1999). "Muscle abnormalities: morphological aspects," in Poultry Meat Science. Poultry Science Symposium Series, Vol. 25, eds R. I. Richardson and G. C. Mead (New York, NY: CABI Publishing), 19-64.

Malila, Y., Carr, K. M., Ernst, C. W., Velleman, S. G., Reed, K. M., and Strasburg, G. M. (2014). Deep transcriptome sequencing reveals differences in global gene expression between normal and pale, soft, exudative turkey meat. J. Anim. Sci. 92, 1250-1260. doi: 10.2527/jas.2013-7293

Mallia, J. G., Barbut, S., Vaillancourt, J. P., Martin, S. W., and McEwen, S. A. (2000). A dark, firm dry-like condition in turkeys condemned for cyanosis. Poult. Sci. 79, 281-285. doi: $10.1093 / \mathrm{ps} / 79.2 .281$

Mudalal, S. (2019). Incidence of white striping and its effect on the quality traits of raw and processed turkey breast meat. Food Sci. Anim. Resour. 39:410. doi: 10.5851/kosfa.2019.e35

OECD/FAO (2016). OECD-FAO Agricultural Outlook 2016-2025. Paris: OECD Publishing.

Orr, J. P., and Riddell, J. R. (1977). Investigation of the vascular supply of the pectoralis muscle of the domestic turkey and comparison of experimentally produced infarcts with naturally occurring deep pectoral myopathy. Am. J. Vet. Res. 38, 1237-1242.

Owens, C. M., Alvarado, C. Z., and Sams, A. R. (2009). Research developments in pale, soft, and exudative turkey meat in North America. Poult. Sci. 88, 1513-1517. doi: 10.3382/ps.2009-2008 
Owens, C. M., Hirschler, E. M., Martinez-Dawson, R., and Sams, A. R. (2000). The characterization and incidence of pale, soft, exudative turkey meat in a commercial plant. Poult. Sci. 79, 553-558. doi: 10.1093/ps/79.4.553

Petracci, M., Bianchi, M., Mudalal, S., and Cavani, C. (2013). Functional ingredients for poultry meat products. Trends Food Sci. Technol. 33, 27-39. doi: $10.1016 /$ j.tifs.2013.06.004

Petracci, M., and Cavani, C. (2012). Muscle growth and poultry meat quality issues. Nutrients 4, 1-12. doi: 10.3390/nu4010001

Petracci, M., Mudalal, S., Babini, E., and Cavani, C. (2014). Effect of white striping on chemical composition and nutritional value of chicken breast meat. Ital. J. Anim. Sci. 13, 179-183. doi: 10.4081/ijas.2014.3138

Petracci, M., Mudalal, S., Soglia, F., and Cavani, C. (2015). Meat quality in fast-growing broiler chickens. World Poult. Sci. J. 71, 363-374. doi: 10.1017/ S0043933915000367

Petracci, M., Soglia, F., Madruga, M., Carvalho, L., Ida, E., and Estévez, M. (2019). Wooden-breast, white striping, and spaghetti meat: causes, consequences and consumer perception of emerging broiler meat abnormalities. Compr. Rev. Food. Sci. Food. Saf. 18, 565-583. doi: 10.1111/1541-4337.12431

Pietrzak, M., Greaser, M. L., and Sosnicki, A. A. (1997). Effect of rapid rigor mortis processes on protein functionality in pectoralis major muscle of domestic turkeys. J. Anim. Sci. 75, 2016-2116.

Radaelli, G., Piccirillo, A., Birolo, M., Bertotto, D., Gratta, F., Ballarin, C., et al. (2017). Effect of age on the occurrence of muscle fiber degeneration associated with myopathies in broiler chickens submitted to feed restriction. Poult. Sci. 96, 309-319. doi: $10.3382 / \mathrm{ps} /$ pew 270

Remignon, H. (2004). "Production of turkeys, geese, ducks and game birds", in Poultry Meat Processing and Quality, ed. G. C. Mead (Duxford: Woodhead Publishing), 211-231. doi: 10.1533/9781855739031.211

Seemann, G., Jones, J. M., Griffiths, N. M., and Grey, T. C. (1986). The influence of storage time-temperature on turkey breast meat quality. Arch. Geflugelkd. 50, 149-153.

Sihvo, H. K., Immonen, K., and Puolanne, E. (2014). Myodegeneration with fibrosis and regeneration in the pectoralis major muscle of broilers. Vet. Pathol. 51, 619-623. doi: 10.1177/0300985813497488

Siller, W. G. (1985). Deep pectoral myopathy: a penalty of successful selection for muscle growth. Poult. Sci. 64, 1591-1595. doi: 10.3382/ps.0641591

Siller, W. G., Wight, A. L., and Martindale, L. (1979). Exercise-induced deep pectoral myopathy in broiler fowls and turkeys. Vet. Sci. Commun. 2, 331-336. doi: $10.1007 /$ bf02291463

Siller, W. G., and Wight, P. A. L. (1978). The pathology of deep pectoral myopathy of turkeys. Avian Pathol. 7, 583-617. doi: 10.1080/03079457808418313

Sirri, F., Maiorano, G., Tavaniello, S., Chen, J., Petracci, M., and Meluzzi, A. (2016). Effect of different levels of dietary zinc, manganese, and copper from organic or inorganic sources on performance, bacterial chondronecrosis, intramuscular collagen characteristics, and occurrence of meat quality defects of broiler chickens. Poult. Sci. 95, 1813-1824. doi: 10.3382/ps/pew064

Soglia, F., Mazzoni, M., and Petracci, M. (2019). Spotlight on avian pathology: current growth-related breast meat abnormalities in broilers. Avian Pathol. 48, 1-3. doi: 10.1080/03079457.2018.1508821

Soglia, F., Baldi, G., Laghi, L., Mudalal, S., Cavani, C., and Petracci, M. (2018). Effect of white striping on turkey breast meat quality. Animal 12, 2198-2204. doi: 10.1017/S1751731117003469

Sosnicki, A. A. (1993). Is the modem turkey prone to pale, soft, exudative (PSE) breast muscle conditions? Meat. Focus Int. 2, 75-78.

Sosnicki, A. A., Cassens, R. G., Mcintyre, D. R., and Vimini, R. J. (1988a). Structural alterations in oedematous and apparently normal skeletal muscle of domestic turkey. Avian Pathol. 17, 775-791. doi: 10.1080/03079458808436501

Sosnicki, A. A., Cassens, R. G., Mcintyre, D. R., Vimini, R. J., and Greaser, M. L. (1988b). Characterization of hypercontracted fibers in skeletal muscle of domestic turkey (Meleagris gallopavo). Food Microst. 7, 147-152.

Sosnicki, A. A., Cassens, R. G., Mcintyre, D. R., Vimini, R. J., and Greaser, M. L. (1989a). Incidence of microscopically detectable degenerative characteristics in skeletal muscle of turkey. Br. Poult. Sci. 30, 69-80. doi: 10.1080/ 00071668908417126
Sosnicki, A. A., Cassens, R. G., Vimini, R. J., and Greaser, M. L. (1989b). Histopathology and morphometry of normal and ischemic muscle in domestic turkey. Poult. Sci. 68:139.

Sosnicki, A. A., Cassens, R. G., Vimini, R. J., and Greaser, M. L. (1991a). Distribution of capillaries in normal and ischemic turkey skeletal muscle. Poult. Sci. 70, 349-357. doi: 10.3382/ps.0700343

Sosnicki, A. A., Cassens, R. G., Vimini, R. J., and Greaser, M. L. (1991b). Histopathological and ultrastructural alterations of turkey skeletal muscle. Poult. Sci. 70, 343-348. doi: 10.3382/ps.0700349

Sosnicki, A. A., Greaser, M. L., Pietrzak, M., and Pospiech, E. (1995). Protein alterations in PSE turkey breast and porcine muscle. Meat Focus Int. 5, 193-195.

Sosnicki, A. A., Greaser, M. L., Pietrzak, M., Pospiech, E., and Sante, V. (1998). PSElike syndrome in breast muscle of domestic turkeys: a review. J. Muscle Foods 9 , 13-23. doi: 10.1111/j.1745-4573.1998.tb00640.x

Sosnicki, A. A., and Wilson, B. W. (1991). Pathology of turkey skeletal muscle: implications for the poultry industry. Food Struct. 10, 317-326.

Strasburg, G. M., and Chiang, W. (2009). Pale, soft, exudative turkey-the role of ryanodine receptor variation in meat quality. Poult. Sci. 88, 1497-1505. doi: $10.3382 /$ ps.2009-2181

Swatland, H. J. (1990). A note on the growth of connective tissues binding turkey muscle fibers together. Can. Inst. Food Sci. Technol. J. 23, 239-241. doi: 10.1016/ S0315-5463(90)70251-70259

Tijare, V. V., Yang, F. L., Kuttappan, V. A., Alvarado, C. Z., Coon, C. N., and Owens, C. M. (2016). Meat quality of broiler breast fillets with white striping and woody breast muscle myopathies. Poult. Sci. 95, 2167-2173. doi: 10.3382/ps/ pew129

Updike, M. S., Zerby, H. N., Sawdy, J. C., Lilburn, M. S., Kaletunc, G., and Wick, M. P. (2005). Turkey breast meat functionality differences among turkeys selected for body weight and/or breast yield. Meat Sci. 71, 706-712. doi: 10.1016/ j.meatsci.2005.05.014

Velleman, S. G. (2015). Relationship of skeletal muscle development and growth to breast muscle myopathies: a review. Avian Dis. 59, 525-531. doi: 10.1637/ 11223-063015-Review.1

Velleman, S. G., Anderson, J. W., Coy, C. S., and Nestor, K. E. (2003). Effect of selection for growth rate on muscle damage during turkey breast muscle development. Poult. Sci. 82, 1069-1074. doi: 10.1093/ps/82.7.1069

Werner, C., Riegel, J., and Wicke, M. (2008). Slaughter performance of four different turkey strains, with special focus on the muscle fiber structure and the meat quality of the breast muscle. Poult. Sci. 87, 1849-1859. doi: 10.3382/ps. 2007-2188

Wilson, B. W., Nieberg, P. S., Buhr, R. J., Kelly, B. J., and Shultz, F. T. (1990). Turkey muscle growth and focal myopathy. Poult. Sci. 69, 1553-1562. doi: $10.3382 /$ ps.0691553

Zaid, A., Abu-Khalaf, N., Mudalal, S., and Petracci, M. (2020). Differentiation between normal and white striped turkey breasts by visible/near infrared spectroscopy and multivariate data analysis. Food Sci. Anim. Resour. 40:96. doi: 10.5851/kosfa.2019.e88

Zampiga, M., Tavaniello, S., Soglia, F., Petracci, M., Mazzoni, M., Maiorano, G., et al. (2019). Comparison of 2 commercial turkey hybrids: productivity, occurrence of breast myopathies, and meat quality properties. Poult. Sci. 98, 2305-2315. doi: 10.3382/ps/pey607

Conflict of Interest: The authors declare that the research was conducted in the absence of any commercial or financial relationships that could be construed as a potential conflict of interest.

Copyright (c) 2020 Zampiga, Soglia, Baldi, Petracci, Strasburg and Sirri. This is an open-access article distributed under the terms of the Creative Commons Attribution License (CC BY). The use, distribution or reproduction in other forums is permitted, provided the original author(s) and the copyright owner(s) are credited and that the original publication in this journal is cited, in accordance with accepted academic practice. No use, distribution or reproduction is permitted which does not comply with these terms. 


\section{OPEN ACCESS}

Edited by:

Krystyna Pierzchala-Koziec,

University of Agriculture in Krakow,

Poland

Reviewed by:

Xiquan Zhang,

South China Agricultural University,

China

Casey M. Owens,

University of Arkansas, United States

*Correspondence:

Yuwares Malila

yuwares.mal@biotec.or.th

Specialty section:

This article was submitted to

Avian Physiology,

a section of the journal

Frontiers in Physiology

Received: 19 December 2019

Accepted: 11 May 2020

Published: 16 June 2020

Citation:

Malila Y, Uengwetwanit T,

Arayamethakorn S, Srimarut $Y$,

Thanatsang KV, Soglia $F$,

Strasburg GM, Rungrassamee $W$ and

Visessanguan W (2020)

Transcriptional Profiles of Skeletal

Muscle Associated With Increasing

Severity of White Striping

in Commercial Broilers.

Front. Physiol. 11:580.

doi: 10.3389/fphys.2020.00580

\section{Transcriptional Profiles of Skeletal Muscle Associated With Increasing Severity of White Striping in Commercial Broilers}

\author{
Yuwares Malila ${ }^{1 *}$, Tanaporn Uengwetwanit' ${ }^{1}$ Sopacha Arayamethakorn', \\ Yanee Srimarut ${ }^{1}$, Krittaporn V. Thanatsang ${ }^{1}$, Francesca Soglia' ${ }^{2}$, Gale M. Strasburg ${ }^{3}$, \\ Wanilada Rungrassamee ${ }^{1}$ and Wonnop Visessanguan ${ }^{1}$
}

\footnotetext{
${ }^{1}$ National Center for Genetic Engineering and Biotechnology (BIOTEC), Thailand Science Park, Pathum Thani, Thailand, ${ }^{2}$ Department of Agricultural and Food Sciences, Alma Mater Studiorum, University of Bologna, Cesena, Italy, ${ }^{3}$ Department of Food Science and Human Nutrition, Michigan State University, East Lansing, MI, United States
}

Development of the white striping (WS) abnormality adversely impacts overall quality of broiler breast meat. Its etiology remains unclear. This study aimed at exploring transcriptional profiles of broiler skeletal muscles exhibiting different WS severity to elucidate molecular mechanisms underlying the development and progression of WS. Total RNA was isolated from pectoralis major of male 7-week-old Ross 308 broilers. The samples were classified as mild $(n=6)$, moderate $(n=6)$, or severe $(n=4)$, based on number and thickness of the white striations on the meat surface. The transcriptome was profiled using a chicken gene expression microarray with onecolor hybridization technique. Gene expression patterns of each WS severity level were compared against each other; hence, there were three comparisons: moderate vs. mild (C1), severe vs. moderate (C2), and severe vs. mild (C3). Differentially expressed genes (DEGs) were identified using the combined criteria of false discovery rate $\leq 0.05$ and absolute fold change $\geq 1.2$. Differential expression of 91,136 , and 294 transcripts were identified in C1, C2, and C3, respectively. There were no DEGs in common among the three comparisons. Based on pathway analysis, the enriched pathways of C1 were related with impaired homeostasis of macronutrients and small biochemical molecules with disrupted $\mathrm{Ca}^{2+}$-related pathways. Decreased abundance of the period circadian regulator suggested the shifted circadian phase when moderate WS developed. The enriched pathways uniquely obtained in C2 were RNA degradation, Ras signaling, cellular senescence, axon guidance, and salivary secretion. The DEGs identified in those pathways might play crucial roles in regulating cellular ion balances and cell-cycle arrest. In C3, the pathways responsible for phosphatidylinositol 3-kinase-Akt signaling, p53 activation, apoptosis, and hypoxia-induced processes were modified. Additionally, pathways associated with a variety of diseases with the DEGs involved in regulation of $\left[\mathrm{Ca}^{2+}\right]$, collagen formation, microtubule-based motor, and immune response were identified. Eight pathways were common to all three comparisons (i.e., calcium signaling, 
Ras-associated protein 1 signaling, ubiquitin-mediated proteolysis, vascular smooth muscle contraction, oxytocin signaling, and pathway in cancer). The current findings support the role of intracellular ion imbalance, particularly $\mathrm{Ca}^{2+}$, oxidative stress, and impaired programmed cell death on WS progression.

Keywords: white striping, commercial broiler, gene expression profile, microarray, myopathy

\section{INTRODUCTION}

White striping (WS) abnormality, as depicted by the appearance of white lines running parallel to muscle fibers on the surface of chicken breast meat, has become of great concern in the poultry industries. Based on their aberrant visual characteristics, the breasts with severe WS condition have reduced appeal to consumers and are downgraded for use as processed meat products, thus resulting in significant economic losses for the poultry industry (Kuttappan et al., 2012, 2016; Petracci et al., 2014). The WS breasts consistently exhibit technological challenges, particularly inferior water holding capacity (Kuttappan et al., 2013; Petracci et al., 2013). The nutritional profile of the WS meat is shifted toward higher fat and lower protein proportions compared to non-WS chicken breasts (Petracci et al., 2014; Zambonelli et al., 2016; Malila et al., 2018).

The specific etiology of the WS abnormality is still under investigation. In order to elucidate the molecular mechanisms underlying development of WS myopathy, Zambonelli et al. (2016) compared the gene expression patterns of pectoralis major muscle from Ross 708 broiler hybrids exhibiting severe WS together with wooden breast (WB) against those without any macroscopic lesions. The differentially expressed genes (DEGs) identified were associated with muscle development, inflammation, polysaccharide metabolism, and calcium signaling. It is worth noting that, in the study of Zambonelli et al. (2016), the abnormal group was affected with both WS and WB conditions. Therefore, the biological pathways directly related with WS development alone could be diluted by the strong effect of WB myopathy and might not be clearly shown in the results. Transcriptome profiles of severe WS (white line thickness $>1 \mathrm{~mm}$ ) in pectoralis major collected from 42-day-old Cobb 500 broilers showed significant increases in transcripts involved in the activation of hypoxia, immune responses, and angiogenesis compared with that of nondefective samples (Marchesi et al., 2019). Dysregulated metabolic processes were also identified at the metabolomic level (Boerboom et al., 2018) with opposite directionalities of some intermediates of tricarboxylic acid cycle as well as incomplete breakdown products of protein catabolism. Recently, using droplet digital polymerase chain reaction (ddPCR), an increased absolute transcript abundance of hypoxia-inducible factor 1 alpha (HIF1A) along with differential expression of HIF1A-related genes were identified in pectoralis major of the WS- and WS+WB-affected Ross 308 broilers (Malila et al., 2019). So far, the growing evidence is pointing toward oxidative stress as a primary contributor to development of WS muscle.

While the previous studies focused on exploring different transcriptomic or metabolomic profiles of severe WS in respect to nondefective muscles, the molecular aspects underlying the progression in severity of WS lesion still remain unclear. The objective of this study was to examine differences in transcriptome profiles and define molecular mechanisms associated with elevating WS severity in pectoralis major collected from 7-week-old male commercial Ross 308 broilers. Our findings regarding the progression of the WS myopathy at molecular level provides complementary information to existing studies concerning the development of WS in commercial broilers. The current findings could lay foundation in the development of an intervention to prevent or delay the progression of WS abnormality.

\section{MATERIALS AND METHODS}

\section{Samples and Sample Collection}

All breast samples were collected within 20 min postmortem from 83 broiler carcasses (7-week-old male Ross 308), raised at a facility of a local poultry meat producer and processed in the industrial processing plant under the routine Halal standard practice. One side of each breast was immediately dissected, snapfrozen, and kept in liquid nitrogen during transportation back to the laboratory (Pathum Thani, Thailand). The muscle samples were stored at $-80^{\circ} \mathrm{C}$ until total RNA isolation. The other side of the breast was dissected, placed in a plastic bag, kept on ice during transportation, and stored at $4^{\circ} \mathrm{C}$ until use.

In this study, all samples were purchased in the form of whole carcasses from the commercial processing plant. Neither experimental treatments or scientific procedures were subjected to the living animals. Thereby, according to BIOTEC Institutional Animal Care and Use Committee, the ethical approval was not required.

\section{White Striping Defect Classification}

The WS abnormality and its severity were inspected using the criteria previously described (Malila et al., 2018). Three WS severity levels (Figure 1), including "mild WS" (1-40 white lines with the thickness of $\leq 0.5 \mathrm{~mm}$ ), "moderate WS" [more than 40 white lines or 1-5 line(s) with the thickness of 1-1.9 mm], and "severe WS" (more than 5 lines with the thickness of 1$1.9 \mathrm{~mm}$ thickness or at least 1 white line with thickness $>2 \mathrm{~mm}$ ), were observed among the samples. The samples showing other abnormalities, e.g., WB, were excluded from this experiment in order to avoid any confounding interpretations from such myopathies. Accordingly, 29 mild, 38 moderate, and 4 severe WS samples were available for this study. Six biological replicates were randomly selected from "mild" and "moderate" groups. For 


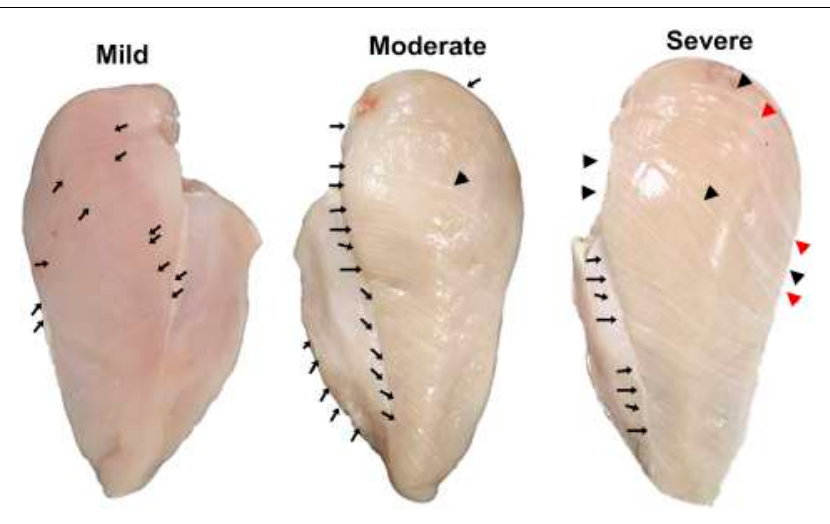

FIGURE 1 | Classification of white striping (WS) abnormality by degree of severity. Mild, 1-40 white lines with the thickness of $\leq 0.5 \mathrm{~mm}$; moderate, more than 40 white lines or 1-5 line(s) with the thickness of 1-1.9 mm; and severe, more than 5 lines with the thickness of 1-1.9 mm thickness or at least 1 white line with thickness $>2 \mathrm{~mm}$. Black arrows, black triangles, and red triangles indicate white lines with thickness $<1.0 \mathrm{~mm}, 1.0-1.9 \mathrm{~mm}$, and $\geq 2.0 \mathrm{~mm}$, respectively.

"severe" samples, all four samples were used. Although our study was initially designed to include the analysis of non-WS samples, there were no nondefective samples (without any macroscopic trait associated with the development of WS) found among our total of 83 samples of the 7 -week-old broilers collected for this trail. This phenomenon emphasized the high prevalence of WS abnormality in 7-week-old commercial broilers.

\section{Total RNA Isolation}

Total RNA was isolated using TRIzol ${ }^{\mathrm{TM}}$ Reagent (Invitrogen), subsequently treated with DNase I (Thermo Scientific, Inc.), and repurified using GeneJET RNA Cleanup and Concentration Micro Kit (Thermo Scientific, Inc.). The samples with an RNA integrity number exceeding 7.0 were analyzed by microarray hybridization at Molecular Genomics Pte. Ltd. (Singapore) and quantitative real-time polymerase chain reaction (qPCR).

\section{Microarray Platform and Experimental Design}

The Agilent SurePrint G3 Custom GE $8 \times 60 \mathrm{~K}$ chicken gene expression microarray (Agilent Technologies, Inc.) used in this study was designed based on the National Center for Biotechnology Information (NCBI) Gallus gallus Annotation Release 103. The detailed information of the array is available at NCBI Gene Expression Omnibus (GEO) with the platform accession GPL24307.

To determine differential gene expression associated with WS severity, expression patterns obtained among the WS levels were compared against one another (Figure 2A). Overall, 16 arrays were hybridized in order to accomplish three comparisons (C1, moderate against mild; C2, severe against moderate; C3, severe against mild).

\section{Microarray Hybridizations}

Total RNA was labeled using one-color low-input Quick Amp labeling kit (Agilent) following the company's instruction. In brief, $100 \mathrm{ng}$ total RNA was reverse transcribed into double-stranded complementary DNA (cDNA) by priming with oligo(dT) primer. The synthesized cDNA was subjected to an in vitro transcription using T7 RNA polymerases to produce cyanine 3-CTP-labeled complementary RNA (cRNA). After purification, $600 \mathrm{ng}$ of the purified cRNA was hybridized onto the Agilent SurePrint array at $65^{\circ} \mathrm{C}$ for $17 \mathrm{~h}$. The array was washed, gently blotted dry, and scanned using an Agilent High-Resolution Microarray Scanner (C Model, Agilent). The TIFF image was saved and analyzed using Agilent Feature Extraction Software version V10.7.1.1 (Agilent).

\section{Microarray Data Analysis and Gene Annotation}

Raw microarray signal values were generated by Agilent Feature Extraction Software version V10.7.1.1 (Agilent). Feature extraction and probe quality control were processed using GeneSpring software. The data were further analyzed in $\mathrm{R}$ version 3.4.0. The signal values of probes deemed to be suspicious or faulty were quarantined using flags and expression values. Flags were categorical indicators including "detected," "compromised," and "not detected" from the scanner. Probes that were flagged as "not detected" and "compromised" were assigned to not available values (NA). Raw signal intensities of probes below 20 for that microarray were assigned as NA values. Probes with missing intensity data were discarded. The filtered raw data were normalized in R using quantile normalization (Bolstad, 2017) and using combat normalization afterward (Müller et al., 2016; Leek et al., 2017). $\log _{2}$ transformation was applied to normalized data for statistical analysis. Statistically significant difference of each transcript between two treatments was identified by analysis of independent two-group $t$ test. The DEGs were identified using combined criteria of false discovery rate $(\mathrm{FDR}) \leq 0.05$ and absolute fold change $(|\mathrm{FC}|) \geq 1$.2. Positive and negative FC values represent increased or decreased expression of a particular gene in the samples exhibiting a greater severity level relative to its counterparts. All microarray data have been submitted to the NCBI GEO repository with GEO accession number GSE107362.

\section{Confirmation of Differential Gene Expression}

Twenty-one genes, resulting in a total of 63 comparison counterparts, were chosen for confirming the microarray data. Of the 63 comparison counterparts, 11 and 18 showed decreased and increased expressions, respectively, with $|\mathrm{FC}| \geq 1.2$, whereas the rest of the genes exhibited $|\mathrm{FC}|<1.2$. Primers (Supplementary Table S1) were designed using Primer-BLAST ${ }^{1}$.

Changes in expression patterns of the selected genes observed in the current microarray analysis were confirmed using qPCR (Malila et al., 2015). Threshold cycle (Ct) was analyzed using BioRad CFX Manager 2.1 software (BioRad).

\footnotetext{
${ }^{1}$ https://www.ncbi.nlm.nih.gov/tools/primer-blast/
} 


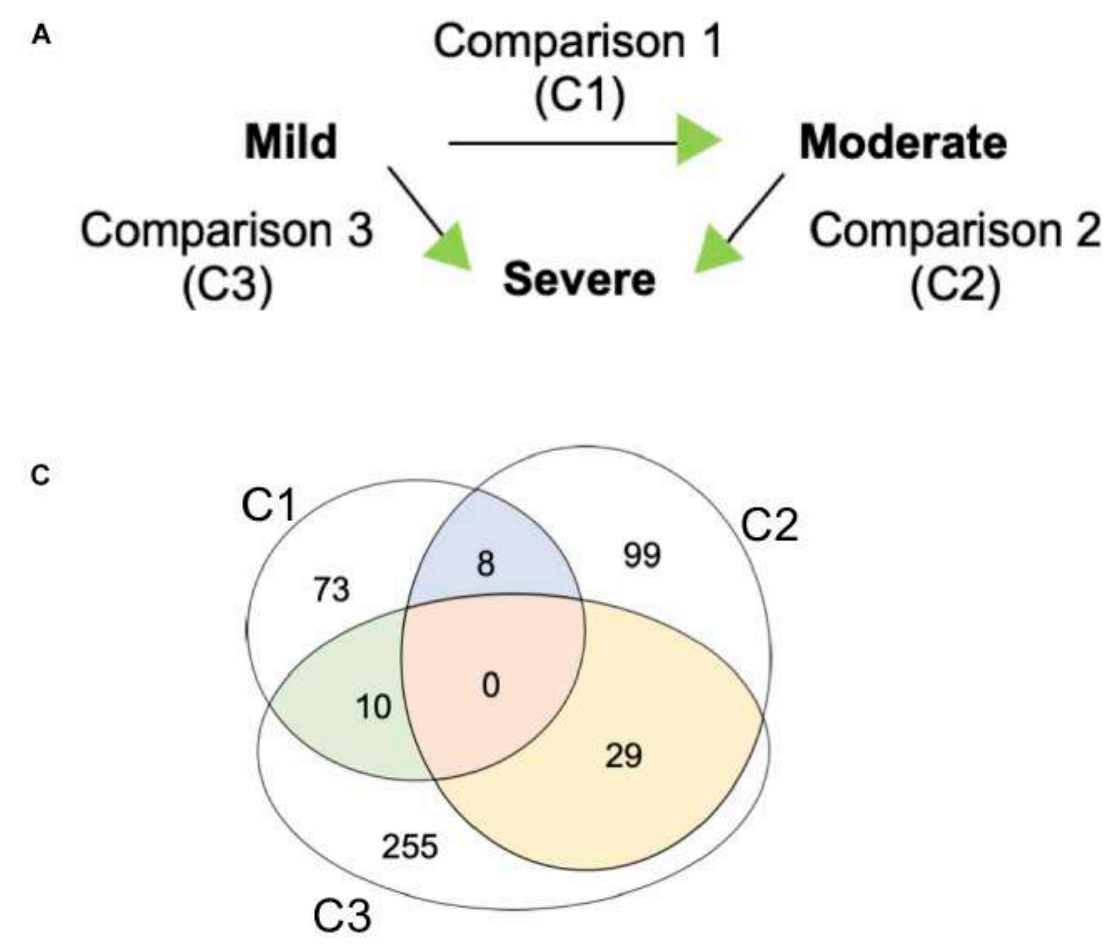

B

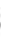

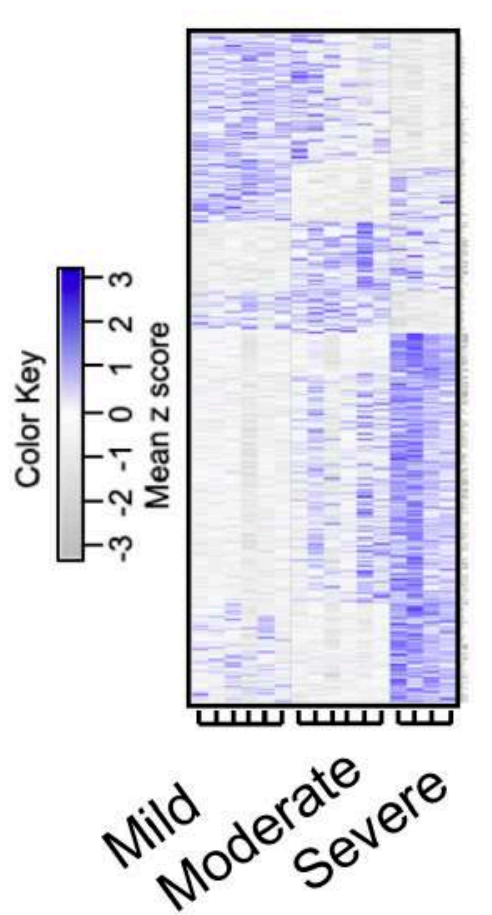

D

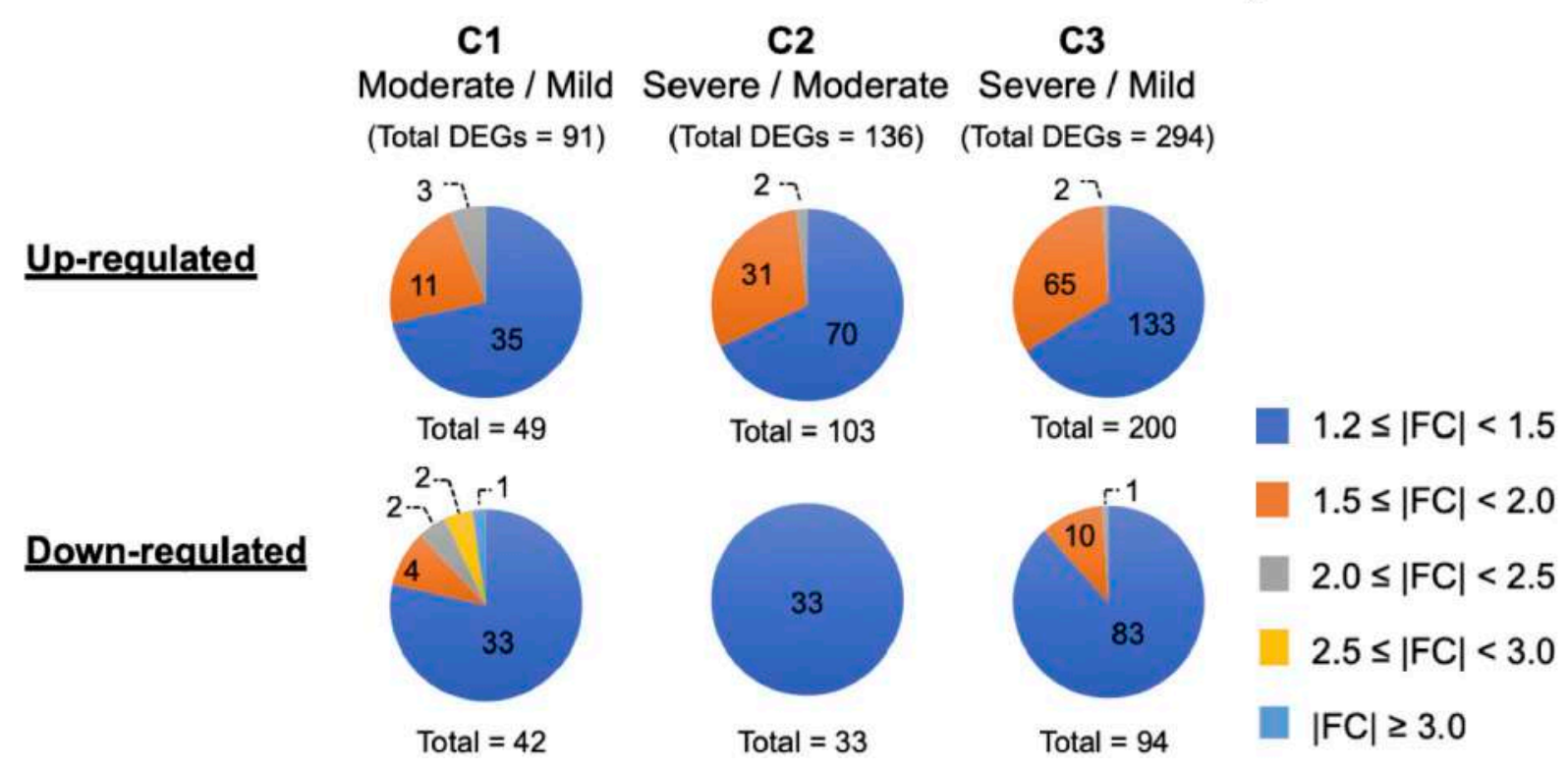

FIGURE 2 | Differential gene expression associated with white striping (WS) severity. (A) Microarray experimental design. The green arrow heads indicate that the particular WS severity level was the numerator in such comparison. (B) Heatmap gene cluster classification for mild $(n=6)$, moderate $(n=6)$, and severe $(n=4)$ WS samples. The expression of each gene is presented in the rows. (C) Venn diagram depicts number of differentially expressed genes (DEGs) obtained from each comparison. The criteria used in identification of DEGs is a combination between false discovery rate $\leq 0.2$ and absolute fold change (|FC|) $\geq 1.2$. (D) Numbers of up- and downregulated DEGs clustered based on |FC|.

Hypoxanthine-guanine phosphoribosyltransferase (HPRT) was chosen as the reference gene due to its unchanged expression across WS groups. Relative messenger RNA (mRNA) abundance of each gene in the sample group relative to its designated counterparts was calculated based on $2^{-\Delta \Delta \mathrm{Ct}}$ method (Livak and
Schmittgen, 2001). To determine statistical difference between two treatments, $\Delta \mathrm{Ct}$ was used in calculation using Student's $t$ test. The significance was set at $p$ value of 0.05 . All relative abundances obtained from qPCR were plotted against $\log _{2} \mathrm{FC}$ analyzed from microarrays. 


\section{Biological Function and Pathway Analysis of Differentially Expressed Genes}

All DEGs of each comparison were grouped into known networks and pathways using Ingenuity Pathway Analysis ${ }^{\circledR}$ (IPA) (trial version, Qiagen) and Kyoto Encyclopedia of Genes and Genomes (KEGG) pathway (Kanehisa et al., 2012). Mapping takes the identifiers from the DEGs and compares them to the database. In IPA, the selected DEGs were mapped to the Ingenuity Knowledge Base database and were then ranked by score, which is calculated based on the hypergeometric distribution and Fisher's exact test. The significance was set at a $p$ value of 0.05 . The enrichment of genes based on KEGG pathways was the number that has highest number of genes mapped on the pathway.

\section{RESULTS}

\section{Differentially Expressed Genes Associated With Increasing WS Severity}

The current microarray analysis revealed 91, 136, and 294 differentially expressed genes in $\mathrm{C} 1, \mathrm{C} 2$, and $\mathrm{C} 3$ comparisons, respectively (Figures 2B,C). The complete DEGs lists for each comparison are available in Supplementary Table S2. No unique genes were common to all three comparisons. However, C1 shared 8 and 10 DEGs with C2 and C3, respectively; for C2 and C3, there were 29 common DEGs (Supplementary Table S3). Approximately $75 \%$ of the DEGs in all comparisons showed $|\mathrm{FC}|$ ranging between 1.2 and 1.5 (Figure 2D), except for the C2 of which all 33 downregulated DEGs fell within such $|F C|$ range. Numbers of the up- and downregulated transcripts were comparable within each comparison. The most up- and downregulated genes in $\mathrm{C} 1$ were chromosome 3 open reading frame $(C 1 O R F 95, F C=2.2)$ and nucleolin-like (LOC107057290, $\mathrm{FC}=-3.3)$, respectively. Mitochondrial translational factor 2 $(M T I F 2, F C=2.3)$ and poly $(\mathrm{A})$-specific ribonuclease regulatory subunit $(P A N 3, \mathrm{FC}=-9.6)$ were the most changed DEGs in C2. For $\mathrm{C} 3$, general transcription factor IIF subunit 1-like $(\mathrm{LOC107051030}, \mathrm{FC}=2.1)$ and neuronal pentraxin $2($ NPTX2, $\mathrm{FC}=-2.5$ ) were the DEGs showing the largest changes.

The changes in gene expression patterns from the present microarray data were further validated using qPCR (Figure 3). Of the 63 comparison counterparts, the changes in expression of 45 counterparts accounted for $71 \%$ of the qPCR data, exhibiting similar trends as that acquired from the microarray platform. As expected, the $|\mathrm{FC}|$ values obtained from qPCR of some genes were greater than those reported in microarray analysis. This is due mainly to better sensitivity of qPCR over microarray (Camarillo et al., 2011).

\section{Functional and Pathway Analysis}

In order to review the enriched biological functions, the DEGs were analyzed using a trial version of IPA. Based on the IPA knowledge base, 50 (55\%), 89 (65\%), and 191 (65\%) DEGs of $\mathrm{C} 1, \mathrm{C} 2$, and $\mathrm{C} 3$, respectively, were recognized and mapped into the respective canonical pathways. Considering the top altered physiological functions (Table 1), the increment of WS severity from mild to moderate $(\mathrm{C} 1)$ and from moderate to severe (C2) similarly impacted organ morphology and development as well as nervous system function. On the other hand, in $\mathrm{C} 3$, the large abundance of DEGs involved cardiovascular system and connective tissue. For cellular functions (Table 2), cell-to-cell

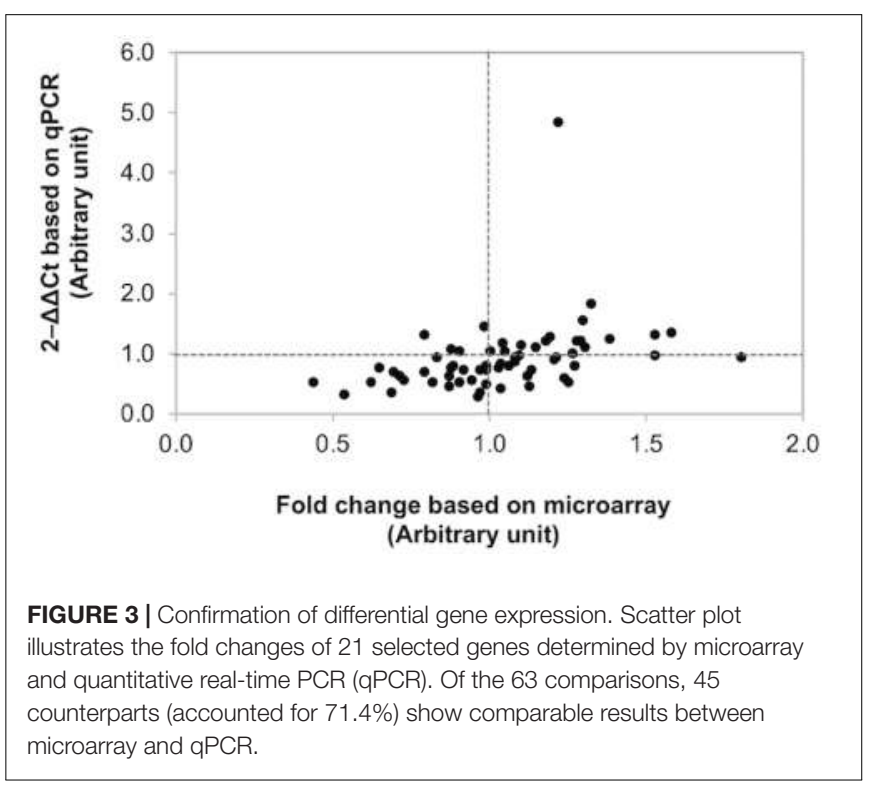

TABLE 1 | The altered physiological functions associated with increasing white striping severity.

\begin{tabular}{|c|c|c|c|}
\hline Physiological functions & \multicolumn{2}{|c|}{$\boldsymbol{P}$ value range } & \#molecules \\
\hline \multicolumn{4}{|l|}{ C1 (Moderate/Mild) } \\
\hline Organ morphology & 4.59E-02 & $9.12 \mathrm{E}-04$ & 6 \\
\hline $\begin{array}{l}\text { Visual system development and } \\
\text { function }\end{array}$ & $9.12 \mathrm{E}-04$ & $9.12 \mathrm{E}-04$ & 2 \\
\hline $\begin{array}{l}\text { Nervous system development and } \\
\text { function }\end{array}$ & 4.39E-02 & $2.13 \mathrm{E}-03$ & 6 \\
\hline Organismal development & 3.15E-02 & $2.13 \mathrm{E}-03$ & 7 \\
\hline $\begin{array}{l}\text { Skeletal and muscular system } \\
\text { development and function }\end{array}$ & 5.00E-02 & $2.13 \mathrm{E}-03$ & 4 \\
\hline \multicolumn{4}{|l|}{ C2 (Severe/Moderate) } \\
\hline $\begin{array}{l}\text { Nervous system development and } \\
\text { function }\end{array}$ & 3.84E-02 & 2.97E-04 & 13 \\
\hline Tissue morphology & 3.49E-02 & 2.97E-04 & 17 \\
\hline $\begin{array}{l}\text { Cardiovascular system } \\
\text { development and function }\end{array}$ & 3.74E-02 & 2.30E-03 & 6 \\
\hline Organ morphology & 3.84E-02 & 2.30E-03 & 14 \\
\hline Organismal development & 3.84E-02 & 2.30E-03 & 19 \\
\hline \multicolumn{4}{|l|}{ C3 (Severe/Mild) } \\
\hline $\begin{array}{l}\text { Cardiovascular system } \\
\text { development and function }\end{array}$ & 1.68E-02 & 7.09E-05 & 18 \\
\hline Organismal development & 1.68E-02 & 7.09E-05 & 30 \\
\hline Tissue morphology & 1.68E-02 & 7.09E-05 & 32 \\
\hline $\begin{array}{l}\text { Nervous system development and } \\
\text { function }\end{array}$ & 1.68E-02 & 2.90E-04 & 29 \\
\hline $\begin{array}{l}\text { Connective tissue development and } \\
\text { function }\end{array}$ & 1.68E-02 & $4.21 \mathrm{E}-04$ & 10 \\
\hline
\end{tabular}


TABLE 2 | The altered cellular functions associated with increasing white striping severity.

\begin{tabular}{|c|c|c|c|}
\hline Cellular functions & \multicolumn{2}{|c|}{$P$ value range } & \#molecule \\
\hline \multicolumn{4}{|l|}{ C1 (Moderate/Mild) } \\
\hline Amino acid metabolism & 3.77E-02 & $1.20 \mathrm{E}-03$ & 3 \\
\hline Small molecule biochemistry & 5.00E-02 & $1.20 \mathrm{E}-03$ & 13 \\
\hline Carbohydrate metabolism & 5.00E-02 & $1.40 \mathrm{E}-03$ & 5 \\
\hline Lipid metabolism & 4.59E-02 & 1.40E-03 & 3 \\
\hline Cell-to-cell signaling and interaction & 5.00E-02 & $2.13 \mathrm{E}-03$ & 6 \\
\hline \multicolumn{4}{|l|}{ C2 (Severe/Moderate) } \\
\hline Cell morphology & 3.91E-02 & 2.97E-04 & 18 \\
\hline Protein synthesis & $3.84 \mathrm{E}-02$ & 8.10E-04 & 6 \\
\hline Cell-to-cell signaling and interaction & 3.46E-02 & $3.31 \mathrm{E}-03$ & 11 \\
\hline Cellular assembly and organization & 3.91E-02 & 3.31E-03 & 18 \\
\hline Carbohydrate metabolism & $3.08 \mathrm{E}-02$ & 3.90E-03 & 9 \\
\hline \multicolumn{4}{|l|}{ C3 (Severe/Mild) } \\
\hline Cellular compromise & $1.68 \mathrm{E}-02$ & $4.21 \mathrm{E}-04$ & 14 \\
\hline Cellular morphology & $1.68 \mathrm{E}-02$ & $5.19 E-04$ & 30 \\
\hline Cellular growth and proliferation & $1.22 \mathrm{E}-02$ & $6.20 \mathrm{E}-04$ & 20 \\
\hline Cell-to-cell signaling and interaction & $1.68 \mathrm{E}-02$ & 8.58E-04 & 25 \\
\hline Cell cycle & 1.68E-02 & 1.04E-03 & 13 \\
\hline
\end{tabular}

signaling and interaction was the overlapping category among the three comparisons. The increasing WS severity from mild to moderate (C1) mainly affected amino acid, carbohydrate, and lipid metabolisms. Although the altered cellular functions in C2 were somewhat similar with those in $\mathrm{C} 1$, the DEGs involved in cellular morphology and cellular assembly and organization were also enriched. As for C3, the large enrichment of DEGs was related with cellular compromise and the changes in cellular growth, proliferation, and cell cycle. All DEGs in the top altered cellular functions are listed in Supplementary Table S3.

To visualize the functional interactions among the DEGs, all DEGs of each comparison were subsequently submitted to KEGG pathway database. The top 30 enriched pathways annotated by KEGG are depicted in Figure 4. Among those enriched pathways, eight pathways, including metabolic pathways, calcium signaling (Figure 5), pathway in cancer (Figure 6), ubiquitin-mediated proteolysis (Supplementary Figure S1), RAP1 signaling (Supplementary Figure S2), adrenergic signaling in cardiomyocytes (Supplementary Figure S3), vascular smooth muscle contraction (Supplementary Figure S4), and oxytocin signaling (Supplementary Figure S5) are overlapped among all severity levels. The altered pathways uniquely defined for each comparison and the overlapping pathways are discussed in detail below.

\section{DISCUSSION}

The severity of the WS abnormality varies among the meat depending on the age, the gender, and the size of the chickens as well as depending on their management, e.g., feeding regime. In our previous investigation (Malila et al., 2018), the incidence of the WS abnormality was as high as $98 \%$ of the samples with broad spectrum of severity. The majority of the abnormal

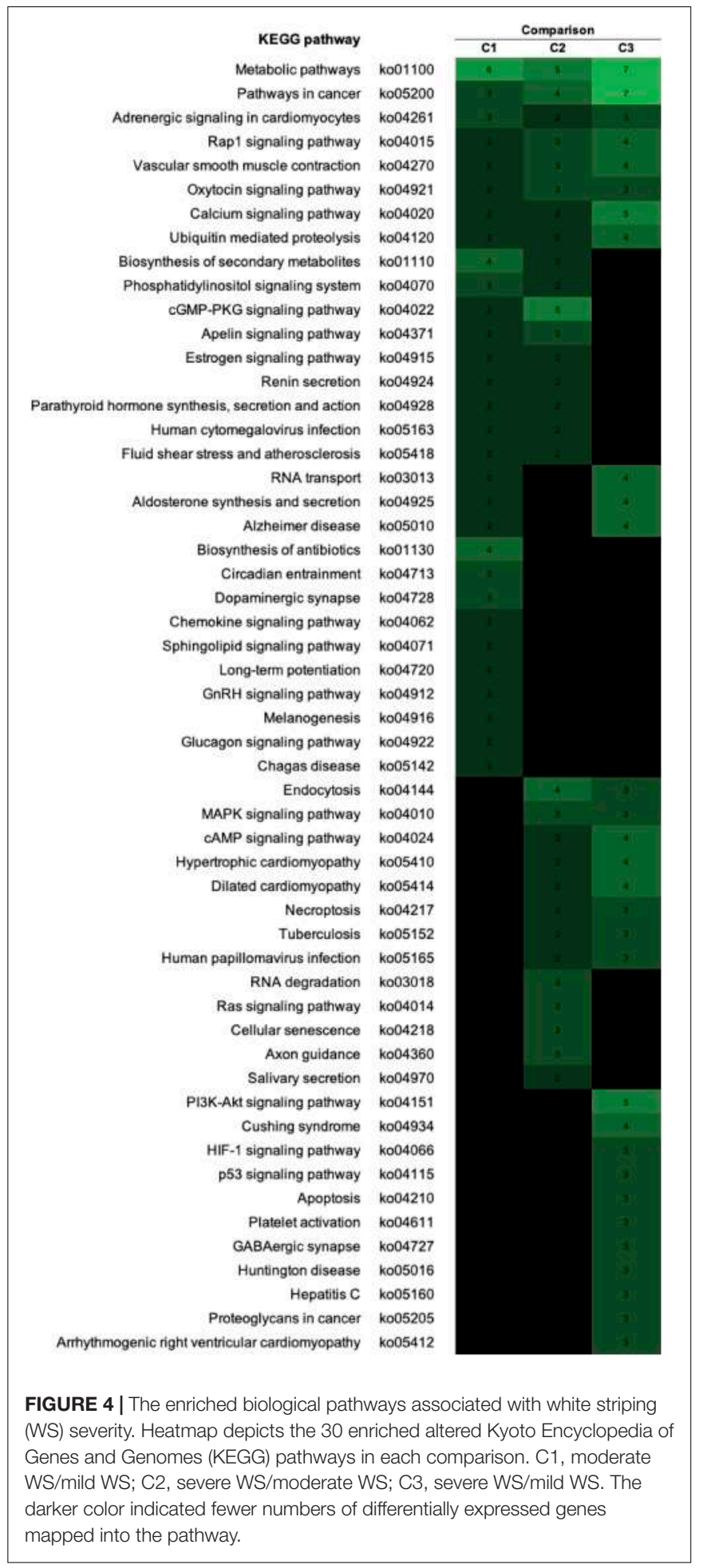

meat samples were categorized as mild (55.7\% of 183 collected broilers) which showed little to no effect on both nutritional and technological properties of the meat. In contrast, severe WS was detected at a small prevalence of $3.3 \%$ but significantly impact the quality of the resulting meat (Malila et al., 2018). Once downgraded and used for manufacturing processed meat 


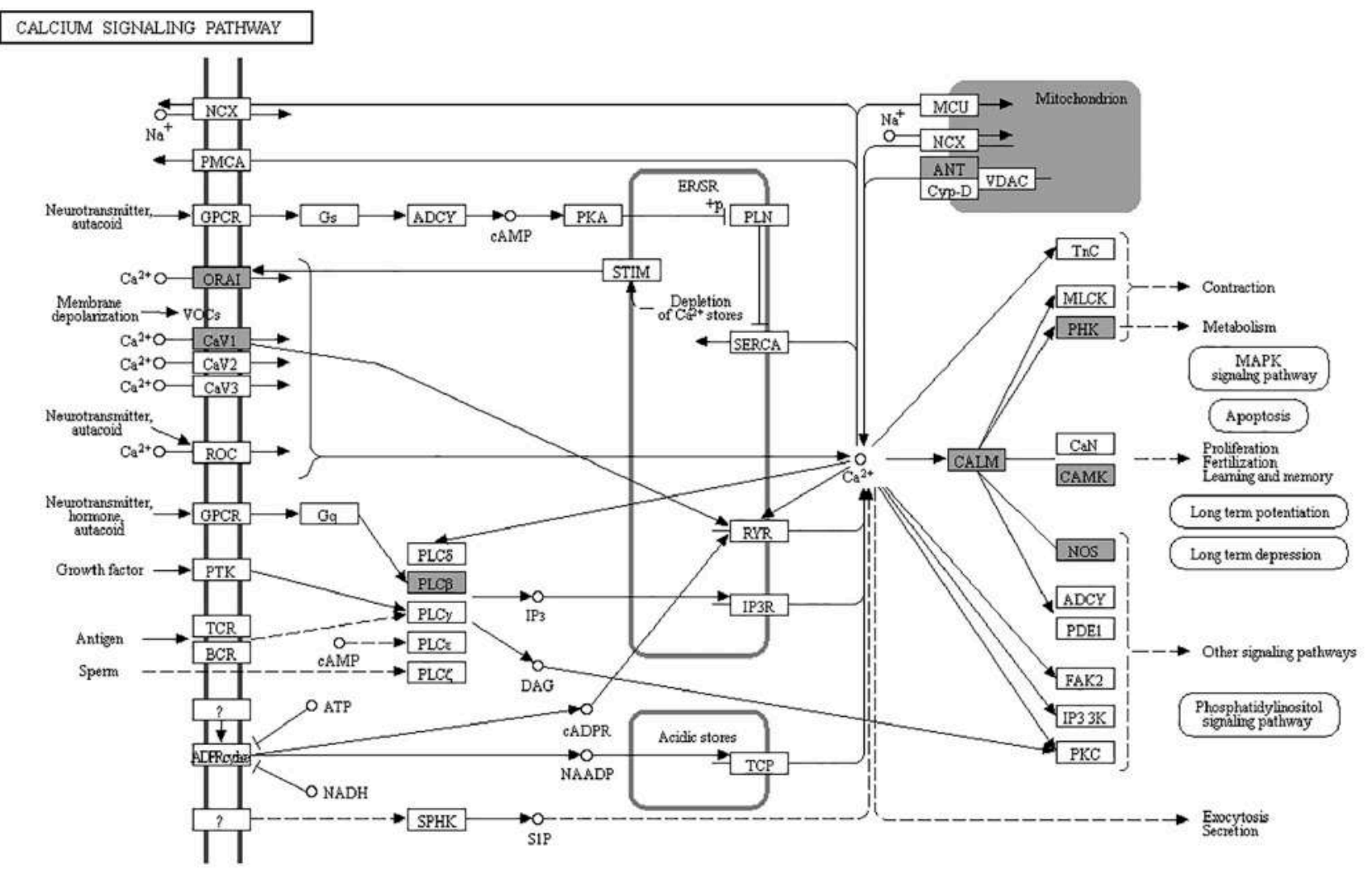

\begin{tabular}{|c|c|c|c|c|c|}
\hline Aliases & ко & KO description & Mild & Moderate & Severe \\
\hline ANT & K05863 & SLC25A4S; solute carrier family 25 (mitochondrial adenine nucleotide translocator), member & 15,497 & 17,527 & 13,370 \\
\hline PHK & K07190 & PHKA_B; phosphorylase kinase alpha/beta subunit & 6.201 & 5.494 & 4,127 \\
\hline CALM & K02183 & CALM; calmodulin & 158 & 121 & 151 \\
\hline PLCB & K05858 & PLCB; phosphatidylinositol phospholipase C, beta [EC:3.1.4.11] & 18 & 24 & 25 \\
\hline NOS & K13242 & NOS3; nitric-oxide synthase, endothelial [EC: 1.14 .13 .39$]$ & 94 & 115 & 127 \\
\hline Cav1 & K04851 & CACNA1D; voltage-dependent calcium channel L type alpha-1D & 204 & 223 & 358 \\
\hline CAMK & K04515 & CAMK2; calcium/calmodulin-dependent protein kinase (CaM kinase) \| [EC:2.7.11.17] & 1,001 & 968 & 1,267 \\
\hline ORAI & K16056 & ORAl1; calcium release-activated calcium channel protein 1 & 1,556 & 1.726 & 2,113 \\
\hline
\end{tabular}

FIGURE 5 | Calcium signaling pathway. The proteins highlighted in gray indicate the differential expression of their encoded genes in at least one comparison. The table indicates fluorescent signal intensity obtained from microarray analysis.

products, the severe WS breasts, even in small proportions, might alter the quality and consistency of an entire batch of processed products (Petracci and Cavini, 2012). In this study, we profiled transcriptomes within the pectoralis major of 7 week-old broilers exhibiting three different WS levels (i.e., mild, moderate, and severe) with the aim of understanding how changes in the transcriptome are associated with increasing WS severity. Since we did not obtain any nondefective breast samples from our sampling sites, the experiment was modified to compare each pair of defective based on their WS severity (Figure 2A).
Regarding the shared DEGs between each comparison pair (Supplementary Table S3), the eight shared DEG responses between $\mathrm{C} 1$ and $\mathrm{C} 2$ exhibited comparable fold changes but in opposite directions. The results indicated that differential gene expression patterns were associated with the progression of the myopathic conditions from mild to moderate, followed by a return to non-significant levels similar to those of the mild samples when the WS severity increased from moderate to a severe degree. These expression patterns suggest the crucial roles of those DEGs in development of moderate WS as well as the potent metabolic switch when the myopathy progressed from 


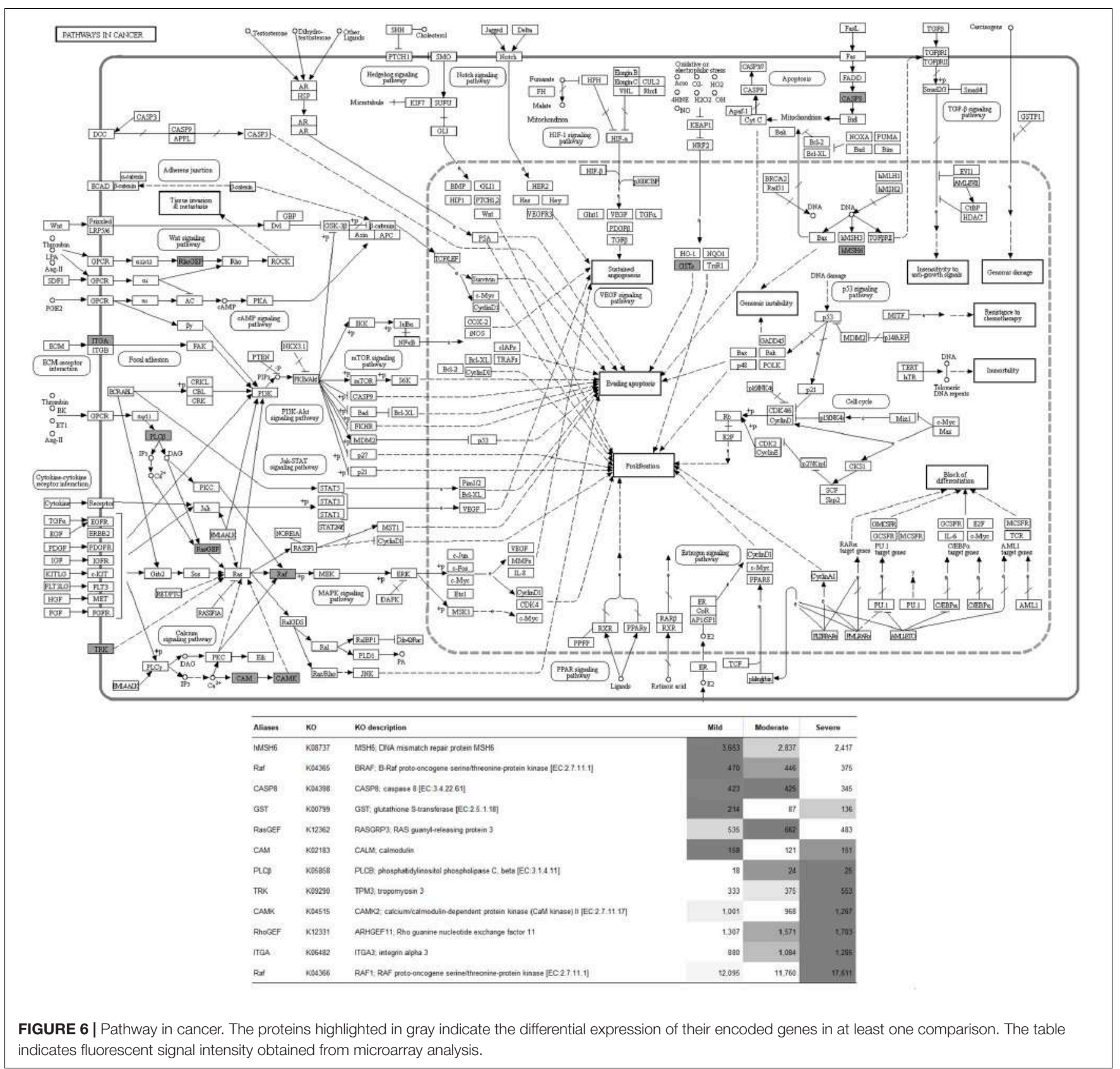

moderate to severe level. Two out of the eight shared DEGs, the transcripts of dimethyladenosine transferase 1 (DIMT1) and zinc finger protein 251 (LOC101750329), are involved in RNA processing. The other two transcripts, the plateletspecific isoform of phosphofructokinase (PFKP) and phytanoylCoA dioxygenase domain-containing protein 1 (LOC100858951), play essential roles in carbohydrate and lipid metabolisms, respectively. The gene $C A L M$ encodes the $\mathrm{Ca}^{2+}$-binding protein calmodulin that mediates a wide range of cellular response to the changes in intracellular $\left[\mathrm{Ca}^{2+}\right]$ (Hamilton et al., 2000). The relevant roles in maintenance of cellular structure of mitochondrial ribosome-associated GTPase 2 ( $m$ tg 2 ) and spastin (SPAST) have been documented (Datta et al., 2005; Roll-Mecak and Vale, 2008). For eva-1 homolog B (EVA1B), although the specific function of this member of EVA1 family is still scarce, the roles of EVA1 in mediating muscle cell extensions were previously demonstrated in the nematode Caenorhabditis elegans (Chan et al., 2014).

Based on the fold changes of the 10 DEGs shared between $\mathrm{C} 1$ and $\mathrm{C} 3$, the expressions of those DEGs were altered when the WS severity progressed from mild to the other severity degrees. Among those 10 shared DEGs, increased expressions of prolyl 4-hydroxylase, transmembrane (P4HTM), and sperm autoantigenic protein 17 (SPA17) were of interest. The P4HTM encodes an endoplasmic reticulum-anchored prolyl 4-hydroxylase possessing a transmembrane domain. Unlike the 
other prolyl 4-hydroxylases that act on collagens, this specific isoform catalyzes hydroxylation of the critical prolines in HIF-1 $\alpha$ but not in type I procollagen, suggesting the crucial participation of this isoform in adaptive response under reduced oxygen availability (Koivunen et al., 2007). Transcription of this gene was induced in human cell lines exposed to hypoxic condition (Koivunen et al., 2007), supporting the greater extent of hypoxia within the moderate and severe WS breasts in comparison to mild WS. As for SPA17, the potential pathogenic role of this transcript, but not the protein, in highly proliferating and neoplastic cells has been addressed (De Jong et al., 2002). The increased SPA17 mRNA abundance in moderate and severe WS might reflect increased cell proliferation within those samples. Considering the DEGs shared between $\mathrm{C} 2$ and $\mathrm{C} 3$, similar fold changes between those two comparisons indicated the upregulation or, in the case of neuronal pentraxin 2 (NPTX2), downregulation only in severe WS samples. Among those 29 shared DEGs between $\mathrm{C} 2$ and $\mathrm{C} 3$, six transcripts were annotated as noncoding RNAs (ncRNA), whereas the other two were annotated as miscellaneous RNAs (miscRNA). The regulatory roles of ncRNAs in oxidative stress response and development of cardiac hypertrophy and apoptosis, the biological pathways frequently identified to be associated with myopathies in broilers, were also addressed (Dong et al., 2019; Turunen et al., 2019). In addition, Zhang et al. (2017) identified a set of ncRNAs involved in regulation of intramuscular preadipocyte differentiation in breast muscle of Chinese indigenous chickens, which was similar to the deposition of fat on connective tissue of the WS breasts. Still, specific biological functions of those ncRNAs associated with WS development remain to be elucidated. Overall, the list of the shared DEGs between the comparisons suggests the key genes partly responsible for each WS severity degree.

\section{Comparison Between Mild and Moderate WS Degrees (C1)}

The small numbers of DEGs in $\mathrm{C} 1$ relative to the other comparisons suggest slight differences at the cellular level between mild and moderate WS degree. Considering the unique enriched biological pathways altered specifically for C1 (Table 3), the majority of the pathways are linked with phosphatidylinositol phospholipase C (PLCB) and CALM. The PLCB-encoding enzyme catalyzes the hydrolysis of phosphatidylinositol 4,5-bisphosphate into two secondary messengers, inositol triphosphate $\left(\mathrm{IP}_{3}\right)$ and diacylglycerol (DAG), which regulate various transduction cascades, particularly homeostasis of macronutrients and intracellular $\left[\mathrm{Ca}^{2+}\right]$ (Kadamar and Ross, 2013). The CALM, the second most differentially expressed gene in $\mathrm{C} 1$, plays roles in a vast diversity of cellular functions (Berchtold and Villalobo, 2014). In this study, an increased PLCB expression coupled with reduced CALM abundance identified in moderate WS compared with those of mild WS appeared to alter metabolisms of macronutrients as well as the biological processes, including circadian clock, dopaminergic synapse, long-term potentiation, gonadotrophin-releasing hormone $(\mathrm{GnRH})$ secretion, melanogenesis, and glucagon signaling (Table 3). Alteration of lipid and amino acid metabolisms could be partly supported by an increase in hepatic aminoadipate aminotransferase (AADAT), currently highlighted in the pathway of antibiotic biosynthesis. Upregulation of AADAT, reported in liver of fattening pigs, was speculated to promote triglyceride biosynthesis from degraded lysine (Kojima et al., 2018).

The association between the dysregulated intracellular $\left[\mathrm{Ca}^{2+}\right]$ and development of moderate WS was emphasized by the 1.4fold decreased chemokine (C motif) ligand (XCL1), encoding lymphotactin as shown in the chemokine signaling pathway (Table 3). Similar to other chemokines, lymphotactin recruits $\mathrm{T}$ and natural killer (NK) cells and induces intracellular $\mathrm{Ca}^{2+}$ mobilization by binding a specific G-protein-coupled receptor (Volkman et al., 2009).

Among the pathways relevant to dopaminergic synapse, sphingolipid signaling, and Chagas disease, transcript abundance of the beta isoform of regulatory subunit 55 of protein phosphatase 2A (PPP2R2B) was decreased 1.2-fold in moderate WS relative to mild samples. Decreased expression of $P P P 2 R 2 B$ has been linked with the promotion of deregulated oncogene signaling pathways required for survival and proliferation of cancer cells (Tan et al., 2010), suggesting the different cell proliferation rates between mild and moderate WS.

Regarding the circadian entrainment, the changes in the clock gene networks not only associated with the disrupted immunity and metabolic disorders (Turek et al., 2005) but also the structure within the muscle (Harfmann et al., 2015). In this study, apart from $P L C B$ and CALM, an increased

TABLE 3 | The unique Kyoto Encyclopedia of Genes and Genomes (KEGG) pathways highlighted in $\mathrm{C} 1$.

\begin{tabular}{|c|c|c|c|c|}
\hline & KEGG pathway & ко & Gene ID & Fold change \\
\hline \multirow[t]{4}{*}{ ko01130 } & Biosynthesis of antibiotics & K00825 & $A A D A T$ & 1.21 \\
\hline & & K00850 & PFKP & 1.21 \\
\hline & & K00869 & MVK & 1.24 \\
\hline & & K11787 & GART & -2.22 \\
\hline \multirow[t]{3}{*}{ ko04713 } & Circadian entrainment & K02183 & CALM & -1.31 \\
\hline & & K05858 & $P L C B$ & 1.32 \\
\hline & & K21945 & PER3 & 2.02 \\
\hline \multirow[t]{3}{*}{ ko04728 } & Dopaminergic synapse & K02183 & CALM & -1.31 \\
\hline & & K04354 & PPP2R2B & -1.20 \\
\hline & & K05858 & PLCB & 1.32 \\
\hline \multirow[t]{2}{*}{ ko04062 } & Chemokine signaling pathway & K05507 & $X C L 1$ & -1.44 \\
\hline & & K05858 & PLCB & 1.32 \\
\hline \multirow[t]{2}{*}{ ko04071 } & Sphingolipid signaling pathway & K04354 & PPP2R2B & -1.20 \\
\hline & & K05858 & $P L C B$ & 1.32 \\
\hline \multirow[t]{2}{*}{ ko04720 } & Long-term potentiation & K02183 & CALM & -1.31 \\
\hline & & K05858 & $P L C B$ & 1.32 \\
\hline \multirow[t]{2}{*}{ ko04912 } & GnRH signaling pathway & K02183 & CALM & -1.31 \\
\hline & & K05858 & $P L C B$ & 1.32 \\
\hline \multirow[t]{2}{*}{ ko04916 } & Melanogenesis & K02183 & CALM & -1.31 \\
\hline & & K05858 & $P L C B$ & 1.32 \\
\hline \multirow[t]{2}{*}{ ko04922 } & Glucagon signaling pathway & K02183 & CALM & -1.31 \\
\hline & & K05858 & PLCB & 1.32 \\
\hline \multirow[t]{2}{*}{ ko05142 } & Chagas disease & K04354 & PPP2R2B & -1.20 \\
\hline & & K05858 & PLCB & 1.32 \\
\hline
\end{tabular}


abundance of period circadian regulator 3 (PER3) was observed. This gene product plays an important role in adipogenesis as indicated by experiments showing that deletion of PER3 promoted adipocyte precursor cells in mouse subcutaneous adipose tissue (Aggarwal et al., 2017). Although the expression of PER3 was upregulated in the $\mathrm{C} 1$ comparison in the current study, differential expression of this gene likely affects adipogenesis in these birds and may be partly responsible for the increased fat content within the moderate WS breasts. As the light is the dominant signal for the biological clock-controlled system, the findings imply that the lighting management might partly influence the entanglement of biological cascades, including circadian clock and metabolic functions during the progression of WS from mild to moderate level. Further investigation is required to elucidate such association.

Overall, the current transcriptome profiling consistently suggested the association between the progression of mild to moderate WS and the dysregulated metabolic processes of macronutrients along with the shifts of $\mathrm{Ca}^{2+}$ homeostasis. The impacts on amino acids, lipids, and carbohydrate were in accordance with the changes in glycolytic enzymes (Kuttappan et al., 2017) and metabolites from tricarboxylic acid cycle (Boerboom et al., 2018) previously reported in pectoralis major of WS + WB samples, and the differential expression of various genes related to carbohydrate and fatty acid metabolisms identified in WS-affected Cobb 500 broilers (Marchesi et al., 2019).

\section{Comparison Between Moderate and Severe WS Degrees (C2)}

Similar to $\mathrm{C} 1$, differential gene expression identified in C2 in which the WS abnormality progressed from moderate to severe levels were related to metabolisms and physiological activities within the abnormal broilers through the signals of phosphatidylinositol, cyclic guanosine monophosphate (cGMP)dependent protein kinase, and apelin (Figure 4). Consistent with $\mathrm{C} 1$, an altered intracellular ion homeostasis, particularly $\mathrm{Ca}^{2+}$, was observed in C2. An increased CALM abundance in severe WS relative to moderate WS might exert relevant roles in several unique enriched biological pathways, including Ras signaling, cellular senescence, and axon guidance (Table 4). In addition to changes in $\left[\mathrm{Ca}^{2+}\right]$, a difference in $\left[\mathrm{K}^{+}\right]$between severe and moderate WS might be hypothesized as a consequence of the 1.5-fold increase in transcriptional level of the calciumactivated potassium channel subunit alpha-1 (KCNMA1). In salivary secretion pathway, the product of KCNMA1 functions as a component of the voltage-gated potassium channel, which is activated by either an increased intracellular $\left[\mathrm{Ca}^{2+}\right]$ or changes in membrane electrical potential (Miller, 2000).

Unlike $\mathrm{C} 1$, the modules containing sequential activities regulating cell proliferation, differentiation, and death, particularly through the pathways of mitogen-activated protein kinase (MAPK) necroptosis, RNA degradation, and cellular senescence (Figure 4), were impacted in C2. In addition, RNA degradation and cellular senescence were among the five unique enriched pathways identified for C2 (Table 4).
TABLE 4 | The unique Kyoto Encyclopedia of Genes and Genomes (KEGG) pathways highlighted in $\mathrm{C} 2$.

\begin{tabular}{|c|c|c|c|c|}
\hline & KEGG Pathway & KO & Gene ID & Fold change \\
\hline \multirow[t]{3}{*}{ ko03018 } & RNA degradation & K00850 & PFKP & -1.29 \\
\hline & & K12572 & PAN3 & -9.57 \\
\hline & & K12584 & DCPS & 1.23 \\
\hline \multirow[t]{3}{*}{ ko04014 } & Ras signaling pathway & K02183 & CALM & 1.25 \\
\hline & & K04366 & $R A F 1$ & 1.50 \\
\hline & & K12362 & RASGRP3 & -1.37 \\
\hline \multirow[t]{3}{*}{ ko04218 } & Cellular senescence & K02183 & CALM & 1.25 \\
\hline & & K04366 & $R A F 1$ & 1.50 \\
\hline & & K05863 & $S L C 25 A 6$ & -1.31 \\
\hline \multirow[t]{3}{*}{ ko04360 } & Axon guidance & K04366 & RAF1 & 1.50 \\
\hline & & K05112 & EPHB3 & 1.52 \\
\hline & & K06820 & PLXNA2 & 1.30 \\
\hline \multirow[t]{2}{*}{ ko04970 } & Salivary secretion & K02183 & CALM & 1.25 \\
\hline & & K04936 & KCNMA1 & 1.53 \\
\hline
\end{tabular}

Considering the complete list of the unique enriched pathways identified in C2 (Table 4), most of them transmit signals in governing cell proliferation and survival. In Ras signaling, Ras functions as a molecular switch for signaling pathways through a cycle of guanidine triphosphates (GTP)- and guanidine diphosphate (GDP)-bound states (Milburn et al., 1990). One of the mechanisms by which GTP-Ras operates includes the recruitment of Raf-1 proteins, encoded by $R A F 1$, to form Raf-1 heterodimers, which in turn stimulate various cellular signaling cascades that control cell proliferation, differentiation, and migration (Zenonos and Kyprianou, 2013). As for the axon guidance, its signal cues non-canonically regulate cell proliferation, cell migration, and gene expression (Russell and Bashaw, 2018). It is also interesting that several DEGs in C2 exhibited consistent fold change with the expression required for proliferation and survival of various cancer cell types as addressed in previous studies. For instance, suppressed Ras guanyl-releasing protein 3 (RASGRP3) was previously identified in transgenic hypertrophic mouse hearts of which malignant mutations of cardiac myosin regulatory light chain (Huang et al., 2016). In the study of Ji et al. (2011), the abundance of ephrin type-B receptor 3 (EPHB3) was positively correlated with severe pathological characteristics, particularly tumor size, in lung cancer cells. Overexpression of KCNMA1 promoted tumor progression in mouse model (Khaitan and Ningaraj, 2019). Taken together, the underlined molecular shifts when the WS abnormality elevating from moderate to severe lesion was more likely related with dysregulated cell proliferation.

In addition to the potential abnormality in cell proliferation, the transcriptional change specific for $\mathrm{C} 2$ exerted more deleterious impact than those observed in $\mathrm{C} 1$. The speculation was supported by the two unique enriched pathways, including RNA degradation and cellular senescence. The RNA degradation is required for monitoring RNA species in living cells. Transcriptional changes of a regulatory subunit of poly(A)nuclease (PAN3, decreased by 9.6-fold) and scavenging decapping enzyme (DCPS, increased by 1.2-fold) suggest 
the fluctuations in RNA degradation pathway to a greater extent in severe WS in compared to moderately affected cases. Even small fluctuations are adequate to initiate cell-to-cell variation and interfere with the precision of cell division cycle (Baudrimont et al., 2019). Knockdown of PFKP stimulates survival of breast cancer cells (Kim et al., 2017). In addition to $P F K P$, a delayed poly (A) tail degradation associated with reduced PAN3 protein level was observed in HeLa cells subjected to oxidative stress (Yamagishi et al., 2014). The findings underline the more pronounced deviation of cell cycle and accelerated cell proliferation with the increment of WS from moderate to severe level. As for cellular senescence, this pathway is an irreversible cell-cycle arrest induced by a number of endogenous and exogeneous stresses arising from oncogene, DNA damage, and oxidative stress (Zhao et al., 2016). Accumulation of the senescent cells interferes with the functions of stem cells, attenuates muscle regeneration, and promotes an imbalance between bone formation and bone resorption (Baar et al., 2018). Sun et al. (2017) reported an increase in senescent biomarkers in association with isoproterenol-induced pathological cardiac hypertrophy, which also exhibited fibrosis similar to those found in severe WS muscle (Kuttappan et al., 2013; Malila et al., 2019).

The findings in the current microarray study may reflect the uncontrolled proliferation of muscle cells and the disruption of cell cycle within the hypertrophic breast affected with severe WS in comparison to moderate level.

\section{Comparison Between Mild and Severe WS Degrees (C3)}

Microarray data of C3 revealed the greatest numbers of DEGs due potentially to the profound distinctive phenotypic characteristics between the severe and mild groups. Approximately twothirds of the DEGs in C3 exhibited positive fold changes. Differences, at gene expression level, in cardiovascular system, connective tissue (Table 1), cell cycle, and cellular compromise (Table 2) are highlighted in between severe and mild cases. The enriched biological pathways specific for C3 (Table 5) could reflect the transcriptional alterations of signaling hubs, including phosphatidylinositol 3-kinase (PI3K)/Akt pathway and p53 cascade, and the downstream outputs of such stress-induced adaptive mechanisms.

Based on the DEGs enlisted in the PI3K/Akt signaling, it is interesting that several DEGs, including angiopoietin 1-receptor tyrosine kinase $(T E K)$, integrin subunit alpha 3 (ITGA3), membrane associated guanylate kinase with inverted orientation (MAGI1), and nitric oxide synthase 3 (NOS3), are found mainly in endothelial cells (Laura et al., 2002; Zhen et al., 2008). The TEK-encoding enzyme regulates vascular morphogenesis and homeostasis (Augustin et al., 2009). Targeted deletion of TEK contributed to postnatal degeneration of newly formed veins and development of hemangioma-like vascular tufts in mouse retina (Chu et al., 2016). Upregulated ITGA3 has been linked with a progression of renal failure (Steenhard et al., 2012) and pathological features of liver cancer (Huang et al., 2018). Depletion of MAGI1 induced upregulation of endoplasmic reticulum (ER) stress-related genes, which in turn
TABLE 5 | The unique Kyoto Encyclopedia of Genes and Genomes (KEGG) pathways highlighted in C3.

\begin{tabular}{|c|c|c|c|c|}
\hline & KEGG Pathway & KO & Gene ID & Fold change \\
\hline \multirow[t]{5}{*}{ ko04151 } & $\begin{array}{l}\text { PI3K-Akt signaling } \\
\text { pathway }\end{array}$ & K05121 & TEK & -1.37 \\
\hline & & K05631 & MAG/1 & -1.45 \\
\hline & & K06482 & ITGA3 & 1.48 \\
\hline & & K13242 & NOS3 & 1.35 \\
\hline & & K16340 & PHLPP2 & -1.23 \\
\hline \multirow[t]{3}{*}{ ko04115 } & $\begin{array}{l}\text { p53 signaling } \\
\text { pathway }\end{array}$ & K04398 & casp8 & -1.22 \\
\hline & & K10143 & RFWD2 & 1.58 \\
\hline & & K10146 & CCNG2 & -1.34 \\
\hline \multirow[t]{3}{*}{ ko04210 } & Apoptosis & K01379 & CTSD & 1.35 \\
\hline & & K04398 & casp8 & -1.22 \\
\hline & & K04724 & CFLAR & -1.28 \\
\hline \multirow[t]{3}{*}{ ko04066 } & $\begin{array}{l}\text { HIF-1 signaling } \\
\text { pathway }\end{array}$ & K04515 & CAMK2D & 1.29 \\
\hline & & K05121 & TEK & -1.37 \\
\hline & & K13242 & NOS3 & 1.35 \\
\hline \multirow[t]{5}{*}{ ko04611 } & Platelet activation & K13242 & NOS3 & 1.35 \\
\hline & & K16056 & ORA/1 & 1.36 \\
\hline & & K19720 & LOC101747382 & 1.53 \\
\hline & & & LOC107055590 & 1.81 \\
\hline & & & LOC107055658 & 1.39 \\
\hline \multirow[t]{3}{*}{ ko04727 } & $\begin{array}{l}\text { GABAergic } \\
\text { synapse }\end{array}$ & K04851 & CACNA1D & 1.82 \\
\hline & & K05175 & GABRA5 & 1.30 \\
\hline & & K13524 & $A B A T$ & -1.35 \\
\hline \multirow[t]{4}{*}{ ko04934 } & Cushing syndrome & K04365 & $B R A F$ & -1.25 \\
\hline & & K04515 & CAMK2D & 1.29 \\
\hline & & K04851 & CACNA1D & 1.82 \\
\hline & & K16056 & ORAl1 & 1.36 \\
\hline \multirow[t]{3}{*}{ ko05160 } & Hepatitis C virus & K04365 & $B R A F$ & -1.25 \\
\hline & & K04398 & casp8 & -1.22 \\
\hline & & K04724 & CFLAR & -1.28 \\
\hline \multirow[t]{3}{*}{ ko05205 } & $\begin{array}{l}\text { Proteoglycans in } \\
\text { cancer }\end{array}$ & K04365 & $B R A F$ & -1.25 \\
\hline & & K04515 & CAMK2D & 1.29 \\
\hline & & K10380 & ANKRD13D & 1.24 \\
\hline \multirow[t]{3}{*}{ ko05016 } & Huntington disease & K04398 & casp8 & -1.22 \\
\hline & & K04648 & DCTN1 & 1.56 \\
\hline & & K10408 & DNAH3 & -1.25 \\
\hline \multirow[t]{3}{*}{ ko05412 } & $\begin{array}{l}\text { Arrhythmogenic } \\
\text { right ventricular }\end{array}$ & K04851 & CACNA1D & 1.82 \\
\hline & cardiomyopathy & K06482 & ITGA3 & 1.48 \\
\hline & & K07610 & $D E S$ & 1.64 \\
\hline
\end{tabular}

signal apoptosis in the endothelial cells (Abe et al., 2019). As demonstrated in vitro and in vivo, NOS3 was highly expressed upon the exposure to reactive oxygen species (Zhen et al., 2008). An increase in synthesis of nitric oxide to increase blood flow due to the limited vascularization within WS/WB affected muscle has been speculated (Petracci et al., 2019). As shown in the study of Christov et al. (2007), endothelial cells stimulate myogenic cell (MPCs) growth, whereas the MPCs 
showed proangiogenic effect upon differentiation. The altered expression of those DEGs supported the hypothesis regarding the imbalanced interplay between the endothelial cells and MPCs within the hypertrophic myofibers in breast muscle of broilers exhibiting severe WS. Still, further studies are necessary to obtain a better understanding underlying specific functions of those DEGs in broiler skeletal muscle.

One of the downstream processes from PI3K-Akt or p53 pathways is the onset of apoptosis with the aim to remove any damaged cells via caspase activity (Chang et al., 2003). Transcript abundance of caspase-8 (CASP8), the principal upstream apoptotic caspase effector of deathinducing receptor signaling (Kruidering and Evan, 2000), was decreased by 1.2 -fold in severe relative to mild WS samples. A reduced mRNA level of CASP8 was recently reported in breast tumors collected from the subjects diagnosed with breast cancer (Aghababazadeh et al., 2017). Apart from CASP8, the decreases of caspase 8 and Fas-associated death domain-like apoptosis regulator (CFLAR) along with the increased cathepsin D (CTSD) abundance were identified in severe WS compared to mild samples. The reduced CFLAR expression accelerates inflammation, ER stress, and cell death in ischemia-induced mouse brain tissues (Wang et al., 2019), whereas overexpression of CTSD was linked with various diseases, e.g., type 2 diabetes (Liu et al., 2017), acute coronary syndromes (Gonçalves et al., 2016), and malignant epithelial tumor (Zhang et al., 2018). Additionally, the extensive alteration of apoptosis in severe WS is emphasized by the increased abundance of dynactin subunit 1 (DCTN1) coupled with decreased axonemal heavy chain 3 of dynein (DNAH3), highlighted in Huntington disease pathway (Table 5). Differential expression of those two genes inhibits apoptotic membrane trafficking during apoptosis (Lane et al., 2001). Overall, the modulated programmed cell death could be anticipated in severe WS.

The importance of altered intracellular $\left[\mathrm{Ca}^{2+}\right]$ in the development of WS was again underlined by the DEGs identified in C3. The $\mathrm{Ca}^{2+}-$ /calmodulin-dependent protein kinase type II delta chain $(C A M K 2 D)$ is part of a complex that regulates ER-mediated $\mathrm{Ca}^{2+}$ influx in either skeletal or cardiac muscles. The $\mathrm{Ca}^{2+}$ voltage-gated channel subunit alpha 1D (CACNA1D) is specifically part of the channel responsible for conferring low-voltage activation and slowly inactivating $\mathrm{Ca}^{2+}$ currents. $\mathrm{The} \mathrm{Ca}^{2+}$ release-activated calcium channel protein 1 (ORAI1), a $\mathrm{Ca}^{2+}$-selective channel particularly in $\mathrm{T}$ cells, mediates $\mathrm{Ca}^{2+}$ influx following the depletion of intracellular $\mathrm{Ca}^{2+}$ stores (Gwozdz et al., 2012). Overexpression of CAMK2D and CACNA1D induces cardiac enlargement, with mild fibrosis resembling the severe WS lesion, in transgenic mice (Zhang et al., 2003). In addition, a decreased expression of the aminobutyrate aminotransferase $(A B A T)$, highlighted in GABAergic signaling, is associated with increased intracellular $\left[\mathrm{Ca}^{2+}\right]$, leading to an increased $\mathrm{Ca}^{2+}$-sensing nuclear translocation associated with aggressive breast cancer (Chen et al., 2019). The changes in transcription of those DEGs likely lead to the excessive $\mathrm{Ca}^{2+}$ influx in severe WS samples (Kuttappan et al., 2013; Zambonelli et al., 2016; Marchesi et al., 2019).
Increased abundances of LOC101747382, LOC107055590, and LOC107055658, encoding type I collagen, coupled with upregulated NOS3 and ORAI1, were highlighted under the pathway of platelet activation. The pathway is responsible primarily for coagulation cascades upon the interaction between platelet membrane glycoproteins and exposed collagens and secondarily for intercellular communication that mediates inflammatory and immunomodulatory activities, particularly with the triggers from increasing intracellular $\left[\mathrm{Ca}^{2+}\right]$ and extracellular matrix (Yun et al., 2016). Generally, the ratio between types 1 and 3 determines mechanical properties of collagen matrix, hence the tissues. An increase in proportion of collagen type 1 has been demonstrated in healing wounds, hypertensive arteries, and fibrotic tissues (Friedman et al., 1993), which was in accordance with fibrotic characteristics of severe WS previously described (Trocino et al., 2015).

Regarding the arrhythmogenic right ventricular cardiomyopathy, the pathological characteristics of this heart muscle disease includes progressive myocyte loss and fibrofatty replacement, which were resembled to the histological lesions of severe WS. Under this enriched pathway, increased transcript abundance of desmin (DES) was significant in severe WS in comparison to that of mild WS. This observation was consistent with the previous report on the induced DES at the protein levels in WS/WB breast muscle samples compared with nondefective ones (Zambonelli et al., 2016; Kuttappan et al., 2017; Soglia et al., 2020). Based on the functions of desmin in structural organization of myofibers during myogenesis, the greater DES in severe WS suggested the occurrence of extensive muscle regeneration within the severe WS in comparison with those of found in mild WS samples.

The majority of the DEGs enlisted in the unique pathways of $\mathrm{C} 3$ were also associated with growth and proliferation of cancer cells as observed in $\mathrm{C} 1$ and $\mathrm{C} 2$. Apart from those DEGs mentioned above, transcript abundance of plekstrin homology domain leucine-rich repeat-containing protein phosphatase 2 (PHLPP2), a negative regulator of PI3K/Akt pathway, was decreased by 1.2 -fold. A loss of PHLPP2 is associated with progression of pancreatic (Smith et al., 2016) and prostatic cancer (Nowak et al., 2019). Differential expressions of cyclin G (CCNG) and increased RING finger and WD repeat domain protein 2 (RFWD2), the two proteins involved in negative autoregulatory feedback loops of the p53 response observed in this study, were concomitant with the modified transcriptional direction addressed in gastric cancer tissues and in ovarian and breast cancer tissues, respectively (Dornan et al., 2004; Gao et al., 2018). Decreased expression of the gene encoding B-Raf (BRAF) by 1.2fold in severe compared to mild WS might imply the impaired proliferative arrest in the severe WS.

HIF-1 signaling is also highlighted in C3. The pathway, consistently identified in the WS/WB myopathies (Malila et al., 2019; Marchesi et al., 2019; Pampouille et al., 2019), stimulates an adaptive mechanism via an activation of transcription factor HIF1 when cells sense low oxygen availability. Despite no difference in expression of HIF-1, the shifts of TEK and NOS3 expression suggested modified angiogenesis and antioxidant activities under hypoxia within the severe WS compared with those in mild WS 
samples. The current results correspond well with our previous report (Malila et al., 2019) in which an increased HIF1A was only detected when absolute transcription level of WS and WS + WB muscle were compared with that of nondefective ones; however, no change was observed among the defective samples. This might explain the absence of HIF1A in the DEGs obtained in this report.

Collectively, the changes in gene expression patterns combined with the unique biological pathways in C3 were in the same direction related to development and proliferation of tumor and cancer in several tissue types. The molecular resemblance underlined the dysregulation of damaged cells as well as the aberrant cell proliferation potentially during muscle regeneration within the muscle exhibiting severe WS myopathy.

\section{Altered Biological Pathways in Common Among All Comparisons}

Most of the overlapping enriched pathways comprised the same set of the DEGs discussed in each comparison, suggesting the importance of those DEGs in the progression of WS condition. The shared altered pathways emphasize the impact of dysregulated intracellular ion homeostasis and modulated cellular cascades that remove damaged cells in relevance with WS abnormality. In this study, transcript abundances of $P L C B$, CACNA1D, CAMK2, and ORAI1 gradually increased during WS progression. The findings were consistent with previous studies of Zambonelli et al. (2016) and Marchesi et al. (2019) identifying upregulation of those genes in WS-related muscle compared with those of nondefective ones. Increases in expression of those genes have been related with elevated intracellular $\left[\mathrm{Ca}^{2+}\right]$. As $\mathrm{Ca}^{2+}$ is one of the crucial secondary messengers associated with phosphoinositol system, $\left[\mathrm{Ca}^{2+}\right]$ disturbance may affect both the molecular functions and the viability of the cell. Elevated intracellular $\left[\mathrm{Ca}^{2+}\right]$ modulates apoptosis and protein degradation, compromises mitochondrial function, and interferes with cell membrane integrity (Florea et al., 2005; Mutryn et al., 2015; Petracci et al., 2015). This could explain the extensive muscle protein degradation observed in WS samples collected from Cobb 500 broilers in the previous report of Vignale et al. (2017). Likewise, NOS3 was increased as WS severity increased. This result supported the speculation of Petracci et al. (2019), suggesting the elevated hypoxic condition as WS severity elevated. In addition, the gene encoding solute carrier family 25 (SLC25A4) slightly decreased as the myopathy progressed. Expression of this gene promoted oxidative stress and apoptosis (Lena et al., 2010). A progressive decrease in PHKB was detected, which agreed well with the previous findings of Marchesi et al. (2019), suggesting an unusual muscle contraction process within the affected muscle.

Consistent with the observation in $\mathrm{C} 2$ and $\mathrm{C} 3$, the numbers of the DEGs obtained in the overlapped pathways were changed in the direction that facilitated proliferation and progression of cancer cells and tumors (Figure 6). Apart from the DEGs discussed earlier, the DNA mismatch repair protein, encoded by MSH6, gradually decreased while the WS severity increased. Previously, suppressed MSH6 was detected under an experimental hypoxic condition (Koshiji et al., 2005). The shifts of glutathione (GST)-encoding gene among the WS severity levels, corresponding with our recent ddPCR results (Malila et al., 2019), implied responsive mechanism of the muscle cells against increased oxidative stress. In addition to impaired apoptosis, ubiquitination-mediated proteolysis (Supplementary Figure S1) was disrupted. An accumulation of the unrepaired oxidative damaged biomolecules could induce a cascade of molecular events that ultimately contribute to promotion of cancer or tumor growth (Conde-Pérezprina et al., 2012). The current microarray results strengthen the case for oxidative stress-induced damage within the WS breast that induce a cascade of molecular events contributing to aberrant cell proliferation.

Although the actual etiology regarding development of WS abnormality remains under investigation, the current transcriptome analyses ascertained an association between disturbances of calcium homeostasis during progression of WS severity. In complement to the previous report, our results underlined the major shifts of biological processes while the abnormality progressed. When WS abnormality advanced from mild to moderate WS, the microarray data revealed transcriptional changes of the key upstream mediators of $\mathrm{Ca}^{2+}$. The impacts of imbalanced intracellular $\left[\mathrm{Ca}^{2+}\right]$ coupled with alterations of the other secondary messengers may exert an overall metabolic shift and inflammation. As the WS myopathy progresses to the severe degree, a more profound perturbation of intracellular $\left[\mathrm{Ca}^{2+}\right]$ along with oxidative stress could be anticipated. One of the major origins of the oxidative stress might potentially be the limited capillarization. The differences in metabolisms of macronutrients and small biochemical molecules might be shadowed by the extensive results of impaired cellcycle arrest and cell proliferation. With the unrepaired DNA mismatches along with modulated programmed cell death, accumulation of abnormal genomic materials and intermediate metabolites could trigger dysregulation of physiological functions and excessive cell proliferation. The aberrant muscle cells underwent necrosis as supported by the previous histological reports (Kuttappan et al., 2013; Malila et al., 2018; Marchesi et al., 2019) and ultimately developed severe WS characteristics.

In conclusion, the current transcriptome analysis strengthens the association between development of WS abnormality in commercial broilers and aberrant molecular mechanisms responsible for controlling ion homeostasis and muscle repair process. The transcriptional alteration, when WS progressed from mild to moderate, also influenced metabolisms of macronutrients and small biomolecules. In the development of severe WS lesion, besides the potential leak of the ions, the changes in expression of several genes resemble those found in highly proliferating cells. In addition to the link with an intensive selection for fast-growing and enlarged muscle mass, this study has shown an alteration of the circadian clock system at transcriptional level. The current findings offer evidence that both the intensive, selective breeding and overall management of the modern broiler may play roles in development and severe progression of this myopathy. Optimizing rather than maximizing growth performance of broilers should be prioritized to ensure security of this inexpensive protein source and the sustainability of poultry industry. 


\section{DATA AVAILABILITY STATEMENT}

The datasets generated for this study can be found in the NCBI GEO Series GSE107362 (https://www.ncbi.nlm.nih.gov/ geo/query/acc.cgi?acc=GSE107362), Platform GPL24307 (https: //www.ncbi.nlm.nih.gov/geo/query/acc.cgi?acc=GPL24307).

\section{ETHICS STATEMENT}

All samples were purchased in the form of whole carcasses from the commercial processing plant. Neither experimental treatments nor scientific procedures were subjected to the living animals. Thereby, according to BIOTEC Institutional Animal Care and Use Committee, the ethical approval was not exempted.

\section{AUTHOR CONTRIBUTIONS}

$\mathrm{YM}, \mathrm{WR}$, and $\mathrm{WV}$ conceived and designed the experiments as well as acquired the funding. YM, TU, YS, SA, and KT performed the experiments and analyzed the data. YM, TU, WR, FS, and GS drafted the manuscript. All authors read and approved the final manuscript.

\section{REFERENCES}

Abe, J. I., Ko, K. A., Kotla, S., Wang, Y., Paez-Mayorga, J., Shin, I. J., et al. (2019). MAGI1 as a link between endothelial activation and ER stress drives atherosclerosis. JCI Insight. 4:e125570. doi: 10.1172/jci.insight.125570

Aggarwal, A., Costa, M. J., Rivero-Gutiérrez, B., Ji, L., Morgan, S. L., and Feldman, B. J. (2017). The circadian clock regulates adipogenesis by a Per3 crosstalk pathway to Klf15. Cell Rep. 21, 2367-2375. doi: 10.1016/j.celrep.2017.11.004

Aghababazadeh, M., Dorraki, N., Javan, F. A., Fattahi, A. S., Gharib, M., and Pasdar, A. (2017). Downregulation of caspase 8 in a group of Iranian breast cancer patients - a pilot study. J. Egypt. Natl. Canc. Inst. 29, 191-195. doi: 10.1016/j. jnci.2017.10.001

Augustin, H. G., Koh, G. Y., Thurston, G., and Alitalo, K. (2009). Control of vascular morphogenesis and homeostasis through the angiopoietin-Tie system. Nat. Rev. Mol. Cell Biol. 10, 165-177. doi: 10.1038/nrm2639

Baar, M. P., Perdiguero, E., Muñoz-Cánoves, P., and de Keizer, P. L. (2018). Musculoskeletal senescence: a moving target ready to be eliminated. Curr. Opin. Pharmacol. 40, 147-155. doi: 10.1016/j.coph.2018.05.007

Baudrimont, A., Jaquet, V., Wallerich, S., Voegeli, S., and Becskei, A. (2019). Contribution of RNA degradation to intrinsic and extrinsic noise in gene expression. Cell Rep. 26, 3752-3761. doi: 10.1016/j.celrep.2019.03.001

Berchtold, M. W., and Villalobo, A. (2014). The many faces of calmodulin in cell proliferation, programmed cell death, autophagy, and cancer. BBA Mol. Cell. Res. 1843, 398-435. doi: 10.1016/j.bbamcr.2013.10.021

Boerboom, G., van Kempen, T., Navarro-Villa, A., and Pèrez-Bonilla, A. (2018). Unraveling the cause of white striping in broilers using metabolomics. Poult. Sci. 97:3977. doi: 10.3382/ps/pey266

Bolstad, B. (2017). PreprocessCore: A Collection Of Pre-Processing Functions. $R$ Package Version 1.40.0. Available at: https:/github.com/bmbolstad/ preprocessCore (accessed July 23, 2018).

Camarillo, C., Swerdel, M., and Hart, R. P. (2011). Comparison of microarray and quantitative real-time PCR methods for measuring MicroRNA levels in MSC cultures. Methods Mol. Biol. 698, 419-429. doi: 10.1007/978-1-60761-999-4_30

Chan, K. K. M., Seetharaman, A., Bagg, R., Selman, G., Zhang, Y., Kim, J., et al. (2014). EVA-1 functions as an UNC-40 Co-receptor to enhance attraction to

\section{FUNDING}

This research was financially supported by Cluster and Program Management, National Science and Technology Development Agency (NSTDA, Thailand) toward YM, YS, TU, SA, WR, and WV (project number P15-50668), by Thailand Research Fund toward YM and WV (Grant Number TRG 5980007) and by Office of the Permanent Secretary, Ministry of Higher Education, Science, Research and Innovation (Thailand; P20-50946).

\section{ACKNOWLEDGMENTS}

The authors would like to express their sincere gratitude toward Dr. Massimiliano Petracci for sacrificing his time providing critical review on this manuscript. The authors are thankful for assistance from Dr. Juthawut U-chupaj and Mr. Premsak Chaiwiwattrakul during sample collection.

\section{SUPPLEMENTARY MATERIAL}

The Supplementary Material for this article can be found online at: https://www.frontiersin.org/articles/10.3389/fphys. 2020.00580/full\#supplementary-material

the MADD-4 guidance cue in Caenorhabditis elegans. PLoS Genet. 10:e1004521. doi: 10.1371/journal.pgen.1004521

Chang, F., Lee, J. T., Navolanic, P. M., Steelman, L. S., Shelton, J. G., Blalock, W. L., et al. (2003). Involvement of PI3K/Akt pathway in cell cycle progression, apoptosis, and neoplastic transformation: a target for cancer chemotherapy. Leukemia 17, 590-603. doi: 10.1038/sj.leu.2402824

Chen, X., Cao, Q., Liao, R., Wu, X., Xun, S., Huang, J., et al. (2019). Loss of ABATmediated GABAergic system promotes basal-like breast cancer progression by activating Ca2+-NFAT1 axis. Theranostics 9, 34-47. doi: 10.7150/thno.29407

Christov, C., Chrétien, F., Abou-Khalil, R., Bassez, G., Vallet, G., Authier, F. J., et al. (2007). Muscle satellite cells and endothelial cells: close neighbors and privileged partners. Mol. Biol. Cell. 18, 1397-1409. doi: 10.1091/mbc.e06-080693

Chu, M., Li, T., Shen, B., Cao, X., Zhong, H., Zhang, L., et al. (2016). Angiopoietin receptor Tie2 is required for vein specification and maintenance via regulating COUP-TFII. eLife 5:e21032. doi: 10.7554/eLife.21032

Conde-Pérezprina, J. C., León-Galván, M. Á, and Konigsberg, M. (2012). DNA mismatch repair system: repercussions in cellular homeostasis and relationship with aging. Oxid. Med. Cell. Longev. 2012:728430. doi: 10.1155/2012/728430

Datta, K., Fuentes, J. L., and Maddok, J. R. (2005). The yeast GTPase Mtg2p is required for mitochondrial translation and partially suppresses an rRNA methyltransferase mutant, mrm2. Mol. Biol. Cell. 16, 954-963. doi: 10.1091/ mbc.e04-07-0622

De Jong, A., Buchili, R., and Robbins, D. (2002). Characterization of sperm protein 17 in human somatic and neoplastic tissue. Cancer Lett. 186, 201-209. doi: 10.1016/s0304-3835(02)00350-6

Dong, Y., Xu, W., Liu, C., Liu, P., Li, P., and Wang, K. (2019). Reactive oxygen species related noncoding RNAs as regulators of cardiovascular diseases. Int. J. Biol. Sci. 15, 680-687. doi: 10.7150/ijbs.30464

Dornan, D., Bheddah, S., Newton, K., Ince, W., Frantz, G. D., Dowd, P., et al. (2004). COP1, the negative regulator of $\mathrm{p} 53$, is overexpressed in breast and ovarian adenocarcinomas. Cancer Res. 64, 7226-7230. doi: 10.1158/0008-5472. CAN-04-2601

Florea, A. M., Yamoah, E. N., and Dopp, E. (2005). Intracellular calcium disturbances induced by arsenic and its methylated derivatives in relation 
to genomic damage and apoptosis induction. Environ. Health Perspect. 113, 659-664. doi: 10.1289/ehp.7634

Friedman, D. W., Boyd, C. D., Norton, P., Greco, R. S., Boyarsky, A. H., Mackenzie, J. W., et al. (1993). Increases in type III collagen gene expression and protein synthesis in patients with inguinal hernias. Ann. Surg. 218, 754-760. doi: 10. 1097/00000658-199312000-00009

Gao, J., Zhao, C., Liu, Q., Hou, X., Li, S., Xing, X., et al. (2018). Cyclin G2 suppresses Wnt/ $\beta$-catenin signaling and inhibits gastric cancer cell growth and migration through Dapper1. J Exp. Clin. Cancer Res. 37:317. doi: 10.1186/s13046-0180973-2

Gonçalves, I., Hultman, K., Dunér, P., Edsfeldt, A., Hedblad, B., Fredrikson, G. N., et al. (2016). High levels of cathepsin D and cystatin B are associated with increased risk of coronary events. Open Heart 3:e000353. doi: 10.1136/openhrt2015-000353

Gwozdz, T., Dutko-Gwozdz, J., Schafer, C., and Bolotina, V. M. (2012). Overexpression of Orail and STIM1 proteins alters regulation of store-operated Ca2+ entry by endogenous mediators. J. Biol. Chem. 287, 22865-22872. doi: 10.1074/jbc.M112.356626

Hamilton, S. L., Serysheva, I., and Strasburg, G. M. (2000). Calmodulin and excitation-contraction coupling. News Physiol. Sci. 15, 281-284. doi: 10.1152/ physiologyonline.2000.15.6.281

Harfmann, B. D., Schroder, E. A., and Esser, K. A. (2015). Cirdadian rhythms, the molecular clock, and skeletal muscle. J. Biol. Rhythms 30, 84-94. doi: 10.1177/ 0748730414561638

Huang, W., Kazmierczak, K., Zhou, Z., Aguiar-Pulido, V., Narasimhan, G., and Szczesna-Cordarya, D. (2016). Gene expression patterns in transgenic mouse models of hypertrophic cardiomyopathy caused by mutations in myosin regulatory light chain. Arch. Biochem. Biophys. 601, 121-132. doi: 10.1016/j.abb. 2016.02.022

Huang, Y., Kong, Y., Zhang, L., He, T., Zhou, X., Yan, Y., et al. (2018). High expression of ITGA3 promotes proliferation and cell cycle progression and indicates poor prognosis in intrahepatic cholangiocarcinoma. BioMed. Res. Int. 2018:2352139. doi: 10.1155/2018/2352139

Ji, X. D., Li, G., Feng, Y. X., Zhao, J. S., Li, J. J., Sun, Z. J., et al. (2011). EphB3 is overexpressed in non-small-cell lung cancer and promotes tumor metastasis by enhancing cell survival and migration. Cancer Res. 71, 1156-1166. doi: 10.1158/0008-5472.CAN-10-0717

Kadamar, G., and Ross, E. M. (2013). Mammalian phospholipase C. Annu. Rev. Physiol. 75, 127-154. doi: 10.1146/annurev-physiol-030212-183750

Kanehisa, M., Goto, S., Sato, Y., Furumichi, M., and Tanabe, M. (2012). KEGG for integration and interpretation of large-scale molecular data sets. Nucleic Acids Res. 40, D109-D114. doi: 10.1093/nar/gkr988

Khaitan, D., and Ningaraj, N. (2019). Evidence of calcium-activated potassium channel subunit alpha-1 as a key promoter of glioma growth and tumorigenicity. Glioma 2, 46-54. doi: 10.4103/glioma.glioma_44_18

Kim, N. H., Cha, Y. H., Lee, J., Lee, S. H., Yang, J. H., Yun, J. S., et al. (2017). Snail reprograms glucose metabolism by repressing phosphofructokinase PFKP allowing cancer cell survival under metabolic stress. Nat. Commun. 8:14374. doi: $10.1038 /$ ncomms 14374

Koivunen, P., Tiainen, P., Hyvärinen, J., Williams, K. E., Sormunen, R., Klaus, S. J., et al. (2007). An endoplasmic reticulum transmembrane prolyl 4 -hydroxylase is induced by hypoxia and acts on hypoxia-inducible factor $\alpha$. J. Biol. Chem. 282, 30544-30552. doi: 10.1074/jbc.M704988200

Kojima, M., Nakajima, I., Arakawa, A., Mikawa, S., Matsumoto, T., Uenishi, H., et al. (2018). Differences in gene expression profiles for subcutaneous adipose, liver, and skeletal muscle tissues between Meishan and Landrace pigs with different backfat thicknesses. PLoS One 13:e0204135. doi: 10.1371/journal.pone. 0204135

Koshiji, M., To, K. K., Hammer, S., Kumamoto, K., Harris, A. L., Modrich, P., et al. (2005). HIF- $1 \alpha$ induces genetic instability by transcriptionally downregulating MutS $\alpha$ Expression. Mol. Cell. 17, 793-803. doi: 10.1016/j.molcel.2005. 02.015

Kruidering, M., and Evan, G. I. (2000). Caspase- 8 in apoptosis: the beginning of "the end"? IUBMB Life 50, 85-90. doi: 10.1080/713803693

Kuttappan, V. A., Bottje, W., Ramnathan, R., Hartson, S. D., Coon, C. N., Kong, B. W., et al. (2017). Proteomic analysis reveals changes in carbohydrate and protein metabolism associated with broiler breast myopathy. Poult. Sci. 96, 2992-2999. doi: 10.3382/ps/pex069
Kuttappan, V. A., Hargis, B. M., and Owens, C. M. (2016). White striping and woody breast myopathies in the modern poultry industry: a review. Poult. Sci. 95, 2724-2733. doi: 10.3382/ps/pew216

Kuttappan, V. A., Lee, Y. S., Erf, G. F., Meullenet, J. F., McKee, S. R., and Owens, C. M. (2012). Consumer acceptance of visual appearance of broiler breast meat with varying degrees of white striping. Poult. Sci. 91, 1240-1247. doi: 10.3382/ ps.2011-01947

Kuttappan, V. A., Shivaprasad, H. L., Shaw, D. P., Valentine, B. A., Hargis, B. M., Clark, F. D., et al. (2013). Pathological changes associated with white striping in broiler breast muscles. Poult. Sci. 92, 331-338. doi: 10.3382/ps.201202646

Lane, J. D., Vergnolle, M. A., Woodman, P. G., and Allan, V. J. (2001). Apoptotic cleavage of cytoplasmic dynein intermediate chain and p150(Glued) stops dynein-dependent membrane motility. J. Cell Biol. 153, 1415-1426. doi: 10. 1083/jcb.153.7.1415

Laura, R. P., Ross, S., Koeppen, H., and Lasky, L. A. (2002). MAGI-1: a widely expressed, alternatively spliced tight junction protein. Exp. Cell Res. 275, 155170. doi: 10.1006/excr.2002.5475

Leek, J., McShane, B. B., Gelman, A., Colquhoun, D., Nuijten, M. B., and Goodman, S. N. (2017). Five ways to fix statistics. Nature 551, 557-559. doi: 10.1038/ d41586-017-07522-z

Lena, A., Rechichi, M., Salvetti, A., Vecchio, D., Evangelista, M., Rainaldi, G., et al. (2010). The silencing of adenine nucleotide translocase isoform linduces oxidative stress and programmed cell death in ADF human glioblastoma cells. FEBS J. 277, 2853-2867. doi: 10.1111/j.1742-4658.2010.07702.x

Liu, L., Chen, B., Zhang, X., Tan, L., and Wang, D. W. (2017). Increased cathepsin $\mathrm{D}$ correlates with clinical parameters in newly diagnosed type 2 diabetes. Dis. Markers 2017:5286408. doi: 10.1155/2017/5286408

Livak, K. J., and Schmittgen, T. D. (2001). Analysis of relative gene expression data using real-time quantitative PCR and the 2(-Delta Delta C(T) Method. Methods 25, 402-408. doi: 10.1006/meth.2001.1262

Malila, Y., Srimarut, Y., U-chupaj, J., Strasburg, G., and Visessanguan, W. (2015). Monitoring of chicken RNA integrity as a function of prolonged postmortem duration. Asian Austr. J. Anim. Sci. 28, 1649-1656. doi: 10.5713/ajas.15.0167

Malila, Y., Thanatsang, K., Arayamethakorn, S., Uengwetwanit, T., Srimarut, Y., Petracci, M., et al. (2019). Absolute expressions of hypoxia-inducible factor-1 alpha (HIF1A) transcript and the associated genes in chicken skeletal muscle with white striping and wooden breast myopathies. PLoS One 14:e0220904. doi: 10.1371/journal.pone.0220904

Malila, Y., U-chupaj, J., Srimarut, Y., Chaiwiwattrakul, P., Uengwetwanit, T., Arayamethakorn, S., et al. (2018). Monitoring of white striping and wooden breast cases and impacts on quality of breast meat collected from commercial broilers (Gallus gallus). Asian Austr. J. Anim. Sci. 31, 1807-1817. doi: 10.5713/ ajas. 18.0355

Marchesi, J. A. P., Ibelli, A. M. G., Peixoto, J. O., Cantão, M. E., Pandolfi, J. R. C., Marciano, C. M. M., et al. (2019). Whole transcriptome analysis of the pectoralis major muscle reveals molecular mechanisms involved with white striping in broiler chickens. Poult. Sci. 98, 590-601. doi: 10.3382/ps/pey429

Milburn, M., Tong, L., deVos, A., Brunger, A., Yamaizumi, Z., Nishimura, S., et al. (1990). Molecular switch for signal transduction: structural differences between active and inactive forms of protooncogenic ras proteins. Science 247, 939-945. doi: $10.1126 /$ science. 2406906

Miller, C. (2000). An overview of the potassium channel family. Genome Biol. 1:reviews0004.1. doi: 10.1186/gb-2000-1-4-reviews0004

Müller, C., Schillert, A., Röthemeier, C., Trégouët, D. A., Proust, C., and Binder, H. (2016). Removing batch effects from longitudinal gene expression - quantile normalization plus ComBat as best approach for microarray transcriptome data. PLoS One 11:e0156594. doi: 10.1371/journal.pone.0156594

Mutryn, M. F., Brannick, E. M., Fu, W., Lee, W. R., and Abasht, B. (2015). Characterization of a novel chicken muscle disorder through differential gene expression and pathway analysis using RNA-sequencing. BMC Genomics 16:399. doi: 10.1186/s12864-015-1623-0

Nowak, D. G., Katsenelson, K. C., Watrud, K. E., Chen, M., Mathew, G., D’Andrea, V. D., et al. (2019). The PHLPP2 phosphatase is a druggable driver of prostate cancer progression. J. Cell Biol. 218, 1943-1957. doi: 10.1083/jcb.201 902048

Pampouille, E., Hennequet-Antier, C., Praud, C., Juanchich, A., Brionne, A., Godet, E., et al. (2019). Differential expression and co- expression gene network 
analyses reveal molecular mechanisms and candidate biomarkers involved in breast muscle myopathies in chicken. Sci. Rep. 9:14905. doi: 10.1038/s41598019-51521-1

Petracci, M., and Cavini, C. (2012). Muscle growth and poultry meat quality issues. Nutrients 4, 1-12. doi: 10.3390/nu4010001

Petracci, M., Mudalal, S., Babini, E., and Cavani, C. (2014). Effect of white striping on chemical composition and nutritional value of chicken breast meat. Ital. J. Anim. Sci. 13, 178-183. doi: 10.4081/ijas.2014.3138

Petracci, M., Mudalal, S., Soglia, F., and Cavani, C. (2015). Meat quality in fastgrowing broiler chickens. Worlds Poult. Sci. J. 71, 363-374. doi: 10.1017/ S0043933915000367

Petracci, M., Sirri, F., Mazzoni, M., and Meluzzi, A. (2013). Comparison of breast muscle traits and meat quality characteristics in 2 commercial chicken hybrids. Poult. Sci. 92, 2438-2447. doi: 10.3382/ps.2013-03087

Petracci, M., Soglia, F., Madruga, M., Carvalho, L., Ida, E., and Estévez, M. (2019). Wooden-breast, white striping, and spaghetti meat: causes, consequences and consumer perception of emerging broiler meat abnormalities. Compr. Rev. Food Sci. 18, 565-583. doi: 10.1111/1541-4337.12431

Roll-Mecak, A., and Vale, R. D. (2008). Structural basis of microtuble severing by the hereditary spastic paraplegia protein spastin. Nature 451, 363-367. doi: 10.1038 /nature 06482

Russell, S. A., and Bashaw, G. J. (2018). Axon guidance pathways and the control of gene expression. Dev. Dyn. 247, 571-580. doi: 10.1002/dvdy.24609

Smith, A. J., Wen, Y. A., Stevens, P. D., Liu, J., Wang, C., and Gao, T. (2016). PHLPP negatively regulates cell motility through inhibition of Akt activity and integrin expression in pancreatic cancer cells. Oncotarget 7, 7801-7815. doi: 10.18632/oncotarget.6848

Soglia, F., Mazzoni, M., Zappaterra, M., Di Nunzio, M., Babini, E., Bordini, M., et al. (2020). Distribution and expression of vimentin and desmin in broiler pectoralis major affected by the growth-related muscular abnormalities. Front. Physiol. 10:1581. doi: 10.3389/fphys.2019.01581

Steenhard, B. M., Vanacore, R., Friedman, D., Zelenchuk, A., Stroganova, L., Isom, K., et al. (2012). Upregulated expression of integrin $\alpha 1$ in mesangial cells and integrin $\alpha 3$ and vimentin in podocytes of Col4a3-null (Alport) mice. PLoS One 7:e50745. doi: 10.1371/journal.pone.0050745

Sun, R., Zhu, B., Xiong, K., Sun, Y., Shi, D., Chen, L., et al. (2017). Senescence as a novel mechanism involved in $\beta$-adrenergic receptor mediated cardiac hypertrophy. PLoS One 12:e0182668. doi: 10.1371/journal.pone.0182668

Tan, J., Lee, P. L., Li, Z., Jiang, X., Lim, Y. C., Hooi, S. C., et al. (2010). B55 $\beta$-associated PP2A complex controls PDK1-directed Myc signaling and modulates rapamycin sensitivity in colorectal cancer. Cancer Cell 18, 459-471. doi: 10.1016/j.ccr.2010.10.021

Trocino, A., Piccirillo, A., Birolo, M., Radaelli, G., Bertotto, D., Filiou, E., et al. (2015). Effect of genotype, gender and feed restriction on growth, meat quality and the occurrence of white striping and wooden breast in broiler chickens. Poult. Sci. 94, 2996-3004. doi: 10.3382/ps/pev296

Turek, F. W., Joshu, C., Kohsaka, A., Lin, E., and Ivanova, G. (2005). Obesity and metabolic syndrome in circadian clock mutant mice. Science 308, 1043-1045. doi: 10.1126/science. 1108750

Turunen, T. A., Roberts, T. C., Laitinen, P., Väänänen, M. A., Korhonen, P., Malm, T., et al. (2019). Changes in nuclear and cytoplasmic microRNA distribution in response to hypoxic stress. Sci. Rep. 9:10332. doi: 10.1038/s41598-019-46 841-1
Vignale, K., Caldas, J. V., England, J. A., Boonsinchai, N., Magnuson, A., Pollock, E. D., et al. (2017). Effect of white striping myopathy on breast muscle (Pectoralis major) protein turnover and gene expression in broilers. Poult. Sci. 96, 886-893. doi: $10.3382 / \mathrm{ps} /$ pew315

Volkman, B. F., Liu, T. Y., and Peterson, F. C. (2009). Lymphotactin structural dynamics. Methods Enzymol. 461, 51-70. doi: 10.1016/S0076-6879(09)05403-2

Wang, X., Zhao, J., Guo, H., and Fan, Q. (2019). CFLAR is a critical regulator of cerebral ischaemia-reperfusion injury through regulating inflammation and endoplasmic reticulum (ER) stress. Biomed. Pharmacother. 117:109155. doi: 10.1016/j.biopha.2019.109155

Yamagishi, R., Hosoda, N., and Hoshino, S. (2014). Arsenite inhibits mRNA deadenylation through proteolytic degradation of Tob and Pan3. Biochem. Biophys. Res. Commun. 455, 323-331. doi: 10.1016/j.bbrc.2014.11.015

Yun, S. H., Sim, E. H., Goh, R. Y., Park, J. I., and Han, J. Y. (2016). Platelet activation: the mechanisms and potential biomarkers. Biomed. Res. Int. 2016:9060143. doi: 10.1155/2016/9060143

Zambonelli, P., Zappaterra, M., Soglia, F., Petracci, M., Sirri, F., Cavani, C., et al. (2016). Detection of differentially expressed genes in broiler pectoralis major muscle affected by white striping - wooden breast myopathies. Poult. Sci. 95, 2771-2785. doi: 10.3382/ps/pew268

Zenonos, K., and Kyprianou, K. (2013). RAS signaling pathways, mutations and their role in colorectal cancer. World J. Gastrointest. Oncol. 5, 97-101. doi: 10.4251/wjgo.v5.i5.97

Zhang, M., Wu, J. S., Yang, X., Pang, X., Li, L., Wang, S. S., et al. (2018). Overexpression cathepsin $\mathrm{D}$ contributes to perineural invasion of salivary adenoid cystic carcinoma. Front. Oncol. 8:492. doi: 10.3389/fonc.2018.00492

Zhang, T., Maier, L. S., Dalton, N. D., Miyamoto, S., Ross, J. Jr., Bers, D. M., et al. (2003). The $\delta \mathrm{C}$ isoform of CaMKII is activated in cardiac hypertrophy and induces dilated cardiomyopathy and heart failure. Circ. Res. 92, 912-919. doi: 10.1161/01.RES.0000069686.31472.C5

Zhang, T., Zhang, X., Han, K., Zhang, G., Wang, J., Xie, K., et al. (2017). Analysis of long noncoding RNA and mRNA using RNA sequencing during the differentiation of intramuscular preadipocytes in chicken. PLoS One 12:e0172389. doi: 10.1371/journal.pone.01172389

Zhao, M., Chen, L., and Qu, H. (2016). CSGene: a literature-based database for cell senescence genes and its application to identify critical cell aging pathways and associated diseases. Cell Death Dis. 7:e2053. doi: 10.1038/cddis. 2015.414

Zhen, J., Lu, H., Wang, X. Q., Vaziri, N. D., and Zhou, X. J. (2008). Upregulation of endothelial and inducible nitric oxide synthase expression by reactive oxygen species. Am. J. Hypertens. 21, 28-34. doi: 10.1038/ajh.2007.14

Conflict of Interest: The authors declare that the research was conducted in the absence of any commercial or financial relationships that could be construed as a potential conflict of interest.

Copyright (c) 2020 Malila, Uengwetwanit, Arayamethakorn, Srimarut, Thanatsang, Soglia, Strasburg, Rungrassamee and Visessanguan. This is an open-access article distributed under the terms of the Creative Commons Attribution License (CC BY). The use, distribution or reproduction in other forums is permitted, provided the original author(s) and the copyright owner(s) are credited and that the original publication in this journal is cited, in accordance with accepted academic practice. No use, distribution or reproduction is permitted which does not comply with these terms. 
OPEN ACCESS

Edited by:

Sandra G. Velleman, The Ohio State University, United States

Reviewed by:

Walter Gay Bottje, University of Arkansas, United States

Michael Babak Papah, University of Pennsylvania, United States

Francesca Soglia,

University of Bologna, Italy

*Correspondence:

Tomohito Iwasak

iwasaki@rakuno.ac.jp

Takafumi Watanabe

t-watanabe@rakuno.ac.jp

Specialty section: This article was submitted to Avian Physiology, a section of the journal

Frontiers in Physiology

Received: 29 March 2020 Accepted: 11 May 2020

Published: 16 June 2020

Citation:

Hosotani M, Kawasaki T,

Hasegawa Y, Wakasa Y, Hoshino M,

Takahashi N, Ueda H, Takaya T,

Iwasaki T and Watanabe T (2020)

Physiological and Pathological Mitochondrial Clearance Is Related

to Pectoralis Major Muscle

Pathogenesis in Broilers With

Wooden Breast Syndrome.

Front. Physiol. 11:579.

doi: 10.3389/fphys.2020.00579

\section{Physiological and Pathological} Mitochondrial Clearance Is Related to Pectoralis Major Muscle Pathogenesis in Broilers With Wooden Breast Syndrome

\author{
Marina Hosotani', Takeshi Kawasaki², Yasuhiro Hasegawaㄹ, Yui Wakasa', \\ Maki Hoshino ${ }^{1}$, Naoki Takahashi' ${ }^{1}$ Hiromi Ueda ${ }^{1}$, Tomohide Takaya ${ }^{4}$, Tomohito Iwasaki ${ }^{*}$ \\ and Takafumi Watanabe ${ }^{1 *}$ \\ ${ }^{1}$ Department of Veterinary Anatomy, School of Veterinary Medicine, Rakuno Gakuen University, Ebetsu, Japan, ${ }^{2}$ Research \\ Office Concerning the Health of Humans and Birds, Abashiri, Japan, ${ }^{3}$ Department of Food Science and Human Wellness, \\ College of Agriculture, Food and Environment Science, Rakuno Gakuen University, Ebetsu, Japan, ${ }^{4}$ Department \\ of Agricultural and Life Science, Faculty of Agriculture, Shinshu University, Nagano, Japan
}

Wooden breast syndrome (WB) constitutes an emerging myopathy in the pectoralis major muscle (PM) of broiler chickens, characterized by myofiber hypertrophy and degeneration along with severe fibrosis. WB pathogenesis has been considered to involve hypoxia induced by rapid growth of the PM. In this study, we focused on mitochondrial morphology and dynamics in the myofibers, as these organelles are sensitive to damage by hypoxia, and examined the effects on WB pathogenesis. Specifically, the PMs of a flock of 35 broilers at 50 days of age were evaluated. First, the severity of disease in each bird was determined by measuring histopathological indices including the fibrotic area (FA) in the muscle and circularity of myofibers (CM). These values were $29.4 \pm 9.6 \%$ and $0.70 \pm 0.042$, respectively, showing variety among the flock. Myofiber vacuolization was observed in all birds including numerous small- or large-rimmed vacuoles, with the former consisting of ultrastructurally autophagosome-like vacuoles engulfing degenerated mitochondria. The large-rimmed vacuoles frequently occurred in the PMs with more severe FA and $\mathrm{CM}$, indicating a relationship between altered autophagy/mitophagy and WB severity. Next, the expression levels of hypoxia-adaptive and mitochondrial dynamics-related genes were analyzed, and their correlations with the histopathological indices were examined. The histopathological indices were negatively correlated with the expression of vascular endothelial growth factor A (VEGFA), indicating that less angiogenesis owing to weakened hypoxia-inducible factor signaling induces more severe WB pathology. In addition, the observed negative correlation with mitochondrial dynamics-related genes implied that WB pathology deteriorates concomitant with reduced mitochondrial dynamics. Furthermore, the expression of mitochondrial dynamics-related genes showed strong positive correlation with that of VEGFA and autophagy-/mitophagyrelated genes. These results revealed that the PMs of broilers possess the mechanism 
of physiological clearance of mitochondria damaged by the hypoxia resulting from the continuous mitochondrial dynamics and autophagy/mitophagy accompanying rapid PM growth. In turn, the altered mitochondrial clearance induced by chronic hypoxia and the accumulation of damaged mitochondria likely underly the severe pathological features of WB.

Keywords: wooden breast syndrome, pathology, hypoxia, mitochondria, autophagy, mitophagy

\section{INTRODUCTION}

In poultry production, meat quality impairment following the selective breeding of fast-growing broiler chickens necessary for supplying the large global demands of chicken meat represents an emerging problem worldwide (Kuttappan et al., 2013; Petracci et al., 2015). Specifically, genetic selection strategies have aimed to increase the yield of the pectoralis major muscle (PM), which constitutes the most superficial breast muscle (Bailey et al., 2015). However, the PMs of some broilers exhibit severe hardness, pale color, and small hemorrhages upon palpation and macroscopic observation in the poultry slaughterhouse (Sihvo et al., 2014) and ultimately cannot be utilized, which produces large economic losses (Abasht et al., 2016). This emerging myopathy, termed Wooden breast syndrome (WB) (Petracci et al., 2019), are characterized by histopathological features such as hypertrophy, necrosis and regeneration of myofibers, macrophage infiltration, and fibrosis (Sihvo et al., 2014; Clark and Velleman, 2016; Kawasaki et al., 2018). Consequently, the achievement of both high production efficiency of PM yield and reduction in the WB presentation is a highly desirable goal in the poultry industry.

In particular, broilers with the same genetics can show either no, mild, or severe WB symptoms at the same age even if raised under identical circumstances (Papah et al., 2018). WB severity is more highly associated with muscle development than with the overall growth of the body (Griffin et al., 2018). WBaffected PMs start to deteriorate after approximately 2 or 3 weeks (Radaelli et al., 2017; Kawasaki et al., 2018), such that a flock of broilers at later life would include birds at both initial and chronic stages of WB.

Wooden breast syndrome pathogenesis is considered to derive from insufficient angiogenesis during the rapid growth of the PMs leading to the production of oxidative stress and hypoxia (i.e., reduced oxygen availability) in the PMs, resulting in their myopathies (Mutryn et al., 2015; Malila et al., 2019). Hypoxia-inducible factor 1 (HIF1), which constitutes the primary transcriptional regulator produced in response to hypoxia in both physiological and pathological contexts, can increase vascularization and maintain oxygen homeostasis (Ziello et al., 2007; Thomas and Ashcroft, 2019). Specifically, HIF1-mediated cellular adaptations under hypoxic conditions regulate the metabolism, morphology, mass, and distribution of mitochondria through their fusion, fission, and autophagy/mitophagy (Thomas and Ashcroft, 2019). Notably, it has been reported that the expression of HIF1 transcripts and related genes is upregulated in the PMs of broilers with both moderate and severe WB compared to that of unaffected birds (Malila et al., 2019), with the first molecular signs of WB development being observed from 2 weeks of age (Papah et al., 2018). Nevertheless, different birds may exhibit individual susceptibilities to the development of such defects, as some are likely able to better counteract the development of hypoxic conditions giving rise to the cascade of events responsible for symptom development. As oxygen is essential for mitochondrial metabolism, we thus hypothesized that the damage to mitochondria mediated by hypoxia serves as the first step in WB pathogenesis, followed by muscle pathology such as hypertrophy and fibrosis. To evaluate the effect of mitochondrial damage on WB pathogenesis, in this study, we therefore performed PM histopathology and mitochondrial morphology assessments and analyzed the expression levels of mitochondrial dynamics-related genes in a flock of broilers including those with moderate to severe WB.

\section{MATERIALS AND METHODS}

\section{Animals and Specimens}

Animal experimentation was approved by the Rakuno Gakuen University Institutional Animal Care and Use Committee (No. VH18A6) in accordance with the Act on Welfare and Management of Animals of the Japanese government. A total of 36 male chicks (Ross308) hatched in the university farm were raised until day 50 . All birds were observed daily by animal stock technicians, and clinical conditions were checked by a poultry veterinarian as necessary during the rearing period. The birds were weighed and determined as clinically unaffected or exhibiting signs of WB by palpating the breast and via the simple method of wing lift examination, which can be performed in live birds by gently lifting up the wings of each bird to assess the ability to achieve back-to-back wing contact (Kawasaki et al., 2016) at 15, 44, and 50 days of age (just prior to euthanasia). The birds were housed in clean concrete-floored pen $(1.8 \times 1.2 \mathrm{~m})$ covered with clean soft sawdust litter under suitable air conditioning and hygiene management and had free access to commercial corn-based diet and water. At 50 days of age, 35 birds weighing over 3,300 g were selected. The body weights of selected birds were $4,090 \pm 478 \mathrm{~g}$. All birds were euthanized by exsanguination under deep anesthesia induced using $20-30 \mathrm{mg} / \mathrm{kg}$ pentobarbital sodium into the radial vein with sterilized disposable $2.5-\mathrm{ml}$ syringes and 23 -gauge needles. The muscle samples were collected from one-third of the anterior part of the PM where fibrosis frequently occurs (Clark and Velleman, 2016; Hasegawa et al., 2020), fixed with Bouin's fixative (Polysciences, Inc., Warrington, PA, United States) at $4^{\circ} \mathrm{C}$ for 
histological analysis, fixed with half-Karnovsky's fixative [2.5\% glutaraldehyde, $2 \%$ paraformaldehyde, $0.1 \mathrm{M}$ cacodylate buffer (CB), $\mathrm{pH} 7.4]$ at $4^{\circ} \mathrm{C}$ for ultrastructural analysis, or immersed in RNAlater (Qiagen, Hilden, Germany) at $4^{\circ} \mathrm{C}$ for the analysis of gene expression. The PMs fixed with Bouin's fixative were embedded in paraffin and cut into $4-\mu \mathrm{m}$-thick sections, which were then used for hematoxylin-eosin (HE) and Azan staining.

\section{Histoplanimetry of the PM}

To evaluate the indices of muscle pathology, fibrotic area (FA) in the muscle and circularity of myofibers (CM) were measured. Several previous studies have reported that the primary histopathological characteristics in WB include collagen deposition (fibrosis) in the interstitium and lack of polygonality of myofibers (Sihvo et al., 2014; Kawasaki et al., 2018). Therefore, we chose the FA and $\mathrm{CM}$ as representative histopathological parameters to show the degree of WB-mediated deterioration. To calculate the FA, the area of myofibers $(a)$ and the whole area of muscle $(b)$ in four different randomly selected regions of individual Azan-stained histological sections of each specimen were measured using Image Pro software V10.0.04 (Media Cybernetics, Inc., Rockville, MD, United States). The FA was calculated as follows: FA $(\%)=100 \times$ [whole area of the muscle $(b)$ - area of myofibers $(a)] /$ whole area of the muscle $(b)$. According to the FA value, the birds were divided into stages IVI by groups of $5 \%$ units; i.e., stage I $(<20 \%, n=6)$, stage II $(20-25 \%, n=5)$, stage III $(25-30 \%, n=6)$, stage IV $(30-35 \%$, $n=5)$, stage $\mathrm{V}(35-40 \%, n=9)$, and stage VI $(>40 \%, n=4)$. To calculate the CM, 200 myofibers on four different randomly selected regions of individual HE-stained histological sections of each specimen were manually traced, and their CMs were calculated using ImageJ ver1.52a (National Institutes of Health, Bethesda, MD, United States). The formula for CM calculation is $4 p \times$ area of myofiber/(perimeter of myofiber $)^{2}$.

\section{Ultrastructural Analysis}

For transmission electron microscope (TEM) analysis, the PMs of sample numbers $1,2,6,9,10,15,17,19$, and $33(n=9)$ fixed by half-Karnovsky's fixative were used for specimen preparation. The contrast of the mitochondrial membrane structures was enhanced for TEM by heavy metal block staining, as described previously (Thai et al., 2016). Briefly, the fixed PMs were washed four times with a solution containing $0.1 \mathrm{M} \mathrm{CB}(\mathrm{pH}$ 7.4) and $2 \% \mathrm{OsO}_{4}$ (TAAB Laboratories Equipment Ltd., Berks, United Kingdom) in $0.15 \% \mathrm{~K}_{4}(\mathrm{CN})_{6}$ (Nacalai Tesque, Inc., Kyoto, Japan) for $4 \mathrm{~min}$ each at $4^{\circ} \mathrm{C}$ and soaked in the same solution for $1 \mathrm{~h}$. After four washes with distilled water (4 min each), the samples were immersed in $0.1 \%$ thiocarbohydrazide (Sigma Aldrich Co. LLC., St. Louis, MO, United States) for $20 \mathrm{~min}$ at room temperature, then washed again with distilled water and immersed in $2 \% \mathrm{OsO}_{4}$ for $30 \mathrm{~min}$ at room temperature. Following further distilled water washing, the samples were immersed in $1 \%$ uranyl acetate at $4^{\circ} \mathrm{C}$ overnight, washed again in distilled water, and immersed in Walton's lead aspartate solution at $60^{\circ} \mathrm{C}$ for $30 \mathrm{~min}$. Finally, the samples were dehydrated through an ethanol series, transferred to QY-1, and embedded in epoxy resin (Quetol 812; Nissin EM Co. Ltd., Tokyo, Japan). The tissue block was sliced into 80 -nm-thick sections perpendicular to the long axis of the myofiber using an ultramicrotome (JUM-7; JEOL Ltd., Tokyo, Japan). The sections were collected on a 100-mesh copper grid, and the structures were observed by TEM (HT-7700; Hitachi High Technology Co., Tokyo, Japan) at an acceleration voltage of $80 \mathrm{kV}$.

\section{Reverse Transcription and Quantitative Real-Time Polymerase Chain Reaction}

The PMs of broilers were homogenized using a BioMasher (Nippi, Inc., Tokyo, Japan), and total RNA from samples was purified using TRIzol reagent (Life Technologies, Carlsbad, CA, United States) according to the manufacturer's instructions. The purified total RNA was used as a template to synthesize complementary DNA (cDNA) using ReverTra Ace qPCR RT Master Mix (Toyobo Co., Ltd., Osaka, Japan). Quantitative realtime PCR (qPCR) analysis was performed on the cDNA using THUNDERBIRD SYBR qPCR Mix (Toyobo Co., Ltd.) and genespecific primers (Table 1, Sigma-Aldrich). The qPCR cycling conditions were $95^{\circ} \mathrm{C}$ for $1 \mathrm{~min}$, followed by 40 cycles of $95^{\circ} \mathrm{C}$ for $15 \mathrm{~s}$, and $60^{\circ} \mathrm{C}$ for $45 \mathrm{~s}$. Data were normalized against the expression level of actin, beta $(A C T B)$ and analyzed using the $\Delta \mathrm{Ct}$ method for correlation analysis and the $\Delta \Delta \mathrm{Ct}$ method for stage-related comparison of gene expression.

\section{Statistical Analysis}

Results were expressed as the mean \pm standard error (SE). Data among three or more groups were compared using the Tukey test $(P<0.05)$. Data between two groups were compared using the Student's $t$ test $(P<0.05)$. Correlations between two parameters were analyzed using Spearman's correlation test $(P<0.05)$.

\section{RESULTS}

\section{Histopathological Observation of the PM}

As shown in Figure 1, the histological sections of the PMs stained with Azan and HE showed both mild and severe fibrotic pathology, as revealed by the collagen distribution stained blue with Azan, and myofiber hypertrophy. The values of FA and CM in the PMs of each broiler are shown in Table 2; mean values in all birds were $29.4 \pm 9.6 \%$ and $0.70 \pm 0.042 \%$, respectively. According to FA, the birds were grouped into six stages. The CM tended to increase with higher stage (Table 2) and significantly positively correlated with the FA [Spearman's correlation coefficient $(\rho)=0.6246, P<0.0001]$. In PMs with relatively low levels of FA and $\mathrm{CM}$ among all broilers (stage I), not only the normal polygonal myofibers but also myofibers with numerous small vacuoles were observed (Figures 1A-C). In comparison, in PMs with relatively high levels of FA and CM (stage VI), several types of myofibers with pathology could be seen: myofibers with numerous small vacuoles and large-rimmed vacuoles, degenerated myofibers, myofibers with small caliber, and split fibers (Figures 1D,E). During the observation period, we obtained clinical information including body weight at 15, 44, and 50 days, weight gain ratio from 15 to 50 days, and results 
TABLE 1 | List of primers used in the study.

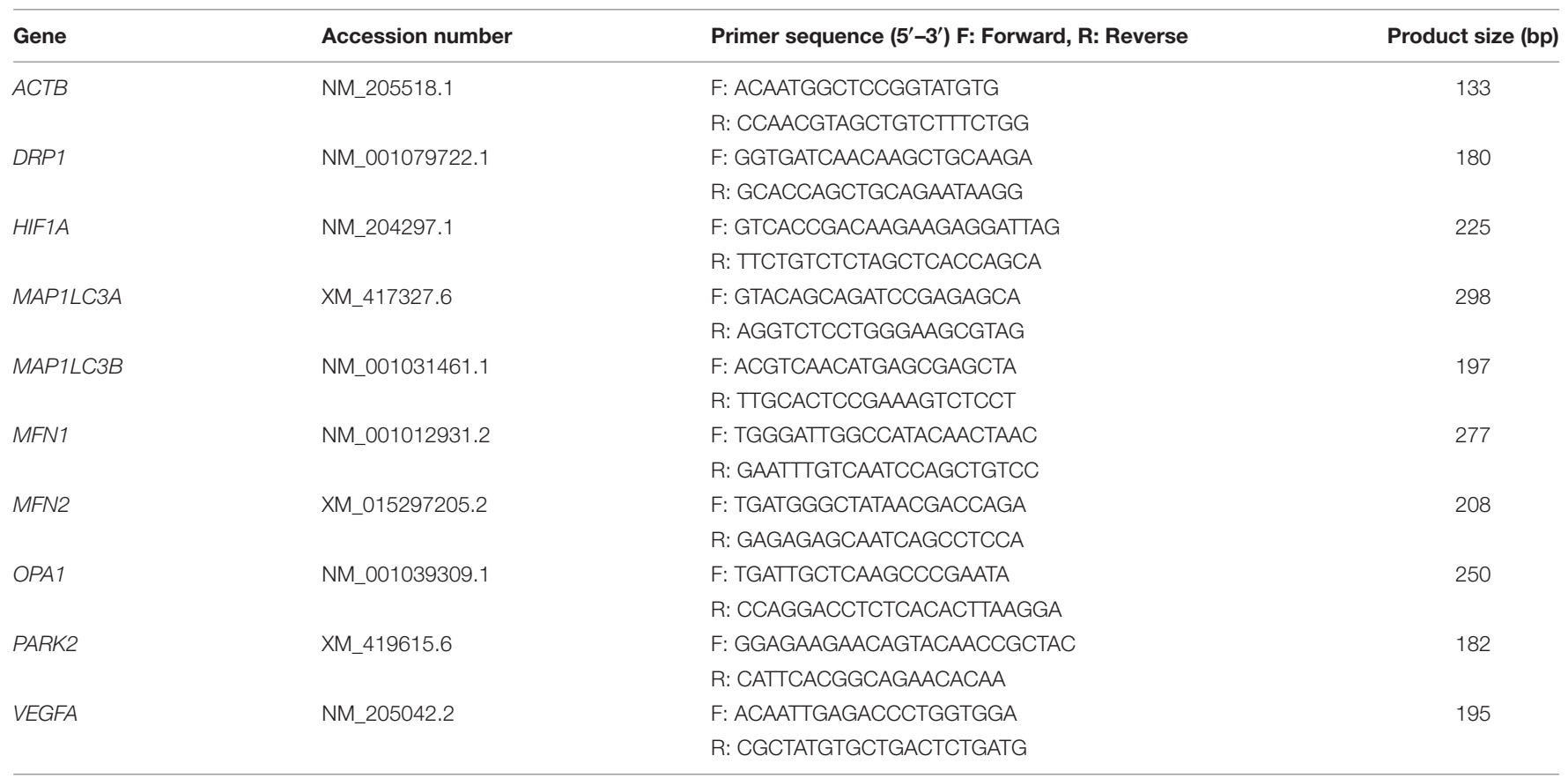

ACTB, actin, beta; DRP1, dynamin-related protein 1; HIF1A, hypoxia inducible factor 1 subunit alpha; MAP1LC3A, B, microtubule associated protein 1 light chain 3 alpha, beta; MFN1, 2, mitofusion 1, 2; OPA1, OPA1, mitochondrial dynamin like GTPase; PARK2, parkin RBR E3 ubiquitin protein ligase; VEGFA, vascular endothelial growth factor $A$.

of palpation and wing lift examination (Supplementary Table 1). Comparison and correlation analysis between PM histopathology and these clinical data were performed; however, no significant associations were detected (Supplementary Tables 2, 3).

\section{Ultrastructural Observation of PM Myofibers}

In healthy myofibers, both normal, small mitochondria containing clear intrastructures and swelled round mitochondria were localized within the intact myofibrils, which are composed of myosin (Figure 2A). In the myofibers with numerous small vacuoles, spherical structures with a single-membrane containing degraded mitochondria, reflecting autophagosome-like vacuoles, were observed (Figure 2B). The myofibrils around the vacuole clearly showed intact morphology. Myofiber regeneration by fusion of multiple myoblasts was also observed (Figure 2C). In the cytosol of the myoblasts, spherical structures with a double-membrane containing normal mitochondria and other organelles such as endoplasmic reticulum, reflecting typical autophagosomes, were observed (Figure 2C).

\section{Relationship Between Hypoxia and Histopathological Observations in the PM}

To examine the association of the muscle hypoxic conditions in WB with the histopathological observations such as fibrosis and hypertrophy in the myofibers, the correlations between the hypoxia-adaptive genes $H I F 1 A$ and vascular endothelial growth factor A (VEGFA), and the muscle histopathological indices FA and CM were analyzed (Table 3). Although the expression of HIF1A did not show significant correlations with either histopathological index, that of VEGFA was significantly negatively correlated with both pathological indices.

\section{Relationship Between Mitochondrial Dynamics and Pathological Observations, Hypoxia, or Autophagy/Mitophagy in the PM}

To reveal the association of the mitochondrial dynamics with the histopathological and hypoxic conditions in the PMs, the correlations between the expression of mitochondrial fusion[mitofusion 1, 2 (MFN1, MFN2) and mitochondrial dynaminlike GTPase (OPA1)] and fission [dynamin-related protein 1 (DRP1)]-related genes and the histopathological indices (FA and $\mathrm{CM}$ ) or the expression of hypoxia-adaptive genes HIF1A and VEGFA were analyzed (Table 4). The expression of MFN1 and MFN2 was significantly negatively correlated with CM and both FA and CM, respectively. Alternatively, DRP1 and HIF1A expression significantly positively correlated. In addition, the expression of all mitochondrial dynamics-related genes including MFN1, MFN2, OPA1, and DRP1 showed strong significantly positive correlation with that of VEGFA.

Based on the ultrastructural observations of the active metabolism of mitochondria as revealed by autophagylike vacuoles, the correlations between the expression of mitochondrial dynamics-related genes and that of autophagy/mitophagy-related genes were also analyzed (Table 4). Specifically, the microtubule-associated protein 1 light chain 


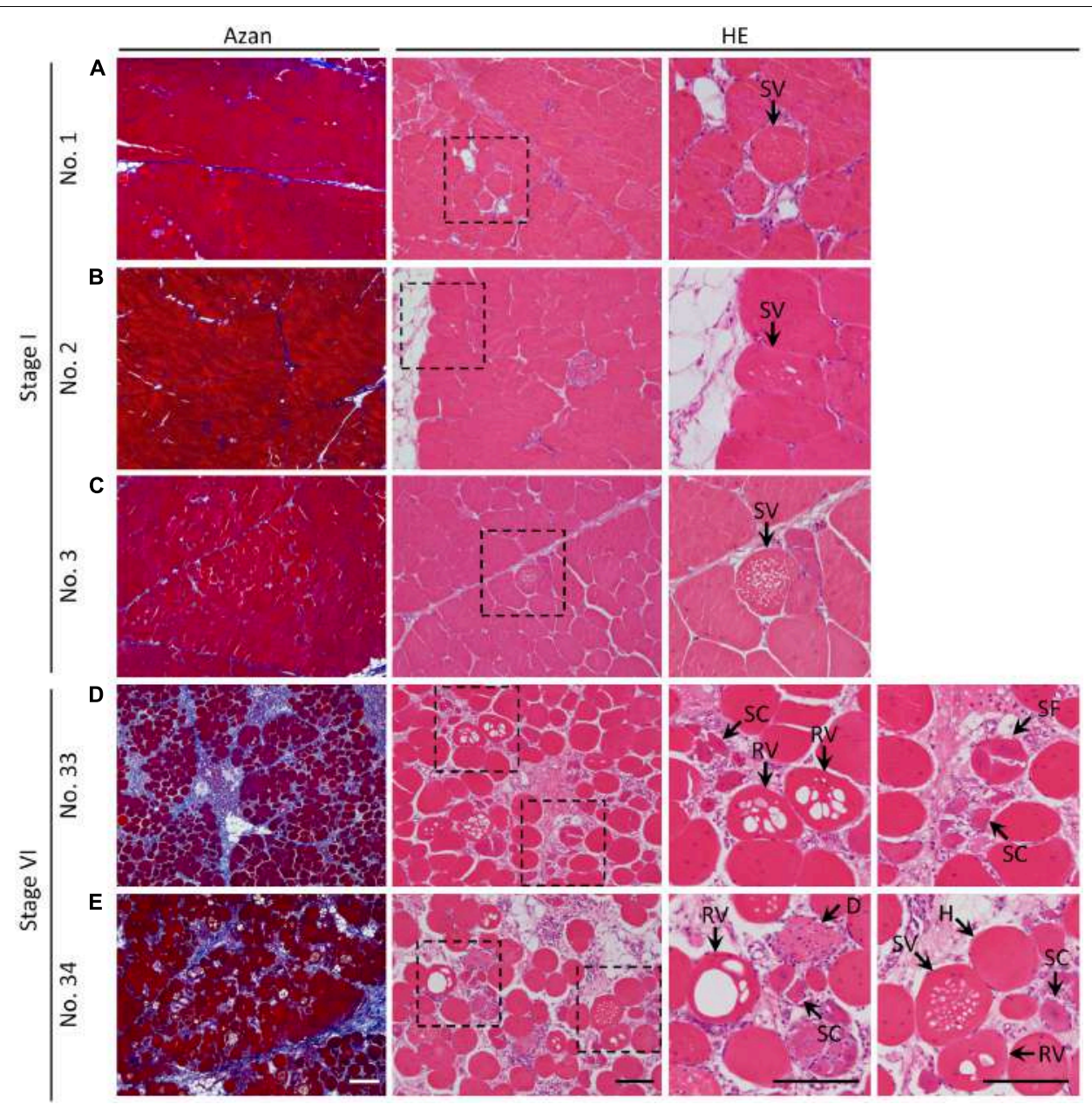

FIGURE 1 | Histology of the pectoralis major muscle of broilers stained with Azan and hematoxylin-eosin. (A-E) Histological observations of the pectoralis major muscle of (A) sample number 1 (stage I; FA, 7.24\% and CM, 0.70), (B) 2 (stage I; FA, 9.06\% and CM, 0.69), (C) 3 (stage I; FA, 13.25\% and CM, 0.68), (D) 33 (stage $\mathrm{VI}$; FA, 40.90\% and CM, 0.70), and (E) 34 (stage VI; FA, 40.97\% and CM, 0.76). The squares indicated by dashed lines are magnified on the right. White bar = 200 mm, black bar = 100 mm. SV, myofiber with numerous small vacuoles; RV, myofiber with large-rimmed vacuoles; H, hypertrophy of myofiber; SC, myofiber with small caliber; SF, split fiber; D, myofiber degeneration; FA, fibrotic area in the muscle; CM, circularity of myofibers.

3 alpha, beta $(M A P 1 L C 3 A, B)$ genes, also termed LC3A and $\mathrm{LC} 3 \mathrm{~B}$, constitute the primary genes related to autophagosome development and maturation (Schaaf et al., 2016), whereas parkin RBR E3 ubiquitin protein ligase (PARK2) acts as a specific regulator of mitophagy (Sato et al., 2018). We found that the expression of MFN1, MFN2, OPA1, and DRP1 was significantly positively correlated with that of all three autophagy-/mitophagy-related genes.

We further compared the expression levels of all examined genes among the six stages. No significant stage-related differences were observed in the expression of any of the examined genes (Figures 3A-C).

\section{DISCUSSION}

The histology of broiler PMs revealed myofiber hypertrophy and internal fibrosis, consistent with previous reports (Sihvo et al., 2014; Velleman and Clark, 2015), whereas the values of FA and CM histopathological indices differed among individuals, revealing that WB severity in the broilers varies during later life. Myofiber vacuolization was observed in all PMs analyzed in this study. Myofibers with numerous small vacuoles were apparent in the PMs with both mild and more severe FA and CM, whereas those with large-rimmed vacuoles frequently occurred in the PMs with more severe index scores. These vacuoles are thus termed autophagic vacuoles (AVs) in skeletal muscle (Malicdan and Nishino, 2012), and their appearance therein constitutes the pathognomonic morphological hallmark of autophagic vacuolar myopathy, which is a group of human hereditary myopathies including Pompe and Danon disease (Nishino, 2006; Cho and Noguchi, 2013; Castets et al., 2016). It has been reported that rimmed vacuoles are outcomes of impaired normal autophagy and represent frequent pathological characteristics in the chronic muscle damage of atrophy and myopathies (Suzuki et al., 2002; Sato et al., 2018). During autophagy, the accumulation of 
TABLE 2 | The fibrotic area (FA) in muscle, circularity of myofibers (CM), and stage classified by the FA in each bird.

\begin{tabular}{|c|c|c|c|c|c|}
\hline Sample No. & FA (\%) & CM & Stage & Mean \pm SE $(\%)$ of FA in each stage & Mean \pm SE of $\mathrm{CM}$ in each stage \\
\hline 1 & 7.24 & 0.70 & I & $13.45 \pm 1.81^{\| l, I I, I V, V, V I}$ & $0.67 \pm 0.02^{V}$ \\
\hline 2 & 9.06 & 0.69 & I & - & - \\
\hline 3 & 13.25 & 0.68 & I & - & - \\
\hline 4 & 16.31 & 0.67 & 1 & - & - \\
\hline 5 & 17.10 & 0.68 & 1 & - & - \\
\hline 6 & 17.76 & 0.57 & I & - & - \\
\hline 7 & 21.53 & 0.67 & $\|$ & $23.59 \pm 0.58^{\mathrm{IV}, \mathrm{V}, \mathrm{VI}}$ & $0.68 \pm 0.01$ \\
\hline 8 & 23.28 & 0.70 & $\|$ & - & - \\
\hline 9 & 24.06 & 0.67 & $\|$ & - & - \\
\hline 10 & 24.13 & 0.67 & $\|$ & - & - \\
\hline 11 & 24.92 & 0.68 & $\|$ & - & - \\
\hline 12 & 25.86 & 0.61 & III & $27.05 \pm 0.64^{\mathrm{IV}, \mathrm{V}, \mathrm{VI}}$ & $0.68 \pm 0.02$ \\
\hline 13 & 26.15 & 0.74 & III & - & - \\
\hline 14 & 26.19 & 0.66 & III & - & - \\
\hline 15 & 26.28 & 0.74 & III & - & - \\
\hline 16 & 27.95 & 0.70 & III & - & - \\
\hline 17 & 29.86 & 0.66 & III & - & - \\
\hline 18 & 31.14 & 0.73 & IV & $31.96 \pm 0.51^{\vee, \mathrm{Vl}}$ & $0.70 \pm 0.01$ \\
\hline 19 & 31.18 & 0.69 & IV & - & - \\
\hline 20 & 31.35 & 0.68 & IV & - & - \\
\hline 21 & 32.05 & 0.70 & IV & - & - \\
\hline 22 & 34.08 & 0.72 & IV & - & - \\
\hline 23 & 36.92 & 0.74 & V & $38.15 \pm 0.35$ & $0.74 \pm 0.01$ \\
\hline 24 & 37.00 & 0.74 & V & - & - \\
\hline 25 & 37.37 & 0.74 & V & - & - \\
\hline 26 & 37.80 & 0.75 & V & - & - \\
\hline 27 & 37.93 & 0.69 & V & - & - \\
\hline 28 & 38.05 & 0.75 & V & - & - \\
\hline 29 & 39.21 & 0.75 & V & - & - \\
\hline 30 & 39.47 & 0.74 & V & - & - \\
\hline 31 & 39.61 & 0.71 & V & - & - \\
\hline 32 & 40.65 & 0.70 & VI & $40.88 \pm 0.08$ & $0.72 \pm 0.01$ \\
\hline 33 & 40.90 & 0.70 & $\mathrm{VI}$ & - & - \\
\hline 34 & 40.97 & 0.76 & VI & - & - \\
\hline 35 & 41.00 & 0.72 & VI & - & - \\
\hline
\end{tabular}

Significant differences among stages are indicated by superscripted stage no. (I, II, III, IV, V, and VI) $(P<0.05$, Tukey test).

protein aggregates disturbs the maturation and fusion steps of autophagosome formation and enlarges the autophagic vesicles, where the rimmed vacuoles originate (Castets et al., 2016). Therefore, the rimmed vacuoles frequently observed in the PMs of broilers with more severe FA and CM implies the pathological condition of autophagy, indicating the close relationship between the altered autophagy and WB severity.

The ultrastructural observations of mitochondrial morphology confirmed that both physiological and pathological autophagies occur in the broiler skeletal muscles. Even in the histologically healthy myofibers, not only normal but also swelled and degenerated mitochondria are distributed within the myofibrils. In the myofibers exhibiting numerous small vacuoles, the AVs appeared to engulf the degenerated mitochondria. Moreover, because these AVs were enveloped by a single membrane, it was inferred that they were in the degradation stage during autophagy, in which the autophagosome double membrane is degraded by lysosomes as an autolysosome (Eskelinen et al., 2011). In mouse studies, deficiency of apolipoprotein B messenger RNA (mRNA) editing enzyme catalytic polypeptide 2 (APOBEC2), a member of the zincdependent cytidine deaminase protein family, has been reported to lead to a loss of skeletal muscle mass and atrophy (Sato et al., 2009). Notably, APOBEC2 deficiency does not affect the sarcomeric structure but rather causes mitochondrial morphological abnormalities in the murine skeletal myofibers, with the abnormal mitochondria being surrounded by AVs and removed by mitophagy (Sato et al., 2018). In comparison, in the myofibers of broilers analyzed in the present study, the myofibrils surrounding the AVs were ultrastructurally healthy and intact, indicating that the engulfment and digestion of these vacuoles is limited to the mitochondria likely through 

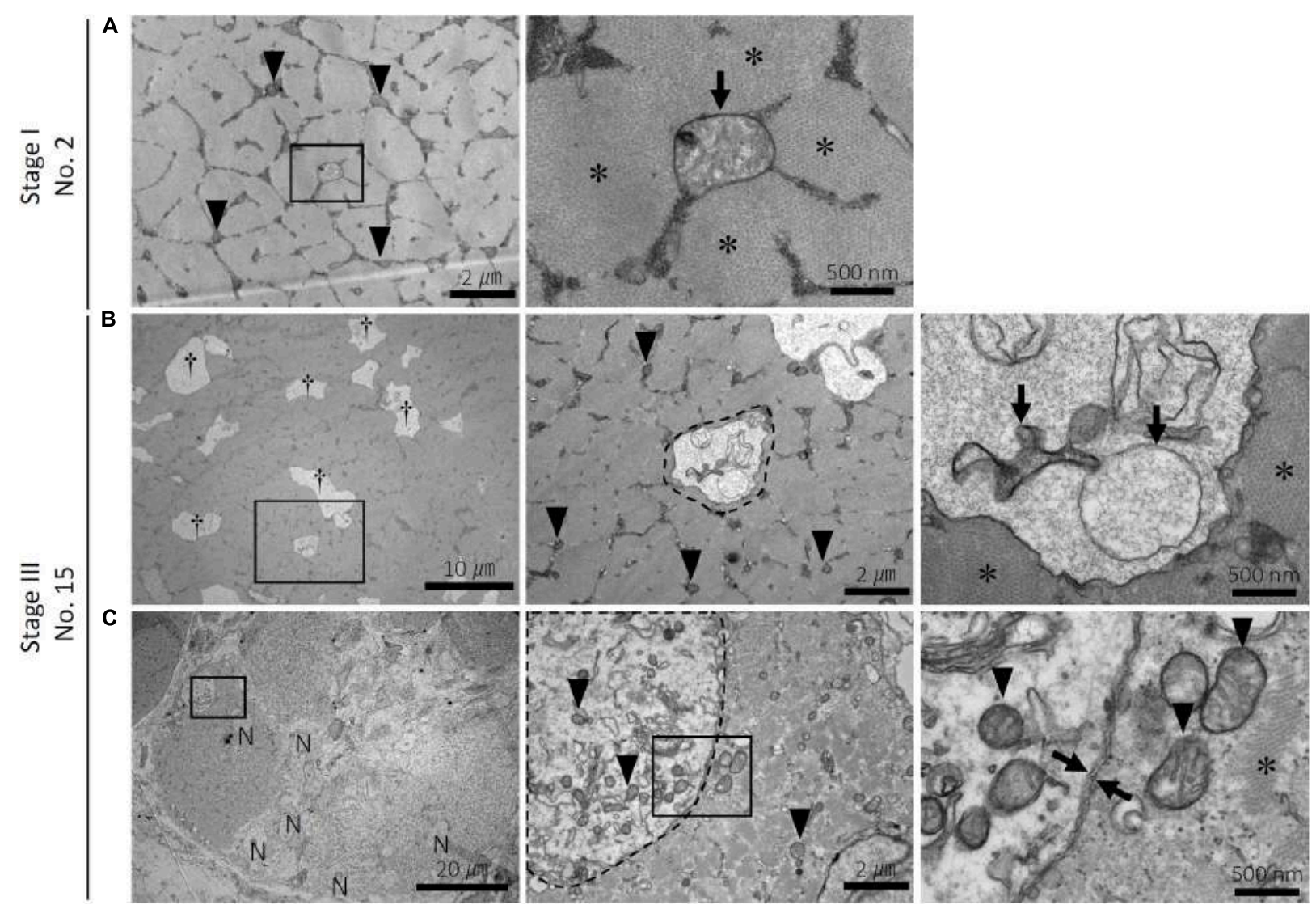

FIGURE 2 | Ultrastructure of the myofibers in the pectoralis major muscle of broilers. Ultrastructure of the pectoralis major muscle of (A) sample number 2 (stage I; FA, 9.06\% and CM, 0.69) and (B,C) 15 (stage III; FA, 26.28\% and CM, 0.74). FA, fibrotic area in the muscle; CM, circularity of myofibers. (A) Distribution of small and clear mitochondria (arrowheads) and a swelled mitochondrion (arrow) within myofibrils (asterisks) in the normal myofiber. The square indicated by black line is magnified on the right. (B) Numerous small vacuoles (daggers) are distributed within myofibrils (asterisks) in the myofibers. The vacuole (surrounded by a dashed line) envelops the swelled and degenerated mitochondria (arrows). The square indicated by black line is magnified on the right. Arrowheads: small and clear mitochondria. (C) Multiple myoblasts accumulated in the pectoralis major muscle. The autophagosome with a double membrane (between arrows) in the myoblast envelops organelles and the small and clear mitochondria (arrowheads). The squares indicated by black line are magnified on the right. N, nuclei in the myoblast; asterisks, myofibrils.

the mitophagy process. Specifically, to maintain skeletal muscle homeostasis, muscle repair and remodeling promotes the proliferation of satellite cells as myoblasts, which activates their myogenic differentiation (Sin et al., 2016). The autophagy and/or mitophagy in myoblasts are physiologically required in order to regenerate the skeletal muscle by myoblast differentiation (Sin et al., 2016; Tokutake et al., 2019). Herein, we observed typical autophagosomes with double membranes engulfing organelles in the myoblasts of the broiler PM. Therefore, we consider that, in the broilers, the autophagy/mitophagy that occurs via AVs in the myofibers and via autophagosomes in the myoblasts has different biological interpretations.

Hypoxia-inducible factorl is a heterodimer composed of an alpha and a beta subunit, which are respectively induced by hypoxia and constitutively expressed (Greijer and van der Wall, 2004). HIF $1 \alpha$ mediates the primary transcriptional response to hypoxia by binding to conserved sequences in the promoter regions of various hypoxia-adaptive genes (Majmundar et al., 2010; Thomas and Ashcroft, 2019). VEGF is the principal
TABLE 3 | Spearman's correlation coefficient ( $\rho$ ) between the hypoxia-adaptive gene expression and muscle histopathological indices.

\begin{tabular}{lccc}
\hline \multirow{2}{*}{ Gene } & \multicolumn{2}{c}{ Histopathological index } \\
\cline { 3 - 4 } & & FA & CM \\
\hline HIF1A $(n=35)$ & $\rho$ & 0.2616 & 0.2392 \\
VEGFA $(n=35)$ & $P$ & 0.129 & 0.1664 \\
& $\rho$ & -0.4476 & -0.584 \\
& $P$ & $0.007^{\star *}$ & $0.0002^{\star \star \star}$
\end{tabular}

HIF1A, hypoxia inducible factor 1 subunit alpha; VEGFA, vascular endothelial growth factor $A ; F A$, fibrotic area in the muscle; $C M$, circularity of myofibers. ${ }^{* *} P<0.01,{ }^{* * *} P<0.001$

transcriptional target of HIF1 $\alpha$ and induces angiogenesis in the hypoxic condition, which increases the oxygen supply (Majmundar et al., 2010). In skeletal muscles, the hypoxia consequent to exercise and ischemia is known to induce angiogenesis through HIF1-VEGF signaling (Ohno et al., 2012). 


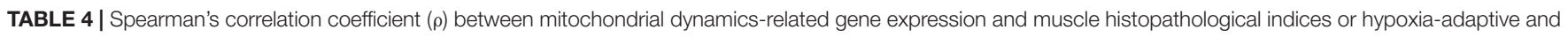
autophagy-/mitophagy-related gene expression.

\begin{tabular}{|c|c|c|c|c|c|c|c|c|}
\hline \multirow[t]{3}{*}{ Gene } & & \multicolumn{7}{|c|}{ Parameter } \\
\hline & & \multicolumn{2}{|c|}{ Histopathological index } & \multicolumn{2}{|c|}{ Hypoxia-adaptive gene expression } & \multicolumn{3}{|c|}{ Autophagy-/mitophagy-related gene expression } \\
\hline & & FA & $\mathrm{CM}$ & HIF1A & VEGFA & MAP1LC3A & MAP1LC3B & PARK2 \\
\hline MFN1 & $\rho$ & -0.279 & -0.3364 & 0.2501 & 0.6804 & 0.5882 & 0.5832 & 0.565 \\
\hline$(n=35)$ & $P$ & 0.1046 & $0.0482^{*}$ & 0.1473 & $<0.0001^{\star \star \star}$ & $0.0002^{\star \star \star}$ & $0.0002^{\star \star \star}$ & $0.0004^{\star \star \star}$ \\
\hline MFN2 & $\rho$ & -0.3734 & -0.4451 & 0.0482 & 0.7555 & 0.6384 & 0.6563 & 0.4866 \\
\hline$(n=35)$ & $P$ & $0.0271^{\star}$ & $0.0074^{\star \star}$ & 0.7834 & $<0.0001^{\star \star \star}$ & $<0.0001^{\star \star \star}$ & $<0.0001^{\star \star \star}$ & $0.003^{\star \star}$ \\
\hline OPA1 & $\rho$ & -0.1499 & -0.2574 & 0.2714 & 0.5644 & 0.6339 & 0.5933 & 0.3854 \\
\hline$(n=35)$ & $P$ & 0.3902 & 0.1355 & 0.1147 & $0.0004^{\star \star \star}$ & $<0.0001^{\star \star \star}$ & $0.0002^{\star \star \star}$ & $0.0222^{*}$ \\
\hline DRP1 & $\rho$ & -0.2734 & -0.237 & 0.3353 & 0.6204 & 0.688 & 0.6796 & 0.5232 \\
\hline$(n=35)$ & $P$ & 0.112 & 0.1705 & $0.049^{\star}$ & $<0.0001^{\star \star \star}$ & $<0.0001^{\star \star \star}$ & $<0.0001^{\star \star \star}$ & $0.0013^{\star *}$ \\
\hline
\end{tabular}

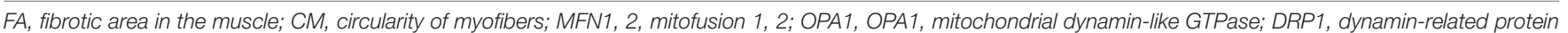

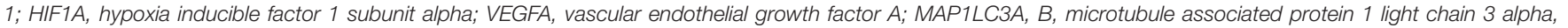
beta; PARK2, parkin RBR E3 ubiquitin protein ligase. ${ }^{*} P<0.05,{ }^{* *} P<0.01,{ }^{* * *} P<0.001$.

A

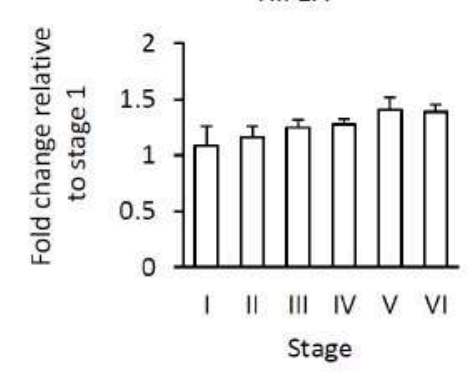

B

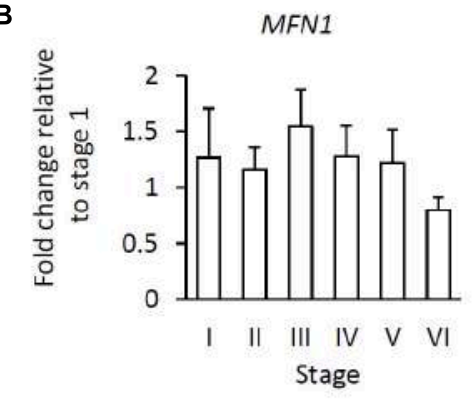

C

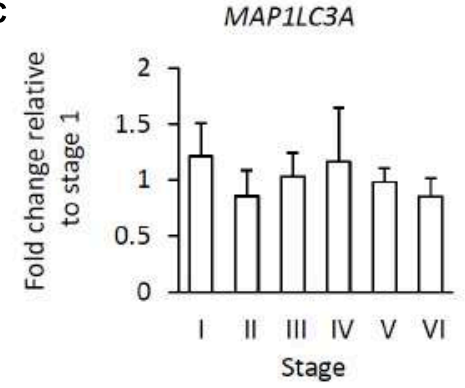

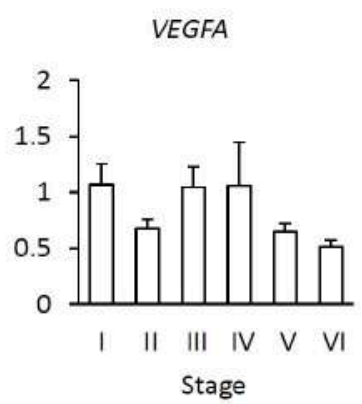

MFN2

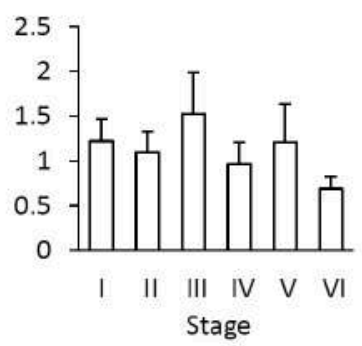

MAP1LC3B

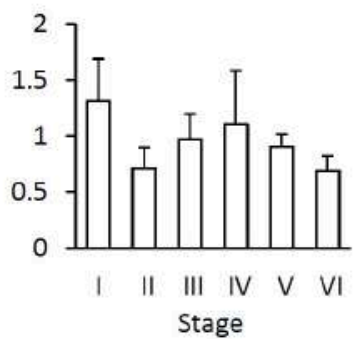

OPA1

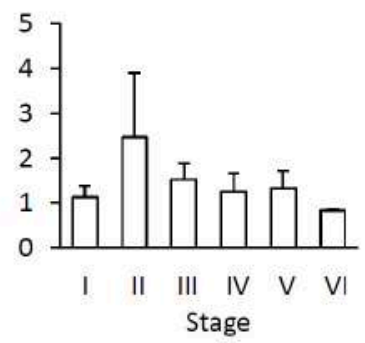

PARK2

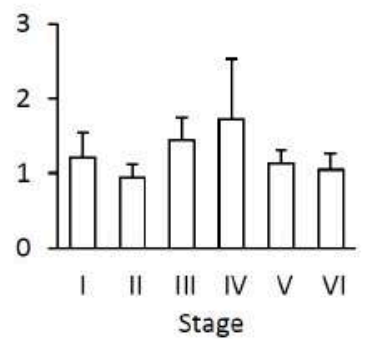

FIGURE 3 | Relationship of gene expression levels in the various stages to fibrotic area in the muscle. (A) Hypoxia-adaptive gene expression including hypoxia inducible factor 1 subunit alpha (HIF1A) and vascular endothelial growth factor A (VEGFA). (B) Mitochondrial dynamics-related gene expression including mitofusion 1, 2 (MFN1, 2), OPA1, mitochondrial dynamin-like GTPase (OPA1), and dynamin-related protein 1 (DRP1). (C) Autophagy-/mitophagy-related gene expression including microtubule-associated protein 1 light chain 3 alpha, beta (MAP1LC3A, B) and parkin RBR E3 ubiquitin protein ligase (PARK2). Data represent the means \pm SE [stage I $(n=6)$, stage II $(n=5)$, stage III $(n=6)$, stage IV $(n=5)$, stage $\mathrm{V}(n=9)$, and stage VI $(n=4)$, Tukey test $(P<0.05)$ ]. 
In the PMs of the broilers, VEGFA expression was significantly negatively correlated with the muscle pathological indices FA and $\mathrm{CM}$, indicating that the decreased angiogenesis and oxygen supply in the skeletal muscle worsened the symptoms of WB. As hypoxia-mediated HIF1 induction occurs at the level of protein stability whereas HIF1A mRNA expression remains unchanged (Greijer and van der Wall, 2004), our correlation analysis results suggest that HIF1A expression and WB severity are not related in the broiler PMs.

Rather, the small caliber myofibers and split fibers observed in the PMs with more severe FA and CM, in which the angiogenesis is insufficient for supplying oxygen, are considered to be the outcomes of impaired myofiber regeneration consequent to the severe hypoxia. In general, the hypoxia-mediated impairment of skeletal muscle regeneration promotes the loss of muscle mass and atrophy (Neel et al., 2013; Romanello and Sandri, 2016). However, in broilers, the skeletal muscles develop fibrosis within the degenerated myofibers instead of decreasing their mass, resulting in the retention of high PM mass. In comparison, in the field of cancer medicine, it is recognized that following repeated and chronic periods of hypoxia, selection for resistance to the hypoxia-induced HIF1 response may occur, which exacerbates hypoxia-adaptive pathologies (Greijer and van der Wall, 2004). We consider that the chronic hypoxia in the broiler PMs might similarly alter the reactivity to HIF signaling and attenuate PM adaptability to the hypoxia.

Mitochondria undergo continuous cycles of fusion and fission, referred as mitochondrial dynamics, to maintain their physiological functions (Tilokani et al., 2018). Mitochondrial fusion, whereby one mitochondrion is produced from the union of two mitochondria, promotes the reduction and dilution of damage in each mitochondria by mixing their matrix contents for complementation (Byrne et al., 2019). MFN1, MFN2, and OPA1 encode key factors for mediating mitochondrial fusion (Hall et al., 2014). Alternatively, mitochondrial fission, whereby one mitochondrion is divided into two daughter mitochondria, not only regulates the amount and distribution of mitochondria but also plays a role in disposing their deteriorated contents by autophagy or mitophagy. Mitochondrial fission is regulated mainly by DRP1 expression (Balog et al., 2016; Byrne et al., 2019).

Mitochondrial quality control is necessary in highly structured skeletal muscles (Favaro et al., 2019). In the PMs of broilers,

\section{PM of healthy broiler or mild WB}
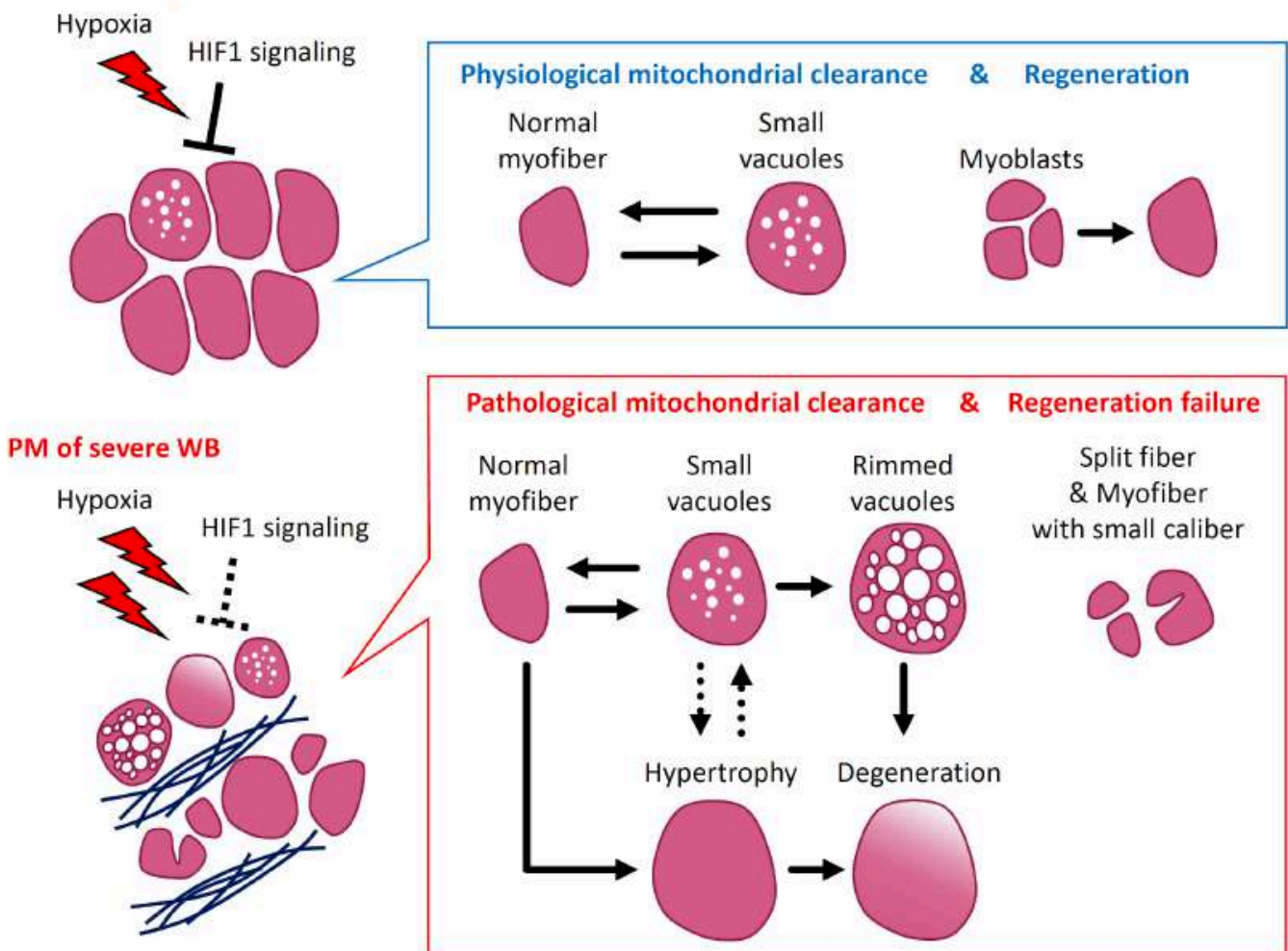

Pathological mitochondrial clearance \& Regeneration failure
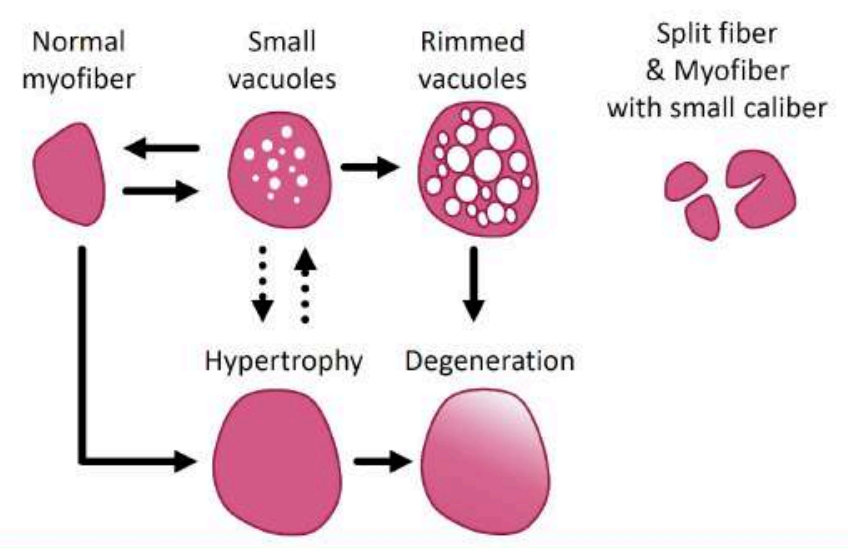

FIGURE 4 | Scheme of wooden breast syndrome (WB) pathogenesis focusing on mitochondrial clearance and myofiber pathology. In pectoralis major muscles (PM) with no or mild WB, the adaptation to hypoxia by HIF1 signaling induces physiological mitochondrial clearance mediated by mitochondrial fusion, fission, and autophagy/mitophagy, which corresponds with the appearance of myofibers with small vacuoles. At this stage, PM repair appears to occur through the differentiation of myoblasts into myofibers. Alternatively, in the muscles exhibiting severe WB, the acquired resistance to hypoxia alters the regulation of mitochondrial clearance. The accumulated damage in mitochondria exacerbates the various pathological characteristics such as myofibers with rimmed vacuoles, along with their hypertrophy and degeneration. Under severe hypoxia, the regeneration of myofibers fails, which corresponds with the frequent appearance of split fibers and myofibers with small caliber. HIF1, hypoxia-inducible factor 1. 
the expression of these mitochondrial dynamics-related genes showed strong positive correlation with that of VEGFA, suggesting that active and continuous mitochondrial dynamics affords the maintenance and clearance of mitochondria damaged by hypoxia at the early stage of $\mathrm{WB}$, during which active angiogenesis occurs. The negative correlation between mitochondrial dynamics-related genes with histopathological indices, especially that of MFN1 and MFN2 with FA and/or CM, indicated that WB pathology deteriorates in conjunction with reduced mitochondrial dynamics, especially fusion.

The series of mitochondrial fusion, fission, and mitophagy is integrated into the sequential mechanism of damaged mitochondria maintenance; i.e., the reorganization and segregation of damaged mitochondrial contents into daughter mitochondria that are eliminated by autophagy and/or mitophagy (Twig et al., 2008). In particular, the muscle specific loss of Drp1 in mouse resulted in myofiber death, atrophy, and degeneration of skeletal muscles, involving inhibition of autophagy and mitophagy (Favaro et al., 2019). The strong positive correlation between the expression of mitochondrial dynamics- and autophagy-/mitophagy-related genes observed in the present study suggested that the maintenance of mitochondrial health is highly controlled by this sequential mechanism in broiler skeletal muscles as well. Specifically, whereas the active maintenance of mitochondrial health by their fusion, fission, and autophagy/mitophagy is triggered in response to the hypoxia at the early stage of $\mathrm{WB}$, the acquired resistance to the HIF1 signaling under conditions of chronic hypoxia appears to lead to the attenuated regulation of mitochondrial maintenance by appropriate mitochondrial dynamics and autophagy/mitophagy. In addition, the broilers cannot voluntarily regulate energy balance and exhibit hyperphagia, resulting in excessive accumulation of energy (Richards, 2003). In the situation of decreased energy demand and increased supply, mitochondria progress through fission and fragmentation to adapt to the nutrient excess, which is associated with impaired mitochondrial function (Liesa and Shirihai, 2013). The damaged mitochondria in PMs mediated by excess nutrients may therefore also be screened out through the process of mitochondrial clearance in broilers.

In conclusion, our findings demonstrate that the PMs of broilers support the mechanism of physiological mitochondrial clearance, which is defined as the mechanism for maintaining mitochondria damaged by hypoxia as mediated by their continuous fusion, fission, and autophagy/mitophagy. In broilers in which the muscle is constantly exposed to hypoxia from early life owing to a genetic background that facilitates rapidly increasing PM mass, the HIF1-mediated response to hypoxia induces physiological mitochondrial clearance.

\section{REFERENCES}

Abasht, B., Mutryn, M. F., Michalek, R. D., and Lee, W. R. (2016). Oxidative stress and metabolic perturbations in wooden breast disorder in chickens. PLoS One 11:e0153750. doi: 10.1371/journal.pone.015 3750
However, the repeated and chronic hypoxia in the PMs weakens HIF1 signaling, exacerbating the oxygen deficiency and reducing physiological mitochondrial clearance. Eventually, the accumulation of damaged mitochondria would induce myofiber hypertrophy, degeneration, and reduced regeneration in the PMs along with fibrosis, reflecting the pathological characteristics of severe WB (Figure 4). This study revealed the essential role of physiological and pathological mitochondrial clearance in skeletal muscle in the pathogenesis of severe WB. Thus, our findings suggest that suppressing hypoxic stress from the initial stage may be crucial to prevent the development of severe WB.

\section{DATA AVAILABILITY STATEMENT}

The raw data supporting the conclusions of this article will be made available by the authors, without undue reservation, to any qualified researcher.

\section{ETHICS STATEMENT}

The animal study was reviewed and approved by the Rakuno Gakuen University Institutional Animal Care and Use Committee.

\section{AUTHOR CONTRIBUTIONS}

MarH, YH, YW, and MakH acquired the data. MarH, YW, and $\mathrm{MakH}$ analyzed the data. MarH, TI, and TW interpreted the results. TK, TT, TI, and TW designed the study. NT and HU provided technical assistance with transmission electron microscopy. MarH and TW wrote the first draft of the manuscript. All authors approved the final version of the manuscript.

\section{FUNDING}

This work was supported by Grant-in-Aid Scientific Research (C) from Japan Society for the Promotion of Science (\#18K05941, TW and \#17K08067, TI).

\section{SUPPLEMENTARY MATERIAL}

The Supplementary Material for this article can be found online at: https://www.frontiersin.org/articles/10.3389/fphys. 2020.00579/full\#supplementary-material

Bailey, R. A., Watson, K. A., Bilgili, S. F., and Avendano, S. (2015). The genetic basis of pectoralis major myopathies in modern broiler chicken lines. Poult. Sci. 94, 2870-2879. doi: 10.3382/ps/pev304

Balog, J., Mehta, S. L., and Vemuganti, R. (2016). Mitochondrial fission and fusion in secondary brain damage after CNS insults. J. Cereb. Blood Flow Metab. 36, 2022-2033. doi: 10.1177/0271678X16671528 
Byrne, J. J., Soh, M. S., Chandhok, G., Vijayaraghavan, T., Teoh, J. S., Crawford, S., et al. (2019). Disruption of mitochondrial dynamics affects behaviour and lifespan in Caenorhabditis elegans. Cell. Mol. Life Sci. 76, 1967-1985. doi: 10. 1007/s00018-019-03024-5

Castets, P., Frank, S., Sinnreich, M., and Rüegg, M. A. (2016). "Get the balance right": pathological significance of autophagy perturbation in neuromuscular disorders. J. Neuromuscul. Dis. 3, 127-155. doi: 10.3233/JND-16 0153

Cho, A., and Noguchi, S. (2013). "Autophagy in GNE myopathy," in Autophagy - A Double-Edged Sword - Cell Survival or Death?, ed. Y. Bailey (London: InTech), doi: $10.5772 / 55223$

Clark, D. L., and Velleman, S. G. (2016). Spatial influence on breast muscle morphological structure, myofiber size, and gene expression associated with the wooden breast myopathy in broilers. Poult. Sci. 95, 2930-2945. doi: 10.3382/ps/ pew243

Eskelinen, E. L., Reggiori, F., Baba, M., Kovács, A. L., and Seglen, P. O. (2011). Seeing is believing: the impact of electron microscopy on autophagy research. Autophagy 7, 935-956. doi: 10.4161/auto.7.9.15760

Favaro, G., Romanello, V., Varanita, T., Andrea Desbats, M., Morbidoni, V., Tezze, C., et al. (2019). DRP1-mediated mitochondrial shape controls calcium homeostasis and muscle mass. Nat. Commun. 10:2576. doi: 10.1038/s41467019-10226-9

Greijer, A. E., and van der Wall, E. (2004). The role of hypoxia inducible factor 1 (HIF-1) in hypoxia induced apoptosis. J. Clin. Pathol. 57, 1009-1014. doi: 10.1136/jcp.2003.015032

Griffin, J. R., Moraes, L., Wick, M., and Lilburn, M. S. (2018). Onset of white striping and progression into wooden breast as defined by myopathic changes underlying pectoralis major growth. Estimation of growth parameters as predictors for stage of myopathy progression. Avian Pathol. 47, 2-13. doi: 10.1080/03079457.2017.1356908

Hall, A. R., Burke, N., Dongworth, R. K., and Hausenloy, D. J. (2014). Mitochondrial fusion and fission proteins: novel therapeutic targets for combating cardiovascular disease. Br. J. Pharmacol. 171, 1890-1906. doi: 10. 1111/bph.12516

Hasegawa, Y., Hara, T., Kawasaki, T., Yamada, M., Watanabe, T., and Iwasaki, T. (2020). Effect of wooden breast on postmortem changes in chicken meat. Food Chem. 315:126285. doi: 10.1016/j.foodchem.2020.126285

Kawasaki, T., Iwasaki, T., Yamada, M., Yoshida, T., and Watanabe, T. (2018). Rapid growth rate results in remarkably hardened breast in broilers during the middle stage of rearing: a biochemical and histopathological study. PLoS One 13:e0193307. doi: 10.1371/journal.pone.0193307

Kawasaki, T., Yoshida, T., and Watanabe, T. (2016). Simple method for screening the affected birds with remarkably hardened pectoralis major muscles among broiler chickens. J. Poult. Sci. 53, 291-297. doi: 10.2141/jpsa.016 0036

Kuttappan, V. A., Huff, G. R., Huff, W. E., Hargis, B. M., Apple, J. K., Coon, C., et al. (2013). Comparison of hematologic and serologic profiles of broiler birds with normal and severe degrees of white striping in breast fillets. Poult. Sci. 92, 339-345. doi: 10.3382/ps.2012-02647

Liesa, M., and Shirihai, O. S. (2013). Mitochondrial dynamics in the regulation of nutrient utilization and energy expenditure. Cell Metab. 17, 491-506. doi: 10.1016/j.cmet.2013.03.002

Majmundar, A. J., Wong, W. J., and Simon, M. C. (2010). Hypoxia-inducible factors and the response to hypoxic stress. Mol. Cell 40, 294-309. doi: 10.1016/j.molcel. 2010.09.022

Malicdan, M. C. V., and Nishino, I. (2012). Autophagy in lysosomal myopathies. Brain Pathol. 22, 82-88. doi: 10.1111/j.1750-3639.2011. 00543.x

Malila, Y., Thanatsang, K., Arayamethakorn, S., Uengwetwanit, T., Srimarut, Y., Petracci, M., et al. (2019). Absolute expressions of hypoxia-inducible factor-1 alpha (HIF1A) transcript and the associated genes in chicken skeletal muscle with white striping and wooden breast myopathies. PLoS One 14:e0220904. doi: 10.1371/journal.pone.0220904

Mutryn, M. F., Brannick, E. M., Fu, W., Lee, W. R., and Abasht, B. (2015). Characterization of a novel chicken muscle disorder through differential gene expression and pathway analysis using RNA-sequencing. BMC Genomics 16:399. doi: 10.1186/s12864-015-1623-0
Neel, B. A., Lin, Y., and Pessin, J. E. (2013). Skeletal muscle autophagy: a new metabolic regulator. Trends Endocrinol. Metab. 24, 635-643. doi: 10.1016/j.tem. 2013.09.004

Nishino, I. (2006). Autophagic vacuolar myopathy. Semin. Pediatr. Neurol. 13, 90-95. doi: 10.1016/j.spen.2006.06.004

Ohno, H., Shirato, K., Sakurai, T., Ogasawara, J., Sumitani, Y., Sato, S., et al. (2012). Effect of exercise on HIF-1 and VEGF signaling. J. Phys. Fit. Sport. Med. 1, 5-16. doi: 10.7600/jpfsm.1.5

Papah, M. B., Brannick, E. M., Schmidt, C. J., and Abasht, B. (2018). Gene expression profiling of the early pathogenesis of wooden breast disease in commercial broiler chickens using RNA-sequencing. PLoS One 13:e0207346. doi: 10.1371/journal.pone.0207346

Petracci, M., Mudalal, S., Soglia, F., and Cavani, C. (2015). Meat quality in fastgrowing broiler chickens. Worlds Poult. Sci. J. 71, 363-374. doi: 10.1017/ S0043933915000367

Petracci, M., Soglia, F., Madruga, M., Carvalho, L., Ida, E., and Estévez, M. (2019). Wooden-breast, white striping, and spaghetti meat: causes, consequences and consumer perception of emerging broiler meat abnormalities. Compr. Rev. Food Sci. 18, 565-583. doi: 10.1111/1541-4337. 12431

Radaelli, G., Piccirillo, A., Birolo, M., Bertotto, D., Gratta, F., Ballarin, C., et al. (2017). Effect of age on the occurrence of muscle fiber degeneration associated with myopathies in broiler chickens submitted to feed restriction. Poult. Sci. 96, 309-319. doi: 10.3382/ps/pew270

Richards, M. P. (2003). Genetic regulation of feed intake and energy balance in poultry. Poult. Sci. 82, 907-916. doi: 10.1093/ps/82. 6.907

Romanello, V., and Sandri, M. (2016). Mitochondrial quality control and muscle mass maintenance. Front. Physiol. 6:422. doi: 10.3389/fphys.2015. 00422

Sato, Y., Ohtsubo, H., Nihei, N., Kaneko, T., Sato, Y., Adachi, S., et al. (2018). Apobec2 deficiency causes mitochondrial defects and mitophagy in skeletal muscle. FASEB J. 32, 1428-1439. doi: 10.1096/fj.20170 0493R

Sato, Y., Shimizu, M., Mizunoya, W., Wariishi, H., Tatsumi, R., Buchman, V. L., et al. (2009). Differential expression of sarcoplasmic and myofibrillar proteins of rat soleus muscle during denervation atrophy. Biosci. Biotechnol. Biochem. 73, 1748-1756. doi: 10.1271/bbb.90085

Schaaf, M. B. E., Keulers, T. G., Vooijs, M. A., and Rouschop, K. M. A. (2016). LC3/GABARAP family proteins: autophagy-(un)related functions. FASEB J. 30, 3961-3978. doi: 10.1096/fj.201600698R

Sihvo, H.-K., Immonen, K., and Puolanne, E. (2014). Myodegeneration with fibrosis and regeneration in the pectoralis major muscle of broilers. Vet. Pathol. 51, 619-623. doi: 10.1177/0300985813497488

Sin, J., Andres, A. M., Taylo, R., Weston, T., Hiraumi, Y., Stotland, A., et al. (2016). Mitophagy is required for mitochondrial biogenesis and myogenic differentiation of C2C12 myoblasts. Autophagy 12, 369-380. doi: 10.1080/ 15548627.2015.1115172

Suzuki, T., Nakagawa, M., Yoshikawa, A., Sasagawa, N., Yoshimori, T., Ohsumi, Y., et al. (2002). The first molecular evidence that autophagy relates rimmed vacuole formation in chloroquine myopathy. J. Biochem. 131, 647-651. doi: 10.1093/oxfordjournals.jbchem.a003147

Thai, T. Q., Nguyen, H. B., Saitoh, S., Wu, B., Saitoh, Y., Shimo, S., et al. (2016). Rapid specimen preparation to improve the throughput of electron microscopic volume imaging for three-dimensional analyses of subcellular ultrastructures with serial block-face scanning electron microscopy. Med. Mol. Morphol. 49, 154-162. doi: 10.1007/s00795-016-0134-7

Thomas, L. W., and Ashcroft, M. (2019). Exploring the molecular interface between hypoxia-inducible factor signalling and mitochondria. Cell. Mol. Life Sci. 76, 1759-1777. doi: 10.1007/s00018-019-03039-y

Tilokani, L., Nagashima, S., Paupe, V., and Prudent, J. (2018). Mitochondrial dynamics: overview of molecular mechanisms. Essays Biochem. 62, 341-360. doi: 10.1042/EBC20170104

Tokutake, Y., Yamada, K., Hayashi, S., Arai, W., Watanabe, T., and Yonekura, S. (2019). IRE1-XBP1 pathway of the unfolded protein response is required during early differentiation of C2C12 myoblasts. Int. J. Mol. Sci. 21:E182. doi: $10.3390 /$ ijms 21010182 
Twig, G., Hyde, B., and Shirihai, O. S. (2008). Mitochondrial fusion, fission and autophagy as a quality control axis: the bioenergetic view. Biochim. Biophys. Acta 1777, 1092-1097. doi: 10.1016/j.bbabio.2008. 05.001

Velleman, S. G., and Clark, D. L. (2015). Histopathologic and myogenic gene expression changes associated with wooden breast in broiler breast muscles. Avian Dis. 59, 410-418. doi: 10.1637/11097-042015Reg.1

Ziello, J. E., Jovin, I. S., and Huang, Y. (2007). Hypoxia-inducible factor (HIF)-1 regulatory pathway and its potential for therapeutic intervention in malignancy and ischemia. Yale J. Biol. Med. 80, 51-60.
Conflict of Interest: The authors declare that the research was conducted in the absence of any commercial or financial relationships that could be construed as a potential conflict of interest.

Copyright (c) 2020 Hosotani, Kawasaki, Hasegawa, Wakasa, Hoshino, Takahashi, Ueda, Takaya, Iwasaki and Watanabe. This is an open-access article distributed under the terms of the Creative Commons Attribution License (CC BY). The use, distribution or reproduction in other forums is permitted, provided the original author(s) and the copyright owner(s) are credited and that the original publication in this journal is cited, in accordance with accepted academic practice. No use, distribution or reproduction is permitted which does not comply with these terms. 


\section{OPEN ACCESS}

Edited by:

Sandra G. Velleman,

The Ohio State University,

United States

Reviewed by:

Francesca Soglia,

University of Bologna, Italy

Yuwares Malila,

National Center for Genetic

Engineering and Biotechnology

(BIOTEC), Thailand

*Correspondence:

Cecile Berr

cecile.berri@inrae.fr

Specialty section:

This article was submitted to

Avian Physiology,

a section of the journal

Frontiers in Physiology

Received: 07 April 2020 Accepted: 18 May 2020

Published: 24 June 2020

Citation:

Praud C, Jimenez J, Pampouille E, Couroussé N, Godet E Le Bihan-Duval E and Berri C (2020) Molecular Phenotyping of White Striping and Wooden Breast

Myopathies in Chicken.

Front. Physiol. 11:633.

doi: 10.3389/fphys.2020.00633

\section{Molecular Phenotyping of White Striping and Wooden Breast Myopathies in Chicken}

\author{
Christophe Praud', Justine Jimenez', Eva Pampouille², Nathalie Couroussé1, \\ Estelle Godet ${ }^{1}$, Elisabeth Le Bihan-Duval ${ }^{1}$ and Cecile Berri ${ }^{1 *}$
}

${ }^{1}$ INRAE, Université de Tours, UMR BOA, Nouzilly, France, ${ }^{2}$ Institut Technique de l'Aviculture, Paris, France

The White Striping (WS) and Wooden Breast (WB) defects are two myopathic syndromes whose occurrence has recently increased in modern fast-growing broilers. The impact of these defects on the quality of breast meat is very important, as they greatly affect its visual aspect, nutritional value, and processing yields. The research conducted to date has improved our knowledge of the biological processes involved in their occurrence, but no solution has been identified so far to significantly reduce their incidence without affecting growing performance of broilers. This study aims to follow the evolution of molecular phenotypes in relation to both fast-growing rate and the occurrence of defects in order to identify potential biomarkers for diagnostic purposes, but also to improve our understanding of physiological dysregulation involved in the occurrence of WS and WB. This has been achieved through enzymatic, histological, and transcriptional approaches by considering breast muscles from a slow- and a fast-growing line, affected or not by WS and WB. Fast-growing muscles produced more reactive oxygen species (ROS) than slow-growing ones, independently of WS and WB occurrence. Within fast-growing muscles, despite higher mitochondria density, muscles affected by WS or WB defects did not show higher cytochrome oxidase activity (COX) activity, suggesting altered mitochondrial function. Among the markers related to muscle remodeling and regeneration, immunohistochemical staining of FN1, NCAM, and $\mathrm{MYH1} 15$ was higher in fast- compared to slow-growing muscles, and their amount also increased linearly with the presence and severity of WS and WB defects, making them potential biomarkers to assess accurately their presence and severity. Thanks to an innovative histological technique based on fluorescence intensity measurement, they can be rapidly quantified to estimate the injuries induced in case of WS and WB. The muscular expression of several other genes correlates also positively to the presence and severity of the defects like TGFB1 and CTGF, both involved in the development of connective tissue, or Twist1, known as an inhibitor of myogenesis. Finally, our results suggested that a balance between TGFB1 and PPARG would be essential for fibrosis or adiposis induction and therefore for determining WS and WB phenotypes.

Keywords: White Striping, Wooden Breast, mitochondria, muscle remodeling, molecular phenotype 


\section{INTRODUCTION}

In a context of global population and consumption increase,the world poultry meat supplies have increased from 15 million tons in 1970 to 122.3 million tons in 2017 (FAO, 2019). Chicken alone accounts for $90 \%$ of world poultry production. The relative increase in chicken meat consumption is explained by several reasons. It is a coveted source of animal protein, since it is quick and inexpensive to produce. It is not affected by religious prohibitions, and therefore, its consumption is growing in most countries. Its nutritional qualities are of interest, particularly that of the breast meat, which is low in calories, low in fat, and high in protein. Like pork, chicken meat is widely used for processing, although it is also largely consumed as cuts. The main part of poultry meat comes from the production of fast-growing strains whose breast meat yield is high, generally ranging from 20 to $25 \%$ depending on age and weight at slaughter (Baéza et al., 2012). These chicken lines are the result of intense genetic selection, mainly based on improving feed efficiency and animal growth, but also increasing their breast meat yields. Less than 10 years ago, the poultry industry had seen the emergence of new quality defects whose incidence has been growing since. Among these, two are predominant: the White Striping (WS) and the Wooden Breast (WB), which are both similar to myopathies.

The WS defect corresponds to the appearance of white striations parallel to the muscle fiber axis. This is mainly observed on the breast pectoralis major (PM) muscle and less frequently on other thigh muscles. The WS condition has been first reported in 2012 by Kuttappan et al. (2012b), who proposed a three-point scale classification based on the macroscopic evaluation of the frequency and thickness of white striations present at the surface of the PM muscle. The normal WS0 class regroups muscles with no apparent white striations; the moderate WS1 class, muscles in which white striations are easily recognizable and the severe WS2 class, muscles in which very noticeable and large white striations are observed. The prevalence of WS defect and its progression have been different according countries. The first prevalence estimations were made by American (Kuttappan et al., 2013a), Italian (Petracci et al., 2013), and Brazilian (Ferreira et al., 2014) research teams, in countries known for their very high chicken production performance. According to these studies, the incidence ranged from 10 to 56\%. However, this prevalence is likely underestimated, since Trocino et al. (2015) reported a huge difference between the percentage of normal muscles within a broiler population classified by visual observation (18.7\%) and that based on microscopic observation of muscle cross-sections (3\%). At the microscopic level, muscles affected by WS are characterized by an increase in adipose tissue deposition, fibrosis, inflammatory infiltrates, increased number of internalized nuclei (Kuttappan et al., 2013c), fiber necrosis, and fiber size variability (Mazzoni et al., 2015). The protein content significantly decreases, while the water, collagen, and lipid contents increase between normal and severely affected muscles (Kuttappan et al., 2013b,c; Petracci et al., 2014). Linked with these changes, alteration of protein turnover toward more protein degradation and fat deposition have been reported in WS muscles (Vignale et al., 2017). Evidence of alterations of glucose metabolism, calcium signaling, hypoxia, cell death, and muscle remodeling have been also recently described (Boerboom et al., 2018; Marchesi et al., 2019), as well as the identification of several QTL and candidate genes involved in muscle metabolism, remodeling, or causal in human myopathies (Pampouille et al., 2018).

The WB defect corresponds to a hard and out-bulging muscle condition that principally affects the breast PM muscle. Even though there is no evidence of a common etiology, the WB condition is often associated with the WS one. Although the prevalence of the WB defect has never been precisely described, it seems to be much less than that of WS. WB mainly concerns the production of heavy broilers primarily used for processing. A Finnish team (Sihvo et al., 2013) first described this condition. Macroscopically, the PM muscle appears hard, curved, viscous, and pale. Histologically, WB muscles are characterized by several cellular abnormalities, such as fiber splitting and necrosis, which are associated with excessive adipose tissue deposition, accumulation of interstitial connective tissue and collagen, and inflammatory cells infiltrates (Sihvo et al., 2013; Velleman and Clark, 2015; de Brot et al., 2016; Soglia et al., 2016). There is also strong evidence of muscle cell regeneration associated with increased expression of several myogenic regulatory factors (Clark and Velleman, 2016). High-throughput metabolomic or transcriptomic approaches have also revealed a great number of dysregulated molecular pathways involved in the response to oxidative stress, altered glucose utilization and lipid metabolism, muscle degeneration and repair, inflammatory responses, and extracellular matrix (ECM) remodeling (Abasht et al., 2016, 2019; Kuttappan et al., 2017; Papah et al., 2018; Pampouille et al., 2019). In addition, the WB condition was recently associated with muscle weakness (Norring et al., 2018).

The occurrence of these breast muscle myopathies clearly represents a limit to the sustainability of chicken meat production because of its important economic consequences and unfavorable impact on consumer acceptability. Since the emergence of these defects, numerous research studies have been carried out in order to understand their etiology, but also to propose nutritional and breeding strategies to limit their incidence. There is a consensus today, which links the appearance of these defects to the efficiency and rapid growth of animals and the increase in their breast meat yield (Kuttappan et al., 2012a, 2013b; Kawasaki et al., 2018), and so far, no genetic or rearing solution has made it possible to reduce drastically the occurrence of these defects without affecting growth performance of animals. Another limitation to the search for solutions to reduce the incidence of muscle myopathies in chickens is the lack of quantitative methods to assess the presence and severity of defects. Indeed, most studies are based on visual or palpation estimation of meat defects, even though recent studies have tested the use of spectral methods to detect defects at slaughterhouse (Traffano-Schiffo et al., 2017; Wold et al., 2017). The present study aims to propose histological and molecular tools allowing for precise quantification of the different lesions present in muscles affected by WS or WB. This will ultimately allow progress in understanding the etiology of these defects but also by refining the diagnosis of injuries accelerate the 
development of non-invasive prediction tools at the service of breeders and producers.

\section{MATERIALS AND METHODS}

\section{Animals and Muscles}

The muscles used in the present study are issued of a trial already described in Pampouille et al. (2019). Briefly, PM muscles were sampled on 42-day-old chickens originating from two chicken lines: an experimental slow-growing (SG) line showing no lesions related to WS and WB defects $(n=8)$ and a commercial fast-growing (FG) one, in which both WS and WB defects were observed at different degrees $(n=32)$. Within the fast-growing line, WS and WB defects were qualified using a three-point scale for both: 0 in case of absence of defect, 1 when the defect was moderate, and 2 when it was severe. Then, four groups of eight animals each were constituted corresponding to the FG-C muscles that exhibited no defect (WS0 and WB0), the FG-WS muscles that were only affected by a severe WS condition (WS2 and WB0), the FG-WB muscles only affected by WB (balanced between WB1 and WB2 and WS0), and the FG-WSWB muscles severely affected by both defects (WS2 and WB2). Two samples by animal were collected $15 \mathrm{~min}$ after slaughter, parallel to the axis of the muscle fibers on the antero-superior part of the PM muscle. The sample dedicated to histology was snap-frozen in isopentane cooled in liquid nitrogen; the other, dedicated to molecular biology, was directly frozen in liquid nitrogen. All muscle samples were stored at $-80^{\circ} \mathrm{C}$ before use.

\section{Histology and Imaging Procedures}

10- $\mu \mathrm{m}$-thick cross-sections were performed using a Leica CM3050 S cryostat (Leica, Nanterre, France) and deposited on Superfrost plus slides (Thermo Scientific, Montigny-le-bretonneux, France). All 40 muscles were analyzed on a total of eight slides, each of them containing one muscle of each group, representing five muscles by slide that were randomly dropped based on chicken identification number. All cross-sections were stored at $-80^{\circ} \mathrm{C}$ before staining or labeling.

Several stainings were performed, such as hematoxylin and eosin (HE), Gomori trichrome modified by Engel and Cunningham (TG), and reduced nicotinamide adenine dinucleotide-tetrazolium reductase (NADH-TR), following classical methods (Dubowitz et al., 2013) in order to assess the morphological and histochemical properties of the muscle cross-sections.

Regarding immunolabeling, five primary antibodies were obtained from the Developmental Studies Hybridoma Bank, created by the NICHD of the NIH and maintained at The University of Iowa, Department of Biology, Iowa City, IA. They were developed by Douglas M. Fambrough, for the anti-fibronectin (FN1, B3/D6) and the anti-Neural Cell Adhesion Molecule (NCAM, 5e) antibodies; by Everett Bandman, for the anti-ventricular myosin heavy chain 15 (MYH15, HV11) antibody; and by Frank E. Stockdale, for the slow developmental myosin heavy chain 6 (MYH6, S46) and the slow myosin heavy chain 7B (MYH7B, S58) antibodies. Secondary antibodies obtained from Southern Biotech (Birmingham, AL, United States) were Goat anti-mouse IgG H + L biotinylated, Goat anti-mouse IgG1 biotinylated, Goat anti-mouse IgA biotinylated, or Goat anti-mouse IgG1 conjugated with Texas Red. Streptavidin-Cy2 conjugated was obtained from Southern Biotech and streptavidin-Alexa Fluor ${ }^{\circledR}$ 680 (A680), conjugated from Life Technologies (Thermo Scientific, Montigny-le-Bretonneux, France). All primary and secondary antibodies were diluted in goat serum diluted in PBS (Sigma, Saint-Quentin-Fallavier, France) at 1\% v/v. Streptavidin were diluted in PBS. All washing steps $(3 \times 5 \mathrm{~min})$ were performed in PBS.

Muscle cross-sections were first blocked for $30 \mathrm{~min}$ in goat serum (Sigma, Saint-Quentin-Fallavier, France) diluted in PBS at $10 \% \mathrm{v} / \mathrm{v}$. Then, primary antibodies were incubated $2 \mathrm{~h}$ at 1/30 for anti-MYH15, anti-MYH6, and anti-MYH7B; and 1/100 for anti-NCAM and anti-FN1. After washing, goat anti-mouse secondary antibodies were incubated for $1 \mathrm{~h}$ : IgG1, biotinylated 1/500 for NCAM and MYH15; IgG H + L, biotinylated 1/1,000 for FN1; IgA, biotinylated 1/500 for anti-MYH7B; and IgG1 Texas Red, conjugated for anti-MYH6. When using biotinylated secondary antibodies, sections were then incubated after washing with streptavidin conjugated to either Cy2 or A680.

To quantify adiposis, muscle sections were incubated with Bodipy 493 (4,4'-Difluoro-1,3,5,7,8-Pentamethyl-4-Bora3a, 4a-Diaza-s-Indacene, 1/3,000, Sigma, Saint-Quentin-Fallavier, France) that is a fluorescent probe staining neutral lipids.

Slides were dehydrated and mounted with Canada balsam (HE and TG) or Moviol (Sigma, Saint-Quentin-Fallavier, France) (NADH-TR and immunohistological labelings), then stored at $4^{\circ} \mathrm{C}$ protected from light before imaging.

For Bodipy 493 imaging, six images selected randomly for each sample (at $10 \times$ magnification) were acquired using a Leica MC170 color camera on a Leica DMRB epifluorescence microscope (Leica Microsystems SAS, Nanterre, France). The mean area occupied by the fluorescence signal (Bodipy 493 and Cy2) as the percentage on the total area observed was then calculated using Image J 1.44p software (NIH, United States). For all other labeling procedures using A680, slides were scanned using an infrared Odyssey CLX imager (LICOR Biosciences, Bad Homburg, Germany), and the fluorescence intensity was measured on a region of interest covering at least $90 \%$ of the area of each muscle sections.

\section{Cytochrome Oxidase Activity (COX) and Reactive Oxygen Species (ROS) Assays}

Both assays were performed from a single sample of $200 \mathrm{mg}$ of $\mathrm{PM}$ muscle that was crushed in phosphate buffer $50 \mathrm{mM}$ $\left(\mathrm{KH}_{2} \mathrm{PO}_{4} 50 \mathrm{mM}, \mathrm{K}_{2} \mathrm{HPO}_{4} 50 \mathrm{mM}\right.$, pH 7.0) with an Ultra-Turrax (IKA, Staufen, Germany) and centrifuged $30 \mathrm{~min}$ at $10^{\circ} \mathrm{C}$ to collect supernatant that was further used for the two assays. For cytochrome oxidase activity (COX), enzymatic reaction was done from $100 \mu \mathrm{g}$ of proteins in phosphate buffer $\left(\mathrm{KH}_{2} \mathrm{PO}_{4} 50 \mathrm{mM}\right.$ and $\mathrm{K}_{2} \mathrm{HPO}_{4} 50 \mathrm{mM}, \mathrm{pH}$ 7.0) in the presence of cytochrome $0.1 \mathrm{mM}$, n-dodecylmaltoside $0.15 \%$, and DTT $5 \mathrm{mM}$ (Sigma, Saint-Quentin-Fallavier, France). The reduction of cytochrome c 
was measured at $550 \mathrm{~nm}$ using a Tecan M200 microplate reader (Tecan, Lyon, France). For reactive oxygen species (ROS) assay, $100 \mu \mathrm{g}$ of proteins was diluted in a reaction buffer $(250 \mathrm{mM}$ sucrose, $50 \mathrm{mM} \mathrm{KCl,} 25 \mathrm{mM}$ Tris-base, $10 \mathrm{mM} \mathrm{K}_{2} \mathrm{HPO}_{4}$, $\mathrm{pH} 7.4$ ). The oxidation of the 2,7-dichlorodihydrofluorescein diacetate $5 \mathrm{mM}$ by oxygen reactive species was measured at $528 \mathrm{~nm}$ using a Spectramax Gemini EM microplate reader (Molecular Devices, Sunnyvale, CA, United States).

\section{RNA Extraction and RT-qPCR Analysis}

Total RNA was extracted from $100 \mathrm{mg}$ of PM muscle with a commercial kit (RNA Now, Ozyme, Saint-Cyr-l'École, France) as described previously (Pampouille et al., 2019). Reverse transcription was done with 10 micrograms of RNA using SuperScript II reverse transcriptase (Invitrogen, Montigny-le-Bretonneux, France), random primers (Promega, Charbonnières-les-Bains, France), and RNAse Out (Invitrogen, Montigny-le-Bretonneux, France) according to manufacturer recommendations. Gene expression quantification was done by qPCR using a Light Cycler 480 (Roche, Meylan, France). Primers were purchased from Eurogentec (Le Tremblay, France) and their sequence are presented in Table 1. PCR conditions were as follow: 5 min of pre-incubation at $95^{\circ} \mathrm{C}$, then 45 amplification cycles of $10 \mathrm{~s}$ at $95^{\circ} \mathrm{C}, 20 \mathrm{~s}$ at $60^{\circ} \mathrm{C}$, and $10 \mathrm{~s}$ at $72^{\circ} \mathrm{C}$. $18 S$ ribosomal $R N A$ was used as housekeeping gene, and its expression was invariant between groups. The calculation of absolute mRNA levels was based on the PCR efficiency (E) and the threshold cycle (CT) deviation of an unknown cDNA versus the control cDNA according to the equation proposed by Pfaffl (2001): absolute mRNA level of a target gene $=\left(E_{\text {target }}\right)^{\Delta}$ CTtarget(control-sample). To account for variations due to mRNA extraction and reverse-transcription reaction, absolute mRNA levels of targeted genes were corrected for $18 \mathrm{~S}$ rRNA levels to give a relative mRNA level.

\section{Statistical Analysis}

Data are presented as mean \pm SEM and analyzed using Statview 5.0 Software (SAS Institute, Inc.). One-way ANOVA was performed in case of normally distributed and homoscedastic variance. Statistical significance $(P \leq 0.05)$ was determined using the post hoc Fisher test. Data that did not meet assumptions of normality and homoscedasticity were analyzed and statistical significance $(P \leq 0.05)$ was determined using Kruskal-Wallis and Mann-Whitney non-parametric tests, respectively. Pearson's

TABLE 1 | Accession numbers and sequences of the forward and reverse primers used to quantify gene ${ }^{1}$ expression by RT-qPCR.

\begin{tabular}{|c|c|c|c|}
\hline Gene & Accession number & Forward sequence & Reverse sequence \\
\hline $18 S$ & XR_003078044.1 & TCCAGCTAGGAATAATGGAATAGGA & CCGGCCGTCCCTCTTAAT \\
\hline$A D I P O Q$ & NM_206991.1 & GCCAGGTCTACAAGGTGTCA & CCATGTGTCCTGGAAATCCT \\
\hline ANKRD1 & NM_204405.1 & GCACAATCATCTGGACACTGG & CACTGAACTGCTGCTCGGTTA \\
\hline ANKRD46 & XM_423967.6 & GCTAGAAGTACTTACAGCCCTCT & AACCTCTGGCTTAAGAAATGAGA \\
\hline ANTXR1 & XM_425758.6 & TTGGCGGCATCAAGAGAATG & GTACCACTTCCGAGGAGAGG \\
\hline CD36 & NM_001030731 & CTGTाTCTCПTGTGGCCПTG & CGTGAGAGAAGCTGTATGGAGG \\
\hline CTGF & NM_204274.1 & TGAGTGGAGTGCTTGCTCCAAGA & GCAGCCAGACAGCTCAAACTTGAT \\
\hline$D A G 1$ & NM_001097540.1 & TCCGAAGCTGGGAAGGAGTCTITG & CGTGGTCACCGAGATGTAGTGGA \\
\hline DYSF & XM_025149877.1 & GGTCGGGATGAGCCAAACAT & GTTCGGGAAGGCGTAGATGA \\
\hline ENPP2 & NM_001198662.1 & ACTGAACGAAATGGAGTCAATGT & TGGTCCATCACACTTGTCGG \\
\hline FABP4 & NM_204290.1 & CAAGCTGGGTGAAGAGTITGATGAG & AGCAGGTTCCCATCCACCACTाT \\
\hline FASN & NM_205155.2 & TCTCGATCTGGCATACGAACTG & CAACTGGTCCGAGCTTCAAAG \\
\hline FBN1 & XM_015291934.1 & AATGCTTCCCTGGCCTTGCAGTA & TGTGCTGGACAGGGCTGACAA \\
\hline MTX3 & NM_001079483.1 & GCATTTGAAACAGCTTACCAACC & GCGTTGTTATCTTCCGAGGG \\
\hline MYH13 & XM_025141815.1 & GGAATGACAACTCCTCACGCT & GCTAGCTGGAAAGTCACTCTGG \\
\hline MYF5 & NM_001030363.1 & ACTCCCCAAAGTGGAGATCCTG & CAGTCCGCCATCACATCG \\
\hline MYOD1 & NM_002478.5 & GGAATCACCAAATGACCCAAAG & TCACTCAGGTITCCTCCTTCCT \\
\hline MYOG & NM_204184.1 & CGGAGGCTGAAGAAGGTGAAC & AGGCGCTCGATGTACTGGAT \\
\hline PCNA & NM_204170.1 & CATGGGCGTCAACCTAAAC & TCCACATCGAGGTCCATAAG \\
\hline PDEЗB & NM_001031182.1 & CACCATCTCAGCGAAAATCACA & TCTCCTAGCTGCCGCTCATCTT \\
\hline PDGFRA & NM_204749.2 & CTTGGAGCTGTTACTCGGGAACTGA & ACCTTGGCTTCTGTTCCAGATGA \\
\hline PITX2 & NM_205010.1 & GTCTGGACCAACCTCACCG & TGCCCAGTTGTTGTACGAGT \\
\hline PLIN2 & NM_001031420.1 & GCCTACATCACCACGAAGGATAACC & CAACCTTGTCAGTGGGTTGATTCAG \\
\hline PNPLA7 & NM_001130742.1 & ПTCACCATCAAGGCCAATCG & GTCCAGCCTCAACTTCCATCCA \\
\hline PPARG & NM_001001460.1 & CACTGCAGGAACAGAACAAAGAA & TCCACAGAGCGAAACTGACATC \\
\hline$S G C B$ & NM_001031155.1 & TGTAGAGCGCAGGAACGTCAAT & AATCACCGCCCAGATAACGAG \\
\hline TGFB1 & NM_001318456.1 & ACCCGATGAGTATTGGGCCAAAGA & GCGGGACACGTTGAACACGAA \\
\hline TUBB4B & NM_001080860.2 & CAGACCGGATTATGAACACCTT & CACCGTATGTTGGCGTAGTT \\
\hline TWIST1 & NM_204739.1 & CGCAGTCCCTGAACGAAGCПT & CACACCGAGAAGGCGTAGCTGA \\
\hline
\end{tabular}

${ }^{1}$ Primers used to measure the expression of genes not listed in this table are described in Pampouille et al. (2019). 
correlation coefficients were calculated between the signal quantification obtained by microscopy and scanner technology and between values of mRNA expression of each gene studied.

\section{RESULTS}

\section{Histological Description}

Muscle characterization was first performed on $\mathrm{HE}-$ and TG-stained cross-sections in order to have an overview of muscle structure and the kind of damages according to the group. SG muscles are made of polygonal fibers containing both internalized and peripheral nuclei. Muscle fibers are surrounded by normal thick endomysium and perimysium without any detectable damage or additional deposit of intermuscular adipose tissue (adiposis) (Figure 1A). By comparison, FG-C muscles contain fibers of more variable sizes and exhibit a slightly extended endomysium and perimysium, first signs of adiposis, some necrotic and regenerating fibers, and rare hypercontracted fibers and vacuoles within muscle fibers (Figure 1B). The presence of internalized nuclei was observed in almost all fibers of FG muscles, whether WS or WB defects were present or not. Muscles only affected by WS (FG-WS) exhibit round fibers of variable size and greater extension of endomysium and perimysium compared to FG-C. These muscles show adiposis essentially in the perimysium, some necrotic and regenerated fibers, and some hypercontracted fibers and rare inflammatory cells foci (Figure 1C). In muscles only affected by $\mathrm{WB}$ (FG-WB), the number of round fibers increases, the extension of endomysium and perimysium is more pronounced, while adiposis decreases compared to FG-WS muscles. Events of fiber necrosis, regeneration, hypercontracted fibers, and vacuoles also increase (Figure 1D). Finally, muscles affected by both defects (FG-WSWB) look like FG-WB muscles, with an increased adiposis (Figure 1E). In muscles affected by WB (FG-WB and FG-WSWB) but not in those affected by WS, some fibers looked segmented.

To explore the metabolic status of muscle fibers, an NADH-TR staining was performed. All SG muscle fibers were weakly stained (Figure 1F). Moreover, they did not present any immunoreactivity, with MYH6 and MYH7B staining, respectively, a slow developmental and a slow myosin heavy chain (data not shown). Some fibers showed a higher staining on NADH-TR in FG-C (Figure 1G) and in FG-WS (Figure 1H) muscles that was usually associated with an immunoreactivity with MYH6, but not with MYH7B (data not shown). The proportion of high NADH-TR stained fibers increased in FG-WB (Figure 1I) and in FG-WSWB (Figure 1J) muscles. This was associated with an increase in MYH6 but not MYH7B positive fibers (data not shown).

To improve description and quantify the different features of WS and WB conditions, we applied several stainings using either specific antibodies against fibronectin, MYH15 and NCAM, or the fluorescent primer BODIPY493 (Figures 1, 2). In SG muscles, adiposis was absent or very rare (Figure 1K), fibronectin was only present around capillaries and in perimysium (Figure 2A), and NCAM was expressed in mononuclear and endothelial cells (Figure 2F). SG muscles did not express MYH15 (Figure 2K). Comparatively, fibronectin was also detected in the endomysium (Figure 2B), NCAM in the cytoplasm of few small fibers (Figure 2G), and MYH15 was detected in few small fibers and mononuclear cells (Figure 2L) in FG-C muscles. Within affected muscles, although small adiposis areas were present in FG-WB (Figure 1N), their size and number greatly increased in

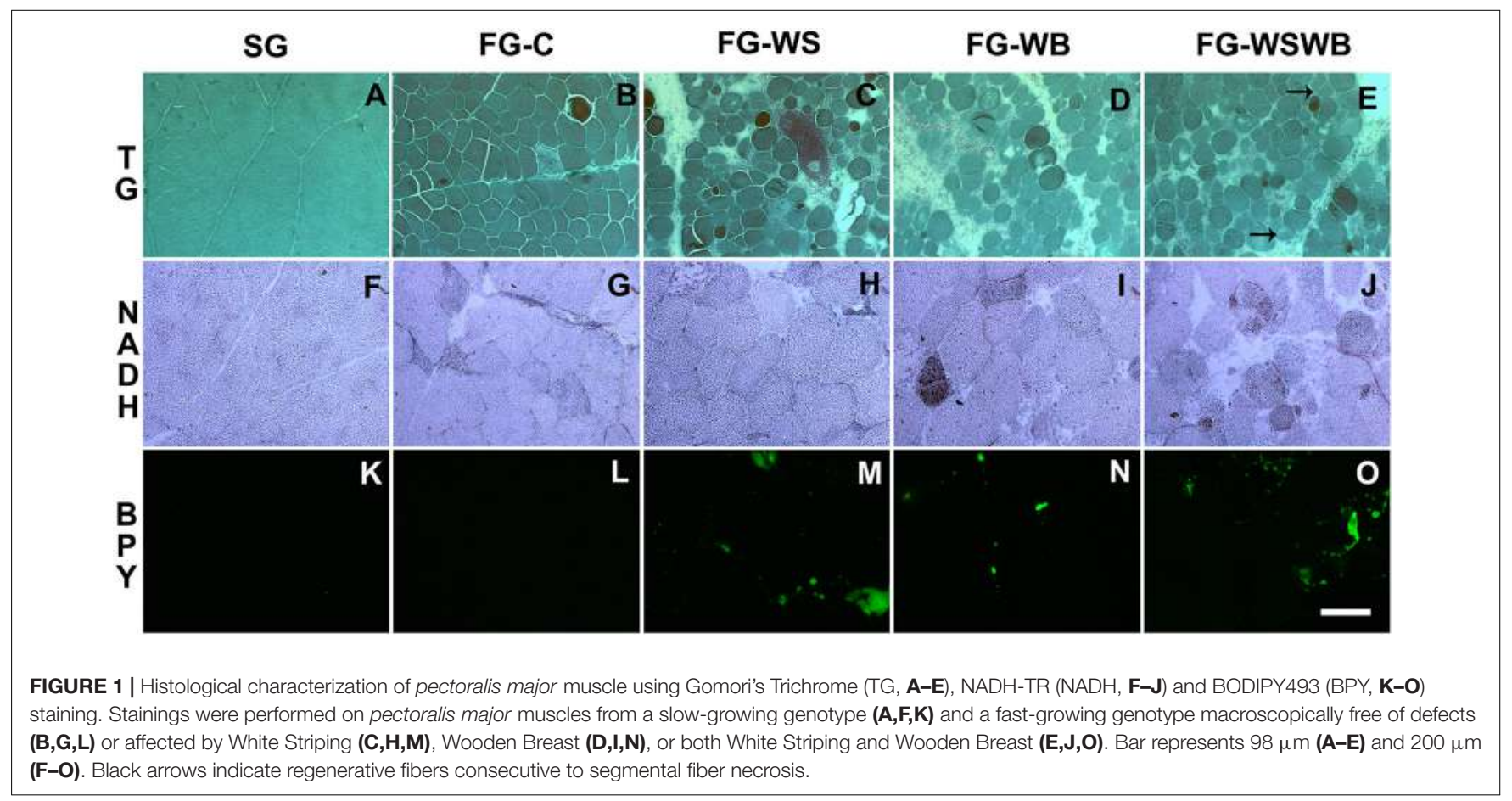




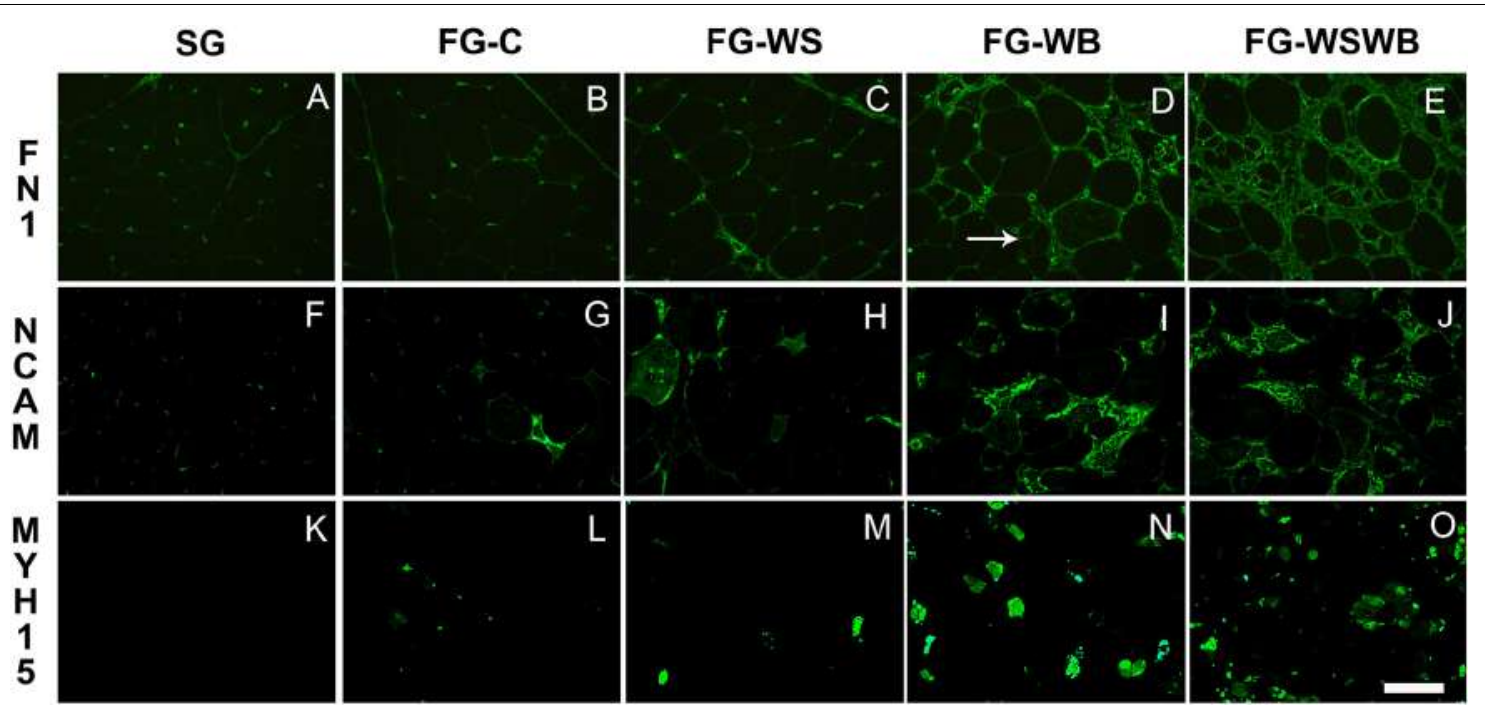

FIGURE 2 | Immunoreactivity for fibronectin (FN1, A-E), NCAM (F-J) and MYH15 (K-O). It was assessed on pectoralis major muscles from a slow-growing

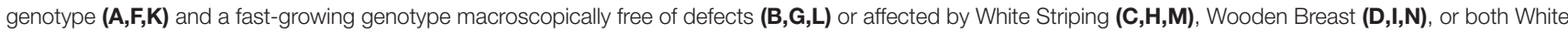
Striping and Wooden Breast (E,J,O). Bar represents $200 \mu \mathrm{m}(\mathbf{A}-\mathbf{J})$ and $98 \mu \mathrm{m}(\mathbf{K}-\mathbf{O})$. White arrow indicates regenerative fiber consecutive to segmental fiber necrosis.

FG-WS (Figure 1M) and in FG-WSWB (Figure 10). Fibronectin labeling extended in both the endomysium and perimysium in FG-WS (Figure 2C), but especially in FG-WB (Figure 2D) and FG-WSWB (Figure 2E). It was also present around small fibers formed after segmental necrosis in FG-WB muscles (Figure 2D). Finally, the number of fibers expressing NCAM (Figure 2I) and MYH15 (Figure 2N) strongly increased in FG-WB muscles, and even more in FG-WSWB, in which the size of the MYH15 marked fibers was also larger (Figures 2J,O).

\section{Quantification of Histological Phenotypes}

The quantification of Bodipy 493 and FN1 labeling was performed on images classically obtained by microscopy and analyzed with the ImageJ software to measure percentage of fluorescent area. The area percentage labeled by Bodipy493 was largely higher in FG compared to SG muscles (Figure 3A). It was about 10 times higher in FG-C and around 30 times higher in muscles affected by WS (FG-WS and FG-WSWB), FG-WB muscles being intermediate between muscles affected by WS and control ones. The percentage area occupied by FN1 was lower in SG compared to FG muscles (Figure 3B). Among FG muscles, the highest value was observed in muscles affected by both WS and WB defects (FG-WSWB), FG-C and FG-WS muscles exhibiting the lowest values, and FG-WB being intermediate.

To quantify NCAM and MYH15 signals, we developed a tissue section imaging protocol based on immunohistochemistry (IHC) fluorescent signal measurement using an Odyssey CLX imager. The interest in developing such a method is time saving for image acquisition and the possibility of analyzing the whole muscle cross-section (Figure 4A). Unfortunately, the implementation of this technique has not yet been possible for the labeling of adipose tissue by the Bodipy 493, as this probe is not adapted to the acquisition wavelength of our scanner. Before deploying this method, we needed to validate that the quantification of the fluorescent signal obtained using the scanner correlated with area calculation performed on images obtained under the microscope. Thus, the fluorescent signals of 10 samples from the different groups (corresponding to two slides) were analyzed using both a classical epifluorescent microscope and scanner technology. Pearson correlations between the signal quantification obtained by the two methodologies were very high: $0.969,0.873$, and $0.977(P \leq 0.001)$ for NCAM, fibronectin, and MYH15 signals, respectively.

The fluorescent signal for the three markers studied obtained using the scanner was very low for SG muscles (Figure 4A), as also observed with the epifluorescence microscope (see Figure 1 for fibronectin). The gross observation of images acquired using the scanner technology revealed that whatever the labeling the intensity of fluorescence increased with the increase of damages observed, especially in FG-WB and FG-WSWB (Figure 4A). Fibronectin labeling allowed observing distinctly perimysium in FG-C and FG-WS, but not in SG muscles, where it was poorly developed, and in FG-WB and FG-WSWB muscles, where endomysium was the most extended, compared to other groups. Likely because of their localization in mononuclear and muscle fiber cells, increased fluorescent signal due to alterations revealed by MYH15 and NCAM labeling in muscles affected by WB was more evident than in fibronectin. FN1 signal quantification showed a similar pattern to that obtained by microscopy, except for an absence of significant difference between SG and FG-C and FG-WS muscles (Figure 4B). Like for fibronectin, NCAM and MYH15 signals progressively increased with the intensity of defect, SG and/or FG-C muscles showing the lowest values and FG-WSWB the highest (Figure 4C). For all markers, FG-WS and 

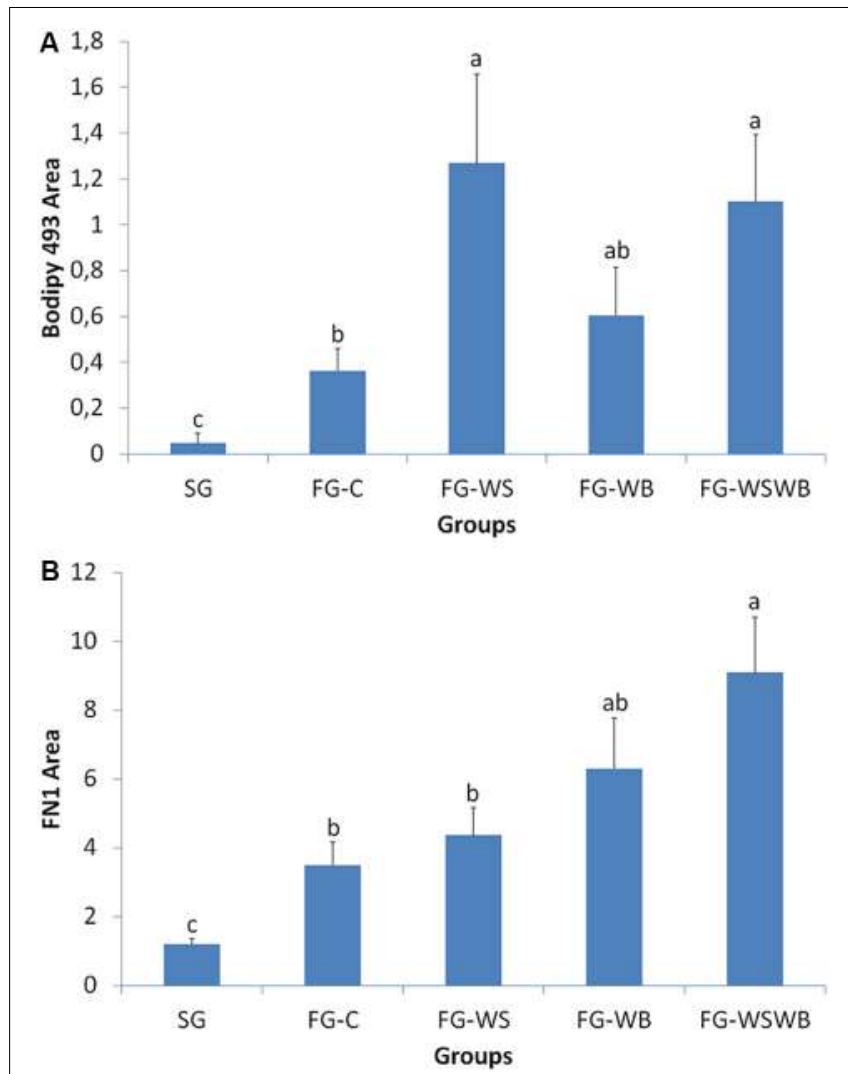

FIGURE 3 | Quantification of the percentage area labeled by Bodipy493 (A) and fibronectin (FN1, B) on pectoralis major muscle cross-sections using classical microscopy. Labeled areas were assessed in a slow-growing genotype (SG) and a fast-growing genotype macroscopically free of defects (FG-C) or affected by White Striping (FG-WS), Wooden Breast (FG-WB), or both White Striping and Wooden Breast (FG-WSWB). Bodipy-493-labeled area increased mostly in muscles affected by White Striping (FG-WS and FG-WSWB). FN1-labeled area increased linearly with the occurrence and severity of both White Striping and Wooden Breast defects. For each labeling, different letters indicate significant differences between muscle groups $(P \leq 0.05)$.

FG-WB muscles exhibited intermediate values (Figures 4B,C). The Pearson correlation coefficient between MYH15 and NCAM signal was very high: $0.915(P \leq 0.001)$.

\section{COX Activity and ROS Assay}

We assessed mitochondrial activity of muscles by measuring the COX enzymatic activity. COX activity was significantly higher in SG than in FG muscles, except for the FG-WSWB group, whose variability of activity between individuals was more important than for other groups (Figure 5A). By contrast, the production of ROS was lower in SG than in FG muscles, in which the FG-C and FG-WS muscles exhibited the highest values and the FG-WSWB muscles the lowest one (Figure 5B).

\section{Gene Expression}

A set of 39 genes were selected for expression analysis, based on several criteria related to their expressional pattern already

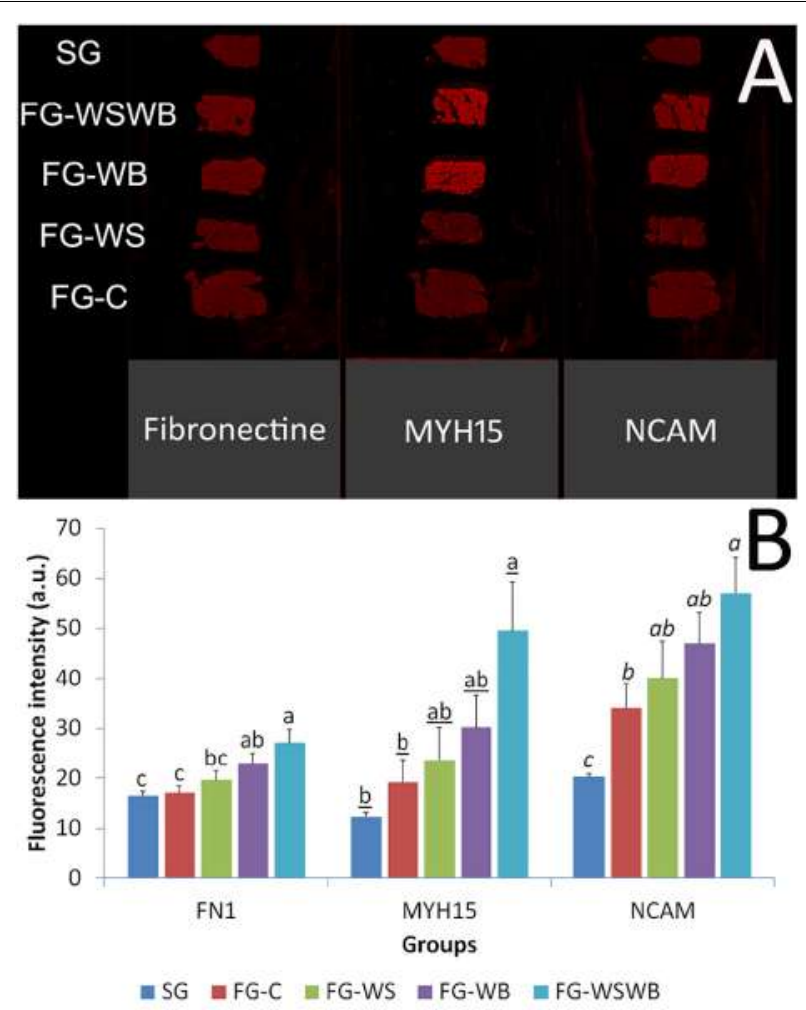

FIGURE 4 | Quantification of the fluorescence intensity following fibronectin, myosin heavy chain 15 (MYH15), and Neural Cell Adhesion Molecule (NCAM) immunostaining using an infrared scanner Odyssey CLX. Labeling were performed on pectoralis major muscles from a slow-growing genotype (SG) and a fast-growing genotype macroscopically free of defects (FG-C) or affected by White Striping (FG-WS), Wooden Breast (FG-WB), or both White Striping and Wooden Breast (FG-WSWB) (A). The fluorescence intensity increased linearly with the occurrence and severity of both White Striping and Wooden Breast defects for Fibronectin, MYH15, and NCAM (B). For each marker, different letters indicate significant differences between muscle groups $(P \leq 0.05)$

described in SG, FG-C, FG-WSWB (Pampouille et al., 2019), their belonging to QTL regions controlling WS or breast muscle yield in chicken (Pampouille et al., 2018), and/or their role in mammalian myopathies or in regeneration or myogenesis processes. Their levels of expression relative to $18 \mathrm{~S}$ ribosomal RNA are presented in Table 2 and in Figures 6-9. Pearson's correlations between the relative mRNA levels of genes differentially expressed in PM muscle are presented in Supplementary File 1. Of the 39 studied genes, 11 were not differential between groups: DAG1, SGCB, DYSF, ADIPOQ, FBN1, MYOD1, MYF5, PITX2, ANKRD1, LRSAM, and PNPLA7 (Table 2). The 28 differential genes could be grouped into six clusters with specific expression profiles. A first cluster (cluster 1) regroups seven genes whose expression is higher in $S G$ than FG muscles. It includes MYH13, MYH1E, MYH1F, ANKRD46, $M Y O C D$ (Table 2), $C A P N 3$, and $P P P 1 R 3 A$ (Figure 6A), despite $M Y H 1 F$ being only numerically upregulated $(P=0.09)$ in FG-C compared to SG. In addition to being more expressed in SG muscles, some of them were also less expressed in muscles 


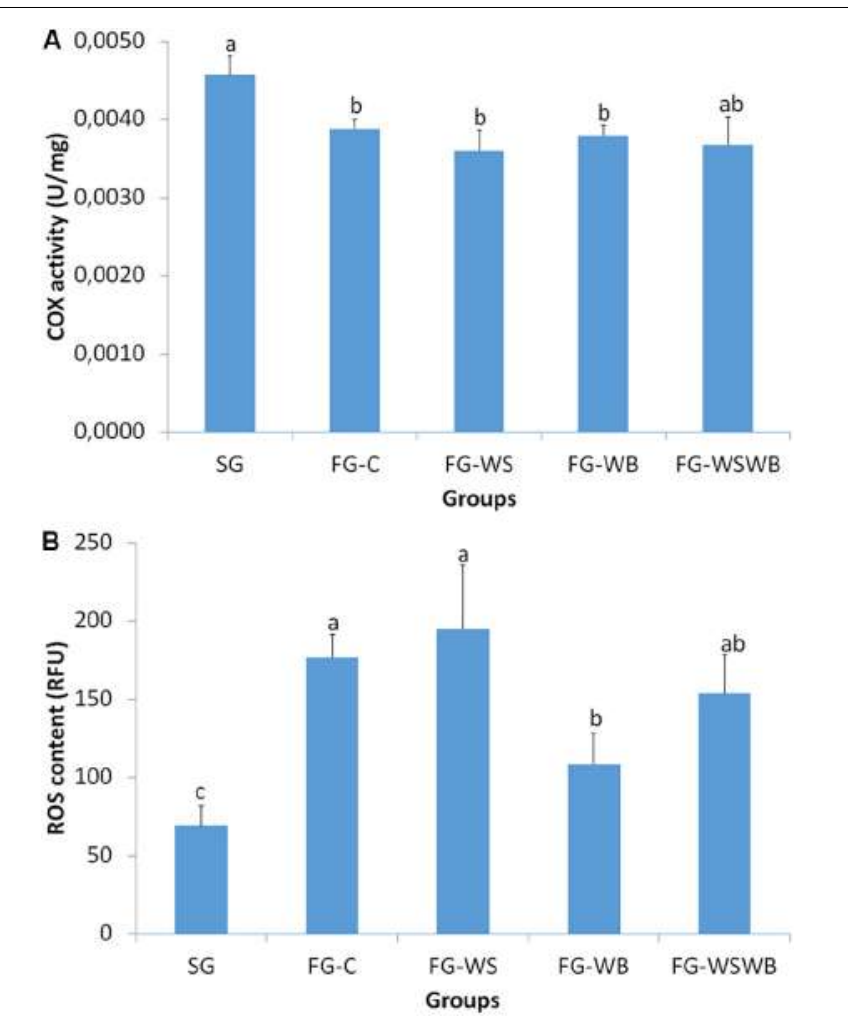

FIGURE 5 | Quantification of the cytochrome oxidase (COX) activity and the production of reactive oxygen species (ROS). These parameters were assessed in pectoralis major muscles from a slow-growing genotype (SG) and a fast-growing genotype macroscopically free of defects (FG-C), or affected by White Striping (FG-WS), Wooden Breast (FG-WB), or both White Striping and Wooden Breast (FG-WSWB). Fast-growing muscles exhibited lower COX activity than slow-growing muscles, independently of the defect occurrence and severity (A). ROS content was higher in fast- than in slow-growing muscles, especially those affected by White Striping (B). For each parameter, different letters indicate significant differences between groups $(P \leq 0.05)$.

affected by WB than in fast-growing control ones (MYH13, MYH1E, ANKRD46, MYOCD; Table 2). Among genes belonging to cluster 1, the expressions of MYOCD, CAPN3, PPP1R3A, and $M Y H 1 E$ were highly positively correlated (Supplementary File 1). The second cluster (cluster 2) regroups genes whose expression was higher in FG than in SG muscles, independently of the presence or severity of the defects. This is the case of PLIN2 and CAV3 (Figure 6B), MYH1B, and TUBB4B (Table 2). A third cluster (cluster 3) brings together two genes, FABP4 and $C D 36$, whose expression was particularly upregulated in muscles affected by WS, even if it is not necessarily different from other FG groups (Figure 7). The Pearson correlation coefficient between the expression of FABP4 and CD36 was $0.81(P \leq 0.001)$ suggesting common regulation (Supplementary File 1). The fourth cluster (cluster 4) groups genes whose expression is only significantly increased in the presence of $\mathrm{WB}$ and even more when WB is associated with WS (compared to FG-C). This is the case of MYH15 and TGFB1 (Figure 8A), TWIST1 and MYOG (Figure 8B), and CTGF and FN1 (Table 2). The fifth cluster (cluster 5) regroups genes whose expression is systematically higher in muscles affected by both WS and WB (FG-WSWB) compared to other groups, although differences between other groups sometimes exists. This is the case for PDGFRA, ENPP2 (Figure 9A), COL6A3, FASN, PPARG, and ANTXR1 (Table 2). Among genes of clusters 4 and 5, MYH15 appeared to be very positively correlated $(\mathrm{R}>0.7)$ with several genes involved in the processes of fibrosis (CTGF, FN1, COL6A3) and adiposis (PDGFRA, PPARG, FASN) (Supplementary File 1). Finally, a sixth cluster (cluster 6) brings together genes like $M T X 3, P D E 3 B$ (Figure 9B), and PCNA (Table 2) that, despite an expression higher in SG compared to control FG muscles, were also highly expressed in muscles severely affected by both WS and WB (Figure 9). Ratio of expression TGFB1/PPARG was calculated to evaluate its potential role in the development of fibrotic or adipogenic phenotypes. It was the lowest in SG and the highest in FG-WB muscles, other groups showing intermediate values (Figure 10).

\section{DISCUSSION}

White Striping (WS) and Wooden Breast (WB) have been widely characterized, especially at the histological level. One of the objectives of the present study was to propose additional innovative histological methodologies to better quantify the different injuries usually associated with these two defects. For that purpose, we used a set of 40 muscles, which were first characterized both macroscopically and microscopically to precisely define the severity of each of the two defects. Among them, eight muscles were from a slow-growing line whose average body weight and breast meat yield at 42 days were much lower than that of the fast-growing line to which it was compared (Pampouille et al., 2019). The aim of including slow-growing muscles was to have control muscles with no lesions typical of WS and WB defects, since all the fast-growing muscles, including normal ones, had them. As already observed (Kuttappan et al., 2013c; Sihvo et al., 2013), we confirmed that muscles affected by WS exhibit adiposis, i.e., an intermuscular adipose tissue deposition, fibrosis, fiber necrosis and regeneration, and some inflammatory infiltrates, and that increased occurrence of fiber necrosis, fibrosis and adipose tissue infiltration are well correlated with the severity of WS (Russo et al., 2015). Our results also supported that damages like fibrosis, hyaline muscle degeneration, and inflammatory cell infiltrate worsen in muscles affected by WB compared to WS, but not necessarily adiposis (Velleman and Clark, 2015; de Brot et al., 2016; Soglia et al., 2016). We also observed an increased number of basophilic fibers, which are known as hypercontracted fibers (Dubowitz et al., 2013), and of hyaline vacuoles in fiber sarcoplasm. Finally, our observations revealed an alteration of the fiber regeneration process in $\mathrm{WB}$ muscle as it seemed preferentially performed consecutive to a segmental fiber necrosis (arrows in Figures 1, 2).

\section{Improving WS and WB Diagnosis Using Histological Phenotypes}

Since their appearance less than 10 years ago, most of studies classified WS and WB muscles according to macroscopic visual 
TABLE 2 | Effect of muscle group ${ }^{1}$ on the relative ${ }^{2}$ mRNA expression measured in pectoralis major muscle.

\begin{tabular}{|c|c|c|c|c|c|c|}
\hline Gene (cluster) & SG & FG-C & FG-WS & FG-WB & FG-WSWB & $P$ \\
\hline MYH13 (1) & $2.10 \pm 0.45(\mathrm{a})$ & $0.98 \pm 0.25(\mathrm{~b})$ & $0.54 \pm 0.13(\mathrm{bc})$ & $0.30 \pm 0.07$ (c) & $0.29 \pm 0.05$ (c) & 0.005 \\
\hline MYH1E (1) & $2.49 \pm 0.22(a)$ & $0.66 \pm 0.20(b)$ & $0.29 \pm 0.08(\mathrm{bc})$ & $0.17 \pm 0.05(\mathrm{c})$ & $0.21 \pm 0.04(\mathrm{c})$ & 0.0002 \\
\hline MYH1F (1) & $1.39 \pm 0.24(\mathrm{a})$ & $0.86 \pm 0.15(a b)$ & $0.60 \pm 0.11(b)$ & $0.47 \pm 0.18(b)$ & $0.56 \pm 0.13(b)$ & 0.02 \\
\hline ANKRD46 (1) & $713.6 \pm 222.7(a)$ & $5.72 \pm 2.86(b)$ & $1.86 \pm 0.45(\mathrm{bc})$ & $1.36 \pm 0.61$ (c) & $0.86 \pm 0.16(\mathrm{c})$ & $\leq 0.0001$ \\
\hline MYOCD (1) & $1.87 \pm 0.13(\mathrm{a})$ & $0.77 \pm 0.19(b)$ & $0.53 \pm 0.06(b)$ & $0.34 \pm 0.03$ (c) & $0.55 \pm 0.09(\mathrm{bc})$ & 0.0002 \\
\hline TUBB4B (2) & $0.57 \pm 0.07(b)$ & $0.85 \pm 0.13(\mathrm{a})$ & $0.93 \pm 0.09(\mathrm{a})$ & $1.15 \pm 0.17(\mathrm{a})$ & $1.03 \pm 0.12(\mathrm{a})$ & 0.01 \\
\hline MYH1B (2) & $0.05 \pm 0.01(b)$ & $0.96 \pm 0.28(a b)$ & $0.75 \pm 0.17(\mathrm{a})$ & $0.57 \pm 0.16(\mathrm{a})$ & $1.26 \pm 0.18(\mathrm{a})$ & $\leq 0.0001$ \\
\hline CTGF (4) & $0.11 \pm 0.03(\mathrm{~d})$ & $0.30 \pm 0.05(\mathrm{c})$ & $0.61 \pm 0.16(\mathrm{bc})$ & $0.86 \pm 0.12(a b)$ & $1.46 \pm 0.27(\mathrm{a})$ & $\leq 0.0001$ \\
\hline FN1 (4) & $0.11 \pm 0.02(\mathrm{~d})$ & $0.45 \pm 0.21$ (c) & $0.62 \pm 0.14(\mathrm{bc})$ & $1.18 \pm 0.25(a b)$ & $1.74 \pm 0.24(\mathrm{a})$ & $\leq 0.0001$ \\
\hline FASN (5) & $0.31 \pm 0.03(\mathrm{c})$ & $0.61 \pm 0.12(b)$ & $0.87 \pm 0.14(a b)$ & $0.91 \pm 0.11(a b)$ & $1.41 \pm 0.38(\mathrm{a})$ & 0.0007 \\
\hline ANTXR1 (5) & $1.00 \pm 0.14(b)$ & $0.70 \pm 0.22(b)$ & $0.73 \pm 0.19(\mathrm{~b})$ & $0.85 \pm 0.18(b)$ & $2.04 \pm 0.48(\mathrm{a})$ & 0.04 \\
\hline PPARG (5) & $0.31 \pm 0.03(\mathrm{c})$ & $0.69 \pm 0.23(\mathrm{bc})$ & $0.70 \pm 0.16(b)$ & $0.60 \pm 0.22(\mathrm{bc})$ & $2.04 \pm 0.56(a)$ & 0.002 \\
\hline COL6A3 (5) & $0.39 \pm 0.05$ (c) & $0.71 \pm 0.18(\mathrm{bc})$ & $0.82 \pm 0.12(b)$ & $1.05 \pm 0.13(b)$ & $1.53 \pm 0.15(\mathrm{a})$ & 0.0005 \\
\hline PCNA (6) & $1.19 \pm 0.13(\mathrm{c})$ & $0.78 \pm 0.12(\mathrm{bc})$ & $0.79 \pm 0.12(b)$ & $1.01 \pm 0.13(b)$ & $1.30 \pm 0.20$ (a) & 0.05 \\
\hline MyoD & $0.84 \pm 0.08$ & $0.71 \pm 0.09$ & $0.84 \pm 0.20$ & $0.82 \pm 0.11$ & $0.75 \pm 0.11$ & NS \\
\hline MYF5 & $1.25 \pm 0.19$ & $1.63 \pm 0.34$ & $1.08 \pm 0.15$ & $1.53 \pm 0.41$ & $1.76 \pm 0.38$ & NS \\
\hline PITX2 & $0.67 \pm 0.20$ & $0.82 \pm 0.17$ & $1.19 \pm 0.33$ & $1.65 \pm 0.40$ & $1.06 \pm 0.17$ & NS \\
\hline DAG1 & $1.05 \pm 0.13$ & $0.89 \pm 0.13$ & $0.86 \pm 0.09$ & $0.80 \pm 0.10$ & $1.13 \pm 0.40$ & NS \\
\hline PNPLA7 & $1.14 \pm 0.12$ & $0.81 \pm 0.10$ & $0.74 \pm 0.06$ & $0.85 \pm 0.07$ & $0.91 \pm 0.09$ & NS \\
\hline LRSAM & $0.75 \pm 0.08$ & $0.78 \pm 0.10$ & $0.91 \pm 0.10$ & $0.95 \pm 0.11$ & $1.07 \pm 0.14$ & NS \\
\hline$A D I P O Q$ & $0.84 \pm 0.18$ & $0.68 \pm 0.10$ & $0.77 \pm 0.04$ & $0.62 \pm 0.08$ & $0.95 \pm 0.27$ & NS \\
\hline FBN1 & $1.12 \pm 0.26$ & $0.55 \pm 0.12$ & $0.66 \pm 0.08$ & $0.64 \pm 0.09$ & $1.35 \pm 0.55$ & NS \\
\hline DYSF & $0.99 \pm 0.09$ & $0.86 \pm 0.06$ & $0.82 \pm 0.06$ & $0.76 \pm 0.04$ & $0.88 \pm 0.10$ & NS \\
\hline ANKRD1 & $0.51 \pm 0.11$ & $0.73 \pm 0.18$ & $0.68 \pm 0.20$ & $0.68 \pm 0.17$ & $1.17 \pm 0.23$ & NS \\
\hline
\end{tabular}

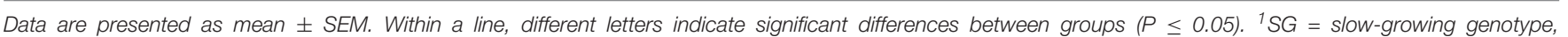

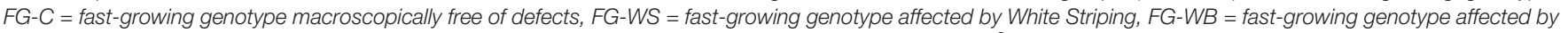

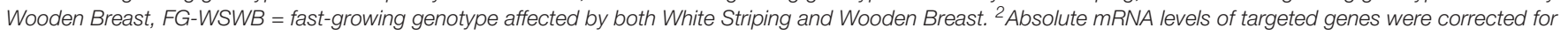
18 S ribosomal RNA levels to give a relative $M R N A$ level.

and/or palpation scores, which are subjective criteria, likely dependent of the person performing them. The limits of these methods were raised by Trocino et al. (2015), and more recently by Pampouille et al. (2019), who revealed discrepancies between muscle categorization made by visual notation or using histological criteria. This last study was the first one to propose quantitative histological phenotype based on fluorescent labeling of adiposis and fibrosis to refine WS and WB diagnosis (Pampouille et al., 2019). In the present study, we proposed other quantitative histological traits able to assess more features of WS and WB conditions, including connective tissue deposition, fiber necrosis, and regeneration, by using antibodies against fibronectin, NCAM, and MYH15. Fibronectin is a component of the ECM surrounding muscle fibers. MYH15 is specific of a chicken ventricular myosin heavy chain that is known to be expressed in developing and regenerating avian skeletal muscles (Camoretti-Mercado et al., 1993). Both fibronectin and MYH15 were chosen because their gene expression was much higher $(\times 8$ for $F N 1$ and $\times 12$ for $M Y H 15)$ in fast-growing muscles affected by both WS and WB than in normal FG muscles (Pampouille et al., 2019). They are also located in QTL regions controlling WS (Pampouille et al., 2018). The neural cell adhesion molecule (NCAM), also called CD56, is a glycoprotein expressed in skeletal muscle regenerative fibers and is considered a good index of muscle regeneration in dystrophin-deficient MDX mouse (Dubois et al., 1994). The present study revealed that fibronectin is clearly more expressed in the most fibrotic muscles and that the area percentage occupied by fibronectin on muscle cross-sections progressively increased from SG to FG-WSWB muscles (SG $<$ FG-C $<$ FG-WS $<$ FG-WB $<$ FG-WSWB; Figures 1, 3). MYH15 labeling showed that it was expressed in regenerating fibers of various size, the fluorescent signal being, however, more intense in the smallest fibers and in some mononuclear cells, which could be myoblasts entering differentiation (Figure 1). This was consistent with the fact that MYH15 is expressed in developing and regenerating muscles (Camoretti-Mercado et al., 1993). As expected, the surface occupied by NCAM labeling greatly increased in muscle showing signs of muscle regeneration, especially those affected by WB (Figure 1). Differences observed between MYH15 and NCAM were likely due to the higher persistence of NCAM expression in more mature fibers than of MYH15, which is consistent with the greatest size of NCAM expressing fibers on immunohistochemistry.

Because the quantification of the fluorescent signal by conventional microscopy is long and not necessarily compatible with the study of large numbers, we developed a rapid method to quantify fluorescence intensity, based on the use of infrared emitting fluorochrome analyzable by a scanner. This methodology was first validated by checking 


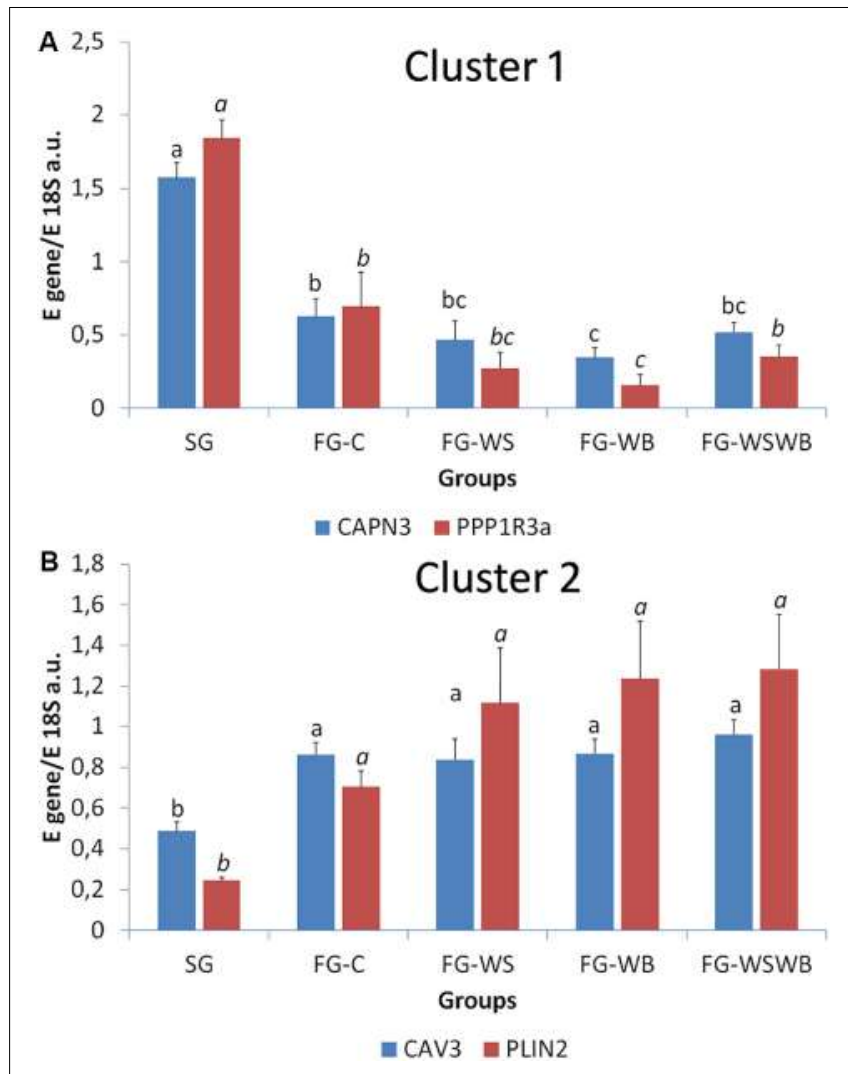

FIGURE 6 | Relative mRNA expression of genes coding for calpain 3 (CAPN3), protein phosphatase 1 regulatory subunit 3A (PPP1R3A), caveolin 3 (CAV3), and perilipin 2 (PLIN2). mRNA expression was assessed in Pectoralis major muscles from a slow-growing genotype (SG) and a fast-growing genotype macroscopically free of defects (FG-C) or affected by White Striping (FG-WS), Wooden Breast (FG-WB), or both White Striping and Wooden Breast (FG-WSWB). Expression of CAPN3 and PPP1R3A was higher in slow-compared to fast-growing muscles (A). CAV3 and PLIN2 expressed more in fast- than in slow-growing muscles (B). Absolute mRNA levels of targeted genes were corrected for $18 S$ ribosomal RNA levels to give a relative mRNA level. For each gene, different letters indicate significant differences between groups $(P \leq 0.05)$.

that the fluorescent intensity measured with the scanner was well correlated to the measurement done on images obtained by microscopy. Correlations were high, comprising between 0.87 and 0.98 according to the marker. Even if this methodology was less sensitive on weak fluorescent signals than microscopy (see Figures 3, 4 for fibronectin), it allowed us to similarly rank FG muscles according to the occurrence of the WS and WB defects, i.e., for the three markers FG-C $<$ FG-WS $<$ FG-WB $<$ FG-WSWB. However, the low sensitivity of the scanner method makes it difficult to compare SG and FG muscles, certainly because of the very different fiber size likely to bias the signal analysis. This was the case for fibronectin, which appeared about three times less expressed in SG than FG-C muscles when analyzed by microscopy (Figure 3), while no significant difference was observed using the scanning technology (Figure 4). This was also observed for MYH15 (Figure 4). Therefore, we conclude that measuring fluorescence

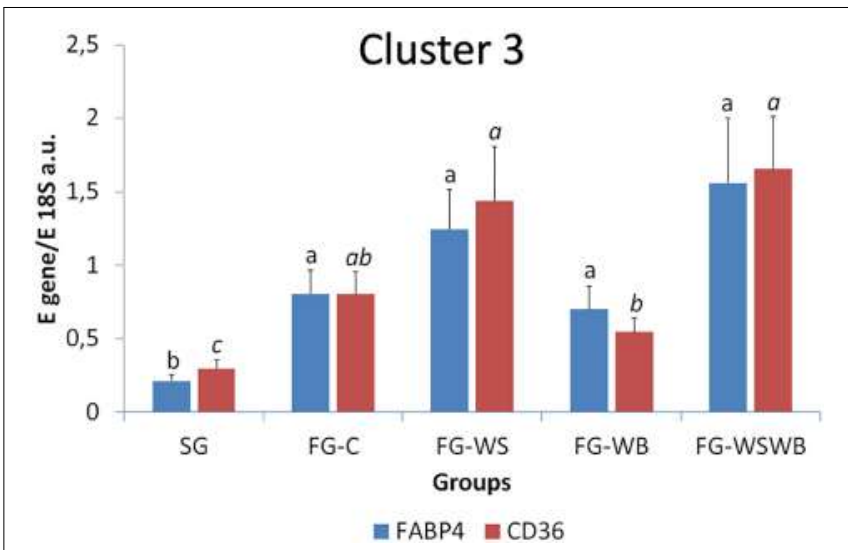

FIGURE 7 | Relative mRNA expression of genes coding for fatty acid binding protein 4 (FABP4) and cluster of differentiation 36 (CD36). mRNA expressions were assessed in pectoralis major muscles from a slow-growing genotype (SG) and a fast-growing genotype macroscopically free of defects (FG-C) or affected by White Striping (FG-WS), Wooden Breast (FG-WB), or both White Striping and Wooden Breast (FG-WSWB). FABP4 and CD36 expressed also more in fast- than in slow-growing muscles, especially in muscles affected by White Striping. Absolute mRNA levels of targeted genes were corrected for $18 S$ ribosomal RNA levels to give a relative mRNA level. For each gene, different letters indicate significant differences between groups $(P \leq 0.05)$.

intensity using a scanner on muscle cross-sections may be useful to quickly and precisely diagnosing injuries involved in the occurrence of both WS and WB defects within a population of broilers exhibiting similar growth rate. In addition, the scan methodology used markers involved in fibrosis and muscle fiber regeneration, which are two processes involved in both WS and WB defects. It would be interesting to identify specific markers either of the WS or of the WB, which are compatible with the use of a scanner. Indeed, we have shown that Bodipy 493 is a very good marker of adiposis (Figure 3 ) and therefore of WS condition, but unfortunately incompatible with the use of our scan technology. Such development will widen the possibilities of fast, specific, and reproducible muscle phenotyping in the future. In the meantime, the histological phenotyping of the muscles can be useful to refine a first visual or spectral phenotyping that easily identifies the presence of WS or WB.

\section{Which Molecular Processes Differentiate or Are Common to WS and WB Conditions?}

By combining histological, expressional, and enzymatic approaches, our study aimed to contribute improving knowledge on the etiology of both WS and WB defects. To achieve this goal, we compared muscle groups that were free from WS and WB or affected by one or the other or both at the same time.

\section{Pathways Involved in Muscle Metabolism and Contractile Function}

As discussed above, adiposis is a good marker of WS and Bodipy 493 a good histological marker to quantify its occurrence and severity. Among genes we measured, only FABP4 and CD36 


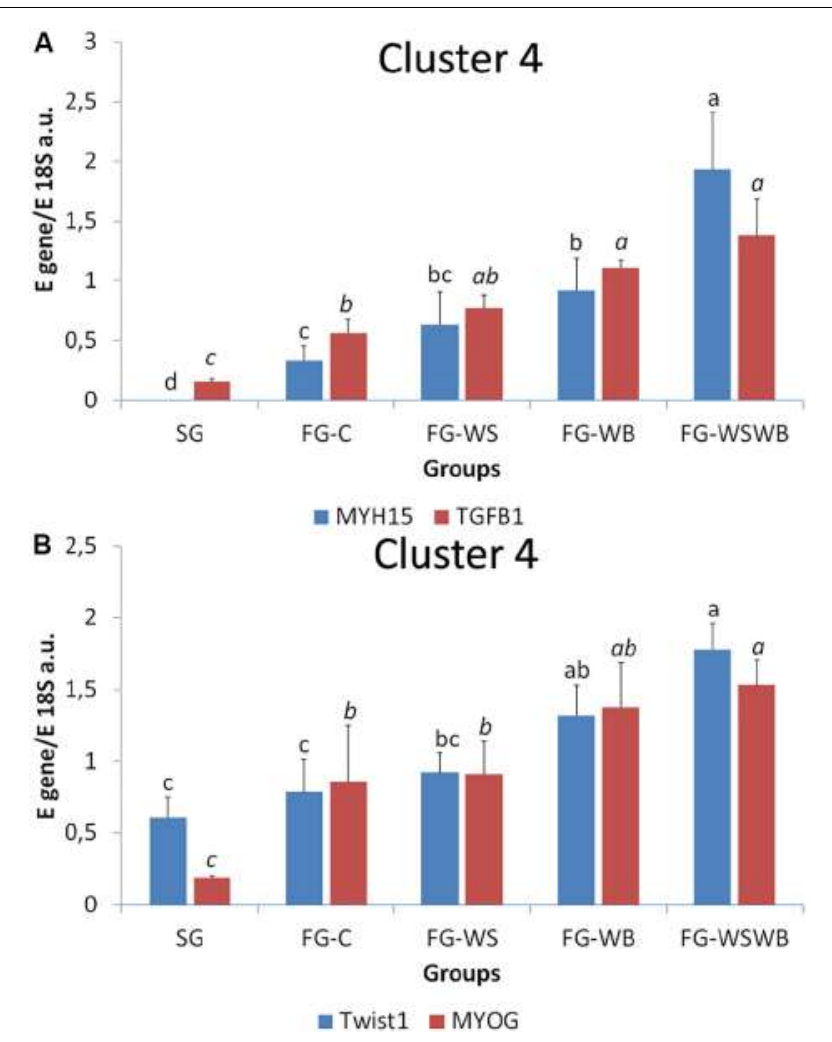

FIGURE 8 | Relative mRNA expression of genes coding for myosin heavy chain 15 (MYH15), transforming growth factor beta 1 (TGFB1), Twist basic helix-loop-helix transcription factor 1 (Twist1), and myogenin (MYOG). mRNA expressions were assessed in pectoralis major muscles from a slow-growing genotype (SG) and a fast-growing genotype macroscopically free of defects (FG-C) or affected by White Striping (FG-WS), Wooden Breast (FG-WB), or both White Striping and Wooden Breast (FG-WSWB). Expression of MYH15, TGFB1 (A), Twist1, and MYOG (B) increased linearly with the occurrence of White Striping and Wooden Breast defects. Absolute mRNA levels of targeted genes were corrected for $18 \mathrm{~S}$ ribosomal RNA levels to give a relative mRNA level. For each gene, different letters indicate significant differences between groups $(P \leq 0.05)$.

expression tended to be higher in muscles affected by WS alone or associated with WB. Upregulation of FABP4 and CD36 genes was already observed by Papah et al. (2018) and Lake et al. (2019) during the early steps of WB development. Their results suggested that dysregulation of FABP4,CD36, and $L P L$ are likely to be associated with fat deposition in muscle. FABP4 encodes a fatty acid-binding protein involved in lipid transport in adipocytes. CD36 codes for the cluster of differentiation 36, a cell surface protein that imports fatty acids inside cells. CD36 binds many ligands including collagen, lipoprotein, phospholipids, and long-chain fatty acids. In relation with their respective function, it is likely that these two proteins participate to recruitment of lipids by adipocytes during their differentiation (Arrighi et al., 2015). PLIN2 coding an adipose differentiation related protein (perilipin-2) was first characterized as an mRNA molecule that expresses early in adipocyte differentiation (Jiang and Serrero, 1992). FASN codes a protein implied in long-chain saturated fatty acids synthesis. Their greater expression in FG compared to SG

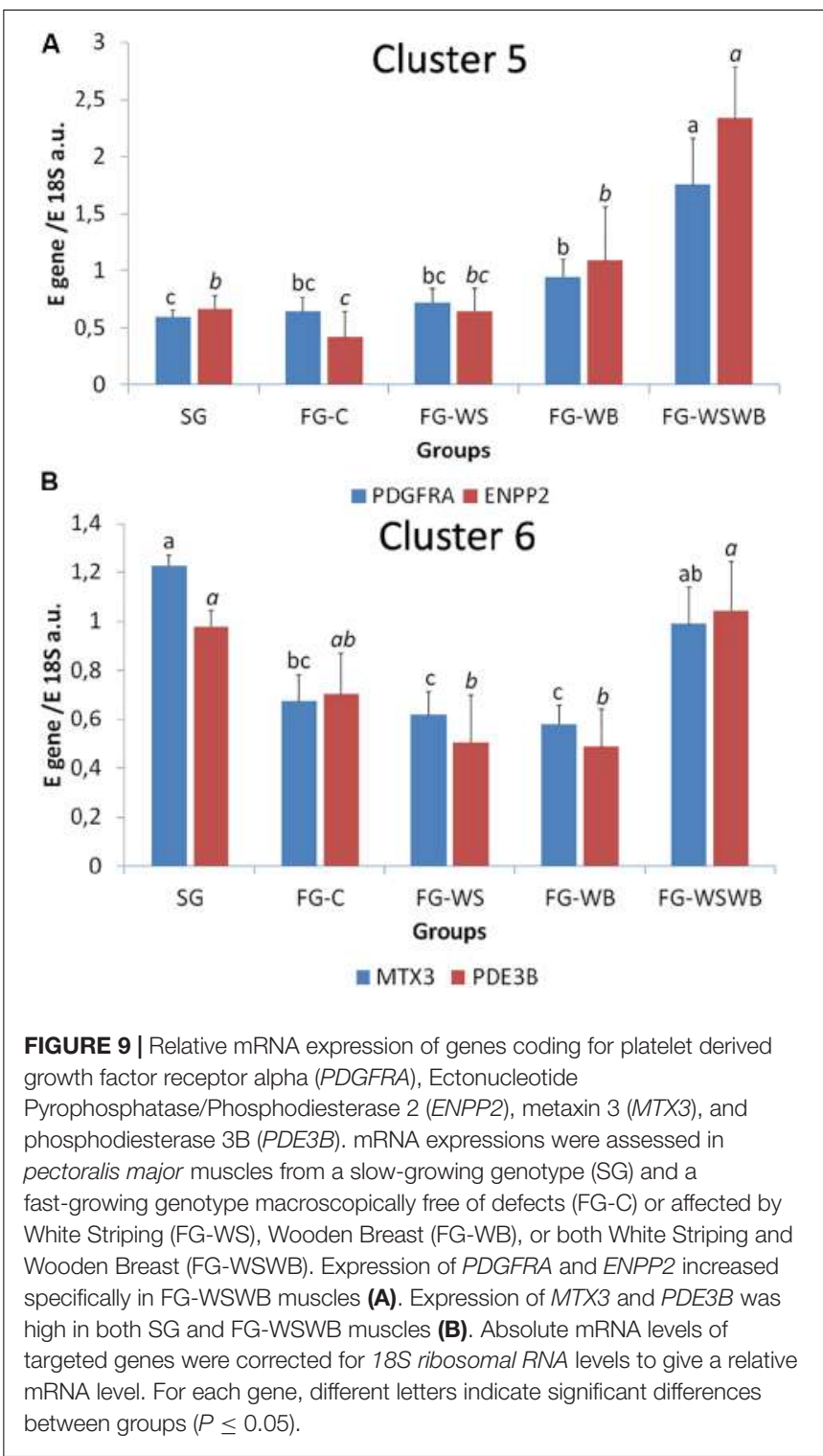

muscles suggests perilipin-2 and FASN may contribute to the higher adiposis observed in all FG muscles, including normal ones (Figure 3). We also measured the expression of ADIPOQ gene coding adiponectin that enhances glucose utilization and fatty-acid combustion. Its expression was not altered by the presence of the defects, which is not in favor of a potential role in their establishment. However, recent results showed that adiponectin content may change following posttranscriptional regulation (Van Pelt et al., 2020).

Among the genes tested, several were specifically regulated in muscles affected with WB compared to normal ones (Table 2). Four of them were downregulated in the presence of WB and more expressed in SG than in FG muscles. They include MYH1E, which codes the main form of myosin expressed in adult chicken fast-twitching breast muscle (Bader et al., 1982), and MYH13, coding the superfast-twitching, myosin mediating the high-velocity and low-tension contractions of 


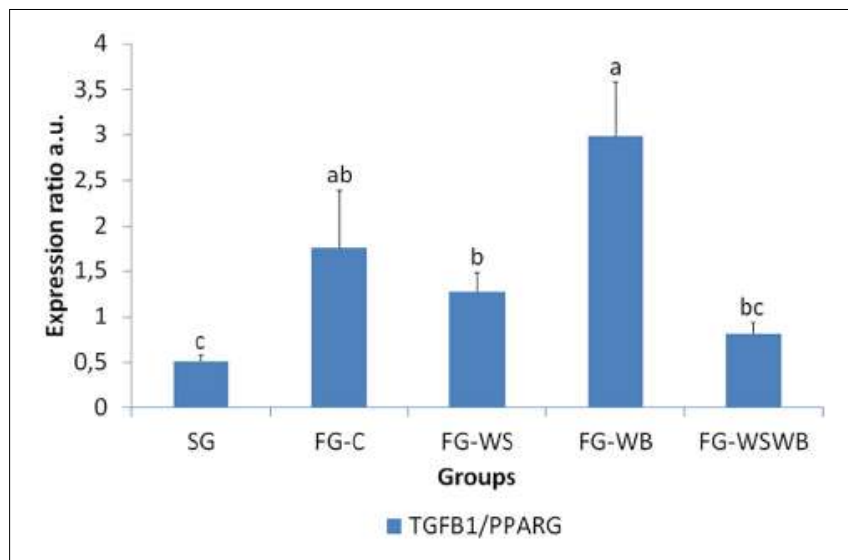

FIGURE 10 | Ratio TGFB1 on PPARG relative mRNA expression. It was assessed in pectoralis major from a slow-growing genotype (SG) and a fast-growing genotype macroscopically free of defects (FG-C) or affected by White Striping (FG-WS), Wooden Breast (FG-WB), or both White Striping and Wooden Breast (FG-WSWB). TGFB1/PPARG ratio was the lowest in SG and the highest in FG-WB muscles, suggesting that a high ratio would favor the development of a fibrotic rather than adipogenic phenotype. Absolute mRNA levels of targeted genes were corrected for $18 S$ ribosomal RNA levels to give a relative $\mathrm{mRNA}$ level. Different letters indicate significant differences between groups $(P \leq 0.05)$.

specific striated muscles (Briggs and Schatchat, 2002). On the contrary, MYH15 coding a slow-twitch ventricular-like myosin heavy chain expressed during regeneration of avian skeletal muscles (Camoretti-Mercado et al., 1993) was significantly upregulated in WB muscles, compared to FG-C; while it was almost not expressed in SG muscles (Figure 8A), as already observed in muscles affected by both WS and WB (Pampouille et al., 2019). Consistently, our study revealed an increased MYH6 signal that it is specific to a slow developmental myosin isoform derived from PM muscle (Miller et al., 1985) in both WS and WB muscles (not shown), arguing in favor of a shift from fast- to slow-twitching as already suggested (Papah and Abasht, 2019). However, the mature form of slow myosin heavy chain (MYH7B, Miller et al., 1985) was not expressed in presence of WS or WB defects (not shown), suggesting that contractile shift induced is mainly due to the replacement of fast IIb toward fast IIa fibers, as observed usually in muscular dystrophies on predominant IIb muscles (Barnard et al., 1982; Pette and Staron, 2001). As revealed by NADH-TR reaction, muscle fiber shift was associated with increased mitochondria density in both WS and WB conditions compared to FG-C muscles (Figure 2). Surprisingly, the increased mitochondria density observed in affected muscle was not accompanied by increased mitochondria COX activity (Figure 5). It was significantly lower in FG than in SG muscles, but not affected by the presence of the defects. in contrast, we observed greater production of ROS in FG than in SG muscles (Figure 5). Altogether, these observations suggest impaired mitochondrial functions in fast-growing muscles, even not affected by WS or WB defects, compared to slow-growing muscles. Another muscle-specific gene, MYOCD, was less expressed in FG muscles, especially in the case of WB. It codes myocardin, which is expressed in cardiac muscle and tissues containing smooth muscle cells, in which it may play a crucial role in cardiogenesis and smooth muscle differentiation (van Tuyn et al., 2005). Myocardin has been recently proposed to play a role in the vascularization reduction in the case of severe defects (Pampouille et al., 2019). The last downregulated gene is ANKRD46 whose function is not known, particularly in connection with myopathies. However, other ankyrins are known to be involved in the costamere, the structure that is responsible for linking sarcomere to the sarcolemma (Maiwailidan et al., 2011). The huge difference in expression (about 125 times; Table 2) that exists between the SG and FG muscles raises questions about its function in chicken muscle and will require further study.

An interesting point is the pattern of expression of MTX3 that is more expressed in SG than in FG muscles, except in very severely affected FG-WSWB muscles (Figure 9). MTX3 encodes metaxin 3, which is involved in the protein transport into the mitochondrion. It contains glutathione S-transferase domain (Adolph, 2004) and may therefore participate to detoxification of reactive electrophile species (RES). RES stimulate the expression of cell survival genes, as well as many other genes commonly upregulated in case of stress and pathogenesis. There is evidence that, although excess RES production can lead to cell damage, lower levels of RES may modulate the expression of cell survival genes and by this way contribute to survival during severe stress (for review, see Farmer and Davoine, 2007). Therefore, the upregulation of MTX3 may contribute to cell survival in chicken muscles severely affected by WS and WB, in coordination with other the pathways such as oxygen transport, axon guidance, and neuromuscular junction repair already reported in these types of muscle (Pampouille et al., 2019).

\section{Pathways Involved in Cell Regeneration and Extracellular Matrix Development}

Of the genes overexpressed in affected muscles, some were only upregulated when WB was diagnosed. They are mainly involved in connective tissue deposition. FN1 codes fibronectin, which plays a key role in the adhesion of cells to the ECM. FN1 expression followed a similar profile as fibronectin histological quantification, suggesting transcriptional regulation (Table 2 and Figure 3). Fibronectin was described as a seric biomarker of Duchenne muscular dystrophy (DMD) (Cynthia Martin et al., 2014), and its expression during fibrosis has been shown to be regulated by TGFB1 and CTGF (Vial et al., 2008), whose expression patterns follow those of FN1 in our study (Table 2 and Figure 8). FN1 has been recently proposed as upstream regulator involved in ECM remodeling, which begins at 3 weeks in chickens affected by Wooden Breast (Papah et al., 2018). By contrast, the expression of FBN1 that codes another ECM glycoprotein fibrillin was not altered by muscle growth or WS or WB condition.

Among the gene-coding proteins of the ECM, some were only upregulated in muscles severely affected by both WS and WB. This is the case of COL6A3 and ANTXR1 (Figure 7 and Table 2), whose expression positively correlated ( $R$ of $0.68 ; P \leq 0.001)$. ANTXR1 encodes a type I transmembrane protein that interacts with collagen types 1 and 6 alpha 3 (coded by COL6A3) and may have a role in ECM homeostasis 
(Hotchkiss et al., 2005). PDGFRA encodes platelet-derived growth factor receptor A, also termed PDGFR $\alpha$, and is described as a marker of fibro-adipogenic progenitors (FAPs), which are required for proper skeletal muscle development, regeneration, and maintenance (Uezumi et al., 2011). However, FAPs are also responsible for fibro-fatty scar deposition following chronic damage (Arrighi et al., 2015; Contreras et al., 2016). In cell culture, PDFGR $\alpha$ positive cells treated by TGF $\beta 1$ expressed fibrosis marker like collagen and CTGF contrary to myoblasts (Uezumi et al., 2011). Recently, it was shown that TGF $\beta$ inhibits PDFGR $\alpha$ expression in FAPs and promotes myofibroblast differentiation of FAPs but inhibit their adipogenicity (Contreras et al., 2019). The concomitant overexpression of TGF $\beta$, CTGF, and PDFGR $\alpha$ especially in WB muscles argue for their role in controlling FAPs differentiation toward fibroblasts or adipocytes.

Our histological observation brought evidence of cell regeneration with the expression of MYH15 and NCAM proteins in many fibers of muscles affected by WB (Figure 2). We also reported increased expression of several genes involved in myogenesis or cell proliferation in WB muscles. This is the case of $M Y O G$, involved in myogenesis and skeletal muscle repair (Figure 7), and of ENPP2 (Figure 8), whose expression increased during myogenic differentiation of $\mathrm{C} 2 \mathrm{C} 12$ murine myoblasts and inhibition prevents the differentiation of myoblasts (Sah et al., 2020). In our study, ENPP2 expression was positively correlated to that of MYH15 ( $R$ of $0.70 ; P \leq 0.001$ ) and of Twist1 ( $R$ of $0.72 ; P \leq 0.001)$. Twist 1 gene inhibits muscle differentiation by repressing muscle-specific gene activation (Hebrok et al., 1994), which suggests antagonist process either activating or repressing muscle differentiation in WB muscle. However, as described recently, Twistl has a key role in maintenance of skeletal muscle progenitors (Choi et al., 2020), but also in adipogenesis (Ren et al., 2016). The overexpression of Twist1 in chicken muscle affected by WB is also consistent with the fact that WB condition has been often associated with a lack of glycogen (Abasht et al., 2016). Indeed, overexpression of Twist 1 and Twist 2 genes reduced total glycogen content in mouse muscle without altering glucose uptake (Mudry et al., 2015). Overexpression of these two genes also increases gene expression of inflammatory cytokines like IL6,TNF $\alpha$, and IL1 $\beta$ (Mudry et al., 2015). However, in the literature, expression of inflammatory cytokines seems to show some discrepancies, as IL1 $\beta$ is downregulated in WB muscle (Zambonelli et al., 2016) while IL2RG and TNFRSF1A are upregulated (Papah et al., 2018). Further experiments are needed to understand Twist1 role in WB pathogenesis. Increased mitotic activity was also recently reported in muscle affected by WB from 25-day-old chicken, indicating that a regeneration process was underway (Meloche et al., 2018). Accordingly, we reported an overexpression of PCNA, specifically expressed in proliferating cells, confirming that cell regeneration was associated with these defects (Figure 9). Interestingly, PCNA was also highly expressed in SG muscles, probably because they are at an earlier physiological stage of development than fast-growing muscles. Obviously, regeneration involved in WB disease did not affect MYF5 and MYOD mRNA expressions, the main myogenic factors implied in myogenesis (not shown). Altogether, our observations highlighted several robust markers of the WB condition, most of them being associated with molecular pathways involved in cell proliferation and muscle differentiation and repair following chronic damage. We also pointed out possible antagonist pathways that are either inhibiting or activating myogenic differentiation, whose balance possibly determines muscle progenitor cell fate.

Among genes involved in lipid metabolism, PPARG and FASN were only significantly upregulated in muscles severely affected by both WS and WB. PPAR $\gamma$ is known as a regulator of adipocyte differentiation, and its expression is inhibited by TGF $\beta 1$ in FAPs (Contreras et al., 2019). To evaluate the relevance of the balance between TGF $\beta 1$ and PPAR $\gamma$ signaling, we calculated the ratio of TGFB1 on PPARG expression. This ratio was significantly modified depending on the group studied $(P \leq 0.01)$ and was the lowest in SG and the highest in FG-WB muscles (Table 2), suggesting that a high ratio TGFB1/PPARG would favor the development of a fibrotic rather than adipogenic phenotype. This hypothesis is strengthened because, among FG muscles, lower ratios were observed in muscles exhibiting WS (Table 2), and expression of $P D E 3 B$, a well-known target of PPAR $\gamma$ (Resnyk et al., 2013; Jahansouz et al., 2018), increased when the TGFB1/PPARG ratio decreased ( $R$ of $-0.60 ; P \leq 0.001$ ). Up-regulation of FASN and $P D E 3 B$ in a chicken model with higher visceral adiposity supported their potential role on regulation of muscle adiposity (Resnyk et al., 2013).

\section{Pathways and Genes Involved in Human Dystrophy}

Several genes selected for mRNA expression studies are known to be causative of muscular dystrophies in human. Among them, DYSF, SGCB, and COL6A3 are also included in QTL regions controlling WS in chicken breast muscle (Pampouille et al., 2018). In context of mammalian muscular dystrophies involving COL6A3 mutations, its expression is usually deficient (Pan et al., 2013). In our study, COL6A3 was highly expressed in fast-growing muscles affected by both WS and WB, probably because of the large amount of fibrosis observed in these muscles (Figure 2). Similarly, $C A V 3$, coding caveolin 3, is at least two-times more expressed in FG than in SG muscles. Previously, a 3 - to 5-fold increase in caveolin 3 induced an increased vesicular trafficking that was associated with cell necrosis and regeneration and decreased expression of sarcolemmal and subsarcolemmal proteins (Galbiati et al., 2000). However, in the present study, muscle groups did not affect genes coding sarcolemmal proteins SGBC and DAG1 (not shown). Only expression of CAPN3 was lower in FG compared to SG muscles. CAPN3 deficiency is associated with limb-girdle muscular dystrophy (Fardeau et al., 1996; Fakhfakh et al., 2012), and its expression is also reduced in degenerating-regenerating mice model and during regeneration process (Stockholm et al., 2001). Its reduced expression in fast-growing compared to slow-growing muscles is therefore consistent with their greater number of degenerating and regenerating cells (Figures 1, 2). Interestingly, CAPN3 expression is highly correlated $(R=0.93 ; P \leq 0.001)$ with that of $P P P 1 R 3 A$, a major gene controlling the glycogen content in chicken muscles between the high- and the low-pHu line (Le Bihan-Duval et al., 2018). The decrease of its expression in the high-pHu line is associated with lower glycogen content 
and higher susceptibility to WS defect. Disruption of the PPP1R3A gene in mice, encoding the glycogen targeting subunit of protein phosphatase 1, causes substantial lowering of the glycogen synthase activity and decrease in the glycogen levels in skeletal muscle (Delibegovic et al., 2003). It also induces impairment of glucose tolerance and insulin resistance in skeletal muscle associated with increased weight gain and massive abdominal and other fat depositions, likely as a consequence of impaired blood glucose utilization in skeletal muscle. It is therefore suggested that dysfunction of the protein coded by PPP1R3A may contribute to the pathophysiology of human type 2 diabetes. This can be related to recent findings in chicken that suggested etiologic similarities between Wooden Breast and type 2 diabetes despite, phenotypic disparities between birds and mammals, likely due to major difference in skeletal muscle glucose transport (Lake and Abasht, 2020). Despite many phenotypic similarities, there is no obvious evidence from our results of common etiology between mammalian dystrophies and myopathies observed in chicken breast muscle. However, genetic and protein studies are underway to explore whether mutations or posttranslational regulations may be involved in chicken myopathies, independently of transcriptional regulations.

\section{CONCLUSION}

Based on a multi-level biological approach, the present study provided new knowledge and molecular tools useful to characterize WS and WB defects in chicken muscles. Among the most interesting molecular markers identified are those linked to muscle contractile properties and fiber regeneration or involved in the development of fibrosis and adiposis. Our study also highlighted several regulatory pathways that could be decisive for the myogenic, adipogenic, or fibrogenic fate of muscle precursors and key molecules controlling both muscle glycogen and fiber cell regeneration that would deserve further study. In order to improve research on avian myopathies, our study offers the scientific community new rapid histological analysis techniques, based on the use of robust and specific markers, capable of precisely quantifying the injuries induced in the event of WS and WB. Such measurements will allow for a fine assessment the impact of rearing or selection strategies on chicken breast muscle myopathies, but may also serve to provide reliable phenotypes for the future development of non-invasive phenotyping tools based on non-destructive methodologies that can be used on live animals.

\section{DATA AVAILABILITY STATEMENT}

The raw data supporting the conclusions of this article will be made available by the authors, without undue reservation.

\section{REFERENCES}

Abasht, B., Mutryn, M. F., Michalek, R. D., and Lee, W. R. (2016). Oxidative stress and metabolic perturbations in wooden breast disorder

\section{ETHICS STATEMENT}

The animal study was reviewed and approved by the Ethical Committee for Animal Experimentation in Val de Loire (C2EA-19).

\section{AUTHOR CONTRIBUTIONS}

$\mathrm{CP}$ and $\mathrm{CB}$ supervised the study, defined the experimental design and supervised the animal phenotyping with EL and EP. JJ, EG, and NC were in charge of the laboratory analysis under the supervision of CP. CP performed the statistical analyses and drafted the first version of the manuscript under the supervision of $\mathrm{CB}$. All the authors read and approved the final manuscript.

\section{FUNDING}

The authors declare that this study received funding from the Bretagne and Pays de la Loire regions (France) through the TECNOVIA project. The funders were not involved in the study design, collection, analysis, and interpretation of data, the writing of this article or the decision to submit it for publication.

\section{ACKNOWLEDGMENTS}

The authors thank the staff of PEAT INRA Poultry Experimental Facility where experimentation was conducted (2018, https://doi.org/10.15454/1.5572326250887292E12) and of the Avian Biology and Poultry Research Unit (UMR BOA, INRAE, Université de Tours, Nouzilly, France) for their technical assistance.

\section{SUPPLEMENTARY MATERIAL}

The Supplementary Material for this article can be found online at: https://www.frontiersin.org/articles/10.3389/fphys. 2020.00633/full\#supplementary-material

FILE S1 | Pearson's correlations between the relative mRNA levels of genes differentially expressed between pectoralis major muscles from a slow-growing genotype (SG) and a fast-growing genotype macroscopically free of defects (FG-C) or affected by White Striping (FG-WS), Wooden Breast (FG-WB), or both White Striping and Wooden Breast (FG-WSWB). Differentially expressed genes are regrouped by cluster (C1-C6). Absolute mRNA levels of targeted genes were corrected for 18 S ribosomal RNA levels to give a relative mRNA level. Pearson's correlation is indicated when statistically significant $(P \leq 0.05)$ or crossed out with a black cross if it is not.

in chickens. PLoS One 11:e0153750. doi: 10.1371/journal.pone.015 3750

Abasht, B., Zhou, N., Lee, W. R., Zhuo, Z., and Peripolli, E. (2019). The metabolic characteristics of susceptibility to wooden breast disease in chickens 
with high feed efficiency. Poult. Sci. 98, 3246-3256. doi: 10.3382/ps/ pez183

Adolph, K. W. (2004). The zebrafish metaxin 3 gene ( $\mathrm{mtx} 3)$ : cDNA and protein structure, and comparison to zebrafish metaxins 1 and 2. Gene 330, 67-73. doi: 10.1016/j.gene.2004.01.005

Arrighi, N., Moratal, C., Clement, N., Giorgetti-Peraldi, S., Peraldi, P., Loubat, A., et al. (2015). Characterization of adipocytes derived from fibro/adipogenic progenitors resident in human skeletal muscle. Cell Death Dis. 6:e1733. doi: 10.1038 /cddis. 2015.79

Bader, D., Masaki, T., and Fischman, D. A. (1982). Immunochemical analysis of myosin heavy chain during avian myogenesis in vivo and in vitro. J. Cell Biol. 95, 763-770. doi: 10.1083/jcb.95.3.763

Baéza, E., Arnould, C., Jlali, M., Chartrin, P., Gigaud, V., Mercerand, F., et al. (2012). Influence of increasing slaughter age of chickens on meat quality, welfare, and technical and economic results. J. Anim. Sci. 90, 2003-2013. doi: 10.2527/jas.2011-4192

Barnard, E. A., Lyles, J. M., and Pizzey, J. A. (1982). Fiber types in chicken skeletal muscles and their changes in muscular dystrophy. J. Physiol. 331, 333-354.

Boerboom, G., van Kempen, T., Navarro-Villa, A., and Perez-Bonilla, A. (2018). Unraveling the cause of white striping in broilers using metabolomics. Poult Sci. 97, 3977-3986. doi: 10.3382/ps/pey266

Briggs, M. M., and Schatchat, F. (2002). The superfast extraocular myosin (MYH13) is localized to the innervation zone in both the global and orbital layers of rabbit extraocular muscle. J. Exp. Biol. 205, 3133-3142.

Camoretti-Mercado, B., Dizon, E., Jakovcic, S., and Zak, R. (1993). Differential expression of ventricular-like myosin heavy chain mRNA in developing and regenerating avian skeletal muscles. Cell Mol. Biol. Res. 39, 425-437.

Choi, I. Y., Lim, H., Cho, H. J., Oh, Y., Chou, B. K., Bai, H., et al. (2020). Transcriptional landscape of myogenesis from human pluripotent stem cells reveals a key role of TWIST1 in maintenance of skeletal muscle progenitors. eLife 3:e46981. doi: 10.7554/eLife.46981

Clark, D. L., and Velleman, S. G. (2016). Spatial influence on breast muscle morphological structure, myofiber size, and gene expression associated with the wooden breast myopathy in broilers. Poult. Sci. 95, 2930-2945. doi: 10.3382/ps/ pew243

Contreras, O., Cruz-Soca, M., Theret, M., Soliman, H., Tung, L. W., Groppa, E., et al. (2019). The cross-talk between TGF-beta and PDGFRalpha signaling pathways regulates stromal fibro/adipogenic progenitors' fate. J. Cell Sci. 132:jcs232157. doi: $10.1242 /$ jcs.232157

Contreras, O., Rebolledo, D. L., Oyarzun, J. E., Olguin, H. C., and Brandan, E. (2016). Connective tissue cells expressing fibro/adipogenic progenitor markers increase under chronic damage: relevance in fibroblast-myofibroblast differentiation and skeletal muscle fibrosis. Cell Tissue Res. 364, 647-660. doi: 10.1007/s00441-015-2343-0

Cynthia Martin, F., Hiller, M., Spitali, P., Oonk, S., Dalebout, H., Palmblad, M., et al. (2014). Fibronectin is a serum biomarker for Duchenne muscular dystrophy. Proteomics Clin. Appl. 8, 269-278. doi: 10.1002/prca.201300072

de Brot, S., Perez, S., Shivaprasad, H. L., Baiker, K., Polledo, L., Clark, M., et al. (2016). Wooden breast lesions in broiler chickens in the UK. Vet. Rec. 178:141.

Delibegovic, M., Armstrong, C. G., Dobbie, L., Watt, P. W., Smith, A. J., and Cohen, P. T. (2003). Disruption of the striated muscle glycogen targeting subunit PPP1R3A of protein phosphatase 1 leads to increased weight gain, fat deposition, and development of insulin resistance. Diabetes Metab. Res. Rev. 52, 596-604. doi: 10.2337/diabetes.52.3.596

Dubois, C., Figarella-Branger, D., Pastoret, C., Rampini, C., Karpati, G., and Rougon, G. (1994). Expression of NCAM and its polysialylated isoforms during mdx mouse muscle regeneration and in vitro myogenesis. Neuromuscul. Disord. 4, 171-182. doi: 10.1016/0960-8966(94)90018-3

Dubowitz, V., Sewry, C. A., and Oldfors, A. (2013). Muscle Biopsy A Practical Approach Saunders. Amsterdam: Elsevier.

Fakhfakh, R., Lamarre, Y., Skuk, D., and Tremblay, J. P. (2012). Losartan enhances the success of myoblast transplantation. Cell Transplant. 21, 139-152. doi: $10.3727 / 096368911 \times 576045$

FAO (2019). Food Outlook - Biannual Report on Global Food Markets. Rome: CC BY-NC-SA 3.0 IGO.

Fardeau, M., Hillaire, D., Mignard, C., Feingold, N., Feingold, J., Mignard, D., et al. (1996). Juvenile limb-girdle muscular dystrophy. Clinical, histopathological and genetic data from a small community living in the Reunion Island. Brain 119(Pt 1), 295-308. doi: 10.1093/brain/119. 1.295

Farmer, E. E., and Davoine, C. (2007). Reactive electrophile species. Curr. Opin. Plant Biol. 10, 380-386. doi: 10.1016/j.pbi.2007.04.019

Ferreira, T. Z., Casagrande, R. A., Vieira, S. L., Driemeier, D., and Kindlein, L. (2014). An investigation of a reported case of white striping in broilers. J. Appl. Poult. Res. 23, 1-6.

Galbiati, F., Volonte, D., Chu, J. B., Li, M., Fine, S. W., Fu, M., et al. (2000). Transgenic overexpression of caveolin-3 in skeletal muscle fibers induces a Duchenne-like muscular dystrophy phenotype. Proc. Natl. Acad. Sci. U.S.A. 97, 9689-9694. doi: 10.1073/pnas.160249097

Hebrok, M., Wertz, K., and Fuchtbauer, E. M. (1994). M-twist is an inhibitor of muscle differentiation. Dev. Biol. 165, 537-544. doi: 10.1006/dbio.1994.1273

Hotchkiss, K. A., Basile, C. M., Spring, S. C., Bonuccelli, G., Lisanti, M. P., and Terman, B. I. (2005). TEM8 expression stimulates endothelial cell adhesion and migration by regulating cell-matrix interactions on collagen. Exp. Cell Res. 305, 133-144. doi: 10.1016/j.yexcr.2004.12.025

Jahansouz, C., Xu, H., Hertzel, A. V., Kizy, S., Steen, K. A., Foncea, R., et al. (2018). Partitioning of adipose lipid metabolism by altered expression and function of PPAR isoforms after bariatric surgery. Int. J. Obes. 42, 139-146. doi: 10.1038/ijo.2017.197

Jiang, H. P., and Serrero, G. (1992). Isolation and characterization of a full-length cDNA coding for an adipose differentiation-related protein. Proc. Natl. Acad. Sci. U.S.A. 89, 7856-7860. doi: 10.1073/pnas.89.17.7856

Kawasaki, T., Iwasaki, T., Yamada, M., Yoshida, T., and Watanabe, T. (2018). Rapid growth rate results in remarkably hardened breast in broilers during the middle stage of rearing: a biochemical and histopathological study. PLoS One 13:e0193307. doi: 10.1371/journal.pone.0193307

Kuttappan, V. A., Bottje, W., Ramnathan, R., Hartson, S. D., Coon, C. N., Kong, B. W., et al. (2017). Proteomic analysis reveals changes in carbohydrate and protein metabolism associated with broiler breast myopathy. Poult. Sci. 96, 2992-2999. doi: 10.3382/ps/pex069

Kuttappan, V. A., Brewer, V. B., Apple, J. K., Waldroup, P. W., and Owens, C. M. (2012a). Influence of growth rate on the occurrence of white striping in broiler breast fillets. Poult. Sci. 91, 2677-2685. doi: 10.3382/ps.2012-02259

Kuttappan, V. A., Brewer, V. B., Mauromoustakos, A., McKee, S. R., Emmert, J. L., Meullenet, J. F., et al. (2013a). Estimation of factors associated with the occurrence of white striping in broiler breast fillets. Poult. Sci. 92, 811-819. doi: 10.3382/ps.2012-02506

Kuttappan, V. A., Huff, G. R., Huff, W. E., Hargis, B. M., Apple, J. K., Coon, C., et al. (2013b). Comparison of hematologic and serologic profiles of broiler birds with normal and severe degrees of white striping in breast fillets. Poult. Sci. 92, 339-345. doi: 10.3382/ps.2012-02647

Kuttappan, V. A., Lee, Y. S., Erf, G. F., Meullenet, J. F., McKee, S. R., and Owens, C. M. (2012b). Consumer acceptance of visual appearance of broiler breast meat with varying degrees of white striping. Poult. Sci. 91, 1240-1247. doi: 10.3382/ps.2011-01947

Kuttappan, V. A., Shivaprasad, H. L., Shaw, D. P., Valentine, B. A., Hargis, B. M., Clark, F. D., et al. (2013c). Pathological changes associated with white striping in broiler breast muscles. Poult. Sci. 92, 331-338. doi: 10.3382/ps.2012-02646

Lake, J. A., and Abasht, B. (2020). Glucolipoxicity: a proposed etiology for wooden breast and related myopathies in commercial broiler chickens. Front. Physiol. 11:69. doi: 10.3389/fphys.220.00169

Lake, J. A., Papah, M. B., and Abasht, B. (2019). Increased expression of lipid metabolism genes in early stages of wooden breast links myopathy of broilers to metabolic syndrome in humans. Genes 10:746. doi: 10.3390/genes101 00746

Le Bihan-Duval, E., Hennequet-Antier, C., Berri, C., Beauclercq, S. A., Bourin, M. C., Boulay, M., et al. (2018). Identification of genomic regions and candidate genes for chicken meat ultimate $\mathrm{pH}$ by combined detection of selection signature and QTL. BMC Genomics 19:294. doi: 10.1186/s12864-0184690- 1

Maiwailidan, Y., Klauza, I., and Kordeli, E. (2011). Novel interactions of ankyrins$\mathrm{G}$ at the costameres: the muscle specific Obscurin/Titin-binding related domain (OTBD) binds plectin and filamin C. Exp. Cell Res. 317, 724-736. doi: 10.1016/ j.yexcr.2011.01.002

Marchesi, J. A. P., Ibelli, A. M. G., Peixoto, J. O., Cantao, M. E., Pandolfi, J. R. C., Marciano, C. M. M., et al. (2019). Whole transcriptome analysis of the pectoralis 
major muscle reveals molecular mechanisms involved with white striping in broiler chickens. Poult. Sci. 98, 590-601. doi: 10.3382/ps/pey429

Mazzoni, M., Petracci, M., Meluzzi, A., Cavani, C., Clavenzani, P., and Sirri, F. (2015). Relationship between pectoralis major muscle histology and quality traits of chicken meat. Poult. Sci. 94, 123-130. doi: 10.3382/ps/peu043

Meloche, K. J., Dozier, W. A. III, Brandebourg, T. D., and Starkey, J. D. (2018). Skeletal muscle growth characteristics and myogenic stem cell activity in broiler chickens affected by wooden breast. Poult Sci. 97, 4401-4414. doi: 10.3382/ps/ pey287

Miller, J. B., Crow, M. T., and Stockdale, F. E. (1985). Slow and fast myosin heavy chain content defines three types of myotubes in early muscle cell cultures. J. Cell Biol. 101, 1643-1650. doi: 10.1083/jcb.101.5.1643

Mudry, J. M., Massart, J., Szekeres, F. L., and Krook, A. (2015). TWIST1 and TWIST2 regulate glycogen storage and inflammatory genes in skeletal muscle. J. Endocrinol. 224, 303-313. doi: 10.1530/JOE-14.0474e

Norring, M., Valros, A., Valaja, J., Sihvo, H. K., Immonen, K., and Puolanne, E. (2018). Wooden breast myopathy links with poorer gait in broiler chickens. Animal 17, 1-6.

Pampouille, E., Berri, C., Boitard, S., Hennequet-Antier, C., Beauclercq, S. A., Godet, E., et al. (2018). Mapping QTL for white striping in relation to breast muscle yield and meat quality traits in broiler chickens. BMC Genomics 19:202. doi: 10.1186/s12864-018-4598-9

Pampouille, E., Hennequet-Antier, C., Praud, C., Juanchich, A., Brionne, A., Godet, E., et al. (2019). Differential expression and co-expression gene network analyses reveal molecular mechanisms and candidate biomarkers involved in breast muscle myopathies in chicken. Sci. Rep. 9:14905. doi: 10.1038/s41598019-51521-1

Pan, T. C., Zhang, R. Z., Markova, D., Arita, M., Zhang, Y., Bogdanovich, S., et al. (2013). COL6A3 protein deficiency in mice leads to muscle and tendon defects similar to human collagen VI congenital muscular dystrophy. J. Biol. Chem. 288, 14320-14331. doi: 10.1074/jbc.m112.433078

Papah, M. B., and Abasht, B. (2019). Dysregulation of lipid metabolism and appearance of slow myofiber-specific isoforms accompany the development of wooden breast myopathy in modern broiler chickens. Sci. Rep. 9:17170. doi: 10.1038/s41598-019-53728-8

Papah, M. B., Brannick, E. M., Schmidt, C. J., and Abasht, B. (2018). Gene expression profiling of the early pathogenesis of wooden breast disease in commercial broiler chickens using RNA-sequencing. PLoS One 13:e0207346. doi: 10.1371/journal.pone.0207346

Petracci, M., Mudalal, S., Babini, E., and Cavani, C. (2014). Effect of white striping on chemical composition and nutritional value of chicken breast meat. Ital. J. Anim. Sci. 13, 179-183.

Petracci, M., Mudalal, S., Bonfiglio, A., and Cavani, C. (2013). Occurrence of white striping under commercial conditions and its impact on breast meat quality in broiler chickens. Poult. Sci. 92, 1670-1675. doi: 10.3382/ps.2012-03001

Pette, D., and Staron, R. S. (2001). Transitions of muscle fiber phenotypic profiles. Histochem. Cell Biol. 115, 359-372. doi: 10.1007/s0041800100268

Pfaffl, M. W. (2001). A new mathematical model for relative quantification in real-time RT-PCR. Nucleic Acids Res. 29:e45. doi: 10.1093/nar/29.9.e45

Ren, R., Chen, Z., Zhao, X., Sun, T., Zhang, Y., Chen, J., et al. (2016). A possible regulatory link between Twist1 and PPAR $\gamma$ gene regulation in 3T3-L1 adipocytes. Lipids Health Dis. 8:189. doi: 10.1186/s12944-0161361-x

Resnyk, C. W., Carre, W., Wang, X., Porter, T. E., Simon, J., Le Bihan-Duval, E., et al. (2013). Transcriptional analysis of abdominal fat in genetically fat and lean chickens reveals adipokines, lipogenic genes and a link between hemostasis and leanness. BMC Genomics 14:557. doi: 10.1186/1471-2164-14-557

Russo, E., Drigo, M., Longoni, C., Pezzotti, R., Fasoli, P., and Recordati, C. (2015). Evaluation of White Striping prevalence and predisposing factors in broilers at slaughter. Poult. Sci. 94, 1843-1848. doi: 10.3382/ps/pev172

Sah, J. P., Hao, N. T. T., Han, X., Tran, T. T. T., McCarthy, S., Oh, Y., et al. (2020). Ectonucleotide pyrophosphatase 2 (ENPP2) plays a crucial role in myogenic differentiation through the regulation by WNT/beta-Catenin signaling. Int. J. Biochem. Cell Biol. 118:105661. doi: 10.1016/j.biocel.2019.105661

Sihvo, H. K., Immonen, K., and Puolanne, E. (2013). Myodegeneration with fibrosis and regeneration in the pectoralis major muscle of broilers. Vet. Pathol. 51, 619-623. doi: 10.1177/0300985813497488

Soglia, F., Mudalal, S., Babini, E., Di Nunzio, M., Mazzoni, M., Sirri, F., et al. (2016). Histology, composition, and quality traits of chicken Pectoralis major muscle affected by wooden breast abnormality. Poult. Sci. 95, 651-659. doi: $10.3382 / \mathrm{ps} / \mathrm{pev} 353$

Stockholm, D., Herasse, M., Marchand, S., Praud, C., Roudaut, C., Richard, I., et al. (2001). Calpain 3 mRNA expression in mice after denervation and during muscle regeneration. Am. J. Physiol. Cell Physiol. 280, C1561-C1569.

Traffano-Schiffo, M. V., Castro-Giraldez, M., Colom, R. J., and Fito, P. J. (2017). Development of a spectrophotometric system to detect white strping physiopathy in whole chicken carcasses. Sensors 17:E1024. doi: 10.3390/ s17051024

Trocino, A., Piccirillo, A., Birolo, M., Radaelli, G., Bertotto, D., Filiou, E., et al. (2015). Effect of genotype, gender and feed restriction on growth, meat quality and the occurrence of white striping and wooden breast in broiler chickens. Poult Sci. 94, 2996-3004. doi: 10.3382/ps/pev296

Uezumi, A., Ito, T., Morikawa, D., Shimizu, N., Yoneda, T., Segawa, M., et al. (2011). Fibrosis and adipogenesis originate from a common mesenchymal progenitor in skeletal muscle. J. Cell Sci. 124(Pt 21), 3654-3664. doi: 10.1242/ jcs.086629

Van Pelt, D. W., Kharaz, Y. A., Sarver, D. C., Eckhardt, L. R., Dzierzawski, J. T., Disser, N. P., et al. (2020). Multiomics analysis of the $\mathrm{mdx} / \mathrm{mTR}$ Mouse Model Of Duchenne Muscular Dystrophy. bioRxiv [Preprint]. doi: 10.1101/589424

van Tuyn, J., Knaän-Shanzer, S., van de Wattering, M. J., de Graaf, M., van des Laarse, A., Schalij, M. J., et al. (2005). Activation of cardiac and smooth muscle-specific genes in primary human cells after forced expression of human myocardin. Cardiovasc. Res. 67, 245-255. doi: 10.1016/j.cardiores.2005.04.013

Velleman, S. G., and Clark, D. L. (2015). Histopathologic and myogenic gene expression changes associated with wooden breast in broiler breast muscles. Avian. Dis. 59, 410-418. doi: 10.1637/11097-042015-Reg.1

Vial, C., Zuniga, L. M., Cabello-Verrugio, C., Canon, P., Fadic, R., and Brandan, E. (2008). Skeletal muscle cells express the profibrotic cytokine connective tissue growth factor (CTGF/CCN2), which induces their dedifferentiation. J. Cell. Physiol. 215, 410-421. doi: 10.1002/jcp.21324

Vignale, K., Caldas, J. V., England, J. A., Boonsinchai, N., Magnuson, A., Pollock, E. D., et al. (2017). Effect of white striping myopathy on breast muscle (Pectoralis major) protein turnover and gene expression in broilers. Poult. Sci. 96, 886-893. doi: 10.3382/ps/pew315

Wold, J. P., Veiseth-Kent, E., Host, V., and Lovland, A. (2017). Rapid online detection and grading of wooden breast myopathy in chicken fillets by near-infrared spectroscopy. PLoS One 12:e0173384. doi: 10.1371/journal.pone. 0173384

Zambonelli, P., Zappaterra, M., Soglia, F., Petracci, M., Sirri, F., Cavani, C., et al. (2016). Detection of differentially expressed genes in broiler pectoralis major muscle affected by white striping-wooden breast myopathies. Poult. Sci. 95, 2771-2785. doi: 10.3382/ps/pew268

Conflict of Interest: The authors declare that the research was conducted in the absence of any commercial or financial relationships that could be construed as a potential conflict of interest.

Copyright ๑ 2020 Praud, Jimenez, Pampouille, Couroussé, Godet, Le Bihan-Duval and Berri. This is an open-access article distributed under the terms of the Creative Commons Attribution License (CC BY). The use, distribution or reproduction in other forums is permitted, provided the original author(s) and the copyright owner $(s)$ are credited and that the original publication in this journal is cited, in accordance with accepted academic practice. No use, distribution or reproduction is permitted which does not comply with these terms. 
OPEN ACCESS

Edited by:

Sandra G. Velleman,

The Ohio State University,

United States

Reviewed by:

Giulia Baldi,

University of Bologna, Italy

Casey M. Owens,

University of Arkansas, United States

${ }^{*}$ Correspondence: Amit Morey

azm0011@auburn.edu

Specialty section:

This article was submitted to

Avian Physiology,

a section of the journal

Frontiers in Physiology

Received: 04 March 2020

Accepted: 18 June 2020

Published: 10 July 2020

Citation:

Morey A, Smith AE, Garner LJ and Cox MK (2020) Application of Bioelectrical Impedance Analysis to Detect Broiler Breast Filets Affected

With Woody Breast Myopathy.

Front. Physiol. 11:808

doi: 10.3389/fphys.2020.00808

\section{Application of Bioelectrical Impedance Analysis to Detect Broiler Breast Filets Affected With Woody Breast Myopathy}

\author{
Amit Morey ${ }^{1 *}$, Avery E. Smith ${ }^{1}$, Laura Jewell Garner ${ }^{1}$ and Marlin K. Cox ${ }^{2}$ \\ ${ }^{1}$ Department of Poultry Science, Auburn University, Auburn, AL, United States, ${ }^{2}$ CQ Foods, Inc., Juneau, AK, United States
}

Woody breast (WB) myopathy in modern broilers is causing major meat quality issues and consumer complaints. The poultry industry is sorting out WB filets through the inconsistent manual hand-palpation method. Bioelectrical impedance analysis (BIA) method was evaluated as a rapid and objective WB detection method. Freshly deboned broiler breast filets ( 15 filets $\times 2$ categories $\times 3$ trials) were sorted (hand-palpation) into severe woody (SW) and normal (N) categories were analyzed for BIA values, cook loss, texture (BMORS) method. SW filets had significantly $(P<0.05)$ higher resistance and reactance compared to $N$ indicating $B I A$ can be used to detect WB filets. In another experiment, we determined the ability of the BIA to differentiate between four WB severity levels using the whole filet. Significant differences were observed in resistance and reactance of normal and other WB categories, however, there were no significant differences among mild, moderate and severe WB categories. Segmental BIA of those filets indicated that BIA can be used to separate cranial, medial and caudal region of the breast filet based on the presence of WB myopathy. Accidental discovery of spaghetti breast in the samples demonstrated the significance of compounding different factors in analyzing WB meat using BIA.

Keywords: woody breast, bioelectric impedance, resistance, reactance, broiler - chicken

\section{INTRODUCTION}

Woody breast (WB) myopathy in modern broilers is causing major meat quality issues and consumer complaints and this problem is further exasperated by industry not having a reasonable objective means to detect it. Convenience, versatility, variety, and health benefits have increased the rate of consumed chicken in the United States from $36 \mathrm{lbs} /$ capita/year in 1965 to approximately $94 \mathrm{lbs} / \mathrm{capita} /$ year in 2018 (National Chicken Council, 2020a). To satisfy the growing demand, the broiler industry has developed fast-growing big-broiler strains ( $>6$ lbs live wt.) that culminated in over 42 billion lbs in 2018 of ready-to-cook poultry meat (National Chicken Council, 2020b).

The fast-growing broiler strains have developed a degenerative muscle myopathy in the pectoralis major termed as woody breast myopathy WB which was first reported by Dr. S. Bilgili at Auburn University in 2013 (Bilgili, 2013). Tijare et al. (2016) reported that the incidence of WB in United States broilers was $96.1 \%$, with $48 \%$ exhibiting mild WB, $28 \%$ exhibiting moderate, and $20 \%$ exhibiting severe WB. Filets with WB are hard (very dense) to the touch with varying degrees of 
hardness due to collagen infiltration along the ventral area of the breast. WB condition is difficult to detect because it can vary in the degree of hardness, be focal or diffuse within the breast and, be randomly distributed within a broiler flock (Tijare et al., 2016). Histology of WB indicates muscle fiber fragmentation, hyalinization, swelling of myofibers, necrotic muscle fiber replacement with connective tissue (fibrosis), macrophage infiltration, and presence of irregular patches of adipose tissue (Bilgili, 2013; Velleman and Clark, 2015). Collagen infiltration is a major causes of $\mathrm{WB}$ hard texture and the infiltration pattern differs among broiler strains (Sihvo et al., 2014; Velleman and Clark, 2015; Tijare et al., 2016). Biochemical analysis of filets indicates higher moisture (4.5\%) lower protein (4\%) content than normal filets (Wold et al., 2017).

Woody breast is apparent to average consumers as well as to culinary experts. Breasts exhibiting WB characteristics have a hard texture and are both visually and texturally unappealing leading to low overall consumer acceptance (Petracci et al., 2015). Filets affected with WB have approximately $50 \%$ lower marinade uptake and approximately 27\% higher cook loss compared to normal breast meat (Mudalal et al., 2015). As a result, poultry processors have to either deal with rejected orders and complaints or if WB is identified prior to sale, offer the product as lower quality product for a reduced price and absorb significant profit losses (Mudalal et al., 2015; Petracci et al., 2015).

Woody breast myopathy is a meat quality issue faced by the global poultry industry. Although established and identified in the United States, WB is now being detected in other countries including the Italy, Denmark, United Kingdom, and Finland (Sihvo et al., 2014; Trocino et al., 2015; Brot et al., 2016; Larsen et al., 2016; Tijare et al., 2016). A study from Italy (Trocino et al., 2015) observed that males from two broiler genotypes had 3-times higher occurrence of WB than females.

Detection methods used by industry are primarily hand palpation which are subjective, problematic, and unreliable. Typically, a plant will train employees to hand palpate the breast filets as they are passing on a conveyor to sense filet hardness and subsequently classify them into ranked severities on processor specific thresholds making industry wide protocols non-existent (Table 1). Personal observations and discussions with United States poultry processors indicate that handpalpation is a subjective evaluation method, can give falsepositive/negative WB scores, pass-on WB to customers, and remove high-valued normal filets thus affecting the profits and quality. Hand-palpation is also costly since 8-10 additional trained personnel will have to be employed per line to sort WB filets. A standard objective measure is needed for industry. Other technologies are available that can provide data rich objective measures of products at the cellular level and these may bridge the gap needed for industry to utilize an industry wide objective measure. Recently, Wold et al. (2017) reported the successful application of near infrared spectroscopy as an on-line method to detect WB in processing plants.

Bioelectrical Impedance Analysis (BIA) is a technology that has been proven to measure many different properties at the cellular level and has been used extensively on a number of organisms ranging from fish to humans and may
TABLE 1 | Various scales used to categorize woody breast.

\begin{tabular}{|c|c|c|}
\hline 2-point scale & 3-point scale & $\begin{array}{l}\text { 4-point scale Tijare et al., } \\
2016\end{array}$ \\
\hline $\begin{array}{l}0 \text { - Normal (no woody } \\
\text { breast or toughness) }\end{array}$ & $\begin{array}{l}0 \text { - Normal (no woody } \\
\text { breast or toughness) }\end{array}$ & $\begin{array}{l}0 \text { - Normal (filets flexible } \\
\text { throughout) }\end{array}$ \\
\hline \multirow[t]{3}{*}{$\begin{array}{l}1-\text { Woody breast (tough } \\
\text { filet) }\end{array}$} & $\begin{array}{l}1 \text { - Medium woody } \\
\text { (upto } 50 \% \text { woody) }\end{array}$ & $\begin{array}{l}1 \text { - Mild (filets hard mainly } \\
\text { in the cranial region but } \\
\text { flexible otherwise) }\end{array}$ \\
\hline & $\begin{array}{l}2 \text { - Severe woody } \\
\text { (>50\% woody) }\end{array}$ & $\begin{array}{l}2 \text { - Moderate (filets } \\
\text { extremely hard and rigid } \\
\text { through from cranial region } \\
\text { of caudal tip filets that were } \\
\text { hard throughout but flexible } \\
\text { in mid-to caudal region) }\end{array}$ \\
\hline & & $\begin{array}{l}3 \text { - Severe (>50\% of filet } \\
\text { area is woody) }\end{array}$ \\
\hline
\end{tabular}

provide a solution for WB detection. It provides instant results, is easy to use and objective. BIA measures the electrical properties of both living and harvested plants and animals. Electrical properties measured are resistance and reactance which dependend on changes in cell membrane/wall integrity and extraand intracellular fluids (Kyle et al., 2004). Although initially designed as a human medical device, BIA has been used to extensively to non-invasively measure proximate composition, health, and freshness of fish and meats (Swatland, 2002; Cox and Hartman, 2005; Chevalier et al., 2006). A new application of BIA may be to detect WB myopathy. The objective of this study was to conduct a proof-of-concept research to evaluate if WB leads to alteration in bioelectrical properties such that BIA can be used to detect filets. Since processors differentiate breast filets based on varying severity levels of WB myopathy, experiments were conducted to determine if BIA could differentiate between those severity levels. It would also be beneficial for the processors to detect which segment of the breast filet has WB so that it can be excised, and the rest of the filet sold at a higher price. Segmental BIA analysis of the cranial, medial and caudal segments of the intact filets was conducted to determine if BIA can differentiate between normal and WB categories.

\section{MATERIALS AND METHODS}

\section{Experiment 1 Proof-of-Concept}

Freshly deboned breast filets were sorted into normal and severe WB meat and analyzed for bioelectrical impedance (resistance and reactance), cook loss, and texture.

\section{Broiler Breast Meat}

Freshly deboned ( $2-3 \mathrm{~h}$ post-mortem) butterfly breast filets from 8-wk broilers (all male; Ross 708; and 8-9 lbs live weight) were obtained from a local broiler processor. All samples were transported to the Department of Poultry Science, Auburn University under refrigeration and analyzed (except texture) within 2-3 h. The left filet from each the butterfly filet was cut 
and were immediately separated into normal (no WB) and severe WB using hand-palpation method (Tijare et al., 2016).

\section{Bioelectrical Impedance Analysis}

Left-side filet was used to measure bioelectric properties using the hand-held BIA equipment (Seafood Analytics, Clinton Town, MI, United States) on the dorsal side of the filet (Figure 1). The BIA unit consists of two signal electrodes and two detecting electrodes that introduce an $800 \mu \mathrm{A}, 50 \mathrm{kHz}, \mathrm{AC}$ current capable of voltage changes between $3.75-10.60 \mathrm{~V}$. The four electrodes are connected to the product using food grade stainless compression electrodes (RJL Systems, Detroit, MI, United States). A fourelectrode array is used to approximate parallel field lines within the tissue, negate product electrode interfaces, and approximate a cylindrical shape. Once the electrodes are in contact with the product, the circuit is connected, and the device takes two measures, resistance and reactance. Data was collected on the dorsal surface (feather side) of the filets weighing $464 \pm 66 \mathrm{~g}$.

\section{Cook Loss}

Left-side filet used for BIA measurement was further used for cook loss analysis immediately $(<15 \mathrm{~min})$ after BIA measurement (approximately $5 \mathrm{~h}$ post-mortem). Cook loss is expressed as weight loss after cooking the thigh relative to its initial weight. Briefly, individual filet were weighed, placed on a raised stainless steel wire rack in a stainless-steel pan $(53.02 \times 32.54 \times 10.16 \mathrm{~cm}$; Vollrath Co., LLC, Sheboygan, WI, United States), covered with aluminum foil and cooked in a pre-heated $\left(176.6^{\circ} \mathrm{C}\right)$ forced air convection oven (Vulcan HEC5D, Troy, $\mathrm{OH}$, United States) to an internal temperature of $74^{\circ} \mathrm{C}$ measured using a stainless-steel digital thermometer (Taylor 1470FS Digital cooking thermometer and Kitchen Timer, Las Cruces, NM, United States). After cooking, the filets were cooled to room temperature $\left(22 \pm 2^{\circ} \mathrm{C}\right)$ in the covered pans

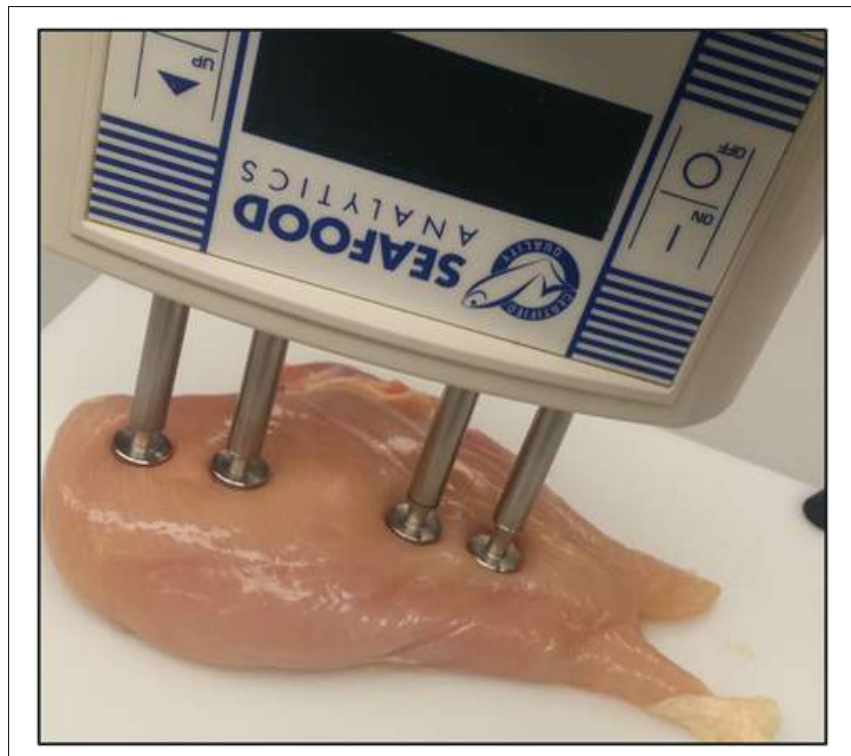

FIGURE 1 | Bioelectrical impedance analysis of whole breast filet. and then reweighed. Cook loss was calculated using the following formula:

$$
\begin{aligned}
& \text { Cook loss }(\%) \\
& =100 \times \frac{(\text { Initial weight of thigh }- \text { cooked weight of thigh) }}{(\text { Initial weight of thigh) }}
\end{aligned}
$$

(Kennedy-Smith et al., 2017).

\section{Texture Analysis}

The cooked filet used for cook loss analysis was placed individually in separate zip-loc bags and stored overnight at $4^{\circ} \mathrm{C}$. The filets were tempered at room temperature for 3$4 \mathrm{~h}$ and analyzed for texture using the Blunt Mullenet-Owens Razor Shear (BMORS) method Texture Analyzer (Model TXXT2i, Technologies, Scarsdale, NY, United States; Lee et al., 2008; Morey and Johnson, 2019).

\section{Statistical Analysis}

Breast filets (15 filets/severity/trial) belonging to normal and severe WB category were obtained and the experiment was conducted in 3 separate replicate trials (15 filets $\times 2$ severity levels $\times 3$ replicate trials $=90$ filets) were used throughout the study. Replications were tested using one-way ANOVA with Tukeys HSD $(p<0.05)$. to determine significant differences between replications. In the absence of significant differences in replications, the data from all replications was combined and analyzed together. Data was analyzed one-way ANOVA with Tukeys HSD to determine significant differences at $p<0.05$.

\section{Experiment 2}

\section{Segmental Bioelectrical Impedance Analysis}

Freshly deboned chicken breast filets were randomly collected (without sorting into WB categories; all male; Ross 708; 89 lbs live weight) from the same poultry processor. The filets were stored for $18 \mathrm{~h}$ at $4^{\circ} \mathrm{C}$ prior to the analysis. Each filet was sorted into one of the WB categories $(0,1,2$, and 3$)$ and the cranial segment was pinched to evaluate the turgor to determine the presence/absence of spaghetti meat. The filet was then analyzed for bioelectrical impedance (resistance and reactance) as per the method above. Each filet was visually divided into three segments, cranial, medial, and caudal region and hand-palpated to determine if the segment was normal (no perceivable hardness) and woody (perceived hardness). Each segment was then analyzed for bioelectrical impedance (resistance and reactance) using two sets of small $(5.08 \mathrm{~cm}$ ) compression electrodes with $1 \mathrm{~cm}$ between signaling and receiving electrode (Figure 2).

\section{Statistical Analysis}

Freshly processed 120 random filets were collected on three separate processing days and analyzed for bioelectrical impedance. Data was combined from all processing days and analyzed for differences in resistance and reactance of different WB categories of the whole filet and the segments with and without spaghetti meat using one-way ANOVA with 


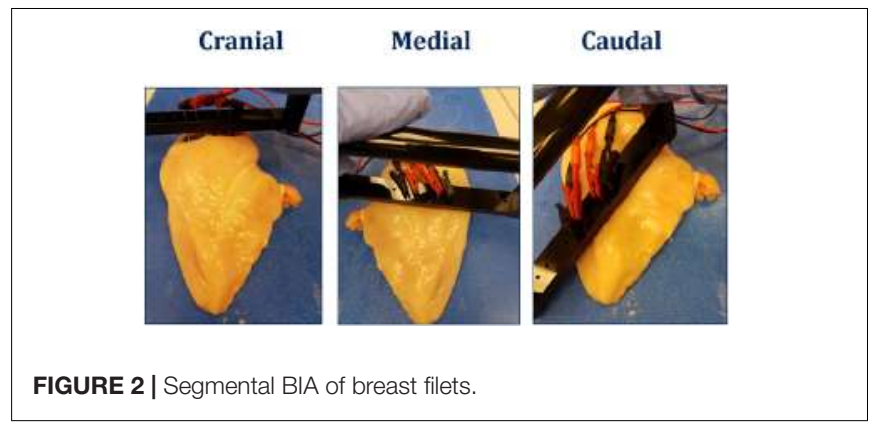

Tukey's HSD to separate means at $p<0.05$. The segmental BIA data (resistance and reactance) on the cranial and caudal region with and without spaghetti meat was analyzed using linear discriminant analysis (LDA) with $60 \%$ of the data used for training and $40 \%$ was used for validation. The prediction accuracy (\%) and error (\%) of the model to differentiate each segment into normal and woody was determined (Wold et al., 2017).

\section{RESULTS}

\section{Experiment 1}

The differences in the quality characteristics and the bioelectrical impedance parameters is given in Table 2. Breast filets affected with severe woody (SW) breast myopathy WB have a significantly higher $(p \leq 0.05)$ cook loss $(41.17 \%)$ compared to normal breast filets $(35.16 \%)$ indicating that WB leads to reduced water holding capacity of the meat (Table 2). BMORS texture analysis indicated that WB meat had significantly higher $(p \leq 0.05)$ peak counts (10.77) compared to normal breast meat (5.45). However, the peak shear force and total energy for normal meat were higher $(p \leq 0.05)$ than severe WB meat. Bioelectrical properties (resistance and reactance) were measured using the hand-held CQ Reader (Table 2). Overall, data indicates that there was a significant difference $(p \leq 0.05)$ in resistance (Normal: $72.18 \Omega$ vs Woody: $78.27 \Omega$ ), and reactance (Normal: $28.04 \Omega$ vs Woody: $37.54 \Omega$ ) of breast meat affected by WB myopathy.

\section{Experiment 2}

Freshly procured breast filets were hand-palpated to separate them into varying WB categories (Tijare et al., 2016; Table 1) and detected for the presence of spaghetti meat. Out of the total 360 filets analyzed, there were $15.56 \%$ normal, $22.5 \%$ mild, $35.56 \%$ moderate, and $35.56 \%$ severe (Table 3 ). The resistance and reactance values were overall significantly different. Normal and WB meat had significantly different resistance (74.19 vs 69.43 , respectively), and reactance ( 19.58 vs $23.49 \Omega$, respectively; Table 3). However, there were not significant differences $(p>0.05)$ in the resistance between mild, moderate and severe WB. No significant differences in reactance were observed between normal, mild and moderate WB (Table 3). Similar data trends in resistance and reactance were observed when the data of whole filets without spaghetti meat was removed (Table 3). A total of 73 filets out of 360 had spaghetti breast myopathy.
TABLE 2 | Differences in cook loss, texture (peak count, shear force, and shear energy) and BIA values (resistance and reactance) of normal and severe woody breast filets (mean \pm std dev).

\begin{tabular}{lccc}
\hline Variable & Normal $(\boldsymbol{n}=\mathbf{4 5})$ & Severe woody $(\boldsymbol{n}=\mathbf{4 5})$ & $\boldsymbol{p}$-value \\
\hline Cook loss $(\%)$ & $35.16 \pm 3.09$ & $41.17 \pm 4.36$ & $<0.0001$ \\
Peak count & $5.45 \pm 2.32$ & $10.77 \pm 2.33$ & $<0.0001$ \\
Peak shear force $(N)$ & $21.66 \pm 3.43$ & $18.17 \pm 4.90$ & 0.0004 \\
Total energy $(N . \mathrm{mm})$ & $277.47 \pm 39.50$ & $242.07 \pm 64.77$ & 0.0043 \\
Resistance $(R ; \Omega)$ & $72.18 \pm 12.86$ & $78.27 \pm 11.14$ & 0.028 \\
Reactance $\left(X_{\mathrm{C}} ; \Omega\right)$ & $28.04 \pm 10.22$ & $37.54 \pm 9.86$ & $<0.0001$ \\
\hline
\end{tabular}

TABLE 3 | BIA analysis of whole breast filets analyzed with and without the filets containing spaghetti meat.

\begin{tabular}{|c|c|c|c|c|c|c|}
\hline \multirow[b]{2}{*}{$\begin{array}{l}\text { Woody breast } \\
\text { score }\end{array}$} & \multicolumn{3}{|c|}{$\begin{array}{l}\text { Whole filets with } \\
\text { spaghetti meat }\end{array}$} & \multicolumn{3}{|c|}{$\begin{array}{l}\text { Whole filets without } \\
\text { spaghetti meat }\end{array}$} \\
\hline & $n$ & Resistance & Reactance & $n$ & Resistance & Reactance \\
\hline Normal & 55 & $74.19^{A}$ & $19.58^{\mathrm{B}}$ & 31 & $70.71^{A}$ & $19.10^{\mathrm{B}}$ \\
\hline Mild & 80 & $66.09^{B}$ & $17.62^{\mathrm{B}}$ & 50 & $65.67^{\mathrm{B}}$ & $18.11^{\mathrm{B}}$ \\
\hline Moderate & 93 & $66.37^{\mathrm{B}}$ & $20.60^{A B}$ & 79 & $66.18^{\mathrm{B}}$ & $20.59^{B}$ \\
\hline Severe & 129 & $69.43^{\mathrm{B}}$ & $23.49^{A}$ & 124 & $66.12^{A B}$ & $23.36^{A}$ \\
\hline$p$-value & & 0.0002 & $<0.0001$ & & 0.0013 & $<0.0001$ \\
\hline CV $(\%)^{*}$ & & 13.38 & 32.04 & & 13.69 & 31.94 \\
\hline
\end{tabular}

Superscripts $A-B$ indicate significant differences $(p<0.05)$ between woody breast scores in each column. ${ }^{*}$ Coefficient of Variation (\%).

Overall, the normal and $\mathrm{WB}$ occurrence in the cranial region was 27.78 and $72.22 \%$, respectively, medial region 40.56 and $31.67 \%$, respectively, and caudal regions was 50 and $50 \%$, respectively. Spaghetti breast was prevalent mostly (24-32\%) in the normal segments while its occurrence was lower in the woody segment $(10-18 \%)$. Contrary to the whole filet resistance and reactance, the normal segments had a lower resistance compared to the woody segments. Resistance and reactance of the cranial and medial segments, respectively, were significantly different while they were not significantly different for the caudal region. Removal of the resistance and reactance data from spaghetti meat segments did not alter the differences in normal and WB meat (Table 4). However, LDA of the cranial segment data using resistance and reactance could accurately predict normal and woody segments at 68.69 and $57.75 \%$, respectively. However, when the spaghetti meat data was removed, the prediction accuracy of both normal and woody segments increased by approx. 3\%. When resistance and reactance data from the cranial segment affected by spaghetti meat was analyzed, the model was able to predict normal and WB at 52-54\% accuracy (Table 5).

\section{DISCUSSION}

\section{Experiment 1}

Woody breast myopathy is a major meat quality issue in the poultry industry. At the macro level, raw WB meat is hard to touch while Aguirre et al. (2018) reported that taste panelists described cooked WB meat crunchy and fibrous. At 
TABLE 4 | BIA Analysis of breast filet segments analyzed with and without filets containing spaghetti meat.

\begin{tabular}{|c|c|c|c|c|c|c|c|}
\hline \multirow[t]{2}{*}{ Breast filet segment } & \multirow[t]{2}{*}{ Woody breast presence } & \multicolumn{3}{|c|}{ Filet segments with spaghetti meat } & \multicolumn{3}{|c|}{ Filet segments without spaghetti meat } \\
\hline & & $n$ & Resistance $(\Omega)$ & Reactance $(\Omega)$ & $n$ & Resistance $(\Omega)$ & Reactance $(\Omega)$ \\
\hline \multirow[t]{4}{*}{ Cranial } & Normal & 100 & $74.96^{\mathrm{B}}$ & $18.24^{\mathrm{B}}$ & 56 & $74.76^{\mathrm{B}}$ & $17.47^{\mathrm{B}}$ \\
\hline & Woody & 260 & $84.20^{\mathrm{A}}$ & $21.42^{\mathrm{A}}$ & 228 & $85.95^{\mathrm{A}}$ & $22.20^{\mathrm{A}}$ \\
\hline & $p$-value & & $<0.0001$ & 0.0047 & & $<0.0001$ & 0.0013 \\
\hline & $\mathrm{CV}(\%)^{\star}$ & & 17.71 & 45.88 & & 17.10 & 45.58 \\
\hline \multirow[t]{4}{*}{ Medial } & $\mathrm{N}$ & 146 & $67.47^{\mathrm{B}}$ & $15.63^{\mathrm{B}}$ & 97 & $67.37^{\mathrm{B}}$ & $15.71^{\mathrm{B}}$ \\
\hline & W & 114 & $76.82^{\mathrm{A}}$ & $18.33^{\mathrm{A}}$ & 86 & $78.06^{\mathrm{A}}$ & $18.34^{\mathrm{A}}$ \\
\hline & $p$-value & & $<0.0001$ & 0.0013 & & $<0.0001$ & 0.0075 \\
\hline & $\mathrm{CV}(\%)^{*}$ & & 17.33 & 44.98 & & 17.34 & 44.74 \\
\hline \multirow[t]{4}{*}{ Caudal } & $\mathrm{N}$ & 180 & $80.13^{A}$ & $18.40^{\mathrm{A}}$ & 122 & $80.85^{\mathrm{A}}$ & $18.26^{\mathrm{A}}$ \\
\hline & W & 180 & $81.38^{A}$ & $18.60^{A}$ & 161 & $83.00^{A}$ & $18.78^{A}$ \\
\hline & $p$-value & & 0.4148 & 0.8345 & & 0.2167 & 0.6227 \\
\hline & $\mathrm{CV}(\%)^{*}$ & & 18.00 & 47.62 & & 17.55 & 46.74 \\
\hline
\end{tabular}

Superscripts ${ }^{A-B}$ indicate significant differences $(p<0.05)$ between normal and woody breast for each segment (cranial, medial, and caudal). ${ }^{\star}$ Coefficient of Variation (\%).

the molecular level, WB is characterized with the conformational changes in muscle protein lead to higher extra-myofibrillar water in WB compared to normal meat (Tasoniero et al., 2017). Previous research conducted in our lab using 7-T Magnetic Resonance Imaging of woody and normal breast filets showed that the WB filets had had significantly higher interstitial water compared to normal filets (Kennedy-Smith et al., 2017) which potentially alters the bioelectrical properties of breast filets.

The differences in the intra- and extra-cellular water can impact meat quality parameters such as cook loss. Higher cook loss in WB filets may also indicate higher levels of free water that can be easily removed from meats due to cooking. Higher levels of free water can be a result of increased accumulation of collagen in the WB filets preventing the binding of free water to the myofibrillar proteins (Kennedy-Smith et al., 2017). Textural differences in WB (Table 2) can be attributed to the alternation in muscle architecture. Histological analysis of WB indicates fibrosis, perimysial thickening, proliferation of connective tissue and a higher collagen content (Soglia et al., 2016) which can potentially contribute to differences in texture of the WB meat. Similar to the current study Solo (2016) also observed that the total shear energy increased as the severity of the filets increased from normal to severe WB. Moreover, the author stated that the normal filets had a peak count of 5.73 which was significantly

TABLE 5 | Discriminate analysis of woody and normal classification in filet segments to determine accuracy and error.

\begin{tabular}{lllllll}
\hline & \multicolumn{2}{c}{ Normal } & & \multicolumn{2}{c}{ Woody } \\
\cline { 2 - 3 } \cline { 6 - 7 } & & \% Accuracy & \% Error & & \% Accuracy & \% Error \\
\hline Cranial segment (all filets) & 68.69 & 31.31 & & 57.75 & 42.25 \\
Cranial segment (no spaghetti) & 72.73 & 27.27 & & 61.33 & 38.67 \\
Cranial segment (only spaghetti) & 52.27 & 47.73 & & 54.55 & 45.45 \\
Medial segment (all data) & 70.55 & 29.45 & & 54.42 & 45.58 \\
Medial segment (no spaghetti) & 73.53 & 26.47 & & 59.59 & 50.44 \\
Medial segment (only spaghetti) & 57.14 & 42.86 & & 11.11 & 88.89
\end{tabular}

lower than severe WBs (8.79) which was similar to the current research. Peak count measurements obtained through BMORS method can distinguish between normal and WB filets.

Cook loss and texture analysis are destructive methods to differentiate between normal and WB. Moreover, these methods are laboratory intensive and are not suitable for in-plant application. Hence the hand-held BIA equipment was used to determine if there are bioelectrical differences between normal and WB meat such that those difference can be further used to detect WB from normal breast meat.

Resistance $\left(R_{\mathrm{S}}\right)$ measures the ability of a substance to conduct electricity while reactance $\left(X_{\mathrm{c}}\right)$ measures its ability to hold a charge (Lukaski, 1987) which are influenced by the biochemical composition of food (Kyle et al., 2004; Hafs and Hartman, 2011). As observed in the cook loss data (Table 2), the changes in the WB muscle architecture can influence the disposition of water within the tissue which can result in alteration in the electrical properties of the meat. It was observed that normal breast filets had lower Rs and Xc (72.18 $\Omega$ and $28.04 \Omega$, respectively) compared to severe WB filets (78.27 $\Omega$ and $37.54 \Omega$, respectively). The resistance is impacted by intra- and extracellular water while reactance mainly arises from cell membranes (Kyle et al., 2004). Cells do not conduct electricity at low frequencies and hence act as insulators forcing the current to pass through the extracellular fluid, which was higher in severe WB (see section "Cook Loss" Table 2), thus increasing the resistance of the muscle (Kyle et al., 2004). Alternatively, Kyle et al. (2004) also state that increase in the suspended non-conducting material will increase the resistance of the conducting water. In case of WB breast myopathy, the nonconducting material could be connective tissue infiltration and granulation tissue (Sihvo et al., 2014; Velleman, 2015; Soglia et al., 2016) which increases the resistance of the meat. These changes in the muscle combined with differences in intra- and extra-cellular water influence the differences in the resistance and reactance of WB compared to the normal meat.

The proof-of-concept research indicates that BIA has the potential to be used as an effective tool to detect severe WB filets at a processing plant. 


\section{Experiment 2}

Tijare et al. (2016) noted that based on the severity, WB can be classified into normal, mild, moderate and severe (Table 2). Poultry processors have favored the 4-tier classification as they can remediate their losses by staggering the price or the utilization of breast meat depending on the severity level. It would be further beneficial for the poultry processors to determine which section of the breast filet has woody characteristics so that they can salvage the remaining breast filet and sell it at a higher cost. Since hand-palpation is very laborious and subjective, and that based on Experiment 1, we had demonstrated that BIA can be used to differentiate between severe and normal WB, a study was conducted to determine if BIA can be used to detect varying WB severities as well as segments of the breast filet that are affected by WB.

When compared to experiment 1 , the normal breast filet in experiment 2 had similar resistance values, however, WB resistance reduced to approx. $69 \Omega$. This difference could be explained by the differences in the experimental setup. Compared to experiment 1 where the filets were analyzed within 6 -h postslaughter, in experiment 2 the filets were stored in the refrigerator for $18 \mathrm{~h}$. Given the fact that the WB filets have higher extracellular water and modified muscle architecture, it exhibits higher drip loss during the first $24 \mathrm{~h}$ compared to the normal meat (Mudalal et al., 2015; Tasoniero et al., 2017; Sun et al., 2018) resulting in lower resistance in the stored WB meat in experiment 2 . Significantly higher resistance of normal breast filet, similar to experiment 1 , indicates that normal meat had a higher water holding capacity even after storage compared to WB. Higher reactance in $\mathrm{WB}$ meat can be attributed to the connective tissue and fibrosis, acting as insulators (Kyle et al., 2004). Accidental findings during the project indicated that presence of spaghetti breast myopathy (loose muscle fibers) can influence the resistance of the meat indicating that the lose muscle fibers in spaghetti meat act as insulators thus increasing the resistance values. The overlap in the varying severity levels can be explained by the design of the CQ Reader which has 2-sets of electrodes at set distances wherein each set takes replicate readings which are averaged and then presented as the data for the whole filet. However, with the varying severity WB levels, different regions in the breast filet may or may not have woody tissue and averaging the data for the entire filet can mask the effect of the differences in electrical properties of those regions. BIA measurement of segments of breast filet can provide a clearer picture of the presence of WB in each segment. The other major reason for the overlap could also be the difficulty in accurately detecting WB severities by hand-palpation especially after $18 \mathrm{~h}$ post-slaughter storage which impacts texture and inter- and extra-cellular water of the meat (Sun et al., 2018).

Segmental BIA of the cranial segment had the lowest percentage $(27.78 \%)$ of normal breast followed by caudal $(50 \%)$ and medial segments (40\%). This shows that if the woody segment in the cranial region is accurately detected, the processor can remove that segment and still utilize the remaining breast filet. Significant differences were observed between normal and woody segments in terms of resistance and reactance. Contrary to the whole breast filet BIA, segmental BIA indicates higher resistance for woody than normal while the reactance pattern remains the same. Segmental BIA measures electrical properties of a localized area and had higher reactance indicating increased non-conducting material (fibers, connective, and granular tissue; Sihvo et al., 2014; Velleman, 2015; Soglia et al., 2016) in the water which would have resulted in higher resistance.

Discriminant analysis conducted using resistance and reactance values indicated that BIA can be used to differentiate between the normal and woody - cranial and medial segments. Although the resistance and reactance data with and without spaghetti were similar for each segment, removal of data of the spaghetti meat affected cranial and medial segments increased the prediction accuracy of normal and woody meat by approximately 3\% (Table 5). Accidental finding of spaghetti breast meat and its electrical properties could be of high interest to the poultry industry. Significant differences in the BIA properties of breast meat with and without spaghetti meat indicate that the new myopathy should be taken into consideration while developing predictive models for WB myopathy using bioelectrical properties.

Bioelectrical impedance analysis can be used as a tool to differentiate between normal and severe breast meat. However, additional efforts are needed to further define and increase the accuracy of BIA to differentiate between varying severity levels. Increased sample number can potentially improve the discrimination ability of the model. Segmental BIA could be used as a tool to detect woody segments in the filets thus providing more granular data as well as better understanding of the spread of the myopathy in the muscle. The accidental finding on the interference of spaghetti breast meat in detecting WB can open new research areas to explore the ability of BIA to detect spaghetti breast myopathy. The research also demonstrates that BIA can alter with the freshness of the filets and each processor must develop the resistance and reactance threshold values based on their process. In its current state, the hand-held device can be used as a near-line technology by quality assurance departments to detect WB prevalence. Moreover, the data obtained by the processors can be used to study WB prevalence between flocks, different nutrition regime, as well as management practices. Further processors can use BIA technology to separate WB meat from the normal meat. Overall, the hand-held BIA technology can help in reducing consumer compliants due to WB.

\section{DATA AVAILABILITY STATEMENT}

The datasets generated for this study are available on request to the corresponding author.

\section{AUTHOR CONTRIBUTIONS}

AM is the lead PI who conceptualized the idea of using bioelectrical impedance analysis to detect woody breast, secured funding, and actively conducted the research. AS conducted the proof-of-concept experiment (Experiment 1) mentioned in the manuscript. LG conducted experiments, collected data 
and analyzed it. MC is the co-developer of the patented CQ Reader used for BIA experiments throughout the project, and supported with data analysis, technical knowledge, and writing manuscript. All authors contributed to the article and approved the submitted version.

\section{REFERENCES}

Aguirre, M. E., Owens, C. M., Miller, R. K., and Alvarado, C. Z. (2018). Descriptive sensory and instrumental texture profile analysis of woody breast in marinated chicken. Poult. Sci. 97, 1456-1461. doi: 10.3382/ps/pex428

Bilgili, S. (2013). Broiler Chicken Myopathies: II. Woody Breast? Available at: http: //poul.auburn.edu/wpcontent/uploads/sites/13/2014/09/WOGSAPR13.pdf. (accessed February 8, 2020)

Brot, S., Perez, S., Shivaprasad, H. L., Baiker, K., Polledo, L., Clark, M., et al. (2016). Short communication: wooden breast lesions in broiler chickens in the UK. Vet. Record 178:141.

Chevalier, D., Ossart, F., and Ghommidh, C. (2006). Development of a nondestructive salt and moisture measurement method in salmon (Salmo salar) fillets using impedance technology. Food Control 17, 342-347. doi: 10.1016/j. foodcont.2004.11.009

Cox, M. K., and Hartman, K. J. (2005). Nonlethal estimation of proximate composition in fish. Canad. J. Fish. Aquat. Sci. 62, 269-275. doi: 10.1139/f0 4-180

Hafs, A. W., and Hartman, K. J. (2011). Influence of electrode type and location upon bioelectrical impedance analysis measurements of brook trout. Trans. Am. Fish. Soc. 140, 1290-1297. doi: 10.1080/00028487.2011.620482

Kennedy-Smith, A., Johnson, M. L., Bauermeister, L. J., Cox, M. K., and Morey, A. (2017). Evaluating a novel bioelectric impedance analysis technology for the rapid detection of wooden breast myopathy in broiler breast filets. Int. Poultry Sci. 96:252.

Kyle, U. G., Bosaeus, I., De Lorenzo, A. D., Deurenberg, P., Elia, M., Gómez, J. M. et al. (2004). Bioelectrical impedance analysis-part I: review of principles and methods. Clin. Nutr. 23, 1226-1243. doi: 10.1016/j.clnu.2004.06.004

Larsen, H. D., Blaabjerg, L. O., Brandt, P., Young, J. F., Rasmussen, M. K., Pedersen, J. R. et al. (2016). The occurrence of wooden breast in a danish flock of broiler chickens. In Proceeding of the XXV World's Poultry Congress (WPC2016). Beijing: World's Poultry Congress.

Lee, Y. S., Owens, C. M., and Meullenet, J. F. (2008). The meullenet-owens razor shear (mors) for predicting poultry meat tenderness: its applications and optimization. J. Texture Stud. 39, 655-672. doi: 10.1111/j.1745-4603.2008. 00165.x

Lukaski, H. C. (1987). Methods for the assessment of human body composition: traditional and new. Am. J. Clin. Nutr. 46, 537-556. doi: 10.1093/ajcn/46.4.537

Morey, A., and Johnson, M. L. (2019). Effects of extended deboning time on the breast meat quality of fast-growing big broilers. MOJ Food Process Technol. 7, 121-126.

Mudalal, S., Lorenzi, M., Soglia, F., Cavani, C., and Petracci, M. (2015). Implications of white striping and wooden breast abnormalities on quality traits of raw and marinated chicken meat. Animal 9, 728-734. doi: 10.1017/ s175173111400295x

National Chicken Council (2020a). Per Capita Consumption of Poultry and Livestock, 1965 to Forecast 2020, in Pounds. Available at: http: //www.nationalchickencouncil.org/about-the-industry/statistics/per-capitaconsumption-of-poultry-and-livestock-1965-to-estimated-2012-in-pounds/. (accessed, February, 03, 2020)

National Chicken Council (2020b). U.S. Broiler Production. http://www. nationalchickencouncil.org/about- the-industry/statistics/u-s-broilerproduction/. (accessed, February, 03, 2020)

\section{FUNDING}

The authors would like to thank the US Poultry and Egg Association and the Auburn University's LAUNCH Innovation Grants Program for providing research funding.

Petracci, M., Mudalal, S., Soglia, F., and Cavani, C. (2015). Meat quality in fastgrowing broiler chickens. Worlds Pou. Sci. J. 71, 363-374. doi: 10.1017/ s0043933915000367

Sihvo, H. K., Immonen, K., and Puolanne, E. (2014). Myodegeneration with fibrosis and regeneration in the pectoralis major muscle of broilers. Vet. Pathol. 51, 619-623. doi: 10.1177/0300985813497488

Soglia, F., Mudalal, S., Babini, E., Di Nunzio, M., Mazzoni, M., Sirri, F., et al. (2016). Histology, composition, and quality traits of chicken pectoralis major muscle affected by wooden breast abnormality. Poult. Sci. 95, 651-659. doi: $10.3382 / \mathrm{ps} / \mathrm{pev} 353$

Solo, J. (2016). Meat Quality and Sensory Analysis of Broiler Breast Fillets with Woody Breast Muscle Myopathy. M.S. Thesis, Univ. Arkansas, Fayetville.

Sun, X., Koltes, D. A., Coon, C. N., Chen, K., and Owens, C. M. (2018). Instrumental compression force and meat attribute changes in woody broiler breast fillets during short-term storage. Poult. Sci. 97, 2600-2606. doi: 10.3382/ ps/pey107

Swatland, H. J. (2002). “On-line monitoring of meat quality," in Pages 193-216 in Meat Processing: Improving Quality, eds J. Kerry, J. Kerry, and D. Ledward (Cambridge: CRC Press).

Tasoniero, G., Bertram, H. C., Young, J. F., Dalle Zotte, A., and Puolanne, E. (2017). Relationship between hardness and myowater properties in wooden breast affected chicken meat: a nuclear magnetic resonance study. LWT 86, 20-24. doi: 10.1016/j.lwt.2017.07.032

Tijare, V. V., Yang, F. L., Kuttappan, V. A., Alvarado, C. Z., Coon, C. N., and Owens, C. M. (2016). Meat quality of broiler breast fillets with white striping and woody breast muscle myopathies. Poult. Sci. 95, 2167-2173. doi: 10.3382/ps/pew129

Trocino, A., Piccirillo, A., Birolo, M., Radaelli, G., Bertotto, D., Filiou, E., et al. (2015). Effect of genotype, gender and feed restriction on growth, meat quality and the occurrence of white striping and wooden breast in broiler chickens. Poult. Sci. 94, 2996-3004. doi: 10.3382/ps/pev296

Velleman, S. G. (2015). Relationship of skeletal muscle development and growth to breast muscle myopathies: a review. Avian. Dis. 59, 525-531. doi: 10.1637/ 11223-063015-review.1

Velleman, S. G., and Clark, D. L. (2015). Histopathologic and myogenic gene expression changes associated with wooden breast in broiler breast muscles. Avian. Dis. 59, 410-418. doi: 10.1637/11097-042015-reg.1

Wold, J. P., Veiseth-Kent, E., Høst, V., and Løvland, A. (2017). Rapid online detection and grading of wooden breast myopathy in chicken fillets by near-infrared spectroscopy. PloS One 12:e0173384. doi: 10.1371/journal.pone. 0173384

Conflict of Interest: MC was employed by the company CQ Foods.

The remaining authors declare that the research was conducted in the absence of any commercial or financial relationships that could be construed as a potential conflict of interest.

Copyright (c) 2020 Morey, Smith, Garner and Cox. This is an open-access article distributed under the terms of the Creative Commons Attribution License (CC BY). The use, distribution or reproduction in other forums is permitted, provided the original author(s) and the copyright owner(s) are credited and that the original publication in this journal is cited, in accordance with accepted academic practice. No use, distribution or reproduction is permitted which does not comply with these terms. 
OPEN ACCESS

Edited by:

Walter Gay Bottje,

University of Arkansas, United States

Reviewed by:

Paweł Tomasz Maækowiak,

Poznań University of Life Sciences,

Poland

Carl Joseph Schmidt,

University of Delaware, United States

*Correspondence:

Gale M. Strasburg

stragale@msu.edu

†These authors have contributed equally to this work

Specialty section:

This article was submitted to

Avian Physiology,

a section of the journa

Frontiers in Physiology

Received: 31 March 2020

Accepted: 28 July 2020

Published: 14 August 2020

Citation:

Horton $K A$, Sporer $K R B$,

Tempelman RJ, Malila Y, Reed KM,

Velleman SG and Strasburg GM

(2020) Knockdown

of Death-Associated Protein

Expression Induces Global

Transcriptome Changes in Proliferating and Differentiating

Muscle Satellite Cells.

Front. Physiol. 11:1036.

doi: 10.3389/fphys.2020.01036

\section{Knockdown of Death-Associated Protein Expression Induces Global Transcriptome Changes in Proliferating and Differentiating Muscle Satellite Cells}

Katherine A. Horton ${ }^{1 t}$, Kelly R. B. Sporer ${ }^{2 \dagger}$, Robert J. Tempelman², Yuwares Malila ${ }^{3}$, Kent M. Reed ${ }^{4}$, Sandra G. Velleman ${ }^{5}$ and Gale M. Strasburg ${ }^{1 *}$

${ }^{1}$ Department of Food Science and Human Nutrition, Michigan State University, East Lansing, MI, United States, ${ }^{2}$ Department of Animal Science, Michigan State University, East Lansing, MI, United States, ${ }^{3}$ National Center for Genetic Engineering and Biotechnology (BIOTEC), Thailand Science Park, Pathum Thani, Thailand, ${ }^{4}$ Department of Veterinary and Biomedical Sciences, University of Minnesota, Saint Paul, MN, United States, ${ }^{5}$ Department of Animal Sciences, Ohio Agricultural Research and Development Center, The Ohio State University, Wooster, OH, United States

Death-associated protein (DAP) undergoes substantial changes in expression during turkey skeletal muscle development, decreasing from the 18 day embryonic stage to 1 day posthatch, and again from 1 day posthatch to 16 weeks of age. These changes suggest that DAP plays an important role at critical stages of the developmental process. The objective of this study was to elucidate the role of DAP in muscle development by examining the effect of reduced DAP expression on global gene expression in proliferating and differentiating turkey pectoralis major muscle satellite cells. Small interfering RNA was used to knock down expression of DAP and the transcriptome was subsequently profiled using a turkey skeletal muscle long oligonucleotide microarray. Microarray data were corroborated using quantitative real-time PCR. In proliferating cells, 458 loci, resulting in 378 uniquely annotated genes, showed differential expression (false discovery rate, FDR < 0.05). Pathway analysis highlighted altered eukaryotic translational initiation factors (elFs) signaling, protein ubiquitination, sirtuin signaling, and mechanistic target of rapamycin (mTOR) signaling as the primary pathways affected in the knockdown proliferating cells. The findings underpinned the potential DAP involvement in cell proliferation of turkey satellite cells through the coordination between protein synthesis and cell cycle. In differentiating cells, 270 loci, accounting for 189 unique genes, showed differential expression (FDR $<0.05$ ). Decreased expression of genes encoding various myofibrillar proteins and proteins involved in sarcoplasmic reticulum calcium flux suggests that DAP may affect regulation of calcium homeostasis and cytoskeleton signaling. This study provides the first evidence that reduced expression of DAP significantly alters the transcriptome profile of pectoralis major muscle satellite cells, thereby reducing proliferation and differentiation.

Keywords: satellite cells, muscle development, death-associated protein, global gene expression, microarray, muscle, turkey 


\section{INTRODUCTION}

Death-associated protein (DAP) is a highly conserved, 15$\mathrm{kDa}$, proline-rich protein with two phosphorylation sites (Deiss et al., 1995; Koren et al., 2010). It was first identified by Deiss et al. (1995) in studies aimed at identifying novel genes with functional relevance in apoptosis. Subsequent cell culture studies found that in nutrient-rich conditions, DAP is a substrate for phosphorylation by the mechanistic target of rapamycin (mTOR) (Koren et al., 2010). When cells are subjected to amino acid starvation, mTOR activity is down-regulated and DAP undergoes dephosphorylation. Analysis of cells grown under nutrient-rich and nutrient-deprived conditions suggests that the phosphorylated form is functionally silent, whereas dephosphorylated DAP is the active form that acts as an inhibitor of cell autophagy (Koren et al., 2010). Subsequently, others have shown that DAP is involved in regulation of autophagy and apoptosis induced by subtilase cytotoxin (SubAB) of some strains of Shiga-toxigenic E. coli (Yahiro et al., 2014). Reduced expression of DAP is also associated with adverse clinical outcomes for human breast (Wazir et al., 2012) and colorectal cancer (Jia et al., 2014) owing to altered regulation of apoptosis and autophagy.

In previous studies, our group investigated changes in gene expression in turkey pectoralis major muscle as a function of temporal development (Sporer et al., 2011b). Based on the following criteria, DAP was one of several differentially expressed (DE) genes selected for further studies. First, DAP expression changed substantially, being highest in embryonic muscle undergoing hyperplasia, followed by a five-fold decrease in neonatal muscle undergoing hypertrophy, and decreased further in muscle from 16-wk market-age turkeys (Sporer et al., 2011b). Second, little was known about the function of DAP, and most importantly, there was no previous evidence for its role in muscle development.

In subsequent studies, knockdown of DAP expression in turkey pectoralis major muscle satellite cells by small interfering RNA (siRNA) resulted in reduction of proliferation by up to $50 \%$ and nearly complete inhibition of the differentiation of satellite cells into myotubes (Velleman et al., 2012). Chicken satellite cells transfected with DAP cDNA showed that overexpression of DAP resulted in increased proliferation, differentiation, and myotube diameter, but had no effect on satellite cell apoptosis (Shin et al., 2013a). These studies were the first to suggest that DAP may play an important role in pectoralis major muscle development.

The objective of the current study was to initiate studies to define the role of DAP in the early stages of muscle growth and development by testing the hypothesis that altered $D A P$ expression modulates expression of other genes that are critical to pectoralis major muscle development. siRNA was used to reduce DAP expression in proliferating and differentiating turkey myogenic satellite cells. Transcriptome differences between control and knockdown cell cultures were measured using a turkey skeletal muscle long oligonucleotide microarray (TSKLMO) (Sporer et al., 2011a). Our results provide the first evidence of global gene expression changes upon knockdown of DAP expression. Pathway analysis suggests potential involvement of $D A P$ in protein synthesis, calcium homeostasis, and muscle mass accumulation.

\section{MATERIALS AND METHODS}

\section{Isolation of Turkey Myogenic Satellite Cells}

Randombred Control Line 2 (RBC2) Pectoralis major muscle satellite cells, previously harvested according to McFarland et al. (1988) and stored in liquid nitrogen, were used for this study. The RBC2 line represents a 1967 commercial line turkey that has been maintained at The Ohio State University, Agricultural Research and Development Center Poultry Research Unit, without conscious selection for any traits (Nestor et al., 1969; Nestor, 1977). An ethics approval statement was not required because no animals were sacrificed for the current study. The cells were analyzed for proliferation and differentiation characteristics as previously described (Velleman et al., 2000, 2012).

\section{Cell Culture}

Growth of control and DAP-knockdown satellite cells were performed at The Ohio State University as described in detail by Velleman et al. (2012). Briefly, satellite cells were transfected with siRNA targeted against DAP or were mock-transfected (without siRNA) $24 \mathrm{~h}$ after plating (Figure 1). Proliferation was induced as previously described (Velleman et al., 2006, 2012) and the extent of proliferation was measured by DNA concentration (McFarland et al., 1995). After $72 \mathrm{~h}$ of proliferation, cell differentiation was induced by changes in growth media as described by Velleman et al. (2012) (Figure 1), The differentiation assay was adapted from Florini (1989) and Yun et al. (1997) as described by Velleman et al. (2012). The extent of differentiation was determined as a function of muscle-specific creatine kinase levels at $48 \mathrm{~h}$ after induction of differentiation.

\section{RNA Isolation}

Satellite cell cultures were frozen at $-80^{\circ} \mathrm{C}$ after $72 \mathrm{~h}$ of proliferation or $48 \mathrm{~h}$ of differentiation and shipped on dry ice

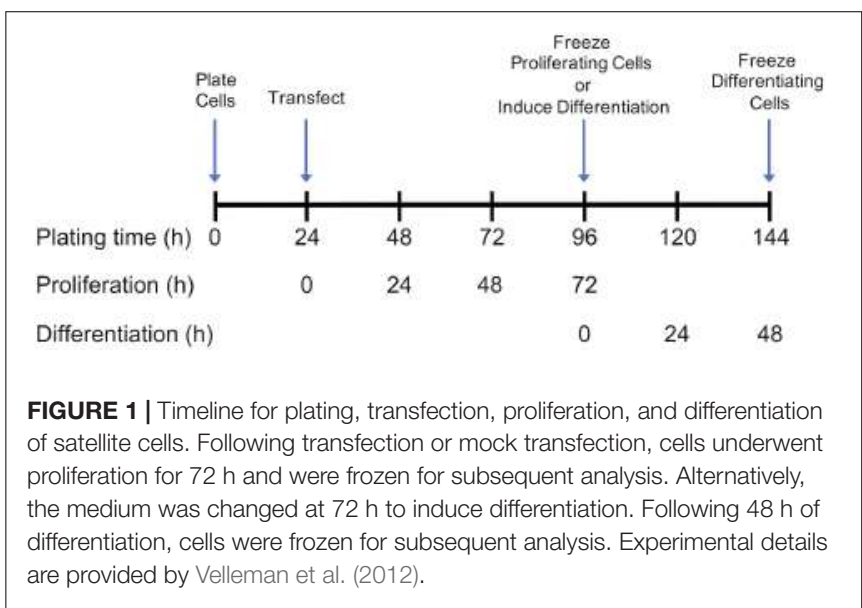


to Michigan State University for RNA isolation and subsequent microarray hybridization. Frozen cells were thawed on ice for 5 min prior to isolation of total RNA with TRIReagent (Molecular Research Center, Inc., Cincinnati, OH, United States) following manufacturer instructions. Contaminating genomic DNA was removed by treatment with RNA-free DNase (Ambion Inc., Austin, TX, United States). Total RNA was quantified using a Nanodrop ND-1000 spectrophotometer (Thermo Fisher Scientific, Waltham, MA, United States) and sample integrity determined with an Agilent 2100 Bioanalyzer (Santa Clara, CA, United States). Samples with an RNA Integrity Number (RIN) of $>8.0$ were considered acceptable for use with the microarray.

\section{Microarray Experimental Design}

Design, construction, and validation of the TSKMLO array are described in Sporer et al. (2011a). The microarray was constructed based on cDNA libraries gathered at critical stages in turkey skeletal muscle development (Reed et al., 2008). Information regarding the TSKMLO platform can be found at the National Center for Biotechnology (NCBI) Gene Expression Omnibus (GEO) database (platform accession: GPL9788). In the current study, RNAs isolated from DAP knockdown samples were directly compared to those of the control treatment. A total of four replicates was used for each experiment (proliferation or differentiation) and dye swaps were included in the experimental design to minimize dye bias, resulting in eight arrays per experiment and 16 arrays for the entire knockdown study.

\section{RNA Amplification and Microarray Hybridization}

Total RNA was amplified, dye-coupled, and purified using the Amino Allyl MessageAmp ${ }^{\text {TM }}$ II aRNA Amplification Kit (Ambion, Inc.) according to the manufacturer's protocol. Due to low levels of RNA isolated from cell culture samples, two rounds of amplification were performed for all samples. Microarray preparation and hybridization were carried out as described in Sporer et al. (2011a). All spot intensities were exported as GenePix Results (GPR) files for statistical analysis.

\section{Microarray Statistical Analysis and Gene Annotation}

The GPR files were subjected to statistical analysis as described in Sporer et al. (2011b). Briefly, dye intensity bias was normalized using the "normexp" background correction method based on Ritchie et al. (2007). Normalized data were described as $\log _{2}$ fluorescent intensities ratio $(\mathrm{Cy} 5 / \mathrm{Cy} 3)$ or $M$-value, and statistically analyzed with a linear model using LIMMA (Smyth, 2005) based on overall intercept, the dye assignment for samples from the DAP knockdown treatment, and the random effect of cells derived from the treatment. Mismatch and negative control spots on the microarray were used to confirm that hybridization to the array was specific (Sporer et al., 2011a). Raw Cy5 and Cy3 intensities, $M$-value, and LOESS-normalized $\log _{2}$ average intensities (A) were submitted with original GPR files to the NCBI GEO (platform accession: GPL9788, series accession: GSE35660).
Differences in expression levels between DAP knockdown and control were considered significant with an estimated false discovery rate $(\mathrm{FDR})<0.05$. In this experiment, the comparison of interest was overall differences between DAP knockdown treatment and control within each developmental stage (proliferation or differentiation). Fold change was defined as the ratio of expression of particular gene in DAP-knockdown cells relative to control cell culture. Gene annotation of the array probes was conducted by iterative NCBI BLAST searches (blast.ncbi.nlm.nih.gov) of the original cDNA sequences used to design the array probes. Sequences were first queried against the turkey refseq gene list (genome annotation 103), then the NCBI refseq database, followed by the turkey genome (v5.1) and the NCBI NR database. Sequences that failed to identify significant matches were designated as novel transcripts. Several sequences aligned to introns within described genes in the turkey genome BLAST searches. These were assigned to the described genes. Sequences with significant alignment to the turkey genome outside of described genes and without matches to other databases were designated as undescribed transcripts.

\section{Confirmation of Gene Expression by Quantitative Real-Time PCR}

Six genes from the proliferation experiment and 9 genes from the differentiation experiment were chosen for gene expression analysis using quantitative real-time PCR (qPCR). Genes were chosen based on function and comparably high levels of differential expression as seen in the microarray analysis. Primers (Supplementary Table S1) were designed using Primer Express 3.0 software (Applied Biosystems, Foster City, CA, United States) and synthesized by Operon, Inc. (Huntsville, AL, United States). Sample Amino Allyl-aRNAs (2 $\mu \mathrm{g})$, prepared for microarray hybridization using the Amino Allyl MessageAmp ${ }^{\mathrm{TM}}$ II aRNA Amplification Kit (Ambion, Inc.), were reverse-transcribed into cDNA using Superscript III (Invitrogen). Synthesized cDNA was quantified using a Nanodrop. Reactions were run using 610 ng cDNA template, $1.2 \mu \mathrm{M}$ primer mix, and Power SYBR Green PCR Master Mix (Applied Biosystems) in an ABI Prism 7900HT Sequence Detection System (Applied Biosystems). Data were analyzed using the $2^{-\Delta \Delta \mathrm{Ct}}$ method (Livak and Schmittgen, 2001). Based on data indicating no change in $C_{t}$ for the described treatment conditions (data not shown), UNC-5 homolog B (UNC5B) and Integral Membrane Protein 2C (IMP2C) were used as endogenous control genes for proliferation and differentiation, respectively. Statistical analysis was performed using a $t$-test (SigmaPlot, San Jose, CA, United States) and results are expressed as a fold change of the DAP knockdown relative to the control.

\section{Functional Analysis}

DE transcripts (FDR $<0.05)$ were subjected to pathway analysis using Qiagen Ingenuity Pathway Analysis (IPA) software (Qiagen, Redwood City, CA, United States). Canonical pathways, indicative of cell signaling and metabolic pathways relevant to the DE genes, were obtained from IPA analysis based on algorithms developed by Krämer et al. (2014). 


\section{RESULTS}

\section{Differential Expression of Satellite Cell Genes}

A combined total of 647 loci (representing up to 559 genes) was DE between DAP knockdown and control treatments in the turkey satellite cells (FDR $<0.05)$. In the proliferating cells, 458 loci (378 unique genes) were DE (Supplementary Table S2), whereas 270 loci (189 unique genes) were DE in differentiation (Figure 2). During proliferation, more genes were down-regulated (244) than up-regulated (214) upon knockdown of DAP (Supplementary Table S2).

As with knockdown of proliferating satellite cells, more genes in the differentiating satellite cells were down-regulated (179) than up-regulated (91) (Supplementary Table S3). As expected, expression of most of the myofibrillar proteins were decreased. Those include two loci of alpha actin (ACTA1, FC $=-19$ and -12$)$, various isoforms of tropomyosin (TPM, FC ranging from -4 to -15$)$, troponin $\mathrm{C}(T N N C, \mathrm{FC}$ ranging between -2 and -18 ), troponin I (TNNI, FC ranging from -2.6 to -13 ), troponin $\mathrm{T}$ (TNNT, FC ranging from -1.9 to -35$)$, nebulin (LOC109368623, FC $=-4)$, and titin $(T T N, \mathrm{FC}=-3.5)$. In addition, the gene for obscurin $(O B S C N)$, a protein linking the myofibril to the sarcoplasmic reticulum (SR), was downreglated 2.2-fold.

Eighty loci showed differential expression in both proliferation and differentiation knockdown experiments (Table 1). Expression of 72 loci was directionally consistent between both the proliferating and differentiating cells. Differences in fold change between proliferation and differentiation ranged from 0.009 to 23.9 with an average of 2.6 (Standard Deviation $=3.61$ ). The largest FC deviation was observed for eukaryotic translation initiation factor 3 subunit $\mathrm{E}(\mathrm{eIF} 3 \mathrm{E})$ with $\mathrm{FC}=29.3$ and 5.3 in proliferation and differentiation, respectively. This initiation factor is a component of the initiation factor 3 (eIF-3) complex, required in the initiation of protein synthesis. In contrast, FC

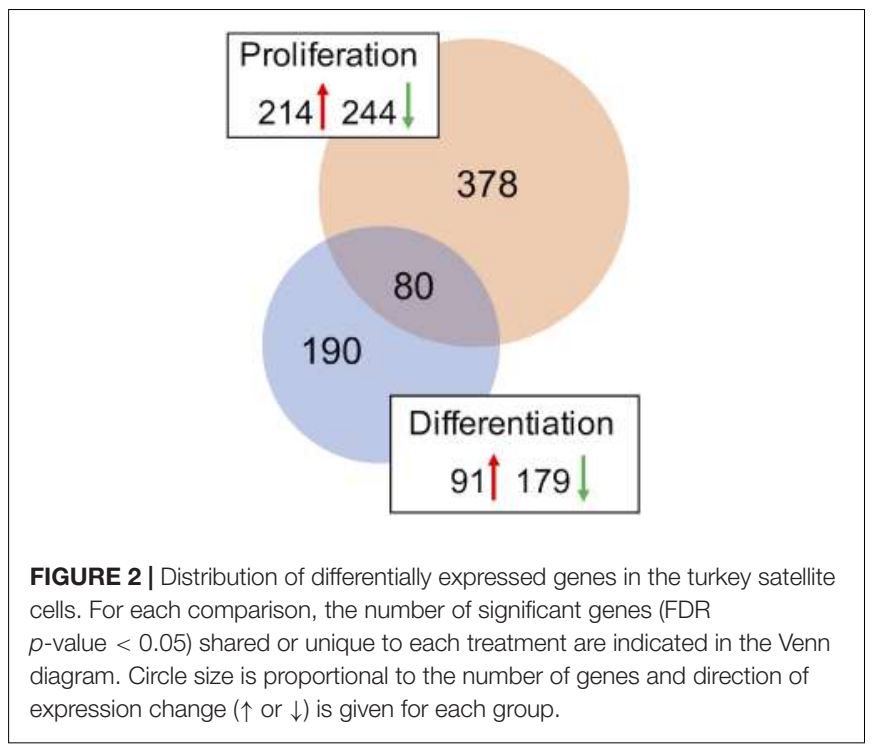

TABLE 1 | Differentially expressed transcripts identified in both proliferation and differentiation knockdown experiments.

\begin{tabular}{|c|c|c|c|c|c|}
\hline \multirow[t]{2}{*}{ Accession } & \multirow[t]{2}{*}{ Gene } & \multicolumn{2}{|c|}{ Proliferation } & \multicolumn{2}{|c|}{ Differentiation } \\
\hline & & FC & FDR & FC & FDR \\
\hline & Repetitive element & -24.5 & 0.004 & -15.0 & 0.002 \\
\hline XM_031555067.1 & PCOLCE2 & -6.4 & 0.022 & 5.1 & 0.033 \\
\hline XM_010716979.3 & KPNA4 & -5.7 & 0.011 & -4.5 & 0.009 \\
\hline NM_001257204 & CSNK2B & -5.0 & 0.022 & -3.8 & 0.043 \\
\hline XM_010721879.2 & POLDIP2 & -4.6 & 0.010 & -4.1 & 0.004 \\
\hline XM_031554139.1 & $\mathrm{HIBCH}$ & -4.5 & 0.010 & -2.3 & 0.040 \\
\hline XM_003207995.4 & ANTXRL & -4.2 & 0.014 & -3.7 & 0.010 \\
\hline NM_205055 & $A K T 1$ & -4.2 & 0.014 & -3.2 & 0.017 \\
\hline XM_003204431.4 & ELOVL5 & -4.0 & 0.016 & -2.8 & 0.035 \\
\hline XM_010719061.2 & RAB24 & -3.7 & 0.014 & -2.5 & 0.035 \\
\hline XM_003211678.4 & АТР2АЗ & -3.6 & 0.017 & -2.7 & 0.035 \\
\hline XM_031554765.1 & LOC100543527 & -3.6 & 0.017 & -2.4 & 0.050 \\
\hline XM_021383972.1 & LOC110392031 & -3.5 & 0.017 & -2.8 & 0.029 \\
\hline XM_010712676.1 & COL1A2 & -3.3 & 0.015 & -3.3 & 0.010 \\
\hline XM_031556345.1 & ZNF207 & -3.3 & 0.014 & -2.1 & 0.048 \\
\hline XM_010716570.3 & NDUFA9 & -3.1 & 0.014 & -2.0 & 0.044 \\
\hline XM_021406417.1 & RAC1 & -3.0 & 0.035 & -3.9 & 0.022 \\
\hline XM_010719149.3 & ANXA6 & -2.9 & 0.014 & -2.9 & 0.007 \\
\hline XM_010726642.2 & ANKRA2 & -2.9 & 0.015 & -2.8 & 0.012 \\
\hline XM_010718524.2 & LOC100549893 & -2.7 & 0.017 & -2.0 & 0.047 \\
\hline XM_010713593.3 & LOC104911671 & -2.6 & 0.023 & -4.9 & 0.004 \\
\hline XM_031556251.1 & OLFML2A & -2.5 & 0.022 & -3.9 & 0.005 \\
\hline XM_010719995.3 & GOLGA3 & -2.5 & 0.020 & -3.4 & 0.005 \\
\hline \multirow[t]{2}{*}{ XM_010706418.3 } & $K L C 4$ & -2.5 & 0.040 & -5.8 & 0.004 \\
\hline & Novel transcript & -2.3 & 0.037 & -2.4 & 0.037 \\
\hline XM_417509 & NFATC2 & -2.2 & 0.025 & 2.5 & 0.016 \\
\hline XM_003205518.3 & SLC25A4 & -2.2 & 0.037 & -5.3 & 0.003 \\
\hline XM_003209143.4 & LOC100551374 & -2.2 & 0.036 & 2.4 & 0.035 \\
\hline XM_010712411.3 & LARP4B & -2.1 & 0.025 & -3.7 & 0.003 \\
\hline XM_027462211.1 & BIN1 & -2.0 & 0.042 & -4.0 & 0.005 \\
\hline XM_010710737.2 & RCN1 & -2.0 & 0.031 & -1.9 & 0.048 \\
\hline XM_010712472.1 & LOC104911367 & -1.9 & 0.045 & -10.3 & 0.001 \\
\hline XM_010725012.3 & RPS15 & 2.0 & 0.048 & 2.7 & 0.023 \\
\hline NM_205166 & S100A11 & 2.1 & 0.047 & 5.3 & 0.003 \\
\hline XM_031603746.1 & SERP1 & 2.1 & 0.047 & 3.7 & 0.009 \\
\hline XM_019617414.2 & LOC100546408 & 2.2 & 0.039 & 3.0 & 0.016 \\
\hline XM_031612939.1 & $C E B P D$ & 2.3 & 0.043 & 2.8 & 0.029 \\
\hline XM_010712938.3 & CDV3 & 2.4 & 0.036 & 2.4 & 0.048 \\
\hline XR_793827.3 & LOC104911327 & 2.4 & 0.025 & 2.3 & 0.042 \\
\hline XM_031606119.1 & CHRDL2 & 2.6 & 0.016 & 2.1 & 0.032 \\
\hline XM_019619377.1 & ARPP19 & 2.7 & 0.024 & 6.4 & 0.003 \\
\hline XM_031555334.1 & AKAP13 & 2.8 & 0.017 & -2.3 & 0.034 \\
\hline XM_031554279.1 & ZСЗН15 & 2.8 & 0.014 & 3.2 & 0.005 \\
\hline XM_003208952.3 & IWS1 & 2.9 & 0.020 & 2.3 & 0.045 \\
\hline XM_010719535.3 & HAGH & 2.9 & 0.017 & 2.1 & 0.044 \\
\hline XM_019614125.2 & ZFAND1 & 3.3 & 0.011 & 2.2 & 0.027 \\
\hline XM_010712335.3 & INS/G1 & 3.3 & 0.018 & 2.7 & 0.031 \\
\hline XM_031603746.1 & SERP1 & 3.5 & 0.011 & 4.0 & 0.003 \\
\hline XM_019617349.1 & FN1 & 3.5 & 0.016 & 5.9 & 0.003 \\
\hline XM_025145468.1 & TMBIM6 & 3.6 & 0.019 & 2.7 & 0.042 \\
\hline XM_003202376.4 & HSP9OB1 & 3.8 & 0.031 & 4.2 & 0.032 \\
\hline X60226 & $P G-M$ & 3.9 & 0.026 & 7.9 & 0.006 \\
\hline
\end{tabular}


TABLE 1 | Continued

\begin{tabular}{|c|c|c|c|c|c|}
\hline \multirow[t]{2}{*}{ Accession } & \multirow[t]{2}{*}{ Gene } & \multicolumn{2}{|c|}{ Proliferation } & \multicolumn{2}{|c|}{ Differentiation } \\
\hline & & FC & FDR & FC & FDR \\
\hline XM_010716510.2 & HDLBP & 3.9 & 0.017 & 3.1 & 0.030 \\
\hline XM_010706345.3 & SPTBN1 & 4.1 & 0.014 & 4.0 & 0.009 \\
\hline XM_010712619.3 & HACD1 & 4.4 & 0.015 & -3.3 & 0.022 \\
\hline ADX43874.1 & ND5 & 4.8 & 0.010 & 4.0 & 0.005 \\
\hline XM_025150460.1 & $R T K N$ & 5.0 & 0.017 & 3.3 & 0.039 \\
\hline NM_205086 & FTH1 & 5.1 & 0.029 & 6.3 & 0.025 \\
\hline XM_010725081 & TPM4 & 5.2 & 0.018 & -4.1 & 0.029 \\
\hline XM_010715351.1 & LOC100548808 & 5.3 & 0.009 & 5.7 & 0.002 \\
\hline XM_010720244.3 & SART3 & 5.7 & 0.008 & 4.8 & 0.003 \\
\hline XM_003210090.4 & IP6K2 & 5.9 & 0.010 & 2.6 & 0.031 \\
\hline XM_010707921.3 & ADAM17 & 6.4 & 0.014 & 3.6 & 0.033 \\
\hline XM_010711943.3 & EIF5 & 6.5 & 0.018 & 4.5 & 0.034 \\
\hline XM_019616511.2 & TAX1BP1 & 6.6 & 0.023 & 5.4 & 0.035 \\
\hline XR_003078040.1 & 28S RNA & 6.6 & 0.009 & 3.1 & 0.016 \\
\hline XM_010706942.1 & LOC104909666 & 7.1 & 0.009 & 6.7 & 0.003 \\
\hline NM_001303217.1 & PRKAA1 & 7.8 & 0.008 & 2.9 & 0.016 \\
\hline NM_001198750.1 & EEF1G & 8.3 & 0.014 & 4.2 & 0.032 \\
\hline XM_010706798.1 & SDE2 & 9.0 & 0.010 & 3.0 & 0.036 \\
\hline NM_001010842 & HSP25 & 9.1 & 0.025 & -19.8 & 0.009 \\
\hline XM_031555617.1 & MITF & 9.4 & 0.009 & 3.6 & 0.018 \\
\hline XM_010711927.3 & HSP90AA1 & 9.8 & 0.019 & 5.9 & 0.042 \\
\hline XM_019616075.1 & FTH1 & 10.1 & 0.003 & 5.7 & 0.002 \\
\hline XM_010709442.2 & ETFDH & 11.1 & 0.003 & 3.5 & 0.004 \\
\hline XM_010716375.3 & PPP1R2 & 11.3 & 0.010 & 6.4 & 0.010 \\
\hline XM_031554584.1 & LOC100541517 & 16.0 & 0.010 & 4.2 & 0.037 \\
\hline XM_019616075.2 & FTH1 & 17.2 & 0.008 & 10.0 & 0.003 \\
\hline JF275060.1 & 16S RNA & 21.7 & 0.015 & 10.9 & 0.028 \\
\hline XM_010709216.2 & EIF3E & 29.2 & 0.010 & 5.3 & 0.050 \\
\hline
\end{tabular}

$F C$, fold change, positive and negative FC indicates increased and decreased, respectively, abundance in DAP-knockdown satellite cells. FDR, false discovery rate. Green highlighting indicates down-regulated genes; red highlighting indicates up-regulated genes.

values were essentially identical for $C D V 3$ (2.37 vs 2.36 ). Protein CDV3 homolog, also known as carnitine deficiency-associated gene expressed in ventricle 3 , encodes the protein Histone $\mathrm{H} 4$.

Four gene loci that were up-regulated during proliferation were down-regulated in differentiation (Table 1). These loci include heat shock protein 25 (HSP25, FC $=9.1$ vs -19.8$)$, tropomyosin 4 (TPM4, FC $=5.2$ vs -4.1$)$, 3-hydroxyacylCoA dehydratase 1 (HACD1, $\mathrm{FC}=4.3$ vs -3.3 ), and A-kinase anchoring protein 13 ( $A K A P 13, \mathrm{FC}=2.8$ vs -2.2 ). Conversely, three gene loci that were down-regulated during proliferation were up-regulated in differentiation. These include deoxyribodipyrimidine photo-lyase-like (LOC100551374, $\mathrm{FC}=-2.19 \mathrm{vs} 2.35$ ), nuclear factor of activated T-cells (NFATC2, $\mathrm{FC}=-2.20$ vs 2.53), and procollagen C-endopeptidase enhancer $2($ PCOLCE2, $\mathrm{FC}=-6.4$ vs 5.1).

\section{Confirmation of Gene Expression}

Differential gene expression identified by microarray analysis was corroborated by performing $\mathrm{qPCR}$ on six genes from the proliferation experiment and nine genes from the differentiation experiment (Supplementary Table S1). All genes selected show similar overall trends in differential expression in both microarray and in qPCR results for proliferation (Figure 3) and differentiation (Figure 4).

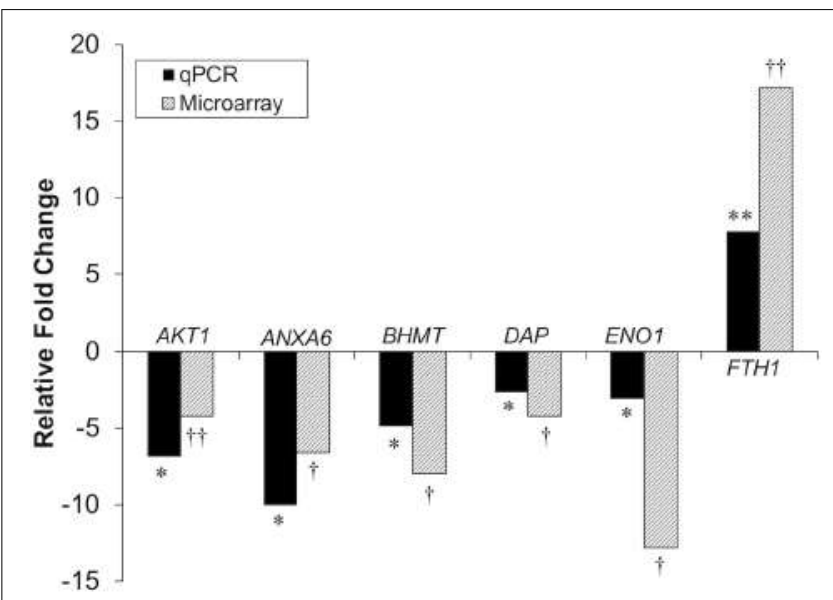

FIGURE 3 | Microarray and GPCR data show similar trends of gene expression in proliferation experiments. Fold change is defined as the change in gene expression in the DAP knockdown samples relative to control samples. Bars below the origin indicate lower expression (down-regulation) of the gene in the $D A P$ knockdown samples; bars above the origin indicate higher expression (up-regulation). ${ }^{\star} P<0.05,{ }^{\star \star} P<0.01$, ${ }^{\dagger} \mathrm{FDR}<0.05$, and ${ }^{\dagger \dagger} \mathrm{FDR}<0.01$. AKT1, Akt1; ANXA6, Annexin A6; BHMT,

Betaine-homosysteine $S$-methyltransferase; $D A P$, death-associated protein; ENO1, enolase 1; FTH1, Ferritin heavy polypeptide 1.

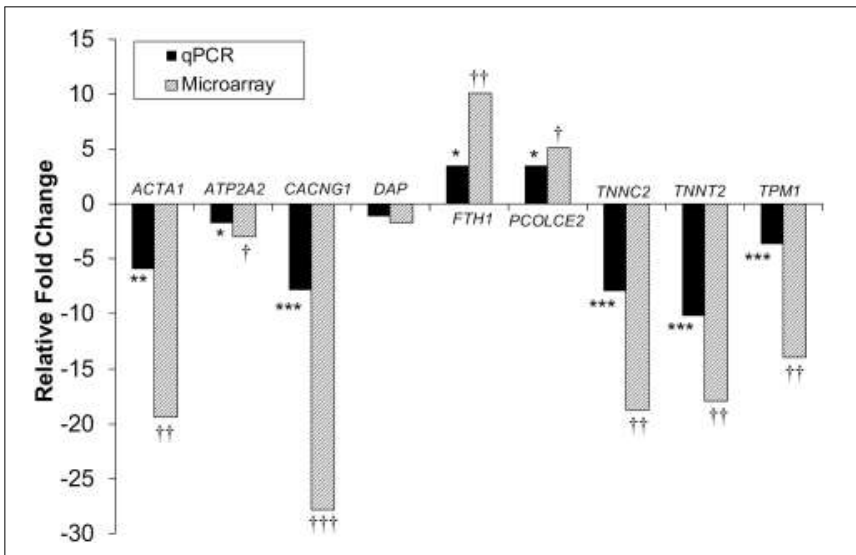

FIGURE 4 | Microarray and qPCR data show similar trends of expression differences in differentiation experiments. Fold change is described as change in gene expression in the DAP knockdown samples relative to controls. Bars below the origin indicate lower expression (down-regulation) of the gene in the $D A P$ knockdown samples; bars above the origin indicate higher expression (up-regulation). ${ }^{\star} P<0.05,{ }^{\star \star} P<0.01,{ }^{* \star *} P<0.001,{ }^{\dagger} \mathrm{FDR}<0.05$,

${ }^{\dagger \dagger} \mathrm{FDR}<0.01$, and ${ }^{\dagger \dagger} \mathrm{FDR}<0.001$. ACTA1, actin alpha 1 skeletal; ATP2A2, sarcoplasmic/endoplasmic reticulum ATPase 2 cardiac; CACNG1, voltage dependent calcium channel gamma 1; DAP, death-associated protein; FTH1, ferritin heavy polypeptide 1; PCOLCE2, procollagen C-endopeptidase enhancer; TNNC2, Troponin C type 2 fast skeletal; TNNT2, Troponin T type 2 cardiac; TPM1, Tropomyosin 1. 


\section{Functional Pathway Analysis}

DE transcripts (FDR < 0.05) were subjected to IPA analysis using their official gene names and canonical pathways considered in interpreting results. The most affected canonical pathways within $D A P$-knockdown proliferating and differentiation satellite cells are shown in Table 2. Knockdown of DAP in turkey satellite cells during proliferation altered expression of several eukaryotic initiation factor (eIF) genes as well as mTOR signaling genes (Figure 5). During differentiation, the calcium signaling pathway (Table 2, Figure 6) was the primary canonical pathway altered by $D A P$ knockdown. In addition, actin cytoskeleton signaling, integrin-linked kinase (ILK) signaling, regulation of actin-based motility by Rho and protein kinase $\mathrm{A}$ are also revealed by IPA as affected canonical pathways (Table 2). IPA calculates activation $(z)$ scores that infer the activation states of genes with predicted functional interactions within molecular networks. All of these enriched pathways exhibited a negative $z$-score, indicating a predicted inactivation. The impact of differential expression on molecular functions include aspects of cell morphology, cellular organization, and cellular maintenance (Table 3). Many of the genes showing significant changes in expression (FDR $<0.05)$ are translated into proteins comprising the myofibrillar architecture including myosin, actin, tropomyosin, the troponin subunits, nebulin, and titin corresponding with the observed failure to form myotubes in our previous study on DAP-knockdown in satellite cells (Velleman et al., 2012).

Pathway analysis of the 80 DE loci common to both proliferation and differentiation knockdown experiments showed the most affected canonical pathways include nitric oxide signaling in the cardiovascular system, neuregulin signaling, mTOR signaling, glucocorticoid receptor signaling and unfolded protein response (Table 4). In an IPA comparison analysis, the largest activation score was observed for nitric oxide signaling in the cardiovascular system (activated, $z=1.34$, $\left.p=8.79 \times 10^{-6}\right)$. This result is based primarily on expression of heatshock proteins (HSP90B1, HSP90AA1, and HSP25), AKT1, and AMPK (PRKAA1).

\section{DISCUSSION}

Myocyte proliferation and differentiation are the result of tightly regulated, temporal changes in gene expression (Buckingham et al., 2003). The processes are not only required for skeletal muscle growth and development, but also for muscle regeneration to maintain integrity of mature adult skeletal muscle. Biological pathways associated with muscle development have been intensively studied; however, many genes involved in this process are as of yet unidentified or their specific roles are not fully elucidated. In a previous transcriptome analysis to characterize temporal changes in gene expression, our group found that DAP transcription was highest in embryonic pectoralis major muscle undergoing hyperplasia, followed by a substantial progressive decline in expression as muscle development shifted to hypertrophy in the 1-day-old hatchling (Sporer et al., 2011b). Transcript levels of DAP continued to
TABLE 2 | Top five canonical pathways affected by DAP knockdown in proliferating and differentiating satellite cells.

\begin{tabular}{llc}
\hline Stage & Canonical pathways & $\boldsymbol{p}$-value \\
\hline Proliferation & EIF2 signaling & $9.68 \times 10^{-11}$ \\
& Protein ubiquitination pathway & $9.98 \times 10^{-8}$ \\
& Sirtuin signaling pathway & $2.69 \times 10^{-7}$ \\
& Regulation of elF4 and p70S6K signaling & $1.56 \times 10^{-6}$ \\
& mTOR signaling & $1.62 \times 10^{-6}$ \\
Differentiation & Calcium signaling & $7.40 \times 10^{-15}$ \\
& Actin cytoskeleton signaling & $3.14 \times 10^{-8}$ \\
& ILK signaling & $4.99 \times 10^{-7}$ \\
& Regulation of actin-based motility by Rho & $1.05 \times 10^{-6}$ \\
& Protein kinase A signaling & $1.07 \times 10^{-6}$ \\
\hline
\end{tabular}

decline between 1 day and 16 weeks of age. Subsequently, we performed siRNA knockdown experiments to elucidate the role of DAP in proliferating and differentiating of pectoralis major muscle satellite cells. Velleman et al. (2012) observed that DAP knockdown resulted in substantial inhibition of both satellite cell proliferation and differentiation in vitro. Similar results were observed in chicken satellite cell cultures (Shin et al., 2013a). Conversely, overexpression of DAP in chicken satellite cells resulted in increased myotube diameter and higher rates of proliferation and differentiation, further suggesting a role for DAP in muscle development based on results from this cell culture model.

The current study was designed to elucidate the molecular mechanisms by which DAP mediates skeletal muscle growth and development. Transcriptomes of DAP-knockdown turkey pectoralis major muscle satellite cells were profiled during proliferating and differentiating states against those of nontreated control cells. To our knowledge, these results provide the first evidence that reduced $D A P$ expression dramatically alters global gene expression in both proliferating and differentiating turkey satellite cells.

Knockdown of DAP in proliferating satellite cells, as demonstrated by microarray analysis, showed expression of this gene was reduced approximately three-fold compared to the control cells. In complementary experiments, an approximate five-fold reduction of DAP was observed by qPCR (Figure 3). However, in the differentiating cells, no significant difference in $D A P$ abundance was observed between treated cells and controls by either microarray or qPCR studies. The absence of DAP differential expression during differentiation was likely the result of the transient transfection system used for these experiments. As described previously (Velleman et al., 2012), satellite cells were first transfected with siRNA, grown to $65 \%$ confluence (about $72 \mathrm{~h}$ ), and then induced to differentiate over a period of $48 \mathrm{~h}$. The half-life of DAP transcripts remains unknown and the treatment conditions may have provided enough time for the cells to transcribe sufficient levels of DAP such that levels were not significantly different between the knockdown and control cells by the $48 \mathrm{~h}$ differentiation timepoint. It is also important to note that changes in transcript abundance due to siRNA transfection do not necessarily result in changes in protein levels. 


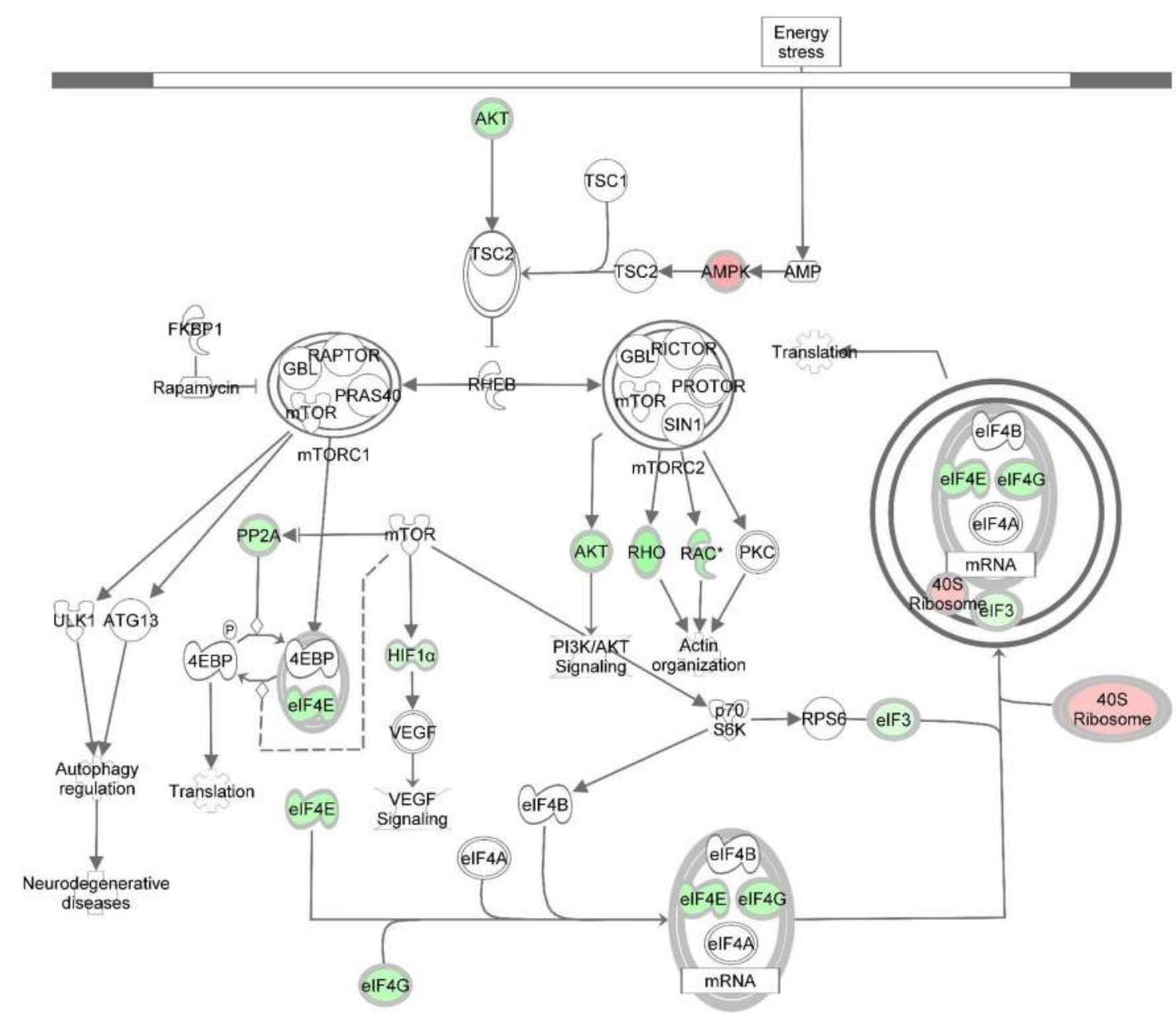

FIGURE 5 | Knockdown of DAP alters gene expression of the mTOR signaling pathway during proliferation of turkey satellite cells. Intermolecular connections between genes are as defined by IPA analysis. *FDR < 0.05. 4EBP, 4E binding protein; ATG13, autophagy related 13; elF3, eukaryotic translation initiation factor 3; elF4A, eukaryotic translation initiation factor 4A; elF4B, eukaryotic translation initiation factor 4B; elF4G, eukaryotic translation initiation factor 4G; FKBP1, FK506 binding protein 1A; GBL, G protein beta subunit like; HIF1 $\alpha$ = hypoxia inducible factor 1 alpha subunit; mTOR, mechanistic target of rapamycin; mTORC1, mechanistic target of rapamycin complex 1; mTORC2, mechanistic target of rapamycin complex 2; p70S6K, p70S6 kinase; PI3K, phosphoinositide 3-kinase; PKC, protein kinase C; PP2A, protein phosphatase 2; catalytic subunit, alpha isozyme; PRAS40, proline-rich Akt substrate 40; RAC, Ras-related C3 botulinum toxin substrate; Rheb, Ras homolog enriched in brain; Rho, Rhodopsin; RPS6, ribosomal protein S6; TSC1, tuberous sclerosis protein 1; TSC2, tuberous sclerosis protein 2; VEGF, vascular endothelial growth factor. Red highlighting indicates up-regulated genes; green highlighting indicates down-regulated genes. This pathway was generated through the use of Ingenuitay Pathway Analysis (QIAGEN, Inc. https://digitalinsights.qiagen.com/products-overview/discovery-insights-portfolio/ analysis-and-visualization/qiagen-ipa/).

\section{Proliferation}

In proliferating turkey pectoralis major muscle satellite cells, a majority of the affected genes were down-regulated compared to the control. The microarray data were corroborated with qPCR, with all of the examined genes showing similar overall trend in fold change. Pathway analysis (Table 2) suggested that knockdown of DAP in proliferating turkey satellite cells altered the cellular signals regulating posttranscriptional processes, in large part, through the modification of eIFs and protein turnover. The deviation of such canonical signals would affect cellular activities, particularly protein synthesis, and eventually in extreme cases, lead to cell death (Table 3).
The mTOR signaling pathway was one of the most significantly affected canonical pathways in response to $D A P$ knockdown. The mTOR enzyme is a serine-threonine protein kinase that regulates cell growth, proliferation, and development in response to nutritional and environmental cues (Hay and Sonenberg, 2004; Wullschleger et al., 2006). This pathway is regulated in part by DAP which is a substrate for phosphorylation (Koren et al., 2010). Studies suggest that mTOR is important in regulating the ultimate growth and size of skeletal muscle (Bodine et al., 2001; Pallafacchina et al., 2002; Ohanna et al., 2005). This is due, in large part, to control of the underlying mechanisms of protein synthesis (Wullschleger et al., 2006; Zanchi and Lancha, 2008; Sandri, 2010). 


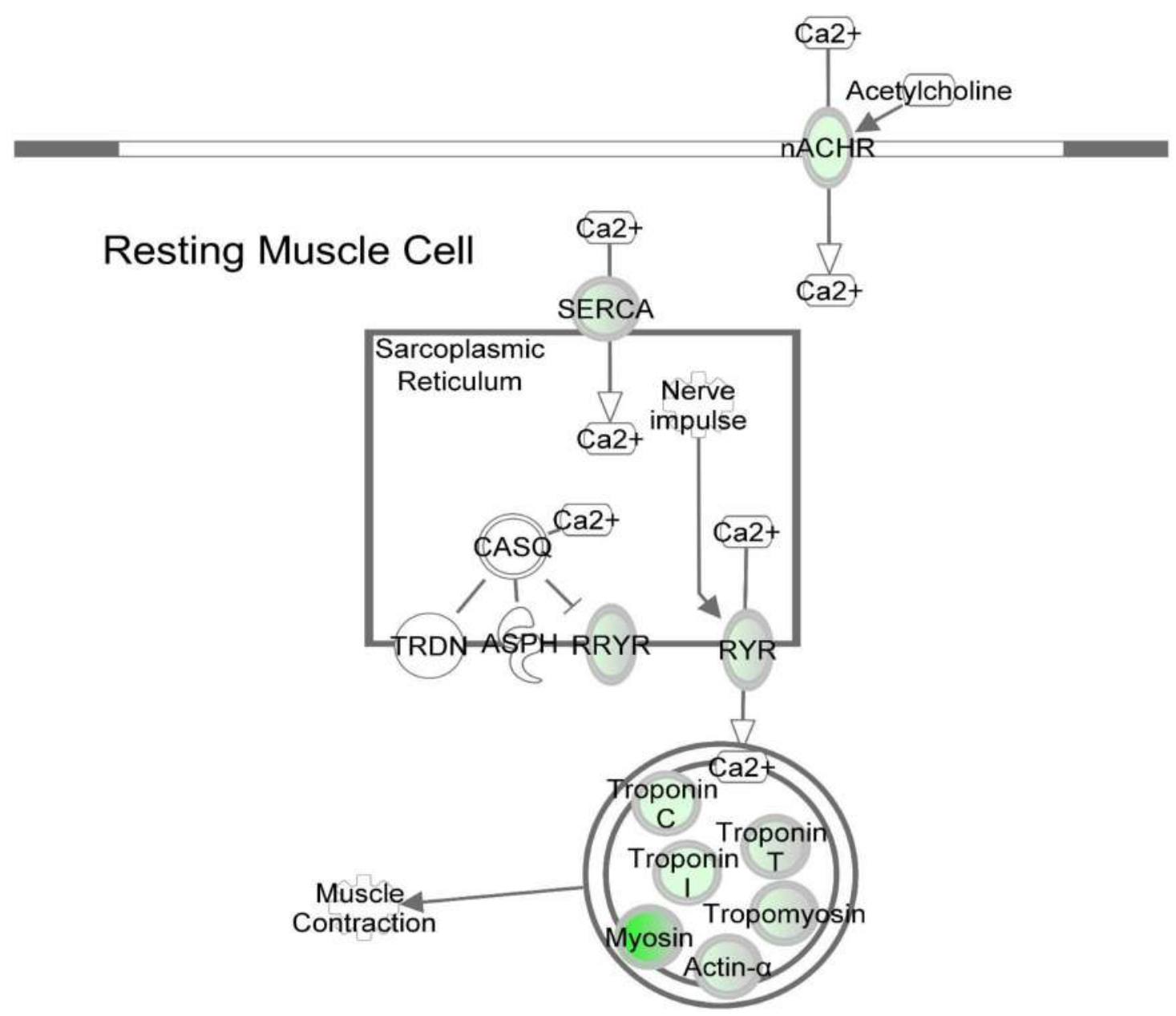

FIGURE 6 | DAP knockdown alters expression of calcium signaling genes during differentiation of turkey satellite cells. Intermolecular connections between genes are as defined by IPA analysis. "FDR $<0.05$, ${ }^{\star *} F D R<0.01$, and ${ }^{* * *} F D R<0.001$. ASPH, aspartate beta-hydroxylase; CASQ, calsequestrin; DHPR, dihydropyridine receptor; nACHR, nicotinic cholinergic receptor gamma subunit; RYR, ryanodine receptor; RRYR, RYR interaction network; SERCA, sarcoplasmic/endoplasmic reticulum $\mathrm{Ca}^{2+}$-ATPase; TRDN, Triadin. Symbols are defined as in Figure 4. Red highlighting indicates up-regulated genes; green highlighting indicates down-regulated genes. This pathway was generated through the use of Ingenuitay Pathway Analysis (QIAGEN, Inc. https://digitalinsights.qiagen.com/productsoverview/discovery-insights-portfolio/analysis-and-visualization/qiagen-ipa/).

In this study, several eIFs, essential for initiation of eukaryotic translation (i.e., protein synthesis), showed altered gene expression as a result of $D A P$ knockdown in proliferating satellite cells (Supplementary Table S2 and Figure 4). Fold change of nine initiation factors affected by $D A P$ knockdown (eIF2S1, eIF3A, eIF3D, eIF3E, eIF3L, eIF3J, eIF4E, eIF4G2, and eIF5) ranged from 0.22 to 29.22. Altered expression of these initiation factors can affect satellite cell proliferation and differentiation (Ohanna et al., 2005; Wullschleger et al., 2006). For example, eIF4E was down-regulated more than fourfold $($ FDR $<0.05)$ in proliferating turkey satellite cells upon DAP knockdown. The translational protein eIF4E recognizes and binds to the $5^{\prime}$ cap of mRNA, aiding in the recruitment of mRNA to the ribosomal complex (Aitken and Lorsch, 2012). A second eIF (eIF4G2), also commonly known as $p 97$ or DAP5, was downregulated nearly fourfold $(\mathrm{FDR}<0.05)$. Reduced expression of eIF4G2 by siRNA in HEK293 cells led to decreased overall translation rate and inhibition of cell proliferation (Lee and McCormick, 2006). Other eIFs affected by DAP knockdown include the eIF3s that are necessary for formation of the pre-initiation complex with the $40 \mathrm{~S}$ ribosomal subunit during translational initiation (Aitken and Lorsch, 2012).

In the present study, differential expression of several eiF3 subunits was observed with DAP knockdown (FDR < 0.05). One subunit $(e I F 3 D)$ was down-regulated more than twofold while, the other four, including $e I F 3 A, e I F 3 E, e I F 3 J$, and $e I F 3 L$, were up-regulated. Among these four, $e I F 3 E$ was the most DE in the proliferating cells with FC $>29$-fold. Differential expression changes of $e I F 3 A$ and $e I F 3 L$ were comparable ( $\sim$ fivefold) whereas eIF3J was increased more than ninefold. Because all other eIFs showed a decrease in expression upon knockdown of DAP, it is proposed that expression of the up-regulated $e I F 3 s$ may be a compensatory mechanism for loss of translational control at early stages during the translation initiation process. Consistent with 
TABLE 3 | Top five altered molecular and cellular functions affected by DAP knockdown in proliferating and differentiating satellite cells.

\begin{tabular}{llc}
\hline Stage & Altered functions & \multicolumn{1}{c}{$\boldsymbol{p}$-value range } \\
\hline Proliferation & Protein synthesis & $7.64 \times 10^{-6}-6.36 \times 10^{-15}$ \\
& Cell death and survival & $8.69 \times 10^{-6}-2.59 \times 10^{-9}$ \\
& Cellular assembly and organization & $4.35 \times 10^{-8}-4.35 \times 10^{-8}$ \\
& DNA replication, recombination, & $4.35 \times 10^{-8}-4.35 \times 10^{-8}$ \\
& and repair & \\
& Cellular movement & $9.06 \times 10^{-7}-1.11 \times 10^{-7}$ \\
Differentiation & Cell morphology & $3.04 \times 10^{-4}-5.88 \times 10^{-19}$ \\
& Cellular assembly and organization & $3.07 \times 10^{-4}-2.02 \times 10^{-16}$ \\
& Cellular function and maintenance & $2.77 \times 10^{-4}-8.89 \times 10^{-16}$ \\
& Cellular development & $3.37 \times 10^{-4}-3.69 \times 10^{-15}$ \\
& Cellular growth and proliferation & $3.37 \times 10^{-4}-3.69 \times 10^{-15}$ \\
\hline
\end{tabular}

TABLE 4 | Top five canonical pathways with shared genes affected by DAP knockdown in proliferating and differentiating satellite cells based on $p$-value.

\begin{tabular}{lc}
\hline Canonical pathways & $\boldsymbol{p}$-value \\
\hline Nitric oxide signaling in the cardiovascular system & $8.79 \times 10^{-6}$ \\
Neuregulin signaling & $1.56 \times 10^{-4}$ \\
mTOR signaling & $3.14 \times 10^{-4}$ \\
Glucocorticoid receptor signaling & $3.63 \times 10^{-4}$ \\
Unfolded protein response & $5.30 \times 10^{-4}$ \\
\hline
\end{tabular}

our findings, yeast cells with reduced expression of eIFs display altered cell cycle profile with an accumulation of the cells in G1 phase (Yu et al., 2006).

Another critical gene significantly altered by DAP knockdown during proliferation was $A K T 1$, down-regulated $>4$-fold relative to control cells (FDR $<0.05)$. This gene encodes the AKT1 protein (also termed protein kinase B) that plays important roles in cell metabolism, proliferation, and growth. AKT1 exerts an anti-apoptotic role in cell survival, and mediates cell growth through the mTOR pathway (Manning and Cantley, 2007; Sandri, 2010). In mice, targeted knockdown of $A K T 1$ in C2 myoblasts blocked differentiation, although with no apparent effect on cell proliferation (Rotwein and Wilson, 2009). Conversely, HéronMilhavet et al. (2006) found AKT1 to play a critical role in proliferation of C2.7 myoblasts and 3T3 fibroblasts. Goncalves et al. (2010) showed that Akt-knockout mice had reduced muscle mass, grip strength, and contractile force. Thus, our results indicating reduced $A K T 1$ expression associated with the $D A P$ knockdown are consistent with impaired proliferation and development. In addition, as noted by Sandri (2010) AKT1 inhibits autophagosome formation and lysosome-directed protein degradation. Thus, it is reasonable to hypothesize that the degree of suppression of $A K T 1$ expression in the DAP knockdown cells may result in increased degradation of muscle proteins due to the absence of the inhibitory effect of AKT1.

The current findings highlight the impaired protein biosynthesis, particularly via modified translation initiation and mTOR signaling, in the DAP-knockdown satellite cells. Such molecular alteration might in turn restrict progression of the cells to initiate a new round of cell division, hence diminishing cell proliferation in the knockdown cells (Polymenis and Aramayo, 2015).

\section{Differentiation}

Based on pathway analysis, the calcium signaling pathway was the top altered canonical pathway reported by IPA (Table 2 and Figure 6). A total of 13 genes in this pathway displayed differential expression upon $D A P$ knockdown with expression ranging from 34 -fold decrease to 2.5 -fold increase. Proteins in the calcium signaling pathway include SR calcium regulators that are responsible for calcium uptake and release, and myofibrillar proteins that respond to changes in $\left[\mathrm{Ca}^{2+}\right]$ to effect muscle contraction. Of the SR calcium regulators, the voltage dependent calcium channel subunit gamma 1 (CACNG1) and both isoforms of avian ryanodine receptors, i.e., RYR 1 and RYR3 were down-regulated approximately 28-fold, 2-fold, and 6-fold, respectively. The CACNG1 gene encodes the dihydropyridine receptor (DHPR) gamma subunit, one of the five subunits of the L-type calcium channel (Rossi and Dirksen, 2006). The DHPR is located in the transverse tubule and associates with RYR1 to elicit calcium release from the SR into the sarcoplasm (Rossi and Dirksen, 2006; Huang et al., 2011). The gamma subunit of the DHPR, in particular, may act as a calcium antagonist during times of cell stress (Andronache et al., 2007). Both RYR1 and RYR3 are calcium channel proteins that open upon activation to allow diffusion of $\mathrm{Ca}^{2+}$ from SR lumen to the cytosol. The sarco/endoplasmic reticulum-Ca ${ }^{2+}$ ATPase is responsible for reducing cytosolic $\mathrm{Ca}^{2+}$ by active transport, coupled with the hydrolysis of ATP, back into the SR (Rossi and Dirksen, 2006). The SERCA gene ATP2A2 showed nearly threefold down-regulation (FDR $<0.05$ ) upon DAP knockdown. Although SERCA2 is more commonly associated with slowtwitch and cardiac muscle, SERCA2a, the more prominent of the SERCA2 isoforms, has been detected in fast-twitch skeletal muscle and neonatal muscle in humans (Zarain-Herzberg and Alvarez-Fernandez, 2002). Proteins that comprise most of the mass of the muscle myofibril were also downregulated, including the structural proteins titin and nebulin, the contractile proteins myosin and actin, and the $\mathrm{Ca}^{2+}$-sensitive regulatory proteins tropomyosin and the three troponin subunits. In addition, obscurin, which may play a role in initiating myofibril assembly (Carlsson et al., 2008), was also downregulated, potentially reducing myotube development observed in the knockdown experiments (Velleman et al., 2012).

It is widely recognized that $\mathrm{Ca}^{2+}$ acts as a second messenger responsible for regulating cascades of signal transduction. Differential expression of the crucial $\mathrm{Ca}^{2+}$-regulatory proteins responsible for release and uptake of cytosolic $\mathrm{Ca}^{2}$ in differentiating satellite cells suggests aberrant cytosolic $\left[\mathrm{Ca}^{2+}\right]$ homeostasis with profound downstream consequences for cellular development. In myogenesis, precise control of $\left[\mathrm{Ca}^{2+}\right]$ is required for activating muscle-specific transcription factors that regulate the fusion of myoblasts to form multinucleated myotubes (Fu et al., 2015). Dysregulated $\left[\mathrm{Ca}^{2+}\right]$ in the DAPknockdown cells may interfere with numerous cellular processes including the calcium-mediated pathways that are critical for myotube formation. 
The other top altered canonical pathways (Table 2) function in a coordinated manner to regulate cytoskeletal reorganization largely required for cell differentiation. The cytoskeleton, recognized as a highly dynamic scaffold, comprises different molecules, e.g., actin and tubulin, arranged into filaments distributed throughout the cytoplasm. Reorganization of such structures induced by extracellular stimuli, activates intracellular signaling, including Rho-family small GTPases through the links with extracellular matrix adhesion molecules such as integrins and syndecans (Shin et al., 2013b; Yen et al., 2014; Ambriz et al., 2018). In this study, two main cytoskeletal proteins, including actin and tubulin, were substantially down-regulated. Within the DAP-knockdown cells, decreased transcripts include two loci corresponding to alpha actin (ACTA1, FC $=-19$ and -12$)$, cardiac actin (ACTC1, FC $=-16)$ and three loci of alpha tubulin (LOC100545462, FC ranging from -5.5 to -9.2). In addition, expression of integrin beta-1 (ITGB1), an integrin complex subunit, and filamin (FLN2), that serves as a linking protein between two actin filaments, were decreased 4.9fold and 3.5-fold, respectively. In contrast, syndecan-2 (SDC2) increased 4.3-fold. Two loci of the non-regulatory myosin light chain (MYL1) were the most highly down-regulated at $>143$-fold and $>59$-fold. Structurally and functionally different from myosin heavy chain, myosin light chain resides in the neck region of myosin and interacts with Rho A effectors, hence mediating assembly of actomyosin complex. With altered cytoskeleton signaling, the formation of multinucleated myotubes could be disrupted, thus preventing the differentiation of the knockdown cells.

There were $80 \mathrm{DE}$ loci common to both proliferation and differentiation. Of the top five canonical pathways affected (Table 4), the most dramatically affected pathway, Nitric Oxide Signaling in the Cardiovascular System, is of particular interest. The DE genes within this pathway include upregulation of the heat shock proteins (HSP90AA1 and HSP90B), the catalytic subunit of $A M P K$ (PRKAA1), and downregulation of AKT1. It is well established that AKT1 plays a critical role in regulating proliferation, differentiation, size, and viability of muscle cells in part through changes in phosphorylation status (Yun and Matts, 2005). The protein HSP90 plays multiple roles in the developing muscle cell. Like most heat shock proteins, it is involved in protein folding and in refolding of denatured proteins (Sato et al., 2000). In addition, HSP90 binds to AKT1 and maintains a balanced phosphorylation state of AKT1 by reducing dephosphorylation through protein phosphatase 2A (PP2A) (Sato et al., 2000; Yun and Matts, 2005). The substantial downregulation of AKT1 coupled with upregulation of HSP90AA1 and $H S P 90 B$ could modulate this balance. Moreover, HSP90 is a target of $S$-nitrosylation that results in loss of its ATP-dependent protein folding activity (Martínez-Ruiz et al., 2005), and could result in accumulation of misfolded proteins accelerating autophagy or apoptosis. Finally, the upregulation of PRKAA1 is associated with decreased protein synthesis through phosphorylation of mTOR and tuberous sclerosis complex 2 (TSC2), thereby increasing autophagy (Thompson, 2018). AMPK also phosphorylates key regulators of autophagy including ULK1, ATG9, and beclin-1.
Early studies of DAP in HeLa cells suggest it is an important positive regulator of cytokine-mediated apoptosis (Deiss et al., 1995). Subsequently, Koren et al. (2010) showed that DAP is phosphorylated under nutrient-rich conditions but functionally silent under starvation conditions where DAP is dephosphorylated and acts as a suppressor of autophagy. The process of autophagy is implicated in skeletal muscle development and renewal as a method for efficiently recycling and removing proteins from cells (Sandri, 2010). $\mathrm{Ca}^{2+}$, acting as a second messenger, regulates the processes of autophagy and apoptosis through complex spatiotemporal mechanisms that may either promote or inhibit these pathways (Harr and Distelhorst, 2010; Sun et al., 2016). Increased or decreased intracellular $\left[\mathrm{Ca}^{2+}\right]$ may disrupt regulation of these pathways, leading to altered cellular development. The results of the current study suggest that DAP plays a central role in turkey muscle growth and development, and perhaps in other cell types, by regulating autophagy as well as calcium signaling and protein translation.

In conclusion, this study demonstrated global changes in the transcriptome following knockdown of DAP in developing turkey satellite cells. The findings underline the essential roles of DAP in proliferation, potentially through the transduction signals associated with translation initiation and mTOR signaling. During differentiation, DAP knockdown altered the biological pathways responsible for controlling $\left[\mathrm{Ca}^{2+}\right]$ and cytoskeleton organization. Future studies will focus on mechanisms by which changes in DAP expression, and consequent changes in the level of cellular DAP protein, alter cellular homeostasis.

\section{DATA AVAILABILITY STATEMENT}

Raw Cy5 and Cy3 intensities, M-value, and LOESS-normalized $\log 2$ average intensities (A) were submitted with original GPR files to the NCBI GEO (platform accession: GPL9788, series accession: GSE35660).

\section{AUTHOR CONTRIBUTIONS}

GS, SV, and KR designed the experiments and acquired the funding for this project. SV performed the cell culture work including DAP knockdown experiments. $\mathrm{KH}$ and $\mathrm{KS}$ isolated RNA and performed the microarray and qPCR experiments. RT analyzed the raw microarray data. KR annotated the microarray oligonucleotides and conducted the IPA analysis. $\mathrm{KH}$ made the initial draft of the manuscript with major revisions provided by YM, KR, SV, and GS. All authors read, revised, and approved the final manuscript.

\section{FUNDING}

This project was supported by a grant from USDA National Institute of Food and Agriculture Award number 2005-3560415628 to GS, SV, KR, and RT. 


\section{ACKNOWLEDGMENTS}

We would like to acknowledge Cindy Coy for technical assistance on the in vitro satellite cell culture work and Juan Abrahante for bioinformatic assistance.

\section{REFERENCES}

Aitken, C. E., and Lorsch, J. R. (2012). A mechanistic overview of translation initiation in eukaryotes. Nat. Struct. Mol. Biol. 19, 568-576. doi: 10.1038/nsmb. 2303

Ambriz, X., de Lanerolle, P., and Ambrosio, J. R. (2018). The mechanobiology of the actin cytoskeleton in stem cells during differentiation and interaction with biomaterials. Stem Cells Int. 2018:2891957. doi: 10.1155/2018/289 1957

Andronache, Z., Ursu, D., Lehnert, S., Freichel, M., Flockerzi, V., and Melzer, W. (2007). The auxiliary subunit gamma 1 of the skeletal muscle L-type Ca2+ channel is an endogenous Ca2+ antagonist. Proc. Natl. Acad Sci. U.S.A. 104, 17885-17890. doi: 10.1073/pnas.0704340104

Bodine, S. C., Stitt, T. N., Gonzalez, M., Kline, W. O., Stover, G. L., Bauerlein, R., et al. (2001). Akt/mTOR pathway is a crucial regulator of skeletal muscle hypertrophy and can prevent muscle atrophy in vivo. Nat. Cell. Biol. 3, 10141019. doi: $10.1038 /$ ncb1101-1014

Buckingham, M., Bajard, L., Chang, T., Daubas, P., Hadchouel, J., Meilhac, S., et al. (2003). The formation of skeletal muscle: from somite to limb. J. Anat. 202, 59-68. doi: 10.1046/j.1469-7580.2003.00139.x

Carlsson, L., Yu, J. G., and Thornell, L. E. (2008). New aspects of obscurin in human striated muscles. Histochem. Cell. Biol. 130, 91-103. doi: 10.1007/s00418-0080413-z

Deiss, L. P., Feinstein, E., Berissi, H., Cohen, O., and Kimchi, A. (1995). Identification of a novel serine/threonine kinase and a novel $15-\mathrm{kD}$ protein as potential mediators of the $\gamma$ interferon-induced cell death. Genes Dev. 9, 15-30. doi: $10.1101 /$ gad.9.1.15

Florini, J. R. (1989). Assay of creatine kinase in microtiter plates using thio-NAD to allow monitoring at $405 \mathrm{~nm}$. Anal. Biochem. 182, 399-404. doi: 10.1016/00032697(89)90614-3

Fu, X., Wang, H., and $\mathrm{Hu}, \mathrm{P}$. (2015). Stem cell activation in skeletal muscle regeneration. Cell. Mol. Life Sci. 72, 1663-1677. doi: 10.1007/s00018-0141819-5

Goncalves, M. D., Pistilli, E. E., Balduzzi, A., Birnbaum, M. J., Lachey, J., Khurana, T. S., et al. (2010). Akt deficiency attenuates muscle size and function but not the response to ActRIIB inhibition. PLoS One. 5:e12707. doi: 10.1371/journal. pone.0012707

Harr, M. W., and Distelhorst, C. W. (2010). Apoptosis and autophagy: decoding calcium signals that mediate life or death. Cold Spring Harb. Perspect. Biol. 2:a005579. doi: $10.1101 /$ cshperspect.a005579

Hay, N., and Sonenberg, N. (2004). Upstream and downstream of mTOR. Genes Dev. 18, 1926-1945. doi: 10.1101/gad.1212704

Héron-Milhavet, L., Franckhauser, C., Rana, V., Berthenet, C., Fisher, D., Hemmings, B. A., et al. (2006). Only Akt1 is required for proliferation while Akt2 promotes cell cycle exit through p21 binding. Mol. Cell Biol. 26, $8267-$ 8280. doi: 10.1128/MCB.00201-06

Huang, C. L., Pedersen, T. H., and Fraser, J. A. (2011). Reciprocal dihydropyridine and ryanodine receptor interactions in skeletal muscle activation. J. Muscle Res. Cell Motil. 32, 171-202. doi: 10.1007/s10974-0119262-9

Jia, Y., Ye, L., Ji, K., Toms, A. M., Davies, M. L., Ruge, F., et al. (2014). Death associated protein 1 is correlated with the clinical outcome of patients with colorectal cancer and has a role in the regulation of cell death. Oncol. Rep. 31, 175-182. doi: 10.3892/or.2013.2866

Koren, I., Reem, E., and Kimchi, A. (2010). DAP1, a novel substrate of mTOR, negatively regulates autophagy. Curr. Biol. 20, 1093-1098. doi: 10.1016/j.cub. 2010.04.041

Krämer, A., Greenn, J., Pollard, J. Jr., and Tugendreich, S. (2014). Causal analysis approaches in ingenuity pathway analysis. Bioinformatics 30, 523-530. doi: 10.1093/bioinformatics/btt703

\section{SUPPLEMENTARY MATERIAL}

The Supplementary Material for this article can be found online at: https://www.frontiersin.org/articles/10.3389/fphys. 2020.01036/full\#supplementary-material

Lee, S. H., and McCormick, F. (2006). p97/DAP5 is a ribosome-associated factor that facilitates protein synthesis and cell proliferation by modulating the synthesis of cell cycle proteins. EMBO J. 25, 4008-4019. doi: 10.1038/sj.emboj. 7601268

Livak, K. J., and Schmittgen, T. D. (2001). Analysis of relative gene expression data using real-time quantitative PCR and the 2- $\Delta \Delta$ Ct method. Methods 25, 402-408. doi: 10.1006/meth.2001.1262

Manning, B. D., and Cantley, L. C. (2007). AKT/PKB signaling: navigating downstream. Cell 129, 1261-1274. doi: 10.1016/j.cell.2007.06.009

Martínez-Ruiz, A., Villanueva, L., González de Orduña, C., López-Ferrer, D., Higueras, M. A., Tarín, C., et al. (2005). S-nitrosylation of Hsp90 promotes the inhibition of its ATPase and endothelial nitric oxide synthase regulatory activities. Proc. Nat. Acad. Sci. U.S.A. 102, 8525-8530. doi: 10.1073/pnas. 0407294102

McFarland, D. C., Doumit, M. E., and Minshall, R. D. (1988). The turkey myogenic satellite cell: optimization of in vitro proliferation and differentiation. Tissue Cell 20, 899-908. doi: 10.1016/0040-8166(88)90031-6

McFarland, D. C., Gilkerson, K. K., Pesall, J. E., Walker, J. S., and Yun, Y. (1995). Heterogeneity in growth characteristics of satellite cell populations. Cytobios 82, 21-27.

Nestor, K. E. (1977). The stability of two randombred control populations of turkeys. Poult. Sci. 56, 54-57. doi: 10.3382/ps.0560054

Nestor, K. E., McCartney, M. G., and Bachev, N. (1969). Relative contributions of genetics and environment to turkey improvement. Poult. Sci. 48, 1944-1949. doi: $10.3382 /$ ps.0481944

Ohanna, M., Sobering, A. K., Lapointe, T., Lorenzo, L., Praud, C., Petroulakis, E., et al. (2005). Atrophy of S6k1(-/-) skeletal muscle cells reveals distinct mTOR effectors for cell cycle and size control. Nat. Cell Biol. 7, 286-294. doi: 10.1038/ ncb1231

Pallafacchina, G., Calabria, E., Serrano, A. L., Kalhovde, J. M., and Schiaffino, S. (2002). A protein kinase B-dependent and rapamycin-sensitive pathway controls skeletal muscle growth but not fiber type specification. Proc. Natl. Acad. Sci. U.S.A. 99, 9213-9218. doi: 10.1073/pnas.142166599

Polymenis, M., and Aramayo, R. (2015). Translate to divide: control of the cell cycle by protein synthesis. Microb. Cell 2, 94-104. doi: 10.15698/mic2015.04.198

Reed, K. M., Mendoza, K. M., Juneja, B., Fahrenkrug, S. C., Velleman, S., Chaing, W., et al. (2008). Characterization of expressed sequence tags from turkey skeletal muscle. Anim. Genet. 39, 635-644. doi: 10.1111/j.1365-2052.2008. 01787.x

Ritchie, M. E., Silver, J., Oshlack, A., Holmes, M., Diyagama, D., Holloway, A., et al. (2007). A comparison of background correction methods for twocolour microarrays. Bioinformatics 23, 2700-2707. doi: 10.1093/bioinformatics/ btm 412

Rossi, A. E., and Dirksen, R. T. (2006). Sarcoplasmic reticulum: the dynamic calcium governor of muscle. Muscle Nerve 33, 715-731. doi: 10.1002/mus. 20512

Rotwein, P., and Wilson, E. M. (2009). Distinct actions of Akt1 and Akt2 in skeletal muscle differentiation. J. Cell Physiol. 219, 503-511. doi: 10.1002/jcp.21692

Sandri, M. (2010). Autophagy in skeletal muscle. FEBS Lett. 584, 1411-1416. doi: 10.1016/j.febslet.2010.01.056

Sato, S., Fujita, N., and Tsuruo, T. (2000). Modulation of Akt kinase activity by binding to Hsp90. Proc. Nat. Acad. Sci. U.S.A. 97, 10832-10837. doi: 10.1073/ pnas. 170276797

Shin, J., McFarland, D. C., Strasburg, G. M., and Velleman, S. G. (2013a). Function of death-associated protein 1 in proliferation, differentiation, and apoptosis of chicken satellite cells. Muscle Nerve 48, 777-790. doi: 10.1002/mus.23832

Shin, J., McFarland, D. C., and Velleman, S. G. (2013b). Migration of turkey muscle satellite cells is enhanced by the syndecan- 4 cytoplasmic domain through the activation of RhoA. Mol. Cell. Biochem. 375, 115-130. doi: 10.1007/s11010-0121534-1 
Smyth, G. K. (2005). "LIMMA: linear models for microarray data," in Bioinformatics and Computational Biology Solutions using $R$ and Bioconductor, eds R. Gentleman, V. Carey, S. Dudoit, R. Irizarry, and W. Huber (New York, NY: Springer), 397-420. doi: 10.1007/0-387-29362-0_23

Sporer, K. R. B., Chiang, W., Tempelman, R. J., Ernst, C. W., Reed, K. M., Velleman, S. G., et al. (2011a). Characterization of a $6 \mathrm{~K}$ oligonucleotide turkey skeletal muscle microarray. Anim. Genet. 42, 75-82. doi: 10.1111/j.1365-2052.2010. 02085.x

Sporer, K. R. B., Tempelman, R. J., Ernst, C. W., Reed, K. M., Velleman, S. G., and Strasburg, G. M. (2011b). Transcriptional profiling identifies differentially expressed genes in developing turkey skeletal muscle. BMC Genomics 12:143. doi: 10.1186/1471-2164-12-143

Sun, F., Xu, X., Wang, X., and Zhang, B. (2016). Regulation of autophagy by Ca2+. Tumor Biol. 37, 15467-15476. doi: 10.1007/s13277-016-5353-y

Thompson, D. M. (2018). The role of AMPK in the regulation of skeletal muscle size, hypertrophy, and regeneration. Int. J. Mol. Sci. 19:3125. doi: 10.3390/ ijms 19103125

Velleman, S. G., Liu, C., Coy, C. S., and McFarland, D. C. (2006). Effects of glypican-1 on turkey skeletal muscle cell proliferation, differentiation and fibroblast growth factor 2 responsiveness. Dev. Growth Differ. 48, 271-276. doi: 10.1111/j.1440-169X.2006.00860.x

Velleman, S. G., Liu, X., Nestor, K. E., and McFarland, D. C. (2000). Heterogeneity in growth and differentiation characteristics in male and female satellite cells isolated from turkey lines with different growth rates. Comp. Biochem. Physiol. Pt. A Mol. Integr. Physiol. 125, 503-509. doi: 10.1016/s1095-6433(00)00178-1

Velleman, S. G., Sporer, K. R. B., Ernst, C. W., Reed, K. M., and Strasburg, G. M. (2012). Versican, matrix Gla protein, and death-associated protein expression affect muscle satellite cell proliferation and differentiation. Poult. Sci. 91, 19641973. doi: 10.3382/ps.2012-02147

Wazir, U., Jiang, W. G., Sharma, A. K., and Mokbel, K. (2012). The mRNA expression of DAP1 in human breast cancer: correlation with clinicopathological parameters. Cancer Genom. Proteom. 9, 199-201.

Wullschleger, S., Loewith, R., and Hall, M. N. (2006). TOR signaling in growth and metabolism. Cell 124, 471-484. doi: 10.1016/j.cell.2006. 01.016
Yahiro, K., Tsutsuki, H., Ogura, K., Nagasawa, S., Moss, J., and Noda, M. (2014). DAP1, a negative regulator of autophagy, controls SubAB-mediated apoptosis and autophagy. Infect. Immun. 82, 4899-4908. doi: 10.1128/IAI.02213-14

Yen, C. F., Wang, H. S., Lee, C. L., and Liao, S. K. (2014). Roles of integrin-linked kinase in cell signaling and its perspectives as a therapeutic target. Gynecol. Minimally Invasive Ther. 3, 67-72. doi: 10.1016/j.gmit.2014. 06.002

Yu, L., Castillo, L. P., Mnaimneh, S., Hughes, T. R., and Brown, G. W. (2006). A survey of essential gene function in the yeast cell division cycle. Mol. Biol. Cell 17, 4736-4747. doi: 10.1091/mbc.e06-04-0368

Yun, B.-G., and Matts, R. L. (2005). Hsp90 functions to balance the phosphorylation state of Akt during $\mathrm{C} 2 \mathrm{C} 12$ myoblast differentiation. Cell. Signal. 17, 1477-1485. doi: 10.1016/j.cellsig.2005.03.006

Yun, Y., McFarland, D. C., Pesall, J. E., Gilkerson, K. K., Vander Wal, L. S., and Ferrin, N. H. (1997). Variation in response to growth factor stimuli in satellite cell populations. Comp. Biochem. Physiol. Part A. Physiol. 117, 463-470. doi: 10.1016/s0300-9629(96)00404-5

Zanchi, N. E., and Lancha, A. H. Jr. (2008). Mechanical stimuli of skeletal muscle: implications on mTOR/p70s6k and protein synthesis. Eur. J. Appl. Physiol. 102, 253-263. doi: 10.1007/s00421-007-0588-3

Zarain-Herzberg, A., and Alvarez-Fernandez, G. (2002). Sarcoendo plasmic reticulum $\mathrm{Ca} 2+-\mathrm{ATPase}-2$ gene: structure and transcriptional regulation of the human gene. Sci. World J. 2, 1469-1483. doi: 10.1100/ tsw. 2002.228

Conflict of Interest: The authors declare that the research was conducted in the absence of any commercial or financial relationships that could be construed as a potential conflict of interest.

Copyright (c) 2020 Horton, Sporer, Tempelman, Malila, Reed, Velleman and Strasburg. This is an open-access article distributed under the terms of the Creative Commons Attribution License (CC BY). The use, distribution or reproduction in other forums is permitted, provided the original author(s) and the copyright owner(s) are credited and that the original publication in this journal is cited, in accordance with accepted academic practice. No use, distribution or reproduction is permitted which does not comply with these terms. 


\section{The Potential of Guanidino Acetic} Acid to Reduce the Occurrence and Severity of Broiler Muscle Myopathies

\author{
Edgar Orlando Oviedo-Rondón* and Hernan Alejandro Córdova-Noboa \\ Prestage Department of Poultry Science, North Carolina State University, Raleigh, NC, United States
}

Guanidinoacetic acid (GAA) is the biochemical precursor of creatine, which, in its phosphorylated form, is an essential high-energy carrier in the muscle. Although creatine has limited stability in feed processing, GAA is well established as a source of creatine in the animal feed industry. Published data demonstrate beneficial effects of GAA supplementation on muscle creatine, energy compounds, and antioxidant status, leading to improvements in broiler body weight gain, feed conversion ratio, and breast meat yield. Although increases in weight gain and meat yield are often associated with wooden breast $(\mathrm{WB})$ and other myopathies, recent reports have suggested the potential of GAA supplementation to reduce the occurrence and severity of WB while improving breast meat yield. This disorder increases the hardness of the Pectoralis major muscle and has emerged as a current challenge to the broiler industry worldwide by impacting meat quality. Genetic selection, fast-growth rates, and environmental stressors have been identified to be the main factors related to this myopathy, but the actual cause of this disorder is still unknown. Creatine supplementation has been used as a nutritional prescription in the treatment of several muscular myopathies in humans and other animals. Because GAA is a common feed additive in poultry production, the potential of GAA supplementation to reduce broiler myopathies has been investigated in experimental and commercial scenarios. In addition, a few studies have evaluated the potential of creatine in plasma and blood enzymes related to creatine to be used as potential markers for WB. The evidence indicates that GAA could potentially minimize the incidence of WB. More data are warranted to understand the factors affecting the potential efficacy of GAA to reduce the occurrence and severity of myopathies.

Keywords: guanidino acetic acid, myopathy, wooden breast, white striping, broiler chicken, meat yield, meat quality

\section{INTRODUCTION}

Genetic selection in poultry has progressed continuously since the early 1960s, resulting in faster growth rates and higher meat production (Havenstein et al., 2003a,b). Over the past 60 years, body weight gain has increased fourfold from 1957 to 2005 with a simultaneous $50 \%$ reduction in feed conversion ratio and $79 \%$ higher Pectoralis major yield in males and $85 \%$ in females 
(Zuidhof et al., 2014). At the same time, the growth, development, structure, and overall metabolism of muscles have been modified by such selection, which has probably resulted in modifications affecting biochemical and sensory characteristics of meat (Petracci et al., 2015, 2019). Several studies have documented that fast-growing strains exhibit higher incidences of idiopathic or inflammatory myopathies and a greater susceptibility for stress-induced myopathies (Bailey et al., 2015; Chen et al., 2019; Montagna et al., 2019). These myopathies include muscle disorders, such as white striping (Kuttappan et al., 2013a,b), spaghetti muscle (Baldi et al., 2018; Montagna et al., 2019; Petracci et al., 2019), and wooden breast (WB; Owens et al., 2009; Petracci and Cavani, 2012; Bailey et al., 2015; Russo et al., 2015; Trocino et al., 2015), which significantly compromise chicken meat quality. WB is a pectoral myopathy in broilers that has been reported worldwide in the poultry industry. WB is macroscopically characterized by hardness of the Pectoralis major muscle often accompanied by pale color, greater drip, and cooking losses, and shear force in comparison to unaffected breast samples (Mudalal et al., 2015; Trocino et al., 2015; Sihvo et al., 2017; Sihvo, 2019). Affected filets are downgraded and have to be transformed into processed meat products, causing considerable economic losses (Mudalal et al., 2015; Petracci et al., 2015, 2019; Cruz et al., 2017). It has been proposed that some white striping lesions progress to become WB (Griffin et al., 2018).

The specific origin of these myopathies has not been well elucidated. Recent findings have linked their onset with hypoxia (Malila et al., 2019) due to reduced vascularization (Sihvo et al., 2018), phlebitis (Papah et al., 2017; Chen et al., 2019), and glucolipotoxicity (Mutryn et al., 2015; Abasht et al., 2016; Lake and Abasht, 2020). The final result is a muscle with defective energy-generating pathways combined with a deficiency and/or dysfunction of tissue ATPases, having consequences in myodegeneration and on muscle fiber contraction degree (Baldi et al., 2020). Genetics and factors related to early development, environment, and nutrition could be involved in the onset of these conditions (Bailey et al., 2015; Montagna et al., 2019; Petracci et al., 2019).

Currently, nutritional strategies to reduce the incidence of myopathies in high-yielding broiler chickens have shown sparse or minimal success. Generally, the reduction in the severity of myopathies has been associated with growth rate reduction (Petracci et al., 2015, 2019; Aviagen, 2019). Recent studies have reported that the dietary inclusion of guanidino acetic acid (GAA) partially ameliorated the occurrence and severity of WB myopathy while maintaining or improving live performance and breast meat yield (Córdova-Noboa et al., 2018a,b; Aviagen, 2019; Vargas, 2019). GAA is a metabolite precursor of creatine, a central energy molecule in muscles. However, only one experiment showed significant effects on white striping, and no effects were observed on spaghetti muscle. Several studies evaluating the use of creatine as a treatment for muscular dystrophies, and neurodegenerative diseases in humans have demonstrated positive outcomes (Hultman et al., 1996; Pearlman and Fielding, 2006; Kley et al., 2013). The hypothesized mechanisms by which GAA could be used to prevent broiler myopathies are revised throughout this review.

\section{ENDOGENOUS PRODUCTION OF GAA AND CREATINE BIOSYNTHESIS}

The chemical nomenclature for guanidino acetic acid (GAA) is $\mathrm{N}$-[aminoiminomethyl]-glycine, also known as glycocyamine or guanidinoacetate. This compound was first isolated from the urine of dogs and humans and has been used as a therapeutic agent since the 1950s (Borsook and Borsook, 1951). Figure 1 describes the general metabolism of GAA with enzymes, amino acids, and vitamins involved. GAA is endogenously produced from arginine (Arg) and glycine (Gly) by a reaction catalyzed by the enzyme L-arginine:glycine amidinotransferase (AGAT; EC 2.1.4.1). Even though this reaction mainly occurs in the kidney and pancreas, some studies have reported that certain endogenous synthesis of GAA outside of the kidneys could be considerable (Ostojic, 2016). After being transported to the liver and pancreas, the enzyme guanidinoacetate methyltransferase (GAMT; EC 2.1.1.2) catalyzes the methylation reaction between GAA and S-adenosyl-methionine (SAM), yielding creatine and S-adenosyl-homocysteine also known as SAH (Wyss and Kaddurah-Daouk, 2000). SAM is generated from methionine (Met) by the enzyme methionine adenosyltransferase (MAT; EC 2.5.1.6). SAH is converted to adenosine and homocysteine by S-adenosyl-homocysteine hydrolase (SAHH; EC 3.3.1.1, also called adenosylhomocysteinase). Although $\mathrm{SAH}$ is recycled back to SAM via homocysteine and methionine in a reaction catalyzed by folic acid and vitamin $\mathrm{B}_{12}$, creatine is transported to the target cells, mainly the muscle, brain, and testes. In these tissues, it is phosphorylated by creatine kinase (CK; EC 2.7.3.2) to produce phosphocreatine. The latter is a high-energy phosphate store for skeletal muscles and the brain (Ostojic, 2015a) to immediately replenish adenosine triphosphate (ATP) from adenosine diphosphate (ADP) at times of ATP depletion.

The formation of GAA catalyzed by AGAT is considered the rate-limiting step for creatine biosynthesis because AGAT is subject to feedback inhibition by elevated blood creatine and ornithine concentrations (Wyss and Kaddurah-Daouk, 2000; De Groot et al., 2018). This inhibition is most likely in place to avoid unnecessary metabolic burden as well as to spare valuable amino acids used for creatine synthesis, namely Gly, Arg, and Met. However, Arg supplementation can increase muscle creatine in chickens, but additional Gly or Met does not have this effect (Khajali et al., 2020). There is evidence proposing that AGAT expression is upregulated when the body is deficient in folic acid, hence causing a shortage of SAM followed by low serum concentration of creatine (Wyss and Kaddurah-Daouk, 2000). Conversely, a different response is expected when the higher serum concentration of creatine is present due to either an endogenous source or by dietary uptake, which results in a reduction of the mRNA content and enzyme concentration, consequently reducing the enzymatic activity of AGAT. The evidence suggests that regulation of AGAT expression occurs 


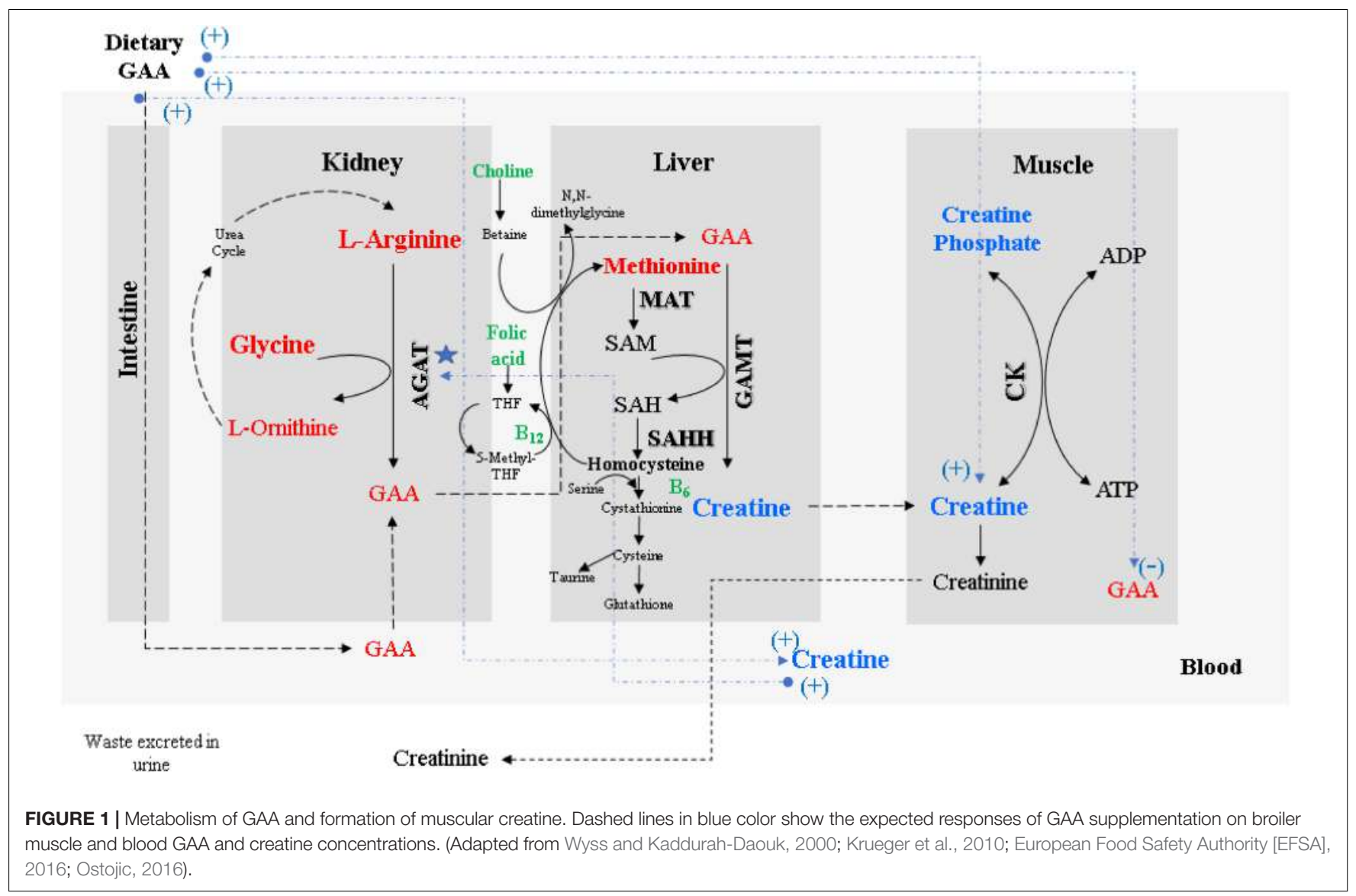

at a pre-translational level (Van Pilsum et al., 1972; Stead et al., 2001; Derave et al., 2004). The creatine biosynthesis pathway is essential due to the role of phosphocreatine in cellular metabolism.

Phosphocreatine supplies energy for cellular activities and moderates the accumulation of ADP from ATP during high rates of cellular metabolism (Wallimann et al., 2011). Creatine and phosphocreatine degrade irreversibly to creatinine, which is excreted in the urine. The magnitude of this daily degradation has been estimated to roughly $1.7 \%$ of the total body pool of creatine and phosphocreatine (Wyss and Kaddurah-Daouk, 2000; Post et al., 2019). However, Tossenberger et al. (2016) suggest a lower daily degradation in modern broilers based on balance studies. Therefore, creatine must be refilled regularly from the diet or produced de novo from GAA, particularly in younger animals in which the requirement is higher due to the expanding muscle mass (Wyss and Kaddurah-Daouk, 2000; Tossenberger et al., 2016). Khajali et al. (2020) proposes that a 21-day-old chicken of $985 \mathrm{~g}$ and average body weight with a daily weight gain of $75 \mathrm{~g}$ would require $169 \mathrm{mg}$ of creatine. Muscle creatine content is a good indicator of the incorporation of dietary creatine. Generally, more than $95 \%$ of the creatine pool is present in the muscles, and the remaining portion is located in the heart and brain. Broiler muscles contain $4.5 \mathrm{~g} / \mathrm{kg}$ of creatine (Tossenberger et al., 2016; Majdeddin et al., 2018). During pathological situations, the creatine balance might be disturbed by metabolic disorders or renal failure (Post et al., 2019).

\section{Role of GAA and Creatine in Muscle Development and Activity}

Guanidinoacetic acid has been related to muscle function mainly by its role in the formation of creatine. Nevertheless, an in vitro study conducted by Wang et al. (2018) using C2C12 cells, which are an immortalized mouse myoblast cell line, indicated that GAA stimulated myogenic differentiation 1 (MyoD) and myogenin (MyoG) mRNA expression increasing the myotube fusion rate. Myoblast fusion is a mechanism of increasing muscle mass without increasing muscle myofiber number. Additionally, GAA supplementation promoted myotube growth through an increase in total myosin heavy chain (MyHC) protein level, myotube thickness, and gastrocnemius muscle cross-sectional area. Finally, GAA promoted myoblast differentiation through MicroRNA (miR) -133a-3p and miR-1a-3p-induced activation of the AKT/mTOR/S6K signaling pathway. These miR are posttranscriptional regulators that play a crucial role in nutrientmediated myogenesis (Wang et al., 2018). In another in vitro study conducted by Deldicque et al. (2005), supplementation with creatine to the same $\mathrm{C} 2 \mathrm{C} 12$ cells increased insulin growth factor, IGF-I, and IGF-II mRNA expression by 30 and 40\%, respectively. It has been shown that IGFs activate the PI3K-Akt-mTOR, p38 MAPK, and Erk1/2 MAPK pathways (Jiao et al., 2013). 
Skeletal muscle contraction has a high-energy demand, and ATP is the immediate energy source. During this process, ATP is hydrolyzed to ADP and must be continuously replenished. With the fast increase in energy demand, the high-energy storage compound phosphocreatine is broken down to provide phosphate to ADP restoring ATP (Wyss and Kaddurah-Daouk, 2000). The creatine/phosphocreatine equilibrium is catalyzed by the enzyme CK. Phosphocreatine is accumulated in the muscles at times of rest and provision of phosphate to refuel ATP from ADP at times of energy demand (Balsom et al., 1994; Nabuurs et al., 2013). Creatine is transported freely in the blood and is mainly absorbed into muscle, brain, and testes (Guimbal and Kilimann, 1993; Wyss and Kaddurah-Daouk, 2000).

In addition to acting as a temporary energy buffer, the creatine/phosphocreatine system also serves other functions in skeletal muscle metabolism. For example, creatine may play a part in regulating muscle protein metabolism (Parise et al., 2001; Nabuurs et al., 2013). Phosphocreatine acts as a carrier, transporting high energy ATP from the mitochondria (production site) to various ATPase sites in the cytosol, especially in tissues of high-energy needs, such as brain and muscles (Balsom et al., 1994; Wyss and Kaddurah-Daouk, 2000). This process is also called the phosphocreatine "shuttle" system (Guimarães-Ferreira, 2014).

\section{GAA and Creatine in Muscle Disorders}

In the early 1900s, it was observed that human patients with muscle diseases retained less creatine than healthy individuals (Hunter, 1928). In 1977, Fitch found that patients with muscle disease had lower cytosolic levels of creatine and phosphocreatine and suggested that this condition might be caused by a failure in the mechanism that retains creatine in the muscle (Fitch, 1977; Nabuurs et al., 2013). Another study also found that patients with neuromuscular disorders can have lower phosphocreatine and creatine levels in skeletal muscle than healthy subjects (Tarnopolsky et al., 2001). Consequently, dietary supplementation of creatine has been used as a pharmacological treatment for a variety of neuromuscular diseases (Tarnopolsky, 2007; Nabuurs et al., 2013). In human trials, creatine in muscles increased with creatine supplementation, and the magnitude of the increase appeared to be greater in patients with low endogenous stores of creatine (Harris et al., 1992; Tarnopolsky, 2007; Nabuurs et al., 2013). Reports indicate that short- and/or medium-term creatine supplementation recovers functional performance and strength in muscular dystrophy and idiopathic inflammatory myopathy (Tarnopolsky, 2007; Kley et al., 2013).

Broilers with WB have a dramatic reduction in ATP concentrations in the early postmortem period. This finding indicated a faulty ATP-generating pathway that might be related to the higher ultimate $\mathrm{pH}$ in WB samples (Baldi et al., 2020). In muscles with low ATP and creatine, GAA can be a direct substrate and thoroughly saturate $\mathrm{CK}$ and serve as compensatory phosphagen. Under these circumstances, phosphorylated GAA may play a role as a phosphocreatine mimetic and a substitute energy donor (Ostojic, 2015b, 2016). This mechanism could be the first mode of action of GAA to mitigate the development of myopathies. However, when the availability of creatine is unhindered, the potential of GAA to act as a substrate for CK is likely negligible. At normal physiological levels, GAA competes with creatine, but it is known that the flux in vitro through the $\mathrm{CK}$ reaction is $\sim 100$ times lower for GAA as compared to creatine. The low affinity of CK for GAA is probably due to the lack of the $\mathrm{N}$-methyl group, which is considered an essential structural feature for the CK reaction (Ostojic, 2015a).

Recently, Ostojic (2015a, 2016) summarized some other physiological roles of GAA in humans that might be relevant as well in animals to reduce the incidence of current poultry myopathies. GAA might stimulate insulin secretion and insulin sensitivity; spare dietary Arg and facilitate its use in protein synthesis and nitric oxide production; stimulate insulin, IGFI, and glucagon release and vasodilation; modulate gammaaminobutyric acid utilization and function; and affect oxidantantioxidant balance. These effects are significant to mitigate myopathies observed in poultry. Wooden breast and other related broiler myopathies have been linked to metabolic disorders mainly due to glucolipotoxicity (Mutryn et al., 2015; Abasht et al., 2016; Lake and Abasht, 2020) and hypoxia (Malila et al., 2019). Insulin resistance causes low-grade inflammation, oxidative stress, and reduces vascularization in muscles (D'Souza et al., 2013), and consequently, increasing insulin sensitivity with GAA could be positive to mitigate myopathies. Higher dietary Arg levels (Arg:Lys ratio: $+30 \%$ in respect to the current recommendations) may play a role in reducing breast muscle abnormalities in broilers by increasing nitric oxide production, vascularization, and oxygenation and even helping with creatine metabolism (Zampiga et al., 2019). In contrast, Zampiga et al. (2018) observed that slight Arg increases were not adequate to mitigate myopathies. However, in practical terms of feed formulation, higher Arg:Lys ratios mean either higher dietary protein content or considerable supplementation of crystalline Arg. These factors impose environmental and economic limitations on implementing higher Arg levels in commercial poultry production.

Nevertheless, nitric oxide is a potent vasodilator compound that causes enhanced blood flow and oxygen supply to the muscle, as well as the removal of harmful catabolites (Khajali and Wideman, 2010), thereby possibly providing positive outcomes on myopathy occurrence (Zampiga et al., 2019). Finally, Amiri et al. (2019) and Nasiroleslami et al. (2018) concluded that GAA could improve the antioxidant status of broilers subject to heat or cold stress, respectively. In these studies, GAA supplementation improved the activity of antioxidant enzymes, such as superoxide dismutase and glutathione peroxidase, measured in serum and liver and decreased plasma levels of malondialdehyde. The antioxidant response should be preserved under stress conditions to minimize susceptibility to myopathy development (Lake and Abasht, 2020).

\section{GAA USED AS FEED ADDITIVE FOR POULTRY}

In poultry nutrition, feed additives that improve energy utilization and naturally enhance muscle development are well 
TABLE 1 | Concentration of guanidino acetic acid (GAA), creatine, and creatinine in $\mathrm{mg} / \mathrm{kg}$ dry matter in different sources.

\begin{tabular}{|c|c|c|c|c|}
\hline Ingredient & GAA & Creatine & Creatinine & References \\
\hline & & - & & \\
\hline Raw chicken breast & & 4,231 & & 3 \\
\hline Boiled (60 min) chicken breast & & 2,973 & & 3 \\
\hline Raw stewing beef & & 3,642 & & 3 \\
\hline Boiled (60 min) stewing beef & & 2,856 & & 3 \\
\hline Raw ox heart & & 2,948 & & 3 \\
\hline Boiled (60 min) ox heart & & 2,017 & & 3 \\
\hline Raw ox liver & & 309 & & 3 \\
\hline Boiled (60 min) ox liver & & 202 & & 3 \\
\hline Cod & & 3,000 & & 4 \\
\hline Herring & & $6,500-10,000$ & & 4 \\
\hline Beef & & 4,500 & & 4 \\
\hline Meat meal $(n=20)$ & & 885 & & 8 \\
\hline Meat and bone meal & $2.4 \pm 1$ & 89 (54-447), 885 & $868.8 \pm 565$ & 5 \\
\hline Meat and bone meal $(n=6)$ & & 199 & & 8 \\
\hline Blood meal $(n=8)$ & & 67 & & 8 \\
\hline Poultry by-products & $4.0 \pm 1$ & $201.2 \pm 108$ & $1,467.1 \pm 1,014$ & 5 \\
\hline Poultry by-products $(n=2)$ & & 156 & & \\
\hline Fish meal & $2.0 \pm 1$ & $1,110.5 \pm 808$ & $2,406.5 \pm 2,330$ & 5 \\
\hline Fish meal & & 1,146 & & 8 \\
\hline Unprocessed bone and raw food $\operatorname{diet}^{1}(n=90)$ & & $1,318-8,548$ & & 6 \\
\hline Unprocessed prey ${ }^{2}(n=15)$ & & $2,735-12,977$ & & 6 \\
\hline Corn & ND & ND & ND & 5,7 \\
\hline Wheat & ND & ND & ND & 5 \\
\hline Sorghum & ND & ND & ND & 5 \\
\hline Canola meal & ND & ND & ND & 5 \\
\hline
\end{tabular}

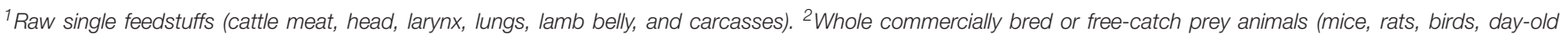

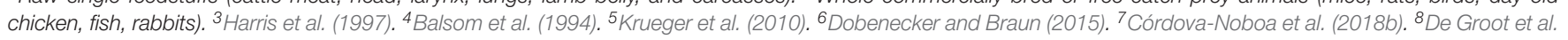
(2019).

accepted. Creatine could be one of these compounds. Creatine is found mostly in fresh meat, fish, and other animal products but is scarce in processed animal proteins (Balsom et al., 1994; Harris et al., 1997; Krueger et al., 2010; Dobenecker and Braun, 2015; De Groot et al., 2019; Khajali et al., 2020) and absent in plants (Khan and Cowen, 1977; Gábor et al., 1984; Krueger et al., 2010; Table 1). As a result, all-vegetable diets entirely lack creatine, and feeds containing animal proteins contain only a little of this semiessential nutrient (Michiels et al., 2012; De Groot et al., 2019; Khajali et al., 2020). The need for high-energy compounds such as phosphocreatine to support the fast growth rate of current broiler breeds is likely compromised as animal proteins are becoming less common in poultry diets.

Creatine is not an ideal feed additive due to its instability and cost (Baker, 2009). Consequently, GAA became attractive to the feed industry as a precursor of creatine due to its industrial properties as a compound with high stability in aqueous solutions (Vranes et al., 2017), and feed processing stability during pelleting and extruding (Van der Poel et al., 2018). Currently, GAA is an approved source of creatine in Europe (European Food Safety Authority [EFSA], 2009, 2016; EC(VO) 1768, 2016) as well as in the United States (FDA 21 CFR \$ 573.496).

The supplementation of GAA at 600 or $1200 \mathrm{mg} / \mathrm{kg}$ to broiler diets has promoted growth, enhanced breast meat yield and other carcass traits, and improved feed conversion ratio (Michiels et al., 2012; Mousavi et al., 2013; Heger et al., 2014; Tossenberger et al., 2016; De Groot et al., 2018; Khajali et al., 2020). The most consistent effect of GAA supplementation has been observed in feed conversion ratio with 4.5- and 8.8-point improvement in broilers supplemented with 600 and $1200 \mathrm{mg} / \mathrm{kg}$, respectively (Khajali et al., 2020).

Dietary GAA has obtained interest to promote higher muscle creatine levels (Majdeddin et al., 2018; Ibrahim et al., 2019), which, in turn, have been associated with improved energetics (Ostojic, 2017; Sadra et al., 2018) and significant increases of high phosphate energy metabolites in broilers supplemented with GAA (Michiels et al., 2012; De Groot et al., 2018; Majdeddin et al., 2018). Improvements in energy utilization have been related to better feed utilization (Khajali et al., 2020). Heger et al. (2014) and Ale Saheb Fosoul et al. (2018) reported increases in energy utilization when GAA was supplemented to broiler diets with variable energy levels, which resulted in a better feed conversion ratio. Similar enhancements in feed and energy efficiency due to GAA supplementation have been observed by Mousavi et al. (2013) and Majdeddin et al. (2018).

The initial research (Beard and Barnes, 1931) conducted in young rats indicated that feeding $1 \mathrm{~g}$ of GAA for 4-6 $\mathrm{h}$ increased about $49 \%(5.94 \pm 0.8 \mathrm{mg}$ of creatine per $\mathrm{g}$ of 
muscle) the creatine concentration in muscle tissue from the hind legs over the control value $(4.0 \pm 0.2 \mathrm{mg}$ of creatine per $g$ of muscle). Compared to other supplements and purified amino acids studied (Ala, Gly, Aspartic acid, glutamic acid, Phe, Tyr, Leu, choline hydrochloride, creatine, His, casein, Val, Cys), GAA yielded the highest increment in creatine above control levels. Even when equivalent concentrations of creatine were supplemented to other treatment groups, the response was better for GAA (Beard and Barnes, 1931). In broilers fed diets with variable energy content and supplemented with GAA, Tabatabaei Yazdi et al. (2017) observed increased concentrations of phosphocreatine, ATP/ADP, and phosphocreatine/ATP ratios in breast muscle. Likewise, Córdova-Noboa et al. (2018b) found that the dietary GAA supplementation (600 g/ton) increased the serum concentrations of creatine and GAA in broiler chickens by 1.6 and 9.2 times, respectively, compared to non-supplemented broilers (Figure 2). In the same way, Nasiroleslami et al. (2018) had reported higher CK activity in GAA-supplemented broilers than in the control group. In a more recent publication, Ibrahim et al. (2019) demonstrate that dietary supplementation of GAA plus $0.4 \%$ methionine $(\mathrm{GAA}+0.4 \%$ Met) increased carcass and breast meat yield and improved plasma and muscle creatine more than dietary creatine itself in mulard ducks.

In addition to the effects in muscle energetics, GAA also has Arg-sparing impacts as reported by Baker (2009); Dilger et al. (2013), Michiels et al. (2012), Ahmadipour et al. (2018a; 2018b; 2018c), and De Groot et al. (2018, 2019). Poultry are uricotelic animals, lacking enzymes to synthesize enough Arg de novo and, therefore, need a sufficient supply of Arg for creatine and nitric oxide production. Arg is especially required under metabolic, environmental, and immunological stress conditions (Khajali and Wideman, 2010). GAA is a source of creatine and, consequently, can spare Arg and, hence, is an excellent candidate to reduce myopathy occurrence and severity. GAA can leave more Arg available to improve muscle regeneration, vascularization, and vasodilatation for better oxygenation necessary to minimize WB (Sihvo et al., 2018; Sihvo, 2019). The Arg-sparing effect has been proven to be an efficient strategy to "supply" Arg in low protein diets and under heat stress (Amiri et al., 2019). This Arg-sparing effect is also important when feeding diets with high inclusion levels of feed ingredients with low Arg content or digestibility, such as wheat, canola meal, distiller's dried grains with solubles, and sorghum (Khajali et al., 2020).

\section{THE POTENTIAL OF GAA TO REDUCE MYOPATHIES IN POULTRY}

Only a few studies have explored the feasibility of alleviating myopathies by supplementing broiler diets with GAA (CórdovaNoboa et al., 2018a,b; Aviagen, 2019; Vargas, 2019). In two separate experiments with male broilers, Córdova-Noboa et al. $(2018 \mathrm{a}, \mathrm{b})$ demonstrated that supplementing with GAA (600 g/ton) improved breast meat yield and ameliorated WB myopathy in male Ross 708 broilers. However, no significant

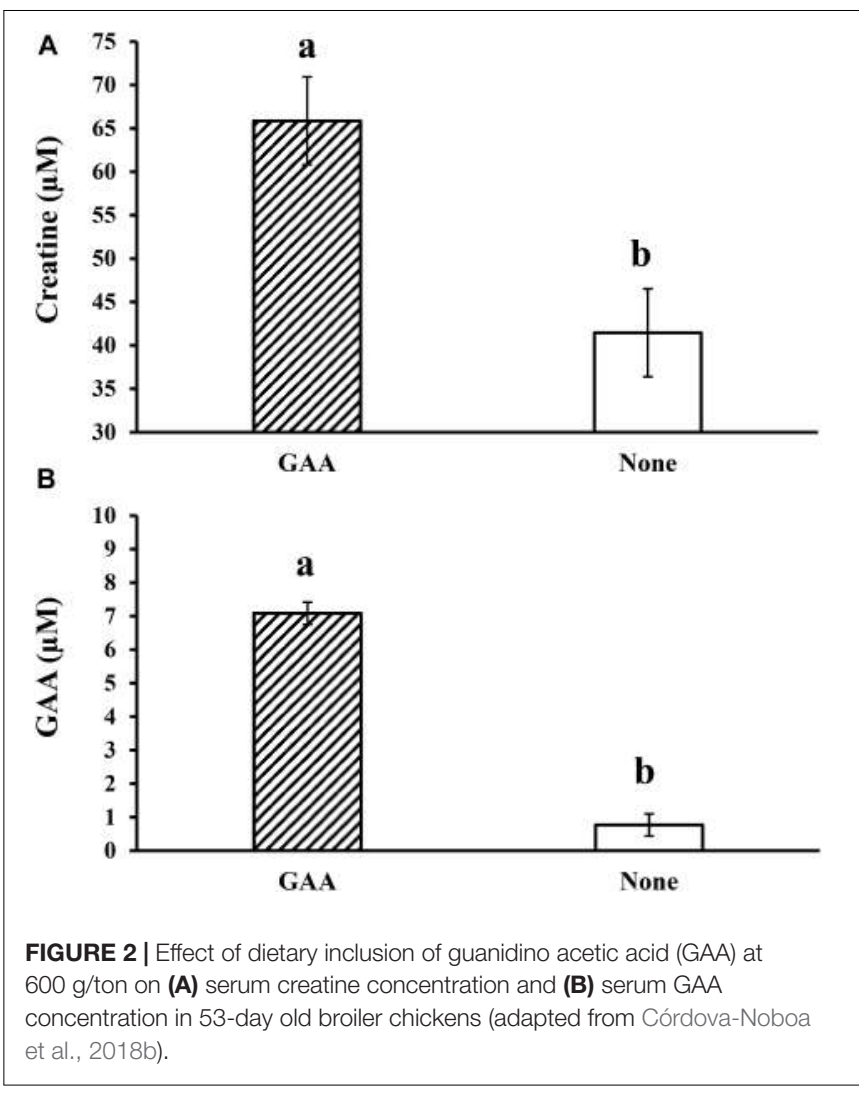

effects on white striping and spaghetti muscle were detected in both experiments. The positive results on WB incidence were mainly due to increasing the number of breast filets with low WB scores and decreasing the filets with more severe WB. The WB severity was evaluated by using the four-level scale (Table 2) developed by Tijare et al. (2016).

In the first experiment with corn or sorghum diets (CórdovaNoboa et al., 2018a), 40 breast samples per treatment, four per each experimental pen were evaluated. The addition of GAA roughly doubled the probability of normal breasts (score 0 ) from approximately 15 to $30 \%$ at 51 days of age (Figure 3 ) although the breast meat yield increased from 38.19 to $39.15 \%$ at 55 days of age only in broilers fed corn-based diets. No significant effect of GAA supplementation was detected on white striping and spaghetti muscle in both evaluations, and no significant impact of GAA

TABLE 2 | Wooden breast (WB) scoring system and characteristics of broiler breast filets detected by palpation at 55 day of age (adapted from Tijare et al., 2016).

\begin{tabular}{lcl}
\hline WB gross classification & Score & Characteristics \\
\hline Normal & 1 & $\begin{array}{l}\text { No hardness detected and flexible } \\
\text { through } \\
\text { Low }\end{array}$ \\
Moderate & 2 & $\begin{array}{l}\text { Mild hardness specially in cranial } \\
\text { region, flexible in caudal region } \\
\text { Hardness throughout with some }\end{array}$ \\
Severe & 3 & $\begin{array}{l}\text { flexibility in mid to caudal region } \\
\text { Marked hardness, rigid throughout }\end{array}$
\end{tabular}




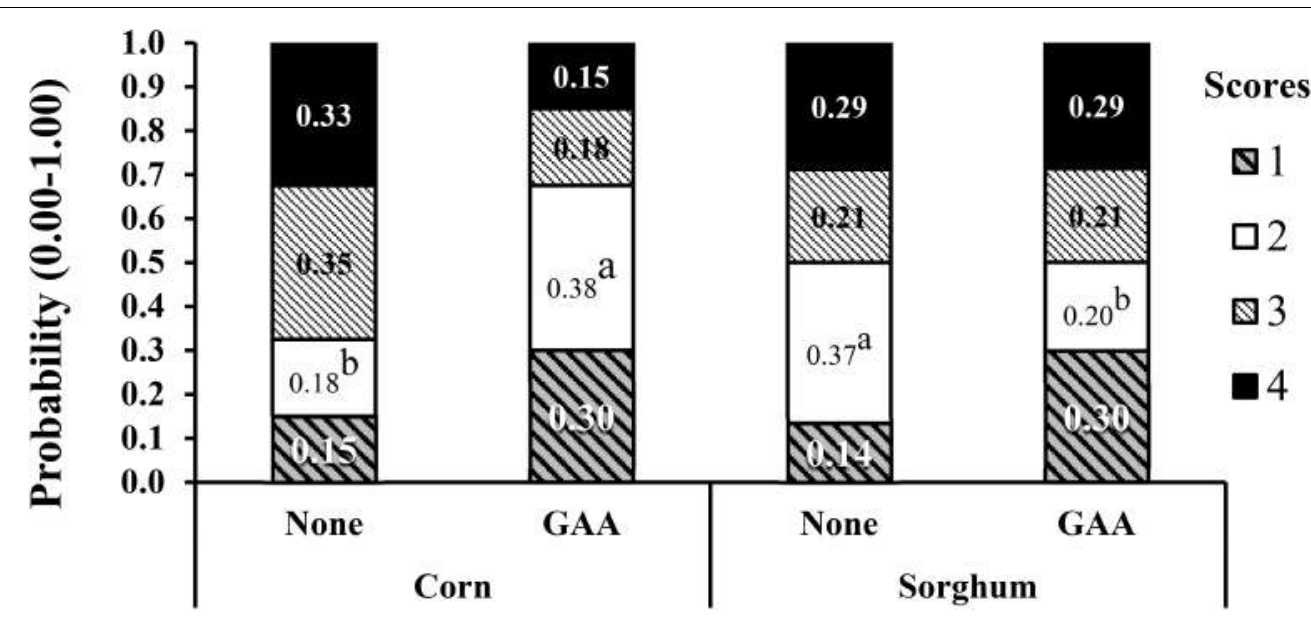

FIGURE 3 | Effect of GAA supplementation (600 g/ton) in diets based on either corn or sorghum on the probability distribution for each wooden breast severity score in Ross-708 male broilers at 51 day of age. Means not sharing a common superscript (a-b) are significantly different $(P<0.05)$ by Tukey's test. Each value represents the probability $(0-1)$ of developing each severity score. Woody breast scores are based on a four-point scale $(1=$ normal, $2=$ low, $3=$ moderate, 4 = severe). Reproduced from Córdova-Noboa et al. (2018a) with the authors' permission.

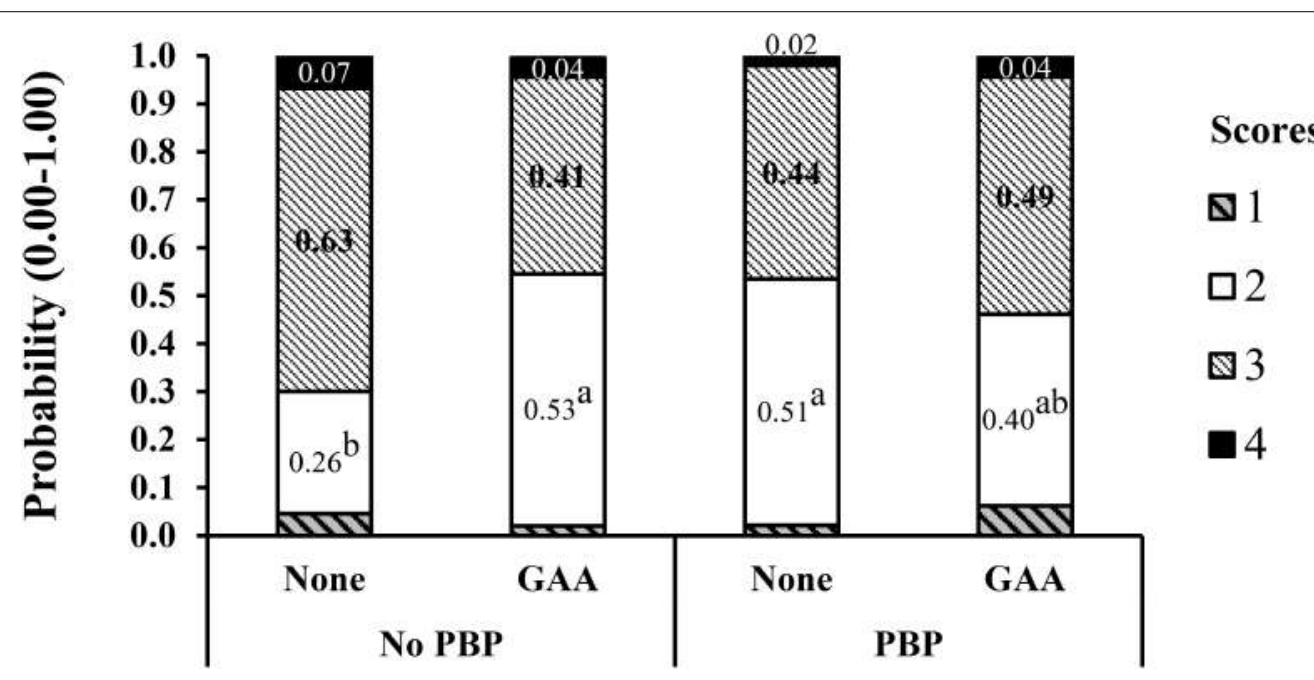

FIGURE 4 | Interaction effect of dietary inclusion of poultry by-products (PBP) and GAA supplementation (600 g/ton) on the probability distribution for each wooden breast severity score in Ross-708 male broilers at 56 day of age. Means not sharing a common superscript (a-b) are significantly different $(P<0.05)$ by Tukey's test. Each value represents the probability $(0.0-1.0)$ of developing each severity score. Scores are based on a four-point scale $(1=$ normal, $2=$ low, $3=$ moderate, 4 = severe). Reproduced from Córdova-Noboa et al. (2018b) with authors' permission.

was detected at 55 days of age in any of the myopathies evaluated. Heat stress during the last days of life affected the variability in the data, and only effects related to the type of grain were detected in that experiment.

In a similar way, in a second experiment (Córdova-Noboa et al., 2018b) in corn-soybean meal diets with or without poultry by-products (PBP), 64 breast meat samples per treatment, four from each experimental pen were scored. The improvement in WB score at 56 days of age with GAA supplementation was more evident in male broilers fed diets without PBP and probably lower in some digestible amino acids (Figure 4). In this case, the number of breast filets with low WB (score 2) doubled, and that of score 3 halved in the GAA-supplemented treatment only for broilers fed diets containing no PBP, reducing the more severe scores of 3 and 4 . The breast meat yield increased significantly in the GAA-supplemented diets with PBP compared to the nonsupplemented (32.35 vs. $31.74 \%$ ).

In both experiments reported by Córdova-Noboa et al. (2018a,b), results were slightly different depending on dietary composition. Carvalho et al. (2013) reported that in cornsoybean meal diets with or without meat and bone meal, GAA supplementation only improved body weight gain when diets also contained 5\% blood meal. Most likely, amino acid levels played a more prominent role than creatine in these diets because blood meal has even lower creatine content than meat and bone meal. Boney et al. (2019) observed that the 


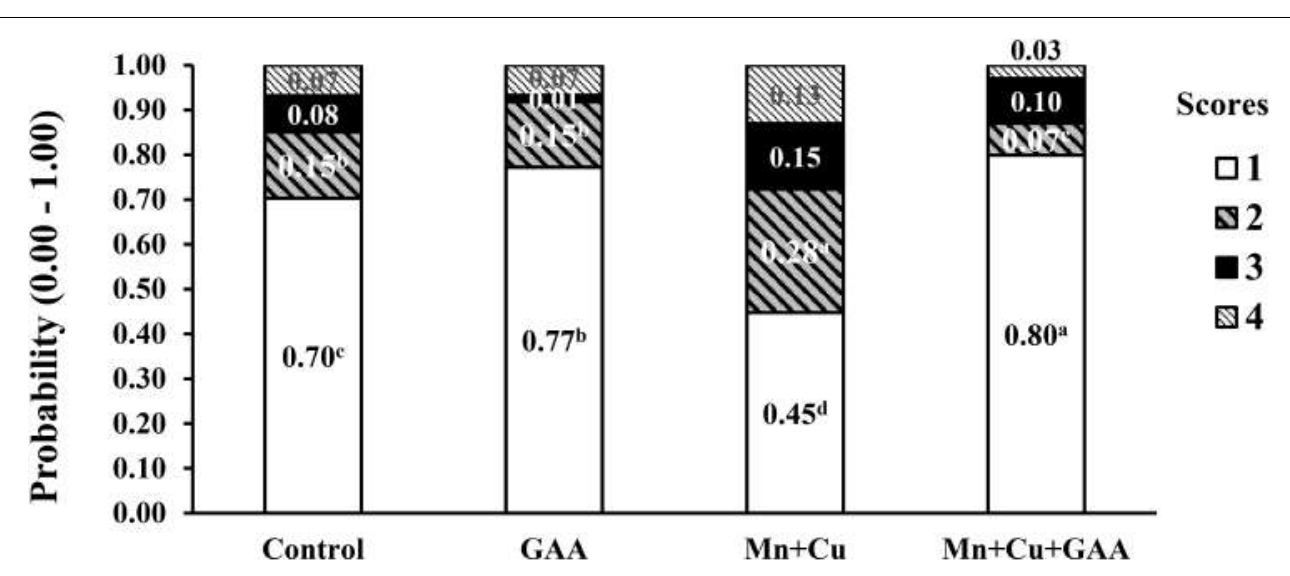

FIGURE 5 | Effect of guanidinoacetic acid (GAA) and organic trace minerals (manganese, $\mathrm{Mn}$, and copper, $\mathrm{Cu}$ ) on the probability distribution for each wooden breast severity score in Ross-308 broilers at 42 day of age (adapted from Vargas, 2019).

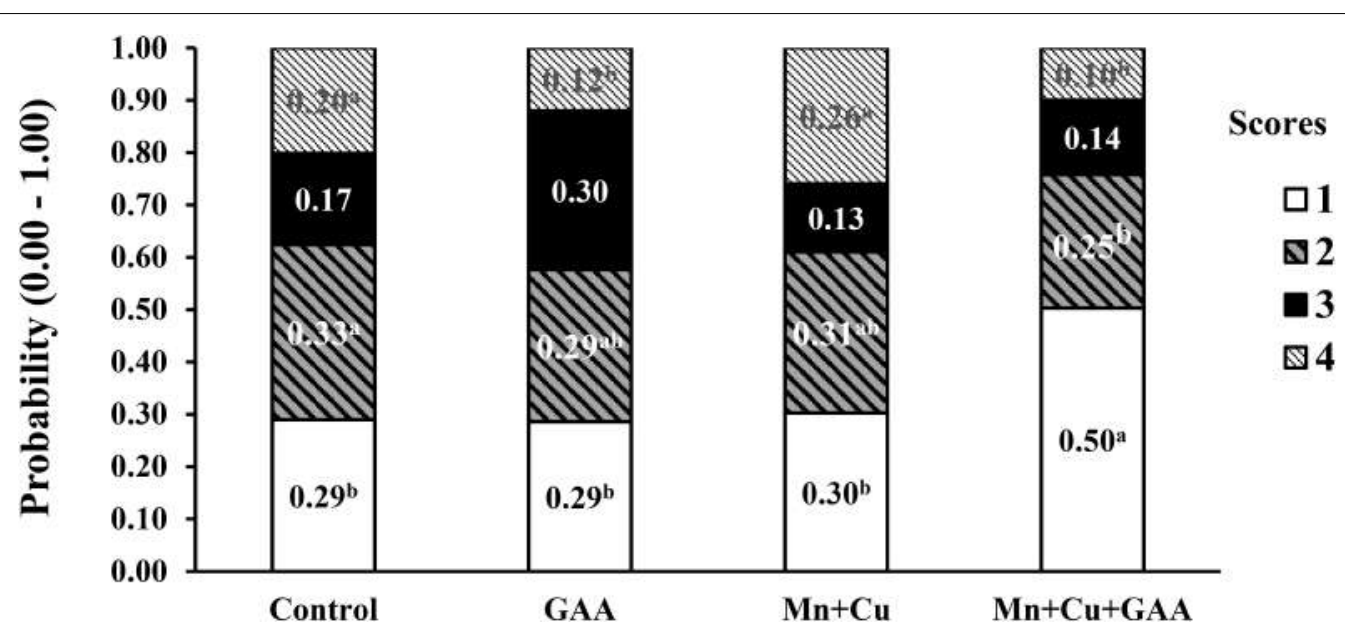

FIGURE 6 | Effect of guanidino acetic acid (GAA) and organic trace minerals (manganese, Mn, and copper, $\mathrm{Cu}$ ) on the probability distribution for each white striping severity score in Ross-308 broilers at 42 day of age (adapted from Vargas, 2019).

inclusion of GAA in non-animal-protein diets did not affect breast meat yield; however, breast meat yield was reduced by 1.78 percentage points when broilers were provided the diet containing PBP meal devoid of GAA. Therefore, the variable effects in these experiments could be more related to specific nutrient digestibility than to feed ingredients or origin of the protein perse.

The degree of responses to GAA supplementation in live performance, breast meat yield, and muscle myopathy have varied by dietary levels of energy (Abudabos et al., 2014; Sadra et al., 2018) and nutrients such as Arg, Met, folic acid, vitamin E (Wyss and Kaddurah-Daouk, 2000; Dilger et al., 2013; De Groot et al., 2019; Ibrahim et al., 2019; Majdeddin et al., 2019; Khajali et al., 2020) and minerals (Vargas, 2019). The GAA or creatine action always depends on the dietary level of other nutrients to influence muscle metabolism due to its regulatory pathways (Figure 1). However, as was expected, the PBP-diets did not contain detectable levels of creatine due to the low creatine level in the PBP itself. Strikingly, both experiments indicated that GAA increased breast meat yield significantly in large broilers without augmenting the incidence of $\mathrm{WB}$ or other myopathies.

In a more recent experiment, Vargas (2019) evaluated the dietary supplementation GAA (600 g/ton) and chelated trace minerals $(\mathrm{Mn}$ and $\mathrm{Cu})$ in Cobb 500 up to 42 days of age on performance and muscle myopathies (WB and white striping). Broilers were fed corn-soybean meal diets with PBP, soybean oil, and meat and bone meal (1.8 to $2.4 \%$ ) in all diets and feather meal $(1.50 \%)$ in the last feeding phase. A total of 120 carcasses per each treatment were evaluated for carcass and cutup part yields and WB and white striping incidence, among other carcass quality parameters, in a commercial processing plant using a similar scoring system as described by Tijare et al. (2016). The presence of spaghetti muscle was not evaluated. Results indicated that GAA supplementation did not significantly affect live performance, carcass or meat yield, or physicochemical parameters of breast meat compared to the control treatment. The dietary inclusion of GAA $+\mathrm{Mn}+\mathrm{Cu}$ increased the number of normal filets from $70.8 \%$ in the control treatment to $80.4 \%$ 
while reducing severe WB from 6.79 to $2.94 \%$ (Figure 5). More evident benefits on reducing WB were observed with GAA than with alternative supplements such as nucleotides and organic $\mathrm{Mn}$ and $\mathrm{Cu}$ minerals alone. The supplementation of GAA had the most significant reduction in WB score 2 compared to control (1.44 vs. $8.13 \%)$. White striping was also reduced by the combined inclusion of GAA $+\mathrm{Mn}+\mathrm{Cu}$ (Figure 6). Normal filets in this treatment were $50.83 \%$ compared to $29.46 \%$ in the control treatment. Filets scored as severe white striping were $20.13 \%$ in the control treatment; the supplementation with GAA reduced them to $11.97 \%$, and the addition of trace minerals GAA $+\mathrm{Mn}+\mathrm{Cu}$ reduced them further to 9.98\%. The data reported by Vargas (2019) indicated once again the relationship between GAA supplementation and the presence of other nutrients, in this case, trace minerals. In a larger sample size than Córdova-Noboa et al. (2018a,b), results from Vargas (2019) evidenced moderate positive effects of WB and white striping.

The early results reported by Córdova-Noboa et al. (2018a,b) generated interest in replicating these experiments on a commercial scale. A recent report by Aviagen (2019) described the use of GAA (600 g/ton) in three commercial-scale trials. The research, conducted in the United States demonstrated slight improvements (decrease) in WB severity at 49 days of age, but not at 56 days of age. The other two tests were conducted in Europe and showed a 17 and $31 \%$ decrease in the incidence of WB. These

TABLE 3 | Plasma creatine kinase (CK) and L-aspartate aminotransferase (AST) concentrations in broilers with dorsal cranial myopathy or woody breast.

\begin{tabular}{lcc}
\hline Parameter & Affected & Unaffected \\
\cline { 2 - 3 } & \multicolumn{1}{c}{---- IU/L----- } \\
\hline Dorsal cranial myopathy & & \\
CK $^{\star}$ & $54,091^{a}$ & $35,203^{\mathrm{b}}$ \\
AST $^{\star *}$ & $409^{\mathrm{a}}$ & $320^{\mathrm{b}}$ \\
Woody breast $^{2}$ & & \\
CK $^{\star \star}$ & $42,360^{\mathrm{a}}$ & $10,164^{\mathrm{b}}$ \\
AST $^{\star *}$ & $356^{\mathrm{a}}$ & $131^{\mathrm{b}}$ \\
\hline
\end{tabular}

${ }^{1}$ Adapted from Amaral et al. (2017). $n=28$ broilers processed at 42 days of age. ${ }^{2}$ Adapted from Kawasaki et al. (2018). $n=10$ broilers sacrificed at 20 and 29 days of age. ${ }^{*} P<0.05 ;{ }^{* *} P<0.01 ;{ }^{a, b}$ Mean values in the same line followed by different letter superscripts are significantly different $(P<0.05)$. studies also indicate that growth performance and breast meat yield were increased in chickens fed GAA-diets while reducing WB incidence. However, there was no indication that white striping was affected in these commercial trials, and spaghetti muscle is not discussed in this report. In summary, GAA, as a feed additive, enhanced productivity of broilers and meat yield without exacerbating breast muscle myopathies.

These beneficial results of GAA on WB incidence and severity could be explained by its role on reducing insulin resistance, sparing Arg to increase vasodilation, improving antioxidant capacity (Ostojic, 2015a), and the energetic balance in muscles due to an increase in muscle creatine and as such help supply ATP and proper muscle function during growth (Baldi et al., 2020). Ibrahim et al. (2019) observed that GAA $+0.4 \%$ Met was superior to creatine to enhance gene expression of insulin growth factor-1, growth hormone, and muscle myogenin and downregulate myostatin in mulard ducks. These two hormones and two muscle transcription factors are affected in myopathies (Vignale et al., 2017; Brothers et al., 2019). Therefore, the positive effects of GAA on its regulation could aid in mitigating moderate WB. Ibrahim et al. (2019) also indicated that a possible shortage of methyl groups could be induced by long-term GAA supplementation in ducks, which could impair protein synthesis unless more methionine is added to the feed. In diets based on sorghum, the lower Arg, and Met digestibility as a consequence of kafirin and tannin presence could reduce the efficacy of GAA (CórdovaNoboa et al., 2018a). Therefore, attention to dietary factors seems essential, and more research is warranted to validate positive results with GAA.

\section{CREATINE AS A MARKER FOR WOODEN BREAST MYOPATHY?}

Finally, it is relevant to highlight that creatine and CK have been selected as markers of numerous myopathies in several species. The CK, alanine transaminase (ALT), and lactate dehydrogenase (LDH) concentrations in blood have been widely studied as blood markers to screen individuals for suspected muscle damage and necrosis in dogs (Tvarijonaviciute et al., 2017), horses (Söderqvist et al., 2013), and humans (Hathout et al., 2016). Therefore, it is already well established that blood concentrations of these enzymes are suitable biomarkers for myopathies.

TABLE 4 | Serum ALT, AST, LDH, and CK concentrations of broilers affected by different WB severity.

\begin{tabular}{|c|c|c|c|c|c|c|}
\hline \multirow[t]{2}{*}{ Enzymes (U/L) } & \multicolumn{4}{|c|}{ Wooden breast score ${ }^{1}$} & \multirow[t]{2}{*}{ SEM } & \multirow{2}{*}{$\begin{array}{c}\text { Pairwise correlation with WB } \\
r^{*}\end{array}$} \\
\hline & 1 & 2 & 3 & 4 & & \\
\hline$N$ & 8 & 25 & 32 & 13 & & \\
\hline $\mathrm{ALT}^{*}$ & $5.50^{\mathrm{b}}$ & $5.42^{b}$ & $6.58^{\mathrm{b}}$ & $8.89^{a}$ & 0.50 & 0.54 \\
\hline $\mathrm{AST}^{\star}$ & $451.67^{b}$ & $511.05^{b}$ & $653.79^{b}$ & $888.25^{\mathrm{a}}$ & 54.78 & 0.63 \\
\hline $\mathrm{LDH}^{*}$ & $1,803^{b}$ & $1,714^{b}$ & $2,175^{b}$ & $4,670^{a}$ & 470 & 0.46 \\
\hline $\mathrm{CK}^{\star}$ & $26,740^{c}$ & $45,490^{c}$ & $67,826^{b}$ & $109,520^{a}$ & 10,459 & 0.53 \\
\hline
\end{tabular}

${ }^{\star} P<0.001 ;{ }^{a-c}$ Mean values in the same line followed by different letter superscripts are significantly different (P < 0.001). Adapted from Córdova-Noboa (2015). 
In the case of broiler chickens, Kawasaki et al. (2018) found roughly three to four times greater, concentrations of plasma CK and L-aspartate aminotransferase (AST) in 20-day old chickens with severe WB than in chickens with normal breast filets (Table 3). Plasma CK and AST might also be helpful in detecting WB in young chickens, but the concentration of these enzymes is also increased in other broiler myopathies. Amaral et al. (2017) concluded that serum CK and AST concentrations were the only blood parameters that differed between broilers with dorsal cranial myopathy and healthy broilers. In that, myopathy of the anterior latissimus dorsi muscle is affected (Table 3).

A study conducted by Córdova-Noboa (2015) compared the serum concentrations for different metabolites in 78 Ross 708 male broilers processed at 55 days of age. The results presented in Table 4 show that broilers affected with severe WB yielded higher blood concentrations of ALT, AST, LDH, and CK in comparison with samples from broilers that had normal, low, and moderate WB scores. The levels of LDH and CK almost doubled in broilers with severe (score 4) WB compared to broilers with no WB. In addition, the pairwise correlations indicate a moderate $(r=0.43-$ $0.63)$ positive correlation $(P<0.001)$ of enzyme concentrations in plasma with WB scores (Table 4). Therefore, the levels of enzymes such as CK, LDH, AST, and ALT are potential markers of $\mathrm{WB}$, and the accuracy for combined use in the detection of myopathies should be further evaluated. These biomarkers indicate once again the importance of muscle creatine and $\mathrm{CK}$ in WB myopathy and the relevance of GAA as potential feed additive to reduce its occurrence.

\section{CONCLUSION}

There is some evidence in the literature from three studies conducted at the experimental level (Córdova-Noboa et al., 2018a,b; Vargas, 2019) and three commercial scenarios (Aviagen, 2019), indicating that dietary supplementation of guanidino acetic acid (GAA) at $600 \mathrm{~g} /$ ton reduces the occurrence and ameliorates the severity of wooden breast in broilers. However, only one experiment indicated that GAA can significantly reduce the incidence or severity of white striping. There is no evidence that spaghetti muscle incidence or severity is affected by GAA supplementation, but these have not been evaluated extensively. The effects of higher levels of GAA supplementation ( $1200 \mathrm{~g} /$ ton) that have been proven to improve live performance and some muscle characteristics have not been evaluated in the incidence and severity of myopathies. Several research reports have indicated that GAA could support muscle development, function, and regeneration by improving energy homeostasis, increasing physiological levels of creatine in muscles in broilers

\section{REFERENCES}

Abasht, B., Mutryn, M. F., Michalek, R. D., and Lee, W. R. (2016). Oxidative stress and metabolic perturbations in wooden breast disorder in chickens. PLoS One 4:e0153750. doi: 10.1371/journal.pone.0153750

Abudabos, A. M., Saleh, F., Lemme, A., and Zakaria, H. A. H. (2014). The relationship between guanidino acetic acid and metabolisable energy level of and in many other species. Creatine in its phosphorylated form phosphocreatine regenerates ATP, the key molecule in muscle energy balance, contraction, protein metabolism, and development. GAA's support of creatine production is of particular importance because commercial poultry feed is deficient in creatine, and poultry genetics are selected for higher muscle development rates, which makes them susceptible to myopathies. The levels of creatine in the muscle and the CK together with $\mathrm{LDH}$ in blood are closely related to $\mathrm{WB}$, indicating the importance of creatine metabolism in myopathies. Research results have also shown that GAA may also potentially prevent broiler muscle degeneration by several mechanisms that include improving glucose metabolism, releasing IGF's, sparing Arg to enhance protein formation, nitric oxide production, improving vascularization, vasodilatation, and oxygenation. Finally, it has been confirmed that GAA supplementation may stimulate myotube growth, reduce oxidative muscle stress, and allow muscle fiber regeneration.

\section{AUTHOR CONTRIBUTIONS}

EO-R and HC-N worked on the research projects presented and in the literature research conducted.

\section{FUNDING}

The authors declare that two of the research projects described in this review were conducted at North Carolina State University and received funding from Evonik Animal Nutrition. The funder was not involved in the study design, collection, analysis, interpretation of data, the writing of this article or the decision to submit it for publication.

\section{ACKNOWLEDGMENTS}

The authors would like to express their acknowledgments to all collaborators, graduate, and undergraduate students who contributed to the sample and data collection, lab analyses in the experiments. Special gratitude to Dr. John H. Barnes, Emeritus Professor from the College of Veterinary Medicine at North Carolina State University for his contributions to different projects during the past years, and Dr. Ulrike Braun from Alzchem Trostberg GmbH, Germany for the peer review. Additionally, it is essential to recognize the financial support of the Evonik Animal Nutrition and Alzchem Trostberg GmbH. Without their funding, these projects would not be possible.

diets on performance of broiler chickens. Italian J. Anim. Sci. 13:3269. doi: 10.4081/ijas.2014.3269

Ahmadipour, B., Naeini, S. Z., Sharifi, M., and Khajali, F. (2018a). Growth performance and right ventricular hypertrophy responses of broiler chickens to guanidinoacetic acid supplementation under hypobaric hypoxia. J. Poult. Sci. 55, 60-64. doi: 10.2141/jpsa.017 0044 
Ahmadipour, B., Khajali, F., and Sharifi, M. (2018b). Effect of guanidinoacetic acid supplementation on growth performance and gut morphology in broiler chickens. J. Poult. Sci. 6, 19-24.

Ahmadipour, B., Sharifi, M., and Khajali, F. (2018c). Pulmonary hypertensive response of broiler chickens to arginine and guanidinoacetic acid under highaltitude hypoxia. Acta Vet. Hung. 66, 114-124.

Ale Saheb Fosoul, S. S., Azarfar, A., Gheisari, A., and Khosravinia, H. (2018). Energy utilisation of broiler chickens in response to guanidinoacetic acid supplementation in diets with various energy contents. Br. J. Nutr. 120, 131140. doi: 10.1017/S0007114517003701

Amaral, P. C., Zimermann, C., Santos, L. R., Noro, M., Prá, M. D., Pilotto, F., et al (2017). Evaluation of physiological parameters of broilers with dorsal cranial myopathy. Braz. J. Poultry Sci. 19, 69-74. doi: 10.1590/1806-9061-2016-0377

Amiri, M., Ghasemi, H. A., Hajkhodadadi, I., and Khaltabadi Farahani, A. H. (2019). Efficacy of guanidinoacetic acid at different dietary crude protein levels on growth performance, stress indicators, antioxidant status, and intestinal morphology in broiler chickens subjected to cyclic heat stress. Anim. Feed Sci. Technol. 254:114208. doi: 10.1016/j.anifeedsci.2019.114208

Aviagen (2019). Breast Muscle Myopathies. Available online at: http: //en.aviagen.com/assets/Tech_Center/Broiler_Breeder_Tech_Articles/English/ Breast-Muscle-Myopathies-2019-EN.pdf (accessed January 15, 2020).

Bailey, R. A., Watson, K. A., Bilgili, S. F., and Avendano, S. (2015). The genetic basis of pectoralis major myopathies in modern broiler chicken lines. Poult. Sci. 94, 2870-2879. doi: 10.3382/ps/pev304

Baker, D. H. (2009). Advances in protein-amino acid nutrition of poultry. Amino Acids 37, 29-41. doi: 10.1007/s00726-008-0198-3

Baldi, G., Soglia, F., Mazzoni, M., Sirri, F., Canonico, L., Babini, E., et al. (2018). Implications of white striping and spaghetti meat abnormalities on meat quality and histological features in broilers. Animal 12, 164-173. doi: 10.1017/ S1751731117001069

Baldi, G., Yen, C.-N., Daughtry, M. R., Bodmer, J., Bowker, B. C., Zhuang, H., et al. (2020). Exploring the factors contributing to the high ultimate $\mathrm{pH}$ of broiler Pectoralis major muscles affected by wooden beast condition. Front. Physiol. 11:343. doi: 10.3389/fphys.2020.00343

Balsom, P. D., Soderlund, K., and Ekblom, B. (1994). Creatine in humans with special reference to creatine supplementation. Sport. Med. 18, 268-280. doi: 10.2165/00007256-199418040-00005

Beard, H. H., and Barnes, B. O. (1931). The influence of feeding proteins, amino acids, and related substances upon creatine-creatinine metabolism. J. Biol. Chem. 94, 49-69.

Boney, J. W., Patterson, P. H., and Solis, F. (2019). The effect of dietary inclusions of guanidinoacetic acid on D1-42 broiler performance and processing yields. J. Appl. Poult. Res. 29, 220-228. doi: 10.1016/j.japr.2019.10.008

Borsook, M. E., and Borsook, H. (1951). Treatment of cardiac decompensation with betaine and glycocyamine. Ann. West Med. Surg. 5, 830-855.

Brothers, B., Zhuo, Z., Papah, M. B., and Abasht, B. (2019). RNA-Sep analysis reveals spatial and sex differences in Pectoralis major muscle of broiler chickens contributing to difference in susceptibility to wooden breast disease. Front. Physiol. 10:764. doi: 10.3389/fphys.2019.00764

Carvalho, C. M. C., Fernandes, E. A., Carvalho, A. P., Maciel, M. P., Cairess, R. M., and Fagundes, N. S. (2013). Effect of creatine addition in feeds containing animal meals on the performance and carcass yield of broilers. Braz. J. Poultry Sci. 15, 269-275. doi: 10.1590/S1516-635X2013000300015

Chen, L. R., Suyemoto, M. M., Sarsour, A. H., Cordova, H. A., Oviedo-Rondoìn, E. O., Wineland, M., et al. (2019). Temporal characterization of broiler breast myopathy ("Woody Breast") severity and correlation with growth rate and lymphocytic phlebitis in three commercial broiler strains and a random-bred broiler strain. Avian Pathol. 48, 319-328. doi: 10.1080/03079457.2019.1598541

Córdova-Noboa, H. A. (2015). Effects of Guanidinoacetic Acid in Broilers. dissertation, North Carolina State University, Raleigh, NC.

Córdova-Noboa, H. A., Oviedo-Rondón, E. O., Sarsour, A. H., Barnes, J., Ferzola, P., Rademacher-Heilshorn, M., et al. (2018a). Performance, meat quality, and pectoral myopathies of broilers fed either corn or sorghum based diets supplemented with guanidinoacetic acid. Poult. Sci. 97, 2479-2493. doi: 10. 3382/ps/pey096

Córdova-Noboa, H. A., Oviedo-Rondón, E. O., Sarsour, A. H., Barnes, J., Sapcota, D., López, D., et al. (2018b). Effect of guanidinoacetic acid supplementation on live performance, meat quality, pectoral myopathies and blood parameters of male broilers fed corn-based diets with or without poultry by-products. Poult. Sci. 97, 2494-2505. doi: 10.3382/ps/pey097

Cruz, R. F. A., Vieira, S. L., Kindlein, L., Kipper, M., Cemin, H. S., and Rauber, S. M. (2017). Occurrence of white striping and wooden breast in broilers fed grower and finisher diets with increasing lysine levels. Poult. Sci. 96, 501-510. doi: $10.3382 /$ ps/pew310

De Groot, A. A., Braun, U., and Dilger, R. N. (2018). Efficacy of guanidinoacetic acid on growth and muscle energy metabolism in broiler chicks receiving arginine-deficient diets. Poult. Sci. 97, 890-900. doi: 10.3382/ps/pex378

De Groot, A. A., Braun, U., and Dilger, R. N. (2019). Guanidinoacetic acid is efficacious in improving growth performance and muscle energy homeostasis in broiler chicks fed arginine-deficient or arginine-adequate diets. Poult. Sci. 98, 2896-2905. doi: 10.3382/ps/pez036

Deldicque, L., Louis, M., Theisen, D., Nielens, H., Dehoux, H., Thissen, J. P., et al. (2005). Increased IGF mRNA in human skeletal muscle after creatine supplementation. Med. Sci. Sports Exerc. 37, 731-736. doi: 10.1249/01.MSS. 0000162690.39830 .27

Derave, W., Marescau, B., Eede, E. V., Eijnde, B. O., De Deyn, P. P., and Hespel, P. (2004). Plasma guanidino compounds are altered by oral creatine supplementation in healthy humans. J. Appl. Physiol. 97, 852-857. doi: 10.1152/ japplphysiol.00206.2004

Dilger, R. N., Bryant-Angeloni, K., Payne, R. L., Lemme, A., and Parsons, C. M. (2013). Dietary guanidino acetic acid is an efficacious replacement for arginine for young chicks. Poult. Sci. 92, 171-177. doi: 10.3382/ps.2012-02425

Dobenecker, B., and Braun, U. (2015). Creatine and creatinine contents in different diet types for dogs -effects of source and processing. J. Anim. Physiol. An. N. 99, 1017-1024. doi: 10.1111/jpn.12383

D’Souza, D. M., Al-Sajee, D., and Hawke, T. J. (2013). Diabetic myopathy: impact of diabetes mellitus on skeletal muscle progenitor cells. Front. Physiol. 4:379. doi: 10.3389/fphys.2013.00379

European Food Safety Authority [EFSA] (2009). Safety and efficiency of guanidino acetic acid as feed additive for chickens for fattening. EFSA J. 988, $1-30$.

European Food Safety Authority [EFSA] (2016). Scientific opinion on the safety and efficacy of guanidinoacetic acid for chickens for fattening, breeder hens and roosters, and pigs. EFSA J. 14:4394. doi: 10.2903/j.efsa.2016.4394

FDA US Food and Drug Administration (2016). Code of Federal Regulations Title 21. Available online at: https://www.accessdata.fda.gov/scripts/cdrh/cfdocs/ cfcfr/CFRSearch.cfm?fr=573.496 (accessed June 1, 2020).

Fitch, C. D. (1977). "Significance of abnormalities of creatine metabolism," in Pathogenesis of Human Muscular Dystrophies, ed. L. P. Rowland (Amsterdam: Excerpta Medica), 328-340.

Gábor, E., Gáspar, O., and Vámos, E. (1984). Quantitative determination of muscle protein in meat products by measuring creatine content. Acta Aliment. Hung. $13,13-22$.

Griffin, J. R., Moraes, L., Wick, M., and Lilburn, M. S. (2018). Onset of white striping and progression into wooden breast as defined by myopathic changes underlying Pectoralis major growth. Estimation of growth parameters as predictors for stage of myopathy progression. Avian Pathol. 47, 2-13. doi: 10.1080/03079457.2017.1356908

Guimarães-Ferreira, L. (2014). Role of the phosphocreatine system on energetic homeostasis in skeletal and cardiac muscles. Einstein 12, 126-131. doi: 10.1590/ S1679-45082014RB2741

Guimbal, C., and Kilimann, M. W. (1993). A Na(+)-dependent creatine transporter in rabbit brain, muscle, heart, and kidney. cDNA cloning and functional expression. J. Biol. Chem. 268, 8418-8421.

Harris, R. C., Lowe, J. A., Warnes, K., and Orme, C. E. (1997). The concentration of creatine in meat, offal and commercial dog food. Res. Vet. Sci. 62, 58-62. doi: 10.1016/s0034-5288(97)90181-8

Harris, R. C., Soderlund, K., and Hultman, E. (1992). Elevation of creatine in resting and exercised muscle of normal subjects by creatine supplementation. Clin. Sci. 83, 367-374. doi: 10.1042/cs0830367

Hathout, Y., Seol, H., Han, M. H. J., Zhang, A., Brown, K. A., and Hoffman, E. P. (2016). Clinical utility of serum biomarkers in Duchenne muscular dystrophy. Clin. Proteom. 13:9. doi: 10.1186/s12014-016-9109-x

Havenstein, G. B., Ferket, P. R., and Qureshi, M. A. (2003a). Carcass composition and yield of 1957 vs 2001 broilers when fed representative 1957 and 2001 broiler diets. Poult. Sci. 82, 1509-1518. doi: 10.1093/ps/82.10.1509 
Havenstein, G. B., Ferket, P. R., and Qureshi, M. A. (2003b). Growth, livability, and feed conversion of 1957 vs. 2001 broilers when fed representative 1957 and 2001 broiler diets. Poult. Sci. 82, 1500-1508. doi: 10.1093/ps/82.10.1500

Heger, J., Zelenka, J., Machander, V., De La Cruz, C., Lešták, M., and Hampel, D. (2014). Effects of guanidinoacetic acid supplementation to broiler diets with varying energy content. Acta Univ. Agric. Silvic. Mendelianae Brun. 62, 477-485. doi: 10.11118/actaun201462030477

Hultman, E., Soderlund, K., Timmons, J. A., Cederblad, G., and Greenhaff, P. L. (1996). Muscle creatine loading in men. J. Appl. Physiol. 81, 232-237. doi: 10.1152/jappl.1996.81.1.232

Hunter, A. (1928). Creatine and Creatinine. London: Longmans Green and Co.

Ibrahim, D., El Sayeda, R., Abdelfattah-Hassan, A., and Morshedy, A. M. (2019). Creatine or guanidinoacetic acid? Which is more effective at enhancing growth, tissue creatine stores, quality of meat, and genes controlling growth/myogenesis in Mulard ducks. J. Appl. Animal Res. 47, 159-166. doi: 10.1080/09712119.2019. 1590205

Jiao, S., Ren, H., Li, Y., Zhou, J., Duan, C., and Lu, L. (2013). Differential regulation of IGF-I and IGF-II gene expression in skeletal muscle cells. Mol. Cell Biochem. 373, 107-113. doi: 10.1007/s11010-012-1479-4

Kawasaki, T., Iwasaki, T., Yamada, M., Yoshida, T., and Watanabe, T. (2018). Rapid growth rate results in remarkably hardened breast in broilers during the middle stage of rearing?: a biochemical and histopathological study. PLoS One 13:e0193307. doi: 10.1371/journal.pone.0193307

Khajali, F., Lemme, A., and Rademacher-Heilshorn, M. (2020). Guanidinoacetic acid as a feed supplement for poultry. Worlds Poult. Sci. J. 1-2.

Khajali, F., and Wideman, R. F. (2010). Dietary arginine: metabolic, environmental, immunological and physiological interrelationships. Worlds Poult. Sci. J. 66, 751-766. doi: 10.1017/s0043933910000711

Khan, A. W., and Cowen, D. C. (1977). Rapid estimation of muscle proteins in beef-vegetable protein mixtures. J. Agric. Food Chem. 25, 236-238. doi: 10.1021/jf60210a029

Kley, R. A., Tarnopolsky, M. A., and Vorgerd, M. (2013). Creatine for treating muscle disorders. Cochrane Database Syst. Rev. 6:CD004760.

Krueger, K., Damme, K., and Lemme, A. (2010). Bessere mast mit CreAmino. DGS Magazin 26, 10-14.

Kuttappan, V. A., Huff, G. R., Huff, W. E., Hargis, B. M., Apple, J. K., Coon, C., et al. (2013a). Comparison of hematologic and serologic profiles of broiler birds with normal and severe degrees of white striping in breast fillets. Poult. Sci. 92, 339-345. doi: 10.3382/ps.2012-02647

Kuttappan, V. A., Shivaprasad, H. L., Shaw, D. P., Valentine, B. A., Hargis, B. M., Clark, F. D., et al. (2013b). Pathological changes associated with white striping in broiler breast muscles. Poult. Sci. 92, 331-338. doi: 10.3382/ps.2012-02646

Lake, J. A., and Abasht, B. (2020). Glucolipotoxicity: a proposed etiology for wooden breast and related myopathies in commercial broiler chickens. Front. Physiol. 11:169. doi: 10.3389/fphys.2020.00169

Majdeddin, M., Golian, A., Kermanshahi, H., De Smet, S., and Michiels, J. (2018). Guanidinoacetic acid supplementation in broiler chickens fed on corn-soybean diets affects performance in the finisher period and energy metabolites in breast muscle independent of diet nutrient density. Br. Poult. Sci. 59, 443-451. doi: 10.1080/00071668.2018.1476678

Majdeddin, M., Golian, A., Kermanshahi, H., Michiels, J., and De Smet, S. (2019). Effects of methionine and guanidoacetic acid supplementation on performance and energy metabolites in breast muscle of male broiler chickens fed cornsoybean diets. Br. Poult. Sci. 60, 554-563. doi: 10.1080/00071668.2019.1631447

Malila, Y., Thanatsang, K., Arayamethakorn, S., Uengwetwanit, T., Srimarut, Y., Petracci, M., et al. (2019). Absolute expressions of hypoxia-inducible factor-1 alpha (HIF1A) transcript and the associated genes in chicken skeletal muscle with white striping and wooden breast myopathies. PLoS One 14:e0220904. doi: 10.1371/journal.pone.0220904

Michiels, J., Maertens, L., Buyse, J., Lemme, A., Rademacher, M., Dierick, N. A., et al. (2012). Supplementation of guanidinoacetic acid to broiler diets: effects on performance, carcass characteristics, meat quality, and energy metabolism. Poult. Sci. 91, 402-412. doi: 10.3382/ps.2011-01585

Montagna, F. S., Garcia, G., Nääs, I. A., Lima, N. D. S., and Caldara, F. R. (2019). Practical assessment of spaghetti breast in diverse genetic strain broilers reared under different environments. Braz. J. Poultry Sci. 21:eRBCA-2019-0759. doi: 10.1590/1806-9061-2018-0759
Mousavi, S. N., Afsar, A., and Lotfollahian, H. (2013). Effects of guanidinoacetic acid supplementation to broiler diets with varying energy contents. J. Appl. Poult. Res. 22, 47-54. doi: 10.3382/japr.2012-00575

Mudalal, S., Lorenzi, M., Soglia, F., Cavani, C., and Petracci, M. (2015). Implications of white striping and wooden breast abnormalities on quality traits of raw and marinated chicken meat. Animal 9, 728-734. doi: 10.1017/ s175173111400295x

Mutryn, M. F., Brannick, E. M., Fu, W., Lee, W. R., and Abasht, B. (2015). Characterization of a novel chicken muscle disorder through differential gene expression and pathway analysis using RNA-sequencing. BMC Genomics 16:399. doi: 10.1186/s12864-015-1623-0

Nabuurs, C. I., Choe, C. U., Veltien, A., Kan, H. E., van Loon, L. J., Rodenburg, R. J., et al. (2013). Disturbed energy metabolism and muscular dystrophy caused by pure creatine deficiency are reversible by creatine intake. J. Physiol. 591, 571-592. doi: 10.1113/jphysiol.2012.241760

Nasiroleslami, M., Torki, M., Saki, A. A., and Abdolmohammadi, A. R. (2018). Effects of dietary guanidinoacetic acid and betaine supplementation on performance, blood biochemical parameters and antioxidant status of broilers subjected to cold stress. J. Appl. Anim. Res. 46, 1016-1022. doi: 10.1080/ 09712119.2018 .1450751

Ostojic, S. M. (2015a). Advanced physiological roles of guanidinoacetic acid. Eur. J. Nutr. 54, 1211-1215. doi: 10.1007/s00394-015-1050-7

Ostojic, S. M. (2015b). Cellular bioenergetics of guanidinoacetic acid?: the role of mitochondria. J. Bioenerg. Biomembr. 47, 369-372. doi: 10.1007/s10863-0159619-7

Ostojic, S. M. (2016). Guanidinoacetic acid as a performance-enhancing agent. Amino Acids 48, 1867-1875. doi: 10.1007/s00726-015-2106-y

Ostojic, S. M. (2017). Tackling guanidinoacetic acid for advanced cellular bioenergetics. Nutrition 34, 55-57. doi: 10.1016/j.nut.2016.09.010

Owens, C. M., Alvarado, C. Z., and Sams, A. R. (2009). Research developments in pale, soft, and exudative turkey meat in North America. Poult. Sci. 88, 1513-1517. doi: 10.3382/ps.2009-00008

Papah, M. B., Brannick, E. M., Schmidt, C. J., and Abasht, B. (2017). Evidence and role of phlebitis and lipid infiltration in the onset and pathogenesis of Wooden Breast Disease in modern broiler chickens. Avian Pathol. 46, 623-643. doi: 10.1080/03079457.2017.1339346

Parise, G., Mihic, S., MacLennan, D., Yarasheski, K. E., and Tarnopolsky, M. A. (2001). Effects of acute creatine monohydrate supplementation on leucine kinetics and mixed-muscle protein synthesis. J. Appl. Physiol. 91, 1041-1047. doi: 10.1152/jappl.2001.91.3.1041

Pearlman, J. P., and Fielding, R. A. (2006). Creatine monohydrate as a therapeutic aid in muscular dystrophy. Nutr. Rev. 64, 80-88. doi: 10.1111/j.1753-4887.2006. tb00191.x

Petracci, M., and Cavani, C. (2012). Muscle growth and poultry meat quality issues. Nutrients 4, 1-12. doi: 10.3390/nu4010001

Petracci, M., Mudalal, S., Soglia, F., and Cavani, C. (2015). Meat quality in fastgrowing broiler chickens. Worlds Poult. Sci. J. 71, 363-374. doi: 10.1017/ s0043933915000367

Petracci, M., Soglia, F., Madruga, M., Carvalho, L., Ida, E., and Estévez, M. (2019). Wooden-breast, white striping, and spaghetti meat: causes, consequences and consumer perception of emerging broiler meat abnormalities. Compr. Rev. Food Sci. F. 18, 565-583. doi: 10.1111/1541-4337.12431

Post, A., Tsikas, D., and Bakker, S. J. L. (2019). Creatine is a conditionally essential nutrient in chronic kidney disease: a hypothesis and narrative literature review. Nutrients 11:1044. doi: 10.3390/nu11051044

Russo, E., Drigo, M., Longoni, C., Pezzotti, R., Fasoli, P., and Recordati, C. (2015). Evaluation of white striping prevalence and predisposing factors in broilers at slaughter. Poult. Sci. 94, 1843-1848. doi: 10.3382/ps/pev172

Sadra, S., Saheb, A., Azarfar, A., Gheisari, A., and Khosravinia, H. (2018). Energy utilisation of broiler chickens in response to guanidinoacetic acid supplementation in diets with various energy contents. Br. J. Nutr. 308, 131140. doi: 10.1017/S0007114517003701

Sihvo, H. K. (2019). Pathology of Wooden Breast Myopathy in Broiler Chickens. Master's thesis, University of Helsinki, Helsinki.

Sihvo, H.-K., Airas, N., Lindén, J., and Puolanne, E. (2018). Pectoral vessel density and early ultrastructural changes in broiler chicken wooden breast myopathy. J. Comp. Pathol. 161, 1-10. doi: 10.1016/j.jcpa.2018.04.002 
Sihvo, H.-K., Lindén, J., Airas, N., Immonen, K., Valaja, J., and Puolanne, E. (2017). Wooden breast myodegeneration of Pectoralis major muscle over the growth period in broilers. Vet. Pathol. 54, 119-128. doi: 10.1177/0300985816658099

Söderqvist, E., Svanholm, C., Olsen, S. N., and Leifsson, P. S. (2013). Equine polysaccharide storage myopathy. Dansk Veterinaertidsskrift 2, 26-31.

Stead, L. M., Au, K. P., Jacobs, R. L., Brosnan, M. E., and Brosnan, J. T. (2001). Methylation demand and homocysteine metabolism: effects of dietary provision of creatine and guanidinoacetate. Am. J. Physiol. Endoc. M 281, E1095-E1100.

Tabatabaei Yazdi, F., Golian, A., Zarghi, H., and Varidi, M. (2017). Effect of wheat-soy diet nutrient density and guanidine acetic acid supplementation on performance and energy metabolism in broiler chickens. Ital. J. Anim. Sci. 16, 593-600. doi: 10.1080/1828051X.2017.1305260

Tarnopolsky, M., Parshad, A., Walzel, B., Schlattner, U., and Wallimann, T. (2001). Creatine transporter and mitochondrial creatine kinase protein content in myopathies. Muscle Nerve 24, 682-688. doi: 10.1002/mus. 1055

Tarnopolsky, M. A. (2007). "Clinical use of creatine in neuromuscular and neurometabolic disorders," in Creatine and Creatine Kinase in Health and Disease. Subcellular Biochemistry, Vol. 46, eds G. S. Salomons and M. Wyss (Dordrecht: Springer).

Tijare, V. V., Yang, F. L., Kuttappan, V. A., Alvarado, C. Z., Coon, C. N., and Owens, C. M. (2016). Meat quality of broiler breast fillets with white striping and woody breast muscle myopathies. Poult. Sci. 95, 2167-2173. doi: 10.3382/ps/pew129

Tossenberger, J., Rademacher, M., Nemeth, K., Halas, V., and Lemme, A. (2016). Digestibility and metabolism of dietary guanidino acetic acid fed to broilers. Poult. Sci. 95, 2058-2067. doi: 10.3382/ps/pew083

Trocino, A., Piccirillo, A., Birolo, M., Radaelli, G., Bertotto, D., Filiou, E., et al. (2015). Effect of genotype, gender and feed restriction on growth, meat quality and the occurrence of white striping and wooden breast in broiler chickens. Poult. Sci. 94, 2996-3004. doi: 10.3382/ps/pev296

Tvarijonaviciute, A., Barranco, T., and Rubio, M. (2017). Measurement of creatine kinase and aspartate aminotransferase in saliva of dogs: a pilot study. BMC Vet. Res. 13:168. doi: 10.1186/s12917-017-1080- $\mathrm{x}$

Van der Poel, A. F. B., Braun, U., Hendriks, W. H., and Bosch, G. (2018). Stability of creatine monohydrate and guanidinoacetic acid during manufacture (retorting and extrusion) and storage of dog foods. J. Anim. Physiol. Anim. Nutr. 103, 1242-1250. doi: 10.1111/jpn.13103

Van Pilsum, J. F., Stephens, G. C., and Taylor, D. (1972). Distribution of creatine, guanidinoacetate and the enzymes for their biosynthesis in the animal kingdom. Biochem. J. 126, 325-345.

Vargas, L. F. (2019). Efeito Da Adição De Diferentes Nutrientes Na Dieta Sobre a Qualidade Das Carcaças e Prevenção de Peito Madeira e Estrias Brancas em
Frangos De Corte. master's thesis, Universidade Tecnologica Federal do Parana, Medianera.

Vignale, K., Caldas, J. V., England, J. A., Boonsinchai, N., Magnuson, A., Pollock, E. D., et al. (2017). Effect of white striping myopathy on breast muscle (Pectoralis major) protein turnover and gene expression in broilers. Poult. Sci. 96, 886-893. doi: $10.3382 / \mathrm{ps} /$ pew315

Vranes, M., Ostojic, S., Tot, A., Papovic, S., and Gadzuric, S. (2017). Experimental and computational study of guanidinoacetic acid self-aggregation in aqueous solution. Food Chem. 237, 53-57. doi: 10.1016/j.foodchem.2017. 05.088

Wallimann, T., Tokarska-Schlattner, M., and Schlattner, U. (2011). The creatine kinase system and pleiotropic effects of creatine. Amino Acids 40, 1271-1296.

Wang, Y., Ma, J., Qiu, W., Zhang, J., Feng, S., Zhou, X., et al. (2018). Guanidinoacetic acid regulates myogenic differentiation and muscle growth through miR-133a-3p and miR-1a-3p co-mediated Akt/mTOR/S6K signaling pathway. Int. J. Mol. Sci. 19:E2837. doi: 10.3390/ijms19092837

Wyss, M., and Kaddurah-Daouk, R. (2000). Creatine and creatinine metabolism. Physiol. Rev. 80, 1107-1213.

Zampiga, M., Laghi, L., Petracci, M., Zhu, C., Meluzzi, A., Dridi, S., et al. (2018). Effect of dietary arginine to lysine ratios on productive performance, meat quality, plasma and muscle metabolomics profile in fast-growing broiler chickens. J. Anim. Sci. Biotechnol. 9:79. doi: 10.1186/s40104-018-0294-5

Zampiga, M., Soglia, F., Petracci, M., Meluzzi, A., and Sirri, F. (2019). Effect of different arginine to lysine ratios in broiler chicken diets on the occurrence of breast myopathies. Poult. Sci. 9:79. doi: 10.3382/ps/pey284

Zuidhof, M. J., Schneider, B. L., Carney, V. L., Korver, D. R., and Robinson, F. E. (2014). Growth, efficiency, and yield of commercial broilers from 1957, 1978, and 2005. Poult. Sci. 93, 1-13. doi: 10.3382/ps.2014-04291

Conflict of Interest: The authors declare that the research was conducted in the absence of any commercial or financial relationships that could be construed as a potential conflict of interest.

Copyright (c) 2020 Oviedo-Rondón and Córdova-Noboa. This is an open-access article distributed under the terms of the Creative Commons Attribution License (CC BY). The use, distribution or reproduction in other forums is permitted, provided the original author(s) and the copyright owner(s) are credited and that the original publication in this journal is cited, in accordance with accepted academic practice. No use, distribution or reproduction is permitted which does not comply with these terms. 
OPEN ACCESS

Edited by:

Sandra G. Velleman, The Ohio State University,

United States

Reviewed by:

Orna Halevy,

Hebrew University of Jerusalem, Israel Walter Gay Bottje,

University of Arkansas, United States

${ }^{*}$ Correspondence:

Richard A. Bailey rbailey@aviagen.com

Specialty section: This article was submitted to Avian Physiology, a section of the journal Frontiers in Physiology

Received: 31 March 2020

Accepted: 29 July 2020

Published: 20 August 2020

Citation:

Bailey RA, Souza E and Avendano S (2020) Characterising the Influence of Genetics on Breast

Muscle Myopathies in Broiler Chickens. Front. Physiol. 11:1041. doi: 10.3389/fphys.2020.01041

\section{Characterising the Influence of Genetics on Breast Muscle Myopathies in Broiler Chickens}

\author{
Richard A. Bailey ${ }^{1 *}$, Eduardo Souza ${ }^{2}$ and Santiago Avendano ${ }^{1}$ \\ ${ }^{1}$ Aviagen, Newbridge, United Kingdom, ${ }^{2}$ Aviagen, Huntsville, AL, United States
}

This report provides the first estimates of the genetic basis of all key breast muscle myopathies (BMM) in broiler chickens [Deep pectoral myopathy, wooden breast, white striping and spaghetti breast] and their relationship with body weight and breast yield. Data from a pure bred high yielding commercial broiler line were analysed to estimate the genetic parameters using a multivariate animal model with the appropriate fixed effects and permanent environmental effect of the dam. Heritabilities of the BMM ranged from 0.04 to 0.25 and the genetic correlation of the BMM with body weight and breast yield ranged from -0.06 to 0.41 . Here we highlight that the genetic variance of BMM accounts for a low proportion of the phenotypic variance and the BMM have a low genetic relationship with performance traits. The large contribution of residual variance to the phenotypic variance for the BBM was $>71.5 \%$ which indicates the importance of the non-genetic effects on BMM. The data presented also show that the moderate to low genetic influence for the development of BMM can be used, through balanced selection, to reduce the myopathy incidence in the long term. The impact of genetic selection against BMM was tested empirically by comparing the incidence of WB and \% breast yield of a commercial broiler with a high generation (HG) broiler. The HG broiler used represents 2 years of genetic improvement compared to the commercial broiler; the HG broiler had an $18.4 \%$ relative decrease in WB and a 1.02\% relative increase in breast yield compared to the commercial broiler. This paper describes the relationship between the genetic and non-genetic factors influencing BMM highlighting the importance of understanding the non-genetic effects on myopathy incidence. It also shows that the genetic component of BMM can be reduced whilst at the same time improving breast yield as part of balanced breeding goals.

Keywords: broiler, breast muscle, meat quality, myopathy, heritability, genetics, breeding

\section{INTRODUCTION}

Poultry production is one of the most sustainable and efficient systems for producing high quality affordable animal protein for human consumption. The increasing efficiency of poultry production is the result of advances in livestock production systems, nutrition, animal breeding and genetic selection. Significant improvements in breast meat yield, live weights and biological efficiency have

Abbreviations: BMM, Breast muscle myopathies; BW, Body weight; BY, Breast yield; DPM, Deep pectoral myopathy; GGP, Great grandparent; GP, Grandparent; HG, High generation; PS, Parent stock; SB, Spaghetti breast; WB, Wooden breast; WS, White striping. 
been proved to be achieved through genetic selection (Havenstein et al., 2003a,b; Fleming et al., 2007; Mussini, 2012; Zuidhof et al., 2014). While it is possible that selection for production traits can have undesirable consequence on bird fitness (Hocking, 2014), these negative consequences are mitigated through balanced selection where health and welfare traits are included as equally important selection criteria (McKay et al., 2000; Neeteson, 2010; Dawkins and Layton, 2012; Avendaño et al., 2017). This is demonstrated by Kapell et al. (2012a,b) where they show that it has been possible to simultaneously improve both production traits and welfare related traits such as leg defects and contact dermatitis. Carcase yield and live weight are key performance traits for broiler chickens and as part of balanced breeding goals there is also a focus on carcase quality to reduce conditions such as ascites, infections, skeletal deformities and muscle myopathies. The impact of continued improvement of carcase quality, along with improvements of live weight and carcase yield, is evident in data from the United States poultry industry where carcase condemnations (combined post- and ante mortem) have decreased from $2.65 \%$ to $0.54 \%$ over the last 30 years (USDA, 2020). One cause of carcase downgrades or condemnation is breast muscle myopathies (BMM) which can result in economic losses to the poultry meat producers (Mitchell, 1999; Kuttappan et al., 2016; Barbut, 2019). One of the most widely recognised and well characterised myopathies is deep pectoral myopathy which was first reported in chickens in the 1980's (Richardson et al., 1980). This myopathy is the result of ischaemic necrosis of the deep pectoral muscle (Pectoralis minor) as a consequence of a compartment syndrome following exertion (Siller et al., 1978). As blood flows into the deep pectoral muscle during exercise, the inelastic fascia surrounding the muscle causes the intramuscular pressure to increase which in turn impedes venous output from the muscle causing blood pooling (Siller and Wight, 1978; Richardson et al., 1980). The consequence of this ischaemia is rapid necrosis of the muscle tissue and erythrocytes which manifests as haemorrhaging followed by a greenish discolouration as the haemoglobin in the red blood cells is broken down (Bianchi et al., 2006). One of the key triggers for this myopathy is excessive flapping or excitability within the flock and clear management recommendations are available to control the field incidence of this condition (Siller et al., 1978; Bilgili and Hess, 2008; Lien et al., 2012).

Over the last decade there has been a renewed focus on BMM within the poultry industry following increased reports of novel myopathies affecting the Pectoralis major muscle in a number of commercial broiler strains (Kuttappan et al., 2012a, 2013a,c; Ferreira et al., 2014; Mudalal et al., 2015). Within the industry vernacular these myopathies are commonly referred to as 'White striping,' 'Wooden breast' and 'Spaghetti breast' owing to their distinctive presentation on inspection (Figure 1; Kuttappan et al., 2013c; Sihvo et al., 2014; Baldi et al., 2018; Huang and Ahn, 2018; Barbut, 2019). White striping (WS) is characterised by white lines running in parallel to the muscle fibres on the surface of the muscle; there is bird to bird variation in the quantity and thickness of these lines which has enabled severity scoring when it

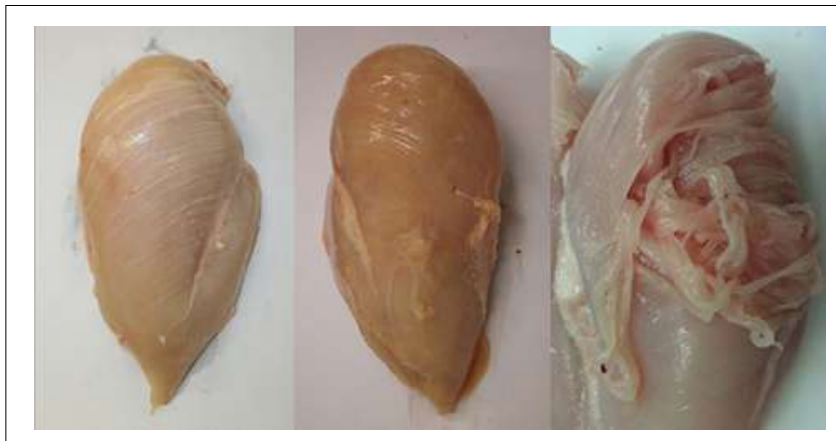

FIGURE 1 | Breast fillets showing the novel myopathies white striping (WS; left), wooden breast (WB; middle), and spaghetti breast (SB; right).

comes to recording of this myopathy (Bailey et al., 2015; Kuttappan et al., 2016). These white lines have, through extensive histological and chemical analysis, been shown to be composed of adipose tissues (Kuttappan et al., 2013a; Bailey et al., 2015). Wooden Breast (WB) manifests as a hardening of the breast fillet which has been proposed to be either due to increased collagen crosslinking (Velleman and Clark, 2015) or due to increased water being trapped within the muscle myofibrillar space (Tasoniero et al., 2017). The hardening of the muscle can occur as a singular focal lesion or multiple focal lesions, and in more severe cases can affect the whole fillet (Sihvo et al., 2017; Huang and Ahn, 2018). Depending on the severity of the condition there can be additional macroscopic features such as a pale colour, surface haemorrhaging and the presence of a sterile transudate on the surface of the muscle. Histological analysis of affected muscles show ongoing degeneration with active regeneration of muscle fibres, infiltration of immune cells along with increased deposition of adipose and connective tissues (Sihvo et al., 2014; Velleman and Clark, 2015). Spaghetti breast (SB), so called because of its resemblance to spaghetti pasta (Baldi et al., 2018), is characterised by a loss of integrity of the muscle fibres in the breast muscle leading to friability and loosening of the muscle tissue (Barbut, 2019). As with the other myopathies the severity of SB is variable, ranging from only a small part of the breast muscle being affected to the whole muscle showing the condition. Histological analysis of SB reveals a disorganised distribution of large and small muscle fibres interspersed with diffuse connective tissue.

Unlike DPM, the aetiology of these novel myopathies is not fully understood nor is the relationship they have with each other. It has been suggested that they are a progression of the same condition and other opinions suggest that they are distinct conditions. Figure 2 shows the incidence of the myopathies in a batch of affected fillets; it can be seen that they can occur individually or in combination with each other. The concurrent occurrence of the myopathies must be taken into account when selecting fillets to investigate each of the myopathies to ensure that it is just one myopathy under investigation. Failure to do so could result in wrongly assigning a biological or biochemical feature of one myopathy 


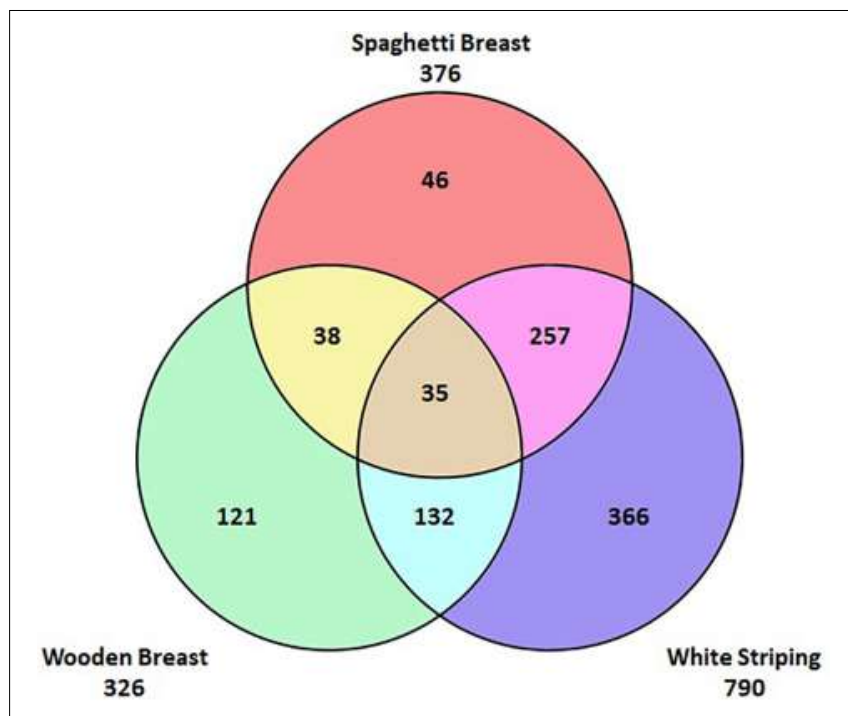

FIGURE 2 | This venn diagram demonstrates the incidence of the myopathies in 995 myopathy affected fillets. It can be seen that the myopathies can occur individually or be comorbid with each other.

to its comorbid counterpart. There have been a number of studies aiming to understand the pathogenesis of the myopathies through the use of gene expression along with proteomic and metabolomic analyses (Mutryn et al., 2015; Velleman and Clark, 2015; Zambonelli et al., 2016; Kuttappan et al., 2017; Boerboom et al., 2018; Cai et al., 2018). Results from gene expression studies show that muscles affected by the myopathies have an increased expression of a range of genes associated with metabolic (e.g. hypoxia, oxidative stress, calcium metabolism, fat metabolism, inflammation), anatomical, and structural biological processes (Mutryn et al., 2015; Zambonelli et al., 2016; Papah et al., 2018; Soglia et al., 2020). Comprehensive studies looking at birds of different ages and different locations in the muscle have highlighted early perturbations in affected muscle and localised changes in metabolic activity within the muscle which can be linked to the development of myopathies (Clark and Velleman, 2017; Papah et al., 2018; Brothers et al., 2019; Lake et al., 2019). Through proteomics, Kuttappan et al. (2017) reported that levels of proteins involved in cellular movement, carbohydrate metabolism, protein synthesis and protein maturation were significantly different in breast fillets with WB compared to breast muscles without WB. Boerboom et al. (2018) identified biological pathways which may explain how WS manifests; they reported significant differences in carbohydrate metabolism and fatty acid composition of the WS fillets compared to unaffected fillets. In addition they confirmed the findings of the gene expression studies reporting evidence of hypoxia and oxidative stress within the affected fillets. Both hypoxia and oxidative stress have been proposed as causes of the histological abnormalities seen in affected muscle tissue (Sihvo et al., 2014; Velleman and Clark, 2015) which could be linked to discord between muscle fibre size and vascular supply leading to a compartment syndrome
(Lilburn et al., 2019). Overall, these studies indicate that there are differences in a range of metabolic, anatomical and structural biological processes within the muscle of affected birds when compared to unaffected breast muscles. These findings are invaluable as they help to reveal the underlying biology and ongoing metabolic processes allowing for the development of biomarkers for further mapping of the pathophysiology of the myopathies. However, whilst these studies inform on the differences in biology between the breast muscles of affected and unaffected birds it is still unclear as to what is causing the initial disruption within the tissues. It is an attractive option to speculate about a potential singular causes for the myopathies based on the outcome of these studies alone; however, there is a need to understand what factors trigger the onset of these conditions leading to the biological changes detected in the studies mentioned above.

A popular theory for the cause of the myopathies is the genetic selection for increased body weight and breast yield (Sihvo et al., 2014; Papah et al., 2018; Petracci et al., 2019; Soglia et al., 2019); to date there is limited information on the genetic basis of the myopathies in broiler populations and their correlations with each other and production traits. A previous analysis of two pure bred commercial broiler genotypes showed low to moderate heritability for WB (0.024-0.097) and WS (0.1850.338 ) indicating that whilst there is a genetic component to the myopathies the non-genetic factors play a much greater role in the manifestation of WB and WS (Bailey et al., 2015). In both broiler populations analysed, WB and WS both had modest genetic correlations with body weight and breast yield $(-0.027-0.248)$. This indicates that selection for body weight and breast yield alone do not explain the manifestation of the myopathies and more research is needed to fully understand how the myopathies initiate and develop. A further study by Alnahhas et al. (2016) showed a higher estimate of heritability of 0.65 for WS in divergently selected broiler lines. However, as the authors rightly described, they measured WS on an underlying continuous scale which can result in a higher heritability estimate compared to the 4 point scale used by Bailey et al. (2015). According to data published by Dempster and Lerner (1950), a heritability of 0.65 would correspond to a heritability of 0.41 on the observed categorical scale which would bring it closer to the heritability for WS described by Bailey et al. (2015). A further difference in these two studies is the fitting of the effect of the common maternal environment; Alnahhas et al. (2016) did not fit this effect to WS therefore this effect would overestimate the genetic variance and heritability estimate in their analysis. Conversely, Bailey et al. (2015) accounted for the common maternal environment effect contributing to the total variance of the myopathies therefore allowing a full discrimination from the additive genetic variance. To date there is no information published on the genetic basis of SB; the aim of this study is to extend the work by Bailey et al. (2015) to further characterise the genetic basis of BMM by estimating heritabilities for DPM, WB, WS and SB along with their genetic correlations with body weight and breast yield. In addition, we will empirically illustrate the impact of genetic selection for reducing WB whilst continuing to improve breast yield as part of a balanced breeding goal. 


\section{MATERIALS AND METHODS}

\section{Study 1: Estimation of Genetic Parameters of Breast Muscle Myopathies}

Birds, Housing and Management

The data analysed in this study comes from the routine meat quality traits recording on a pure bred commercial broiler line from within the Aviagen (Tennessee, United States) breeding program. The phenotypic data spans four generations collected over 3 years from 105 flocks, with the inclusion of an extra generation of pedigree relationships for the estimation of the genetic parameters. Within this study the key phenotypic traits of interest were broiler body weight (BW), breast meat yield (BY), deep pectoral myopathy (DPM), WB, SB and WS (Table 1). The birds were housed within environmentally controlled pedigree broiler farms in Crossville Tennessee, United States; a detailed description of environmental parameters can be found in Table 2 . The birds were all housed in pens with wood shavings provided as the litter substrate with ad libitum access to food and water. All birds were incubated in the same hatchery with the same incubation conditions, after hatch all birds received the required vaccinations and were tagged with a unique barcoded wingband for identification.

\section{Recording of Traits}

All birds were given a uniquely barcoded wingband after hatch and all traits measured were recorded to this number. The birds were individually weighed at 40 days of age and then taken to the processing plant. Following plucking and evisceration, the

TABLE 1 | Number of records for each trait used in the analysis for the estimation of genetic parameters in the pure line broiler population in study 1 .

\begin{tabular}{lc}
\hline Trait & Number of records \\
\hline Body weight (BW) & 154781 \\
Breast yield (BY) & 34794 \\
Deep pectoral myopathy (DPM) & 35394 \\
Wooden breast (WB) & 38730 \\
Spaghetti breast (SB) & 38773 \\
White striping (WS) & 38780 \\
\hline
\end{tabular}

TABLE 2 | Environmental parameters for all farms with birds under examination for both studies reported in this paper.

\begin{tabular}{ll}
\hline Environmental parameter & Target \\
\hline Feed days: 0-10 & Starter (259 g CP/kg; $12.34 \mathrm{MJ}$ ME/kg) \\
Feed days: $11-25$ & Grower (234 g CP/kg; $12.84 \mathrm{MJ} \mathrm{ME/kg)}$ \\
Feed days: 25-final weighing & Finisher (212 g CP/kg; $13.04 \mathrm{MJ} \mathrm{ME} / \mathrm{kg}$ ) \\
Stocking density & 34.1 to $36 \mathrm{~kg}$ bird weight per m2 \\
Temperature & Gradually reduced from 29 to $20^{\circ} \mathrm{C}$ \\
Photoperiod day 0-7 & 23L:1D \\
Photoperiod day 8-final weighing & 20L:4D \\
Light intensity day 0-7 & 40 lux \\
Light intensity day 8-final weighing & Gradually reduced from 20 to 10 lux
\end{tabular}

carcasses were air chilled with a target to reach $3^{\circ} \mathrm{C}$ within $8_{-}^{-}$ $10 \mathrm{~h}$ of kill. the carcases were deboned $24 \mathrm{~h}$ after kill and the presence of BMM was then assessed and recorded by a trained team of individuals as previously described in Bailey et al. (2015). DPM was recorded as present or absent, whereas $\mathrm{WB}, \mathrm{SB}$ and WS were all scored on a severity scale. WB severity was based on a four point scale, where score 0 is normal, score 1 is a minor focal lesion of WB, score 2 is multiple focal lesions of WB and score 3 is extensive WB across the whole muscle. SB was scored on a three point scale where score 0 is normal, score 1 is less than $50 \%$ of the breast affected and a score 2 is more than $50 \%$ of the breast affected by SB Similarly, WS was recorded on a 4 point scale of severity; a score of 0 represents no striping, score 1 is noticeable striping covering only part of the breast, score 2 is a breast fillet with extensive striping covering the breast surface and score 3 is extensive coverage with very thick stripes. The scoring criteria for each BMM capture the range of mild to severe phenotypes which were then used for the estimation of genetic parameters.

\section{Statistical Analyses}

The traits BW, PW, BY, DPM, WB, SB and WS were analysed together in the following multivariate animal model to estimate genetic parameters:

$$
y=X b+Z a+W c+e,
$$

Where: $\mathbf{y}$ denotes the vector of observations of the traits, $\mathbf{b}$ is the vector of the fixed effects accounting for the interaction between hatch-week, pen and contributing mating group of each flock. The vector of additive genetic effects is shown by a, the vector of permanent environmental effects of the dam (PEM) is represented by $\mathbf{c}$, and e denotes the vector of residuals. $\mathbf{X}, \mathbf{Z}$ and $\mathbf{W}$ represent incidence matrices relating the vectors $\mathbf{b}, \mathbf{a}$, and $\mathbf{c}$ to y. The assumed (co)variance structure was:

$$
\mathrm{V}\left[\begin{array}{l}
\mathbf{a} \\
\mathbf{c} \\
\mathbf{e}
\end{array}\right]=\left[\begin{array}{ccc}
\mathbf{A} \otimes \mathbf{G} & \mathbf{0} & \mathbf{0} \\
\mathbf{0} & \mathbf{I} \otimes \mathbf{C} & \mathbf{0} \\
\mathbf{0} & \mathbf{0} & \mathbf{I} \otimes \mathbf{R}
\end{array}\right]
$$

Where: A represents the additive and genetic relationship matrix and I represents identity matrix. The variance and covariance matrices of additive genetic effects, PEM and residual effects are denoted by $\mathbf{G}, \mathbf{C}$ and $\mathbf{R}$, respectively. All variance component analyses were performed by REML using VCE (Groeneveld et al., 2008).

\section{Study 2: Empirical Testing of Genetic Selection to Reduce Myopathies Birds, Housing and Management}

The commercial broiler chicken is the result of a four way cross of pure bred broiler lines. The crossing of lines takes place over multiple generations requiring four parental generations, namely: Pedigree Elite Stock, Great grandparents (GGP), Great parents (GP) and Parent Stock (PS) as shown in Figure 3. Hence, it takes at least 4 years for the impact of genetic selection to reach the commercial broiler from the elite pedigree 


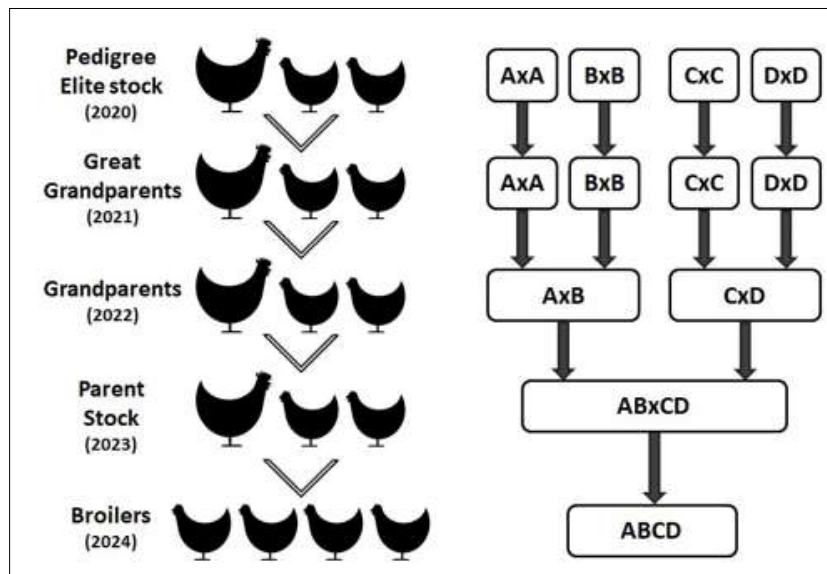

FIGURE 3 | The commercial broiler is a four way cross of pure broiler lines (represented by A, B, C, and D) as demonstrated in this image. The movement of genetic material from the pedigree pure lines to the commercial broiler population takes around 4 years through great grandparent, grandparent, and parent multiplier flocks, i.e. the impact of any genetic selection made in 2020 will reach the broiler population in 2024. population. As part of Aviagen's ongoing strategy to reduce the genetic propensity for the expression of BMM every selection candidate of all lines is assessed and scored on farm for WB through palpation, while their siblings are assessed and scored for the presence of all BMM through carcase evaluation following processing. This data is then used to select against individuals with a higher propensity for the BMM. A higher propensity for BMM is based on predicted breeding values; a higher propensity for BMM would be considered those individuals which have a higher than average breeding value for BMM. In order to empirically demonstrate the impact of this genetic selection we generated a 'high generation' (HG) broiler by fast tracking the generation of PS from the mating of birds from the GGP generation as described in Figure 4. The impact of genetic selection against the genetic propensity to WB can be then assessed through comparing the performance of the HG and the current commercial broiler. In this study, hatching eggs for commercial Ross 708 broilers were incubated alongside hatching eggs for HG Ross 708 broilers as per the Aviagen recommendations ${ }^{1}$. The chicks of both groups were reared side by side in separate pens as commercial broilers as per the Aviagen Ross 708 broiler handbook ${ }^{1}$; A total of four consecutives hatches were placed and a sample of the birds of each group, commercial and HG broiler, were sent for processing (Table 3). Hatching eggs for the HG broilers originated from the same parent flock for each of the four consecutive hatches whereas hatching eggs for commercial broilers were obtained from commercial field flocks which were all age matched where possible (Table 3). PS for both commercial and HG broilers were fed feed from the same feed mill and the same management parameters were used during rear and lay as per the Aviagen PS management guide.

${ }^{1}$ www.aviagen.com

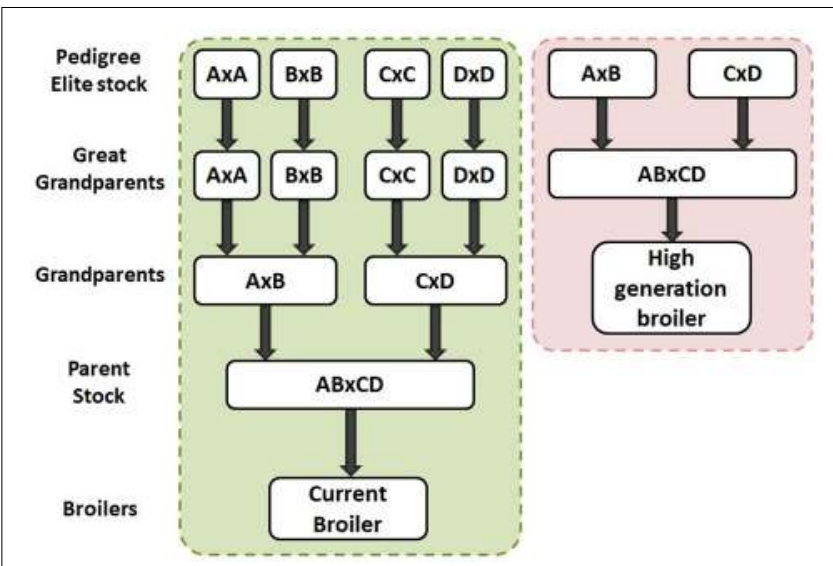

FIGURE 4 | In study 2 a high generation broiler was produced by using great grandparents as parent stock by crossing pedigree pure lines (right dashed box). The high generation broiler can then be compared to the current commercial broiler (left dashed box) to see the impact of genetic selection for a trait of interest such as wooden breast. The generational difference between the two broiler groups represents 2 years of genetic progress.

\section{Recording of Traits}

Birds were processed as outlined in the previous section above. Breast meat was weighed and recorded as a \% of body weight to give breast yield. All breast fillets were scored for WB on the same 4 point scale described above.

\section{Data Analysis}

Due to hatch to hatch variation in performance, as shown by the mean BW in Table 3, the data for breast yield and myopathy incidence were normalised to relative values in order to visualise and compare the impact of genetic selection across all four hatches of birds in study 2. This was performed by the following formula:

$$
\text { Relative value }=1-
$$

(Trait values for current broiler - Trait value for high generation broiler)

$$
\text { Trait value of the current broiler }
$$

Any significance of differences between the two broiler groups in each hatch were determined with a student's $t$-test.

\section{RESULTS}

\section{Study 1: Estimation of Genetic Parameters of Breast Muscle Myopathies}

The summary of production traits and the mean scores of BMM for the pure line population can be seen in Table 4. The mean BW was $3.03 \mathrm{~kg}$ and the mean BY was 28.9\%, respectively. For the BMM: WB and WS had mean scores of 0.30 and 0.19 , respectively; both DPM and SB had lower mean scores of 0.16 and 0.04 .

Table 5 displays the heritabilities of the traits analysed in study 1 along with the genetic and phenotypic correlations between 
TABLE 3 | Placement date, parent stock age, broiler age and average (AVG) bodyweight per hatch and number of birds processed and assessed for wooden breast myopathy per group in study 2 comparing commercial Ross 708 broilers and high generation Ross 708 broilers.

\begin{tabular}{|c|c|c|c|c|c|c|}
\hline Hatch $^{1}$ & $\begin{array}{c}\text { Chick } \\
\text { placement } \\
\text { date }\end{array}$ & Age of parent flocks (weeks) ${ }^{2}$ & $\begin{array}{l}\text { Number of birds } \\
\text { placed per group }\end{array}$ & $\begin{array}{c}\text { Age of } \\
\text { processing (days) }\end{array}$ & $\begin{array}{l}\text { AVG Body } \\
\text { weight (g) }\end{array}$ & $\begin{array}{c}\text { Number of birds } \\
\text { processed per group }\end{array}$ \\
\hline $1(\mathrm{M}+\mathrm{F})$ & 21st March & 38 & 600 & 55 & 4337 & 160 \\
\hline $2(\mathrm{M}+\mathrm{F})$ & 18th April & 41 & 640 & 62 & 4600 & 80 \\
\hline $3(\mathrm{M}+\mathrm{F})$ & 30th May & 48 & 480 & 62 & 4300 & 80 \\
\hline 4 (only M) & 11th July & $\begin{array}{c}\text { Com } \\
39\end{array}$ & 150 & 55 & 4331 & 120 \\
\hline
\end{tabular}

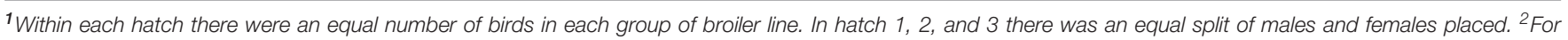

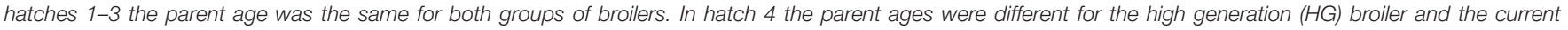
commercial (Com) broiler.

TABLE 4 | Descriptive statistics for the traits used in study 1 for the estimation of genetic parameters in the pure broiler line.

\begin{tabular}{lcc}
\hline Trait & Mean & SD \\
\hline Body weight Kg (BW) & 3.03 & 0.37 \\
\% Breast Yield (BY) $^{1}$ & 28.9 & 2.30 \\
Deep pectoral myopathy (DPM) $^{2}$ & 0.15 & 0.11 \\
Wooden breast (WB) $^{2}$ & 0.19 & 0.10 \\
Spaghetti breast (SB) $^{2}$ & 0.04 & 0.03 \\
White striping (WS) $^{2}$ & 0.30 & 0.11 \\
\hline
\end{tabular}

${ }^{1}$ Breast yield is expressed as a percentage of body weight. ${ }^{2}$ The myopathies show the mean score based on the severity score mentioned in the materials and methods section.

TABLE 5 | Genetic parameter results for study 1.

\begin{tabular}{lccccc}
\hline BW & BY & DPM & WB & SB & WS \\
\hline $\mathbf{0 . 3 1}_{(\mathbf{0 . 0 3})}$ & $-0.06_{(0.02)}$ & $0.14_{(0.03)}$ & $0.20_{(0.04)}$ & $-0.06_{(0.03)}$ & $0.23_{(0.04)}$ \\
0.15 & $\mathbf{0 . 4 0}_{(\mathbf{0 . 0 2})}$ & $0.23_{(0.02)}$ & $0.41_{(0.03)}$ & $0.36_{(0.02)}$ & $0.31_{(0.01)}$ \\
0.03 & 0.02 & $\mathbf{0 . 0 6}_{(\mathbf{0 . 0 2})}$ & $0.46_{(0.02)}$ & $0.04_{(0.03)}$ & $0.34_{(0.03)}$ \\
0.08 & 0.13 & 0.10 & $\mathbf{0 . 0 7}_{(\mathbf{0 . 0 4})}$ & $-0.04_{(0.02)}$ & $0.74_{(0.04)}$ \\
0.02 & 0.17 & 0.03 & -0.02 & $\mathbf{0 . 0 4}_{(\mathbf{0 . 0 2})}$ & $0.02_{(0.01)}$ \\
0.23 & 0.22 & 0.12 & 0.25 & 0.05 & $\mathbf{0 . 2 5}_{(\mathbf{0 . 0 2})}$
\end{tabular}

Estimates of heritabilities (bold, diagonal), genetic correlations (above diagonal), and phenotypic correlations (below diagonal) for body weight (BW), breast yield (BY), deep pectoral myopathy (DPM), wooden breast (WB), spaghetti breast (SB) and white striping (WS). Standard errors are displayed in parentheses.

the traits. The measures of genetic and phenotypic correlations range from -1 (an antagonistic relationship) to 1 (a positive relationship) and it describes any shared genetic background or phenotypic relationship between two traits.

The phenotypic correlations between the BMM and the production traits $\mathrm{BY}$ and, BW were low to moderate ranging from 0.02 to 0.23 ; this indicates the $\mathrm{BMM}$ are not linked wholly to changes in BW or BY. Equally the phenotypic correlations between the individual myopathies were generally low to moderate ranging from -0.02 to 0.25 ; thus suggesting the individual BMM are independent of each other. The genetic correlation between BY with BW was low with an estimate of 0.06 indicating these two traits are independent of each other.
The genetic correlations between the BMM and BW and BY were low to moderate with estimates ranging from -0.06 to 0.41 . This indicates there is limited shared genetic background between BW and BY with the BMM. The genetic correlations between the myopathies were predominately low to moderate ranging from -0.04 to 0.52 with a higher genetic correlation between WB and WS of 0.74 .

Heritability is the proportion of phenotypic variance explained by genetic variance, and thus provides an indication of the genetic influence on variance on any given trait. The estimated heritabilities for BW and BY were 0.31 and 0.40 , respectively, and the estimated heritabilities for the BMM ranged from low to moderate: 0.04 for SB, 0.06 for DPM, 0.07 for WB and 0.25 for WS. Table 6 shows the proportion of phenotypic variance that was accounted for by the environmental and maternal environment effects. For BW and BY the PEM effect accounted for 0.5 and $1.0 \%$ of the phenotypic variance, respectively, thus accounting for only a very small proportion of the phenotypic variance. For the BMM the PEM similarly accounted for a small proportion of the phenotypic variance ranging from 0.3 to $3.4 \%$. Overall, the results show that the majority of the phenotypic variance of BMM traits analysed in study 1 is explained by the environmental (i.e. residual) variance. The residual variance for BMM accounts for the larger proportion of the phenotypic variance with a range of 71.5-95.2\%. Thus, these results indicate a modest genetic influence on the variation in BMM at 40 days in the line studied; however, there is a much greater variation resulting from the non-genetic factors.

\section{Study 2: Empirical Testing of Genetic Selection to Reduce Myopathies}

The relative and mean $\mathrm{BY}$ and WB incidences for the HG and current commercial broilers across the four hatches are displayed in Figure 5. In each hatch a reduction in the relative incidence of WB can be seen in the HG broiler compared to the current commercial broiler. Simultaneously, in each hatch there is a relative increase in $\mathrm{BY}$ seen in the $\mathrm{HG}$ broiler compared to the current commercial broiler. Overall the higher generation broilers had on average 18.4\% less WB $(p<0.001)$ and $2.4 \%$ more breast yield $(p<0.03)$ relative to the current commercial broiler. These results demonstrate that selection for increased 
TABLE 6 | Phenotypic (PHEN), residual (RES), maternal permanent environmental (PEm) variances and proportions of phenotypic variance accounted for by RES (Prop RES) and PEm (Prop PEm) for body weight (BW), breast yield (BY), deep pectoral myopathy (DPM), wooden breast (WB), spaghetti breast (SB) and white striping (WS) analysed in study 1.

\begin{tabular}{lrrrll}
\hline Trait & PHEN & RES & PEM & Prop RES & Prop PEm \\
\hline BW & 582.11 & 393.01 & 5.93 & $0.675_{(0.007)}$ & $0.010_{(0.001)}$ \\
BY & 3.06 & 1.82 & 0.01 & $0.595_{(0.010)}$ & $0.005_{(0.003)}$ \\
WB & 372.12 & 335.28 & 11.16 & $0.901_{(0.004)}$ & $0.030_{(0.002)}$ \\
SB & 166.42 & 158.50 & 1.14 & $0.952_{(0.003)}$ & $0.007_{(0.002)}$ \\
WS & 605.63 & 432.91 & 20.84 & $0.715_{(0.005)}$ & $0.034_{(0.001)}$ \\
DPM & 1154.06 & 1079.49 & 3.68 & $0.935_{(0.004)}$ & $0.003_{(0.003)}$
\end{tabular}

Standard errors for PropRES and PropPEm are displayed in parentheses.

breast meat in broilers can be achieved whilst selecting against the genetic propensity to develop BMM such as WB. The generational difference of the commercial and HG broilers used in this study represents 2 years. This means an annual realised relative reduction in $\mathrm{WB}$ of $9.2 \%$ and an annual realised relative increase in BY of $1.2 \%$.

\section{DISCUSSION}

The importance of BMM in the poultry industry has been well documented in academic and popular poultry press over the last 10 years. Myopathies can lead to carcase downgrades in severe cases or, in the milder cases they can have a negative influence on the eating quality, ability of meat to take up marinates, cooking losses and consumer acceptance (Kuttappan et al., 2012b; Aguirre et al., 2016; Tijare et al., 2016; Bowker et al., 2018). A recent publication by Gratta et al. (2019) showed that, when compared to unaffected breast fillets, the microbial shelf life of breast fillets with WS or WB was significantly longer. This supports the general consensus that breast fillets affected by the myopathies do not pose a human health concern (Kuttappan et al., 2012b, 2013b). The aetiology of the breast myopathies is still unknown, however, various hypotheses have been proposed; a common hypothesis for the increased incidence of myopathies such as WB, WS and SB in broiler chickens is that they are an unexpected consequence of selection for body weight and breast yield (Zambonelli et al., 2016; Baldi et al., 2018; Petracci et al., 2019). Previous published data have estimated heritabilities and genetic correlations for WS individually (Alnahhas et al., 2016) and WB and WS together with DPM (Bailey et al., 2015). To the knowledge of the authors there are no data published on the genetic basis of SB; thus here we report the first dataset describing the genetic basis of SB. Furthermore, the analysis presented here is the first report characterising the genetic basis of all the BMM together and their relationship with bodyweight and breast yield in broiler chickens. The data presented here show a lower contribution of the BMM genetic variance to the phenotypic variance resulting in low heritability estimates and a large contribution of residual variance. This means, whilst there is a modest genetic component to the variation in myopathies, it

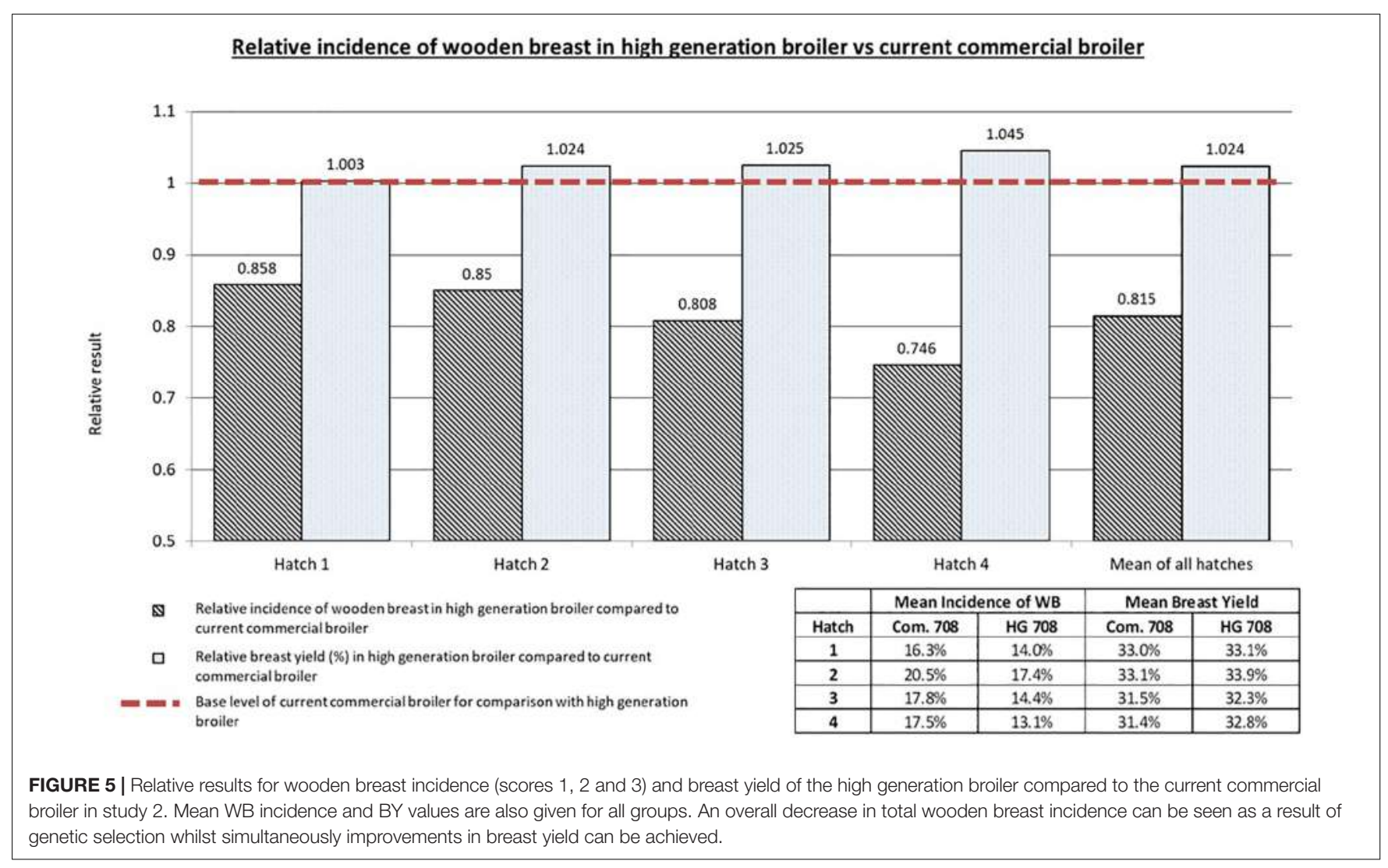


is the non-genetic effects that are of greater importance which is in agreement with previous published results (Bailey et al., 2015).

In this study, BW and BY showed estimates of heritability of 0.31 and 0.40 , respectively, which are similar in magnitude to those found in the literature (Bailey et al., 2015; Alnahhas et al., 2016). The heritabilities for DPM, WB and SB are low with estimates ranging from 0.04 to 0.07 indicating a very strong influence of the non-genetic factors in the variation in manifestation of these myopathies. It is well documented that DPM incidence is heavily influenced by on farm factors (Lien et al., 2012), thus a low heritability estimate for DPM is not unexpected. The heritability estimate for $\mathrm{WB}$ is in line with previous published data (Bailey et al., 2015); this reiterates the importance of understanding the influence of the non-genetic factors on WB. The high residual variance and accompanying low heritability for SB would indicate that non-genetic factors are of significantly greater influence for the variation in expression of this trait; therefore more work is needed to elucidate the risk factors. Compared to the other myopathies, WS had higher heritability estimate of 0.25 which is similar to previous published data where a heritability range of $0.185-0.338$ for WS was reported (Bailey et al., 2015). Despite the higher heritability the residual variance still dominates indicating that the non-genetic effects are of greater importance for the variation seen with WS.

The genetic correlations between the traits suggests that genetic selection for increased BW and BY has not had a concomitant increase in the genetic propensity for the expression of breast myopathies. The genetic correlations between the production traits and the BMM are low to moderate indicating that only a small proportion of the genetic component influencing BW and BY is shared with the genetic component of the BMM. Similarly, the phenotypic correlations between the myopathies and production traits are also low to moderate, ranging from 0.02 to 0.23 . The lack of strong phenotypic correlations indicates that the myopathies can occur in birds of all sizes with a range of BW and BY. These results indicate that selection for increased BW and BY is not the sole cause of the myopathies. A comprehensive study comparing conventional and slower growing broilers by Muth and Valle Zárate (2017) demonstrated there were no detrimental effects on meat quality in the faster growing broilers. Therefore, understanding why BMM occur requires more extensive research into all factors that can impact upon muscle development and subsequent meat quality.

Within this analysis the contribution of the PEM to the phenotypic variance of each trait was estimated. For this dataset the PEM for BW, BY, WB and WS is negligible when compared to the heritabilities and standard errors for those traits. However, for DPM and SB the PEM was slightly larger and had it been ignored in the analysis the heritability for DPM would have increased from 0.06 to 0.09 and SB would have increased from 0.04 to 0.074 . This highlights the importance of fitting the PEM when estimating genetic parameters in chicken datasets that are characterised by families with large sets of full sibs to ensure accurate discrimination of the additive genetic variance.

Despite the modest influence of genetics on the myopathies, the genetic component has not been overlooked by primary breeders as it can be used as part of a holistic approach to reduce the incidence and severity of the myopathies. The results presented here show that, from the perspective of genetic selection, it is possible to continue to improve breast yield and body weight whilst reducing the genetic propensity for the expression of all the BMM as described by Bailey et al. (2015). Since the novel myopathies were first recognised, Aviagen took advantage of the modest genetic component of the three myopathies and incorporated them into a selection strategy. This is the first report showing the impact of this selection strategy and how myopathies can be selected against without impacting upon performance. The comparison of HG broilers with the current commercial broiler empirically shows that the ongoing genetic selection against WB is effective and that it can be done whilst still improving production traits such as BY. One assumption made in the interpretation of the results for study 2 is that the reduction in relative incidence of the myopathies in the HG broilers is due only to genetic selection. It is possible that there are confounding factors which could influence the result especially as the parents of each group of broilers were reared in different environments. However, the PS in both locations were reared under similar management parameters and feed specification to limit these confounding effects. Furthermore, the broiler progeny of the HG and current commercial broilers for each of the four hatches were incubated and subsequently reared together to further limit the impact of environmental factors which could influence the manifestation of WB. In this study there was hatch to hatch variation in bird performance; season is known to impact upon broiler performance (Koknaroglu and Atilgan, 2007; Osti et al., 2017) and as the hatches were placed at different times of the year, season could play a role in the hatch to hatch variation.

The ability to select for increased breast yield whilst reducing the genetic propensity for WB is testament to the low genetic correlation between the two traits. The effect of this selection over time can be seen in Figure 6 which shows the relationship between BY \% and WB \% between 2011 and 2019; each line in this graph represents the relationship between the breeding values for each trait within each year. The broken arrow demonstrates the joint direction of the average breeding value for each trait involved in the trade off. Although it has often been suggested that the only viable options to reduce BMM is to either reduce genetic selection for BW and BY or use slower growing alternatives, here we show that meat quality can be improved whilst improving performance as part of a balanced multi-trait breeding goal.

Whilst the genetic component of the myopathies can be targeted through selection, it must be acknowledged that genetic selection is not the sole solution for reducing the incidence of the myopathies. Again, non-genetic effects have a much greater influence on the variation in BMM and therefore offer a more successful short term opportunity to significantly reduce their incidence. Genetic selection against the myopathies is more of a long term strategy owing to the low heritabilities of the BMM and the time taken for genetic progress to pass from the elite population to the commercial broiler. Ultimately, the successful reduction of myopathies in broiler chickens will be the result 


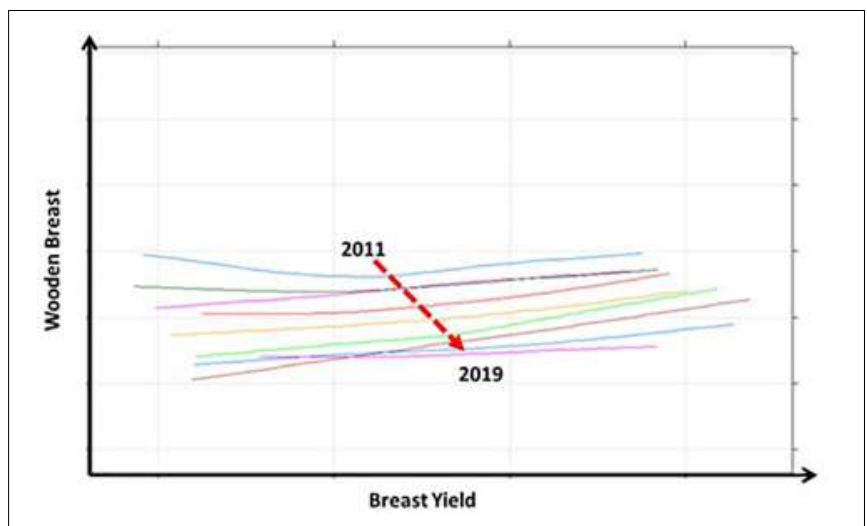

FIGURE 6 | The long term relationship between breast yield (\%) and wooden breast (\%) for the years 2011 to 2019 is displayed in this graph. The different coloured lines each represent the year long relationship between breeding values for each trait for each year. The broken arrow shows the movement of the mean breeding value for each trait from 2011 to 2019. It shows that there has been a yearly decrease in the mean breeding value for wooden breast whilst the mean breeding value for breast yield has increased.

of a holistic approach to understand and account for all the influencing factors.

Flock growth and performance are influenced by a wide range of environmental and management factors such as brooding, nutrition, temperature and ventilation. Understanding how these factors influence bird growth in terms of muscle development may give more insight into the pathophysiology of the myopathies. Muscle growth, development and maintenance are reliant on the satellite cells; these are multipotent stem cells located on the periphery of muscle fibres and provide the required myogenic precursors for muscle fibre growth and repair (Moss and Leblond, 1970). Incubation, brooding and early chick management have all been shown to influence satellite cells and play a role in muscle growth and development thus influencing final breast yield and composition of the breast muscle. For example, thermal manipulations during incubation have been shown to increase myoblast activity leading to increased satellite cell number and increased breast yields in the broiler (Piestun et al., 2009, 2011, 2013; Al-Musawi et al., 2012). Satellite cells are extremely active post hatch and proliferate to increase their population within the muscle, however, as the bird gets older the activity of these cells decrease which could have an impact on their ability to support the muscle later in life (Mann et al., 2011; Harthan et al., 2013; Daughtry et al., 2017). Failure to provide newly hatched chicks with adequate nutrition during brooding can result in reduced satellite cell activity and number (Harthan et al., 2014; Powell et al., 2014). There can be long term consequences of impaired satellite cell function and development with regards to meat quality in the older broiler. Satellite cells in birds which are feed restricted during early life and then have full access to ad lib have activity associated with muscle fibre degeneration and adipogenesis (Velleman et al., 2010) leading to lower protein and higher fat in breast tissue (Zhan et al., 2007). Heat stress during the early life of the chick has been demonstrated to have a negative impact on satellite cell function leading to increased collagen and fat deposition in breast muscle (Piestun et al., 2017; Patael et al., 2019). Thus it is very important to ensure incubation, farm management and feed availability are optimal to encourage early chick growth to promote maximal satellite cell proliferation and activity for supporting muscle development. There have been a number of technical articles from industry (Aviagen Ltd, 2019) and peer reviewed articles highlighting potential nutritional and management related strategies to reduce the myopathies whilst maintaining optimal performance. Nutritional strategies include modifying the broiler growth curve through lysine reduction (Meloche et al., 2018a,b) or increasing phytase levels in the feed to give support to the muscle (Greene et al., 2019). Management and nutritional strategies which successfully reduce BMM incidence provide a great opportunity to further understand the key biological pathways identified by gene expression, metabolomic and proteomic analyses of breast tissue affected by myopathies. This could help distinguish cause and effect when it comes to characterising the underlying aetiology of each of the conditions. The BMM can appear together or individually likely indicating they are separate conditions, however, the biological pathway studies reveal similar key pathways for each BMM.; here we show low phenotypic correlations between the BMM ranging from -0.02 to 0.25 , and the genetic correlations are low to high ranging from -0.04 to 0.74 . It's probable that there is a common origin to the BMM and more research is needed to untangle which factors influence the manifestation of a particular BMM; this may depend upon what the initial trigger is, the age of the bird when it occurs or even the sex of the bird.

The aim of this paper was to characterise the genetic basis of BMM found in broiler chickens and their relationship with body weight and breast yield. Data from a pedigree broiler pure line showed that the residual variance accounted for a greater proportion of the phenotypic variance of the myopathies than the genetic variance. This indicates that the non-genetic factors have a greater impact on the variance of the myopathy traits than the genetic factors. It has been previously described how balanced breeding goals can lead to cumulative improvements in traits that have low genetic basis such as the myopathies (Neeteson, 2010; Kapell et al., 2012a,b; Bailey et al., 2015). Using a HG broiler and the current commercially available broiler we demonstrate the effectiveness of the balanced breeding approach; the results show that BY can be successfully improved whilst at the same time reducing the genetic component of WB. In the long term genetic selection will help reduce the incidence of all myopathies, however, in the shorter term exploring management strategies to capture the non-genetic effects is critical.

\section{DATA AVAILABILITY STATEMENT}

The datasets presented in this article are not readily available because it contains propriety information. Access to the data will be permitted by agreement. Requests to access the datasets should be directed to RB, rbailey@aviagen.com. 


\section{ETHICS STATEMENT}

The data used in this study came from the routine data collection and operation of the Aviagen breeding program where all animals are under the care of the Aviagen Veterinary Department, Huntsville, AL, United States. The data used in this trial is collected from birds going to slaughter as part of normal commercial poultry processing through an abbatoir. These birds were not experimental animals

\section{REFERENCES}

Aguirre, M. E., Owens, C. M., Miller, R. K., and Alvarado, C. Z. (2016). Descriptive sensory and texture profile characterization of marinated woody breast meat. Poult. Sci. Assoc. Annu. Meet. Abstr. 97, 1456-1461. doi: 10.3382/ps/pex428

Al-Musawi, S. L., Stickland, N. C., and Bayol, S. A. M. (2012). In ovo temperature manipulation differentially influences limb musculoskeletal development in two lines of chick embryos selected for divergent growth rates. J. Exp. Biol. 215, 1594-1604. doi: 10.1242/jeb.068791

Alnahhas, N., Berri, C., Chabault, M., Chartrin, P., Boulay, M., Bourin, M. C., et al. (2016). Genetic parameters of white striping in relation to body weight, carcass composition, and meat quality traits in two broiler lines divergently selected for the ultimate $\mathrm{pH}$ of the Pectoralis major muscle. BMC Genet. 17:61. doi: 10.1186/s12863-016-0369-2

Avendaño, S., Neeteson, A. M., and Fancher, B. (2017). "Broiler breeding for sustainability and welfare - are there trade-offs?", in Proceedings of the New Zealand Poultry Beyond 2023 Conference, Queenstown.

Aviagen Ltd (2019). Breast Muscle Myopathies. Newbridge: Aviagen Ltd.

Bailey, R. A., Watson, K. A., Bilgili, S. F., and Avendano, S. (2015). The genetic basis of Pectoralis major myopathies in modern broiler chicken lines. Poult. Sci. 94, 2870-2879. doi: 10.3382/ps/pev304

Baldi, G., Soglia, F., Mazzoni, M., Sirri, F., Canonico, L., Babini, E., et al. (2018). Implications of white striping and spaghetti meat abnormalities on meat quality and histological features in broilers. Animal 12, 164-173. doi: 10.1017/ S1751731117001069

Barbut, S. (2019). Recent myopathies in broiler's breast meat fillets. Worlds Poult. Sci. J. 75, 559-582. doi: 10.1017/s0043933919000436

Bianchi, M., Petracci, M., Franchini, A., and Cavani, C. (2006). The occurrence of deep pectoral myopathy in roaster chickens. Poult. Sci. 85, 1843-1846. doi: $10.1093 / \mathrm{ps} / 85.10 .1843$

Bilgili, S. F., and Hess, J. B. (2008). Green Muscle Disease Reducing the Incidence in Broiler Flocks. Newbridge: Aviagen Ltd.

Boerboom, G., Van Kempen, T., Navarro-Villa, A., and Pérez-Bonilla, A. (2018). Unraveling the cause of white striping in broilers using metabolomics. Poult. Sci. 97, 3977-3986. doi: 10.3382/ps/pey266

Bowker, B. C., Maxwell, A. D., Zhuang, H., and Adhikari, K. (2018). Marination and cooking performance of portioned broiler breast fillets with the wooden breast condition. Poult. Sci. 97, 2966-2970. doi: 10.3382/ps/pey144

Brothers, B., Zhuo, Z., Papah, M. B., and Abasht, B. (2019). RNA-seq analysis reveals spatial and sex differences in Pectoralis major muscle of broiler chickens contributing to difference in susceptibility to wooden breast disease. Front. Physiol. 10:764. doi: 10.3389/fphys.2019.00764

Cai, K., Shao, W., Chen, X., Campbell, Y. L., Nair, M. N., Suman, S. P., et al. (2018). Meat quality traits and proteome profile of woody broiler breast (Pectoralis major) meat. Poult. Sci. 97, 337-346. doi: 10.3382/ps/pex284

Clark, D. L., and Velleman, S. G. (2017). Physiology and reproduction: spatial influence on breast muscle morphological structure, myofiber size, and gene expression associated with the wooden breast myopathy in broilers. Poult. Sci. 95, 2930-2945. doi: 10.3382/ps/pew243

Daughtry, M. R., Berio, E., Shen, Z., Suess, E. J. R., Shah, N., Geiger, A. E., et al. (2017). Satellite cell-mediated breast muscle regeneration decreases with broiler size. Poult. Sci. 96, 3457-3464. doi: 10.3382/ps/pex068

Dawkins, M. S., and Layton, R. (2012). Breeding for better welfare: genetic goals for broiler chickens and their parents. Anim. Welf. 21, 147-155. doi: 10.7120/ 09627286.21.2.147 and there were no experimental procedures carried out on them.

\section{AUTHOR CONTRIBUTIONS}

All authors were involved with the conception of the research. $\mathrm{RB}$ drafted the manuscript which was revised and reviewed by SA and ES.

Dempster, E. R., and Lerner, I. M. (1950). Heritability of threshold characters. Genetics 35, 212-236.

Ferreira, T. Z., Casagrande, R. A., Vieira, S. L., Driemeier, D., and Kindlein, L. (2014). An investigation of a reported case of white striping in broilers. J. Appl. Poult. Res. 23, 748-753. doi: 10.3382/japr.2013-00847

Fleming, E. C., Fisher, C., and McAdam, J. (2007). "Genetic progress in broiler traits - implications for welfare," in Proceedings of the British Society of Animal Science. BSAS Annual Conference, Southport, 50. doi: 10.1017/ s1752756200019530

Gratta, F., Fasolato, L., Birolo, M., Zomeño, C., Novelli, E., Petracci, M., et al. (2019). Effect of breast myopathies on quality and microbial shelf life of broiler meat. Poult. Sci. 98, 2641-2651. doi: 10.3382/ps/pez001

Greene, E., Flees, J., Dadgar, S., Mallmann, B., Orlowski, S., Dhamad, A., et al. (2019). Quantum blue reduces the severity of woody breast myopathy via modulation of oxygen homeostasis-related genes in broiler chickens. Front. Physiol. 10:1251. doi: 10.3389/fphys.2019.01251

Groeneveld, E., Kovac, M., and Mielenz, N. (2008). VCE User's Guide and Reference Manual Version 6.0.

Harthan, L. B., McFarland, D. C., and Velleman, S. G. (2013). The effect of syndecan-4 and glypican-1 expression on age-related changes in myogenic satellite cell proliferation, differentiation, and fibroblast growth factor 2 responsiveness. Comp. Biochem. Physiol. - A Mol. Integr. Physiol 166, 590-602. doi: 10.1016/j.cbpa.2013.09.007

Harthan, L. B., McFarland, D. C., and Velleman, S. G. (2014). The effect of nutritional status and myogenic satellite cell age on turkey satellite cell proliferation, differentiation, and expression of myogenic transcriptional regulatory factors and heparan sulfate proteoglycans syndecan- 4 and glypican1. Poult. Sci. 93, 174-186. doi: 10.3382/ps.2013-03570

Havenstein, G. B., Ferket, P. R., and Qureshi, M. A. (2003a). Carcass composition and yield of 1957 versus 2001 broilers when fed representative 1957 and 2001 broiler diets. Poult. Sci. 82, 1509-1518. doi: 10.1093/ps/82.10.1509

Havenstein, G. B., Ferket, P. R., and Qureshi, M. A. (2003b). Growth, livability, and feed conversion of 1957 versus 2001 broilers when fed representative 1957 and 2001 broiler diets. Poult. Sci. 82, 1500-1508. doi: 10.1093/ps/82.10.1500

Hocking, P. M. (2014). Unexpected consequences of genetic selection in broilers and turkeys: problems and solutions. Br. Poult. Sci. 55, 1-12. doi: 10.1080/ 00071668.2014 .877692

Huang, X., and Ahn, D. U. (2018). The incidence of muscle abnormalities in broiler breast meat - A review. Korean J. food Sci. Anim. Resour. 38, 835-850. doi: 10.5851/kosfa.2018.e2

Kapell, D. N. R. G., Hill, W. G., Neeteson, A. M., McAdam, J., Koerhuis, A. N. M., and Avendano, S. (2012a). Genetic parameters of foot-pad dermatitis and body weight in purebred broiler lines in 2 contrasting environments. Poult. Sci. 91, 565-574. doi: 10.3382/ps.2011-01934

Kapell, D. N. R. G., Hill, W. G., Neeteson, A. M., McAdam, J., Koerhuis, A. N. M., and Avendaño, S. (2012b). Twenty-five years of selection for improved leg health in purebred broiler lines and underlying genetic parameters. Poult. Sci. 91, 3032-3043. doi: 10.3382/ps.2012-02578

Koknaroglu, H., and Atilgan, A. (2007). Effect of season on broiler performance and sustainability of broiler production. J. Sustain. Agric. 31, 113-124. doi: 10.1300/J064v31n02_08

Kuttappan, V. A., Bottje, W., Ramnathan, R., Hartson, S. D., Coon, C. N., Kong, B. W., et al. (2017). Proteomic analysis reveals changes in carbohydrate and protein metabolism associated with broiler breast myopathy. Poult. Sci. 96, 2992-2999. doi: 10.3382/ps/pex069 
Kuttappan, V. A., Brewer, V. B., Apple, J. K., Waldroup, P. W., and Owens, C. M. (2012a). Influence of growth rate on the occurrence of white striping in broiler breast fillets. Poult. Sci. 91, 2677-2685. doi: 10.3382/ps.2012-02259

Kuttappan, V. A., Lee, Y. S., Erf, G. F., Meullenet, J. F. C., Mckee, S. R., and Owens, C. M. (2012b). Consumer acceptance of visual appearance of broiler breast meat with varying degrees of white striping. Poult. Sci. 91, 1240-1247. doi: 10.3382/ps.2011-01947

Kuttappan, V. A., Brewer, V. B., Mauromoustakos, A., McKee, S. R., Emmert, J. L., Meullenet, J. F., et al. (2013a). Estimation of factors associated with the occurrence of white striping in broiler breast fillets. Poult. Sci. 92, 811-819. doi: 10.3382/ps.2012-02506

Kuttappan, V. A., Huff, G. R., Huff, W. E., Hargis, B. M., Apple, J. K., Coon, C., et al. (2013b). Comparison of hematologic and serologic profiles of broiler birds with normal and severe degrees of white striping in breast fillets. Poult. Sci. 92, 339-345. doi: 10.3382/ps.2012-02647

Kuttappan, V. A., Shivaprasad, H. L., Shaw, D. P., Valentine, B. A., Hargis, B. M., Clark, F. D., et al. (2013c). Pathological changes associated with white striping in broiler breast muscles. Poult. Sci. 92, 331-338. doi: 10.3382/ps.201202646

Kuttappan, V. A., Hargis, B. M., and Owens, C. M. (2016). White striping and woody breast myopathies in the modern poultry industry: a review. Poult. Sci. 95, 2724-2733. doi: 10.3382/ps/pew216

Lake, J. A., Papah, M. B., and Abasht, B. (2019). Increased expression of lipid metabolism genes in early stages of wooden breast links myopathy of broilers to metabolic syndrome in humans. Genes 10: 746. doi: 10.3390/genes101 00746

Lien, R. J., Bilgili, S. F., Hess, J. B., and Joiner, K. S. (2012). Induction of deep pectoral myopathy in broiler chickens via encouraged wing flapping. J. Appl. Poult. Res. 21, 556-562. doi: 10.3382/japr.2011-00441

Lilburn, M. S., Griffin, J. R., and Wick, M. (2019). From muscle to food: oxidative challenges and developmental anomalies in poultry breast muscle. Poult. Sci. 97, 4255-4260. doi: 10.3382/ps/pey409

Mann, C. J., Perdiguero, E., Kharraz, Y., Aguilar, S., Pessina, P., Serrano, A. L., et al. (2011). Aberrant repair and fibrosis development in skeletal muscle. Skelet. Muscle 1:21. doi: 10.1186/2044-5040-1-21

McKay, J. C., Barton, N. F., Koerhuis, A. N. M., and McAdam, J. (2000). The challenge of genetic change in the broiler chicken. BSAP Occas. Publ. 27, 1-7. doi: $10.1017 /$ s1463981500040486

Meloche, K. J., Fancher, B. I., Emmerson, D. A., Bilgili, S. F., and Dozier, W. A. (2018a). Effects of quantitative nutrient allocation on myopathies of the Pectoralis major muscles in broiler chickens at 32, 43, and 50 days of age. Poult. Sci. 97, 1786-1793. doi: 10.3382/ps/pex453

Meloche, K. J., Fancher, B. I., Emmerson, D. A., Bilgili, S. F., and Dozier, W. A. (2018b). Effects of reduced digestible lysine density on myopathies of the Pectoralis major muscles in broiler chickens at 48 and 62 days of age. Poult. Sci. 97, 3311-3324. doi: 10.3382/ps/pey171

Mitchell, M. A. (1999). "Muscle abnormalities-pathophysiological mechanisms," in Poultry Meat Science, eds R. Richardson and G. Mead (Oxon: CABI Publishing), 65-98.

Moss, F., and Leblond, C. P. (1970). Nature of dividing nuclei in skeletal muscle of growing rats. J. Cell Biol. 44, 459-462. doi: 10.1083/jcb.44.2.459

Mudalal, S., Lorenzi, M., Soglia, F., Cavani, C., and Petracci, M. (2015). Implications of white striping and wooden breast abnormalities on quality traits of raw and marinated chicken meat. Animal 9, 728-734. doi: 10.1017/ S175173111400295X

Mussini, F. J. (2012). Comparative Response of Different Broiler Genotypes to Dietary Nutrient Levels. Available online at: http://oatd.org/oatd/record? record $=\% 22$ oai:scholarworks.uark.edu:etd- $1470 \% 22$ (accessed July 21, 2017).

Muth, P. C., and Valle Zárate, A. (2017). Breast meat quality of chickens with divergent growth rates and its relation to growth curve parameters. Arch. Anim. Breed. 60, 427-437. doi: 10.5194/aab-60-427-2017

Mutryn, M. F., Brannick, E. M., Fu, W., Lee, W. R., and Abasht, B. (2015). Characterization of a novel chicken muscle disorder through differential gene expression and pathway analysis using RNA-sequencing. BMC Genom. 16:399. doi: 10.1186/s12864-015-1623-0

Neeteson, A. M. (2010). Striking the balance. J. Anim. Breed. Genet. 127, 85-86. doi: $10.1111 /$ j.1439-0388.2010.00866.x
Osti, R., Bhattarai, D., and Zhou, D. (2017). Climatic variation: effects on stress levels, feed intake, and bodyweight of broilers. Rev. Bras. Cienc. Avic. 19, 489-496. doi: 10.1590/1806-9061-2017-0494

Papah, M. B., Brannick, E. M., Schmidt, C. J., and Abasht, B. (2018). Gene expression profiling of the early pathogenesis of wooden breast disease in commercial broiler chickens using RNA-sequencing. PLoS One 13:e207346. doi: 10.1371/journal.pone.0207346

Patael, T., Piestun, Y., Soffer, A., Mordechay, S., Yahav, S., Velleman, S. G., et al. (2019). Early posthatch thermal stress causes long-term adverse effects on pectoralis muscle development in broilers. Poult. Sci. 98, 3268-3277. doi: 10. 3382/ps/pez123

Petracci, M., Soglia, F., Madruga, M., Carvalho, L., Ida, E., and Estévez, M. (2019). Wooden-breast, white striping, and spaghetti meat: causes, consequences and consumer perception of emerging broiler meat abnormalities. Compr. Rev. Food Sci. Food Saf. 2019, 565-583. doi: 10.1111/1541-4337.12431

Piestun, Y., Druyan, S., Brake, J., and Yahav, S. (2013). Thermal manipulations during broiler incubation alter performance of broilers to 70 days of age. Poult. Sci. 92, 1155-1163. doi: 10.3382/ps.2012-02609

Piestun, Y., Halevy, O., Shinder, D., Ruzal, M., Druyan, S., and Yahav, S. (2011). Thermal manipulations during broiler embryogenesis improves post-hatch performance under hot conditions. J. Therm. Biol. 36, 469-474. doi: 10.1016/ j.jtherbio.2011.08.003

Piestun, Y., Harel, M., Barak, M., Yahav, S., and Halevy, O. (2009). Thermal manipulations in late-term chick embryos have immediate and longer term effects on myoblast proliferation and skeletal muscle hypertrophy. J. Appl. Physiol. 106, 233-240. doi: 10.1152/japplphysiol.91090.2008

Piestun, Y., Patael, T., Yahav, S., Velleman, S. G., and Halevy, O. (2017). Early posthatch thermal stress affects breast muscle development and satellite cell growth and characteristics in broilers. Poult. Sci. 96, 2877-2888. doi: 10.3382/ ps/pex065

Powell, D. J., McFarland, D. C., Cowieson, A. J., Muir, W. I., and Velleman, S. G. (2014). The effect of nutritional status on myogenic gene expression of satellite cells derived from different muscle types. Poult. Sci. 93, 2278-2288. doi: 10.3382/ps.2013-03810

Richardson, J. A., Burgener, J., Winterfield, R. W., and Dhillon, A. S. (1980). Deep pectoral myopathy in seven-week-old broiler chickens. Avian Dis. 24, 1054-1059. doi: 10.2307/1589983

Sihvo, H.-K., Airas, N., Lindén, J., Immonen, K., and Puolanne, E. (2017). Vessel and myofibre density within focal wooden breast myopathy. J. Comp. Pathol. 156:81. doi: 10.1016/j.jppa.2016.11.086

Sihvo, H. K., Immonen, K., and Puolanne, E. (2014). Myodegeneration with fibrosis and regeneration in the Pectoralis major muscle of broilers. Vet. Pathol. 51, 619-623. doi: 10.1177/0300985813497488

Siller, W. G., and Wight, P. A. L. (1978). The pathology of deep pectoral myopathy of turkeys. Avian Pathol. 7, 583-617. doi: 10.1080/03079457808418313

Siller, W. G., Wight, P. A. L., and Martindale, L. (1978). Exercise-induced deep pectoral myopathy in broiler fowls and turkeys. Vet. Sci. Commun. 2, 331-336. doi: 10.1007/BF02291463

Soglia, F., Mazzoni, M., and Petracci, M. (2019). Spotlight on avian pathology: current growth-related breast meat abnormalities in broilers. Avian Pathol. 48, 1-3. doi: 10.1080/03079457.2018.1508821

Soglia, F., Mazzoni, M., Zappaterra, M., Di Nunzio, M., Babini, E., Bordini, M., et al. (2020). Distribution and expression of vimentin and desmin in broiler Pectoralis major affected by the growth-related muscular abnormalities. Front. Physiol. 10:1581. doi: 10.3389/fphys.2019.01581

Tasoniero, G., Bertram, H. C., Young, J. F., Dalle Zotte, A., and Puolanne, E. (2017). Relationship between hardness and myowater properties in Wooden Breast affected chicken meat: a nuclear magnetic resonance study. LWT - Food Sci. Technol. 86, 20-24. doi: 10.1016/j.lwt.2017.07.032

Tijare, V. V., Yang, F. L., Kuttappan, V. A., Alvarado, C. Z., Coon, C. N., and Owens, C. M. (2016). Meat quality of broiler breast fillets with white striping and woody breast muscle myopathies. Poult. Sci. 95, 2167-2173. doi: 10.3382/ps/pew129

USDA (2020). Poultry Slaughter Annual Summary. Available online at: https://usda.library.cornell.edu/concern/publications/pg15bd88s (accessed January 30, 2020).

Velleman, S. G., and Clark, D. L. (2015). Histopathologic and myogenic gene expression changes associated with wooden breast in broiler breast muscles. Avian Dis. 59, 410-418. doi: 10.1637/11097-042015-reg.1 
Velleman, S. G., Nestor, K. E., Coy, C. S., Harford, I., and Anthony, N. B. (2010). Effect of posthatch feed restriction on broiler breast muscle development and muscle transcriptional regulatory factor gene and heparan sulfate proteoglycan expression. Int. J. Poult. Sci. 9, 417-425. doi: 10.3923/ijps.2010. 417.425

Zambonelli, P., Zappaterra, M., Soglia, F., Petracci, M., Sirri, F., Cavani, C., et al. (2016). Detection of differentially expressed genes in broiler Pectoralis major muscle affected by White Striping - Wooden Breast myopathies. Poult. Sci. 95, 2771-2785. doi: $10.3382 / \mathrm{ps} /$ pew268

Zhan, X. A., Wang, M., Ren, H., Zhao, R. Q., Li, J. X., and Tan, Z. L. (2007). Effect of early feed restriction on metabolic programming and compensatory growth in broiler chickens. Poult. Sci. 86, 654-660. doi: 10.1093/ps/86.4.654
Zuidhof, M. J., Schneider, B. L., Carney, V. L., Korver, D. R., and Robinson, F. E. (2014). Growth, efficiency, and yield of commercial broilers from 1957, 1978, and 2005. Poult. Sci. 93, 2970-2982.doi: 10.3382/ps.2014-04291

Conflict of Interest: All authors are employed by Aviagen Ltd.

Copyright (๑) 2020 Bailey, Souza and Avendano. This is an open-access article distributed under the terms of the Creative Commons Attribution License (CC BY). The use, distribution or reproduction in other forums is permitted, provided the original author(s) and the copyright owner(s) are credited and that the original publication in this journal is cited, in accordance with accepted academic practice. No use, distribution or reproduction is permitted which does not comply with these terms. 


\section{OPEN ACCESS}

Edited by:

Krystyna Pierzchala-Koziec,

University of Agriculture in Krakow,

Poland

Reviewed by:

Klaus Wimmers,

Leibniz Institute for Farm Animal

Biology (FBN), Germany

H. Van Den Brand,

Wageningen University and Research,

Netherlands

*Correspondence: Edgar Orlando Oviedo-Rondón edgar_oviedo@ncsu.edu; eooviedo@hotmail.com

Specialty section:

This article was submitted to Avian Physiology,

a section of the journal

Frontiers in Physiology

Received: 23 March 2020

Accepted: 19 August 2020

Published: 18 September 2020

Citation:

Oviedo-Rondón EO, Velleman SG and Wineland MJ (2020) The Role of Incubation Conditions in the Onset of Avian Myopathies.

Front. Physiol. 11:545045. doi: 10.3389/fphys.2020.545045

\section{The Role of Incubation Conditions in the Onset of Avian Myopathies}

\author{
Edgar Orlando Oviedo-Rondón ${ }^{1 *}$, Sandra G. Velleman ${ }^{2}$ and Michael J. Wineland ${ }^{3}$ \\ ${ }^{1}$ Prestage Department of Poultry Science, North Carolina State University, Raleigh, NC, United States, ${ }^{2}$ Department \\ of Animal Sciences, The Ohio State University, Columbus, OH, United States, ${ }^{3}$ Hatchery Consultant Inc., Raleigh, NC, \\ United States
}

White striping, wooden breast, and spaghetti muscle have become common myopathies in broilers worldwide. Several research reports have indicated that the origin of these lesions is metabolic disorders. These failures in normal metabolism can start very early in life, and suboptimal incubation conditions may trigger some of the key alterations on muscle metabolism. Incubation conditions affect the development of muscle and can be associated with the onset of myopathies. A series of experiments conducted with broilers, turkeys, and ducks are discussed to overview primary information showing the main changes in breast muscle histomorphology, metabolism, and physiology caused by suboptimal incubation conditions. These modifications may be associated with current myopathies. Those effects of incubation on myopathy occurrence and severity have also been confirmed at slaughter age. The impact of egg storage, temperature profiles, oxygen concentrations, and time of hatch have been evaluated. The effects have been observed in diverse species, genetic lines, and both genders. Histological and muscle evaluations have detected that myopathies could be induced by extended hypoxia and high temperatures, and those effects depend on the genetic line. Thus, these modifications in muscle metabolic responses may make hatchlings more susceptible to develop myopathies during grow out due to thermal stress, high-density diets, and fast growth rates.

Keywords: incubation, myopathies, temperature, hypoxia, metabolism, chickens, ducks, turkeys

\section{INTRODUCTION}

The growth, development, structure, and general metabolism of muscles in poultry species have been modified by genetic selection (Tesseraud et al., 2003; Petracci et al., 2014, 2019). These genetic changes probably resulted in modifications that affect the biochemical and sensory characteristics of meat. Havenstein et al. (1994a; 1994b; 2003a; 2003b) concluded that 85 to $90 \%$ of the differences between today's broiler and the average chicken 60 years ago are due to genetic selection. In recent years, numerous studies showed that fast-growing genetic lines exhibited a high incidence of idiopathic myopathies and greater susceptibility to stress-induced myopathies that have significant implications on meat quality. Nowadays, the poultry industry observes an increased incidence of abnormal conditions such as deep pectoral myopathy also called green muscle disease (Kijowski et al., 2014), exudative meat or little water retention, wooden breast, striated breast and spaghetti muscle in broilers (Owens et al., 2009; Petracci and Cavani, 2012; Bailey et al., 2015; Russo et al., 2015; Petracci et al., 2019), white stripping in turkeys (Soglia et al., 2018) and myopathies in ducks 
(Gopalakrishnakone, 1986). Bailey et al. (2015) indicated the importance of understanding the environmental and management factors that contribute with more than $65 \%$ to the variance of incidence for striated breast and with more than $90 \%$ to the variation in the occurrence of wooden breast and deep pectoral myopathy in broilers.

An essential factor to consider in the management of poultry species is the incubation conditions during embryo development. Avian embryos exhibit phenotypic (De Smit et al., 2006; Branum et al., 2016) and metabolic (Loh et al., 2004; McNabb, 2006) developmental plasticity to temperature, humidity, oxygen, and $\mathrm{CO}_{2}$ concentrations among other environmental factors like light (Halevy et al., 2006b). Phenotypic plasticity includes all interactions of an organism's genotype with its environment (Burggren et al., 2016).

Artificially incubated eggs are influenced by the temperature, ventilation, humidity, and turning that allow tissue growth, extraembryonic membrane development, gas exchange, and moisture loss (Decuypere and Michels, 1992; French, 1997; Molenaar et al., 2010; Boleli et al., 2016). However, the optimum parameters for all embryos in a machine are not the same due breeder genetics and age, egg composition and yolk size, eggshell and albumen properties, storage time and temperature of storage, among other factors (Molenaar et al., 2010; Yalçin et al., 2014; El Sabry et al., 2015; Nangsuay et al., 2017; Okur et al., 2018).

In large incubator machines, embryo development is primarily affected by the physical microenvironment around the egg (French, 1997; Boleli et al., 2016). The temperature sensed by the embryo depends on three factors: (1) the air temperature, (2) the heat exchange between the egg and its microenvironment depending on the speed of airflow, and (3) the heat production of the embryo that increases as it growths (Van Brecht et al., 2005). It has been demonstrated that any variation on incubation parameters especially the long-term high temperatures and low oxygen tension during the last incubation phases in the hatchers may affect negatively almost all tissues and organ development, including muscles (Hulet et al., 2007; Leksrisompong et al., 2007; Molenaar et al., 2010; Janisch et al., 2015; Boleli et al., 2016; Clark et al., 2017). The impact of those developmental changes has been observed during the life post-hatch in growth and meat quality parameters or the incidence and severity of myopathies. The objective of this publication is to present the scientific evidence that shows that incubation conditions may be an essential factor affecting the susceptibility of poultry to myopathies. The first part of this manuscript will describe the muscle development in avian embryos, its regulation, and the effects of the environment. The second portion will provide a brief description of myopathies with an emphasis on the wooden breast. The third section will discuss evidence that incubation can cause myopathy-like effects in chickens, turkeys, and ducks at hatch and processing age.

\section{MUSCLE DEVELOPMENT IN AVIAN EMBRYOS}

Understanding muscle development is essential to recognize the multiple effects of incubation conditions on the onset of myopathies. The development of skeletal muscle depends on myogenesis and, in part, also on adipogenesis from embryogenesis to adulthood. In the embryo, myogenesis starts with myogenic determination activating myoblasts that first proliferate and then differentiate and fuse into multinucleate fibers. Myofiber ontogenesis commences very early during embryonic life, with the presentation of two or three successive waves of myoblasts, which establish the origin of the diverse types of muscle fibers (Picard et al., 2002; Halevy, 2020).

\section{Muscle Cell Development}

In the chick, embryonic myoblasts are most abundant on embryonic day 5 (ED5) during the primary wave of myogenic cells occurring between ED3 and ED7, whereas fetal myoblasts are most abundant between ED8 and ED12 in the second wave of myogenic cells that occurs between ED7 and ED16 (Stockdale, 1992; Picard et al., 2002). From ED15 onward, satellite cells, or also called adult myoblasts, can be distinguished by their morphology and location under the basal membrane of the myofibers (Hartley et al., 1992). These cells are the primary source of myogenic precursors in the post-hatch muscle (Schultz and McCormick, 1994); however, their numbers are reduced to less than $5 \%$ of total myofiber nuclei toward the end of the growth phase, and they become largely quiescent (Halevy et al., 2001, 2004). Nevertheless, satellite cells can reenter the cell cycle in response to several muscular stresses and go through proliferation, followed by the withdrawal from the cell cycle and fusion into existing or newly formed fibers (Schultz and McCormick, 1994; Hawke and Garry, 2001). Short-term changes in incubation temperature can stimulate the development of satellite cells during different time frames of embryonic development. This thermal stimulus has been successful when applied during the first 4 to 12 days of incubation (Maltby et al., 2004; Krischek et al., 2013a,b), in late-term embryos between ED16 and ED18 (Halevy et al., 2006a,c; Piestun et al., 2013) as well as by changes in environmental temperature during early post-hatch development (Halevy et al., 2001, 2006c). Those are the positive effects of incubation temperature on muscle development, but they only occur when applied in these embryonic developmental periods and during short time in what it has been called circadian incubation.

The muscle mass and intramuscular fat are both determined by cell numbers and unit cell size, mainly during embryo development in avian species (Picard et al., 2002). Cell numbers of adipocytes and muscle fibers increase during the embryonic period by hyperplasia, basically until hatch time (Smith, 1963; Sporer et al., 2011). Breast muscle organizational differences among breeds and between sexes begin to occur between ED20 and ED25 in turkeys (Velleman et al., 2007). While the average cross-sectional area and diameter of the fibers peaks at ED27 in ducks (Li et al., 2016). Thus, embryonic muscle development during the perinatal period has an essential impact on the posthatch accumulation of muscle mass and its infiltration with intramuscular fat (Velleman, 2007). Studies of proteomics and metabolomics of the chicken breast muscle from ED12 to D14 (Liu et al., 2017) indicated that the growth of the embryonic muscle in Cobb chickens is mainly a result of sturdier hyperplasia 
process than hypertrophy. The extent of this hyperplasia is the major contributor to the post-hatchling excellent muscle mass accretion in fast-growing Cobb broiler chicks, which has been measured as a 35.9 -fold change from D1 to D14. The main time for intramuscular fat accumulation is from ED17 to day 1 (D1) post-hatch in chickens (Liu et al., 2017), and in mule ducks, the highest level was D1 (Chartrin et al., 2007). Most of the hyperplasia and intramuscular fat accretion happens during the time of higher oxygen demand.

Myogenesis is governed by a family of proteins known as myogenic regulatory factors (MRFs). These basic helix loop helix (bHLH) transcription factors act sequentially in myogenic differentiation. The vertebrate MRF family members include MyoD1, Myf5, myogenin, and MRF4 (Myf6). These transcription factors work together with the myocyte enhancer factor-2 proteins. MyoD1 and myogenin are successively expressed only in activated satellite cells (Hernández-Hernández et al., 2017). The paired-box transcription factor Pax7 is selectively expressed in quiescent and proliferating satellite cells and is essential in their self-renewal. Pax7 is a marker of myogenesis during post-hatch muscle growth. Its expression is preserved by satellite cells in the adult chicken muscle (Halevy et al., 2004; Allouh et al., 2008).

Hypoxia during embryonic development is a potent inhibitor of myoblast differentiation decreasing expression of MyoD, Myf5, myogenin, and myosin heavy chains, which hampers the formation of multinucleated myotubes (Beaudry et al., 2016; Yang et al., 2017). The impact of hypoxia will depend on timing during the muscle maturation process (Halevy, 2020) and the severity of oxygen deprivation (Beaudry et al., 2016). Myoblast can recover their capacity to proliferate and differentiate when normal oxygen levels are restored (Beaudry et al., 2016). This process is mediated by the hypoxia-inducing factor (HIF) proteins with his three $\alpha$ isoforms HIF- $1 \alpha$, HIF- $2 \alpha$, and HIF- $3 \alpha$. The balance between HIF- $1 \alpha$ and HIF- $2 \alpha$ is important for muscle regeneration and satellite cell self-renewal (Yang et al., 2017). Embryos may overcome hypoxia challenges pre-hatch, but mechanisms for post-hatch muscle regeneration could be affected in muscles that have suffered injuries during extended hypoxia. In summary, all these regulatory factors can be affected by incubation conditions leading to different phenotypic and metabolic traits in muscles (Al-Musawi et al., 2012; Harding et al., 2015; Malila et al., 2019).

Satellite cell proliferation and differentiation are affected by several growth factors and hormones. One of them is the muscle-secreted insulin-like growth factor I (IGF-I) isoform that increases myofiber hypertrophy (Adams et al., 2000). Temperature and oxygen concentration affect levels and cellular signaling of IGF in muscles during the perinatal period (Duan et al., 2010). Halevy et al. (2001) demonstrated that elevated levels of IGF-I in the chick muscle in response to mild heat stress $\left(37^{\circ} \mathrm{C}\right)$ for $24 \mathrm{~h}$ at D3 post-hatch, resulted in greater muscle cell proliferation, differentiation and continuous muscle growth in broilers. On the contrary, lower IGF-I muscle concentrations and more significant myostatin production caused by high levels of glucocorticoids have been both confirmed to contribute to the muscle atrophy of fast-twitch or type II muscle fibers, present in breast muscle. Glucocorticoid levels are increased from ED16 until the day of hatch (Wise and Frye, 1973; Tona et al., 2003b).
Higher concentrations of glucocorticoids have been observed in embryos from stored eggs (Tona et al., 2003b), during hypoxia (Giussani et al., 2007; Tong et al., 2015) or on embryos exposed to higher temperatures (von Blumröder and Tönhardt, 2002). Hypoxia also alters the cellular response to IGF signaling by reprogramming the intracellular signal transduction network (Duan et al., 2010).

\section{Myogenesis Regulation and Environmental Effects}

Research on thermal manipulation of chick embryos at critical phases of development has been conducted to improve long-term physiological responses, such as thermoregulation and muscle development (Yahav et al., 2004a,b; Piestun et al., 2008). These thermal manipulations frequently caused noteworthy decay in plasma thyroid hormone concentration (Yahav et al., 2004a,b; Piestun et al., 2008), which has been linked to myopathies and other metabolic disorders (Valentin, 2017). In contrast, thermal manipulations for $3 \mathrm{~h}$ at $38.5^{\circ} \mathrm{C}$ or $39.5^{\circ} \mathrm{C}$ have been sufficient to stimulate muscle development when applied during the period of maximum satellite cell expansion in chick embryos ED16 to ED18. The result in broilers at 42 days of age has been increased Pectoralis muscle weight compared to controls. However, changes in machine temperature during other embryonic periods had no effect. Despite the potential benefits of thermal conditioning, these short periods of thermal modifications are still not standard in the poultry industry worldwide, and constant heat stress due to multi-stage incubation or inadequate management of singlestage profiles are more common (Molenaar et al., 2010, 2013; Boleli et al., 2016).

Significant adverse effects on meat yield and muscle development have been observed with continuous or longterm (more than $6 \mathrm{~h}$ ) cyclic high temperatures $\left(>38^{\circ} \mathrm{C}\right)$ during incubation (Hulet et al., 2007; Naraballobh et al., 2016a,b; Clark et al., 2017). High incubation temperatures increase oxygen consumption of embryos during the late endothermic phase of incubation that occurs in the 3 to 4 days before hatch (Janke et al., 2002; Mortola and Labbè, 2005; Molenaar et al., 2010, 2013). Consequently, high incubation temperatures in the plateau stage (ED17 to ED21) of oxygen consumption (Rahn, 1981) makes embryos more susceptible to all adverse developmental effects of hypoxia during this last phase (Wineland et al., 2006a,b; Oviedo-Rondón et al., 2008a,b, 2009a,b).

Embryos from precocial poultry species face some hypoxia during the hatching process, but the extent of this phase may have detrimental effects on the development of almost all organs and tissues (Tona et al., 2003a; Mortola and Labbè, 2005; De Smit et al., 2006). During this late phase of incubation, the eggshell conductance, frequently nominated as $\boldsymbol{G}$, becomes very important for embryo respiration (Rahn, 1981; Mortola and Labbè, 2005; Oviedo-Rondón et al., 2008a,b, 2009a,b). The $\boldsymbol{G}$ is the eggshell capacity to passively transfer gasses from the environment to the embryo that can also be measured indirectly by loss of metabolic water and gasses from inside the egg to the external environment (Rahn, 1981). Eggs may vary on $\boldsymbol{G}$ depending on specie, genetics, breeder age, and egg size (Rahn, 1981; Visschedijk et al., 1985; Nangsuay et al., 2017). 


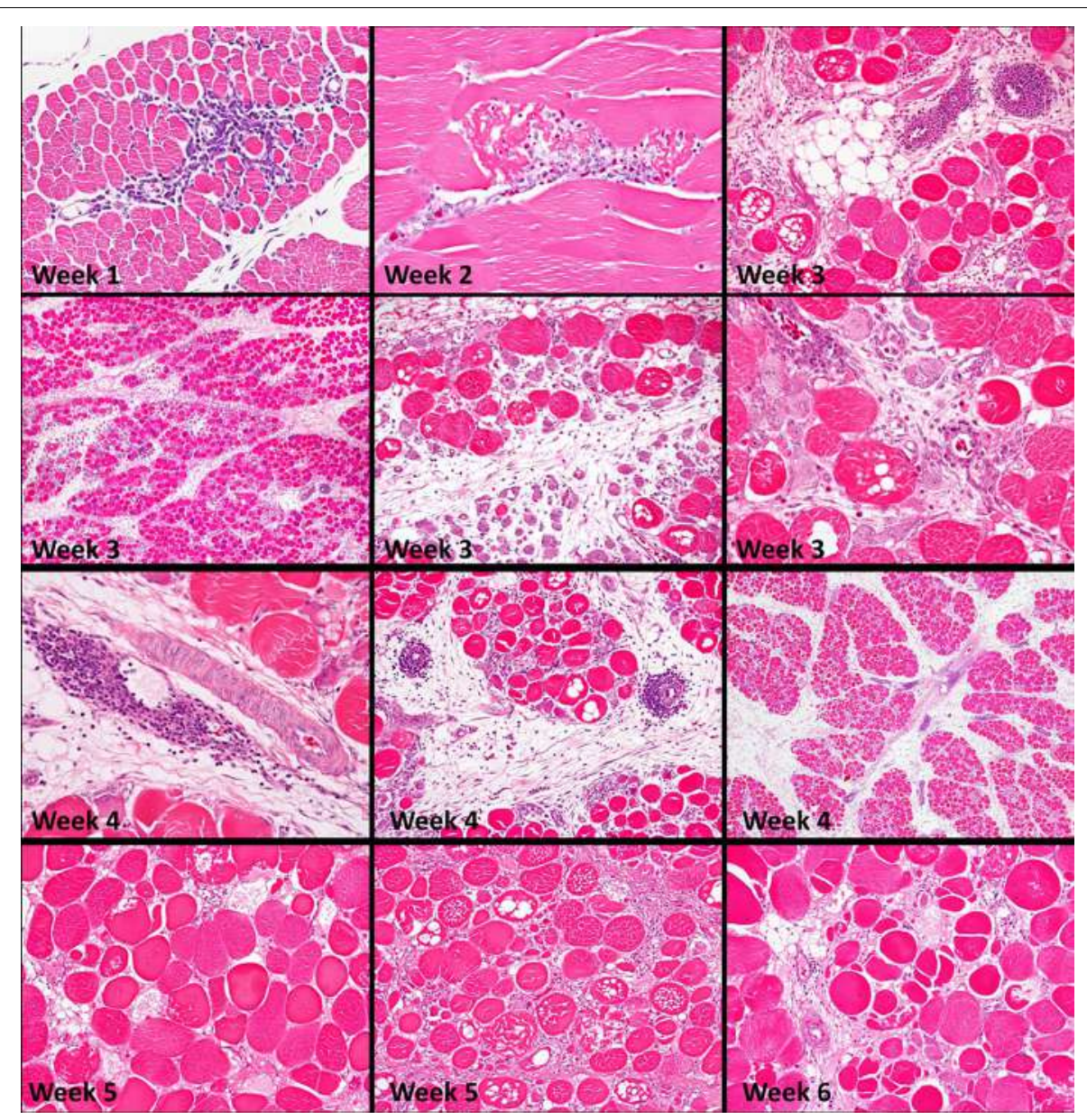

FIGURE 1 | Histopathology, weeks 1 through 6, of superficial pectoral muscles, Study 1. During weeks 1 and 2, the earliest changes are scattered small foci of myofiber degeneration and lymphocytic vasculitis affecting post-capillary venules (phlebitis), which continues to at least six weeks. Muscles are not uniformly affected; lesions are more severe toward the surface of the muscle. Severe muscle degeneration with concurrent regeneration and expansion of the interstitium occurs by three weeks. Lesions intensify during weeks 4 and 5. Vacuolation of affected myofibers is often present, and there is atrophy of muscle fasciculi (bundles). Increasing fibrosis and regeneration begin to predominate through weeks 5 and 6 . Connective tissue and fat, contributing to "white striping," accumulate in interstitial tissues within and between muscle fasciculi. Fiber splitting is prominent at six weeks [Reproduced with permission of Chen et al. (2019)].

Eggs with reduced $\boldsymbol{G}$ may face more severe hypoxia. Taking into consideration that recent findings have linked myopathy onset with hypoxia (Malila et al., 2019), the factors that cause hypoxia during life should receive attention to determine strategies to minimize myopathy incidence.

\section{BREAST MYOPATHIES IN AVIAN SPECIES}

White striping (Kuttappan et al., 2013b; Petracci et al., 2013), wooden breast, green muscle, and spaghetti meat have been well described (Chen et al., 2019; Petracci et al., 2019). Histologically, it is difficult to identify differences between cases of wooden breast and striped breast. Wooden breast muscle has lateral packing of collagen and more significant fat infiltration (Velleman et al., 2017). The muscles affected by wooden breast have poor micro vascularization, myodegeneration, rounded fibers, internalization of the nuclei, lymphocytic infiltration, and fibers in the process of regeneration (Soglia et al., 2016; Sihvo et al., 2018; Petracci et al., 2019). These lesions can be observed (Figure 1) since the first week of age (Chen et al., 2019). There is diffuse thickening of the connective tissue of the endomysium and perimysium associated with granulation tissue and increased deposition of connective tissue (fibrosis) and fat deposition or infiltration. Different degrees of muscle damage occur, which are diffuse and not continuous in the Pectoralis major muscle (Soglia et al., 2016; Clark and Velleman, 2017).

Several studies have shown that wooden breast is related to hypoxia (Malila et al., 2019) and metabolic disturbances of muscles (Mutryn et al., 2015; Abasht et al., 2016; Petracci et al., 2019). The affected muscles have lower glycogen content, impaired glucose metabolism, higher concentrations of metabolites characteristic of oxidative stress, high protein levels, increased hypoxanthine, xanthine, and urates. The lack of glycogen in the muscle leads to greater activation of the ascorbate 
biosynthesis pathway and excessive production of hydrogen peroxide. The excess of free radicals can consume glutathione and exacerbate the oxidative process (Mutryn et al., 2015; Abasht et al., 2016). The wooden breast has been observed very early in life (Sihvo et al., 2018; Chen et al., 2019) and frequently broilers affected have increased blood $\mathrm{CO}_{2}$ and decreased $\mathrm{O}_{2}$ concentrations (Livingston et al., 2019a) related to a state of hypoxia and oxidative stress or low vascularization (Soglia et al., 2016; Sihvo et al., 2018; Chen et al., 2019). Other common findings in broilers affected by wooden breast are increased intracellular calcium (Mutryn et al., 2015) and in white stripping elevated serum levels of creatine kinase, alanine transaminase, aspartate aminotransferase and lactate dehydrogenase (Kuttapan et al., 2013a). Some of these histological, molecular, and metabolic characteristics of myopathies have been observed in poultry hatchlings when incubation conditions have been modified or were suboptimal as it will be discussed in the following sections.

\section{EVIDENCE OF INCUBATION EFFECTS ON MUSCLE DEVELOPMENT AND MYOPATHIES}

In several studies, it has been observed that variations on temperature during incubation affect myoblast proliferation and skeletal muscle hypertrophy in chickens (Collin et al., 2007; Piestun et al., 2009; Janisch et al., 2015; Naraballobh et al., 2016a,b), turkeys (Maltby et al., 2004; Krischek et al., 2013a), and ducks (Hepp et al., 2006; Hopkins et al., 2011; Liu et al., 2015). Most of the studies have evaluated thermal modifications for the short term during different periods of incubation to stimulate muscle growth. However, suboptimal incubation may affect the occurrence of myopathies. Table 1 presents a summary of some publications that have reported the effects of incubation in myopathies. Myopathies in embryos and newly hatched broilers and ducks have been previously reported (Rigdon, 1967; Rigdon et al., 1968; Yamagiwa et al., 1975).

Recently, Nyuiadzi et al. (2020) reported slightly less white striping occurrence at D40, when Ross 308 chicken embryos from 36-wk-old breeders, incubated at constant $37.6^{\circ} \mathrm{C}\left(99.68^{\circ} \mathrm{F}\right)$ and $70 \% \mathrm{RH}$ were exposed to $15^{\circ} \mathrm{C}$ and $81 \% \mathrm{RH}$ for 30 minutes at days 18 and 19. This treatment also had improvements in final body weight (37 grams) compared to the control, but no effects on feed efficiency, carcass and cut up part yields, or meat quality. However, when those chickens were exposed to initial low brooding temperatures $\left(29^{\circ} \mathrm{C}\right)$, compared to control brooding $\left(32^{\circ} \mathrm{C}\right.$ at $\left.\mathrm{D} 1\right)$, the white stripping in males tripled, and the footpad dermatitis quadrupled. The environmental conditions post-hatch may affect the responses of hatchlings to incubation treatments. The treatment reported by Nyuiadzi et al. (2020) was a short cold exposure that could occur when transferring eggs from setters to hatchers. As far as brooding conditions are adequate, it seems reducing embryo metabolic heat during this period (ED18 and ED19) is positive to reduce white striping. Still, adaptation to cooler brooding could be an issue. It remains to be evaluated the effects of appropriate stepdown temperatures in the hatchers, as eggshell temperature raises due to embryo metabolic heat production. The reduction in machine temperature is a common practice in single-stage incubation (Boleli et al., 2016), but not used in multi-stage machines or the experiment reported by Nyuiadzi et al. (2020).

The most common situation under industrial conditions is the overheating and reduced egg air exchange that embryos can encounter during the last phase of incubation. This condition can arise either in multi-stage machines or single-stage machines with management and maintenance issues (French, 1997; Boleli et al., 2016). Under commercial conditions, in large incubators, variations in fan speed and direction and or changes in turning angles of egg trays reduce airflow over the eggs (French, 1997) and airflow direction (Van Brecht et al., 2005). The most frequent overheating occurs when all embryos in a machine have the fastest growth rate, metabolic heat production, higher oxygen demand, and $\mathrm{CO}_{2}$ production during the plateau of oxygen consumption (Rahn, 1981; Wineland et al., 2006a,b; Christensen et al., 2007). Hypoxia in the last 72 to $96 \mathrm{~h}$ of incubation is also more frequent in embryos from eggs stored for more than 5 days due to a delay in development and the extended period between internal and external pipping (Tona et al., 2003a). Livingston et al. (2019b) reported an increased white stripping score (2.58 vs. 3.15) when 42-day-old Ross-708 broilers coming from eggs stored for 8 to 14 days were compared to broilers from eggs stored for 1 to 7 days fed ad libitum.

\section{High Temperatures and Low Oxygen Concentration During Incubation in Poultry Myopathies}

The high temperatures with $>38^{\circ} \mathrm{C}$ of eggshell temperature, and low oxygen concentrations $(<18 \%)$ in the incubators during these last stages of incubation can affect the development and viability of muscle fibers in avian species (Table 1). Mulder et al. (1998) and Crossley et al. (2003) demonstrated that when chicken embryos experience hypoxia during the last 3 days of incubation, there is a rearrangement of blood flow, increasing to heart and brain and reducing to liver, muscle and yolk sac. During acute hypoxia, in the plateau of oxygen consumption (Rahn, 1981), the systemic secretion of catecholamines results in vasodilation of the chorioallantoic membrane (CAM) vessels via $\beta$-adrenoceptors. In the meantime, vasoconstriction is produced in some of the intraembryonic vessels via $\alpha$-adrenergic receptors (Crossley et al., 2003). As CAM blood flow accounts for 20 to 50\% of total cardiac output (Mulder et al., 1998), CAM vasodilation during hypoxia could cause general embryo hypotension and lower tissue oxygenation.

The damages of hypoxia in the breast muscle under high temperatures of incubation have been detected on the first day of age in turkeys (Christensen et al., 2007) and broilers (unpublished data). High machine temperatures $\left(39^{\circ} \mathrm{C}\right.$ vs. $\left.36^{\circ} \mathrm{C}\right)$ and low oxygen concentrations ( $17 \%$ vs. $21 \%$ ) affected negatively pipping and thigh muscle weights, but only the temperature significantly influenced breast muscle weights and fiber diameters. High temperatures $\left(39^{\circ} \mathrm{C}\right)$ increased creatine kinase and lactate dehydrogenase and reduced muscle glycogen. Histologically all signs of muscle degradation (Figure 2) were observed on poults 
TABLE 1 | Overview of literature reporting effects of incubation conditions on myopathy development in chickens, turkeys, and ducks.

\begin{tabular}{|c|c|c|c|c|c|c|c|c|c|c|c|c|}
\hline \multirow[t]{2}{*}{ Reference } & \multirow[t]{2}{*}{ Species } & \multirow[t]{2}{*}{ Genetic line } & \multirow{2}{*}{$\begin{array}{l}\text { Egg } \\
\text { storage } \\
\text { (d) }\end{array}$} & \multicolumn{4}{|c|}{ Incubation treatments } & \multirow[t]{2}{*}{ Sex } & \multicolumn{2}{|c|}{ BW } & \multicolumn{2}{|c|}{ Myopathy } \\
\hline & & & & $\mathrm{T}\left({ }^{\circ} \mathrm{C}\right)$ & RH (\%) & Period & Duration & & Age (d) & Weight (g) & wS & WB \\
\hline \multirow{2}{*}{$\begin{array}{l}{ }^{1} \text { Christensen } \\
\text { et al., } 2007\end{array}$} & Turkeys & Nicholas & 4 & 36.0 & 53 & ED24 - ED28 & 24 h/day & Mixed & 1 & $x$ & $=$ & \\
\hline & & & 4 & 39.0 & 53 & ED24 - ED28 & $24 \mathrm{~h} /$ day & Mixed & 1 & $x$ & $\uparrow^{3}$ & \\
\hline \multirow{12}{*}{$\begin{array}{l}\text { 1Oviedo- } \\
\text { Rondón et al., } \\
\text { 2016, partially } \\
\text { published by } \\
\text { Chen et al., } \\
2019\end{array}$} & Broilers & Cobb MXx & 3 & 37.8 & 50 & EDO - ED21 & $24 \mathrm{~h} /$ day & Males & 56 & 4,345 & $=$ & $=$ \\
\hline & & Cobb 500 Ross & & & & & & Females & & 4,554 & & \\
\hline & & $344 \times$ Ross 708 & & & & & & & & 4,464 & & \\
\hline & & Hubbard M99 x & & & & & & & & & & \\
\hline & & Cobb 500 & & & & & & & & & & \\
\hline & & Ross 344 x & 3 & 37.0 & 50 & EDO - ED3 & 24 h/day & Males & 56 & 4,478 & $=$ & $=$ \\
\hline & & Ross 708 & & & & & & Females & & 4,437 & & \\
\hline & & Hubbard M99 x & & & & & & & & & & \\
\hline & & & & & & & & & & & & \\
\hline & & & & 39.0 & 50 & ED18 - ED21 & 24 h/day & & & & & \\
\hline & & $\begin{array}{l}\text { Cobb MX X } \\
\text { Cobb } 500\end{array}$ & 3 & 37.0 & 50 & EDO - ED3 & 24 h/day & $\begin{array}{l}\text { Males } \\
\text { Females }\end{array}$ & 56 & 4,410 & $=$ & $\uparrow$ \\
\hline & & & & 39.0 & 50 & ED18 - ED21 & 24 h/day & & & & & \\
\hline \multirow{3}{*}{$\begin{array}{l}{ }^{1} \text { Clark et al., } \\
2017\end{array}$} & Broilers & Ross 708 & $x$ & 37.8 & 53 & EDO - ED21 & $24 \mathrm{~h} /$ day & $x$ & 63 & 5,478 & $=$ & \\
\hline & & & & 39.5 & 65 & ED14 - ED18 & $3 \mathrm{~h} /$ day & $x$ & 63 & 5,490 & $=$ & \\
\hline & & & & 39.5 & 65 & ED14 - ED18 & 12 h/day & $x$ & 63 & 5,320 & $\downarrow^{3}$ & \\
\hline \multirow{2}{*}{$\begin{array}{l}{ }^{1} \text { Livingston } \\
\text { et al., 2019b }\end{array}$} & Broilers & Ross 708 & $1-7$ & 37.8 & $x$ & EDO - ED21 & 24 h/day & Males & 42 & 3,056 & $=$ & $=$ \\
\hline & & & $8-14$ & 37.8 & $x$ & ED0 - ED21 & 24 h/day & Males & 42 & 3,056 & $\uparrow$ & $=$ \\
\hline \multirow{2}{*}{$\begin{array}{l}{ }^{2} \text { Nyuiadzi et al., } \\
2020\end{array}$} & Broilers & Ross 308 & 3 & 37.6 & 56 & EDO - ED21 & $24 \mathrm{~h} /$ day & Males & 40 & 2,769 & $=$ & $=$ \\
\hline & & & & 15.0 & 81 & ED18 - ED19 & $30 \mathrm{~min} /$ day & Males & 40 & 2,818 & $\uparrow$ & $=$ \\
\hline \multirow{5}{*}{$\begin{array}{l}\text { Velleman, } \\
\text { Wineland, and } \\
\text { Oviedo (Data } \\
\text { not published) } \\
\text { Da Costa et al., } \\
2016\end{array}$} & Ducks & Maple Leaf & 4 & 37.8 & 50 & ED12 - ED21 & 24 h/day & Males & 35 & 3,211 & $=$ & $=$ \\
\hline & & & & $37.8^{4}$ & 50 & ED12 - ED21 & 24 h/day & Males & 35 & 3,139 & $\uparrow^{3}$ & \\
\hline & & & & & & & & Females & & & & \\
\hline & & & & $37.8-35.5$ & 50 & ED12 - ED21 & 24 h/day & $\begin{array}{l}\text { Males } \\
\text { Females }\end{array}$ & 35 & 3,237 & $\uparrow^{3}$ & \\
\hline & & & & $37.8-35.5^{4}$ & 50 & ED12-ED21 & 24 h/day & $\begin{array}{l}\text { Males } \\
\text { Females }\end{array}$ & 35 & 3,240 & $\uparrow^{3}$ & \\
\hline
\end{tabular}

T; Temperature, RH; Relative Humidity, ED; Embryonic Day, $x ;$ not reported, =; No significant differences, $\uparrow$ and $\downarrow ;$ Increase or decrease incidence or score of myopathies. ${ }^{1}$ Treatments based on eggshell temperature. ${ }^{2}$ Treatments based on machine temperature. ${ }^{3}$ Histological scores of myopathies. ${ }^{4}$ Reduced eggshell conductance at ED 14 by immersing one-third of the egg in warm paraffin to reduce the respiratory surface. 
subjected to high temperatures and low oxygen concentrations. These observations are similar to the ones reported during the onset of muscle myopathies (Papah et al., 2017; Chen et al., 2019).

Recently, Halevy (2020) summarized the hypotheses explaining the effects of thermal stress during the early growth phase. Thermal stress during the perinatal period could cause myodegeneration. In these muscles, increased interstitial fibro-adipogenic progenitors and recruitment of myofibroblasts and adipogenic cells are observed. Finally, there is a reduction in the number of satellite cells leading to ineffective myofiber function (Patael et al., 2019). More fibrous and adipose tissue causes a decrease in the endomysial and perimysial connective tissue space that may restrict muscle vascularization (Dransfield and Sosnicki, 1999). Less capillarity limits nutrient and oxygen supply and hampers the removal of metabolic end products like lactic acid.

The deleterious effects of suboptimal incubation on early muscle development have an impact on the incidence and severity of wooden breast at the time of the processing of broilers (Oviedo-Rondón et al., 2016). Specific details of the experiment that will be discussed in the following lines have been partially published by Chen et al. (2019). Breast myopathies were observed in one experiment conducted to evaluate the effects of the genetic line, temperature incubation profiles, and gender. A total of 1,000 eggs from three genetic line crosses: Cobb MX x Cobb 500, Ross 344 x Ross 708, and Hubbard M99 $\mathrm{x}$ Cobb 500 were randomly distributed into four machines with two incubation temperature profiles. These eggs were collected from three breeder flocks of 48 weeks of age and stored for 3 days before incubation. In two incubators, the standard eggshell temperature was maintained close to $37.8^{\circ} \mathrm{C}\left(100.0^{\circ} \mathrm{F}\right)$ during the whole incubation period to simulate single-stage incubation (Standard). In the other two machines, eggshell temperatures were low $\left(36.9^{\circ} \mathrm{C}\right)$ for the first 3 days and close to $37.8^{\circ} \mathrm{C}$ until the last 3 days when eggs were subjected to elevated $\left(38.9^{\circ} \mathrm{C}\right)$ eggshell temperatures (Low-high), as it is often observed in multi-stage machines. The relative humidity was $50 \%$ for multistage machines and varied from $65 \%$ to $40 \%$ in single-stage incubation. Eggshell temperatures were measured five times per day with pipe-probes and thermistors to avoid opening the machines or affecting ventilation. At hatch, 960 chicks sexed were randomly distributed into 80 pens ( 12 chickens/pen) with five replicate pens per treatment combination. The chicken density was nine chickens $/ \mathrm{m}^{2}$. All chickens were fed typical cornsoybean meal diets ad libitum formulated according to Aviagen recommendations (2014). The lighting program was 23L:1D during the first week and 16L:8D until the end of the experiment. No significant differences $(P>0.05)$ among genetic lines were observed on body weight. At 56 days of age, the average body weight was $4.45 \pm 0.08 \mathrm{~kg}$ for mixed-sex broilers. At 57 days of age, two broilers per pen were processed, wooden breast and white striping myopathies evaluated $16 \mathrm{~h}$ after deboning.

The results indicated that no $(P>0.05)$ three-way interactions were detected. Genetic by temperature profile and genetic by gender interaction effects on white striping $(P<0.05)$ and differences due to genetics $(P<0.001)$ on wooden breast were observed. The most severe scores for wooden breast and white striping were found in Ross 344 x Ross 708 broilers. However, this Ross line had almost $2 \%$ points more of breast meat yield (38.50\%) than the other two lines (36.78 and $35.03 \%$ ) at 57 days of age. The lowest scores for white striping were observed on Hubbard M99 x Cobb 500 when broilers were incubated on the standard temperature profile. The females of this line also had the lowest white striping scores as compared to Ross females or Cobb MX x Cobb 500 males. The low-high incubation treatment had 2.18 times more broilers exhibiting wooden breast score 2 than broilers coming from the standard incubation, independently of the genetic line and sex (Figure 3). In Cobb MX x Cobb 500 broilers, the incidence increased six-fold due to low-high suboptimal incubation ( $0.10 v s$. 0.65$)$. Still, no significant effects of incubation were observed in the other two genetic lines. The differences in results between genetic lines could be due to thicker and less porous eggshells with low $G$ in the Cobb line. It has been demonstrated that eggs from these genetic lines vary on $G$ (Oviedo-Rondón et al., 2008a,b). In conclusion, genetic lines differed on white striping, and wooden breast severity and incubation temperature profiles influenced the incidence of myopathies. Deep pectoral myopathy was only observed in a few broilers with no relation to genetic line, incubation profile, or sex. No spaghetti muscle was detected in this experiment.

In contrast, Clark et al. (2017) evaluated the effects of increasing the setter temperature from $37.8^{\circ} \mathrm{C}\left(100.04^{\circ} \mathrm{F}\right)$ to $39.5^{\circ} \mathrm{C}\left(103.1^{\circ} \mathrm{F}\right)$ for 3 and $12 \mathrm{~h}$ per day from 14 to 18 days of incubation on time of hatch, growth, breast muscle development and myopathies at 63 days of age. During the last 3 days of incubation, the temperature was stepped down to $36.4,36.0$, and $35.8^{\circ} \mathrm{C}$ on $\mathrm{ED} 19,20$, and 21 , respectively. Chickens were raised in floor pens for a final density of $41.5 \mathrm{~kg} / \mathrm{m}^{2}$. During the first week, chicks were subject to $23 \mathrm{~h}$ of light and then $18 \mathrm{~h}$ of light for the remainder of the study. Ambient temperatures were controlled as follows: 31 to $29^{\circ} \mathrm{C}$ for wk 1,29 to $27^{\circ} \mathrm{C}$ for wk 2,27 to $24^{\circ} \mathrm{C}$ for wk 3,24 to $22^{\circ} \mathrm{C}$ for wk 4,22 to $21^{\circ} \mathrm{C}$ for wk 5,21 to $20^{\circ} \mathrm{C}$ for wk 6,20 to $18^{\circ} \mathrm{C}$ for wk 7 , and $18^{\circ} \mathrm{C}$ for wk 8 and 9 . The chicks incubated at an increased temperature for $12 \mathrm{~h}$ per day had reduced $(P<0.01)$ body and carcass weights $(\sim 150 \mathrm{~g}$ less $)$ compared to the $3 \mathrm{~h}$ and control treatments, and worse $(P<0.01)$ feed conversion ratio ( $1 \%$ worse). But, this treatment applied $12 \mathrm{~h}$ per day had fewer broilers with moderate and severe myopathies compared to the control. Myopathies were evaluated by a microscopic myopathy score system based upon the severity of necrotic and fibrotic attributes, as described by Clark and Velleman (2017). The authors concluded that increasing incubation temperature from ED14 to ED18 was a feasible management strategy to reduce myopathies while maintaining meat quality parameters. The authors also evaluated the time of hatch. Chickens that hatched early had a higher occurrence of moderate to severe degenerative changes in the breast muscle compared to those that hatched later. The longer time that these chickens had to reach feed probably affected satellite cells. Even though the eggshell temperature was not measured in that experiment, it is known that machine temperatures higher than $37.6^{\circ} \mathrm{C}$ after 15 days of incubation could cause overheating in the embryos (Molenaar et al., 2013), and this slight overheating 


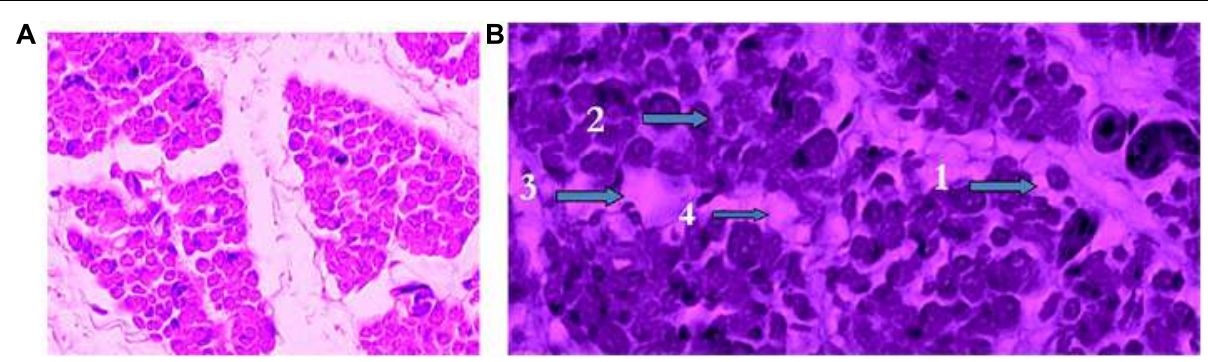

FIGURE 2 | Effect of temperature and oxygen concentration during the last 4 days of incubation on breast muscles of Ross 308 chicks at hatch. (A) Breast muscle of chicks incubated at $36^{\circ} \mathrm{C}$ and $23 \%$ of oxygen exhibiting healthy muscle fibers. (B) Breast muscle of chicks incubated at $39^{\circ} \mathrm{C}$ and $17 \%$ of oxygen with four stages of muscle degeneration: 1. Rounded fibers, 2. Opaque fibers, 3. Degeneration, and 4. Phagocytosis. (Reproduced with permission of V. Christensen and M. Wineland).

B
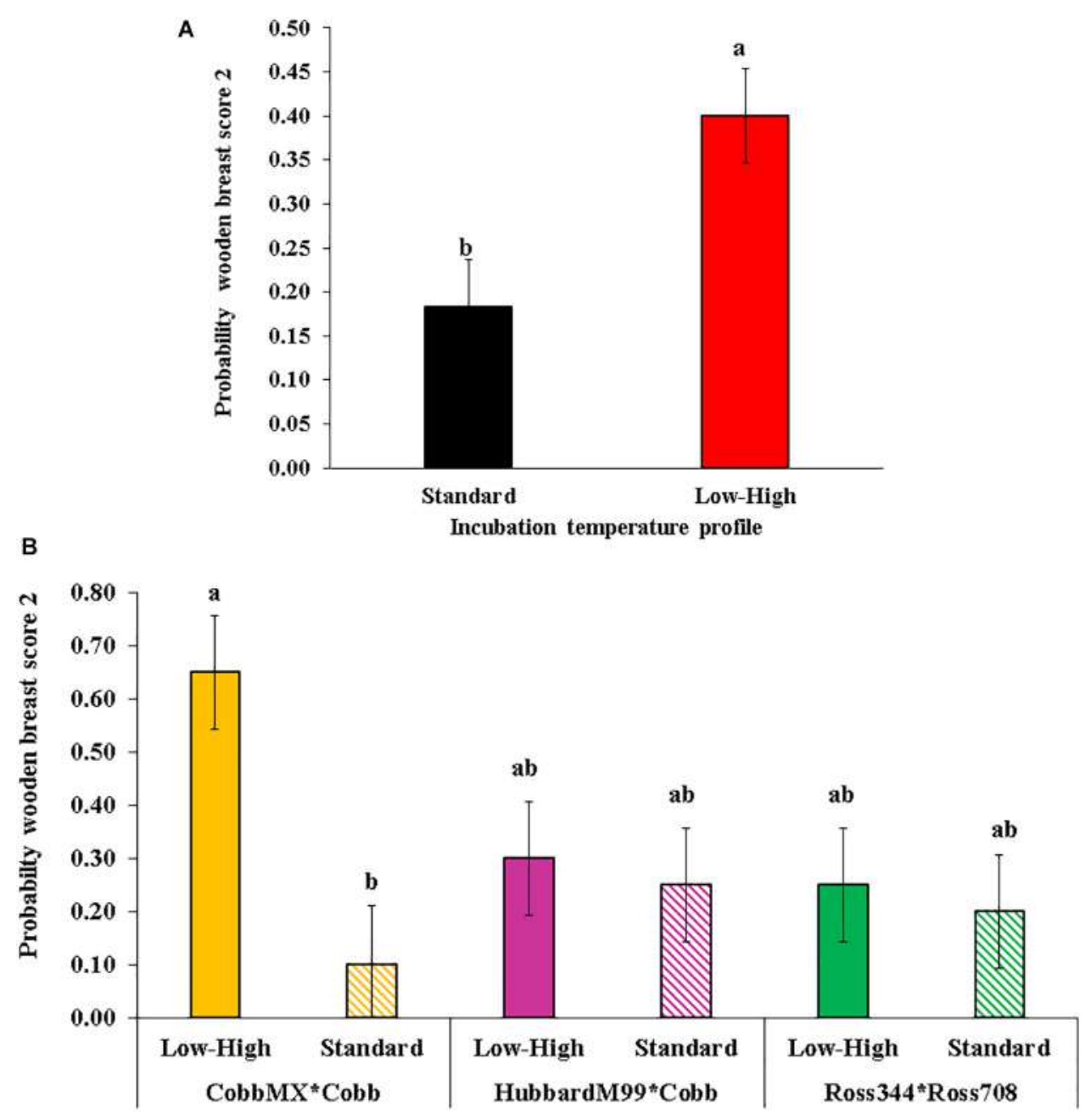

FIGURE 3 | Effect of incubation temperature profile on the probability of observing wooden breast score 2 (moderate severity). (A) Effect of incubation temperature profile independently of the genetic line. (B) Effect of incubation temperature profile by genetic line cross. [Modified from data of Chen et al. (2019)].

could explain the effects on live performance observed in this experiment.

The differences in the responses observed between the two experiments discussed here are related to the length of exposure to higher temperatures ( 3 days vs. $12 \mathrm{~h}$ ), and the timing of application of the heat stress (14 to 18 days vs. 18 to 21 days). Chicken embryos respond differently to heat stress (Molenaar et al., 2013) and hypoxia (Crossley et al., 2003) depending on timing during embryo development, being more critical and deleterious for muscles during the last phase of incubation 
(Hartley et al., 1992; Christensen et al., 2007; Boleli et al., 2016; Halevy, 2020).

\section{High Temperatures and Reduced Eggshell Conductance Effects in Ducks}

Some incubation effects on muscle myopathies have also been observed in Pekin ducks. One experiment was conducted to evaluate the effects of $G$ and temperature profiles during incubation on duck muscle histological characteristics at 35 days of age. The materials and methods and a portion of the results of this experiment were published by Da Costa et al. (2016). Briefly, a total of 10,000 Pekin duck eggs were randomly sorted, equally distributed into four groups, and placed in two single-stage incubators under commercial conditions. Treatments consisted of two $G$, reduced and normal, and two incubation temperature profiles, elevated and normal, after ED12. Eggshell $G$ was reduced by dipping lower one-third of eggs in wax at 14 days of incubation. At hatch, ducklings were placed in a commercial house. At hatch and market age, five drakes and five hens of each treatment combination were selected, weighed, and processed. The histomorphological structure of the muscle was assessed for muscle fiber integrity, perimysial, and endomysial spacing, as described in Velleman et al. (2003). For the histological data, a $2 \times 2$ factorial design with temperature and $G$ as main factors was used. Results (Figure 4) indicated that either elevated $\mathrm{T}$ or reduced $G$ altered $(P<0.05)$ muscle fiber diameter and increased perimysial and endomysial fibrosis. The reduced $G$ treatment had the most detrimental effects with increased collagen deposition typical of fibrosis in the perimysial connective tissue space and the presence of inflammatory cells suggestive of myofiber necrosis. Interestingly, elevated temperature with both hypoxia and without hypoxia caused by variations on eggshell $G$ increased the endomysial spacing between the myofibers compared to the control. Unlike the males, the hens with hypoxia (reduced $G$ ) did not have altered collagen deposition typical of fibrosis. In the hens, the elevated temperature with hypoxia decreased muscle fiber bundle size and the endomysial spacing between individual muscle fibers. Decreases of this nature are associated with reduced muscle mass accretion.

\section{Metabolic Changes in Poultry Embryo Muscle Post Environmental Stress}

Suboptimal environmental conditions during incubation can cause metabolic modifications in the muscle that may continue in the life post-hatch. To understand the relationship between incubation effects and myopathies is indispensable to review the metabolic changes observed in myopathies. The histological (Velleman et al., 2017; Sihvo et al., 2018), metabolic characteristics (Abasht et al., 2016), the gene expression (Mutryn et al., 2015), and the chronological evolution (Chen et al., 2019) of the lesions in the muscles with wooden breast in chickens are very similar to those observed in the muscles of human and diabetic animals (D'Souza et al., 2013). Chronic low-grade inflammation, oxidative stress, lower vascularization, and impaired extracellular matrix remodeling are common denominators for mechanisms underlying deterioration of chicken muscle health and decreased satellite cell functionality similar to the observed in diabetes mellitus type 2 (Lake and Abasht, 2020). These failures in avian muscle development are likely due to disturbances in glucose metabolism, or insulin resistance. The insulin response in avian species differs from metabolism in mammals, with different transport proteins that respond to insulin (Seki et al., 2003) and a cellular system that causes the birds' muscles to be insensitive to insulin despite the high plasma glucose levels (Dupont et al., 2009). This control system could be altered by high levels of corticosterone induced by stressors (Dong et al., 2007; Yuan et al., 2008; Zhao et al., 2009). These high corticosterone levels can be observed during the perinatal period in avian species, and chicken embryos could be induced to have diabetes type I (Shi et al., 2014). The metabolic changes in the muscle caused by other poultry myopathies have not been described with the same detail given to wooden breast.

High temperatures and oxygen concentrations during the last phase of incubation affect the metabolism of the chicks, especially in the glucose and insulin balance (Loyau et al., 2015). Increased hatcher temperature elevates embryonic plasma glucose concentrations compared to controls (Molenaar et al., 2013), which was accompanied by increased plasma glucagon and variations in IGF-I concentrations (Christensen et al., 2001). IGF-I also can be stimulated by oxygen supplementation during incubation and decreased by hypoxia (Christensen et al., 1999). These changes appeared dependent on the genetics of embryos. Embryos selected for fast growth show more fluctuation in response to environmental oxygen and temperature than embryos from genetics selected for egg production. In the same way, the embryos from eggs with low $G$ are more susceptible to changes in environmental conditions (Oviedo-Rondón et al., 2008a,b).

Al-Musawi et al. (2012) demonstrated with molecular and histological methods that increasing incubation temperature from 37.5 to $38.5^{\circ} \mathrm{C}$ between ED 4 and ED7 enhanced motility and body mass in both layer and broiler embryos. However, the muscle responses vary between layers and broilers. In layers, increased mRNAs were observed in hindlimb Myf5 at ED5-8, Pax7 (ED5-10), Bone Morphogenetic Protein 4, BMP4 (ED69) and IGF-I (ED9-10, ED18). These molecular changes led to gastrocnemius muscle hypertrophy, increased fiber and nuclei numbers, and higher nuclei to fiber ratio by ED18. In contrast, broiler embryo hindlimbs had a delay in the peak of mRNA Myf5 expression, increased Pax7 (ED5, ED7-10), and BMP4 (ED6$8)$, increased proliferator-activated receptor gamma (PPAR $\gamma$ ) mRNA (ED7-10) but reduced IGF-I (ED8-10). The result in broilers was a lower gastrocnemius cross-sectional area with fewer fiber and nuclei, no changes in fiber to nuclei ratio (ED18), and increased intramuscular lipid deposition. These authors concluded that incubation temperature modifications affected broiler and layer embryos differently. The molecular regulation of hindlimb myogenic, adipogenic, and growth factor expression varied between these two groups causing different muscle phenotypes. Lipid infiltration and metabolic muscle changes are common in myopathies (Papah et al., 2017). Increasing incubation temperature in one degree Celsius, from ED4 to ED7, created all these metabolic changes in muscles. Heat stress during 
A
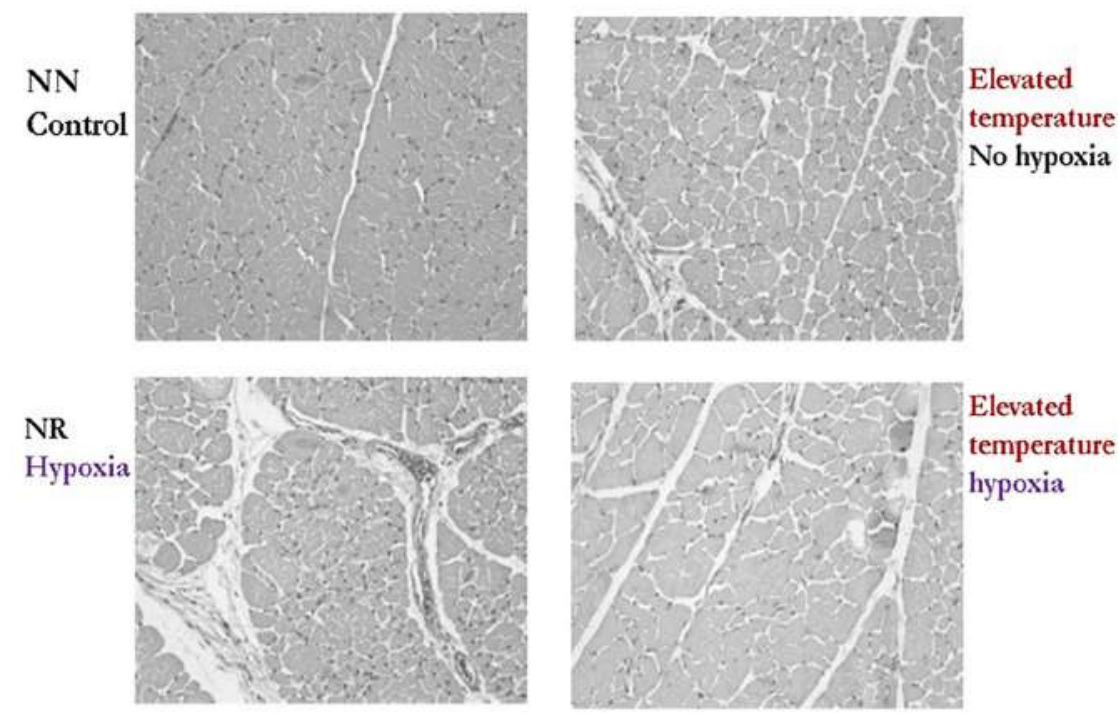

\section{Elevated temperature} hypoxia

B
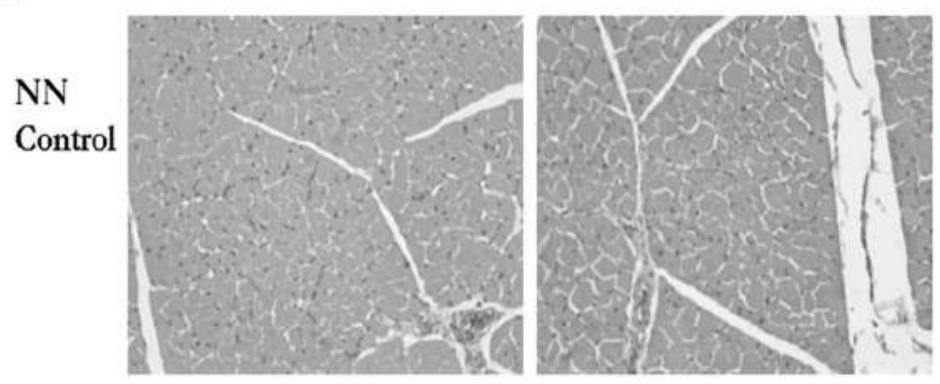

\section{Elevated temperature No hypoxia}
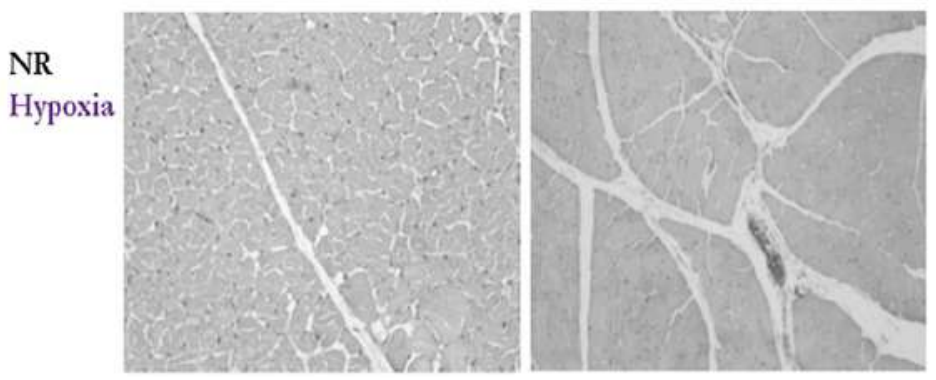

\section{Elevated} temperature hypoxia

FIGURE 4 | Effect of temperature after 12 days of incubation (Normal vs. Elevated) and reduced eggshell conductance (Normal vs Reduced) after 14 days of incubation on breast muscle histomorphology of drake (A) and hen (B) Pekin ducks at 35 days of age. These results demonstrated altered muscle fiber diameter with increased perimysial and endomysial connective spacing compared to the control. (Reproduced with permission of M. Wineland. S. Velleman and E. Oviedo).

the last stage of incubation cause even more significant impacts, because higher metabolic heat release and oxygen demand are critical for muscle development.

Chicken IGF-I has been recognized as a candidate gene responsible for body composition, growth, fat deposition, and other metabolic activities critical for satellite cell proliferation and skeletal muscle hypertrophy (Zanou and Gailly, 2013). The production of IGF-I is highly dependent on oxygen concentrations (Duan et al., 2010). The beneficial effects of IGF-I lowering glucose via inhibition of renal gluconeogenesis (Clemmons, 2007) and sensitizing cells for insulin (Kabir et al., 2010) can aid in glucose metabolism regulation. Low IGF-I, hyperglycemia, and failures of glucose metabolism are frequently observed in myopathies like striated and wooden breast muscle (Abasht et al., 2016; Lake and Abasht, 2020).

Hyperglycemia has a negative effect on vascularization. Larger et al. (2004) demonstrated that hyperglycemia decreases angiogenesis in the chicken CAM model after the 5th day. Two days after inducing hyperglycemia, these authors detected increased apoptosis of endothelial cells and pericytes, using transferase-mediated deoxyuridine triphosphate nick-end 


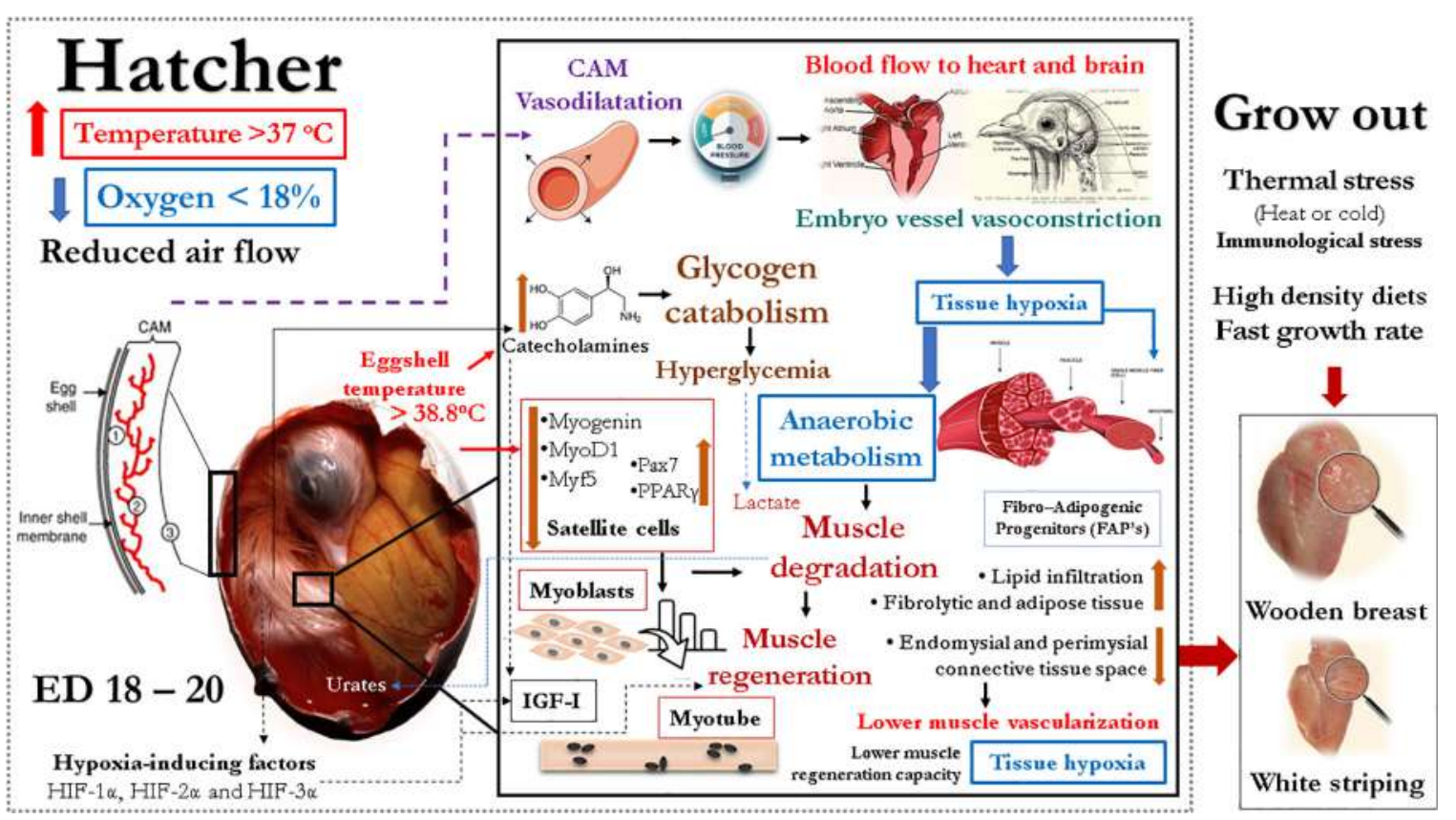

FIGURE 5 | Graphical description of the hypothesis supported by the evidence presented in this manuscript indicating that temperature, oxygen concentrations, and ventilation, especially during the last phase of incubation, affect metabolism of glucose and lipids in the embryonic muscle leading to changes in glucose and fat muscle metabolism, satellite cell number and function, vascularization and capabilities of regeneration. The final result could be susceptibility to develop myopathies during grow out.

labeling assay and electron microscopy. The analysis of bromodeoxyuridine incorporation revealed a reduction in endothelial cell proliferation without altering the gene expression of vascular growth factors. In summary, hyperglycemia can impair angiogenesis through induction of apoptosis and decreased proliferation of endothelial cells. Lack of vascularization in the breast muscle is one of the initial signs of myopathies (Sihvo et al., 2018; Chen et al., 2019; Lake and Abasht, 2020). It remains to be proven that vascularization can be reduced in breast muscle after stressful conditions in the last phase of incubation.

Finally, satellite cells are multipotential stem cells that mediate post-hatch muscle growth and respond differently to temperature based upon aerobic versus anaerobic fiber-type origin. Harding et al. (2015) observed that Pectoralis major and Biceps femoris satellite cells accumulated less lipid when incubated at low temperatures and more fat at elevated temperatures compared to the control. These researchers did not detect significant differences in apoptosis. Breast muscles, which are rich in type IIB fast-twitch glycolytic anaerobic fibers, can be more susceptible to high environmental temperatures and predisposed to adipogenic conversion than other muscles. Satellite cells isolated from the Pectoralis major accumulated more lipid at elevated temperatures compared to Biceps femoris cells. The higher lipid content could be related to the higher expression of adipogenic genes such as CCAAT/enhancer-binding protein $\beta(\mathrm{C} / \mathrm{EBP} \beta)$ and PPAR $\gamma$ in satellite cells isolated from the Pectoralis major compared to those from Biceps femoris (Harding et al., 2015). This experiment demonstrated that breast muscle during embryo development could be more susceptible to develop the fat infiltration observed in breast myopathies (Papah et al., 2017; Sihvo et al., 2018; Chen et al., 2019; Petracci et al., 2019).

In summary, there is evidence in the literature indicating that temperature and oxygen concentrations during incubation could modify muscle glucose metabolism, insulin balance, IGFI release, muscle adipogenesis, vascularization, and satellite cell numbers and functions that are related to myopathies. These modifications in muscle metabolic responses may make hatchlings more susceptible to develop myopathies during grow out due to thermal stress, high-density diets, and fast growth rates (Petracci et al., 2019). Based on the evidence presented, it is hypothesized that incubation conditions may play an important role in the occurrence of myopathies, and the main steps that could lead to myopathies are described in Figure 5.

\section{CONCLUSION}

Variations on temperature during incubation impact muscle development post-hatch. Higher temperatures during short periods of 3, 6 and up to $12 \mathrm{~h}$ during ED 4 to 7 or 14 to 18 have been shown to have positive effects on muscle growth; however, responses in muscle composition and meat properties vary depending on the genetic line. The last phase of incubation in avian species is critical for muscle development. During this period, stressful conditions due to high temperatures or low oxygen concentrations can alter the maturation processes. Embryos coming from eggs stored for periods more extended 
than 5 days may be more susceptible to hypoxia and to develop myopathies due to the extended period between internal and external piping. Higher degrees of stress during these stressful conditions at the end of incubation increase corticosterone, catecholamines, reduce IGF-I, and increased glucose levels due to higher degradation of glycogen and increased insensitivity to insulin. This cascade of reactions could affect satellite cell numbers and ability for regeneration post-hatch, reduce vascularization, increase hypoxia, oxidative stress, inflammation, cause impaired extracellular matrix remodeling, switching of type II muscle fibers, and finally cause some apoptosis increasing adipogenesis in the muscles. The combined results of this developmental plasticity to common environmental stress close to hatch could increase the severity and incidence of myopathies observed in breast meat of broilers, turkeys, and ducks at processing age.

\section{AUTHOR CONTRIBUTIONS}

The information presented was developed during collaborative research with several other groups, including all three authors for

\section{REFERENCES}

Abasht, B., Mutryn, M. F., Michalek, R. D., and Lee, W. R. (2016). Oxidative stress and metabolic perturbations in wooden breast disorder in chickens. PLoS One 11:e0153750. doi: 10.1371/journal.pone.0153750

Adams, G. R., McCue, S. A., Bodell, B. W., Zeng, M., and Baldwin, K. M. (2000). Effects of spaceflight and thyroid deficiency on hindlimb development. I. Muscle mass and IGF-I expression. J. Appl. Physiol. 88, 894-903. doi: 10.1152/ jappl.2000.88.3.894

Allouh, M. Z., Yablonka-Reuveni, Z., and Rosser, B. W. C. (2008). Pax7 reveals a greater frequency and concentration of satellite cells at the ends of growing skeletal muscle fibers. J. Histochem. Cytochem. 56, 77-87. doi: 10.1369/jhc. 7A7301.2007

Al-Musawi, S. L., Stickland, N. C., and Bayo, S. A. M. (2012). In ovo temperature manipulation differentially influences limb musculoskeletal development in two lines of chick embryos selected for divergent growth rates. J. Exp. Biol. 215, 1594-1604. doi: 10.1242/jeb.068791

Bailey, R. A., Watson, K. A., Bilgili, S. F., and Avendano, S. (2015). The genetic basis of pectoralis major myopathies in modern broiler chicken lines. Poult. Sci. 94, 2870-2879. doi: 10.3382/ps/pev304

Beaudry, M., Hidalgo, M., Launay, T., Bello, V., and Darribeİre, T. (2016). Regulation of myogenesis by environmental hypoxia. J. Cell Sci. 129, 2887-2896. doi: $10.1242 /$ jcs. 188904

Boleli, I. C., Morita, V. S., Matos, J. B. Jr., Thimotheo, M. I., and Almeida, V. R. (2016). Poultry egg incubation: integrating and optimizing production efficiency. Braz. J. Poult. Sci. 18, 1-16. doi: 10.1590/1806-9061-20160292

Branum, S. R., Tazawa, H., and Burggren, W. W. (2016). Phenotypic developmental plasticity induced by preincubation egg storage in chicken embryos (Gallus gallus domesticus). Physiol. Rep. 4:e12712. doi: 10.14814/phy2.12712

Burggren, W. W., Santin, J. F., and Rojas Antich, M. (2016). Cardio-respiratory development in bird embryos: new insights from a venerable animal model. R. Bras. Zootec. 45, 709-728. doi: 10.1590/s1806-92902016001100010

Chartrin, P., Bernadet, M. D., Guy, G., Mourot, J., Hocquette, J. F., Rideau, N., et al. (2007). Do age and feeding levels have comparable effects on fat deposition in breast muscle of mule ducks? Animal 1, 113-123. doi: 10.1017/ S1751731107658029

Chen, L. R., Suyemoto, M. M., Sarsour, A. H., Cordova, H. A., Oviedo-Rondoìn, E. O., Wineland, M., et al. (2019). Temporal characterization of broiler breast myopathy ("Woody Breast") severity and correlation with growth rate and several years. EO-R collected all the information and wrote the article. SV and MW reviewed and corrected the manuscript.

\section{FUNDING}

This study was funded by the US Poultry and Egg Association, the NC Agricultural Foundation, Maple Leaf Farms, and Cobb Vantress Inc. without their funding and support these projects would not be possible.

\section{ACKNOWLEDGMENTS}

The authors would like to express their acknowledgments to all collaborators, graduate and undergraduate students, and technicians who contributed to the sample, lab analyses, and data collection in multiple experiments. Special gratitude to Dr. John H. Barnes, Emeritus Professor from the College of Veterinary Medicine at North Carolina State University for his contributions to different projects during the past years.

lymphocytic phlebitis in three commercial broiler strains and a random-bred broiler strain. Avian Pathol. 48, 319-328. doi: 10.1080/03079457.2019.1598541

Christensen, V. L., McMurtry, J. P., Donaldson, W. E., and Nestor, K. E. (1999). Supplemental oxygen affects plasma insulin-like growth factors in embryos from selected lines of turkeys. Poult. Sci. 78, 1606-1610. doi: 10.1093/ps/78.11. 1606

Christensen, V. L., McMurtry, J. P., Donaldson, W. E., and Nestor, K. E. (2001). Incubation temperature affects plasma insulin-like growth factors in embryos from selected lines of turkeys. Poult. Sci. 80, 949-954. doi: 10.1093/ps/80.7.949

Christensen, V. L., Wineland, M. J., Grimes, J. L., Oviedo, E. O., Mozdziak, P. S., Ort, D. T., et al. (2007). Effect of incubator temperature and oxygen concentration at the plateau stage in oxygen consumption on turkey embryo muscle growth and development. Int. J. Poult. Sci. 6, 406-412. doi: 10.3923/ijps. 2007.406.412

Clark, D. L., and Velleman, S. G. (2017). Spatial influence on breast muscle morphological structure, myofiber size, and gene expression associated with the wooden breast myopathy in broilers. Poult. Sci. 95, 2930-2945. doi: 10.3382/ps/ pew243

Clark, D. L., Walter, K. G., and Velleman, S. G. (2017). Incubation temperature and time of hatch impact broiler muscle growth and morphology. Poult. Sci. 96, 4085-4095. doi: 10.3382/ps/pex202

Clemmons, D. R. (2007). Modifying IGF1 activity: an approach to treat endocrine disorders, atherosclerosis and cancer. Nat. Rev. Drug Discov. 6, 821-833. doi: $10.1038 / \mathrm{nrd} 2359$

Collin, A., Berri, C., Tesseraud, S., Rodon, F. E., Skiba-Cassy, S., Crochet, S., et al. (2007). Effects of thermal manipulation during early and late embryogenesis on thermotolerance and breast muscle characteristics in broiler chickens. Poult. Sci. 86, 795-800. doi: 10.1093/ps/86.5.795

Crossley, D. A. II, Burggren, W. W., and Altimiras, J. (2003). Cardiovascular regulation during hypoxia in embryos of the domestic chicken Gallus gallus. Am. J. Physiol. Regul. Integr. Comp. Physiol. 284, 219-226. doi: 10.1152/ajpregu. 00654.2001

Da Costa, M. J., Oviedo-Rondón, E. O., Wineland, M., and Jeffrey, D. (2016). Pathogeny of fatigued walking condition in pekin ducks. Avian Dis. 60, 731738. doi: 10.1637/11292-100315-RegR

De Smit, L., Bruggeman, V., Tona, J. K., Debonne, M., Onagbesan, O., Arckens, L., et al. (2006). Embryonic developmental plasticity of the chick: increased $\mathrm{CO} 2$ during early stages of incubation changes the developmental trajectories during prenatal and postnatal growth. Comp. Biochem. Physiol. A 145, 166-175. doi: 10.1016/j.cbpa.2006.06.046 
Decuypere, E., and Michels, S. G. (1992). Incubation temperature as a management tool: a review. Worlds Poult. Sci. J. 48, 28-38. doi: 10.1079/WPS19920004

Dong, H., Lin, H., Jiao, H. C., Song, Z. G., Zhao, J. P., and Jiang, K. J. (2007). Altered development and protein metabolism in skeletal muscles of broiler chickens (Gallus gallus domesticus) by corticosterone. Comp. Biochem. Phys. A 147, 189-195. doi: 10.1016/j.cbpa.2006.12.034

Dransfield, E., and Sosnicki, A. A. (1999). Relationship between muscle growth and poultry meat quality. Poult. Sci. 78, 743-746. doi: 10.3382/ps/pex20

D'Souza, D. M., Al-Sajee, D., and Hawke, T. J. (2013). Diabetic myopathy: impact of diabetes mellitus on skeletal muscle progenitor cells. Front. Physiol. 4:379. doi: 10.3389/fphys.2013.00379

Duan, C., Ren, H., and Gao, S. (2010). Insulin-like growth factors (IGFs), IGF receptors, and IGF-binding proteins: roles in skeletal muscle growth and differentiation. Gen. Comp. Endocr. 167, 344-351. doi: 10.1016/j.ygcen.2010. 04.009

Dupont, J., Tesseraud, S., and Simon, J. (2009). Insulin signaling in chicken liver and muscle. Gen. Comp. Endocrinol. 163, 52-57. doi: 10.1016/j.ygcen.2008. 10.016

El Sabry, M. I., Yalçin, S., and Turgay-İzzetoğlu, G. (2015). Effect of breeder age and lighting regimen on growth performance, organ weights, villus development, and bursa of fabricius histological structure in broiler chickens. Czech J. Anim. Sci. 60, 116-122. doi: 10.17221/8076-CJAS

French, N. A. (1997). Modeling incubation temperature: the effects of incubator design, embryonic development, and egg size. Poult. Sci. 76, 124-133. doi: 10.1093/ps/76.1.124

Giussani, D. A., Salinas, C. E., Villena, M., and Blanco, C. E. (2007). The role of oxygen in prenatal growth: studies in the chick embryo. J. Physiol. 585, 911-917. doi: 10.1113/jphysiol.2007.141572

Gopalakrishnakone, P. (1986). Muscular dystrophy in white Pekin ducks. Am. J. Pathol. 125, 218-219.

Halevy, O. (2020). Timing is everything - the high sensitivity of avian satellite cells to thermal conditions during embryonic and posthatch periods. Front. Physiol. 11:235. doi: 10.3389/fphys.2020.00235

Halevy, O., Krispin, A., Leshem, Y., McMurtry, J. P., and Yahav, S. (2001). Early-age heat exposure affects skeletal muscle satellite cell proliferation and differentiation in chicks. Am. J. Physiol. Regul. Integr. Comp. Physiol. 281, 302-309. doi: 10.1152/ajpregu.2001.281.1.R302

Halevy, O., Lavi, M., and Yahav, S. (2006a). "Enhancement of meat production by thermal manipulations during embryogenesis of broilers," in New Insights into Fundamental Physiology and Perinatal Adaptation of Domestic Fowl, eds S. Yahav, and B. Tzschentke, (Nottingham: Nottingham University Press), 77-87.

Halevy, O., Piestum, Y., Rozenboim, I., and Yablonka-Reuveni, Z. (2006b). In ovo exposure to monochromatic green light promotes skeletal muscle cell proliferation and affects myofiber growth in posthatch chicks. Am. J. Physiol. Regul. Integr. Comp. Physiol.290, R1062-R1070. doi: 10.1152/ajpregu.00378. 2005

Halevy, O., Yahav, S., and Rozenboim, I. (2006c). Enhancement of meat production by environmental manipulations in embryo and young broilers. World Poult. Sci. J. 62, 485-497. doi: 10.1017/S0043933906001103

Halevy, O., Piestun, Y., Allouh, M., Rosser, B., Rinkevitch, Y., Reshef, R., et al. (2004). The pattern of Pax7 expression during myogenesis in the posthatch chicken establishes a model for satellite cell differentiation and renewal. Dev. Dyn. 231, 489-502. doi: 10.1002/dvdy.20151

Harding, R. L., Clark, D. L., Halevy, O., Coy, C. S., Yahav, S., and Velleman, S. G. (2015). The effect of temperature on apoptosis and adipogenesis on skeletal muscle satellite cells derived from different muscle types. Physiol. Rep. 3:e12539. doi: 10.14814/phy2.12539

Hartley, R. S., Bandman, E., and Yablonka-Reuveni, Z. (1992). Skeletal muscle satellite cells appear during late chicken embryogenesis. Dev. Biol. 153, 206-216. doi: 10.1016/0012-1606(92)90106-q

Havenstein, G. B., Ferket, P. R., and Qureshi, M. A. (2003a). Carcass composition and yield of $1957 v s 2001$ broilers when fed representative 1957 and 2001 broiler diets. Poult. Sci. 82, 1509-1518. doi: 10.1093/ps/82.10.1509

Havenstein, G. B., Ferket, P. R., and Qureshi, M. A. (2003b). Growth, livability, and feed conversion of 1957 vs. 2001 broilers when fed representative 1957 and 2001 broiler diets. Poult. Sci. 82, 1500-1508. doi: 10.1093/ps/82.10.1500

Havenstein, G. B., Ferket, P. R., Scheideler, S. E., and Larson, B. T. (1994a), Growth, livability, and feed conversion of 1957 vs. 1991 broilers when fed typical 1957 and 1991 broiler diets. Poult. Sci. 73, 1785-1794. doi: 10.3382/ps.073 1785
Havenstein, G. B., Ferket, P. R., Scheideler, S. E., and Rives, D. V. (1994b). Carcass composition and yield of 1991 vs. 1957 broilers when fed "typical" 1957 and 1991 broiler diets. Poult. Sci. 73, 1795-1804. doi: 10.3382/ps.0731795

Hawke, T. J., and Garry, D. J. (2001). Myogenic satellite cells: physiology to molecular biology. J. Appl. Physiol. 91, 534-551. doi: 10.1152/jappl.2001.91. 2.534

Hepp, G. R., Kennamer, R. A., and Johnson, M. H. (2006). Maternal effects in Wood Ducks: incubation temperature influences incubation period and neonate phenotype. Funct. Ecol. 20, 308-314. doi: 10.1111/j.1365-2435.2006. 01108.x

Hernández-Hernández, J. M., García-González, E. G., Brun, C. E., and Rudnicki, M. A. (2017). The myogenic regulatory factors, determinants of muscle development, cell identity and regeneration. Semin. Cell Dev. Biol. 72, 10-18. doi: 10.1016/j.semcdb.2017.11.010

Hopkins, B. C., Durant, S. E., Hepp, G. R., and Hopkins, W. A. (2011). Incubation temperature influences locomotor performance in young wood ducks (Aix sponsa). J. Exp. Zool. 315, 274-279. doi: 10.1002/jez.673

Hulet, R., Gladys, D., Hill, D., Meijerhof, R., and El-Shiekh, T. (2007). Influence of egg shell embryonic incubation temperature and broiler breeder flock age on posthatch growth performance and carcass characteristics. Poult. Sci. 86, 408-412. doi: $10.1093 / \mathrm{ps} / 86.2 .408$

Janisch, S., Sharifi, A. R., Wicke, M., and Krischek, C. (2015). Changing the incubation temperature during embryonic myogenesis influences the weight performance and meat quality of male and female broilers. Poult. Sci. 94, 2581-2588. doi: 10.3382/ps/pev239

Janke, O., Tzschentke, B., Höchel, J., and Nichelmann, M. (2002). Metabolic responses of chicken and muscovy duck embryos to high incubation temperatures. Comp. Biochem. Physiol. A 131, 741-750. doi: 10.1016/S10956433(02)00012-0

Kabir, G., Mosaraf, M., Omar, F., Naimul, H., Zahid, H., Quamrun, N., et al. (2010). Association of serum free IGF-1 and IGFBP-1 with insulin sensitivity in impaired glucose tolerance (IGT). Int. J. Diabetes Mellit. 2, 144-147. doi: 10.1016/j.ijdm.2010.09.003

Kijowski, J., Kupińska, E., Stangierski, J., Tomaszewska-Gras, J., and Szablewski, T. (2014). Paradigm of deep pectoral myopathy in broiler chickens. World Poult. Sci. 70, 125-138. doi: 10.1017/S0043933914000117

Krischek, C., Gerken, M., and Wicke, M. (2013a). Effects of a higher incubation temperature between embryonic day 9 and 12 on growth and meat quality characteristics of turkeys. Br. Poult. Sci. 54, 5-11. doi: 10.1080/00071668.2013. 763901

Krischek, C., Kuembet, U., Wicke, M., and Gerken, M. (2013b). A higher incubation temperature between embryonic day 3 and 6 influences growth and meat quality characteristics of broiler after hatch. Arch. Geflügelk. 77, 59-65.

Kuttapan, V. A., Huff, G. R., Huff, W. E., Hargis, B. M., Apple, J. K., Coon, C., et al. (2013a). Comparison of hematologic and serologic profiles of broiler birds with normal and severe degrees of white striping in breast fillets. Poult. Sci. 92, 339-345. doi: $10.3382 /$ ps.2012-02647

Kuttappan, V. A., Shivaprasad, H. L., Shaw, D. P., Valentine, B. A., Hargis, B. M., Clark, F. D., et al. (2013b). Pathological changes associated with white striping in broiler breast muscles. Poult. Sci. 92, 331-338. doi: 10.3382/ps.2012-02646

Lake, J. A., and Abasht, B. (2020). Glucolipotoxicity: a proposed etiology for wooden breast and related myopathies in commercial broiler chickens. Front. Physiol. 11:169. doi: 10.3389/fphys.2020.00169

Larger, E., Marre, M., Corvol, P., and Gasc, J. M. (2004). Hyperglycemia-induced defects in angiogenesis in the chicken chorioallantoic membrane model. Diabetes 53, 752-761. doi: 10.2337/diabetes.53.3.752

Leksrisompong, N., Romero-Sanchez, H., Plumstead, P. W., Brannan, K. E., and Brake, J. (2007). Broiler incubation 1. Effect of elevated temperature during late incubation on body weight and organs of chicks. Poult. Sci. 86, 2685-2691. doi: 10.3382/ps.2007-00170

Li, H., Shu, J., Shan, Y., Chen, W., Song, C., and Xu, W. (2016). Myofiber development during embryonic to neonatal development in duck breeds differing in muscle growth rates. J. Integr. Agric. 15, 403-413. doi: 10.1016/ S2095-3119(14)60949-7

Liu, H., Liu, J., Yan, X., Li, Q., Zhao, Y., Wang, Y., et al. (2015). Impact of thermal stress during incubation on gene expression in embryonic muscle of Peking ducks (Anasplatyrhynchos domestica). J. Therm. Biol. 53, 80-89. doi: 10.1016/j. jtherbio.2015.08.013

Liu, R., Wang, H., Liu, J., Wang, J., Zheng, M., Tan, X., et al. (2017). Uncovering the embryonic development related proteome and metabolome signatures in 
breast muscle and intramuscular fat of fast-and slow-growing chickens. $B M C$ Genomics 18:816. doi: 10.1186/s12864-017-4150-3

Livingston, M. L., Ferket, P. R., Brake, J., and Livingston, K. A. (2019a). Dietary amino acids under hypoxic conditions exacerbates muscle myopathies including wooden breast and white stripping. Poult. Sci. 98, 1517-1527. doi: $10.3382 /$ ps/pey463

Livingston, M. L., Landon, C., Barnes, H. J., and Brake, J. (2019b). White striping and wooden breast myopathies of broiler breast muscle is affected by timelimited feeding, genetic background, and egg storage. Poult. Sci. 98, 217-226. doi: $10.3382 /$ ps/pey333

Loh, B., Maier, I., Winar, A., Janke, O., and Tzschentke, B. (2004). Prenatal development of epigenetic adaptation processes in poultry: changes in metabolic and neuronal thermoregulatory mechanisms. Avian Poult. Biol. Rev. 15, 119-128. doi: 10.3184/147020604783637976

Loyau, T., Bedrani, L., Berri, C., Métayer-Coustard, S., Praud, C., Coustham, V., et al. (2015). Cyclic variations in incubation conditions induce adaptive responses to later heat exposure in chickens: a review. Animal 9, 76-85. doi: $10.1017 /$ S1751731114001931

Malila, Y., Thanatsang, K., Arayamethakorn, S., Uengwetwanit, T., Srimarut, Y., Petracci, M., et al. (2019). Absolute expressions of hypoxia-inducible factor-1 alpha (HIF1A) transcript and the associated genes in chicken skeletal muscle with white striping and wooden breast myopathies. PLoS One 14:e0220904. doi: 10.1371/journal.pone.0220904

Maltby, V., Somaiya, A., French, N. A., and Stickland, N. C. (2004). In ovo temperature manipulation influences post-hatch muscle growth in the turkey. Br. Poult. Sci. 45, 491-498. doi: 10.1080/00071660412331286190

McNabb, F. M. A. (2006). Avian thyroid development and adaptive plasticity. Gen. Comp. Endocrinol. 147, 93-101. doi: 10.1016/j.ygcen.2005.12.011

Molenaar, R., van den Borne, J. J., Hazejager, E., Kristensen, N. B., Heetkamp, M. J., Meijerhof, R., et al. (2013). High environmental temperature increases glucose requirement in the developing chicken embryo. PLoS One 8:e59637. doi: 10.1371/journal.pone.0059637

Molenaar, R. I., Reijrink, I. A. M. I., Meijerhof, R., and Van den Brand, H. (2010). Meeting embryonic requirements of broilers throughout incubation: a review. Braz. J. Poult. Sci. 12, 137-148. doi: 10.1590/S1516-635X2010000300001

Mortola, J. P., and Labbè, K. (2005). Oxygen consumption of the chicken embryo: interaction between temperature and oxygenation. Respir. Physiol. Neurobiol. 146, 97-106. doi: 10.1016/j.resp.2004.10.011

Mulder, A. L. M., van Golde, J. C., Prinzen, F. W., and Blanco, C. E. (1998). Cardiac output distribution in response to hypoxia in the chick embryo in the second half of the incubation time. J. Physiol. 508, 281-287. doi: 10.1111/j.1469-7793. 1998.281br.x

Mutryn, M. F., Brannick, E. M., Fu, W., Lee, W. R., and Abasht, B. (2015). Characterization of a novel chicken muscle disorder through differential gene expression and pathway analysis using RNA-sequencing. BMC Genomics 16:399. doi: 10.1186/s12864-015-1623-0

Nangsuay, A., Meijerhof, R., van den Anker, I., Heetkamp, M. J. W., Kemp, B., and van den Brand, H. (2017). Effects of breeder age, strain, and eggshell temperature on nutrient metabolism of broiler embryos. Poult. Sci. 96, 18911900. doi: $10.3382 /$ ps/pew 417

Naraballobh, W., Trakooljul, N., Muráni, E., Brunner, R., Krischek, C., Janisch, S., et al. (2016a). Immediate and long-term transcriptional response of hind muscle tissue to transient variation of incubation temperature in broilers. BMC Genomics 17:323. doi: 10.1186/s12864-016-2671-9

Naraballobh, W., Trakooljul, N., Muráni, E., Brunner, R., Krischek, C., Janisch, S., et al. (2016b). Transient shifts of incubation temperature reveal immediate and long-term transcriptional response in chicken breast muscle underpinning resilience and phenotypic plasticity. PLoS One 11:e0162485. doi: 10.1371/ journal.pone. 0162485

Nyuiadzi, D., Berri, C., Dusart, L., Travel, A., Méda, B., Bouvarel, I., et al. (2020). Short cold exposures during incubation and postnatal cold temperature affect performance, breast meat quality, and welfare parameters in broiler chickens. Poult. Sci. 99, 857-868. doi: 10.1016/j.psj.2019.10.024

Okur, N., Eleroğlu, H., and Türkoğlu, M. (2018). Impacts of breeder age, storage time and setter ventilation program on incubation and post-hatch performance of broilers. Braz. J. Poult. Sci. 20, 27-36. doi: 10.1590/1806-9061-2017-0550

Oviedo-Rondón, E. O., Cordova, H. A., Saldaña, B., Sarsour, A., Bernal-Arango, L., Mesquita, M., et al. (2016). "Effects of genetic line, incubation temperature profiles and gender on woody breast and white striping at $57 \mathrm{~d}$ of age," in Proceedings of the World's Poultry Congress $C D$, Beijing.

Oviedo-Rondón, E. O., Small, J., Wineland, M. J., Christensen, V. L., Grimes, J. L., Funderburk, S. V. L., et al. (2008a). Effects of incubator temperature and oxygen concentration during the plateau stage of oxygen consumption on turkey embryo long bone development. Poult. Sci. 87, 1464-1470. doi: 10.3382/ ps.2007-00470

Oviedo-Rondón, E. O., Small, J., Wineland, M. J., Christensen, V. L., Mozdziak, P. S., Koci, M. D., et al. (2008b). Broiler embryo bone development is influenced by incubator temperature, oxygen concentration and eggshell conductance at the plateau stage in oxygen consumption. Br. Poult. Sci. 49, 666-676. doi: $10.1080 / 00071660802433149$

Oviedo-Rondón, E. O., Wineland, M. J., Funderburk, S., Small, J., Cutchin, H., and Mann, K. M. (2009a). Incubation conditions affect leg health in large, high-yield broilers. J. Appl. Poult. Res. 18, 640-646. doi: 10.3382/japr.2008-00127

Oviedo-Rondón, E. O., Wineland, M. J., Small, J., Cutchin, H., McElroy, A., Barri, A., et al. (2009b). Effect of incubation temperatures and chick transportation conditions on bone development and leg health. J. Appl. Poult. Res. 18, 671-678. doi: 10.3382/japr.2008-00135

Owens, C. M., Alvarado, C. Z., and Sams, A. R. (2009). Research developments in pale, soft, and exudative turkey meat in North America. Poult. Sci. 88, 1513-1517. doi: 10.3382/ps.2009-00008

Papah, M. B., Brannick, E. M., Schmidt, C. J., and Abasht, B. (2017). Evidence and role of phlebitis and lipid infiltration in the onset and pathogenesis of Wooden Breast Disease in modern broiler chickens. Avian Pathol. 46, 623-643. doi: 10.1080/03079457.2017.1339346

Patael, T., Piestun, Y., Soffer, A., Mordechay, S., Yahav, S., Velleman, S. G., et al. (2019). Early posthatch thermal stress causes long-term adverse effects on pectoralis muscle development in broilers. Poult. Sci. 98, 3268-3277. doi: 10. 3382/ps/pez123

Petracci, M., and Cavani, C. (2012). Muscle growth and poultry meat quality issues. Nutrients 4, 1-12. doi: 10.3390/nu4010001

Petracci, M., Mudalal, S., Babini, E., and Cavani, C. (2014). Effect of white striping on chemical composition and nutritional value of chicken breast meat. Ital. J. Anim. Sci. 13, 179-183. doi: 10.4081/ijas.2014.3138

Petracci, M., Mudalal, S., Bonfiglio, A., and Cavani, C. (2013). Occurrence of white striping under commercial conditions and its impact on breast meat quality in broiler chickens. Poult. Sci. 92, 1670-1675. doi: 10.3382/ps.201203001

Petracci, M., Soglia, F., Madruga, M., Carvalho, L., Ida, E., and Estévez, M. (2019). Wooden-breast, white striping, and spaghetti meat: causes, consequences and consumer perception of emerging broiler meat abnormalities. Compr. Rev. Food Sci. Food Saf. 18, 565-583. doi: 10.1111/1541-4337.12431

Picard, B., Lefaurcheur, L., Berri, C., and Duclos, M. J. (2002). Muscle fibre ontogenesis in farm animal species. Reprod. Nutr. Dev. 42, 415-431. doi: 10. 1051/rnd:2002035

Piestun, Y., Druyan, S., Brake, J., and Yahav, S. (2013). Thermal manipulations during broiler incubation alter performance of broilers to 70 days of age. Poult. Sci. 92, 1155-1163. doi: 10.3382/ps.2012-02609

Piestun, Y., Harel, M., Barak, M., Yahav, S., and Halevy, O. (2009). Thermal manipulations in late-term chick embryos have immediate and longer-term effects on myoblast proliferation and skeletal muscle hypertrophy. J. Appl. Physiol. 106, 233-240. doi: 10.1152/japplphysiol.91090.2008

Piestun, Y., Shinder, D., Ruzal, M., Halevy, O., Brake, J., and Yahav, S. (2008). Thermal manipulations during broiler embryogenesis: effect of acquisition of thermotolerance. Poult. Sci. 87, 1-10. doi: 10.3382/ps.2008-00030

Rahn, H. (1981). Gas exchange of avian eggs with special reference to turkey eggs. Poult. Sci. 60, 1971-1980. doi: 10.3382/ps.0601971

Rigdon, R. H. (1967). Acute myopathy in embryos and newly hatched chicks. Arch. Pathol. 84, 633-637.

Rigdon, R. H., Ferguson, T. M., Trammel, J. L., Couch, J. R., and German, H. L. (1968). Necrosis in the "pipping" muscle of the chick. Poult. Sci. 47, 873-877. doi: $10.3382 /$ ps.0470873

Russo, E., Drigo, M., Longoni, C., Pezzotti, R., Fasoli, P., and Recordati, C. (2015). Evaluation of white striping prevalence and predisposing factors in broilers at slaughter. Poult. Sci. 94, 1843-1848. doi: 10.3382/ps/pev172

Schultz, E., and McCormick, K. M. (1994). Skeletal muscle satellite cells. Rev. Physiol. Biochem. Pharmacol. 123, 213-257. doi: 10.1007/BFb0030904 
Seki, Y., Sato, K., Kono, T., Abe, H., and Akiba, Y. (2003). Broiler chickens (Ross strain) lack insulin-responsive glucose transporter GLUT4 and have GLUT8 cDNA. Gen. Comp. Endocrinol. 133, 80-87. doi: 10.1016/S0016-6480(03)00 $145-\mathrm{X}$

Shi, L., Ko, M. L., Huang, C. C., Park, S. Y., Hong, M. P., Wu, C., et al. (2014). Chicken embryos as a potential new model for early onset type I diabetes. J. Diabetes Res. 2014:354094. doi: 10.1155/2014/354094

Sihvo, H. K., Airas, N., Lindén, J., and Puolanne, E. (2018). Pectoral vessel density and early ultrastructural changes in broiler chicken wooden breast myopathy. J. Comp. Pathol. 161, 1-10. doi: 10.1016/j.jcpa.2018.04.002

Smith, J. H. (1963). Relation of body size to muscle cell size and number in the chicken. Poult. Sci. 42, 283-290. doi: 10.3382/ps.0420283

Soglia, F., Baldi, G., Laghi, L., Mudalal, S., Cavani, C., and Petracci, M. (2018). Effect of white striping on turkey breast meat quality. Animal 12, 2198-2204. doi: $10.1017 /$ S1751731117003469

Soglia, F., Mudalal, S., Babini, E., Di Nunzio, M., Mazzoni, M., Sirri, F., et al. (2016). Histology, composition, and quality traits of chicken Pectoralis major muscle affected by wooden breast abnormality. Poult. Sci. 95, 651-659. doi: $10.3382 / \mathrm{ps} / \mathrm{pev} 353$

Sporer, K. R., Tempelman, R. J., and Ernst, C. W. (2011). Transcriptional profiling identifies differentially expressed genes in developing turkey skeletal muscle. BMC Genomics 12:143. doi: 10.1186/1471-2164-12-143

Stockdale, F. E. (1992). Myogenic cell lineages. Dev. Biol. 154, 284-298. doi: 10. 1016/0012-1606(92)90068-r

Tesseraud, S., Pym, R. A. E., Bihan-Duval, E. L., and Duclos, M. J. (2003). Response of broilers selected on carcass quality to dietary protein supply: live performance, muscle development, and circulating insulin-like growth factors (IGF-I and -II). Poult. Sci. 82, 1011-1016. doi: 10.1093/ps/82.6.1011

Tona, K., Malheiros, R. D., Bamelis, F., Careghi, C., Moraes, V. M., Onagbesan, O., et al. (2003a). Effects of storage time on incubating egg gas pressure, thyroid hormones, and corticosterone levels in embryos and on their hatching parameters. Poult. Sci. 82, 840-845. doi: 10.1093/ps/82.5.840

Tona, K., Onagbesan, O., De Ketelaere, B., Decuypere, E., and Bruggeman, V. (2003b). Effects of turning duration during incubation on corticosterone and thyroid hormone levels, gas pressures in air cell, chick quality, and juvenile growth. Poult. Sci. 82, 1974-1979. doi: 10.1093/ps/82.12.1974

Tong, Q., McGonnell, I. M., Roulston, N., Bergoug, H., Romanini, C. E. B., Garain, P., et al. (2015). Higher levels of $\mathrm{CO} 2$ during late incubation alter the hatch time of chicken embryos. Br. Poult. Sci. 56, 503-509. doi: 10.1080/00071668. 2015.1041097

Valentin, B. (2017). "Skeletal muscle," in Pathologic Basis of Veterinary Disease, ed. J. Zachary, (Saint Louis, MO: Elsevier), 908-953.

Van Brecht, A., Hens, H., Lemaire, J. L., Aerts, J. M., Degraeve, P., and Berckmans, D. (2005). Quantification of the heat exchange of chicken eggs. Poult. Sci. 84, 353-361. doi: $10.1093 / \mathrm{ps} / 84.3 .353$

Velleman, S. G. (2007). Muscle development in the embryo and hatchling. Poult. Sci. 86, 1050-1054. doi: 10.1093/ps/86.5.1050

Velleman, S. G., Anderson, J. W., and Nestor, K. E. (2003). Possible maternal inheritance of breast muscle morphology in turkeys at sixteen weeks of age. Poult. Sci. 82, 1479-1484. doi: 10.1093/ps/82.10.1479

Velleman, S. G., Clark, D. L., and Tonniges, J. R. (2017). Fibrillar collagen organization associated with broiler wooden breast fibrolitic myopathy. Avian Dis. 61, 481-490. doi: 10.1637/11738-080217-Reg.1

Velleman, S. G., Coy, C. S., and McFarland, D. C. (2007). Effect of syndecan1 , syndecan-4, and glypican-1 on turkey muscle satellite cell proliferation, differentiation, and responsiveness to fibroblast growth factor 2. Poult. Sci. 86, 1406-1413. doi: 10.1093/ps/86.7.1406

Visschedijk, A. H. J., Tazawa, H., and Piiper, J. (1985). Variability of shell conductance and gas exchange of chicken eggs. Respir. Physiol. 59, 339-345. doi: 10.1016/0034-5687(85)90137-9 von Blumröder, D., and Tönhardt, H. (2002). Influence of long-term changes in incubation temperature on catecholamine levels in plasma of chicken embryos (Gallus gallusf. domestica). Comp. Biochem. Phys. A 131, 701-711. doi: 10.1016/ S1095-6433(02)00008-9

Wineland, M. J., Christensen, V. L., Yildrum, I., Fairchild, B. D., Ort, D. T., and Mann, K. M. (2006a). Incubator environment interacts with genetic line of broiler at the plateau stage to affect embryo plasma thyroxine and triiodothyronine concentrations. Int. J. Poult. Sci. 5, 714-722. doi: 10.3923/ijps. 2006.714.722

Wineland, M. J., Christensen, V. L., Yildrum, I., Fairchild, B. D., Ort, D. T., and Mann, K. M. (2006b). Incubator temperature and oxygen concentration at the plateau stage in oxygen consumption affects intestinal maturation of broiler chicks. Int. J. Poult. Sci. 5, 229-240. doi: 10.3923/ijps.2006. 229.240

Wise, P. M., and Frye, B. E. (1973). Functional development of the hypothalamohypophyseal-adrenal cortex axis in the chick embryo, Gallus domesticus. J. Exp. Zool. 185, 277-291. doi: 10.1002/jez.1401850302

Yahav, S., Collin, A., Shinder, D., and Picard, M. (2004a). Thermal manipulations during broiler chick embryogenesis: effects of timing and temperature. Poult. Sci. 83, 1959-1963. doi: 10.1093/ps/83.12.1959

Yahav, S., Sasson, R. R., and Shinder, D. (2004b). The effect of thermal manipulations during embryogenesis of broiler chicks (Gallus domesticus) on hatchability, body weight and thermoregulation after hatch. J. Therm. Biol. 29, 245-250. doi: 10.1016/j.jtherbio.2004.03.002

Yalçin, S., Güler, H. C., Yaşa, Ý., Turgay İzzetoğlu, G., and Özkan, S. (2014). Effect of breeder age and slaughter weight on meat quality traits of broiler breast and leg meats. Eur. Poult. Sci. 78. doi: 10.1399/eps.2014.45

Yamagiwa, S., Itakura, C., and Ono, T. (1975). Muscle lesions in embryos and chicks on the day of hatching. Nihon Juigaku Zasshi 37, 1-10. doi: 10.1292/jvms1939. 37.1

Yang, X., Yang, S., Wang, C., and Kuang, S. (2017). The hypoxia-inducible factors HIF $1 \alpha$ and HIF $2 \alpha$ are dispensable for embryonic muscle development but essential for postnatal muscle regeneration. J. Biol. Chem. 292, 5981-5991. doi: $10.1074 /$ jbc.M116.756312

Yuan, L., Lin, H., Jiang, K. J., Jiao, H. C., and Song, Z. G. (2008). Corticosterone administration and high-energy feed results in enhanced fat accumulation and insulin resistance in broiler chickens. Br. Poult. Sci. 49, 487-495. doi: 10.1080/ 00071660802251731

Zanou, N., and Gailly, P. (2013). Skeletal muscle hypertrophy and regeneration: interplay between the myogenic regulatory factors (MRFs) and insulin-like growth factors (IGFs) pathways. Cell. Mol. Life Sci. 70, 4117-4130. doi: 10.1007/ s00018-013-1330-4

Zhao, J. P., Lin, H., Jiao, H. C., and Song, Z. G. (2009). Corticosterone suppresses insulin- and NO-stimulated muscle glucose uptake in broiler chickens (Gallus gallus domesticus). Comp. Biochem. Phys. C 149, 448-454. doi: 10.1016/j.cbpc. 2008.10.106

Conflict of Interest: MW is currently a consultant under Hatchery Consultant Inc.

The remaining authors declare that the research was conducted in the absence of any commercial or financial relationships that could be construed as a potential conflict of interest.

Copyright $\odot 2020$ Oviedo-Rondón, Velleman and Wineland. This is an open-access article distributed under the terms of the Creative Commons Attribution License (CC BY). The use, distribution or reproduction in other forums is permitted, provided the original author(s) and the copyright owner(s) are credited and that the original publication in this journal is cited, in accordance with accepted academic practice. No use, distribution or reproduction is permitted which does not comply with these terms. 


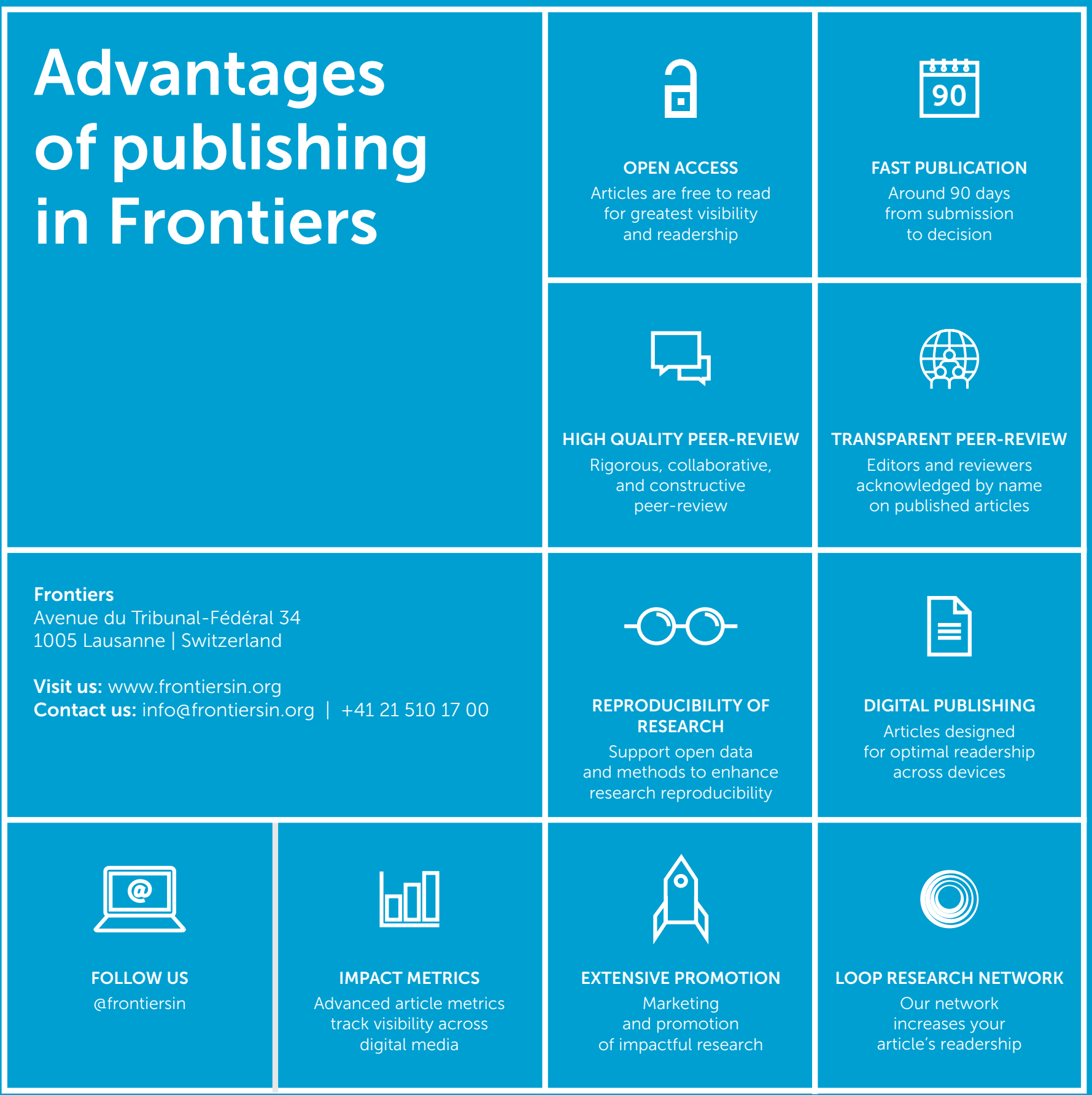

